\title{
Erfolgsfaktoren von Genossenschaftsbanken
}

Eine Analyse auf Basis von Jahresabschlüssen und regionalen Wirtschaftsdaten

\author{
Dissertation \\ zur Erlangung des Grades \\ doctor rerum politicarum (Dr. rer. pol.) \\ der Fakultät für Wirtschaftswissenschaften \\ der Technischen Universität Chemnitz
}

Vorgelegt von:

Betreut von:

Zweitgutachter:

Einreichung:

Disputation und Rigorosum:
Dipl.-Kfm. Thomas Maurer

Prof. Dr. Friedrich Thießen

Professur für Finanzwirtschaft und Bankbetriebslehre

Fakultät für Wirtschaftswissenschaften

Technische Universität Chemnitz

Prof. Dr. Dr. h. c. Dirk Linowski

19.10.2015

08.12.2015 

Titel:

Autor:

Jahr:

Seitenzahl:

Anzahl Literaturquellen:

Abbildungsanzahl:

Tabellenanzahl:

Anlagenanzahl:

Universität:

Fakultät:

Art:

Kurzbeschreibung:

Schlagwörter:

Hinweis:
Erfolgsfaktoren von Genossenschaftsbanken Eine Analyse auf Basis von Jahresabschlüssen und regionalen Wirtschaftsdaten

Dipl.-Kfm. Thomas Maurer

2015

197

213

74

55

2

Technische Universität Chemnitz

Fakultät für Wirtschaftswissenschaften

Dissertation

Der Erfolg von Genossenschaftsbanken ist von einer Vielzahl betriebswirtschaftlicher, volkswirtschaftlicher und demografischer Faktoren abhängig. Die Arbeit untersucht den Einfluss auf den Geschäftserfolg bei über 900 deutschen Instituten. Wesentliche Erfolgsfaktoren sind dabei die Lage (städtisch, halbstädtisch oder ländlich), die Betriebsgröße, die Kreditquote, die Eigenkapitalquote sowie Steigung und Niveau der Zinsstrukturkurve.

Erfolg, Erfolgsfaktoren, Bankerfolg, Geschäftserfolg, Erfolgsmessung, Kostenvorteile, Fusionen, Return-onInvestment-Analyse, Jahresabschlussanalyse

Die Arbeit kann unter www.springer.com als E-Book oder Buch bezogen werden. 

Die Genossenschaftsbanken sind eine tragende Säule des deutschen Bankwesens. Sie sind seit 150 Jahren fester Bestandteil der Versorgung von Privatkunden und Unternehmen mit Bankdienstleistungen. Derzeit stehen Banken vor zahlreichen Herausforderungen. Auf der einen Seite sind umfangreiche regulatorische Anforderungen zu erfüllen. Auf der anderen Seite wenden sich Kunden von ertragreichen Bankprodukten ab und entwickeln neue Verhaltensweisen. Genossenschaftsbanken scheinen in dieser Lage besonders gefährdet. Ihre Betriebsgröße wird als zu gering angesehen, um angemessen auf Veränderungen im Bankenumfeld reagieren zu können. Ihre Verankerung in der Fläche mit regional orientierten Instituten, die nicht wie die Privatbanken unattraktive Gebiete verlassen können, wirke als zusätzlicher Hemmschuh - so die These.

An diesem Punkt setzt die Arbeit von Herrn Maurer ein. Der Autor fragt, wie die Rentabilität von Genossenschaftsbanken mit ihrer regionalen Lage korreliert ist. Mit nahezu einer Vollerhebung aller genossenschaftlichen Institute untersucht er Kenngrößen des externen Rechnungswesens auf ihre Korrelation mit dem Unternehmenserfolg hin.

Die Ergebnisse sind interessant und aufschlussreich. Hier seien nur einige skizziert. Die Erfolge ländlicher und städtischer Banken differieren systematisch. Das Einlage- und Kreditgeschäft weist komplexe, aber systematische Differenzen auf. Ländliche Banken haben leichte, aber hochsignifikante Ertragsvorteile - Bankgeschäft in ländlichen Gebieten ist nicht unattraktiv. Ein Abgleich der Erfolgswerte der Banken mit Daten der Volkswirtschaftlichen Gesamtrechnung zeigt, dass Banken in ärmeren Regionen mit niedrigeren Arbeitnehmereinkommen erfolgreicher sind. Gleichwohl ist auch ein Sachkostennachteil kleinerer Banken in ländlichen Regionen nicht zu verkennen. Die optimale Betriebsgröße liegt oberhalb der Betriebsgrößen von 600 kleinen Volksbanken. Die Konsequenzen daraus werden in der Arbeit abgeleitet.

Insgesamt liegt eine besonders gründliche Arbeit vor, die einen sorgfältig ausgearbeiteten Datensatz einer sehr großen Anzahl von Volksbanken zur Basis hat. Ich wünsche den Lesern des Bandes großen Gewinn und hoffe für die Volksbanken Deutschlands, dass sie die Herausforderungen der Zukunft insbesondere auch im ländlichen Raum bewältigen.

Friedrich Thießen 

Die vorliegende Arbeit entstand im Rahmen meiner Tätigkeit am Lehrstuhl für Finanzwirtschaft und Bankbetriebslehre der Technischen Universität Chemnitz. Hiermit danke ich allen, die mich auf dem Weg zur erfolgreichen Promotion mit Rat und Tat unterstützt haben. Zunächst gilt Prof. Dr. Friedrich Thießen für die Betreuung meiner Dissertation und seinen fachlichen Rat mein Dank. Prof. Dr. Dr. h. c. Dirk Linowski danke ich für seine nützlichen Ratschläge und die Übernahme des Zweitgutachtens. Auch das Doktorandenseminar des HypoVereinsbank-UniCredit Group Stiftungsfonds, an dem ich mehrmals teilnehmen und vortragen durfte, war dem Erfolg meiner Arbeit zuträglich. Den teilnehmenden Professoren und Doktoranden danke ich für ihre wertvollen Hinweise und Vorschläge. Meinen Kollegen am Lehrstuhl, Tommy Jehmlich, Jan Justus Brenger und Rick Hofmann, danke ich für ihre Unterstützung und fachlichen Rat. Da ein derartig umfangreiches Projekt nur mit einer soliden Basis erfolgreich werden kann, danke ich vor allem meiner Familie für den Rückhalt und ihre Unterstützung.

Thomas Maurer 

INHALTSÜBERSICHT

ABBILDUNGSVERZEICHNIS $\quad$ XV

$\begin{array}{lc}\text { TABELLENVERZEICHNIS } & \text { XIX }\end{array}$

ABKÜRZUNGS- UND SYMBOLVERZEICHNIS SXI

1 EINLEITUNG 1

2 EINORDNUNG UND ZIELSETZUNG 3

3 THEORETISCHE ANALYSE 17

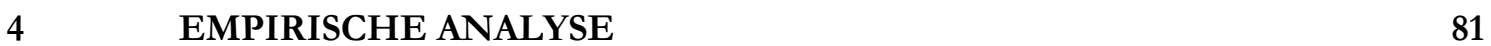

5 SCHLUSSBETRACHTUNG 194

$\begin{array}{ll}\text { ANHANG } & 199\end{array}$

$\begin{array}{ll}\text { LITERATURVERZEICHNIS } & 201\end{array}$ 

$\begin{array}{ll}\text { ABBILDUNGSVERZEICHNIS XV X } & \text { XV }\end{array}$

$\begin{array}{ll}\text { TABELLENVERZEICHNIS } & \text { XIX }\end{array}$

ABKÜRZUNGS- UND SYMBOLVERZEICHNIS XXI

1 EINLEITUNG 1

2 EINORDNUNG UND ZIELSETZUNG $\quad 3$

2.1 STAND DER FORSCHUNG ..........................................................................................

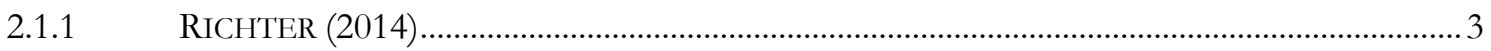

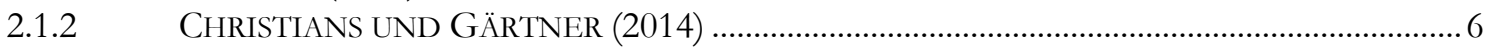

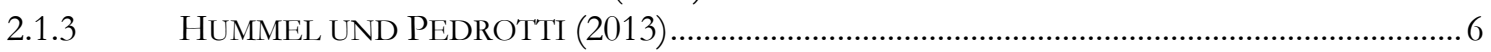

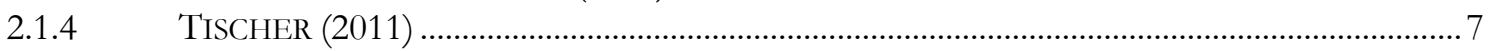

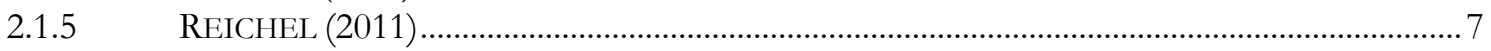

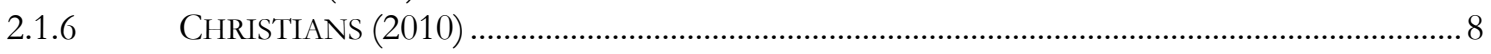

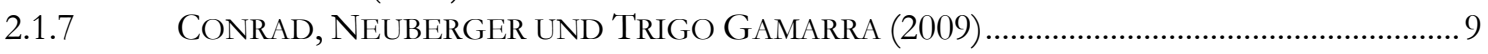

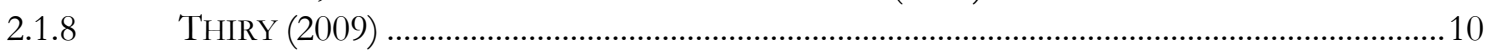

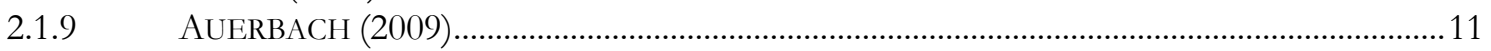

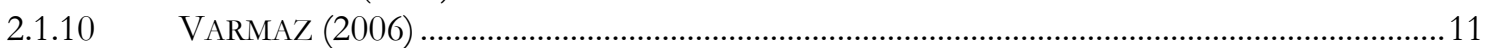

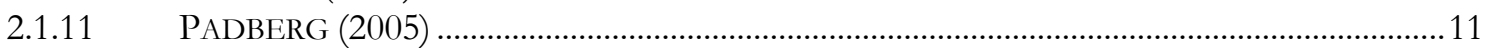

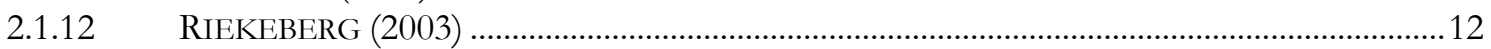

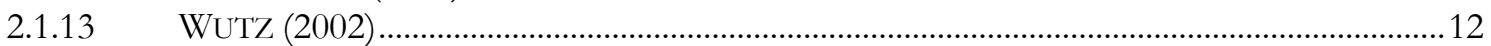

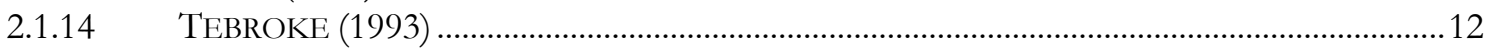

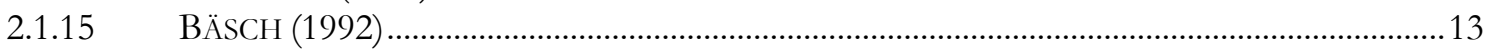

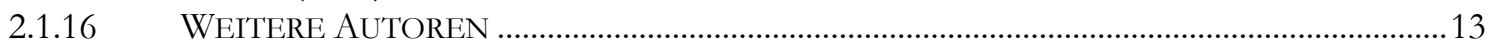

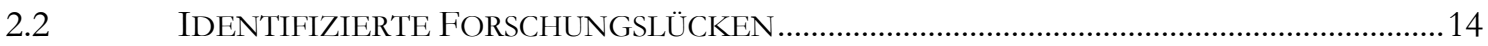

2.3 FORSCHUNGSFRAGEN DIESER UNTERSUCHUNG_........................................................14

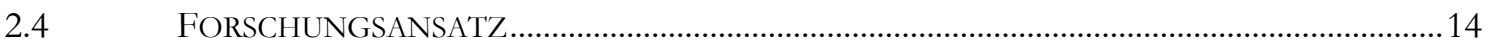

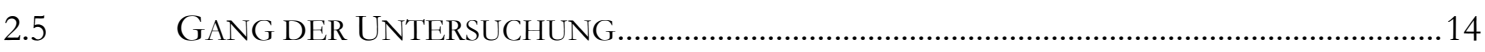

3 THEORETISCHE ANALYSE $\quad 17$

3.1 DEFINITIONEN UND KONZEPTIONELLER BEZUGSRAHMEN...............................................

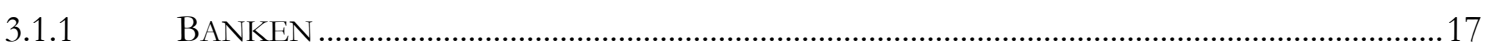

3.1.1.1 DIE GESCHICHTE DER GENOSSENSCHAFTSBANKEN …………………........................... 19

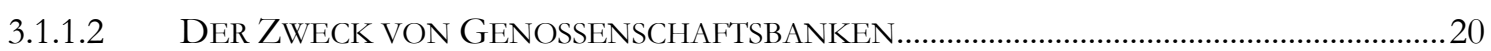

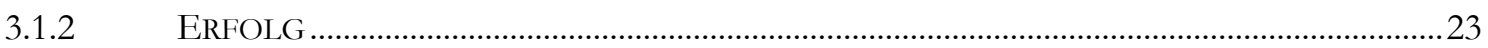

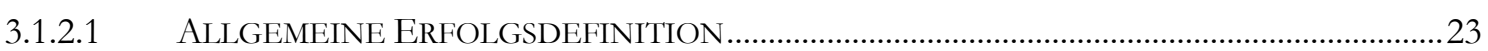

3.1.2.2 ERFOLG VON UNTERNEHMEN ……………………………………………………...2

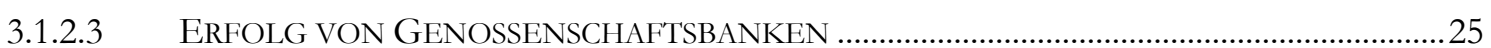

3.1.2.4 FÖRDERWIRTSCHAFTSERFOLG VS. GESCHÄFTSERFOLG ………………...........................26

3.1.3 ERFOLGSFAKTOREN UND ERFOLGSFAKTORENFORSCHUNG ……....................................26

3.1.3.1 HISTORIE DER ERFOLGSFAKTORENFORSCHUNG ……......................................................2

3.1.3.2 ERGEBNISSE DER ERFOLGSFAKTORENFORSCHUNG ……..................................................2 


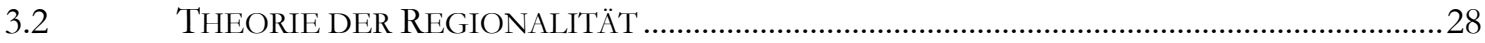

3.2.1 DAS REGIONALPRINZIP VON GENOSSENSCHAFTSBANKEN ……........................................2

3.2.2 DIE REGIONALE IDENTITÄT VON GENOSSENSCHAFTSBANKEN ……………………......29

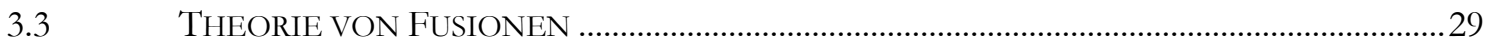

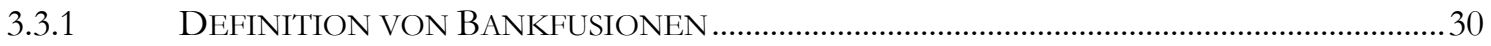

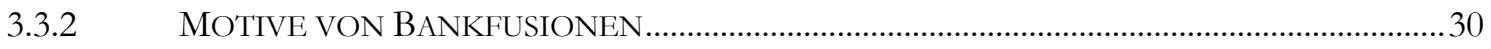

3.3.3 FUSIONSHISTORIE VON GENOSSENSCHAFTSBANKEN ......................................................

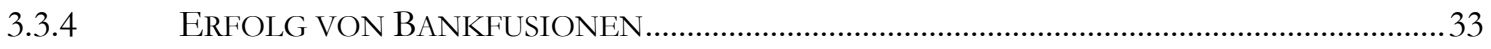

3.3.5 IDENTITÄTSVERLUST NACH FUSIONEN.........................................................................

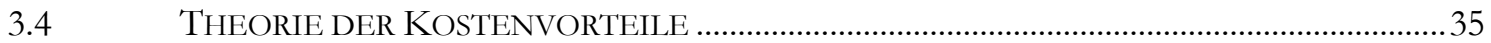

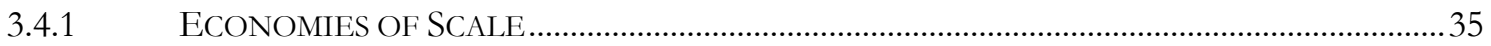

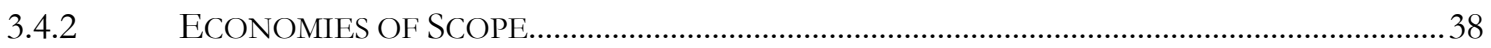

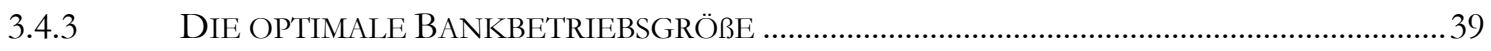

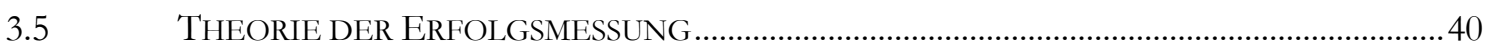

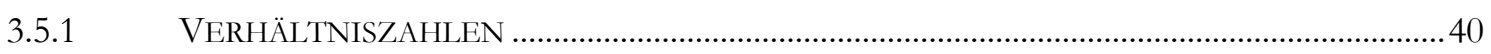

3.5.2 MESSGRÖßEN FÜR DIE BETRIEBSGRÖßE EINER BANK........................................................ 41

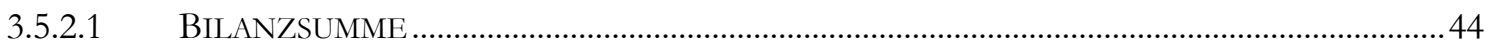

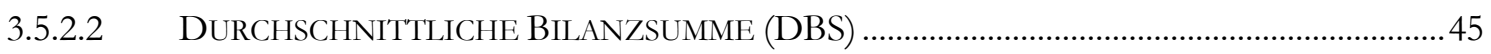

3.5.3 MESSGRÖßEN FÜR DEN ERFOLG EINER BANK ……......................................................... 46

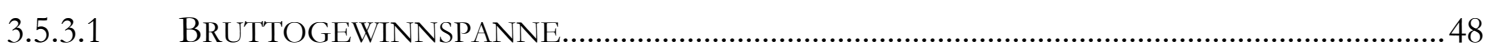

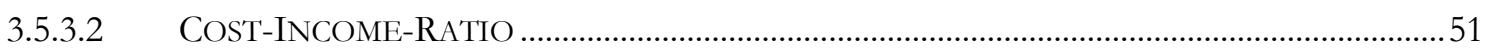

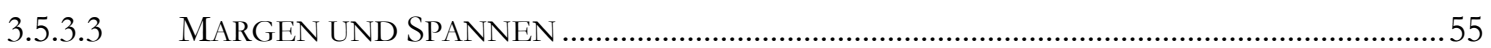

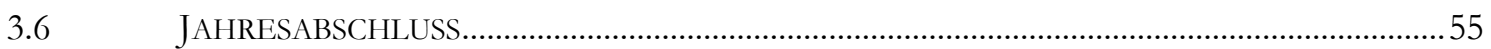

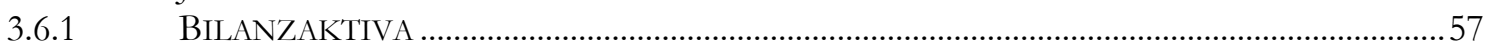

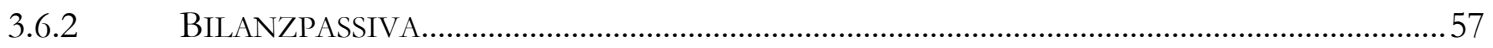

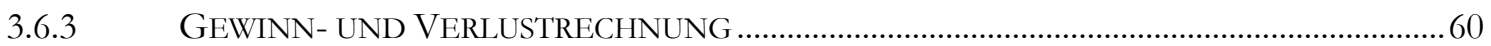

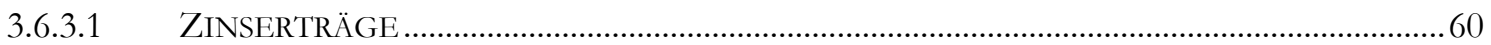

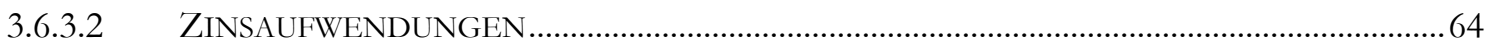

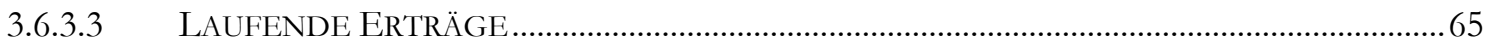

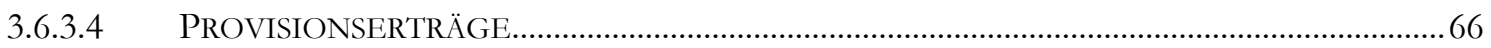

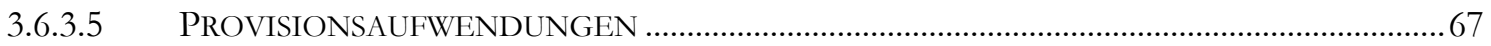

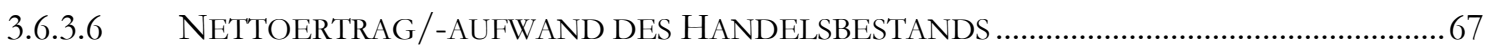

3.6.3.7 PERSONALAUFWAND UND PERSONALAUFWANDSSPANNE ................................................67

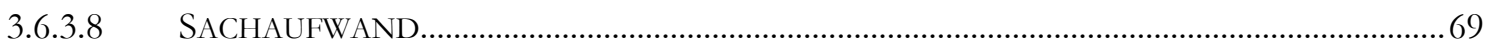

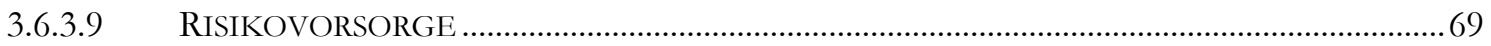

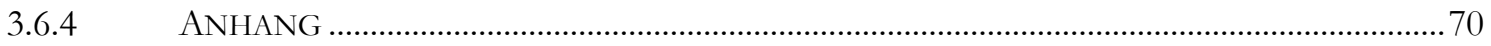

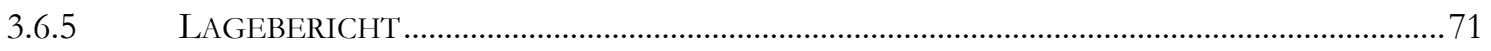

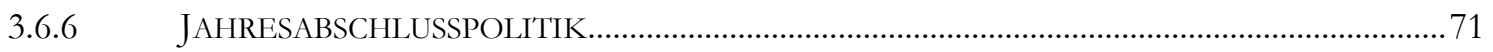

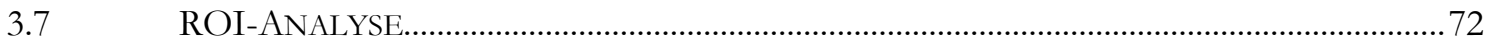

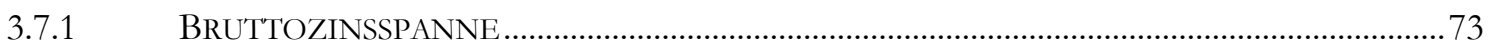

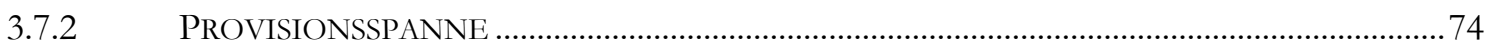

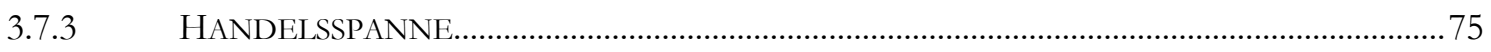

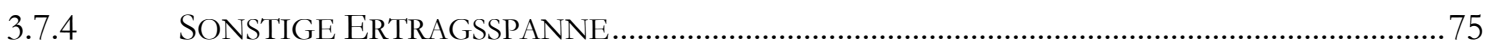

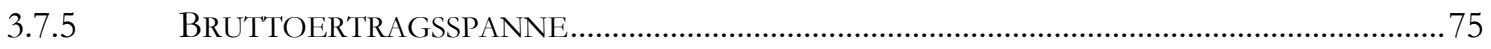

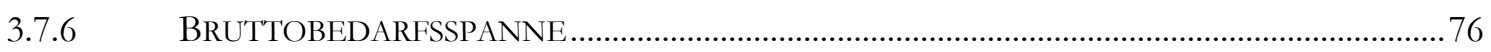

3.7.7 BRUTTOGEWINN AUS NORMALER GESCHÄFTSTÄTIGKEIT_..................................................76

3.7.8 BETRIEBSERGEBNIS AUS NORMALER GESCHÄFTSTÄTIGKEIT_..............................................76

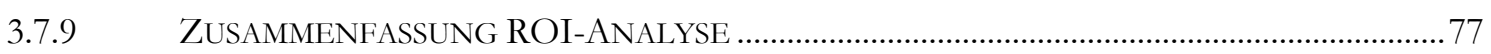

Z.8 ZU UNTERSUCHENDE ERFOLGSFAKTOREN …….............................................................. 
3.9 ABLEITUNG DER FORSCHUNGSHYPOTHESEN................................................................78

3.9.1 HYPOTHESENKATEGORIE A: NACH DER LAGE VON BANKEN.........................................79

3.9.2 HYPOTHESENKATEgORIE B: NACH POSITIONEN DER GEWINN- UND VERLUSTRECHNUNG UND NACH DER BILANZSTRUKTUR …………………………........79

3.9.3 HYPOTHESENKATEGORIE C: NACH DER GRÖßE VON BANKEN ……………………..............

3.9.4 HYPOTHESENKATEGORIE D: NACH REGIONALEN WIRTSCHAFTSDATEN ……............... 80

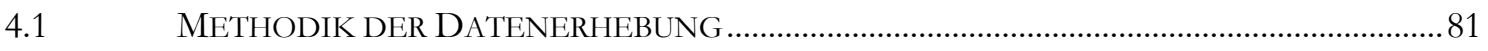

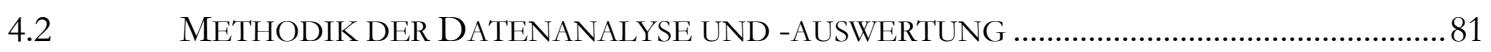

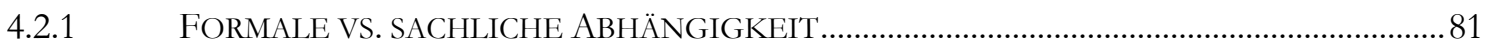

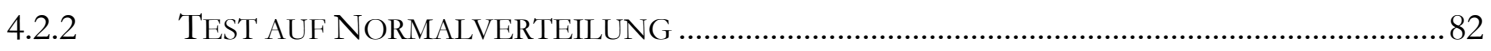

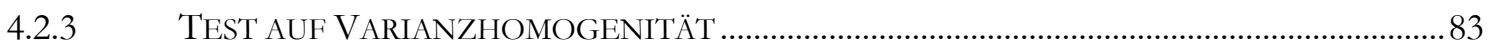

4.2.4 BOXPLOT

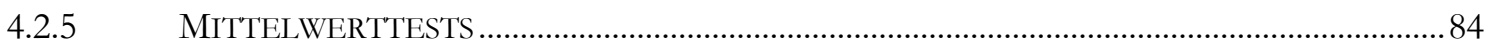

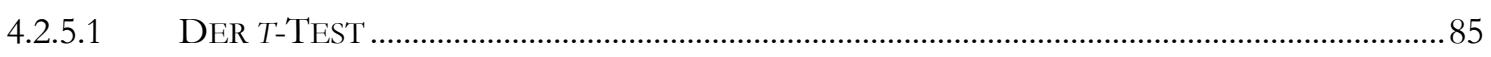

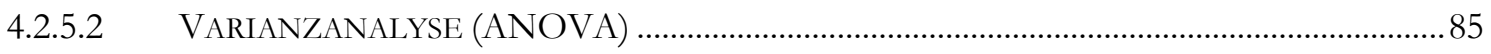

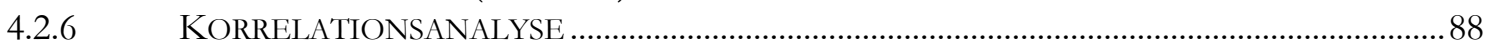

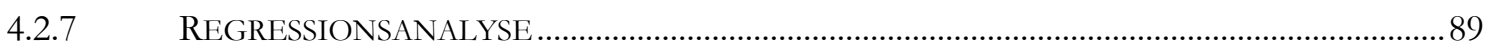

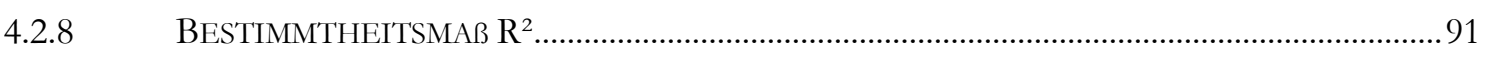

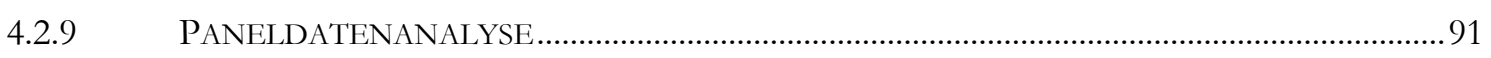

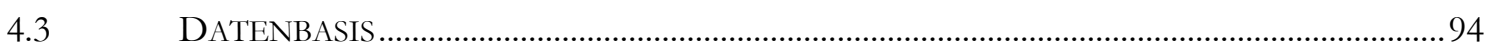

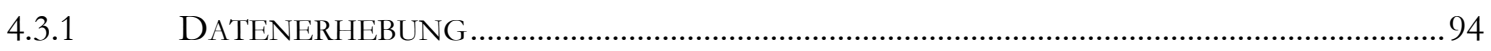

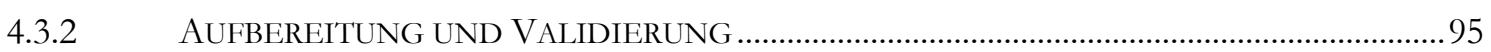

4.3.3 BESCHREIBUNG DER DATENBASIS _.............................................................................. 96

4.3.3.1 GEMEINDEVERZEICHNIS UND REGIONALATLAS................................................................ 96

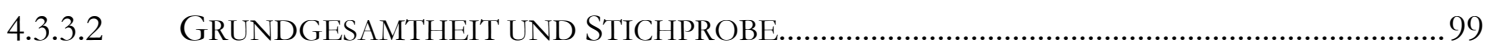

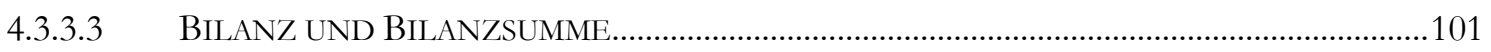

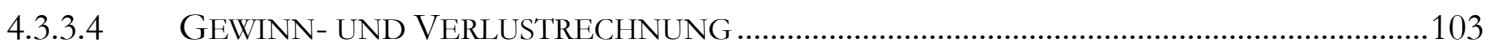

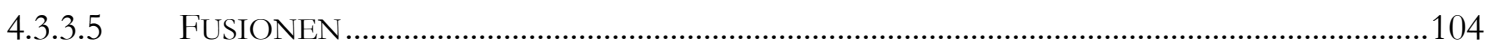

4.3.3.6 VERTEILUNG DER UNTERSUCHTEN BANKEN IN DEUTSCHLAND .................................105

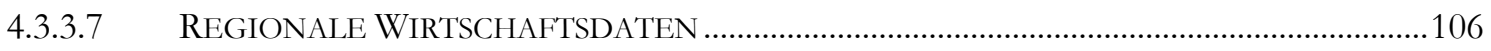

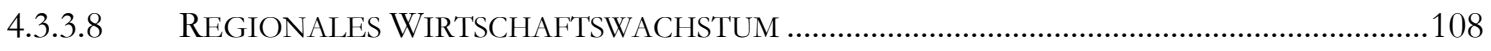

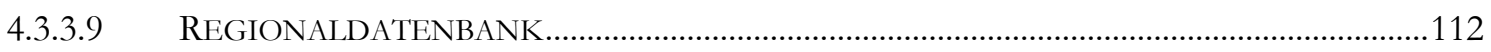

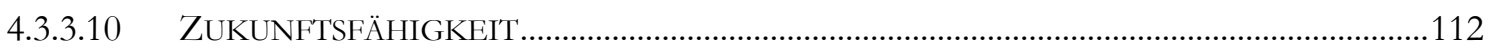

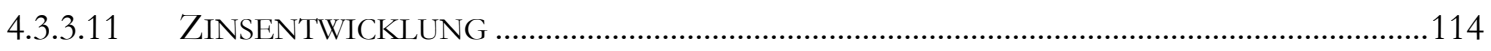

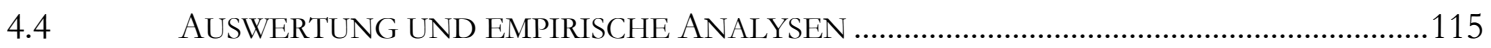

4.4.1 ROI-ANALYSE DES GENOSSENSCHAFTSBANKENSEKTORS...........................................115

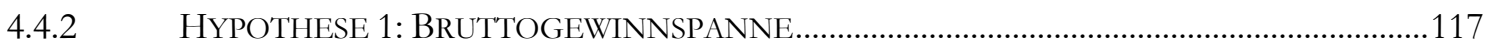

4.4.3 HYPOTHESE 2: ZINSERTRÄGE AUS KREDIT- UND

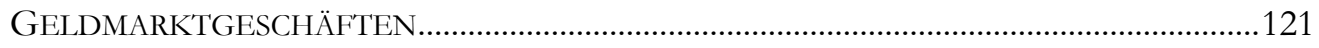

4.4.4 HYPOTHESE 3: ZINSERTRÄGE AUS FESTVERZINSLICHEN
WERTPAPIEREN UND SCHULDBUCHFORDERUNGEN....................................................126

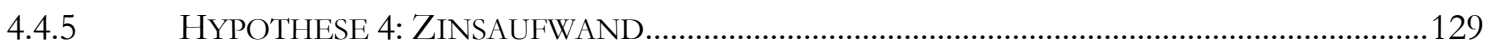

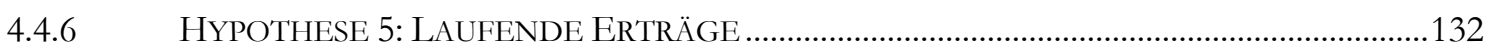

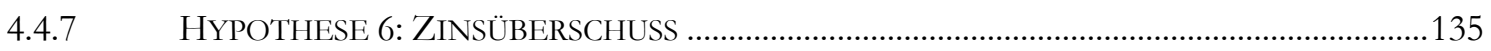

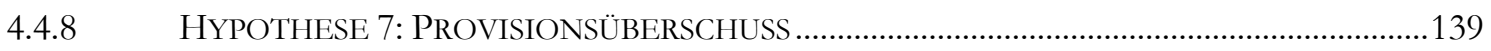

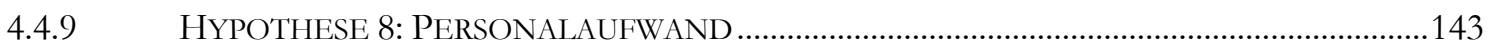

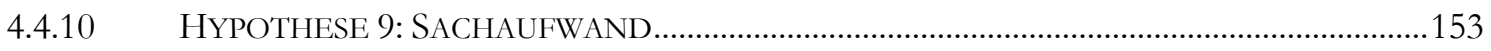

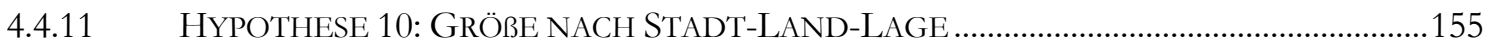

4.4.12 HYPOTHESE 11: LAGE NACH BUNDESLAND ...................................................................157 
4.4.13 HYPOTHESE 12: ABSCHREIBUNGEN AUF FORDERUNGEN ……………........................159

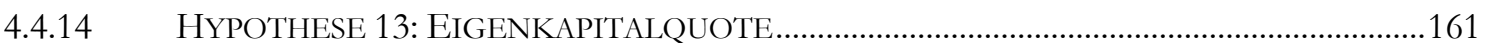

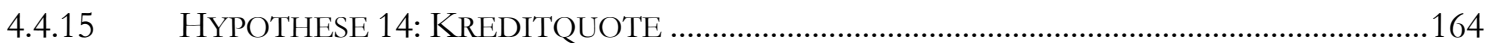

4.4.16 HYPOTHESE 15: EFFIZIENTE BETRIEBSGRÖßE....................................................................167

4.4.17 HYPOTHESE 16: HÄUFUNG KLEINER GENOSSENSCHAFTSBANKEN …............................174

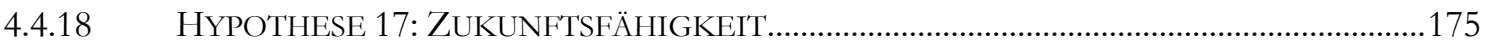

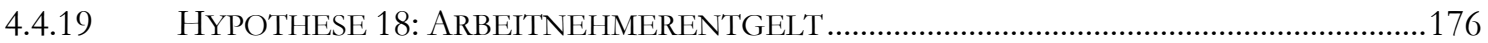

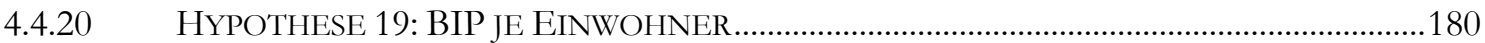

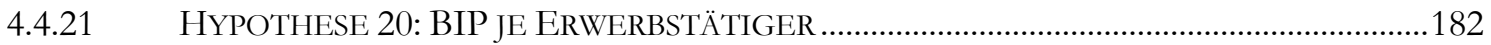

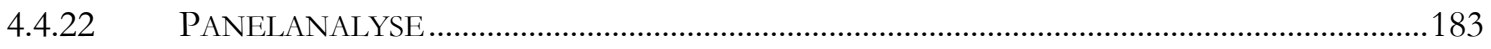

$5 \quad$ SCHLUSSBETRACHTUNG 194

$\begin{array}{ll}\text { ANHANG } & 199\end{array}$ 


\section{ABBILDUNGSVERZEICHNIS}

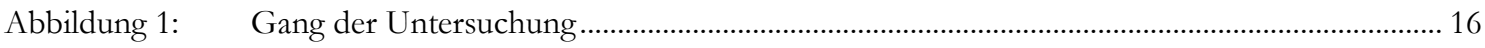

Abbildung 2: Universalbanken im deutschen Bankensystem …................................................................ 18

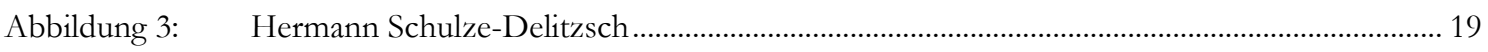

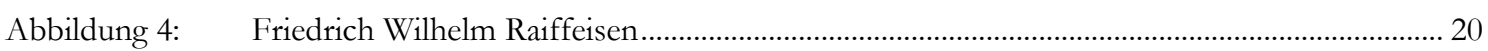

Abbildung 5: $\quad$ Entwicklung der Volksbanken und Raiffeisenbanken ab 1970............................................... 32

Abbildung 6: Fusionshistorie der VR Bank Main-Kinzig-Büdingen eG .................................................... 33

Abbildung 7: Skaleneffekte bei der Produktivität .............................................................................................. 37

Abbildung 8: Inputfaktoren der bankbetrieblichen Leistungserstellung........................................................ 42

Abbildung 9: Ermittlung des Bruttogewinns aus normaler

Geschäftstätigkeit ......................................................................................................................... 50

Abbildung 10: Gliederung der allgemeinen Verwaltungsaufwendungen ......................................................6 68

Abbildung 11: ROI-Grundschema zur Verknüpfung gesamtbankbezogener

Aufwands-, Ertrags- und Ergebniskennzahlen ........................................................................... 73

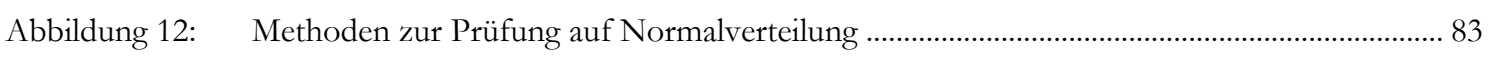

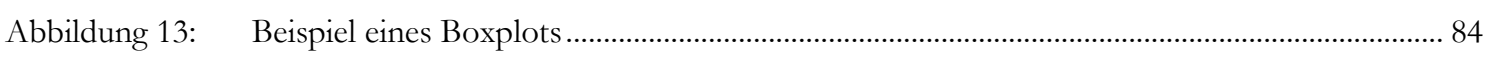

Abbildung 14: Verfahren zum Vergleich von mehr als zwei unabhängigen Stichproben .............................. 88

Abbildung 15: Zeitreihenplot der Verteilung von

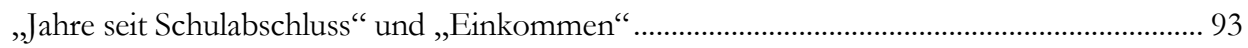

Abbildung 16: Streudiagramm der Verteilung von „Jahre seit

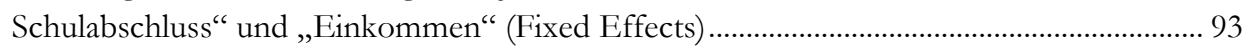

Abbildung 17: $\quad$ Stadt-Land-Gliederung Deutschlands nach Gemeinden........................................................ 98

Abbildung 18: Anteile der Institute an der Grundgesamtheit und an der Stichprobe (2011)

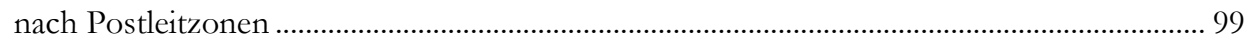

Abbildung 19: Verteilung der untersuchten Banken ...................................................................................... 105

Abbildung 20: Schematische Darstellung der wichtigsten VGR-Begriffe ...................................................... 109

Abbildung 21: Jahresdurchschnittliches BIP-Wachstum 2005-2011 auf Kreisebene .................................... 110

Abbildung 22: Bereiche und Kriterien zur Ermittlung der Zukunftsfähigkeit .............................................. 112

Abbildung 23: Einfluss der Lage auf die Zukunftsfähigkeit......................................................................... 113

Abbildung 24: Beurteilung der Zukunftsfähigkeit auf Kreisebene .............................................................. 114

Abbildung 25: Entwicklung der Bruttoertragsspanne und ihrer vier Komponenten ................................... 116

Abbildung 26: Verteilung der Bruttogewinnspanne nach Lagebereichen................................................... 118

Abbildung 27: Entwicklung der Bruttogewinnspanne nach Lagebereichen ................................................ 119

Abbildung 28: Entwicklung der Zinserträge aus Kredit- und Geldmarktgeschäften nach Lagebereichen.

Abbildung 29: Bilanzanteile ausgewählter Aktiva über alle analysierten

Genossenschaftsbanken (2011)

Abbildung 30: Entwicklung der Anteile risikoreicher Kundenforderungen nach Lagebereichen. 
Abbildung 31: Entwicklung der Zinserträge aus festverzinslichen Wertpapieren und Schuldbuchforderungen nach Lagebereichen.....

Abbildung 32: Entwicklung der Zinserträge aus festverzinslichen Wertpapieren und Schuldbuchforderungen aller Banken

Abbildung 33:

Entwicklung des Zinsaufwands nach Lagebereichen

Abbildung 34:

Bilanzanteile ausgewählter Passiva über alle analysierten

Genossenschaftsbanken (2011)

Abbildung 35:

Entwicklung der laufenden Erträge nach Lagebereichen.

Abbildung 36:

Entwicklung der laufenden Erträge aller Banken

Abbildung 37:

Entwicklung des Zinsüberschusses

exklusive laufender Erträge nach Lagebereichen

Abbildung 38: Entwicklung des Zinsüberschusses inklusive laufender Erträge nach Lagebereichen

Abbildung 39:

Entwicklung des Provisionsüberschusses nach

Lagebereichen

Abbildung 40:

Entwicklung der Provisionsspanne aller Banken

Abbildung 41:

Entwicklung des über alle Banken kumulierten Provisionsüberschusses

Abbildung 42:

Entwicklung des Provisionsüberschusses je MA nach Lagebereichen..

Abbildung 43:

Entwicklung des nach Lagebereichen kumulierten Provisionsüberschusses

Abbildung 44:

Entwicklung der Personalaufwandsspanne

nach Lagebereichen.

Abbildung 45: Entwicklung des Personalaufwands je Mitarbeiter nach Lagebereichen......

Abbildung 46: Entwicklung des Bruttogewinns aus normaler

Geschäftstätigkeit je Mitarbeiter nach Lagebereichen

Abbildung 47: Entwicklung der durchschnittlichen Bilanzsumme je Mitarbeiter nach Lagebereichen.

Abbildung 48: Entwicklung der Personalaufwandsspanne über alle Banken

Abbildung 49:

Lageabhängige Häufigkeitsverteilung der Bankgrößen nach Mitarbeiteranzahl (2011)...... 152

Abbildung 50:

Einfluss der Lage auf die Mitarbeiteranzahl

Abbildung 51:

Entwicklung des Sachaufwands nach Lagebereichen.

Abbildung 52:

Entwicklung der Bankgröße nach Lagebereichen

Abbildung 53

Wachstumsraten der durchschnittlichen Bilanzsumme im Vergleich zum Vorjahr

Abbildung 54:

Aggregierte Bilanzsumme der untersuchten Banken nach den drei Lagebereichen

Abbildung 55:

Einfluss der Lage nach Bundesland auf den Erfolg.

Abbildung 56:

Zusammenhang zwischen Bruttogewinnspanne und Risikovorsorge ...... 160

Abbildung 57:

Entwicklung der mittleren Eigenkapitalquote im Zeitablauf

Abbildung 58:

Einfluss der Eigenkapitalquote auf die Bruttogewinnspanne

Abbildung 59:

Zusammenhang zwischen Kreditquote und 
Abbildung 60: Entwicklung der mittleren Kreditquote im Genossenschaftssektor 165

Abbildung 61: Entwicklung von aggregierter Bilanzsumme und aggregierter Kreditsumme im Genossenschaftssektor 166

Abbildung 62: Entwicklung der mittleren Kreditquote nach Bundesländern 167

Abbildung 63: Histogramm zur Häufigkeit von Banken im jeweiligen Intervall.. 168

Abbildung 64: Boxplot zur Verteilung der Bilanzsummen ... 168

Abbildung 65: Zusammenhang zwischen Bankgröße und Bruttogewinnspanne

Abbildung 66: Erfolgsmaximale Bankgröße nach Jahren in Mio. Euro 171

Abbildung 67: Zusammenhang zwischen Zukunftsfähigkeit und Bruttogewinnspanne 176

Abbildung 68: Einfluss des Arbeitnehmerentgelts auf die Bruttogewinnspanne.

Abbildung 69: Entwicklung des Arbeitnehmerentgelts in Euro nach Lagebereichen. 178

Abbildung 70: Zusammenhang zwischen regionalem BIP je Einwohner und der Bruttogewinnspanne.

Abbildung 71: Entwicklung des regionalen BIP je Einwohner nach Lagebereichen

Abbildung 72: Zusammenhang zwischen der Bruttogewinnspanne und regionalem BIP je Erwerbstätiger.....

Abbildung 73: Zusammenhang zwischen der Lage und regionalem BIP je Erwerbstätiger

Abbildung 74: Einfluss des BIP-Wachstums auf die Bruttogewinnspanne 



\section{TABELLENVERZEICHNIS}

Tabelle 1: $\quad$ Entwicklung der Volksbanken und Raiffeisenbanken ab 1970............................................ 31

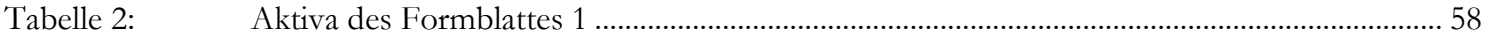

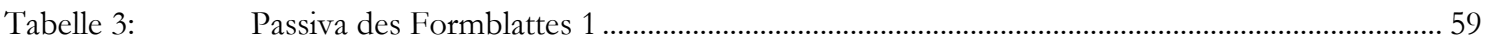

Tabelle 4: $\quad$ Positionen der Gewinn- und Verlustrechnung des Formblattes 3 .........................................6 61

Tabelle 5: $\quad$ Erfolgsspaltung in der Gewinn- und Verlustrechnung sowie Begriffskategorien der ROI-Analyse.................................................................................... 77

Tabelle 6: $\quad$ Von der Untersuchung ausgeschlossene Banken ..................................................................... 96

Tabelle 7: $\quad$ Aufteilung der Fläche Deutschlands nach der Stadt-/Landgliederung .................................. 97

Tabelle 8: $\quad$ Abdeckungsquote der vorliegenden Daten nach Jahren ....................................................... 100

Tabelle 9: $\quad$ Zuordnung von Positionen der Bilanzaktiva zu Positionen des Balance Sheet.................... 101

Tabelle 10: $\quad$ Zuordnung von Positionen der Bilanzpassiva zu Positionen des Balance Sheet................. 102

Tabelle 11: $\quad$ Zuordnung von Positionen der Gewinn- und Verlustrechnung zu Positionen des Income Statements.

Tabelle 12: $\quad$ Landkreise und kreisfreie Städte mit den höchsten durchschnittlichen Wachstumsraten von 2005 bis 2011

Tabelle 13: $\quad$ Landkreise und kreisfreie Städte mit den geringsten durchschnittlichen Wachstumsraten von 2005 bis 2011

Tabelle 14: $\quad$ ROI-Analyse aller Banken über den Betrachtungszeitraum

Tabelle 15: Entwicklung der Beiträge zur Bruttoertragsspanne

Tabelle 16: Entwicklung der Bruttogewinnspanne nach Lagebereichen

Tabelle 17:

Entwicklung der Zinserträge aus Kredit- und Geldmarktgeschäften nach Lagebereichen

Tabelle 18: $\quad$ Einfluss der Lage auf die Zinserträge aus Kredit- und Geldmarktgeschäften (OLS)

Tabelle 19:

Entwicklung der Zinserträge aus festverzinslichen Wertpapieren und

Schuldbuchforderungen nach Lagebereichen

Tabelle 20: $\quad$ Einfluss der Lage auf die Zinserträge aus festverzinslichen Wertpapieren und Schuldbuchforderungen (OLS)

Tabelle 21:

Entwicklung des Zinsaufwands nach Lagebereichen

Tabelle 22:

Einfluss der Lage auf die Zinsaufwandsspanne (OLS)

Tabelle 23:

Entwicklung des Durchschnittszinssatzes der Verbindlichkeiten nach Lagebereichen ..... 131

Tabelle 24: Entwicklung der laufenden Erträge nach Lagebereichen.

Tabelle 25:

Einfluss der Lage auf die laufenden Erträge (OLS)

Tabelle 26:

Entwicklung des Zinsüberschusses exklusive laufender Erträge nach Lagebereichen ....... 136

Tabelle 27:

Berechnung des Zinsüberschusses ...

Tabelle 28:

Entwicklung des Zinsüberschusses inklusive laufender Erträge nach Lagebereichen.....

Tabelle 29:

Einfluss der Lage auf den Zinsüberschuss (OLS)

Tabelle 30:

Entwicklung des Provisionsüberschusses nach Lagebereichen 
Tabelle 31: $\quad$ Einfluss der Lage auf die Provisionsspanne (OLS) ............................................................... 140

Tabelle 32: $\quad$ Entwicklung der Personalaufwandsspanne nach Lagebereichen........................................... 143

Tabelle 33: $\quad$ Einfluss der Lage auf die Personalaufwandsspanne (OLS) .................................................. 144

Tabelle 34: $\quad$ Entwicklung des Personalaufwands je Mitarbeiter nach Lagebereichen ............................... 145

Tabelle 35: $\quad$ Entwicklung der Personalaufwandsspanne über alle Banken ................................................ 149

Tabelle 36: Bilanzsummenwachstum im Vergleich zum Vorjahr.............................................................. 151

Tabelle 37: Durchschnittliche Personalaufwandsveränderung im Vergleich zum Vorjahr ..................... 151

Tabelle 38: Berechnung des erweiterten Sachaufwands ................................................................................. 153

Tabelle 39: $\quad$ Entwicklung des Sachaufwands nach Lagebereichen .............................................................. 154

Tabelle 40: $\quad$ Einfluss der Lage auf die Sachaufwandsspanne (OLS) ..........................................................155

Tabelle 41: $\quad$ Entwicklung der Bankgröße nach Lagebereichen ................................................................... 156

Tabelle 42: $\quad$ Einfluss der Lage nach Bundesland auf den Erfolg .............................................................. 158

Tabelle 43: Bestandteil „Abschreibungen auf Forderungen“ im Betriebsergebnis aus normaler Geschäftstätigkeit ................................................................................................................ 159

Tabelle 44: $\quad$ Zusammenhang zwischen Bruttogewinnspanne und Risikovorsorge ................................... 160

Tabelle 45: Einfluss der Eigenkapitalquote auf die Bruttogewinnspanne................................................. 163

Tabelle 46: $\quad$ Erfolgsmaximale Betriebsgröße in Mio. Euro ……................................................................. 170

Tabelle 47: $\quad$ Korrelationen unterhalb und oberhalb der effizienten Bankgröße ....................................... 172

Tabelle 48: $\quad$ Fläche je Genossenschaftsbank nach Bundesländern ............................................................. 175

Tabelle 49: $\quad$ Entwicklung des Arbeitnehmerentgelts in Euro nach Lagebereichen................................... 178

Tabelle 50: $\quad$ Einfluss des regionalen Arbeitnehmerentgelts auf die Bruttogewinnspanne ....................... 179

Tabelle 51: $\quad$ Einfluss der Lage auf das regionale Bruttoinlandsprodukt je Einwohner ............................. 181

Tabelle 52: $\quad$ Einfluss der Lage auf das regionale Bruttoinlandsprodukt je Erwerbstätiger ....................... 182

Tabelle 53: $\quad$ Ergebnisse der Paneluntersuchung (Fixed Effects) ……....................................................... 184

Tabelle 54: $\quad$ Ergebnisse der Paneluntersuchung (Fixed Effects) ……........................................................ 186

Tabelle 55: $\quad$ Ergebnisse der Paneluntersuchung (Pooled OLS) ……............................................................. 191

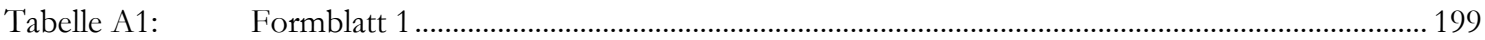

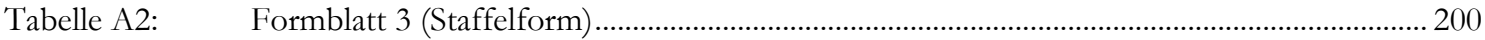




\section{ABKÜRZUNGS- UND SYMBOLVERZEICHNIS}

\begin{tabular}{|c|c|}
\hline ANOVA & Analysis of Variance (Varianzanalyse) \\
\hline ANE & Arbeitnehmerentgelt \\
\hline AOSE-Spanne & außerordentliche und sonstige Ertragsspanne \\
\hline BGS & Bruttogewinnspanne \\
\hline BIP & Bruttoinlandsprodukt \\
\hline BLG & Bruttolöhne und -gehälter \\
\hline BS & Bilanzsumme \\
\hline BVR & Bundesverband der Deutschen Volksbanken und Raiffeisenbanken \\
\hline CIR & Cost-Income-Ratio (Aufwand-Ertrag-Verhältnis) \\
\hline CRS & Constant returns-to-scale (konstante Skalenerträge) \\
\hline DBS & Durchschnittliche Bilanzsumme \\
\hline EDF & Empirical Distribution Function \\
\hline $\mathrm{eG}$ & eingetragene Genossenschaft \\
\hline EKQ & Eigenkapitalquote \\
\hline FE & Fixed Effects \\
\hline GenG & Genossenschaftsgesetz \\
\hline GLS & Generalized Least Squares (Methode der verallgemeinerten kleinsten Quadrate) \\
\hline $\mathrm{GuV}$ & Gewinn- und Verlustrechnung \\
\hline HGB & Handelsgesetzbuch \\
\hline HSD & honestly significant difference (Post-hoc-Test) \\
\hline NUTS & Nomenclature des unités territoriales statistiques \\
\hline LFB & Liquiditätsmäßig-finanzieller Bereich (Wertbereich) \\
\hline MKQ & Methode der kleinsten Quadrate \\
\hline OLS & Ordinary Least Squares (Methode der kleinsten Quadrate) \\
\hline $\mathrm{R}^{2}$ & Bestimmtheitsmaß \\
\hline RE & Random Effects \\
\hline RechKredV & $\begin{array}{l}\text { Verordnung über die Rechnungslegung der Kreditinstitute und } \\
\text { Finanzdienstleitungsinstitute }\end{array}$ \\
\hline ROI & Return on Investment \\
\hline TOB & Technisch-organisatorischer Bereich (Betriebsbereich) \\
\hline VGRdL & Arbeitskreis Volkswirtschaftliche Gesamtrechnungen der Länder \\
\hline VRS & Variable returns-to-scale (variable Skalenerträge) \\
\hline ZA & Zinsaufwand \\
\hline
\end{tabular}



Der Genossenschaftssektor stellt eine der drei Säulen des deutschen Bankensystems dar. ${ }^{1}$ Per Ende 2011, dem Ende des empirischen Betrachtungszeitraumes der vorliegenden Arbeit, existierten 1.121 Kreditgenossenschaften sowie zwei genossenschaftliche Zentralbanken. ${ }^{2}$ Im Durchschnitt jenes Jahres wies der Sektor eine aggregierte Bilanzsumme von $987 \mathrm{Mrd}$. Euro aus, was mit über $13 \%$ einem bedeutenden Anteil an den Universalbanken in Deutschland entspricht. ${ }^{3}$ Der überwiegende Teil der Passivseiten von Kreditgenossenschaften ist geschäftsbedingt auf Kundeneinlagen zurückzuführen. ${ }^{4}$

Durch die Lage in einer bestimmten Region und die häufig kleinen Geschäftsgebiete ist davon auszugehen, dass Zusammenhänge zwischen den örtlichen Gegebenheiten und den Kreditgenossenschaften identifiziert werden können. Aufgabe der Dissertation ist es, betriebswirtschaftliche, volkswirtschaftliche und demografische Größen zu untersuchen, denen ein Einfluss auf den Geschäftserfolg von Genossenschaftsbanken in Deutschland unterstellt wird. Generell spricht man bei diesen Größen von Erfolgsfaktoren. Einige Erfolgsfaktoren sind endogen, das heißt, diese können von den Banken selbst beeinflusst bzw. gesteuert werden. Dazu zählen unter anderem Fusionen, die Bankgröße als auch Aufwendungen, auf die später näher eingegangen wird. Andere Erfolgsfaktoren sind exogen, sie können nicht durch das Wirken der Bank beeinflusst werden, z. B. Arbeitslosigkeit, Bildungsniveau oder vorhandenes Vermögen.

\footnotetext{
${ }^{1}$ Vgl. Abschnitt 3.1.1.

${ }^{2}$ Vgl. Bundesbank (2014c), S. 86.

${ }^{3}$ Vgl. Bundesbank (2014c), S. 86; vgl. Abschnitt 3.1.1.

${ }^{4}$ Vgl. Abbildung 34 auf S. 137.
} 



\subsection{Stand der Forschung}

Die Erforschung von Effizienz und Erfolgsfaktoren im Bankbereich fand bisher in mehreren Untersuchungen Niederschlag. Diese werden hier systematisch dargestellt. Ein spezieller Fokus liegt dabei auf den Genossenschaftsbanken. Bei jeder Studie werden gegebenenfalls bestehende Forschungslücken identifiziert, die weiter unten in konkrete Hypothesen einfließen. ${ }^{5}$

\subsubsection{RICHTER (2014)}

Der Titel des Werkes von RICHTER lautet Produktivität und ibre Einflussfaktoren - Eine empirische Analyse der Kreditgenossenschaften. Er möchte aufklären, welche Faktoren in der Vergangenheit die Cost-Income-Ratio (CIR) beeinflussten. Der Autor bezeichnet die CostIncome-Ratio als das gängige Produktivitätsmaß und benutzt sie in seinem Werk. ${ }^{6}$ Folgende Angaben verwendet er als Datenbasis:

- Detaillierte Bilanz- und GuV-Daten, Informationen zu Struktur und Geschäftslage der Banken (1993-2012, 20.530 Bankenjahre, sehr hohe Abdeckungsquote)

- Marktstellung (Quotient aus Kundenanzahl mit mindestens einem Bankkonto und der Anzahl der Einwohner des Geschäftsgebietes)

- Zukunftsfähigkeit (nach KRÖHNERT ET AL.)

- Euro-Swapsätze für fünf Jahre und zehn Jahre

Als Erfolgsfaktoren ${ }^{8}$ werden untersucht:

- Zinsniveau (in Prozent)

- Steilheit der Zinsstrukturkurve (Differenz von Swapsätzen unterschiedlicher Laufzeiten)

- Schlankheit der Filialstruktur (Zweigstellen pro Kunde)

- Mitarbeiteranzahl pro Kunde

- Zugehörigkeit zum jeweiligen Regionalverband (1 bis 7, anonymisiert)

- Zugehörigkeit zur jeweiligen Rechenzentrale (1 bis 2, anonymisiert)

- Markttyp (1 bis 4, hohe/niedrige Zukunftsfähigkeit, hohe/niedrige Marktstellung)

- Größe der Bank anhand der Bilanzsumme (DBS)

Folgende Ergebnisse hat RICHTER erzielt:

Als Proxy für die Höhe des Zinsniveaus werden durch Richter die Euro-Swapsätze für die Laufzeiten fünf Jahre und zehn Jahre dargestellt. Diese beiden Swapsätze sind jedoch hoch miteinander korreliert, daher können sie nicht zusammen in derselben Analyse untersucht werden. Um dennoch beide Laufzeiten zu berücksichtigen und Multikollinearität zu ver-

\footnotetext{
5 Vgl. Abschnitt 3.9.

${ }^{6}$ Vgl. Richter (2014), S. 417.

${ }^{7}$ Vgl. Kröhnert, Klingholz, Sievers, Großer und Friemel (2011), S. 1 f.

${ }^{8}$ Vgl. Richter (2014), S. 426.
} 
meiden, werden sie einzeln alternativ, das heißt im Austausch untersucht. Multikollinearität bedeutet, dass sich ein Regressor als lineare Funktion eines anderen Regressors darstellen lässt. ${ }^{9}$ Im Ergebnis hat der Swapsatz für fünf Jahre einen hochsignifikanten Einfluss auf die Cost-Income-Ratio, ebenso wie der Swapsatz für zehn Jahre.

Im Vergleich zur absoluten Höhe des Swapsatzes im vorangegangenen Absatz hat die Steilheit der Zinsstrukturkurve einen gemäß Koeffizienten 14-mal größeren, ebenso hochsignifikanten Einfluss. Die Steilheit wurde als Differenz zwischen dem Swapsatz für zehn Jahre und demjenigen für fünf Jahre bestimmt. Vergrößert sich der Abstand zwischen den beiden genannten Swapsätzen um einen Prozentpunkt, so sinkt die CIR um 0,07 Prozentpunkte. Der Median der Kennzahl CIR für 2012 liegt bei 0,6969 bzw. 69,69 \%.

Die Anzahl der Zweigstellen pro Kunde zeigt keinen signifikanten Zusammenhang zur CIR. Folglich lässt sich für die Genossenschaftsbanken in dem untersuchten Zeitraum nicht sagen, dass eine Schließung von Filialen empirisch eine Verbesserung der CIR zur Folge gehabt hätte.

Der Zusammenhang der Mitarbeiter pro Kunde einerseits und der CIR andererseits fällt hochsignifikant aus. Dies liegt auch an dem hohen Anteil, den die Personalaufwendungen an den gesamten allgemeinen Verwaltungsaufwendungen repräsentieren. RICHTER spricht hier von ca. 60 \%. Erhöht sich die Anzahl der Mitarbeiter pro Kunde um einen Prozentpunkt, so verschlechtere sich die CIR um 1,75 Prozentpunkte. Anhand eines Zahlenbeispiels soll dieser Zusammenhang näher betrachtet werden. Der Median der Kennzahl Mitarbeiter pro Kunde liegt für 2012 bei 0,0035970 bzw. bei 0,3597\%. Nach Erhöhung um einen Prozentpunkt läge die Kennzahl bei 1,3597\%, also dem 3,78-fachen des Ausgangswertes. Eine Erhöhung der Personalausgaben um den Faktor 3,78 müsste jedoch die CIR deutlich höher ansteigen lassen, auf Werte weit über 1. Möglicherweise ist eine Interpretation realistischer, nach der bei einer Erhöhung der Mitarbeiter pro Kunde um 1 \% (von 0,3597 \% auf 0,3633 \%) die CIR im Mittel um 1,75 Prozent absinkt.

Die Zugehörigkeit zu einem der sieben Regionalverbände hat nachweislich keinen starken statistisch signifikanten Einfluss auf das Produktivitätsniveau. Lediglich bei Verband 6 ergibt sich eine schwache Signifikanz $(0,0628)$, jedoch gepaart mit einem beachtlichen Koeffizienten von $-0,132^{*}$, das heißt, Banken dieses Verbandes können im Mittel mit einer CIR rechnen, die um 13,2\% unter dem Wert anderer Regionen liegt. ${ }^{10}$ Statt 69,69 \%, dem Mittel für das Jahr 2012, wäre hier mit 56,49 \% zu rechnen. Um welchen Verband es sich dabei handelt, lässt der Autor aus Anonymitätsgründen offen.

Bei der Rechenzentralzugehörigkeit sieht dies ähnlich aus: Ein schwach signifikanter Einfluss bei Rechenzentrale 1 ist nachweisbar. Der Koeffizient lautet $-0,0190^{*}$, das heißt, die CIR von Banken mit Rechenzentrale 1 ist um nahezu 2 Prozentpunkte besser als bei den übrigen Banken. ${ }^{11}$

\footnotetext{
${ }^{9}$ Vgl. Backhaus (2006), S. 89 f.

${ }^{10}$ In Abweichung zur vorliegenden Arbeit verwendet Richter: *** $\mathrm{p}<0,01,{ }^{* *} \mathrm{p}<0,05,{ }^{*} \mathrm{p}<0,10$, Vgl. Richter (2014), S. 428.

11 Vgl. Richter (2014), S. 426 ff.
} 


\section{Kritik und Forschungslücken}

- Es besteht nur ein unscharfer regionaler Bezug über die Zugehörigkeit zu einem der sieben zum Zeitpunkt der Untersuchung existierenden und bisweilen recht groBen Regionalverbände. ${ }^{12}$ Es werden keine weiteren regionalen Wirtschaftsdaten verwendet. RiCHTER schlägt selbst vor, dass eine differenziertere geografische Betrachtung weiterreichende Erkenntnisse liefern kann. ${ }^{13}$

- Die Verwendung der Cost-Income-Ratio zur Beurteilung wird in der Literatur kritisch gesehen. Einige Autoren, wie BURGER ET AL., argumentieren für eine angepasste Variante der Cost-Income-Ratio. ${ }^{14}$ Trotzdem lässt RiCHTER die Umstrittenheit der CIR nicht unreflektiert, sondern stellt kurz die Literaturmeinung dar. ${ }^{15}$

- Auf Basis der Kennzahl Zweigstellen je Kunde verweist Richter auf die Effizienz von Bankfusionen im Genossenschaftssektor. ${ }^{16}$ Bei Fusionen jedoch entstehen größere Banken mit mehr Zweigstellen und auch mehr Kunden. Die Relation Zweigstellen je Kunde bleibt daher von einer Fusion zunächst im Mittel unverändert. Erst bei einer nachgelagerten möglichen Ausdünnung des Filialnetzes könnte sich die genannte Kennzahl ändern. Insofern sollte geprüft werden, welche anderen Kennzahlen zur direkten Beurteilung des Fusionserfolgs zur Anwendung kommen können. Mit Zweigstellen je Kunde ist dies jedenfalls nicht möglich.

- Richter kommt zu dem Resultat, dass die Cost-Income-Ratio mit zunehmender Bilanzsumme sinkt, und erklärt dies mit Skaleneffekten (Economies of Scale). In Bezug auf die Erforschung der Beziehung zwischen Bankgröße und Cost-Income-Ratio geht die Arbeit nicht weitreichend genug ins Detail, ein möglicher nichtlinearer Verlauf bleibt unerforscht.

- Nicht ganz klar wird, warum RICHTER zunächst die OLS-Regression durchführt, die Ergebnisse interpretiert, um dann festzustellen, dass einige Annahmen des OLS-Modells nicht erfüllt sind. ${ }^{17}$ Im nächsten Schritt wird dann die aufgrund von Anwendungsvoraussetzungen geeignetere GLS-Regression durchgeführt (Fixed Effects) und wieder die Ergebnisse interpretiert. ${ }^{18}$ PARK ist für solche Fälle der Meinung, dass nur ein Modell richtig sein kann. ${ }^{19}$

- Fraglich ist, wie es dem Autor gelingt, in einem Fixed Effects-Modell zeitkonstante unabhängige Variablen zu berücksichtigen. Diese werden von dem Modell normalerweise übergangen. ${ }^{20}$ Dem Autor gelingt es trotzdem, einen Koeffizienten samt Signifikanzniveau auszuweisen.

\footnotetext{
${ }^{12}$ Der aktuell größte Regionalverband trägt den Namen Genossenschaffsverband und hat seinen Sitz in Neu-Isenburg bei Frankfurt am Main und betreut Kreditgenossenschaften in allen Bundesländern außer Baden-Württemberg, Bayern und Nordrhein-Westfalen. Durch Fusionen im Jahr 2008 mit dem Genossenschaftsverband Norddeutschland mit Sitz in Hannover und 2012 mit dem Mitteldeutschen Genossenschaffsverband mit Sitz in Chemnitz hat sich die Anzahl der Regionalverbände von 7 auf 5 reduziert. Die übrigen 4 aktuell bestehenden Regionalverbände lauten Baden-Württembergischer Genossenschaftsverband (BWGV), Genossenschaftsverband Bayern (GVB), Genossenschaftsverband Weser-Ems (GVWE) und Rheinisch-Westfälischer Genossenschaftsverband (RWGV).

${ }^{13}$ Vgl. Richter (2014), S. 429.

${ }^{14}$ Vgl. Burger, Frohmüller und Moormann (2008).

15 Vgl. Richter (2014), S. 425.

${ }^{16}$ Vgl. Richter (2014), S. 427 f

17 Ordinary Least Squares (OLS), Methode der kleinsten Quadrate.

${ }^{18}$ Generalized Least Squares (GLS), Methode der verallgemeinerten kleinsten Quadrate, vgl. Backhaus (2006), S. 368.

${ }^{19}$ Vgl. Park (2010), S. 1.

${ }^{20}$ Vgl. Giesselmann und Windzio (2012), S. 44; vgl. Bode und Wilke (2014), S. 171; vgl. Allison (2009), S. 12f; vgl. Kopp und Lois (2009), S. 23.
} 


\subsubsection{CHRISTIANS UND GÄRTNER (2014)}

CHRISTIANS UND GÄRTNER untersuchen in ihrer Arbeit Einfluss regionaler Bankenmärkte auf dezentrale Banken: Demografie, Bankenwettbewerb und Kreditportfolio, warum dezentrale Banken auch in strukturschwachen Regionen in der Regel erfolgreich wirtschaften. Hierbei fanden sie heraus, dass Sparkassen in demografisch schrumpfenden Regionen Ostdeutschlands durch geringen Bankenwettbewerb eine relativ höhere Zinsspanne erzielen. ${ }^{21}$ Außerdem konnten Sie zeigen, dass ein hoher Eigenkapitalanteil sich positiv auf die Gesamtkapitalrendite vor Steuern auswirkt. ${ }^{22}$ Ein Einfluss auf die Nachsteuerrendite wurde nicht untersucht. Möglicherweise ist ein hoher Eigenkapitalanteil hier eher nachteilig, da sich die Aufwendungen für das Eigenkapital nicht steuerreduzierend abziehen lassen, während Institute mit hohem Fremdkapitalanteil von dieser Abzugsfähigkeit profitieren können. Weiter wiesen sie eine positive Wirkung eines hohen Kundeneinlagenüberschusses nach. ${ }^{23}$ Jedoch ist davon auszugehen, dass die Kausalität hier über einen Umweg herzustellen ist. Denn ein hoher derartiger Überschuss deutet darauf hin, dass Einlagen nur zu geringen Anteilen in Form von Darlehen wieder ausgereicht werden können, und folglich mehr margenarmes Kreditersatzgeschäft getätigt werden muss. Für periphere Regionen mit geringer Kreditnachfrage ist daher anzunehmen, dass durch die ebenfalls dort vorhandene geringere Wettbewerbsintensität höhere Margen realisiert werden können.

\subsubsection{Hummel und Pedrotti (2013)}

In ihrem Artikel Zinsmarge und Effizienz von Genossenschaftsbanken untersuchen HuMMEL UND PEDROTTI $^{24}$ die verschiedenen Bankengruppen Genossenschaftsbanken, Sparkassen und private Banken in sieben europäischen Ländern. Verglichen werden hierzu ausgewählte Bilanz- und GuV-Positionen im Zeitraum 1999 bis 2009. ${ }^{25}$ Speziell stellen die Autoren hierbei auf die Nettozinsmarge ab. Sie aggregierten 165 Gruppen zu einem Datensatz, um die gemeinsamen Merkmale der Gruppenmitglieder herauszustellen. Im Ergebnis konnte nachgewiesen werden, dass Genossenschaftsbanken europaweit eine höhere Zinsmarge als Sparkassen oder Kreditbanken erwirtschaften. Begründet wird dies einerseits mit einem vergleichsweise hohen Risiko im kleinteiligen Kreditgeschäft mit dem Mittelstand, andererseits mit einer soliden Refinanzierung durch kostengünstige kurzfristige Einlagen. ${ }^{26}$ Gerade diese Einlagen erlauben eine lukrative Fristentransformation durch Ausreichung in längere Laufzeiten. ${ }^{27}$ Zusätzlich attestieren die Autoren den Genossenschaftsbanken einen effizienteren Produktionsprozess - trotz einer oft kleinen Größe. Besonders würdigen sie die stabilitätsverleihenden Eigenschaften während der Finanzkrise. ${ }^{28}$

Als Ansatz für weitere Forschung nennen die Autoren selbst einen mikroökonomischen Bottom-Up-Ansatz, welcher von der Ebene der einzelnen Institute ausgeht. Das Ziel solle sein, die gruppenspezifischen Eigenschaften der Genossenschaftsbanken zu charakterisie-

\footnotetext{
${ }^{21}$ Vgl. Christians und Gärtner (2014), S. 1.

22 Vgl. Christians und Gärtner (2014), S. 14.

23 Vgl. Christians und Gärtner (2014), S. 15.

24 Vgl. Hummel und Pedrotti (2013).

25 Vgl. Hummel und Pedrotti (2013), S. 347.

${ }^{26}$ Vgl. Hummel und Pedrotti (2013), S. 356.

${ }^{27}$ Vgl. Hummel und Pedrotti (2013), S. 349; vgl. Walter (2013), S. 294; vgl. Rolfes (2008), S. 8; Unter Fristentransformation versteht man generell die Transformation kurzfristiger Passiva in langfristige Aktiva, bzw. vice versa. Neben der Fristentransformation gibt es noch zwei weitere grundlegende Transformationsleistungen von Banken: Die Transformation der Losgröße erklärt die Bündelung von in der Regel kleineren Einlageeinheiten zu in der Regel größeren Krediteinheiten. Die Risikotransformation ermöglicht das Kreditgeschäft; das Kreditrisiko wird durch Kreditzinsen abgebildet, die über den Einlagezinsen liegen.

${ }^{28}$ Vgl. Hummel und Pedrotti (2013), S. 357.
} 
ren. ${ }^{29}$ Zusätzlich solle ein alternativer Effizienzmaßstab zur Cost-Income-Ratio zur Anwendung kommen. ${ }^{30}$ Die vorliegende Arbeit greift diesen Vorschlag auf und setzt ihn - mit eigenen Schwerpunkten - für Deutschlands Kreditgenossenschaften um.

\subsubsection{TISCHER (2011)}

In seiner Dissertationsschrift untersucht TISCHER empirisch die Effizienz der deutschen Sparkassen. Der verwendete Datensatz umfasst den Zeitraum 1994 bis $2008 .^{31}$ Er berücksichtigt regionale und soziodemografische Aspekte. Ziel der Arbeit ist es, Effizienzunterschiede zwischen den einzelnen Sparkasseninstituten herauszustellen und die Unterschiede geschäftspolitisch zu erklären. ${ }^{32}$ Tischer setzt sich kritisch mit der Verwendung der Aufwand-Ertrag-Relation als Effizienzmaß auseinander ${ }^{33}$, lehnt sie jedoch schließlich ab. ${ }^{34}$ In der empirischen Analyse konnte er feststellen, dass die technische Ineffizienz die Skalenineffizienz dominiert. ${ }^{35}$ Die technische Effizienz betrachtet hierbei das Verhältnis von Input zu Output ${ }^{36}$, die Skaleneffizienz beschreibt die mögliche Größenveränderung eines Instituts zur Kostenverringerung. ${ }^{37}$ Zur Generierung desselben Outputs können die Sparkassen ihre Inputs um etwa $20 \%$ reduzieren, während die größenbedingten Steigerungspotenziale nur auf ca. $5 \%$ taxiert werden. ${ }^{38}$ Daher betrachtet er das Erreichen einer optimalen Betriebsgröße als ein nachrangiges Ziel. ${ }^{39}$

\subsubsection{REICHEL (2011)}

Nach dem Artikel von REICHEL zum Thema Der Beitrag der Kreditgenossenschaften zur Finanzstabilität besitzen zwei Faktoren einen wesentlichen Einfluss auf die Höhe des Zinsergebnisses. Es handelt sich um die Zinsstruktur und das Niveau der langfristigen Zinsen. ${ }^{40}$ Flacht sich die Zinsstruktur um einen Prozentpunkt ab, so führt dies zu einer Senkung des Zinsüberschusses um ca. 0,06 Prozentpunkte. ${ }^{41}$ Bei einer Erhöhung der langfristigen Zinsen um einen Prozentpunkt fand er einen Anstieg des Zinsüberschusses von 0,2 Prozentpunkten. ${ }^{42}$ Beispielhaft für das Jahr 2010 gehe der Bundesverband der Deutschen Volksbanken und Raiffeisenbanken (BVR) davon aus, dass ca. ein Drittel des Zinsüberschusses der Genossenschaftsbanken aus der Fristentransformation herrührt. ${ }^{43}$ Die gute Ertragslage der Jahre 2009 und 2010 sei maßgeblich auf den Strukturbeitrag der Fristentransformation zurückzuführen. ${ }^{44}$

Weiter adressiert REICHEL in seinem Artikel die Effekte des regionalen Geschäftsmodells. ${ }^{45}$ Sie tragen zu einer effizienten Finanzintermediation bei, nehmen eine Rolle als Steuerzahler

\footnotetext{
${ }^{29}$ Vgl. Hummel und Pedrotti (2013), S. 357.

${ }^{30}$ Vgl. Hummel und Pedrotti (2013), S. 356.

${ }^{31}$ Vgl. Tischer (2011), S. 52.

32 Vgl. Tischer (2011), S. 197.

${ }_{33}$ Vgl. Tischer (2011), S. 57 f.

${ }^{34}$ Vgl. Tischer (2011), S. 202.

35 Vgl. Tischer (2011), S. 201.

36 Vgl. Conrad (2008), S. 187.

37 Vgl. Conrad, Neuberger und Trigo Gamarra (2009), S. 113; vgl. 3.4.1.

38 Vgl. Tischer (2011), S. 201.

${ }^{39}$ Vgl. Tischer (2011), S. 201.

${ }^{40}$ Vgl. Reichel (2011), S. 36.

${ }^{41}$ Vgl. Reichel (2011), S. 36.

42 Vgl. Reichel (2011), S. 36.

${ }^{43}$ Vgl. Reichel (2011), S. 36.

44 Vgl. Reichel (2011), S. 36.

45 Vgl. Reichel (2011), S. 959.
} 
ein und stoßen wichtige Sekundäreffekte an. ${ }^{46} \mathrm{Zu}$ diesen zählt er auf der Inputseite die Käufe von Vorleistungen, die Investitionsgüternachfrage sowie die Nachfrageeffekte durch Gehälter der Angestellten. ${ }^{47}$ Auf der Outputseite nennt er die Kreditvergabe und die Sicherstellung der Kundenliquidität, durch welche sich stabilisierende Impulse ergeben. ${ }^{48}$

\subsubsection{Christians (2010)}

CHRISTIANS untersucht in seinem Werk Der Erfolg ostdeutscher Kreditinstitute den Einfluss der Region auf die Profitabilität von Sparkassen und Genossenschaftsbanken. ${ }^{49}$ Seine Studie beschäftigt sich genauer mit dem Bankenerfolg selbst und mit den Erfolgsdeterminanten, die ihrerseits Einfluss auf den Erfolg besitzen. ${ }^{50}$ Betrachtet wurden die Jahre 1996 bis 2008. ${ }^{51}$ Aus dieser Basis zieht er viele konkrete Schlussfolgerungen.

Verglichen mit den Sparkassen, können Genossenschaftsbanken in Ostdeutschland höhere Zins- und Provisionsmargen erzielen. Jedoch weisen sie überproportional höhere Betriebsaufwendungen auf, die die Margenvorteile überkompensieren und in sich in geringerer Effizienz niederschlagen. Ihre CIR liegt daher um zehn Prozentpunkte unter der der Sparkassen. Als mögliche Gründe nennt CHRISTIANs kleinere Betriebsgrößen und eine schwächere Mitarbeiterproduktivität. Die geringere Spanne beim Bewertungsergebnis von Genossenschaftsbanken führt er darauf zurück, das weniger erfolgreiche Institute auch weniger Risikovorsorge treffen. Die erfolgreicheren Sparkassen hingegen bildeten 2007 mehr Reserven. Die Banken können durch den Ansatz des Wahlrechts nach \340f HBG einen geringeren Gewinn ausweisen. ${ }^{52}$

Bei Betrachtung der regionalen Parameter fand CHRIsTIANS, dass die Zinsspanne in Städten signifikant erhöht war. Im Detail führt es dies auf den Zinsaufwand zurück und begründet dies mit einem stärkeren Wettbewerb: In Städten müssten die Banken ihren Kunden relativ teure Produkt- und Preisangebote unterbreiten. ${ }^{53}$

Insbesondere für sogenannte überdurchschnittliche Problemregionen zieht CHRISTIANS ein beruhigendes Fazit: Speziell Sparkassen gelingt es auch dort, gute bis überdurchschnittliche Rentabilitätskennziffern zu erzielen. Regionale Banken können also trotz eines wirtschaftlich ungünstigen Standortes profitabel arbeiten. ${ }^{54}$ Im Vergleich zu Genossenschaftsbanken oder Sparkassen besitzen private Banken keinen regionalen Auftrag. Sie konzentrieren sich bei der Markterschließung bzw. beim Rückzug auf Gebiete, in denen sie finanziell lukrative Kunden vermuten. ${ }^{55}$

Beim Bankenvergleich anhand der CIR stellte sich heraus, dass die Unterschiede auf die Betriebskostenspanne zurückzuführen sind, und nicht etwa auf die Ertragsseite. Hohe Zinsen für vergebene Kredite bzw. niedrige Zinsen für entgegengenommene Einlagen sind nicht die Begründungen für Erfolg. Dieser wird indessen regelmäßig durch die Höhe von Personal- und Verwaltungskosten bestimmt. Jedoch sieht er ein reines Absenken des Kos-

\footnotetext{
${ }^{46}$ Vgl. Reichel (2011), S. 959.

47 Vgl. Reichel (2011), S. 959.

48 Vgl. Reichel (2011), S. 959.

${ }^{49}$ Vgl. Christians (2010).

${ }^{50}$ Vgl. Christians (2010), S. 143

51 Vgl. Christians (2010), S. 39.

52 Vgl. Christians (2010), S. 143 und Abschnitt 3.6.3.9.

${ }^{53}$ Vgl. Christians (2010), S. 143 und Abschnitt 3.6.3.9.

54 Vgl. Christians (2010), S. 143 und Abschnitt 3.6.3.9.

55 Vgl. Christians (2010), S. 2.
} 
tenniveaus zur Abhilfe als ungeeignet an. Einen Einfluss der Region auf die CIR schließt er ebenso aus wie einen Einfluss der Bankengröße.

\section{Kritik und Forschungslücken}

- Es ist davon auszugehen, dass sich regionale Unterschiede dennoch auf den Erfolg niederschlagen. Möglicherweise ist das Unterscheidungskriterium der Prosperität einer Region ${ }^{56}$ nicht ideal geeignet, um Verschiedenheiten zu identifizieren.

- Auch die Größe der Institute sollte detaillierter betrachtet werden.

- Eine Ausweitung der Untersuchung auf das gesamte Bundesgebiet wäre wünschenswert.

\subsubsection{CONRAD, NEUberger UND Trigo GamarRa (2009)}

In ihrem Werk Der Einfluss regionaler und demographischer Umfeldfaktoren auf die Kosten- und Ertragssituation von Sparkassen - Eine Effirienzanalyse betrachten CONRAD, NEUBERGER und Trigo Gamarra die Effizienz von Sparkassen im gesamten Bundesgebiet. ${ }^{57}$ Die Arbeit geht von der bisherigen demografischen Entwicklung in Deutschland aus, und bezieht auch die zukünftige prognostizierte Entwicklung mit ein - denn diesbezüglich unterscheiden sich die einzelnen Regionen. Untersucht wurde der Zeitraum 2001 bis 2005 auf Basis von Daten des Betriebsvergleichs von Sparkassen. ${ }^{58}$ Je nach Jahr werden zwischen 433 und 435 Datensätze ausgewertet, dies umfasst nahezu alle Institute des Sparkassensektors. Die Autoren stellen detailliert auf verschiedene Effizienzarten ab und ermitteln diese für jedes Institut. Dazu zählen die Technische, die Kosten-, die Ertrags- und die Skaleneffizienz.

Technische Effizienz betrachtet das Verhältnis von Input zu Output einer Sparkasse. ${ }^{59}$ Kosteneffizienz zieht eine zusätzliche allokative Komponente (Preise der Inputfaktoren) hinzu. ${ }^{60}$ Ertragseffizienz erklärt die Fähigkeit eines Instituts, seine Erträge zu maximieren, wenn die Inputmengen und Outputpreise bereits feststehen. ${ }^{61}$ Schließlich beschreibt die Skaleneffizienz, inwieweit ein Institut seine Betriebsgröße hin zu einer optimalen Größe verändern kann, bei der die geringsten durchschnittlichen Kosten anfallen. ${ }^{62}$

Im Ergebnis konnten sie nachweisen, dass ein Großteil der Sparkassen ein hohes Maß an Wirtschaftlichkeit aufweist. Dies gilt für die Technische Effizienz mit einem durchschnittlichen Effizienzwert von $75 \%$, der Ertragseffizienz mit $75 \%$ und der Skaleneffizienz mit gar $96 \%$. Bei der Kosteneffizienz deckten die Autoren häufig ein Steigerungspotenzial auf. Hier lagen die Durchschnittswerte bei $60 \%$. Zudem nahm die Kosteneffizienz im Zeitraum 2001 bis 2005 stetig ab. ${ }^{63}$ Die genannte Periode liegt zeitlich vor der Finanzkrise.

In Hinblick auf die räumliche Verteilung effizienter Institute konnten die Autoren zeigen, dass relativ viele von diesen in den alten Bundesländern liegen. In den neuen Bundesländern wiesen die Institute hingegen eine geringere Streuung der Effizienz im Vergleich zu

\footnotetext{
${ }^{56}$ Unter Prosperität ist im vorliegenden regionalen Kontext eine Situation zu verstehen, in der eine Bank etwa eine hohe Anzah lukrativer Kunden besitzt, in der andererseits aber auch zahlreiche Wettbewerber für die Verknappung der Margen führen.

57 Vgl. Conrad et al. (2009).

${ }^{58}$ Vgl. Conrad et al. (2009), S. 26.

${ }^{59}$ Vgl. Conrad (2008), S. 187.

${ }^{60} \mathrm{Vgl}$. Conrad et al. (2009), S. 10.

${ }^{61}$ Vgl. Conrad et al. (2009), S. 11.

${ }^{62}$ Vgl. Conrad et al. (2009), S. 113; vgl. Abschnitt 3.4.1.

${ }^{63}$ Vgl. Conrad et al. (2009), S. 65 f.
} 
den alten Bundesländern auf. Die effizientesten Sparkassen liegen häufig in sehr dicht besiedelten Regionen oder in sehr dünn besiedelten, peripheren Regionen. ${ }^{64}$ In den genannten, dicht besiedelten Regionen stehen die Institute aufgrund des Wettbewerbs unter sehr hohem Druck, welcher zu höherer Effizienz führt. Im Gegensatz dazu führt bei Sparkassen auf dem Lande das Fehlen von Wettbewerbern zu auskömmlicheren Margen. Zusammenhänge in der gleichen Wirkungsrichtung sind auch für Genossenschaftsbanken zu erwarten, wie sie in der vorliegenden Arbeit analysiert werden.

In Räumen mit Einwohnerzuwachs bzw. -verlust konnten im Zeitraum 2001 bis 2005 eher keine Effizienzunterschiede nachgewiesen werden. Für Räume mit schwacher wirtschaftlicher Entwicklung und ländliche Räume konnte nachgewiesen werden, dass dort die Sparkassen bezüglich der Effizienz sehr homogen sind, das heißt ähnlich gut oder ähnlich schlecht. Jedoch fiele ihnen eine effiziente Einbeziehung der Ressourcen in den Leistungserstellungsprozess schwerer. ${ }^{65}$

Nachfolgend beschreiben die Autoren schließlich, wie sich die Umfeldfaktoren auf die Effizienz von Sparkassen auswirken. Als relevante Umfeldfaktoren wurden die Einwohnerdichte, die wirtschaftliche Situation (Kaufkraft, Attraktivität, Arbeitslosigkeit, Nachfragepotenzial), die Altersstruktur, die Konkurrenzsituation, die Betriebsgröße sowie die Eigenkapitalausstattung identifiziert. Eine hohe Relevanz und Signifikanz von Zusammenhängen konnte in der Arbeit belegt werden. Die Faktoren erklären Teile der ermittelten Ineffizienzen, welche auch regionale und demografische Gründe aufweisen, und nur teilweise dem Management angelastet werden können. ${ }^{66}$

Mit einer schlüssigen Begründung beantworten die Autoren die Frage, ob Sparkassen in allen Regionen eine Überlebenschance hätten - trotz demografischen Wandels und struktureller Veränderung im Bankenmarkt. Diese Überlebenschance bestehe, da sich die Institute anpassen, etwa an starken Wettbewerb oder an schwierige regionalwirtschaftliche Lage. Durch das Ausnutzen ihrer Potenziale erhöhen die Sparkassen ihre Effizienz und können so ihre Wettbewerbsfähigkeit dauerhaft sichern. ${ }^{67}$

\subsubsection{THIRY (2009)}

THIRY untersucht in seinem Werk Eine empirische Analyse der Marktdisziplinierung deutscher Sparkassen die 450 größtenteils nicht kapitalmarktorientierten deutschen Sparkassen ${ }^{68}$ im Zeitraum 1998 bis $2005 .^{69}$ Er setzt zur Normierung bei der Bildung diverser Spannen ${ }^{70}$ auf die Bilanzsumme, nicht auf die durchschnittliche Bilanzsumme. ${ }^{71}$ Er konnte Anzeichen einer direkten Marktdisziplinierung durch Fremdkapitalgeber nachweisen. ${ }^{72}$ Diese Indizien belegen, dass Sparkassen je nach wirtschaftlicher Lage auf Einlagen basierend sanktioniert werden. ${ }^{73}$ Dazu werden höhere Renditeaufschläge als Risikoprämie gefordert. ${ }^{74}$

\footnotetext{
${ }^{64} \mathrm{Vgl}$. Conrad et al. (2009), S. 66.

${ }^{65}$ Vgl. Conrad et al. (2009), S. 66.

${ }^{66}$ Vgl. Conrad et al. (2009), S. 67.

${ }^{67}$ Vgl. Conrad et al. (2009), S. 67 f.

${ }^{68}$ Vgl. Thiry (2009), S. 280.

${ }^{69}$ Vgl. Thiry (2009), S. 281.

${ }^{70}$ Vgl. Abschnitt 3.5.3.3.

${ }^{71}$ Vgl. Thiry (2009), S. 155, 158, 160

72 Vgl. Thiry (2009), S. 271.

${ }^{73}$ Vgl. Thiry (2009), S. $271 \mathrm{f}$.

${ }^{74}$ Vgl. Thiry (2009), S. 168, 170.
} 


\subsubsection{AUERBACH (2009)}

AUERBACH (2009) untersucht in seinem Werk Fusionen deutscher Kreditinstitute die Erfolgsfaktoren von Fusionen am Beispiel von Sparkassen und Kreditgenossenschaften. ${ }^{75}$ Empirisch betrachtet er dazu 1.682 Fusionen beider Banktypen im Zeitraum 1993 bis $2006 .{ }^{76}$ Dazu vergleicht er fusionierende und nicht fusionierende Institute im Jahr der Verschmelzung sowie in den vier folgenden Jahren. ${ }^{77}$ Er wies nach, dass bei fusionierenden Instituten die Erfolgskennzahlen im Fusionsjahr deutlich schwächer ausfielen als bei nicht fusionierenden. ${ }^{78}$ Der negative Effekt nimmt im Laufe der folgenden Geschäftsjahre ab, einige Institute erreichen dann auch positive Effekte. ${ }^{79}$ Insgesamt weist AUERBACH für Fusionen im Genossenschaftssektor somit gemischte Ergebnisse aus. ${ }^{80}$

\subsubsection{VARMAZ (2006)}

VARMAZ untersuchte in seinem Werk Rentabilität im Bankensektor die Bilanz-, GuV- und Jahresabschlusskennzahlen deutscher Banken im Zeitraum 1990 bis 2004. ${ }^{81}$ Die Zielstellung der Arbeit liegt in der Identifizierung von rentabilitätstreibenden Faktoren im deutschen Bankensektor. ${ }^{82}$ Er fand im Mittel höhere Kreditquoten bei Sparkassen (durchschnittlich $70 \%$ ) und Genossenschaftsbanken (durchschnittlich $68 \%$ ) als bei den Kreditbanken (durchschnittlich 52\%). ${ }^{83}$ Den Sparkassen und Kreditgenossenschaften attestiert ein eine deutlich höhere Rentabilität als den Kreditbanken, bei durchschnittlich kleineren Betriebsgrößen. ${ }^{84}$ Als besonders wichtigen externen Faktor für diese höhere Rentabilität führt er den Wettbewerb an. ${ }^{85}$ Als wichtigen internen Einflussfaktor auf die Rentabilität identifiziert er eine effiziente Kombination von Faktoreinsätzen zu Produkten. ${ }^{86}$ Einen Einfluss der Skaleneffizienz konnte er bei Sparkassen und Genossenschaftsbanken nicht nachweisen. ${ }^{87}$ Systemspezifische Effizienzvorteile kann er am ehesten bei Kreditgenossenschaften nachweisen. ${ }^{88}$ Eine große Betriebsgröße erscheint vorteilhaft, wenn der Markt stark konzentriert ist. ${ }^{89}$

\subsubsection{PADBERg (2005)}

PADBERG analysiert in seinem Werk die Jahresabschlüsse von Genossenschaftsbanken und Sparkassen. In Bezug auf die Zinsmarge konnte er Unterschiede zwischen Instituten in städtischer und ländlicher Lage nachweisen. Bei seinem Vergleich zwischen als ländlich anzusehenden Kreissparkassen und als städtisch geltenden Stadtsparkassen konnte er zeigen, dass die ländlichen Institute eine höhere Zinsmarge erzielten. ${ }^{90}$ Explizit für Genos-

\footnotetext{
${ }^{75} \mathrm{Vgl}$. Auerbach (2009), S. 6.

${ }^{76} \mathrm{Vgl}$. Auerbach (2009), S. 238.

77 Vgl. Auerbach (2009), S. 238.

${ }^{78}$ Vgl. Auerbach (2009), S. 238.

${ }^{79} \mathrm{Vgl}$. Auerbach (2009), S. 238.

${ }^{80}$ Vgl. Auerbach (2009), S. 238.

81 Vgl. Varmaz (2006), S. 95.

82 Vgl. Varmaz (2006), S. 261.

${ }^{83}$ Vgl. Varmaz (2006), S. 96.

${ }^{84}$ Vgl. Varmaz (2006), S. 261.

85 Vgl. Varmaz (2006), S. 121 ff., S. 261.

${ }^{86}$ Vgl. Varmaz (2006), S. 121 ff., S. 261.

87 Vgl. Varmaz (2006), S. 262.

${ }^{88}$ Vgl. Varmaz (2006), S. 263.

${ }^{89}$ Vgl. Varmaz (2006), S. 262.

${ }^{90}$ Vgl. Padberg (2005), S. 25.
} 
senschaftsbanken traf er jedoch keine Aussage. ${ }^{91}$ Eine entsprechende Untersuchung soll in der vorliegenden Arbeit durchgeführt werden.

\subsubsection{RIEKEBERG (2003)}

In seiner Arbeit Erfolgsfaktoren von Sparkassen untersuchte RIEKEBERG eine zur vorliegenden Arbeit verwandte Aufgabenstellung. Die sehr vielschichtige Analyse basiert auf der Auswertung geführter Interviews und setzt lineare Strukturgleichungsmodelle ein. Die Arbeit betrachtet verschiedene Erfolgsfaktoren ${ }^{92}$, wie z. B. die Wettbewerbssituation ${ }^{93}$ oder die Standortattraktivität ${ }^{94}$. RIEKEBERG untersucht die Abhängigkeit der Wettbewerbssituation von der räumlichen Präsenz der Wettbewerber. ${ }^{95}$ Er konnte empirisch nachweisen, dass der Wettbewerb umso höher ist, je näher die Filialen anderer Wettbewerber liegen, je höher die Anzahl anderer Wettbewerber ist und je mehr Bankstellen je Wettbewerber existieren. ${ }^{96}$

\subsubsection{WUTZ (2002)}

WUTZ thematisiert die Auswirkungen der geografischen Umwelt auf die Effizienz von Sparkassen und Genossenschaftsbanken. ${ }^{97}$ Er verwendet Standortfaktoren zur Bewertung der Effizienz von bayrischen Genossenschaftsbanken im Zeitraum von 1993 bis 1997. Durch Fusionen hat sich die Anzahl vorhandener Banken im Betrachtungszeitraum von 717 auf 588 reduziert. Untersucht wurden schließlich 533 Banken. Als Standortfaktoren werden die Größe des Einzugsgebietes, Marktdurchdringung, Kundenstruktur, Einlagensumme pro Kunde, Marktanteil und die Bruttozinsspanne unterschieden. ${ }^{98}$ WUTZ fand heraus, dass kleine Institute oft von positiven Standortfaktoren profitieren. Große Banken hingegen leiden eher an nachteiligen Standortfaktoren. ${ }^{99}$

\subsubsection{TEBROKE (1993)}

In seiner Arbeit Größe und Fusionserfolg von Genossenschaftsbanken untersucht TEBROKE die Bedeutung der Betriebsgröße von Kreditgenossenschaften für ihre Ergebnisstruktur. ${ }^{100}$ Zur Auswertung zieht er insgesamt 31.259 Jahresabschlüsse heran, die den Jahren 1980 bis 1988 entstammen. ${ }^{101}$ Im Mittel pro Jahr werden $93 \%$ aller existierenden Institute abgedeckt. TEBROKE untersucht den Einfluss der Größe auf verschiedene Aufwands- und Ertragspositionen und wendet dazu das ROI-Schema an. ${ }^{102}$ Die Institute werden hierzu nach ihrer Bilanzsumme, die er zur Messung der Größe einsetzt, in 15 Größenklassen eingeteilt. ${ }^{103}$ Die Grenzen dieser Größenklassen werden jedoch nicht anhand von Quantilen bestimmt, sondern willkürlich gesetzt, in der Regel bei Vielfachen von zehn Mio. DM. ${ }^{104}$ Die Grenzen zwischen den einzelnen Größenklassen erhöht er in jedem Jahr mit derselben Wachstumsrate, um welche sich auf die aggregierte Bilanzsumme über alle Ge-

\footnotetext{
91 Vgl. Padberg (2005), S. 26.

92 Vgl. Riekeberg (2003), S. 85 f.

${ }^{3}$ Vgl. Riekeberg (2003), S. 223.

${ }^{94}$ Vgl. Riekeberg (2003), S. 226.

95 Vgl. Riekeberg (2003), S. 223.

${ }^{96}$ Vgl. Riekeberg (2003), S. 233.

${ }^{97}$ Vgl. Wutz (2002).

98 Vgl. Wutz (2002), S. 8 ff.

${ }^{99}$ Vgl. Wutz (2002), S. 17.

100 Vgl. Tebroke (1993), S. 59.

101 Vgl. Tebroke (1993), S. 100.

102 Vgl. Tebroke (1993), S. 98 f.

103 Vgl. Tebroke (1993), S. 100.

104 Vgl. Tebroke (1993), S. 101.
} 
nossenschaftsbanken hinweg vergrößert hat. ${ }^{105}$ TEBROKE fand, dass Kreditinstitute mit wachsender Bilanzsumme leichte Nachteile im Zinsgeschäft hinnehmen müssen. ${ }^{106}$ Dies führt auf die Zinsaufwendungen zurück, welche stärker als die Zinserträge steigen. ${ }^{107}$ Kleinere Institute profitieren hingegen von günstigeren Refinanzierungskosten. ${ }^{108}$ Dank steigender Provisionsüberschüsse und relativ sinkendem Verwaltungsaufwand profitieren größere Genossenschaftsbanken und realisieren deutliche Vorteile im technischorganisatorischen Bereich. ${ }^{109}$ Insgesamt weist TEBROKE so mit wachsender Betriebsgröße eine deutliche Zunahme der Nettozinsspanne nach. ${ }^{110}$ Jedoch traten die Erfolge häufig erst erheblich nach dem Fusionszeitpunkt ein. ${ }^{111}$ Im Mittel konnte er hier einen zeitlichen Nachlauf von etwa drei Jahren identifizieren. ${ }^{112}$

\subsubsection{BÄSCH (1992)}

In seiner Arbeit zur Jahresabschlussanalyse bei Universalbanken stellt BÄSCH die bedeutsamsten externen Einflussfaktoren auf den Bankerfolg vor. Dazu zählt er das Wirtschaftswachstum, die Kreditnachfrage, die Zinsen und Wechselkurse. ${ }^{113}$ Die ersten drei dieser Erfolgsfaktoren werden von der vorliegenden Arbeit aufgegriffen und untersucht. Das Wirtschaftswachstum wird als regionales Bruttoinlandsprodukt in Gestalt der Veränderung zum Vorjahreswert abgebildet. ${ }^{114}$ Die Kreditnachfrage schlägt sich in der Untersuchung der Kreditquote nieder. ${ }^{115}$ Das Niveau und die Steilheit der Zinsstrukturkurve werden im Rahmen einer Panelanalyse untersucht. ${ }^{116}$ Wechselkurse werden hingegen nicht betrachtet.

\subsubsection{Weitere Autoren}

Buehn, Karmann und PedrotTI ${ }^{117}$ (2013) finden in ihrem Aufsatz What determines the interest margin? An analysis of the German banking system heraus, dass speziell bei Genossenschaftsbanken die Zinsspanne wesentlich durch die Marktmacht und durch Ineffizienz bestimmt wird. Die Marktmacht wird in der vorliegenden Arbeit durch das verwandte Konzept der Wettbewerbsintensität abgebildet.

\footnotetext{
105 Vgl. Tebroke (1993), S. 101.

106 Vgl. Tebroke (1993), S. 289.

${ }^{107}$ Vgl. Tebroke (1993), S. 290.

108 Vgl. Tebroke (1993), S. 290.

${ }^{109}$ Vgl. Tebroke (1993), S. 289.

${ }^{110}$ Vgl. Tebroke (1993), S. 290.

111 Vgl. Tebroke (1993), S. 249.

112 Vgl. Tebroke (1993), S. 249.

113 Vgl. Bäsch (1992), S. 296.

$114 \mathrm{Vgl}$. Abschnitt 4.3.3.8.

115 Vgl. Varmaz (2006), S. 96; vgl. Abschnitt 4.4.15.

116 Vgl. Abschnitt 4.4.22.

117 Vgl. Karmann, Buehn und Pedrotti (2013), S. 2.
} 


\subsection{Identifizierte Forschungslücken}

Bei der Analyse der bestehenden Literatur wurden speziell folgende Forschungslücken identifiziert:

1. Berücksichtigung eines präzisen regionalen Bezuges bei der Auswertung sowie beim Vergleich von Jahresabschlüssen im Bankbereich

2. Berücksichtigung der Lage nach städtisch, halbstädtisch und ländlich bei der Auswertung

3. Identifizierung der Lageeinflüsse auf die Erfolgskomponenten

Durch eine systematische Analyse sollen diese Zusammenhänge für den Bereich der Kreditgenossenschaften erforscht werden.

\subsection{Forschungsfragen dieser Untersuchung}

Auf Basis eines Datensatzes zu Jahresabschlüssen von Banken sollen Erfolgsfaktoren identifiziert werden. Der Datensatz umfasst die Geschäftsjahre 2005 bis 2011 und umfasst für das Jahr 2011 insgesamt 963 Genossenschaftsbanken, was einem Anteil von $86 \%$ an allen existierenden Genossenschaftsbanken entspricht. ${ }^{118}$ Die Erfolgsfaktoren können vielfältig sein und aus diversen Bereichen stammen. In Frage kommen etwa die Größe eines Instituts, gemessen an der durchschnittlichen Bilanzsumme, oder seine Lage, eingeteilt nach städtisch, halbstädtisch oder ländlich. Spezielles Augenmerkt legt die vorliegende Arbeit auf den regionalen Bezug der Daten, um eine möglichst kleinteilige und direkte Verknüpfung zu erreichen. Hierzu werden folgende Forschungsfragen gestellt:

1. Welche Hinweise lassen sich finden, wie Erfolgsfaktoren auf den Erfolg von Banken wirken?

2. Welchen Einfluss hat dabei die geografische Lage der Institute?

\subsection{Forschungsansatz}

Der Forschungsansatz besteht im Wesentlichen darin, mehrere erhobene Datensätze zu verknüpfen und anschließend mittels statistischer Methoden auszuwerten. Zur Anwendung kommen die einfache sowie die multiple lineare Regression, die Varianzanalyse und auch die Panelanalyse. Dabei kann die Varianzanalyse signifikante Unterschiede im Mittelwert zweier Gruppen von Merkmalsträgern identifizieren. Die Panelanalyse erlaubt es, Entwicklungen von Werten derselben Merkmalsträger im Zeitverlauf zu beschreiben. Da die Datensätze sehr umfangreich sind, kann der Markt der Genossenschaftsbanken nahezu vollständig abgebildet werden.

\subsection{Gang der Untersuchung}

Kapitel 1 enthält die Einleitung.

Kapitel 2 stellt den Stand der Forschung dar. Aus den identifizierten Forschungslücken werden die Forschungsfragen abgeleitet und der Forschungsansatz aufgebaut. 
Kapitel 3 definiert grundlegende Begriffe wie die Genossenschaftsbank, Erfolg, Erfolgsfaktoren und geht auf die Erfolgsfaktorenforschung ein. Es beinhaltet die theoretische Analyse und reicht von der Theorie von Fusionen über die Theorie der Kostenvorteile und der Erfolgsmessung bis zur Theorie der Regionalität. Theoretisch betrachtet werden der Jahresabschluss und die ROI-Analyse. Aus den zu untersuchenden Erfolgsfaktoren werden die Forschungshypothesen abgeleitet.

In Kapitel 4 wird die empirische Analyse beschrieben. Im Nachgang zur Darlegung der Art und Weise der Datenerhebung folgt die Methodik der Datenanalyse, in deren Rahmen die verwendeten statistischen Methoden erläutert werden. Im Anschluss an die Beschreibung der verwendeten Datensätze erfolgt die Auswertung im Rahmen der Überprüfung der aufgeworfenen Hypothesen. In diesem Kontext wird auch eine Paneldatenuntersuchung durchgeführt. Schließlich werden die Ergebnisse der empirischen Analyse zusammengefasst.

Kapitel 5 fasst die Arbeit zusammen, zieht Schlussfolgerungen für die Genossenschaftsbanken und bietet einen Ausblick. 
Der Gang der Untersuchung wird grafisch in Abbildung 1 dargestellt.

\section{Einleitung}

\section{Einordnung und Zielsetzung}

- 2.1 Stand der Forschung

- 2.2 Identifizierte Forschungslücken

- 2.3 Forschungsfragen dieser Untersuchung

- 2.4 Forschungsansatz

- 2.5 Gang der Untersuchung

\section{Theoretische Analyse}

- 3.1 Definitionen und konzeptioneller Bezugsrahmen

- 3.2 Theorie von Fusionen

- 3.3 Theorie der Kostenvorteile

- 3.4 Theorie der Erfolgsmessung

- 3.5 Theorie der Regionalität

- 3.6 Der Jahresabschluss

- 3.7 ROI-Analyse

- 3.8 Zu untersuchende Erfolgsfaktoren

- 3.9 Ableitung der Forschungshypothesen

\section{Empirische Analyse}

- 4.1 Methodik der Datenerhebung

- 4.2 Methodik der Datenanalyse

- 4.3 Datenbasis

- 4.4 Auswertung und empirische Analyse

- 4.5 Schlussfolgerungen aus der empirischen Analyse

\section{Schlussbetrachtung}

Abbildung 1: Gang der Untersuchung 
In diesem Kapitel der theoretischen Analyse werden die Grundlagen der späteren empirischen Untersuchung gelegt. Ein Teil der vorliegenden Arbeit befasst sich mit der Größe von Genossenschaftsbanken. Diese Größe wird fundamental von Fusionen beeinflusst, welche in Abschnitt 3.3 grundlegend thematisiert werden.

Der Genossenschaftssektor weist eine Struktur aus den einzelnen Kreditgenossenschaften, den genossenschaftlichen Zentralbanken und weiteren Spezialinstituten auf, wie z. B. die Investmentfondsgesellschaft Union Investment oder die Bausparkasse Schwäbisch Hall. Durch diese spezielle Struktur können innerhalb des Genossenschaftssektors Verbundeffekte in hohem Umfang realisiert werden. Die einzelnen Kreditgenossenschaften fungieren hierbei als Eigentümer oder Anteilseigner der Zentralbanken bzw. der Spezialinstitute. Die Übertragung von Aufgaben an jene ist eine probate und praktikable Art des Outsourcings. Die Ausgliederung von Teilbereichen in Tochtergesellschaften wird im Falle der Genossenschaftsbanken häufig im Immobilienbereich realisiert. Es existieren zahlreiche hundertprozentige Tochtergesellschaften von Kreditgenossenschaften, deren Aufgabe im Erwerb, der Verwaltung und der Vermietung von Immobilien besteht. Weitere Ansätze zum Outsourcing von Bankdienstleistungen liefert LINSEISEN bereits im Jahre 1994. ${ }^{119}$ Jedoch stellte sie fest, dass die Möglichkeiten zur Auslagerung von Aufgabenbereichen zum Zeitpunkt ihrer Veröffentlichung kaum genutzt wurden. ${ }^{120}$ Dies führt sie auf die schwere Trennbarkeit von Beschaffung, Produktion und Absatz bei Bankdienstleistungen zurück. ${ }^{121}$ Mittlerweile jedoch hat sich die Situation grundlegend verändert. KIPKER konstatierte im Jahr 2010, dass es einige Jahre zuvor noch undenkbar gewesen sei, über parzielles oder gar vollständiges Outsourcing zu diskutieren. ${ }^{122}$ Durch die Trennung in einzelne Funktionsbanken mit den Aufgaben des Vertriebs, der Steuerung und der Produktion können ausgewählte Leistungen schließlich heute ausgelagert werden. ${ }^{123}$ Eine detaillierte Untersuchung zum Umfang oder zu den Leistungen der Verbundeffekte ist im Rahmen der vorliegenden Arbeit jedoch nicht vorgesehen.

\subsection{Definitionen und konzeptioneller Bezugsrahmen}

\subsubsection{Banken}

Das Gesetz über das Kreditwesen definiert Kreditinstitute als „,...] Unternehmen, die Bankgeschäfte gewerbsmäßig oder in einem Umfang betreiben, der einen in kaufmännischer Weise eingerichteten Geschäftsbetrieb erfordert. “124 Die beiden zentralen und wichtigsten Bankgeschäfte, das Einlagengeschäft und das Kreditgeschäft, werden an gleicher Stelle folgend in Nr. 1 und Nr. 2 aufgeführt und definiert. ${ }^{125}$ Dabei versteht man unter dem Einlagengeschäft „die Annahme fremder Gelder als Einlagen oder anderer unbedingt rückzahlbarer Gelder des Publikums [...], ohne Rücksicht darauf, ob Zinsen vergütet werden. “126 Das Kreditgeschäft wird definiert als die „Gewährung von Gelddarlehen und

\footnotetext{
${ }^{119}$ Vgl. Linseisen (1995), S. 159 ff.

${ }^{120}$ Vgl. Linseisen (1995), S. 159 ff.

${ }^{121}$ Vgl. Linseisen (1995), S. 159 ff.

122 Vgl. Kipker (2010), S. 168.

${ }^{123}$ Vgl. Linseisen (1995), S. $159 \mathrm{ff}$

124 S 1 Abs. 1 KWG.

${ }^{125} \int 1$ Abs. 1 Nr. 1 und Nr. 2 KWG.

126 S 1 Abs. 1 Nr. 1 KWG.
} 
Akzeptkrediten“. ${ }^{127}$ Weitere Bankgeschäfte sind Pfandbriefgeschäft, Diskontgeschäft, Finanzkommissionsgeschäft, Depotgeschäft, Rückerwerb von Darlehensforderungen, Garantiegeschäft, Scheckeinzugsgeschäft, Wechseleinzugsgeschäft, Reisescheckgeschäft, Emissionsgeschäft und die Tätigkeit als zentrale Gegenpartei. ${ }^{128}$

Im deutschen Bankensystem existieren Universalbanken und Spezialbanken. ${ }^{129}$ Die Universalbanken werden dadurch charakterisiert, dass sie eine Vielzahl der in $\ 1$ Abs. $1 \mathrm{KWG}$ genannten Geschäfte durchführen, vor allem jedoch das Einlagen- und das Kreditgeschäft. ${ }^{130}$ Die Universalbanken gliedern sich in drei Säulen: Kreditbanken, Sparkassen und Genossenschaftsbanken. ${ }^{131}$ Eine grafische Darstellung befindet sich in Abbildung 2.

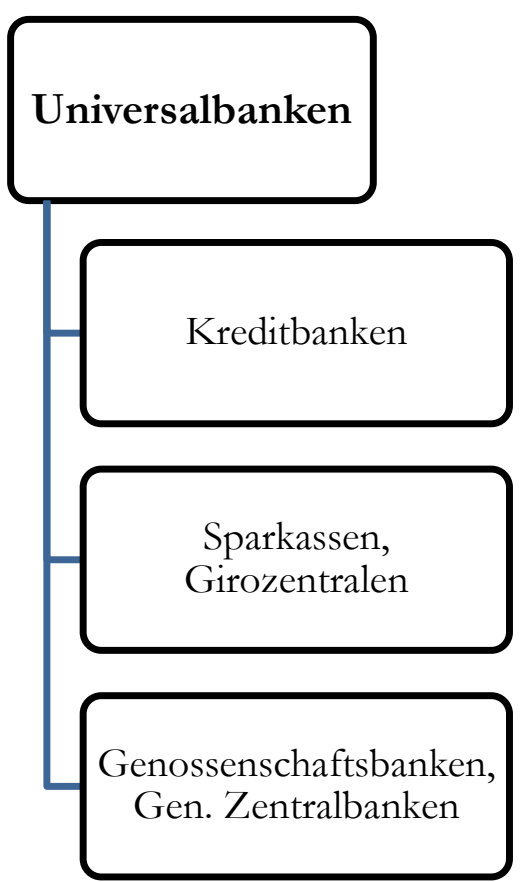

Abbildung 2: $\quad$ Universalbanken im deutschen Bankensystem ${ }^{132}$

Die Kreditbanken, bestehend aus Großbanken, Regionalbanken und sonstigen Kreditbanken sowie Zweigstellen ausländischer Banken, wiesen im Jahresdurchschnitt $2011^{133}$ eine aggregierte Bilanzsumme von 3.826 Mrd. Euro auf. ${ }^{134}$ Der Sparkassensektor besteht aus den Sparkasseninstituten und den Landesbanken, er kam im selben Zeitraum auf eine durchschnittliche Bilanzsumme von 2.583 Mrd. Euro. ${ }^{135}$ Der Genossenschaftssektor wies 987 Mrd. Euro aus. Er steht im speziellen Fokus der vorliegenden Arbeit.

Zu den Spezialbanken zählen Realkreditinstitute, Bausparkassen, Direktbanken, Kapitalanlagegesellschaften, Wertpapiersammelbanken und Kreditinstitute mit Sonderaufgaben. ${ }^{136}$

\footnotetext{
127 . 1 Abs. 1 Nr. 2 KWG.

$128 \int 1$ Abs. 1 KWG.

${ }^{129}$ Vgl. Hartmann-Wendels, Pfingsten und Weber (2010), S. 31; Becker und Peppmeier (2013), S. 83; Richard und Mühlmeyer (2011), S. 17; Grill und Perczynski (2011), S. 51 ff.; Tolkmitt (2007), S. 60 ff.

${ }^{130}$ Vgl. Hartmann-Wendels et al. (2010), S. 31.

131 Vgl. Hartmann-Wendels et al. (2010), S. 31; Becker und Peppmeier (2013), S. 83; Richard und Mühlmeyer (2011), S. 17; Grill und Perczynski (2011), S. 51 ff.; Tolkmitt (2007), S. 60 ff.

132 Eigene Darstellung, in Anlehnung an Hartmann-Wendels et al. (2010), S. 31.

${ }^{133}$ Das Jahr 2011 entspricht dem letzten Jahr des Betrachtungszeitraumes.

134 Vgl. Bundesbank (2014c), S. 86.

135 Vgl. Bundesbank (2014c), S. 86.

${ }^{136}$ Vgl. Hartmann-Wendels et al. (2010), S. 31.
} 
Realkreditinstitute und Banken mit Sonderaufgaben aggregierten im selben Zeitraum jahresdurchschnittlich 1.572 Mrd. Euro. ${ }^{137}$

Der Genossenschaftssektor besteht im Wesentlichen aus den Volksbanken und Raiffeisenbanken sowie den beiden Zentralinstituten DZ BANK und WGZ BANK, welche den Zahlungsverkehr zwischen den einzelnen Instituten abwickeln. ${ }^{138} \mathrm{Im}$ Rahmen der Fusion beider Zentralinstitute im Jahr 2016 geht die WGZ BANK in der DZ BANK auf. Zusätzlich gibt es im sogenannten genossenschaftlichen Verbund noch spezielle Verbundpartner. Diese übernehmen Aufgaben, welche nicht oder zumindest nicht effizient von den Instituten ausgeübt werden können. ${ }^{139}$ Dazu zählen etwa die Bausparkasse Schwäbisch Hall, die Investmentfondsgesellschaft Union Investment oder die genossenschaftlichen Rechenzentralen Fiducia IT AG und GAD eG.

Im Rahmen dieser Arbeit werden die Begriffe Genossenschaftsbank, Kreditgenossenschaft, Kreditinstitut, Institut und Bank synonym verwendet. Werden andere Institute beschrieben, welche keine Genossenschaftsbanken sind, so wird dies gesondert kenntlich gemacht.

\subsubsection{Die Geschichte der Genossenschaftsbanken}

Die Gründung der ersten Genossenschaftsbanken geht zurück auf die Mitte des 19. Jahrhunderts. ${ }^{140}$ Durch die Industrialisierung wurde die Finanzierung kleiner Landwirtschaftsund Gewerbebetriebe zunehmend schwieriger. ${ }^{141}$ HERMAnN SCHUlzE-DELITZSCH gründete im Jahre 1850 in Delitzsch einen Vorschussverein. Dieser verfolgte teils gewerbliche, teils aber auch wohltätige Zwecke. Es handelte sich hierbei um die erste gewerbliche Krediteinrichtung und stellte den Vorläufer der späteren Kreditgenossenschaften dar. Hieraus entstanden die Volksbanken, welche überwiegend über gewerbliche Kunden in Städten verfügten. ${ }^{142}$ HermanN SCHUlze-DelitzsCH gilt als der Gründungsvater der deutschen Volksbanken. ${ }^{143}$ Er wird in Abbildung 3 dargestellt.

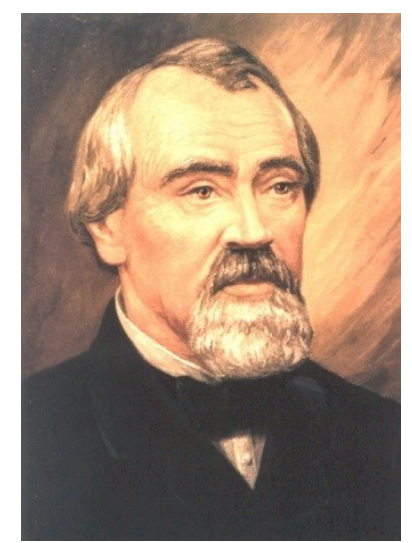

Abbildung 3: Hermann Schulze-Delitzsch ${ }^{144}$

\footnotetext{
137 Vgl. Bundesbank (2014c), S. 86.

138 Vgl. Hartmann-Wendels, Pfingsten und Weber (2015), S. 36.

${ }^{139}$ Vgl. Schax (2008), S. 20.

140 Vgl. Hartmann-Wendels et al. (2010), S. 39.

141 Vgl. Staats (2006), S. 40.

142 Vgl. Staats (2006), S. 40.

143 Stiftung GIZ (2015).

${ }^{144}$ Stiftung GIZ (2015).
} 
FRIEDRICH WILHELM RAIFFEISEN gründete im Jahre 1854 eine teils karitative Einrichtung mit dem Namen „Heddesdorfer-Wohlthätigkeits-Verein““ ${ }^{145}$ Aus dieser entstand 1864 der „Heddesdorfer Darlehnskassen-Verein“, während er bereits 1862 die erste moderne ländliche Genossenschaftsbank mit der Bezeichnung „Darlehenskassenverein Anhausen“ gründete. Zahlreiche weitere Gründungen folgten. ${ }^{146}$ Hieraus entwickelten sich die Raiffeisenbanken, welche vor allem landwirtschaftliche Kunden im ländlichen Raum hatten. ${ }^{147}$ FRIEDRICH WILHELM RAIFFEISEN gilt als der Gründungsvater der deutschen Genossenschaften und insbesondere der Raiffeisenbanken. ${ }^{148}$ Abbildung 4 stellt ein Bild von RAIFFEISEN dar.

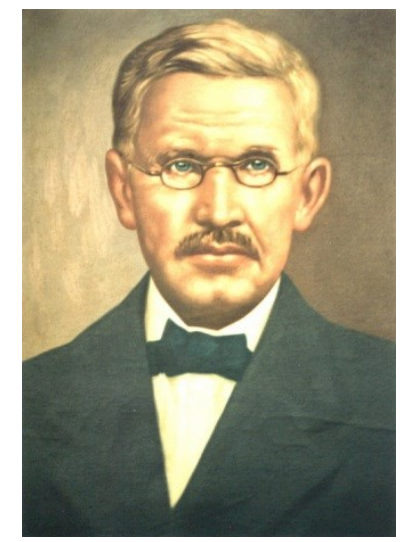

Abbildung 4: $\quad$ Friedrich Wilhelm Raiffeisen ${ }^{149}$

Erst durch die Gründung des gemeinsamen Bundesverbandes der Deutschen Volksbanken und Raiffeisenbanken (BVR) im Jahre 1972 kam es zu einer Annäherung, welche den Beginn der Abschwächung der bis dahin üblichen Differenzierung bedeutete. ${ }^{150}$ Bis dahin blieben beide Banktypen stark voneinander getrennt. ${ }^{151}$ Es existierte eine konsequente Separation zwischen der landwirtschaftlichen Sphäre (Volksbanken) und der kreditwirtschaftlichen Sphäre (Raiffeisenbanken).

Im Jahr 2003 untersuchte KLEIN die Möglichkeiten, Vorteile und Umsetzbarkeit einer Idee, nach der sich gar deutsche Sparkassen zukünftig erfolgreich als eingetragene Genossenschaften organisieren könnten. Dabei lehnt er sich an das französische Vorbild an. ${ }^{152}$

\subsubsection{Der Zweck von Genossenschaftsbanken}

Die Genossenschaft wird im Genossenschaftsgesetz geregelt und definiert die Genossenschaft in $₫ 1$ Abs. 1 allgemein als eine Gesellschaft, ,[...] deren Zweck darauf gerichtet ist, den Erwerb oder die Wirtschaft ihrer Mitglieder oder deren soziale oder kulturelle Belange durch gemeinschaftlichen Geschäftsbetrieb zu fördern [... “ “. ${ }^{153}$ Diese Definition findet Anwendung auf alle Genossenschaften, ganz gleich welcher Art sie sind und welchem

\footnotetext{
145 Vgl. Grüger (2007), S. 1.

146 Vgl. Grüger (2007), S. 1.

${ }^{147}$ Vgl. Staats (2006), S. 40.

148 BVR (2015b).

${ }^{149}$ BVR (2015b).

${ }^{150} \mathrm{Vgl}$. Staats (2006), S. 41.

${ }^{151}$ Vgl. Staats (2006), S. 40.

${ }^{152}$ Vgl. Klein (2003), S. 320-444, insbes. 385 ff.

153 s 1 Abs. 1 GenG.
} 
Zweck sie dienen. Diese allgemeinen Regelungen über Genossenschaften gelten daher auch speziell für Genossenschaftsbanken. Folglich stellt die Förderung ein zentrales Element innerhalb der Aufgaben einer jeden Genossenschaftsbank dar. Zunächst kommen als zu fördernde Elemente in Frage:

- Der Erwerb der Genossenschaftsmitglieder

- Die Wirtschaft der Genossenschaftsmitglieder

- Soziale Belange der Genossenschaftsmitglieder

- Kulturelle Belange der Genossenschaftsmitglieder

Die Ausdehnung des Förderzweckes auf soziale und kulturelle Belange erfolgte erst im Jahre 2006 im Rahmen der Novellierung des Genossenschaftsgesetzes. ${ }^{154}$ Daher stehen der Erwerb und die Wirtschaft der Genossenschaftsmitglieder als Förderelemente zunächst im Zentrum der Betrachtung. ${ }^{155}$ BEUTHIEN unternimmt einen Versuch der definitorischen Trennung beider Begriffe: unter der Förderung des Erwerbs versteht er die Unterstützung der gewerblichen, freiberuflichen oder sonstigen Erwerbstätigkeit. ${ }^{156} \mathrm{Zu}$ Förderung zählt er die Vermehrung von Einnahmen, Verminderung von Ausgaben und andere, nicht direkt monetär messbare Vorteile, wie die Verschaffung von Kredit oder eine Werbetätigkeit zugunsten der Mitglieder. ${ }^{157}$ Hingegen versteht er unter der Förderung der Wirtschaft nicht nur die Förderung der Hauswirtschaft im engeren Sinne, sondern die gesamte private Lebenswirtschaft, die der materiellen und ideellen Daseinsvorsorge dient. ${ }^{158}$ Auch enthalten sind hier die im Genossenschaftsgesetz separat genannten sozialen und kulturellen Förderbereiche. ${ }^{159}$

Der Formulierung „durch gemeinschaftlichen Geschäftsbetrieb“ kommt ebenfalls wesentliche Bedeutung zu. Zu fördern sind die Mitglieder, die gleichzeitig Kunden des von ihnen betriebenen Unternehmens sind. Diese förderwirtschaftliche Einheit von Mitgliedern und Kunden wird Identitätsprinzip genannt. ${ }^{160}$ Zur Förderung bietet die Genossenschaft ihren Genossen sogenannte naturale Förderleistungen an. Zu diesen zählen Waren, Werkleistungen und Dienstleistungen, z. B. durch Einkaufsverbände zur Bündelung der Marktmacht der Genossen bei der Beschaffung von Saatgut. ${ }^{161}$

Das Konzept von Genossenschaften unterscheidet sich grundsätzlich von Kapitalgesellschaften, die darauf angelegt sind, einen möglichst hohen Gewinn zu erwirtschaften. Für Kapitalgesellschaften wird dies z. B. durch das Konzept des Shareholder Value erfasst. ${ }^{162}$ Im Unterschied dazu sind die Mitglieder einer Genossenschaft an einem möglichst hohen Nutzen ihrer Mitgliedschaft interessiert. Der Begriff des Nutzens wird in diesem Zusammenhang in einem erweiterten Sinne verstanden: einerseits erhalten die Genossen eine Rückvergütung des Gewinns, indem ihre Geschäftsanteile verzinst werden. Andererseits bekommen sie auch günstige Konditionen bei Geschäften mit der Genossenschaft gewährt. Genauso wie Kapitalgesellschaften dürfen auch Genossenschaften den nicht thesau-

\footnotetext{
154 Vgl. \ 1 Abs. 1 GenG i. d. F. der Bekanntmachung vom 16.10.2006; BGBl. Teil I, Nr. 47, S. 2232

155 Vgl. Fechtrup (1994), S. 67.

156 Vgl. Beuthien (2013), S. 41.

157 Vgl. Beuthien (2013), S. 41

158 Vgl. Beuthien (2013), S. 42.

${ }^{159}$ Vgl. Beuthien (2013), S. 42.

160 Vgl. Beuthien (2013), S. 9.

161 Vgl. Beuthien (2013), S. 9.

162 Vgl. Rappaport (1998), S. 3
} 
rierten Teil des Gewinns an die Mitglieder ausschütten. Denkbar sind hier einerseits die Zuschreibung zum Anteil des Genossen oder andererseits die direkte Ausschüttung an diesen in bar in Form einer Dividende. Kritikwürdig im Falle der Barausschüttung ist die Tatsache, dass auch jene Genossen profitieren, welche keine Abschlüsse mit dem von ihnen betriebenen Unternehmen getätigt haben. BEUTHIEN argumentiert daher, dass es „genossenschaftlicher“" sei - also eher dem genossenschaftlichen Grundgedanken zuträglich -, wenn die Förderung der Mitglieder durch die Geschäftskonditionen erfolgt, und nicht als Dividende auf Geschäftsguthaben. ${ }^{163}$ Hierbei ist in Kauf zu nehmen, dass sich die Förderung auch auf Nichtmitglieder erstreckt.

Historisch bestand eine Konzentration der Geschäftsbeziehung auf die Mitglieder. ${ }^{164}$ Jedoch sind mittlerweile auch Geschäfte mit Nichtmitgliedern möglich. Laut HARTMANNWENDELS ET AL. steht der historisch gelebte Fördergedanke bei zahlreichen Genossenschaftsbanken heute nicht mehr im Vordergrund. ${ }^{165}$ So ist etwa die Kreditvergabe an Nichtmitglieder seit 1974 möglich. Jedoch wurden viel früher bereits de facto Einlagen von Nichtmitgliedern entgegengenommen. ${ }^{166}$ Geschäfte mit Nichtmitgliedern insgesamt haben mittlerweile eine große Bedeutung erlangt. ${ }^{167}$ Das Genossenschaftsgesetz regelt, dass in die Satzung einer Genossenschaft hierzu eine entsprechende Bestimmung aufzunehmen ist, nach der „die Ausdehnung des Geschäftsbetriebes auf Personen, welche nicht Mitglieder der Genossenschaft sind, zugelassen wird““ ${ }^{168}$ PEEMÖLlER fordert die Gewährung eines sogenannten Förderplus', welches den Mitgliedern einen Vorteil gegenüber Nichtmitgliedern bietet, die er selbst auch als Nur-Kunden bezeichnet. ${ }^{169}$ DuLLENKOPF empfiehlt die Ausdehnung des Nicht-Kundengeschäfts. ${ }^{170}$ BEUTHIEN sieht im Genossenschaftsrecht eine Regelung, nach der das Nichtmitgliedergeschäft jedoch konkret nur ein Nebenzweck bleiben muss. ${ }^{171}$ Ein beliebiger Ausbau dieses Nichtmitgliedergeschäfts ist folglich nicht vorgesehen. Durch den Erwerb von Geschäftsanteilen können jedoch Nichtmitglieder zu Mitgliedern werden. Auf diese Weise - also durch den Erwerb von Genossenschaftsanteilen besteht dann laut BEUTHIEN schlussendlich doch eine Wachstumsmöglichkeit für das Geschäft im Bereich von Nichtmitgliedern.

Die Kreditgenossenschaften wurden aus dem gewerblich-handwerklichen und dem landwirtschaftlichen Mittelstand heraus gegründet. ${ }^{172}$ Es handelt sich um freiwillige Zusammenschlüsse mit gleichberechtigten Teilhabern und folgenden Prinzipien. ${ }^{173}$

1. Selbsthilfe: Gefördert werden soll die Wirtschaft der Mitglieder. ${ }^{174}$

2. Selbstverantwortung: Das Risiko aus seinem Handel trägt jedes Mitglied selbst. ${ }^{175}$

3. Selbstverwaltung: Die Genossenschaftsbank verwaltet sich selbst. ${ }^{176}$

\footnotetext{
163 Vgl. Beuthien (2013), S. 10.

164 Vgl. Grüger (2007), S. 3.

165 Vgl. Hartmann-Wendels et al. (2010), S. 39.

${ }^{166}$ Vgl. Hartmann-Wendels et al. (2010), S. 39.

167 Vgl. Bundesbank (2014b).

168 \& 8 Abs. 1 Nr. 5 GenG.

169 Vgl. Peemöller (2005), S. 408.

170 Vgl. Dullenkopf (2001), S. 247

171 Vgl. Beuthien (2013), S. 10.

172 Vgl. Christians (2010), S. 27.

${ }^{173}$ Vgl. Christians (2010), S. 27 f.

${ }^{174}$ Vgl. Staats (2006), S. 40; vgl. Mathweis (1996), S. 23.

175 Vgl. Staats (2006), S. 40; vgl. Mathweis (1996), S. 24.

176 Vgl. Staats (2006), S. 40; vgl. Mathweis (1996), S. 23 f.
} 
Unter dem Selbsthilfegrundsatz ist zu verstehen, dass die Genossen die beabsichtigten Fördereffekte selbst realisieren, indem sie Geschäfte mit ihrer Genossenschaft abschlieBen. ${ }^{177}$ Hier spiegelt sich das oben genannte Identitätsprinæip wider. Unter der Selbstverantwortung versteht sich die in den Anfängen existierende persönliche Haftung der Genossen für die Verbindlichkeiten der Genossenschaft. ${ }^{178}$ Durch das Genossenschaftsgesetz von 1889 konnte die persönliche Haftung zunächst abgeschwächt werden, während sie seit einer Novelle des Genossenschaftsgesetzes im Jahre 1974 vollständig aufgehoben werden kann. ${ }^{179}$ De facto besteht jedoch häufig eine Haftung in der Höhe des Geschäftsanteils. Unter der Selbstverwaltung ist zu verstehen, dass die Genossen die Geschicke ihrer Genossenschaft selbst regeln. ${ }^{180}$ Dazu setzen sie die vorhandenen gesellschaftsrechtlichen Verwaltungs- und Kontrollrechte ein. ${ }^{181}$ Hierbei sind alle Genossen gleichberechtigt. ${ }^{182}$ Diese Gleichberechtigung äußert sich darin, dass jeder Genosse nur eine Stimme hat, unabhängig von der Höhe seines Geschäftsanteils. ${ }^{183}$ Dieses sogenannte Kopfstimmrecht war bereits im ersten Genossenschaftsgesetz von 1867 enthalten. ${ }^{184}$ Die Abstimmung nach Köpfen unterscheidet sich grundlegend von Kapitalgesellschaften, bei denen sich im Allgemeinen die Anzahl der Stimmrechte aus der Höhe der gehaltenen Anteile ergibt. ${ }^{185}$

Übertragen auf die heutigen Kreditgenossenschaften zählen zu den angebotenen Leistungen das Girokonto zur Teilnahme am bargeldlosen Zahlungsverkehr, die Bereitstellung von Darlehen zur Finanzierung der Geschäftstätigkeit, aber auch darüber hinausgehende Maßnahmen, die der Förderung des Erwerbs bzw. der Wirtschaft der Mitglieder zweckdienlich sind. Dazu zählen Veranstaltungen, Seminare und auch die dadurch gebotenen Möglichkeiten zur Vernetzung und zum Erfahrungsaustausch der Mitglieder.

\subsubsection{Erfolg}

Im folgenden Abschnitt soll erörtert werden, was unter Erfolg allgemein und hiernach, etwas spezieller, unter dem Erfolg eines Unternehmens zu verstehen ist. Schließlich wird der Erfolg der im Fokus der Betrachtung stehenden Genossenschaftsbanken abgegrenzt.

\subsubsection{Allgemeine Erfolgsdefinition}

Naturgemäß existieren zahlreiche unterschiedliche Definitionen des Begriffs Erfolg. Es gibt dazu zahlreiche verschiedene und voneinander abweichende Sichtweisen und Definitionen. JACOBSEN nennt zunächst einige Betrachtungsdimensionen für den Erfolg, wozu die folgenden zählen: ${ }^{186}$

1. Subjektivität vs. Objektivität

2. Bimodal, multimodal oder kontinuierlich

3. Finanziell oder nicht-finanziell

4. Abstraktheit ${ }^{187}$

${ }^{177}$ Vgl. Mathweis (1996), S. 23.

178 Vgl. Mathweis (1996), S. 24.

${ }^{179}$ Vgl. Mathweis (1996), S. 24.

${ }^{180}$ Vgl. Mathweis (1996), S. 23.

181 Vgl. Mathweis (1996), S. 23 f.

${ }^{182}$ Vgl. Mathweis (1996), S. 24.

${ }^{183}$ Vgl. Staats (2006), S. 40.

$184 \mathrm{Vgl}$. Staats (2006), S. 40.

185 Vgl. Mathweis (1996), S. 24; mit Ausnahme von Mehrfachstimmrechten.

186 Vgl. Jacobsen (2003), S. 40.

187 Vgl. Jacobsen (2003), S. 40. 
Erfolg kann subjektiv sein, das heißt, eine Person schätzt die Höhe des Erfolgs oder allein die Tatsache, ob ein Erfolg vorliegt, anders ein als eine weitere Person. In Bezug auf Genossenschaftsbanken kann etwa der Förderwirtschaftserfolg ${ }^{188}$ durch verschiedene Akteure unterschiedlich beurteilt werden. Objektiv hingegen ist ein Erfolg, wenn er anhand objektiv überprüfbarer und vergleichbarer Kriterien ermittelt wurde. Die zur Messung des Erfolgs in Frage kommenden Größen werden in Abschnitt 3.5.3 diskutiert. Eine vollständige Objektivität kann jedoch auch hier nicht garantiert werden - schließlich existieren im Rahmen der unten beschriebenen Jahresabschlusspolitik zahlreiche Wahlmöglichkeiten und Spielräume. ${ }^{189}$

Bimodal unterscheidet lediglich in Erfolg und Misserfolg, während eine multimodale Sichtweise hier mehrere Abstufungen zulässt, etwa sehr hoher, hoher, durchschnittlicher, geringer und sehr geringer Erfolg. Eine quasi kontinuierliche Erfolgsmessung erlauben hingegen beispielsweise die in Euro und Cent angegebenen Maße wie der Bruttogewinn aus normaler Geschäftstätigkeit, der Jahresüberschuss oder eine Kennzahl wie die Cost-Income-Ratio.

Häufig wird der Erfolg in finanziellen Größen gemessen. ${ }^{190}$ Von den üblichen ErfolgsmaBen abweichend, ist auch eine abstrakte Erfolgsbeurteilung denkbar, beispielsweise über die Erreichung bzw. Nichterreichung von Erwartungen. ${ }^{191}$

Insgesamt kann Erfolg als der Grad der Zielerreichung definiert werden. Jedoch unterscheiden sich die Ziele je nach Perspektive beträchtlich. ${ }^{192}$

\subsubsection{Erfolg von Unternehmen}

Auch in Bezug auf ein Unternehmen kann der Erfolg je nach Betrachtung unterschiedlich beurteilt werden. Einerseits existieren verschiedene Erfolgsdimensionen, wie das reine Überleben des Unternehmens, Umsatz, Gewinn, Mitarbeiter, Zweckerfüllung oder Reputation. ${ }^{193}$ Als weitere mögliche Erfolgsmaße kommen z. B. Umsatzwachstum, Gewinnwachstum, Mitarbeiteranzahl, Mitarbeiterwachstum, Profitabilität, Eigenkapitalrendite, Umsatzrendite, Marktanteile oder Zahl der Patente in Frage. ${ }^{194}$ Zusätzlich sind Umsatzstruktur, Kostenstruktur und Dividenden zu nennen. ${ }^{195}$ In der klassischen betriebswirtschaftlichen Theorie erfolgt die Erfolgsmessung häufig anhand des Gewinns, also der Differenz zwischen dem erzielten finanziellen Ertrag und dem dafür erforderlichen finanziellen Aufwand. ${ }^{196}$ Andererseits kann der Erfolg aus verschiedenen Perspektiven beurteilt werden. Aus gesamtwirtschaftlicher Perspektive etwa ist es wünschenswert, dass ein Unternehmen „günstige“ Folgen für die Gesellschaft herbeiführt. ${ }^{197}$ Allgemein ist hierunter die Versorgung der Gesellschaft mit Gütern und Dienstleistungen zur Befriedigung vorhandener und erkennbarer Bedürfnisse zu verstehen. ${ }^{198}$ Die Unternehmen sollen zur Erhaltung und zur

\footnotetext{
${ }^{188} \mathrm{Vgl}$. Abschnitt 3.1.2.4

${ }^{189} \mathrm{Vgl}$. Abschnitt 3.6.5

${ }^{190}$ Vgl. Jacobsen (2003), S. 40; jedoch kommen auch nicht-finanzielle Maße wie etwa die Kundenzufriedenheit, Betriebsklima oder, speziell bei Genossenschaftsbanken, die Zufriedenheit der Mitglieder zum Einsatz.

191 Vgl. Jacobsen (2003), S. 40

192 Vgl. Jacobsen (2003), S. 41.

193 Vgl. Jacobsen (2003), S. 41

194 Vgl. Jacobsen (2003), S. 41.

195 Vgl. Schierenbeck (2003a), S. 63.

196 Vgl. Jacobsen (2003), S. 41.

197 Vgl. Jacobsen (2003), S. 41; ein Unternehmen kann jedoch nur im Rahmen einer Wirtschafts- und Rechtsordnung seiner Tätigkeit nachgehen. Zur Sicherung seiner fortwährenden Existenz darf es zumindest über lange Frist hinweg keinen Verlust machen.

${ }^{198}$ Vgl. Perridon, Steiner und Rathgeber (2012), S. 3.
} 
Erhöhung des Wohlstandes der Volkswirtschaft beitragen. ${ }^{199} \mathrm{Zu}$ den Aufgaben zählen spezieller die Schaffung von Arbeits- und Ausbildungsplätzen, das Angebot sinnvoller Produkte und Dienstleitungen, Innovationsbeiträge, ein möglichst hoher Beitrag zum Bruttoinlandsprodukt und nicht zuletzt die Produktion eines möglichst hohen Steueraufkommens. ${ }^{200}$

\subsubsection{Erfolg von Genossenschaftsbanken}

Generell existiert eine Vielzahl von Maßen für den Erfolg von Unternehmen. In der Regel sind die Unternehmenseigentümer primär an der Gewinnerzielung interessiert. Auf Genossenschaftsbanken kann man dieses Argument nicht ohne weiteres übertragen. Denn Genossenschaften müssen ihrem Förderauftrag nachkommen. ${ }^{201}$ Wendet man die Erfolgsperspektiven von Unternehmen auf Genossenschaftsbanken an, so ist zunächst gesamtwirtschaftlich das Angebot von Bankdienstleistungen zu nennen, um einen Betrag zur Förderung der regionalen Wirtschaft zu leisten, um Arbeits- und Ausbildungsplätze zu schaffen und ein möglichst hohes Steueraufkommen zu produzieren. Jedoch kommt im Rahmen der Erfolgsbetrachtung von Genossenschaftsbanken noch die zusätzliche Perspektive der Mitglieder hinzu. Aus deren Perspektive gehört zu den zentralen Zielen einer Genossenschaftsbank die Förderung derselben, präziser formuliert die Förderung des Erwerbs, der Wirtschaft, der sozialen und kultureller Belange dieser Mitglieder. ${ }^{202}$ Speziell bei Genossenschaftsbanken ist daher von einem Erfolg auszugehen, wenn die Ziele erreicht werden.

Es ist also denkbar, den Grad der Erfolgserzielung an dem Umfang festzumachen, in welchem eine Genossenschaftsbank die Wirtschaft bzw. den Erwerb ihrer Mitglieder gefördert hat. Hiergegen wendet BEUTHIEN ein, dass die Genossenschaft selbst schwer beurteilen könne, welchen Nutzen ihre Mitglieder ihrerseits aus den angebotenen Leistungen ziehen. ${ }^{203}$ Dies führt er zurück auf den fehlenden Einblick in die jeweiligen Einzelwirtschaften der Mitglieder. ${ }^{204}$ Aber selbst wenn derartige Informationen vorlägen, ist davon auszugehen, dass diese weder vollständig von allen Mitgliedern vorhanden sind, noch dass sie untereinander vergleichbar sind. ${ }^{205}$ Spätestens an dieser Stelle ist eine Zusammenfassung und Auswertung durch die Genossenschaft erheblich erschwert und daher nicht realistisch. ${ }^{206}$ SASSEN hält selbst eine verallgemeinerte Bestimmung des Förderzweckes für prinzipiell unmöglich, da dieser Förderzweck in erster Linie vom subjektiven Empfinden jedes einzelnen Mitglieds abhängt. ${ }^{207}$

BEUTHIEN argumentiert weiter, dass auch keine zwingende Notwendigkeit für eine Gewinnmaximierung in der Förderwirtschaft besteht. Die Genossenschaft muss die Förderung lediglich bezwecken und auf ihre erfolgreiche Umsetzung hinwirken. Einen in Euro messbaren Fördererfolg jedoch schuldet sie nicht. ${ }^{208}$ BEUTHIEN und HANRATH präzisieren,

\footnotetext{
${ }^{199}$ Vgl. Perridon, Steiner und Rathgeber (2012), S. 3.

${ }^{200}$ Vgl. Jacobsen (2003), S. 41.

201 Vgl. Abschnitt 3.1.1; gleichwohl wie Unternehmen generell müssen auch Genossenschaftsbanken langfristig einen Gewinn erzielen, um ihren eigenen Fortbestand zu sichern.

$202 \mathrm{Vgl}$. Abschnitt 3.1.1.

203 Vgl. Beuthien (2013), S. 44

204 Vgl. Beuthien (2013), S. 44.

${ }^{205}$ Vgl. Beuthien (2013), S. 44 f.

${ }^{206}$ Vgl. Beuthien (2013), S. 44 f.

${ }^{207}$ Vgl. Sassen (2012), S. 38.

${ }^{208}$ Vgl. Beuthien (2013), S. 45.
} 
dass bereits das Anbieten ihrer Produkte durch die Genossenschaft an ihre Mitglieder als Förderleistung anzusehen sei. Hieraus leiten sie ab, dass höhere Einnahmen oder Ersparnisse, welche die Mitglieder dank der Angebote der Genossenschaft erzielen können, nicht im Zentrum der Förderung von deren Erwerb oder Wirtschaft nach $\int 1$ Abs. 1 GenG stehen. Die Förderung der Mitglieder sei vielmehr bereits darin zu sehen, dass die Genossenschaft überhaupt Leistungen an die Mitglieder bereitstellt, beispielweise über die Vergabe von Krediten. ${ }^{209}$ Eine erfolgreiche Förderung zeichnet sich dadurch aus, dass die Mitglieder die Förderung als solche ansehen und sie bei der Genossenschaft nachfragen. ${ }^{210}$ Von BEUTHIEN wird dies als Mitgliederakzeptanz bezeichnet. ${ }^{211}$

Eine weitere mögliche Sichtweise zur Beurteilung des Erfolgs in der Förderwirtschaft leitet sich ab aus der Betrachtung der Entwicklung der Mitgliederzahlen. Denn denjenigen Mitgliedern, denen der Nutzen aus der Mitgliedschaft nicht ausreichend erscheint, steht die Möglichkeit offen, aus der Genossenschaft auszutreten. Dies schlägt sich ultimativ in einem Rückgang bzw. geringerem Nettowachstum der Mitgliederzahl nieder.

\subsubsection{Förderwirtschaftserfolg vs. Geschäftserfolg}

Selbst eine Genossenschaftsbank, welche in einem Geschäftsjahr überhaupt keinen Jahresüberschuss ausweist, kann trotzdem ihren Förderzweck erstklassig erfüllt haben - indem sie etwa Konditionen für Geschäfte mit ihren Mitgliedern eingeräumt hat, die für eben diese Mitglieder vorteilhaft sind. Die Bank wäre beispielsweise in der Lage gewesen, eine höhere Marge in einem Geschäft zu erzielen. Hierauf verzichtet sie jedoch zugunsten ihrer Mitglieder. Gemäß dieser Argumentation ist die Erzielung eines positiven Jahresüberschusses zunächst per se nicht notwendig. Jedoch ist dieser Jahresüberschuss in der langen Frist Ausdruck eines nachhaltigen Wirtschaftens, welches auch die Bildung von Rücklagen ermöglicht. Diese Rücklagen wiederum bieten die Möglichkeit zur Kompensation eines eventuellen negativen Ergebnisses und bilden die Grundlage für das Fortbestehen der Genossenschaftsbank $^{212}$, und somit auch für vorteilhafte Konditionen für die Mitglieder in Zukunft. Deshalb wird in der vorliegenden Arbeit speziell der Geschäftserfolg von Genossenschaftsbanken untersucht. Der Fördererfolg wird nicht näher betrachtet.

\subsubsection{Erfolgsfaktoren und Erfolgsfaktorenforschung}

Unter den Erfolgsfaktoren versteht man allgemein alle diejenigen Faktoren, die einen nennenswerten Einfluss auf den Erfolg einer Unternehmung haben ${ }^{213}$, die „langfristig wirksam sind und einen Wettbewerbsvorteil gegenüber den Konkurrenten begründen. “214 Dazu zählen sowohl unternehmensinterne als auch -externe Faktoren. Während bei den internen Faktoren eine Beeinflussungs- und Gestaltungsmöglichkeit angenommen wird, werden externe Faktoren weitestgehend als gegeben und nicht beeinflussbar betrachtet. ${ }^{215}$ Die verschiedenen in Betracht kommenden Messgrößen für den Erfolg werden unten in Abschnitt 3.5.3 näher erläutert.

\footnotetext{
${ }^{209}$ Vgl. Beuthien und Hanrath (2008), S. 90.

${ }^{210} \mathrm{Vgl}$. Beuthien und Hanrath (2008), S. 90.

211 Vgl. Beuthien und Hanrath (2008), S. 90.

${ }^{212}$ Ein fortgesetzter Anfall negativer Jahresergebnisse führt zur Aufzehrung des Eigenkapitals und gefährdet den Fortbestand der Bank.

${ }^{213}$ Vgl. Klemm-Bax (2000), S. 9.

214 Vgl. Matz (2007), S. 15.

215 Vgl. Matz (2007), S. 15
} 


\subsubsection{Historie der Erfolgsfaktorenforschung}

Die Erforschung von Erfolgsfaktoren begann bereits im Jahr $1960 .{ }^{216}$ Die Firma General Electric suchte einen systematischen Ansatz zur Eruierung, welche Faktoren einen positiven Einfluss auf den Erfolg ihres Unternehmens haben. ${ }^{217}$ Hiermit wurde die Beratungsfirma Schoeffler and Associates beauftragt. Das aufgesetzte Projekt nannte sich Profit Impact of Market Strategies (PIMS) und führte zu einem großen Interesse in der Geschäftswelt und auch in der akademischen Forschung. Ab 1972 wurde das Projekt durch die Harvard Business School unter Einbeziehung einer höheren Anzahl von Unternehmen weitergeführt ${ }^{218}$. Ab 1975 übernahm schließlich das gemeinnützige Strategic Planning Institute (SPI) die Fortführung des Projektes. ${ }^{219}$ Jedoch wurden im direkten Nachgang der Untersuchungen bei General Electric zunächst - zumindest bis 1978 - nur wenige Daten und Ergebnisse für die wissenschaftliche Forschung veröffentlicht. Das ist darauf zurückzuführen, dass es sich im Wesentlichen um vertrauliche Informationen handelte. ${ }^{220}$ Im Jahre 1977 enthielt die Datenbasis dann bereits Informationen über mehr als 1.000 sogenannte Strategische Geschäftseinheiten (Strategic Business Units, SBUs). Unter einer Strategischen Geschäftseinheit versteht man hierbei eine operative Einheit, die eine abgegrenzte Produktmenge an eine Kundengruppe verkauft und dabei eine definierte Menge an Wettbewerbern hat. ${ }^{221}$ Diese Abgrenzung war notwendig geworden, um die vielfältigen Geschäftsfelder von Großkonzernen zu untersuchen. Ab 1978 wurde mit PIMS Associates ein Beratungsinstitut für Mitgliedsunternehmen aufgebaut. Die erste Niederlassung in Europa wurde 1982 in London gegründet, weitere folgten in Köln und Wien. ${ }^{22}$ In ihrer Studie von 1987 fanden BUZZELL und GALE heraus, dass auch Serviceaspekte bedeutend für den Erfolg des Unternehmens seien. ${ }^{223}$ Eine hohe Leistungsqualität resultiere in stärkerer Kundentreue, häufigeren Wiederholungskäufen, weniger Preiskämpfen bzw. stärkerer Durchsetzbarkeit höherer Preise, niedrigeren Marketingaufwendungen und einer Steigerung des Marktanteils. ${ }^{224}$

\subsubsection{Ergebnisse der Erfolgsfaktorenforschung}

In diesem Abschnitt sollen die wichtigsten Ergebnisse der Erfolgsfaktorenforschung dargestellt und ausgeführt werden. Als bedeutendste Erfolgsfaktoren wurden Marktanteil, Produktqualität, Kundenzufriedenheit, Marktwachstum, Innovationsrate, Produktivität, Investitionstätigkeit und vertikale Integration identifiziert. ${ }^{225}$ Die Marktposition, genauer gesagt der relative Marktanteil, hat einen positiven Einfluss auf den Erfolg. ${ }^{226}$ Je höher der relative Marktanteil ist, desto höher ist der zu erwartende Erfolg. Teilen sich etwa in einem speziellen Fall nur wenige Akteure einen Markt, so entfällt auf den einzelnen Akteur ein höherer Marktanteil, welcher wiederum einen höheren Erfolg erhoffen lässt. ${ }^{227}$ Des Weiteren spiegelt sich die relative Produktqualität im Erfolg nieder. Darunter wird die Produktqualität im

\footnotetext{
${ }^{216}$ Vgl. Anderson und Paine (1978), S. 602.

${ }^{217}$ Vgl. Matz (2007), S. 17; vgl. Weis (2012), S. 140.

${ }^{218}$ Vgl. Weis (2012), S. 140.

${ }^{219}$ Vgl. Buzzell (2004), S. 602; vgl. Buzzell und Gale (1987), S. V.

${ }^{220}$ Vgl. Anderson und Paine (1978), S. 602.

221 Vgl. Anderson und Paine (1978), S. 602 f.

${ }^{222}$ Vgl. Buzzell und Gale (1987), S. V.

223 Vgl. Klee (2002), S. 79.

224 Vgl. Klee (2002), S. 79.

${ }^{225}$ Vgl. Matz (2007), S. 17; vgl. Bergmann (2007), S. 5.

${ }^{226}$ Vgl. Weis (2012), S. 140.

${ }^{227}$ Vgl. Abschnitt 4.4.2.
} 
Vergleich mit den Wettbewerbern verstanden. ${ }^{228}$ Mit einem umso höheren Erfolg kann gerechnet werden, je besser die Qualität der Produkte ist. Auch die Kundenzufriedenheit wird als eine Grundlage für den langfristigen Geschäftserfolg einer Unternehmung angesehen. Sie fungiert auch als ein Indikator für den Grad der Kundenorientierung. ${ }^{229}$ Das Marktwachstum zählt zu den externen Erfolgsfaktoren, die als nicht gestaltbar angenommen werden. Im Gegensatz zum Marktanteil oder zur Produktqualität hat die Intensität von Investitionen eine gegensätzliche Wirkung auf den Erfolg. ${ }^{230}$ Dieser Zusammenhang leitet sich daraus ab, dass bei der Durchführung von Investitionen Aufwendungen entstehen, welche den Erfolg in derselben Periode, beispielsweise bemessen am Jahresüberschuss, sinken lassen. Jedoch können Investitionen langfristig, also mit einer Zeitverzögerung (time lag), zu einem höheren Erfolg führen. Ein hoher Grad von vertikaler Integration, dem letzten genannten Erfolgsfaktor, ermöglicht die Realisation von Verbundeffekten (Economies of $S$ cope), was wiederum in einem höheren Erfolg resultieren kann. ${ }^{231}$

\subsection{Theorie der Regionalität}

\subsubsection{Das Regionalprinzip von Genossenschaftsbanken}

Ein Hauptaugenmerk der vorliegenden Arbeit liegt auf der Betrachtung der Regionalität. Dazu wird untersucht, welchen Einfluss das regionale Umfeld auf die Ertragslage von Banken hat. Insgesamt spielt die Regionalität für Kunden in zahlreichen Wirtschaftsbereichen eine wichtige Rolle. GÄRTNER konnte die räumliche Nähe gar als stabilisierenden Faktor während bzw. nach der Finanzkrise identifizieren. ${ }^{232}$ Dabei versteht er unter räumlicher Nähe, dass die Genossenschaftsbanken gemäß ihrem Auftrag Geschäfte mit ihren Mitgliedern durchführen, die in der unmittelbaren Umgebung des Instituts ansässig sind. Die räumliche Nähe generell kann Informationsasymmetrien in der Kunden-BankBeziehung verringern und sorgt auch dafür, dass vor Ort erwirtschaftetes Kapital in der Region verbleibt. ${ }^{233}$ Speziell durch das Vorhandensein zahlreicher Genossenschaftsbanken und Sparkassen bietet der deutsche Bankenmarkt Hinweise darauf, dass durch den regionalen Bezug Finanzkrisen abgemildert werden können und die Versorgung strukturschwacher Gebiete mit Bankdienstleistungen gesichert wird. ${ }^{234}$ Begründet wird das mit der Existenz regional integrierter Bankenmärkte, der räumlichen Nähe in der Kunden-Bank-Beziehung sowie der Übernahme regionaler Verantwortung. ${ }^{235}$ Auch REICHEL führt die Krisenresistenz der Institute zurück auf das Geschäftsmodell an sich und auf die enge Verzahnung mit der Realwirtschaft in der Region. ${ }^{236}$

Das Regionalprinzip wird in der Literatur auch als horizontale Arbeitsteilung bezeichnet. ${ }^{237}$ Es rührt bei Genossenschaftsbanken von dem Zweck der Institute her, welcher, wie bereits oben beschrieben, auf die Förderung des Erwerbs und der Wirtschaft der Mitglieder gerichtet ist. ${ }^{238}$ Durch die Identität von Mitglied und Kunde ist die Genossenschaftsbank per

\footnotetext{
${ }^{228}$ Vgl. Weis (2012), S. 140.

${ }^{229}$ Vgl. Bergmann (2007), S. 5.

${ }^{230} \mathrm{Vgl}$. Weis (2012), S. 140

231 Vgl. Abschnitt 3.4.2.

232 Vgl. Gärtner (2009), S. 1.

${ }^{233}$ Vgl. Gärtner (2009), S. 4.

234 Vgl. Gärtner (2009), S. 1.

235 Vgl. Gärtner (2009), S. 1.

236 Vgl. Reichel (2011), S. 959.

237 Vgl. Auerbach (2009), S. 236.

238 Vgl. Abschnitt 3.1.1.2.
} 
definitionem fest regional verwurzelt. ${ }^{239}$ CHRISTIANS beschreibt die weitestgehende Festlegung der Geschäftspolitik dieser Institute durch das Regionalprinzip. ${ }^{240}$

Welche Vorteile das Regionalprinzip für die Marktteilnehmer im Geschäftsgebiet einer Bank hat, spricht HANKER in seinem Artikel an. ${ }^{241}$ Um erfolgreich wirtschaften zu können, Arbeits- und Ausbildungsplätze schaffen zu können, brauchen die ansässigen Unternehmen einen verlässlichen Finanzpartner, welchen sie in den Genossenschaftsbanken finden. ${ }^{242}$ Dies bezieht sich nicht nur auf das Angebot fairer Konditionen bei der Kreditvergabe, sondern auch auf die Unterstützung bei strategischen Unterscheidungen. ${ }^{243}$ Die Bank und die Region befinden sich hierbei gewissermaßen in einem Kreislauf: Ist eine Region gut versorgt, so geht es dieser gut und sie kann sich wirtschaftlich entfalten; die regionale Bank wiederum hat durch eine positive regionale Situation ebenso die Möglichkeit, sich erfolgreich zu entwickeln. ${ }^{244}$

REICHLING spricht von lokal orientierten Banken, welche im Rahmen des bestehenden Fusionsdruckes im Begriff sind, zu regionalen Instituten überzugehen. ${ }^{245}$

\subsubsection{Die regionale Identität von Genossenschaftsbanken}

In diesem Abschnitt wird die Bedeutung der regionalen Identität für die Institute erörtert. Diese regionale Identität ist in der Historie der Bankunternehmungen gewachsen. ${ }^{246}$ Bereits im 19. Jahrhundert nahmen zahlreiche Institute ihre Geschäftstätigkeit auf, nachdem sie von selbständigen Gewerbetreibenden oder Landwirten gegründet worden waren. ${ }^{247}$ Durch das Zusammenwirken der Bank mit ihren Kunden entwickelte sich über Generationen hinweg eine Unternehmenskultur, auf welcher schließlich auch die regionale Identität der Bank basiert. ${ }^{248}$ Unter der Unternehmenskultur versteht REICHLING die „Summe von Werten und Normen, die symbolisch über menschliche Handlungsweisen und Produkte kommuniziert werden. ${ }^{\text {(6249 }}$

\subsection{Theorie von Fusionen}

Der Begriff Fusion ist aus dem Lateinischen abgeleitet, wo fundere für gießen oder schmelzen steht. Es handelt sich dabei um eine Verschmelzung von zwei oder mehr Unternehmen, deren Vermögen vereinigt wird. ${ }^{250}$ Unterschieden wird grundsätzlich zwischen einer Verschmelzung durch Aufnahme und einer Verschmelzung durch Neugründung. ${ }^{251}$ Bei der Aufnahme wird das Vermögen inklusive Verbindlichkeiten von einer oder mehr Gesellschaften auf eine bestehende Gesellschaft übertragen. ${ }^{252}$ Bei der Neugründung wird in Deutschland das Vermögen inklusive Verbindlichkeiten auf eine neu gegründete Gesellschaft übertragen. ${ }^{253}$ Die rechtliche Grundlage stellt das Umwandlungsgesetz dar. ${ }^{254} \mathrm{Nach}$

\footnotetext{
${ }^{239} \mathrm{Vgl}$. Abschnitt 3.1.1.2.

${ }^{240}$ Vgl. Christians (2010), S. 2.

241 Vgl. Hanker (2007), S. 25.

${ }^{242}$ Vgl. Hanker (2007), S. 25.

${ }^{243}$ Vgl. Hanker (2007), S. 25.

244 Vgl. Hanker (2007), S. 25.

${ }^{245}$ Vgl. Reichling (2004), S. 150; vgl. Abschnitt 3.3.5.

${ }^{246}$ Vgl. Reichling (2004), S. 152.

247 Vgl. Reichling (2004), S. 152

${ }^{248}$ Vgl. Reichling (2004), S. 152.

${ }^{249}$ Reichling (2004), S. 152.

${ }^{250}$ Vgl. Becker (2012), S. 137.

251 Vgl. Becker (2012), S. 137.

${ }^{252}$ Vgl. Becker (2012), S. 137.

${ }^{253}$ Vgl. Becker (2012), S. 137.
} 
der Fusion stehen die vormals selbständigen Teile unter einer „neuen“ Leitung ${ }^{255}$ und bilden eine wirtschaftliche Einheit. ${ }^{256}$ Zusätzlich bilden sie eine rechtliche Einheit und führen eine gemeinsame Firma. ${ }^{257}$

\subsubsection{Definition von Bankfusionen}

Unter einer Fusion im Bankbereich versteht man den Zusammenschluss von zwei oder mehreren Instituten zu einer größeren Bank. In der Literatur werden zwei übergeordnete Bereiche von Fusionen unterschieden. ${ }^{258}$ Einerseits handelt es sich um originär betriebswirtschaftlich motivierte Fusionen, andererseits um erzwungene Fusionen. ${ }^{259}$ Für außenstehende Betrachter ist häufig nicht erkennbar, aus welchem der beiden Gründe die Fusion stattgefunden hat. Außerdem sind Mischformen möglich, welche Komponenten beider Arten beinhalten. Befindet sich ein Institut in wirtschaftlichen Schwierigkeiten, so fusioniert es in der Regel mit einem intakten Institut mit angrenzendem oder teilweise überlappendem Geschäftsgebiet. ${ }^{260}$ Damit wird eine Schließung vermieden und es entsteht keine Verunsicherung bei den Kunden. Nach außen hin erscheint jedoch alles wie eine wirtschaftlich motivierte Fusion. ${ }^{261}$

\subsubsection{Motive von Bankfusionen}

Im Bereich der tatsächlich wirtschaftlich motivierten Fusionen können drei Hauptziele als Begründung eines Zusammenschlusses angeführt werden. ${ }^{262}$ KALHÖFER nennt hierzu zunächst Wachstumsziele, um die eigene Marktposition zu sichern und auszubauen. ${ }^{263} \mathrm{Als}$ zweites mögliches Ziel geht er auf die unten beschriebenen Skaleneffektete ein, um die Fixkostenanteile zu reduzieren und die kapazitätsinduzierten Leerkosten zu verringern. ${ }^{265}$ Als dritten Punkt nennt er die ebenfalls unten beschriebenen Verbundeffektet ${ }^{266}$ zur Ausnutzung möglicher Synergien. ${ }^{267}$ Generell gilt für Genossenschaftsbanken, dass die horizontale Arbeitsteilung (Regionalprinzip) die organischen Wachstumsmöglichkeiten begrenzt. ${ }^{268}$ Daher kommt den Fusionen als externe Wachstumsoption eine beträchtliche Bedeutung $\mathrm{zu}^{269}$ Dennoch muss konstatiert werden, dass eine wirtschaftlich gesunde Genossenschaftsbank nicht das primäre Ziel hat, rein räumlich zu wachsen. Vielmehr muss ein Institut engagiert darauf hinarbeiten, dass sich das bestehende Einzugsgebiet der Bank positiv entwickelt. Hiervon kann die Bank dann durch Geschäftsabschlüsse wieder profitieren. Des Weiteren trägt diese Strategie zur Bewahrung der regionalen Identität bei. ${ }^{270}$

\footnotetext{
${ }^{254}$ Vgl. $\int 1$ Abs. 1 UmwG.

${ }^{255}$ Nach einer Fusion zweier Banken A und B kann die „,neue“ Leitung aus der Leitung von Bank A, der Leitung von Bank B oder einer Mischung der Leitung beider Altinstitute bestehen.

${ }^{256}$ Vgl. Mathweis (1996), S. 28.

257 Vgl. Mathweis (1996), S. 28.

${ }^{258}$ Vgl. Kalhöfer (2004), S. 79.

${ }^{259}$ Vgl. Kalhöfer (2004), S. 79.

${ }^{260}$ Vgl. Reichling (2004), S. 150; vgl. Kalhöfer (2004), S. 79.

${ }^{261}$ Vgl. Kalhöfer (2004), S. 79; KALHÖFER rechnet mit einer bedeutenden Anzahl von Fusionen im erzwungenen Bereich.

262 Vgl. Kalhöfer (2004), S. 79.

263 Vgl. Fischer und Rolfes (2001), S. 2, zitiert nach Kalhöfer (2004), S. 79.

$264 \mathrm{Vgl}$. Abschnitt 3.4.1, Economies of Scale.

265 Vgl. Fischer und Rolfes (2001), S. 2, zitiert nach Kalhöfer (2004), S. 79.

266 Vgl. Abschnitt 3.4.2, Economies of Scope.

${ }^{267}$ Vgl. Fischer und Rolfes (2001), S. 2, zitiert nach Kalhöfer (2004), S. 79.

${ }^{268}$ Vgl. Auerbach (2009), S. 236.

${ }^{269}$ Vgl. Auerbach (2009), S. 236.

${ }^{270}$ Vgl. Abschnitt 3.3.5.
} 
Im Vorfeld zu einer Fusion bestehen Vorstellungen darüber, welche Größe ein Institut optimaler Weise erlangen sollte. Zur Erreichung dieser optimalen Bankgröße existieren verschiedene Möglichkeiten. ${ }^{271}$ Im Falle einer Vergrößerung des Geschäftsumfanges sind neben dem Wachstum die wichtigsten Varianten die Fusion und die Akquisition. Bei Genossenschaftsbanken handelt es sich in vielen Fällen um kleinere Institute, wodurch der Zusammenschluss die am häufigsten praktizierte Art der Größenveränderung ist. Dennoch ist auch eine Verringerung der Institutsgröße durch verschiedene Maßnahmen denkbar, wie unten in Abschnitt 3.4.1 beschrieben wird.

\subsubsection{Fusionshistorie von Genossenschaftsbanken}

Das Genossenschaftsgesetz sah in seiner Erstfassung von 1889 keine direkte Verschmelzung von Genossenschaften vor, sodass eine Fusion lediglich über den Umweg der Auflösung und einer anschließenden Neugründung zu realisieren war. ${ }^{272}$ Erst durch die Novelle von 1922 wurde die Möglichkeit zur Verschmelzung durch Übernahme geschaffen, und seit der Novelle von 1974 ist schließlich die Verschmelzung durch Neubildung möglich. ${ }^{273}$ Unter den Genossenschaftsbanken gibt es aus den genannten Gründen seit Jahren eine starke Konsolidierungsentwicklung. Die Verringerung der Anzahl der Institute ist nicht auf Schließungen, sondern im Wesentlichen auf Fusionen zurückzuführen. ${ }^{274}$ Die Anzahl der Institute hat sich seit dem Jahr 1970 stetig und stark verringert, sodass von ursprünglich 7.096 im Jahre 1970 per Ende 2011 lediglich noch 1.121 Genossenschaftsbanken ihre Dienstleistungen anbieten. ${ }^{275}$ Dies entspricht einem Rückgang um $84 \%$; die Entwicklung wird in Tabelle 1 dargestellt.

\begin{tabular}{cccccc}
\hline Jahr & $\begin{array}{c}\text { Anzahl der } \\
\text { Banken }\end{array}$ & Jahr & $\begin{array}{c}\text { Anzahl der } \\
\text { Banken }\end{array}$ & Jahr & $\begin{array}{c}\text { Anzahl der } \\
\text { Banken }\end{array}$ \\
\hline 1970 & 7.096 & 1985 & 3.660 & 1999 & 2.034 \\
\hline 1971 & 6.348 & 1986 & 3.600 & 2000 & 1.794 \\
\hline 1972 & 5.753 & 1987 & 3.479 & 2001 & 1.621 \\
\hline 1973 & 5.522 & 1988 & 3.359 & 2002 & 1.489 \\
\hline 1974 & 5.278 & 1989 & 3.223 & 2003 & 1.392 \\
\hline 1975 & 5.196 & 1990 & 3.037 & 2004 & 1.335 \\
\hline 1976 & 5.044 & 1990 & 3.344 & 2005 & 1.290 \\
\hline 1977 & 4.814 & 1991 & 3.145 & 2006 & 1.255 \\
\hline 1978 & 4.607 & 1992 & 2.909 & 2007 & 1.232 \\
\hline 1979 & 4.448 & 1993 & 2.773 & 2008 & 1.197 \\
\hline 1980 & 4.226 & 1994 & 2.658 & 2009 & 1.156 \\
\hline 1981 & 3.935 & 1995 & 2.589 & 2010 & 1.138 \\
\hline 1982 & 3.823 & 1996 & 2.504 & 2011 & 1.121 \\
\hline 1983 & 3.761 & 1997 & 2.417 & 2012 & 1.101 \\
\hline 1984 & 3.711 & 1998 & 2.248 & 2013 & 1.078 \\
\hline
\end{tabular}

Tabelle 1: $\quad$ Entwicklung der Volksbanken und Raiffeisenbanken ab 1970

271 Vgl. Abschnitt 3.4.1.

272 Vgl. Mathweis (1996), S. 30.

${ }^{273}$ Vgl. Mathweis (1996), S. 30.

${ }^{274}$ Vgl. Kalhöfer (2004), S. 77; vgl. Kalhöfer (2004), S. 80f; vgl. Büschgen (2001), S. 16, zitiert nach Kalhöfer (2004), S. 80 f.

275 Vgl. BVR (2015a).

${ }^{276}$ Eigene Darstellung, in Anlehnung an BVR (2015a); Der zweite Wert für das Jahr 1990 beinhaltet die Institute der neuen Bundesländer. 
Andererseits hat sich im selben Zeitraum die Zahl der Mitglieder von 6,2 Mio. auf 17,0 Mio. erhöht, und die konsolidierte Bilanzsumme aller Institute stieg von 40 Mrd. auf 729 Mrd. an. ${ }^{277}$

In Bezug auf den Anstieg der Bilanzsumme muss konstatiert werden, dass die Bilanzsumme der fusionierten Bank nicht zwangsläufig der Summe der Bilanzsummen der vorher existierenden Einzelbanken entsprechen muss. Vielmehr kann die neue Bilanzsumme auch darunter liegen. Dies ist immer dann der Fall, wenn es zwischen den Instituten, die in der Fusion involviert waren, gegenseitige Forderungen bzw. Verbindlichkeiten gibt. Jedoch ist davon auszugehen, dass Genossenschaftsbanken mit benachbartem oder überschneidendem Geschäftsgebiet keine nennenswerten Verflechtungen über Forderungen bzw. Verbindlichkeiten aufweisen.

Die Entwicklung der Anzahl der Institute wird in Abbildung 5 dargestellt.

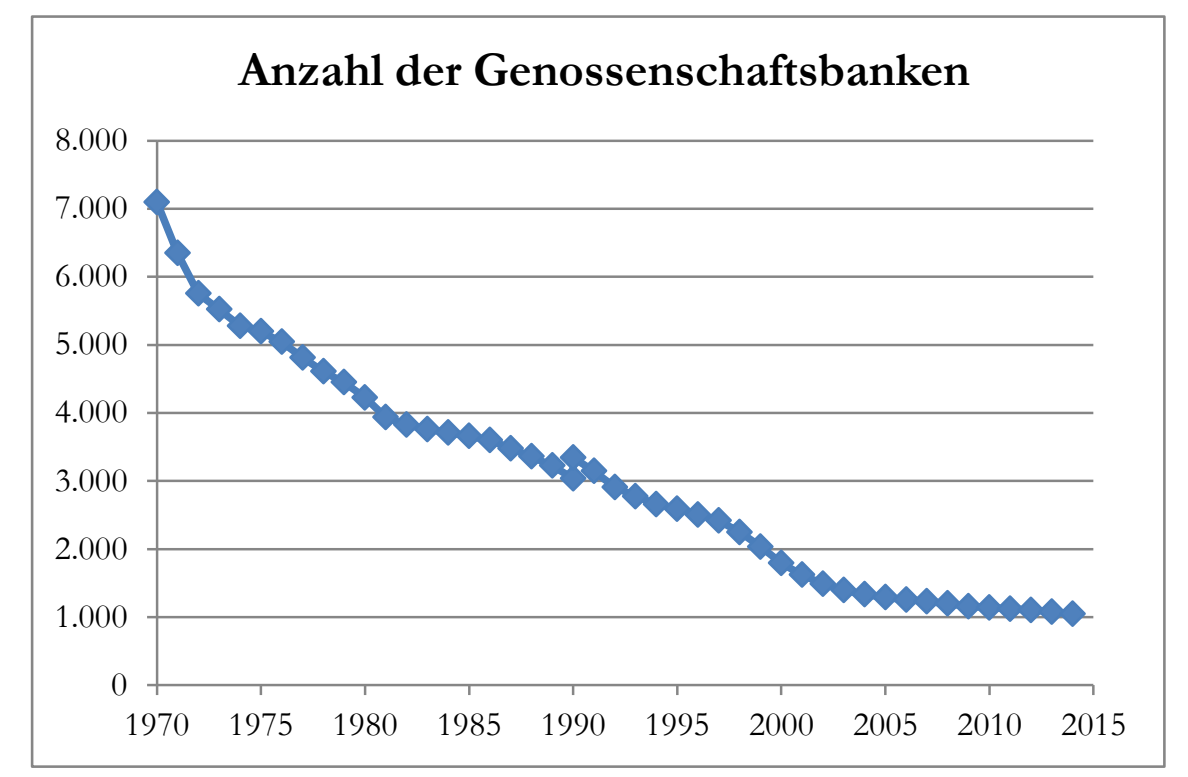

Abbildung 5: Entwicklung der Volksbanken und Raiffeisenbanken ab $1970^{278}$

Die starke Konsolidierung im Genossenschaftssektor führt REICHLING auch auf den vom Genossenschaftsverband ausgeübten Fusionsdruck zurück, welchen er mit missionarischem Eifer betrieben sieht. ${ }^{279}$ Auch STAATS sieht die Volksbanken und Raiffeisenbanken seit längerem einem starken Fusionsdruck ausgesetzt, und führt dies auf die geringe Institutsgröße zurück, welche die Genossenschaftsbanken im Vergleich zu anderen Banken aufweisen. ${ }^{280}$ Nach REICHLING sahen sich andererseits durch die Verschärfung der regulatorischen Vorschriften, insbesondere hinsichtlich des Eigenkapitals, zahlreiche kleine Genossenschaftsbanken dazu gezwungen, ihre Kapital- und Risikostruktur zu verbessern. ${ }^{281}$ Ein wichtiges Fusionsziel des Bundesverbandes der Deutschen Volksbanken und Raiffeisenbanken ist die Wahrung des Grundsatzes „ein Markt - eine Genossenschaftsbank “. ${ }^{282}$

\footnotetext{
277 Vgl. BVR (2015a).

278 Eigene Darstellung, basierend auf BVR (2015a).

${ }^{279}$ Vgl. Reichling (2004), S. 150.

${ }^{280}$ Vgl. Staats (2006), S. 293.

281 Vgl. Abschnitt 3.4.1; vgl. Reichling (2004), S. 150.

${ }^{282}$ Vgl. Staats (2006), S. 295.
} 
Hierdurch soll unnötiger Wettbewerb zwischen den verschiedenen Kreditgenossenschaften vermieden werden, welcher durch Gebietsüberschneidungen entsteht. ${ }^{283}$

Vergleicht man Fusionen von Genossenschaftsbanken mit denen von Sparkassen, so sind einige Unterschiede erkennbar. ${ }^{284}$ Zunächst existiert pro Kreis stets nur ein Sparkasseninstitut, während auf Seite der Kreditgenossenschaften in manchen Fällen sogar mehrere Institute pro Ort anzutreffen sind. ${ }^{285}$ Bei Gebietsreformen wie Kreisfusionen müssen auch die entsprechenden Sparkassen fusionieren, Genossenschaftsbanken werden hierdurch hingegen nicht automatisch zu Fusionen gezwungen. ${ }^{286}$ Dies begründet die Existenz einer Vielzahl sehr kleiner Kreditgenossenschaften. ${ }^{287}$ Am Beispiel der Main-Kinzig-Bank ist in Abbildung 6 die Fusionshistorie zu sehen. Bemerkenswert ist die durchgängig hohe Zahl von Fusionen etwa seit dem Jahr $1960 .{ }^{288}$ Die Bank in ihrer aktuellen Form entstand schließlich im Jahr 2009.

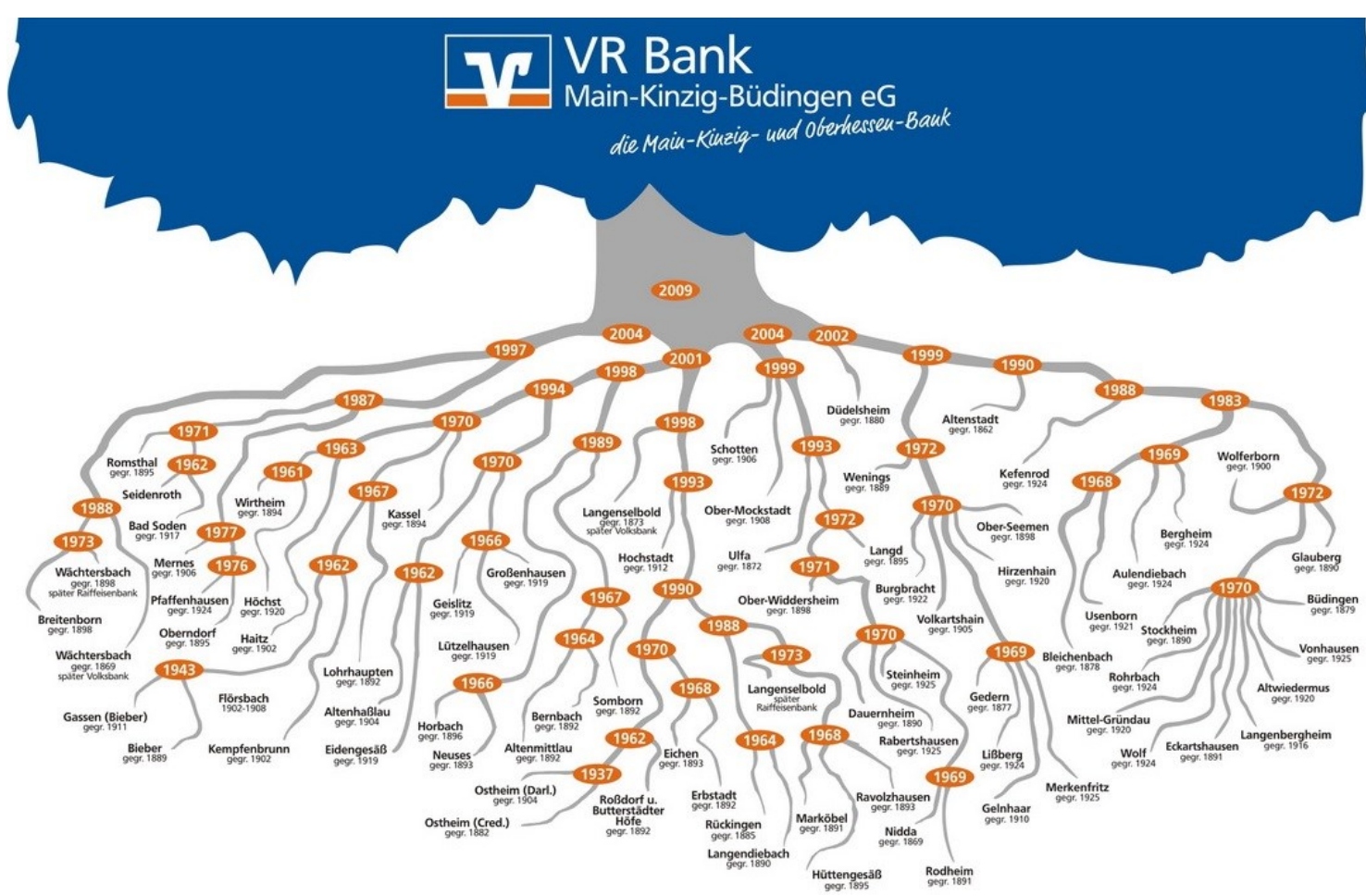

Abbildung 6: Fusionshistorie der VR Bank Main-Kinzig-Büdingen eG ${ }^{289}$

\subsubsection{Erfolg von Bankfusionen}

Der Erfolg von Bankfusionen wurde von mehreren Autoren in der Literatur bewertet. KALHÖFER argumentiert, dass nicht allgemein beantwortet werden könne, ob sich durch eine Konzentration tatsächlich Vorteile aufgrund von Skaleneffekten ergeben. ${ }^{290}$ Hingegen sei nur eine Betrachtung des Einzelfalles möglich. ${ }^{291}$ Er zitiert eine Untersuchung aus dem

${ }^{283}$ Vgl. Staats (2006), S. 295

${ }^{284}$ Vgl. Staats (2006), S. 294

285 Vgl. Staats (2006), S. 294

${ }^{286}$ Vgl. Staats (2006), S. 295

287 Vgl. Staats (2006), S. 295

288 Die Gesamtdaten liegen vom BVR erst ab dem Jahr 1970 vor.

${ }^{289}$ Vgl. VR Bank Main-Kinzig-Büdingen eG (2012), S. 1.

${ }^{290}$ Vgl. Kalhöfer (2004), S. 81.

291 Vgl. Kalhöfer (2004), S. 81 
Sparkassensektor von HAASIS, der einen größenbedingten Kostenvorteil von ca. $20 \%$ der Verwaltungskosten errechnete. ${ }^{292}$ In einem Vergleich über Genossenschaftsbanken, Sparkassen und private Kreditbanken zeigen sich jedoch zunehmende Kostennachteile bei größeren Einheiten. ${ }^{293}$ Die theoretisch gewünschten Effekte von Fusionen ließen sich nur eher selten realisieren. ${ }^{294}$ Gemäß einer Studie von BAIN \& COMPANY seien ein Viertel der Fusionen im Bankbereich Misserfolge, die Hälfte sei nicht klassifizierbar, und nur ein Viertel sei empirisch erfolgreich. ${ }^{295}$

Die Autoren WEIMER und WISSKIRCHEN untersuchten dabei weltweit 50 Fusionen. ${ }^{296}$ Als Kriterien zur Beurteilung des Erfolges bei Bankfusionen setzten sie die relative Aktienperformance, die absolute Entwicklung von Erträgen sowie die Cost-Income-Ratio ein. ${ }^{297}$ TEBROKE ${ }^{298}$ legt mit seinem Werk Größe und Fusionserfolg von Genossenschaftsbanken eine umfangreiche Studie über den Erfolg von Bankfusionen vor und kommt zu dem Schluss, dass sich in etwa vier von zehn Fällen ein Fusionserfolg feststellen lässt. ${ }^{299}$ Der weit überwiegenden Zahl der von ihm untersuchten Fusionsfälle attestiert TEBROKE ein Scheitern. ${ }^{300}$ Hierzu betrachtete er die Entwicklung der Ergebnisstruktur. ${ }^{301}$ Bei einer Messung des Erfolges anhand der Eigenkapitalrentabilität und der Reingewinnspanne ist die Fusion ceteris paribus umso erfolgreicher, je größer die aufnehmende Genossenschaftsbank im Vergleich zur abgebenden ist, je größer ihre horizontale Kundendeckungsquote ist und je geringer ihre Eigenkapitalquote ist. ${ }^{302}$

Die Gründe für den sehr heterogen ausfallenden Erfüllungsgrad der erwarteten Effizienzverbesserungen sind vielschichtig. Im Fusionsjahr selbst sind, durch den Personalbedarf zur Umsetzung der Fusion, zunächst geringere Erfolge zu erwarten. Zeitgleich wird die Gelegenheit einer Fusion von Managern gern dazu verwendet, um hinausgeschobene Abschreibungen vorzunehmen und damit Verluste zu realisieren, die mit der Fusion selbst nicht in Verbindung stehen. Dies führt zusätzlich zu geringeren Ergebnissen.

\subsubsection{Identitätsverlust nach Fusionen}

Durch Fusionen mit benachbarten Genossenschaftsbanken kommt es zwangsläufig zur Ausbildung größerer Geschäftsgebiete. Dabei haben zahlreiche Banken bereits mehrere oder gar viele Fusionen durchlaufen. In einer nicht repräsentativen Studie untersuchte REICHLING, ob Fusionen eine negative Auswirkung auf die Identität der Institute haben könnten. Er konnte zeigen, dass Fusionen eine Gefahr für die regionale Identität eines genossenschaftlichen Kreditinstituts darstellen. ${ }^{303}$ Jedoch löst dabei die erste Verschmelzung den größten Schock in der Identität aus; des Weiteren stellte er fest, dass folgende Fusionen nicht $\mathrm{zu}$ einem weiteren Absinken der Identität eines Instituts führen. ${ }^{304}$ Die

\footnotetext{
292 Vgl. Kalhöfer (2004), S. 81.

${ }^{293}$ Vgl. Kalhöfer (2004), S. 81.

${ }^{294}$ Vgl. Kalhöfer (2004), S. 83.

${ }^{295}$ Vgl. Kositzki (2004), S. 6; vgl. Kalhöfer (2004), S. 83

296 Vgl. Kositzki (2004), S. 6.

297 Vgl. Weimer und Wißkirchen (1999), S. 759

${ }^{298}$ Vgl. Abschnitt 2.1.14.

${ }^{299}$ Vgl. Tebroke (1993), S. 294.

300 Vgl. Tebroke (1993), S. 293.

301 Vgl. Tebroke (1993), S. 293.

302 Vgl. Tebroke (1993), S. 294.

${ }^{303}$ Vgl. Reichling (2004), S. 158

${ }^{304}$ Vgl. Reichling (2004), S. 155
} 
Banken sollten mit der Schaffung geeigneter Kundenbindungsinstrumente reagieren; es lasse sich ein Übergang von der lokalen zur regionalen Identität nachweisen. ${ }^{305}$

\subsection{Theorie der Kostenvorteile}

Die Betrachtung der Kostenvorteile entstammt der neoklassischen Theorie. ${ }^{306}$ Unterstellt werden hier Nachfrage- und Kostenfunktionen, die es erlauben, den gewinnmaximalen Punkt einer Produktion zu bestimmen. ${ }^{307}$ Hieraus lassen sich Economies of Scope und Economies of Scale ableiten, welche nachfolgend beschrieben werden.

\subsubsection{Economies of Scale}

Die sogenannten Skaleneffekte beziehen sich auf Größenvorteile und basieren auf dem Konzept der Erfahrungskurve. ${ }^{308}$ Gemäß diesem Konzept sinken die durchschnittlichen Stückkosten bei einer Erhöhung der Ausbringungsmenge. ${ }^{309}$ HARTMANN-WENDELS ET AL. sprechen dabei von positiven Skaleneffekten. ${ }^{310}$ Anfangs wurden Economies of Scale bei Produktionsprozessen nachgewiesen. ${ }^{311}$ Doch auch bei der Beschaffung, Forschung und Entwicklung, Absatz und Werbung haben sie Einfluss. ${ }^{312}$ Die folgenden Beispiele illustrieren den Zusammenhang in drei Kategorien.

- Technische Gesetzmäßigkeiten: Rechenzentralen arbeiten umso wirtschaftlicher, je mehr Prozesse sie verarbeiten.

- Bei Unteilbarkeiten: Absatzstarke Unternehmen können ihre Marketingkosten auf eine größere Stückzahl verteilen und haben somit pro Stück geringere Aufwendungen. ${ }^{313}$

- Spezialisierungsgewinne: Durch Arbeitsteilung und Spezialisierung kann dieselbe Aufgabe häufig bei geringeren Stückkosten wiederholt werden. Auch im Investmentbanking oder im Beratungsgeschäft können große Firmen Vorteil haben. ${ }^{314}$

Die Spezialisierungsgewinne lassen sich im Wesentlichen auf die bahnbrechenden Erkenntnisse von ADAM SMITH aus dem Jahr 1776 zurückführen. ${ }^{315}$ In seinem Werk $A n$ Inquiry into the Nature and Causes of the Wealth of Nations untersuchte er den Zusammenhang zwischen dem Grad der Spezialisierung und dem Output der Leistungserstellung. ${ }^{316}$ Er identifizierte ein beträchtliches Steigerungspotenzial für die Produktivität, wenn komplexe Tätigkeiten in ihre Bestandteile aufgespalten werden und derart auf die Arbeiter aufgeteilt werden, dass jeder von ihnen lediglich einen oder wenige Fertigungsschritte durchführt. ${ }^{317}$ Hierbei führt er das Beispiel der Stecknadelproduktion an, welche im Wesentlichen aus 18 Arbeitsschritten bestand - von der Herstellung des Drahtfadens bis schließlich zur Verpa-

\footnotetext{
305 Vgl. Reichling (2004), S. 158.

306 Vgl. Swoboda (2005), S. 40.

307 Vgl. Swoboda (2005), S. 40.

308 Vgl. Deelmann (2015), S. 42.

${ }^{309}$ Vgl. Wöhe und Döring (2013), S. 876; vgl. Deelmann (2015), S. 42.

310 Vgl. Hartmann-Wendels et al. (2015), S. 51.

311 Vgl. Grant (2010b), S. 232.

312 Vgl. Grant (2010b), S. 232.

313 Vgl. Grant (2010b), S. 232.

314 Vgl. Grant (2010b), S. 232.

315 Vgl. Hutzschenreuter (2009), S. 413.

316 Vgl. Hutzschenreuter (2009), S. 413.

317 Vgl. Hutzschenreuter (2009), S. 413.
} 
ckung der Nadeln. ${ }^{318}$ SMITH schätzte, dass in einer unspezialisierten Produktion, bei der jeder Arbeiter alle Arbeitsschritte vom ersten bis zum letzten ausführt, zehn Arbeiter insgesamt weniger als 200 Stecknadeln pro Tag schaffen. ${ }^{319}$ Durch die Verteilung der Arbeitsschritte auf zehn spezialisierte Arbeiter lässt sich pro Tag eine Menge von insgesamt 48.000 Stück herstellen. ${ }^{320}$ Im Wesentlichen geht es bei Economies of Scale also darum, den Produktionsprozess in viele einzelne, standardisierte Arbeitsschritte aufzuteilen. Durch diese Spezialisierung findet eine effizientere Produktion statt. Die Produktionsmenge steigt, und die anfallenden Fixkosten können auf eine größere Anzahl von hergestellten Einheiten verteilt werden. ${ }^{321}$ Economies of Scale lassen sich erzielen, solange keine Kapazitätsgrenzen bestehen. Übertragen auf das moderne Bankgeschäft lässt sich aussagen, dass das Wachstumspotenzial heute wesentlich von Investitionen im IT-Bereich abhängt. Die vorliegende Arbeit wird empirisch klären, inwiefern sich Economies of Scale bei Genossenschaftsbanken erzielen lassen. ${ }^{322}$

Fusionen stellen, ähnlich Akquisitionen, eine grundsätzliche Möglichkeit dar, die Größe einer Bank zu erweitern. Betrachtet man die Passivseite, so können Banken noch alternativ durch die Entgegennahme von Einlagen (Fremdkapital) wachsen, oder durch die Erzielung von Gewinnen die Eigenkapitalbasis vergrößern. ${ }^{323}$ Als Beispiel aus dem Bankbereich kann die bereits erwähnte Fusionswelle im Genossenschaftssektor genannt werden. ${ }^{324}$ Die Bildung größerer Geschäftseinheiten soll die Betriebskosten senken und die Effizienz verbessern. ${ }^{325}$ Speziell müssen auch alle regulatorischen Vorschriften umgesetzt werden. Obwohl die einzelnen kleinen Banken Unterstützung von Ihren Verbänden erhalten, haben sie dennoch eine höhere relative Kostenbelastung als größere Institute. ${ }^{326}$

Jedoch werden in der Literatur auch die Grenzen diskutiert, die bei den Skaleneffekten bestehen. GRANT argumentiert, dass kleine und mittlere Unternehmen im Wettbewerb mit deutlich größeren Konkurrenten gut bestehen können. Er zählt als Beispiele einige mittelgroße Automobilhersteller auf, die in jüngster Zeit profitabler als große oder kleine Hersteller waren. Er nennt das Bankgeschäft in den USA und Europa, wo große Häuser weder bei den Kosten noch bei der Profitabilität zu den Besten gehören. ${ }^{327}$ Dabei geht es bei vielen Fusionen gerade um Kostenreduktion. ${ }^{328}$ Kleine und mittlere Unternehmen haben laut GRANT einerseits Flexibilitätsvorteile, während sie andererseits keine Nachteile wegen des Managements großer Strukturen haben, die etwa in den Bereichen der Motivation oder der Koordination auftreten können. ${ }^{329}$ In Bezug auf Genossenschaftsbanken argumentiert KOBMANN mit Transaktionskosten bei größeren Betriebsgrößen, welche aus dann erforderlichen Stabsabteilungen, einer weiteren Hierarchiestufe und einem erweiterten Vorstand resultieren können. ${ }^{330}$ Er rät zu einer Reduktion der Betriebsgröße, da das Optimum bei einer relativ geringen Größe mit weniger Hierarchiestufen zu vermuten sei. ${ }^{331}$ CONRAD ET

\footnotetext{
${ }^{318}$ Vgl. Schneider, Geiger und Scheuring (2008), S. 12.

319 Vgl. Hutzschenreuter (2009), S. 413

320 Vgl. Wieser (2003), S. 310.

${ }^{321}$ Vgl. Watson und Head (2010), S. 444; vgl. Brealey, Myers und Allen (2014), S. 809.

322 Vgl. Abschnitt 4.4.11.

323 Vgl. Hartmann-Wendels et al. (2015), S. 51.

${ }^{324}$ Vgl. Hartmann-Wendels et al. (2015), S. 51.

325 Vgl. Hartmann-Wendels et al. (2015), S. 51.

326 Vgl. Schax (2008), S. 23.

327 Vgl. Grant (2010b), S. 232

${ }^{328}$ Vil. Brealey et al. (2014), S. 809.

${ }^{329}$ Vgl. Grant (2010b), S. 234.

${ }^{330}$ Vgl. Kobmann (1996), S. 143.

331 Vgl. Kobmann (1996), S. 143
} 
AL. beschreiben die Veränderung der Betriebsgröße hin zu einer optimalen Größe. ${ }^{332}$ Damit können zwar Wachstum und Fusionen, aber auch Schrumpfung bzw. Teilung gemeint sein. Auch HaRTMANN-WEndels ET AL. nennen die Aufspaltung des Geschäftsbetriebes als Maßnahme zur Erreichung einer optimalen Betriebsgröße, indem negative Größeneffekte überwunden werden ${ }^{333}$. COELLI ET AL. stellen die Skaleneffekte wie in Abbildung 7 dar.

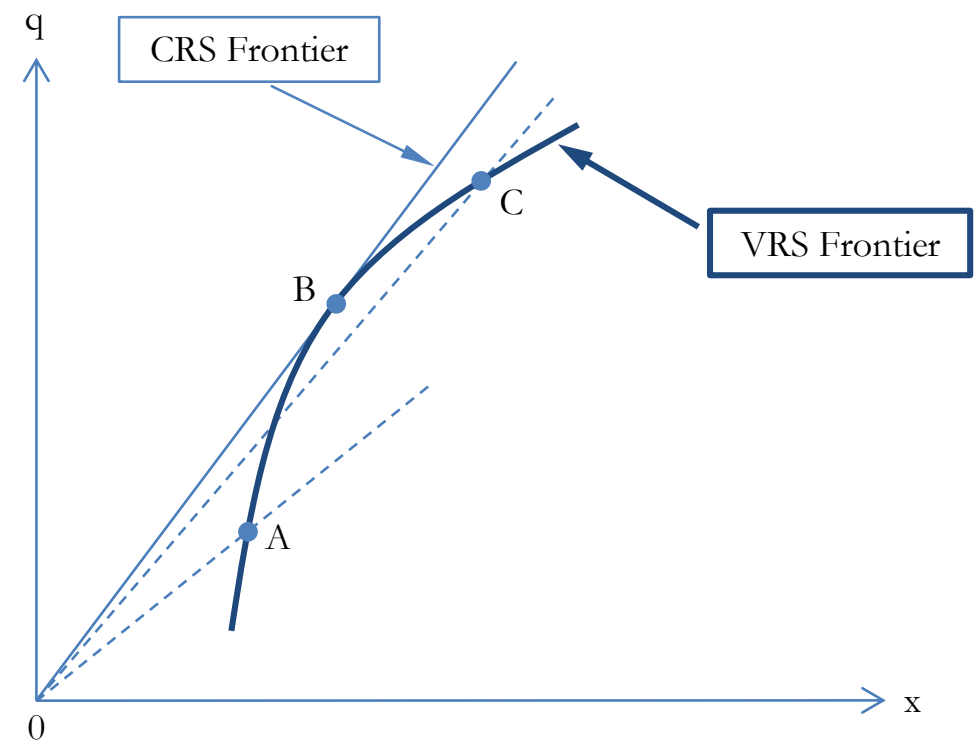

Abbildung 7: Skaleneffekte bei der Produktivität ${ }^{334}$

Das Beispiel in Abbildung 7 bezieht sich auf eine Produktion, in die nur ein Faktor eingeht, und bei der auch nur eine Produktart hergestellt wird. ${ }^{335}$ Auf der Abszissenachse beschreibt $x$ die Einsatzmenge des Inputfaktors, auf der Ordinatenachse kennzeichnet $q$ die Menge an produzierten Einheiten. VRS Frontier kennzeichnet den effizienten Rand einer Produktionsfunktion mit variablen Skalenerträgen (variable returns-to-scale). ${ }^{336}$ Hier ist die Outputmenge von der produzierten Stückzahl abhängig. ${ }^{337}$ Weist die Produktionstechnologie eine solche variable Outputfunktion auf, so sind zweierlei Effekte möglich. Einerseits könnte eine Bank ineffizient sein, weil sie zu klein ist und zu wenige Einheiten produziert, z. B. in Punkt A. Durch eine Erhöhung der Produktionsmenge $x$ kann sie den Punkt B auf dem effizienten Rand erreichen. Hier fallen die geringsten Stückkosten an. Andererseits könnte die Bank aufgrund ihrer Größe skalenineffizient sein, indem sie zu viele Einheiten produziert, ${ }^{338}$ wie es etwa in Punkt $\mathrm{C}$ der Fall wäre. Durch eine Absenkung der Menge $x$ kann die Bank ebenfalls den stückkostenminimalen Punkt B erreichen. Die Skaleneffizienz kann alternativ auch am Steigungswinkel der Strahlen abgelesen werden, welche den jeweiligen Punkt mit dem Koordinatenursprung verbinden. Dieser Winkel ergibt sich aus dem Verhältnis von Output- zu Inputmenge. Hinweis: obwohl die beiden Punkte A und C nicht

\footnotetext{
332 Vgl. Conrad et al. (2009), S. 13.

333 Vgl. Hartmann-Wendels et al. (2015), S. 51.

${ }^{334}$ Eigene Darstellung, in Anlehnung an Coelli, Prasada Rao, O'Donnell und Battese (2005), S. 59.

335 Vgl. Coelli et al. (2005), S. 58.

${ }^{336}$ Vgl. Coelli et al. (2005), S. 58.

337 Vgl. Coelli et al. (2005), S. 58.

338 Vgl. Conrad et al. (2009), S. 13.
} 
skaleneffizient sind, sind sie dennoch technisch effizient, denn sie liegen auf dem effizienten Rand der Produktionsfunktion. ${ }^{339}$

Gehen in die Produktion mehrere Faktoren ein, so wird das Einsatzverhältnis der einzelnen Faktoren bei einer Variation der Menge jedoch beibehalten; variiert wird stets nur die Outputmenge. ${ }^{340}$ In jedem Fall ist eine Produktionsfunktion skaleneffizient, wenn sie konstante Skalenerträge (constant returns-to-scale) liefert. Bei einer Veränderung der Inputmenge verändert sich die Outputmenge proportional. In diesem Fall ist die entsprechende Produktionsfunktion CRS Frontier eine gerade Linie. ${ }^{341}$

Durch das Auftreten der Economies of Scale implizieren HARTMANN-WENDELS ET AL. eine Mindestgröße, als eine minimale Unternehmensgröße, zur Sicherstellung von langfristigem Erfolg. ${ }^{342}$ Andererseits gebe es, jenseits eines optimalen Bereiches, eine Größe, ab welcher die Banken negative Skaleneffekte aufweisen. ${ }^{343}$ Damit sei eine Obergrenze für den optimalen Geschäftsumfang gesetzt. ${ }^{344}$ Im Rahmen der vorliegenden Arbeit werden die Economies of Scale in Bezug auf die optimale Betriebsgröße von Banken angewendet. ${ }^{345}$

\subsubsection{Economies of Scope}

Economies of Scope existieren, wenn der kombinierte Einsatz einer Ressource für verschiedene Zwecke insgesamt weniger verbraucht als die Summe des Einsatzes für die Zwecke einzeln. ${ }^{346}$ Economies of Scope gibt es aus ganz ähnlichen Gründen wie Economies of Scale. ${ }^{347}$ Economies of Scale sind Kosteneinsparungen, wenn eine höhere Anzahl eines Produkts hergestellt wird, Economies of Scope hingegen treten bei der kombinierten Herstellung mehrerer Produkte zu Tage. ${ }^{348}$ Economies of Scope beziehen sich also auf Verbundeffekte. ${ }^{349}$ Hierunter ist die vertikale Erweiterung der Produktionskette zu verstehen. ${ }^{350}$ Ein Unternehmen kann beispielsweise auf der Einkaufsseite dazu übergehen, an Stelle seiner Zulieferer selbst Rohstoffe zu fördern, Teile herzustellen oder Leistungen zu erstellen. ${ }^{351}$ Auf der Verkaufsseite ist gemeint, dass ein Unternehmen selbst den Absatz bis hin zum Endkunden organisiert. ${ }^{352} \mathrm{Ob}$ ein Unternehmen sich für einen derartigen vertikalen Ausbau der Produktions- und Absatzkette entscheidet, hängt maßgeblich von den Transaktionskosten ab. ${ }^{353} \mathrm{Im}$ Bankensektor resultieren die Kostenvorteile aus der Fixkostendegression, Cross-Selling-Möglichkeiten und sogenannten Spill-over-Effekten. ${ }^{354}$ Bei letzteren werden Informationen aus einem Geschäftsbereich vorteilhaft für andere Bereiche eingesetzt. ${ }^{355}$ Neben den Synergieeffekten bzw. Verbundeffekten kann auch ein Knowhow-Transfer angestrebt werden. ${ }^{356}$ Somit lassen sich Kundenverbindungen, Technologien

\footnotetext{
339 Vgl. Coelli et al. (2005), S. 58; vgl. Conrad, Neuberger und Trigo Gamarra (2014), S. 546.

${ }^{340}$ Vgl. Coelli et al. (2005), S. 58.

341 Vgl. Coelli et al. (2005), S. 58

342 Vgl. Hartmann-Wendels et al. (2015), S. 51.

${ }^{343}$ Vgl. Hartmann-Wendels et al. (2015), S. 51.

${ }^{344}$ Vgl. Hartmann-Wendels et al. (2015), S. 51.

345 Vgl. Abschnitt 4.4.16.

346 Vgl. Grant (2010a), S. 409.

347 Vgl. Grant (2010a), S. 409

348 Vgl. Grant (2010a), S. 409.

${ }^{349}$ Vgl. Baxmann (1999), S. 24.

${ }^{350}$ Vgl. Deelmann (2015), S. 43.

${ }^{351}$ Vgl. Deelmann (2015), S. 43

${ }^{352}$ Vgl. Brealey et al. (2014), S. 809; vgl. Deelmann (2015), S. 43.

353 Vgl. Deelmann (2015), S. 43.

354 Vgl. Hockmann und Thießen (2012), S. 53.

355 Vgl. Hockmann und Thießen (2012), S. 53.

356 Vgl. Kalhöfer (2004), S. 79.
} 
und Erfahrungen für die übrigen Geschäftsfelder nutzbar machen. ${ }^{357}$ Idealerweise erhöht sich auch der Kundennutzen, weil verschiedene Leistungen integriert aus einer Hand angeboten werden können. ${ }^{358}$

Als Gegenteil der vertikalen Integration ist das Outsourcing anzusehen. ${ }^{359}$ Wörtlich bedeutet Outsourcing die Nutzung externer Ressourcen. ${ }^{360}$ Hierbei wird der Ansatz verfolgt, Teile von Zulieferern zu beziehen anstatt diese selbst zu produzieren, oder den Vertrieb anderen Unternehmen zu überlassen. ${ }^{361}$ Ein Teil der erzielten Einsparungen resultiert aus der Effizienz des Zulieferers, der seinerseits durch gebündelte Stückzahlen mehrerer Kunden Skaleneffekte erzielen kann, sowie aus dessen Erfahrungen bei der Herstellung der ausgelagerten Teile. Ein weiterer Teil kommt, zumindest in Betrieben der Industrieproduktion, aus der so ermöglichten Umgehung von Tarifverträgen bzw. aus der Verlagerung in Niedriglohnregionen. ${ }^{362}$

\subsubsection{Die optimale Bankbetriebsgröße}

Die Diskussion der optimalen Betriebsgröße wird sowohl im Bereich der Nichtbanken, wie z. B. Industrieunternehmen, als auch im Bereich der Banken intensiv geführt. ${ }^{363}$ HARTMANN-WENDELS ET AL. unterscheiden im Bankbereich zunächst zwischen der optimalen Größe des Gesamtunternehmens und der optimalen Größe einzelner Betriebsstätten wie etwa der Bankfilialen. ${ }^{364}$ Die vorliegende Arbeit konzentriert sich im Wesentlichen auf die Perspektive der Gesamtbank; die Filialgröße wird nur indirekt betrachtet. Den Ausgangspunkt der Argumentation einer optimalen Größe bildet die These der Existenz Economies of Scale. ${ }^{365}$

Das Nachweisen von Economies of Scale im Bankbereich ist nicht unproblematisch, ebenso wie die als ein Ziel der vorliegenden Arbeit gesetzte Quantifizierung einer optimalen Bankgröße. ${ }^{366}$ Dies ist darauf zurückzuführen, dass die hierzu herangezogenen Variablen zunächst präzise definiert werden müssen. ${ }^{367}$ Speziell ist einerseits eine Messgröße zur Abbildung des Geschäftsumfangs vonnöten; andererseits braucht es eine geeignete Darstellung der Relation zwischen den aufgewendeten Inputfaktoren und den als Produkte hergestellten Outputfaktoren. ${ }^{368}$ Die genannten Größen werden weiter unten in den Abschnitten 3.5.2 und 3.5.3 diskutiert.

HELTEN konstatiert hingegen für Versicherungsunternehmen, welche ebenfalls der Finanzbranche zugeordnet werden, dass es allgemein keine optimale Betriebsgröße gibt. ${ }^{369} \mathrm{Er}$ führt dies auf abweichende Zielsysteme zwischen den einzelnen Unternehmen zurück. ${ }^{370}$

\footnotetext{
357 Vgl. Kalhöfer (2004), S. 79.

${ }^{358}$ Vgl. Kalhöfer (2004), S. 79.

${ }^{359}$ Vgl. Brealey et al. (2014), S. 810.

360 Vgl. Disselbeck (2007), S. 162.

361 Vgl. Brealey et al. (2014), S. 810.

${ }^{362}$ Vgl. Disselbeck (2007), S. 163; vgl. Brealey et al. (2014), S. 810.

${ }^{363}$ Vgl. Hartmann-Wendels et al. (2015), S. 51.

364 Vgl. Hartmann-Wendels et al. (2015), S. 51.

365 Vgl. Hartmann-Wendels et al. (2015), S. 51.

366 Vgl. Dymski (1999), S. 55; vgl. Gilligan, Smirlock und Marshall (1984), S. 393; vgl. Mitchell und Onvural (1996), S. 178; vgl. Heffernan (2005), S. 483; vgl. Humphrey (1992), S. 115 f.; vgl. Hartmann-Wendels et al. (2015), S. 51; vgl. Casu, Molyneux und Girardone (2006) S. 15.

367 Vgl. Hartmann-Wendels et al. (2015), S. 52.

${ }^{368}$ Vgl. Hartmann-Wendels et al. (2015), S. 52.

${ }^{369}$ Vgl. Helten (2004), S. 8.

${ }^{370}$ Vgl. Helten (2004), S. 8.
} 
Ungeachtet ihrer Größe müssen die Genossenschaftsbanken ihre regionale Identität bewahren, denn diese stellt eine entscheidende Voraussetzung für einen erfolgreichen Geschäftsbetrieb dar. ${ }^{371}$

\subsection{Theorie der Erfolgsmessung}

\subsubsection{Verhältniszahlen}

Unter Verhältniszahlen versteht man den Quotienten aus zwei Zahlen, welche in einem sinnvollen bzw. sachlogischen Zusammenhang stehen. ${ }^{372}$ Innerhalb der Verhältniszahlen unterscheidet man weiter in Gliederungszahlen, Beziehungszahlen und Messzahlen.

Verhältniszahlen gehören zu den relativen Kennzahlen. Diese werden von absoluten Kennzahlen wie Umsatz, Bilanzsumme oder Eigenkapital abgegrenzt.

\section{Gliederungszahlen}

Gliederungszahlen erlauben es, eine Gesamtmasse in Teilmassen aufzugliedern. Eine Gliederungszahl ergibt sich, indem man eine der Teilmassen in das Verhältnis zur Gesamtmasse setzt. ${ }^{373}$ Ein Beispiel für eine Gliederungszahl ist die Eigenkapitalquote in der folgenden Beziehung, die angibt, welchen Anteil das Eigenkapital am gesamten Kapital ausmacht. ${ }^{374}$

$$
\text { Eigenkapitalquote }=\frac{\text { Eigenkapital }}{\text { Gesamtkapital }}
$$

Ein weiteres Beispiel zeigt den Anteil der durch Grundpfandrechte gesicherten Kundenforderungen am Gesamtbestand der Forderungen an Kunden.

$$
\text { Grundpfandanteil }=\frac{\text { Durch Grundpfandrechte gesicherte Kundenforderungen }}{\text { Gesamtbestand der Forderungen an Kunden }}
$$

Ein drittes Beispiel beziffert den Sparanteil als Anteil der Spareinlagen am Gesamtbestand der Verbindlichkeiten gegenüber Kunden.

$$
\text { Sparanteil }=\frac{\text { Spareinlagen }}{\text { Gesamtbestand der Verbindlichkeiten gegenüber Kunden }}
$$

\section{Beziehungszahlen}

Eine Beziehungszahl entsteht, wenn man zwei verschiedenartige, wesensfremde, aber sachlich sinnvoll zusammenhängende Größen in das Verhältnis zueinander setzt. ${ }^{375} \mathrm{Zu}$ den Nachteilen von Beziehungszahlen zählt, dass Veränderungsursachen nur dann ergründet werden können, wenn auch die Werte vorliegen, welche in die Berechnung der Kennzahl einfließen. ${ }^{376}$ Beispielsweise kann die Erhöhung der Eigenkapitalrendite entweder aus einer Erhöhung des Gewinns oder aber aus der Reduktion von Eigenkapital herrühren. Beispiele stellen die nächsten Ausdrücke dar:

\footnotetext{
${ }^{371}$ Vgl. Abschnitt 3.3.5.

372 Vgl. Bourier (2012), S. 119.

${ }^{373}$ Vgl. Bourier (2012), S. 119 f

374 Vgl. Coenenberg (2012), S. 1020; vgl. Brösel (2012), S. 239, 257; vgl. Küting und Weber (2012), S. 53.

375 Vgl. Bourier (2012), S. 120 f.

${ }^{376}$ Vgl. Coenenberg (2012), S. 1020.
} 


$$
\begin{aligned}
& \text { Verschuldungsgrad }=\frac{\text { Fremdkapital }}{\text { Eigenkapital }} \\
& \text { Eigenkapitalrendite }=\frac{\text { Gewinn }}{\text { Eigenkapital }}
\end{aligned}
$$

$$
\text { Einwohnerdichte }=\frac{\text { Anzahl der Einwohner }}{\text { Fläche in } \mathrm{km}^{2}}
$$

Bei allen Spannen des Bankbetriebs handelt es sich um Beziehungszahlen. Beispielhaft sei hier die Provisionsspanne angeführt.

$$
\text { Provisionsspanne }=\frac{\text { Provisionsüberschuss }}{\text { Durchschnittliche Bilanzsumme }}
$$

\section{Messzahlen}

Von einer Messzahl oder Indexzahl spricht man, wenn zwei sachlich gleiche Größen in Relation gesetzt werden, diese Größen sich jedoch räumlich oder zeitlich unterscheiden. ${ }^{377}$ Ein Wert wird anhand eines anderen gemessen. ${ }^{378}$ Beispielsweise betrage die Bilanzsumme einer bestimmten Bank 100 Mio. Euro per Ende 2010. Für Ende 2011 liegt der Wert bei 105 Mio. Euro. Die Errechnung der Messzahl erfolgt in der nächsten Beziehung.

$$
\frac{\text { Bilanzsumme Ende } 2011}{\text { Bilanzsumme Ende } 2010}=\frac{105 \text { Mio. Euro }}{100 \text { Mio. Euro }}=105 \%
$$

Die folgende Messzahl Älterenverbältnis beziffert, wie viele Einwohner oberhalb einer gewissen Altersgrenze (z. B. 65 Jahre) auf einen Einwohner unterhalb dieser Grenze kommen.

$$
\text { Älterenverhältnis }=\frac{\text { Anzahl Über-65-jähriger }}{\text { Anzahl Unter-65-jähriger }}
$$

Beim Wachstum der Kundeneinlagen handelt es sich ebenso um eine Messzahl.

$$
\frac{\text { Kundeneinlagen Ende } 2011}{\text { Kundeneinlagen Ende } 2010}=\frac{558 \text { Mio. Euro }}{539 \text { Mio. Euro }}=103,5 \%
$$

\subsubsection{Messgrößen für die Betriebsgröße einer Bank}

Es liegt in der Natur der Sache, dass verschiedene Banken unterschiedlich groß sind. Um Banken anhand ihrer Größe vergleichbar zu machen, muss zunächst ein Kriterium zur Messung dieser Größe vorhanden sein. ${ }^{379}$ Die Herleitung eines geeigneten Maßes für die Betriebsgröße wird sehr umfangreich von TEBROKE diskutiert ${ }^{380}$ und wurde von HARTMANN-Wendels ET AL. in ähnlicher Form übernommen. ${ }^{381}$ Den Ausgangspunkt bildet die Definition nach Gutenberg, die für Industriebetriebe ein System von Produktivfaktoren festlegt. ${ }^{382}$ Hierzu zählen Arbeit, Betriebsmittel, Werkstoffe und Führung als

\footnotetext{
377 Vgl. Coenenberg (2012), S. 1021.

378 Vgl. Bourier (2012), S. $121 \mathrm{f}$.

${ }^{379} \mathrm{Vgl}$. Schierenbeck (2003b), S. 426

${ }^{380}$ Vgl. Tebroke (1993), S. 59-71.

381 Vgl. Hartmann-Wendels et al. (2015), S. 52.

382 Vgl. Obst und Hagen (2000), S. 1135; vgl. Tebroke (1993), S. 59.
} 
Inputfaktoren. ${ }^{383}$ Jedoch kann diese Definition für Industriebetriebe nicht ohne weiteres für den Bankbetrieb übernommen werden, da der sehr entscheidende monetäre Faktor für die Bestände an Bar- und Buchgeld fehlt. ${ }^{384}$ Das System wird daher um diesen Faktor erweitert, da er im Rahmen der kreditwirtschaftlichen Produktion eine fundamentale Bedeutung aufweist. ${ }^{385}$

Busse vON COLBE definierte die Betriebsgröße eines Industrieunternehmens inputorientiert als bewertetes Leistungspotenzial pro Zeiteinheit. ${ }^{386}$ Bei der Übertragung des Systems von Produktionsfaktoren auf den Bankbetrieb wird dieser nach DEPPE gedanklich in einen technisch-organisatorischen Bereich (TOB), dem sogenannten Betriebsbereich, und in einen liquiditätsmäßig-finanziellen Bereich (LFB), dem sogenannten Wertbereich, aufgeteilt. ${ }^{387}$ Abbildung 8 stellt die Inputfaktoren grafisch dar.

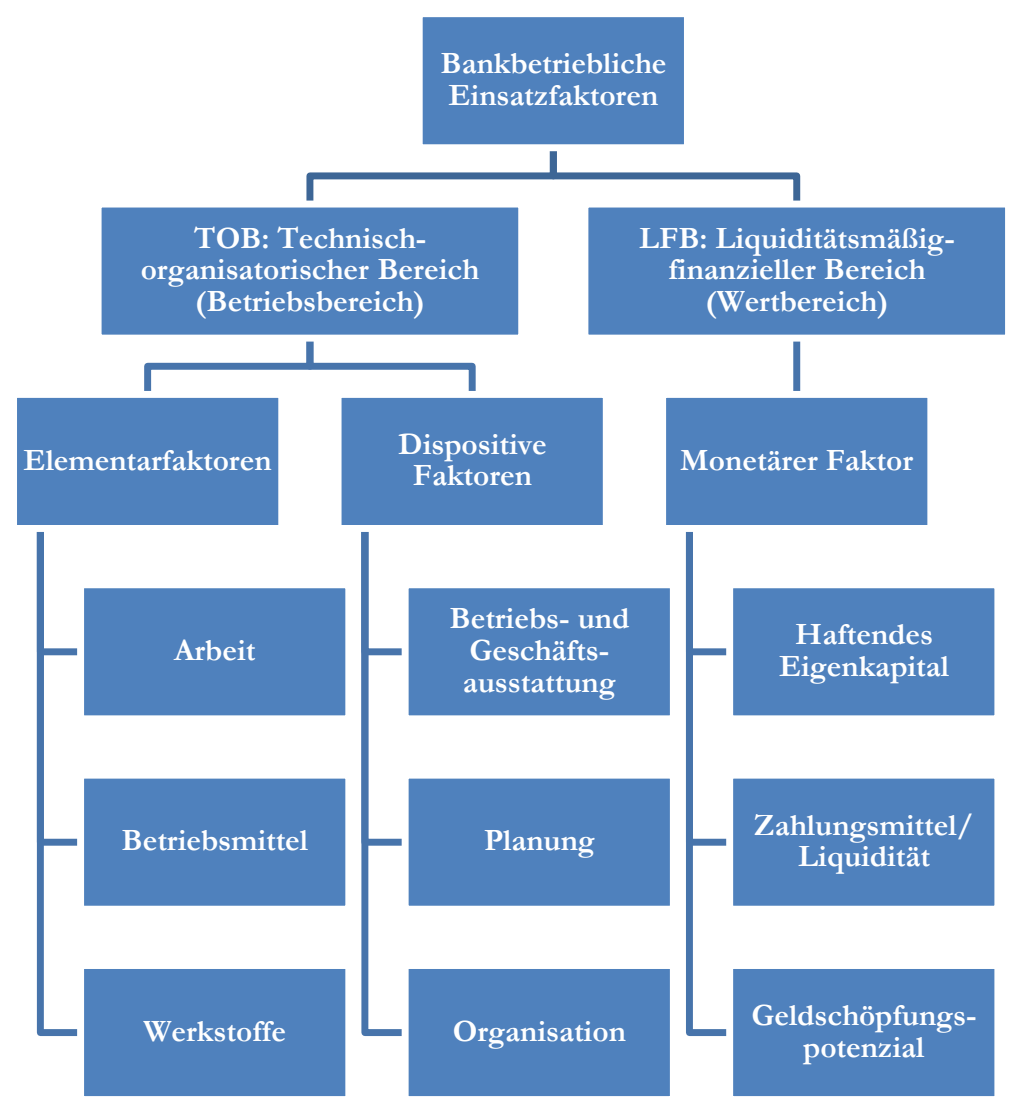

Abbildung 8: Inputfaktoren der bankbetrieblichen Leistungserstellung ${ }^{388}$

Der Betriebsbereich ist die „sichtbare Gestalt“ eines Kreditinstituts und umfasst „die Struktur, das sachliche und räumliche Zusammenwirken der oben unterschiedenen Elementarfaktoren und dispositiven Faktoren“ ${ }^{389}$ Den Wertbereich versteht DEPPE als ein „Abstraktum, das die Schaffung und Auflösung rechtlicher Beziehungen finanzieller Art zwischen dem Kre-

\footnotetext{
383 Vgl. Tebroke (1993), S. 59.

384 Vgl. Hartmann-Wendels et al. (2015), S. 52.

385 Vgl. Tebroke (1993), S. 59.

386 Vgl. Busse von Colbe, Walther (1964), S. 13, zitiert nach Hartmann-Wendels et al. (2015), S. 52.

387 Vgl. Deppe (1969), S. 20, zitiert nach Hartmann-Wendels et al. (2015), S. 52; vgl. Tebroke (1993), S. 79 f.

388 Eigene Darstellung, in Anlehnung an Tebroke (1993), S. 60.

${ }^{389}$ Deppe (1969), S. 20, zitiert nach Tebroke (1993), S. 61.
} 
ditinstitut und der Außenwelt erfasst“ und damit ,im Wesentlichen die Nutzung des monetären Faktors" umschließt. ${ }^{390}$

Häufig findet die Größenmessung von Instituten lediglich anhand des Wertbereiches statt, da dieser für den Leistungserstellungsprozess dominant ist. ${ }^{391}$ Als mögliche Kennzahlen zur Messung der Betriebsgröße finden sich in der Literatur: ${ }^{392}$

- Bilanzpassiva
○ Eigenkapital
- Kundeneinlagen
- Gesamte Einlagen
- Gesamtes Fremdkapital

- Bilanzaktiva

- Vergebene Kredite an andere Kreditinstitute

○ Vergebene Kredite an Kunden, ggfs. gegliedert nach

- der Sicherung durch Grundpfandrechte

- Kommunalkrediten und

- übrigen Forderungen

- Sachanlagen

- Bilanzsumme

- Anzahl der Kunden

Im Folgenden werden die genannten Größen bezüglich ihres Einsatzes für die Messung des Geschäftsvolumens von Banken diskutiert. Zunächst werden mögliche Bilanzpassiva betrachtet, welche die Mittelherkunft abbilden. Die Höhe des bilanziellen Eigenkapitals kann auf Basis einzelner Bilanzpositionen ermittelt werden. Die Tatsache, dass sich rechtsformspezifische Unterschiede beeinflussend auf die Höhe des Eigenkapitals auswirken, kann generell zu Verzerrungen führen. ${ }^{393}$ Jedoch werden im Rahmen der vorliegenden Arbeit ausschließlich Genossenschaftsbanken untersucht und verglichen, die durchweg die Rechtsform der Genossenschaft aufweisen, daher sind hieraus keine nachteiligen Auswirkungen zu erwarten. Viel schwerer wiegt hingegen, dass das Eigenkapital mit in der Regel 2 bis $5 \%$ lediglich einen Bruchteil des monetären Faktors ausmacht. ${ }^{394}$ Zusätzlich limitiert das Eigenkapital nicht die gesamte Geschäftstätigkeit einer Bank, sondern nur ganz bestimmte Aktivgeschäfte. ${ }^{395}$ BAXMANN empfiehlt daher, nicht auf Basis des haftenden Eigenkapitals auf die Betriebsgröße abzustellen, sondern hierfür stattdessen die gesamte Geschäftstätigkeit einzusetzen. ${ }^{396}$ Die Kundeneinlagen sind ein wesentlicher Bestandteil des Fremdkapitals und empfehlen sich daher als Größenkennzahl; insbesondere geben sie

\footnotetext{
${ }^{390}$ Deppe (1969), S. 20, zitiert nach Tebroke (1993), S. 61.

391 Vgl. Hartmann-Wendels et al. (2015), S. 52.

392 Vgl. Hartmann-Wendels et al. (2015), S. 52.

${ }^{393}$ Vgl. Hartmann-Wendels et al. (2015), S. 52.

394 Vgl. Baxmann (1995b), S. 32.

395 Vgl. Baxmann (1995b), S. 32.

${ }^{396}$ Vgl. Baxmann (1995b), S. 33.
} 
Aufschluss über das Kreditschöpfungspotenzial. ${ }^{397}$ Kritisiert wird, dass diese Kennzahl nicht für alle Kreditinstitute sinnvoll einsetzbar ist, da etwa Bürgschaftsbanken oder Landesbanken kein größeres Einlagengeschäft betreiben. Dennoch würde sie für Genossenschaftsbanken grundsätzlich gut funktionieren. ${ }^{398}$ In der Literatur wird auch die Verwendung der gesamten Einlagen oder des gesamten Fremdkapitals als Größenkennzahl diskutiert. ${ }^{399}$ Auch die Verwendung einer mehrdimensionalen Größendefinition wird von BAXMANN hinterfragt, bestehend aus der durchschnittlichen Bilanzsumme (LFB-Merkmal) und der Summe aus Personal- und Sachkosten (TOB-Merkmal). ${ }^{400}$ Jedoch kam auch BAXMANN in Ermangelung eines nachweisbaren Vorteils der mehrdimensionalen Größe zu dem Schluss, der eindimensionalen Betriebsgrößenerfassung den Vorzug zu erteilen. ${ }^{401}$

Die verschiedenen Bilanzaktiva bilden die Mittelverwendung ab. Bei einer Änderung der Mittelallokation würde sich auch die gewählte Größenkennzahl verändern, aus diesem Grund sind die Bilanzaktiva für die Größenmessung ungeeignet. Auch in der Literatur bleiben denkbare Maßzahlen des Betriebsbereiches häufig unberücksichtigt, was auf die engpassorientierte Dominanz des Wertbereiches zurückgeführt wird. ${ }^{402} \mathrm{Zu}$ den Maßzahlen des Betriebsbereiches zählen auch die Sachanlage, die Anzahl der Mitarbeiter oder die Anzahl der Filialen bzw. Zweigstellen.

Aufgrund der Unabhängigkeit von der Bilanzstruktur empfehlen HARTMANN-WENDELS ET AL. schließlich den Einsatz der Bilanzsumme. ${ }^{403}$ Jedoch verweisen sie auch auf die damit verbundenen Schwachpunkte, dass etwa außerbilanzielle Geschäfte wie z. B. das Investmentbanking nicht darin abgebildet werden, oder dass umgekehrt Positionen ohne direkten Bankbezug enthalten sind, zu denen etwa Beteiligungen, Grundstücke oder Gebäude gehören. ${ }^{404} \mathrm{Im}$ Folgenden wird erläutert, warum von allen möglichen Ansätzen in der Regel die Bilanzsumme bzw. die durchschnittliche Bilanzsumme als Messgröße eingesetzt wird.

\subsubsection{Bilanzsumme}

Häufig kommt die Bilanzsumme als Proxy zum Einsatz, um das Geschäftsvolumen bzw. die Größe von Banken zu beurteilen, beispielsweise durch die DeutsChe BundesBanK. ${ }^{405}$ Auch SCHIERENBECK empfiehlt für gesamtbankbezogene ROI-Analysen die Bilanzsumme als Maßstab des Geschäftsvolumens. ${ }^{406}$ Speziell betont er die Konvention, die einfache Bilanzsumme zu verwenden, und nicht etwa Aktiv- und Passivseite addiert als Geschäftsvolumen zu betrachten. ${ }^{407}$ Bei der Bilanzsumme handelt es sich naturgemäß um eine Momentaufnahme, welche die Forderungen und Verbindlichkeiten in einem bestimmten Zeitpunkt zusammenfasst. Der Beginn und das Ende des Geschäftsjahres einer jeden Genossenschaftsbank werden in ihrer Satzung definiert und können pro Institut individuell festgelegt werden. De facto gilt jedoch für alle deutschen Genossenschaftsbanken, dass das Geschäftsjahr am 31.12. eines jeden Jahres endet. Beispielsweise lautet $\ 42$ der Satzung der

\footnotetext{
397 Vgl. Hartmann-Wendels et al. (2015), S. 52.

398 Vgl. Hartmann-Wendels et al. (2015), S. 52.

${ }^{399}$ Vgl. Hartmann-Wendels et al. (2015), S. 52.

${ }^{400}$ Vgl. Baxmann (1995a), S. 470.

401 Vgl. Baxmann (1995a), S. 470.

402 Vgl. Hartmann-Wendels et al. (2015), S. 52.

${ }^{403}$ Vgl. Hartmann-Wendels et al. (2015), S. 52 f

${ }^{404}$ Vgl. Hartmann-Wendels et al. (2015), S. 53.

${ }^{405}$ Vgl. Bundesbank (2013c), S. 21.

406 Vgl. Schierenbeck (2003b), S. 426.

${ }^{407}$ Vgl. Tebroke (1993), S. 70; vgl. Schierenbeck (2003b), S. 426.
} 
Volksbank Stuttgart eG „Das Geschäftsjahr ist das Kalenderjahr.““408 Alle untersuchten Jahresabschlüsse weisen den 31.12. als Ende des Geschäftsjahres auf.

Gerade weil es sich hierbei um eine Momentaufnahme handelt, weist diese einen Schwachpunkt auf: Die Bilanzzusammensetzung wird nur an diesem Stichtag beurkundet. ${ }^{409}$ Jedoch sind Strukturänderungen im Zeitablauf möglich: ${ }^{410}$ Am Vortag und auch bereits am Tag danach können die Bilanzposten - zumindest theoretisch - beträchtlich abweichen. ${ }^{411}$ Auch sind alle enthaltenen Angaben stark vergangenheitsorientiert. ${ }^{412}$ Trotz allem ist der Jahresabschluss häufig die einzige verfügbare und geprüfte Quelle über die Bilanzzusammensetzung für eine externe Bilanzanalyse. ${ }^{413}$ Es steht keine arbeitstäglich aktualisierte und veröffentlichte Alternative zur Verfügung.

Die Bilanzsumme erscheint somit als Instrument zum Größenvergleich zwischen zwei oder mehr Banken zwar geeignet. Es sollte jedoch beachtet werden, dass durch bilanzverlängernde oder -verkürzende Maßnahmen die Höhe der Bilanzsumme - in einigen Grenzen beeinflusst werden kann. Diese Maßnahmen der Jahresabschlusspolitik werden in Abschnitt 3.6.5 beschrieben.

Es gibt auch Autoren, die die Bilanzsumme als Referenzgröße problematisch sehen, wie z. B. GISCHER ${ }^{414}$ oder PADBERG ${ }^{415}$, mit dem Argument, dass nicht alle Positionen zinstragend sind. Die vorliegende Arbeit orientiert sich trotzdem und mangels einer praktikablen Alternative im Wesentlichen an dieser Größe. Abweichend, und damit in diesem Detail präziser, wird jedoch Hypothese 4: Zinsaufwand zusätzlich alternativ geprüft, indem die angefallenen Zinsaufwendungen nur in Relation zu den korrespondierenden Passivpositionen 1, 2,3 und 9 gesetzt werden. ${ }^{416}$

\subsubsection{Durchschnittliche Bilanzsumme (DBS)}

In einigen Fällen geht es primär nicht darum, die Größe einer Bank zu ermitteln: Bei der Analyse von Angaben aus der Gewinn- und Verlustrechnung empfiehlt sich beispielsweise die Verwendung der jahresdurchschnittlichen Bilanzsumme (DBS). Dies wird von der DeUTSCHEN BUNDESBANK befürwortet und auch praktiziert. Als Vorteil wird die bessere Vergleichbarkeit von Größen aus der GuV-Statistik angeführt. ${ }^{417}$ Auch andere Autoren wie RICHTER ${ }^{418}$, CHRISTIANs $^{419}$ und RIEKEBERG ${ }^{420}$ empfehlen die DBS für diesen Anwendungsfall.

Das Konzept der durchschnittlichen Bilanzsumme ist durchaus schlüssig. In den meisten Fällen (85\%) wächst die Bilanzsumme einer Bank vom Bilanzstichtag des Vorjahres zum Bilanzstichtag des Berichtsjahres leicht an. In $15 \%$ der Fälle sinkt sie hingegen leicht. ${ }^{421}$ Alle Aufwendungen und Erträge fallen jedoch über das gesamte Berichtsjahr hinweg an. Daher ist es nicht angebracht, die Bilanzsumme des Berichtsjahres als Bezugsgröße zu

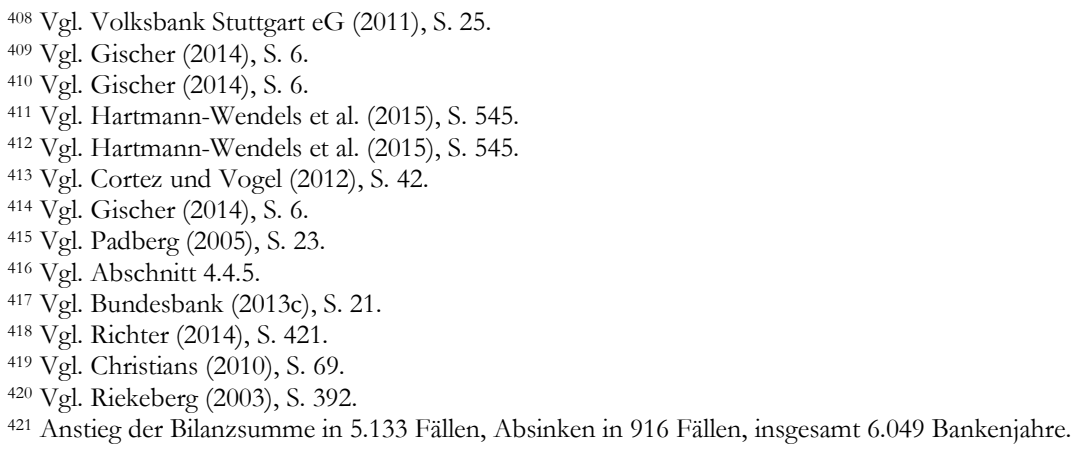


verwenden, denn hierdurch würde die tatsächliche durchschnittliche Bilanzgröße des Bezugszeitraums systematisch überschätzt. Auch bei Verwendung der Bilanzsumme des Vorjahres kommt es zu Verzerrungen, die Bilanzsumme des Bezugszeitraumes würde unterschätzt. Anstatt dieser beiden Varianten verwendet man also die durchschnittliche Bilanzsumme. Diese errechnet sich aus dem arithmetischen Mittel aller arbeitstäglichen Werte eines Jahres für die Bilanzsumme.

Jedoch wird diese durchschnittliche Bilanzsumme in der Regel nicht explizit veröffentlicht. Um dennoch einen validen Wert für die durchschnittliche Bilanzsumme eines Jahres $i$ zu erhalten, wird die folgende Rechenvorschrift verwendet:

$$
D B S_{i}=\frac{B S_{i-1}+B S_{i}}{2}
$$

Die DBS errechnet sich hiernach als das arithmetische Mittel aus der Bilanzsumme des Berichtsjahres $B S_{i}$ und der Bilanzsumme des Berichtsvorjahres $B S_{i-1}{ }^{422}$ Auch SCHIERENBECK empfiehlt die Berechnung der DBS als Durchschnitt von zwei Stichtagsbilanzen, also der Schlussbilanz des betreffenden Jahres und der Schlussbilanz des Vorjahres. $^{423}$

\subsubsection{Messgrößen für den Erfolg einer Bank}

Der Messung des Erfolges kommt in der vorliegenden Arbeit eine zentrale Bedeutung zu. Um den Erfolg der untersuchten Banken vergleichbar zu machen, wird in der vorliegenden Arbeit angestrebt, ein schlüssiges und funktionales Maß hierfür zu finden. Hierbei wird jedoch nicht ein System verschiedener Kennziffern favorisiert. Vielmehr steht eine einzelne Kennziffer zum Ziel, welche in der Folge eine große Menge an Informationen in verdichteter Form enthält. In diesem Falle von nur einer Kennzahl sind naturgemäß Schwächen gegenüber einem System mehrerer Kennzahlen zu erwarten. Trotz allem wird aus Gründen der Vergleichbarkeit die Verwendung einer einzelnen Kennzahl bevorzugt.

In der Literatur werden dazu die in den bankbetrieblichen Produktionsprozess eingehenden Faktoren einerseits (Input) und die hergestellten Bankprodukte andererseits (Output) betrachtet. Es wird darauf verwiesen, dass es bisher noch keine geschlossene Theorie über die Produktion einer Bank gibt. ${ }^{424} \mathrm{Im}$ Wesentlichen werden für empirische Arbeiten zwei verschiedene Ansätze verwendet, ${ }^{425}$ der Production Approach nach GILLIGAN ET AL. ${ }^{426}$ und der Intermediation Approach nach SEALEY und LINDLEY ${ }^{427}$. Beide Ansätze liefern deutlich unterschiedliche Ergebnisse für die Skaleneffizienz und die technische Effizienz. ${ }^{428}$

Der Production Approach zeichnet sich dadurch aus, dass er die Bank als einen Produzenten betrachtet. ${ }^{429}$ Als Produktionsfaktoren (Input) werden lediglich Arbeit und Sachkapital

\footnotetext{
422 Vgl. Riekeberg (2003), S. 419.

${ }^{423}$ Vgl. Schierenbeck (2003b), S. 427.

${ }^{424}$ Vgl. Humphrey (1992), Berger und Humphrey (1997), Berger und Mester (2003), zitiert nach Hartmann-Wendels et al. (2015), S. 53.

${ }^{425}$ Vgl. Hartmann-Wendels et al. (2015), S. 53.

${ }^{426}$ Vgl. Gilligan et al. (1984), S. 394 f.

${ }^{427}$ Vgl. Sealey und Lindley (1977), S. $1251 \mathrm{f}$.

${ }^{428}$ Vgl. Wutz (2000), S. 1.

${ }^{429}$ Vgl. Hartmann-Wendels et al. (2015), S. 53.
} 
eingesetzt. ${ }^{430}$ Die Bank erstellt daraus verschiedene Einlagekonten zur eigenen Refinanzierung und verschiedene Kreditkonten zur Mittelverwendung. ${ }^{431}$ Gemessen wird der Output anhand der Anzahl erstellter Konten oder anhand der Anzahl von Transaktionen je Konto. ${ }^{432}$ Die Höhe der Einlagen und der Kredite geht jedoch nicht in die Berechnung des Outputs ein, ebenso wenig die Höhe der erforderlichen Zinsaufwendungen auf der Refinanzierungsseite. ${ }^{433}$ Der monetäre Faktor wird somit durch dieses Modell nicht berücksichtigt, was im Widerspruch zu Abschnitt 3.5.2 steht; auch Fristen-, Losgrößen- und Risikotransformation werden ausgeklammert. ${ }^{434}$ Was die Messung des Outputs aller Banken auf Basis des Production Approachs schließlich unmöglich macht, ist die Tatsache, dass die zur externen Bilanzanalyse erforderlichen Angaben zur Anzahl der jeweiligen Konten nicht regelmäßig von den Instituten veröffentlicht werden. ${ }^{435}$

Der Intermediation Approach hingegen versteht den Produktionsprozess einer Bank als die Summe der beiden Teilleistungen Transformation und Intermediation. ${ }^{436}$ Als Produktionsfaktoren (Input) werden regelmäßig die Einlagen eingesetzt. ${ }^{437}$ Aus diesen stellt die Bank das Kreditvolumen her und legt einen Wertpapierbestand an. Hierfür fallen zur Refinanzierung Zinsaufwendungen im Wertbereich und Verwaltungsaufwendungen im Betriebsbereich an. Jedoch unterscheiden sich die verschiedenen möglichen Aktivpositionen zum Teil deutlich hinsichtlich des Aufwandes bei Anschaffung und Verwaltung. Als Beispiele seien einerseits eine Bundesanleihe genannt, die leicht erhältlich und verwaltbar ist, und andererseits ein Immobiliendarlehen, bei dem der Aufwand in jeder Hinsicht höher liegen dürfte. ${ }^{438}$ Auch resultieren unterschiedlich hohe Aufwendungen aus der Frage, ob das Kreditportfolio eines Instituts eher granular auf sehr viele Kreditnehmer aufgeteilt ist, oder ob es eher dazu neigt, wenige, aber dafür sehr großvolumige Darlehen zu vergeben. ${ }^{439}$

Die Mehrzahl der Effizienzuntersuchungen im Bankbereich greift auf den Intermediation Approach zurück. Hier sind die auszuwertenden Daten leichter bzw. - im Unterschied zum Production Approach - überhaupt für eine externe Analyse erhältlich. ${ }^{440}$ Auch die vorliegende Arbeit setzt den Intermediation Approach ein. Unberücksichtigt bleiben hier jedoch die einzelnen Transformationsleistungen von Risiko und Fristigkeiten. ${ }^{441}$ Auch einzelne Bankdienstleistungen, welche über das klassische Einlagen- und Kreditgeschäft hinausgehen, können durch den Intermediation Approach nicht erfasst werden. ${ }^{442}$ Aufgrund der steigenden Bedeutung des Dienstleistungsgeschäftes gibt es in der Literatur mittlerweile Ansätze zur Berücksichtigung von Provisionen aus Serviceleistungen und von Erträgen aus dem nichtzinsabhängigen Geschäft. ${ }^{443}$ Selbst die Losgrößentransformation kann durch den Intermediation Approach nicht abgebildet werden, da sowohl die Einlagen auf der Seite der Mittelherkunft als auch die Darlehen auf der Seite der Mittelverwendung lediglich aggregiert vorliegen.

\footnotetext{
${ }^{430}$ Vgl. Hartmann-Wendels et al. (2015), S. 53.

${ }^{431}$ Vgl. Hartmann-Wendels et al. (2015), S. 53.

432 Vgl. Hartmann-Wendels et al. (2015), S. 53.

${ }^{433}$ Vgl. Hartmann-Wendels et al. (2015), S. 53.

${ }^{434}$ Vgl. Hartmann-Wendels et al. (2015), S. 53.

435 Vgl. Hartmann-Wendels et al. (2015), S. 53.

${ }^{436}$ Vgl. Hartmann-Wendels et al. (2015), S. 54.

${ }^{437}$ Vgl. Hartmann-Wendels et al. (2015), S. 54

438 Vgl. Hartmann-Wendels et al. (2015), S. 54.

${ }^{439}$ Vgl. Hartmann-Wendels et al. (2015), S. 54.

${ }^{440}$ Vgl. Hartmann-Wendels et al. (2015), S. 54.

${ }^{441}$ Vgl. Wimmer (2013), S. $111 \mathrm{f}$.

442 Vgl. Wutz (2000), S. 4.

${ }^{443}$ Vgl. Hunter und Timme (1995), S. 165 f.; Lang und Welzel (1996), S. 1003 f.; beide zitiert nach Wutz (2000), S. 4.
} 
Wie bereits oben in Abschnitt 3.1.2 angedeutet, existieren verschiedene Maße für den Erfolg eines Unternehmens. Es soll diskutiert werden, welche Größe zur Beurteilung des geschäftlichen Erfolges von Genossenschaftsbanken zur Anwendung kommt. In der Literatur werden mehrere Größen vorgeschlagen. Selbst ein erwünschtes Bilanzsummenwachstum im Vergleich zum Vorjahr, etwa begründet durch einen gewachsenen Bestand an Einlagen, kann theoretisch als ein Erfolg bewertet werden. Folgend wird ein Kriterienkatalog aufgestellt, um die gesuchte Größe näher einzugrenzen. Idealerweise weist diese Größe zur Erfolgsmessung folgende Eigenschaften auf:

1. Es handelt sich um eine einzelne Größe.

2. Die Größe ist generell geeignet, als Proxy für den geschäftlichen Erfolg zu dienen.

3. Es gibt nur geringe Möglichkeiten, die Höhe der Größe im Rahmen der Jahresabschlusspolitik zu beeinflussen. ${ }^{444}$

4. Eine Vergleichbarkeit der Größe über alle untersuchten Individuen hinweg ist gewährleistet.

\subsubsection{Bruttogewinnspanne}

In der vorliegenden Arbeit soll zur Messung des geschäftlichen Erfolges der Bruttogewinn aus normaler Geschäftstätigkeit verwendet werden. ${ }^{445}$ Es handelt sich dabei um eine Maßzahl aus dem externen Rechnungswesen. Dessen Aufgabe ist es generell, Informationen für Personen oder Institutionen bereitzustellen, welche nicht $\mathrm{zu}$ dem jeweiligen Unternehmen selbst gehören. ${ }^{446}$ In diesem Zuge werden hauptsächlich die Bilanz und die Gewinn- und Verlustrechnung erstellt, wobei umfangreiche gesetzliche Anforderungen zu berücksichtigen sind. ${ }^{447}$ Als Rechnungsgrößen im externen Rechnungswesen generell, und auch zur Ermittlung der Bruttogewinnspanne speziell, werden Aufwendungen und Erträge der Gewinn- und Verlustrechnung herangezogen.

Die Bruttogewinnspanne ähnelt dem Betriebsergebnis vor Bewertung aus dem internen Rechnungswesen der Kreditinstitute. Dieses dient hingegen der Information von Führungskräften und Mitarbeitern des Unternehmens und kann - von einigen Ausnahmen abgesehen - unabhängig von rechtlichen Regelungen durchgeführt werden. ${ }^{448}$ Speziell im hier thematisch verwandten Teilgebiet der Kostenrechnung werden Kosten und Erlöse als Rechnungsgrößen betrachtet. Bei dem Betriebsergebnis vor Bewertung handelt es sich um eine entscheidende Kennziffer für die nachhaltige Ertragskraft eines Instituts. ${ }^{449}$ Der Einsatz des Betriebsergebnisses vor Bewertung als Indikator für den Erfolg von Banken ist in der Praxis und auch in der Literatur sehr gebräuchlich. Verwendet wird es von der BUNDESBANK ${ }^{450}$ zur Erstellung von Statistiken. Auch CHRISTIANs ${ }^{451}$ und RIEKEBERG ${ }^{452}$ setzen das Betriebsergebnis vor Bewertung in das Verhältnis zur durchschnittlichen Bilanzsumme. Die Verwendung dieser Kennzahl zur Erfolgsbeurteilung empfehlen auch GiSCHER ${ }^{453}$ sowie HOLTMANN und MORALES. ${ }^{454}$ Eben-

\footnotetext{
${ }^{444}$ Vgl. Abschnitt 3.6.5.

${ }^{445}$ Vgl. Schierenbeck (2003b), S. 425

446 Vgl. Götze (2010), S. 3.

447 Vgl. Götze (2010), S. 3

448 Vgl. Götze (2010), S. 3.

${ }^{449}$ Vgl. Bremke, Wagener und West (2004), S. 303.

${ }^{450} \mathrm{Vgl}$. Bundesbank (2013c), S. 13.

${ }^{451}$ Vgl. Christians (2010), S. 69.

${ }_{452}$ Vgl. Riekeberg (2003), S. 387 und 392.

${ }^{453}$ Vgl. Gischer (2014), S. 7.
} 
so raten BOTSIS ET AL. zum Einsatz des Betriebsergebnisses vor Bewertung, unter anderem auch als Kreditrisikoindikator. ${ }^{455}$ HUMMEL und PEDROTTI würden einen anderen Effizienzmaßstab als die Cost-Income-Ratio einsetzen, um die Erklärungskraft ihrer Modelle zu erhöhen. ${ }^{456}$ Dies könnte das Betriebsergebnis vor Bewvertung sein.

Neben der Tatsache, dass das Betriebsergebnis vor Bewertung nur durch interne Angaben der Bank (Kosten und Erlöse) ermittelt werden kann, unterscheidet es sich in einigen Punkten von der Bruttogewinnspanne, welche auf Aufwendungen und Erträgen basiert. Zunächst stellen die sogenannten neutralen Erträge und Aufwendungen keine Erlöse und Kosten dar. ${ }^{457}$ Hierzu zählen erstens betriebsfremde (das heißt nicht zum Kerntätigkeitsbereich des Unternehmens gehörige) Erträge und Aufwendungen. Zweitens zählen periodenfremde Erträge und Aufwendungen ebenfalls nicht zu den Erlösen und Kosten ${ }^{458}$, jedoch bleiben diese aufgrund ihrer Periodenfremdheit in der Gewinn- und Verlustrechnung der Bezugsperiode ohnehin unberücksichtigt. ${ }^{459}$ Drittens stellen außerordentliche (das heißt durch einen auBerordentlichen Vorgang oder in außerordentlicher Höhe entstehende) Erträge und Aufwendungen keine Erlöse und Kosten dar. ${ }^{460}$ Andererseits dürfen sogenannte Zusatzerlöse und -kosten nicht als Erträge und Aufwendungen angesetzt werden. ${ }^{461}$ Des Weiteren sind in einigen Fällen unterschiedliche Wertansätze im externen und internen Rechnungswesen möglich, beispielsweise bei Abschreibungen. ${ }^{462}$ Aus Vereinfachungsgründen wird von den eben skizzierten Unterschieden zwischen den Rechengrößen des externen und internen Rechnungswesens abstrahiert. Obgleich damit das Betriebsergebnis vor Bewertung und die Bruttogewinnspanne als Synonyme betrachtet werden könnten, verwendet die vorliegende Arbeit - mit Blick auf die Herkunft der verwendeten Daten - die im externen Rechnungswesen verwurzelte Bruttogewinnspanne.

Es folgt die Beurteilung der Bruttogewinnspanne anhand des in Abschnitt 3.5.3 ausgearbeiteten Kriterienkatalogs.

Ad 1. Die Bruttogewinnspanne lässt sich in einer einzigen Größe ausdrücken.

Ad 2. Die Bruttogewinnspanne beinhaltet sämtliche wesentlichen Erträge und Aufwendungen des Kerngeschäftes. Sie setzt sich ferner aus den in Abbildung 9 dargestellten Komponenten zusammen. Hierbei wurde auf die Nummerierung gemäß Formblatt 3 zur Kreditinstituts-Rechnungslegungsverordnung - Gewinn- und Verlustrechnung in der Staffelform abgestellt. ${ }^{463}$

\footnotetext{
${ }^{454}$ Vgl. Holtmann und Morales (2010), S. 172.

455 Vgl. Botsis, Rock und Kaiser (2012), S. 115

456 Vgl. Hummel und Pedrotti (2013), S. 356.

${ }^{457}$ Vgl. Götze (2010), S. 7.

458 Vgl. Götze (2010), S. 7; vgl. Wöhe und Döring (2013), S. 647 f.

${ }^{459}$ Vgl. Götze (2010), S. 7.

${ }^{460} \mathrm{Vgl}$. Götze (2010), S. 7.

461 Vgl. Götze (2010), S. 7

462 Vgl. Götze (2010), S. 7 f

${ }^{463}$ Vgl. BaFin (2012b).
} 

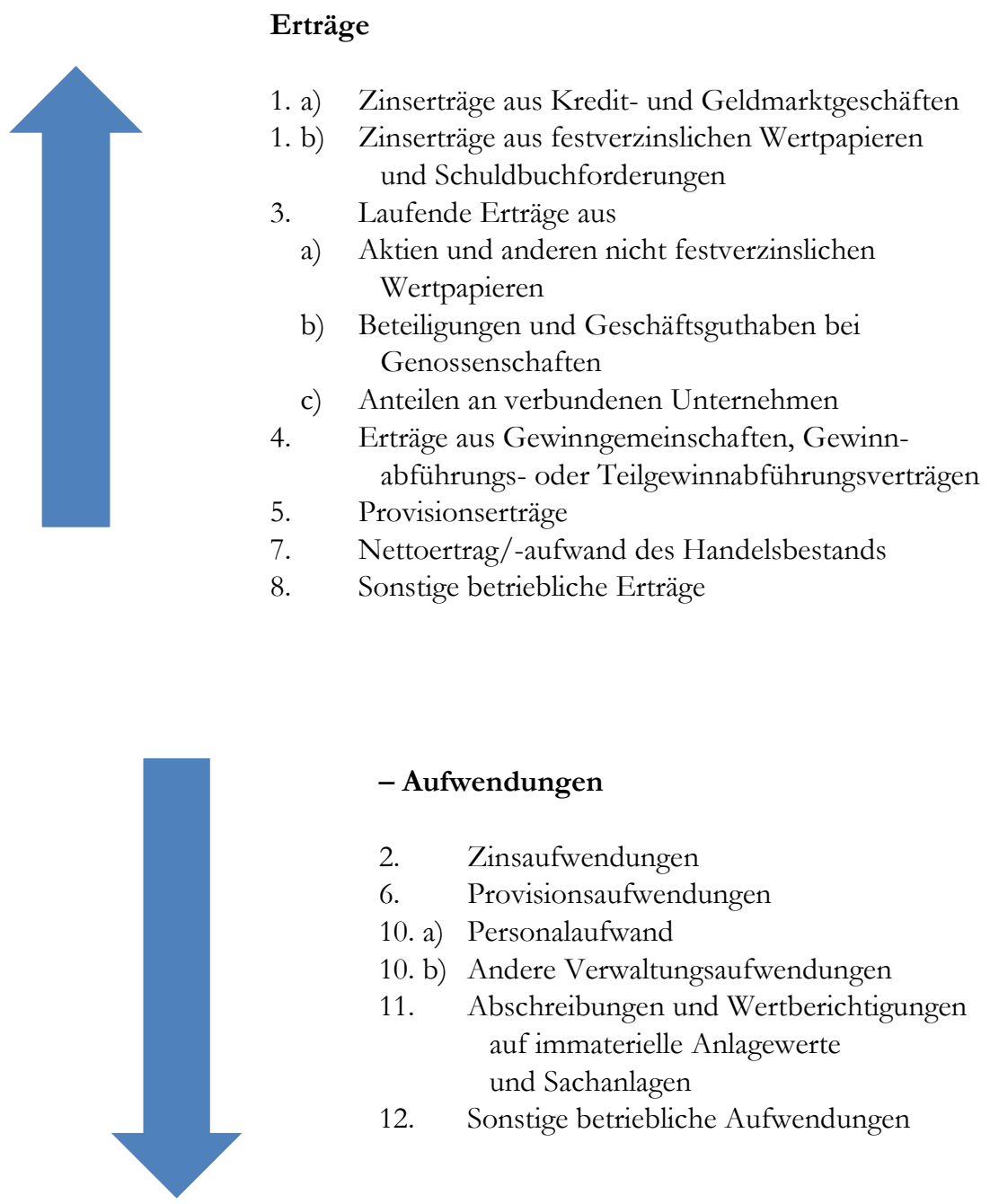

\author{
- Aufwendungen \\ 2. Zinsaufwendungen \\ 6. Provisionsaufwendungen \\ 10. a) Personalaufwand \\ 10. b) Andere Verwaltungsaufwendungen \\ 11. Abschreibungen und Wertberichtigungen \\ auf immaterielle Anlagewerte \\ und Sachanlagen \\ 12. Sonstige betriebliche Aufwendungen
}

\title{
Abbildung 9: Ermittlung des Bruttogewinns aus normaler Geschäftstätigkeit
}

Ad 3. Um eine möglichst hohe Vergleichbarkeit zwischen den einzelnen Instituten herzustellen, sollen für die Größe möglichst geringe Beeinflussungsmöglichkeiten durch die einzelne Bank bestehen. Daher sind folgende Positionen ausdrücklich nicht in der Berechnung der Bruttogewinnspanne enthalten:

- 13. Abschreibungen und Wertberichtigungen auf Forderungen und bestimmte Wertpapiere sowie Zuführungen zu Rückstellungen im Kreditgeschäft

- 14. Erträge aus Zuschreibungen zu Forderungen und bestimmten Wertpapieren sowie aus der Auflösung von Rückstellungen im Kreditgeschäft

- 15. Abschreibungen und Wertberichtigungen auf Beteiligungen, Anteile an verbundenen Unternehmen und wie Anlagevermögen behandelte Wertpapiere

- 16. Erträge aus Zuschreibungen zu Beteiligungen, Anteilen an verbundenen Unternehmen und wie Anlagevermögen behandelten Wertpapieren

- 17. Aufwendungen aus Verlustübernahme 
Ad 4. Die Bruttogewinnspanne wird im Rahmen dieser Arbeit in das Verhältnis zur jeweiligen durchschnittlichen Bilanzsumme gesetzt. Auch werden andere Positionen der Gewinn- und Verlustrechnung und der Bilanz in das Verhältnis zur durchschnittlichen Bilanzsumme bzw. zur Bilanzsumme gesetzt. DBS bzw. Bilanzsumme dienen bei der Auswertung als Normierungsinstrument. Die hierbei ermittelten Relationen werden auch als Verhältniszahlen bezeichnet. ${ }^{464}$

Die Bruttogewinnspanne (BGS) als Verhältniszahl setzt den Bruttogewinn aus normaler Geschäftstätigkeit in Relation zur durchschnittlichen Bilanzsumme. ${ }^{465}$

$$
\text { Bruttogewinnspanne }=\frac{\text { Bruttogewinn aus normaler Geschäftstätigkeit }}{D B S}
$$

Alternative gebräuchliche Bezeichnungen für die Bruttogewinnspanne sind laut BOTSIS Teilbetriebsergebnisspanne oder Ertragsspanne. ${ }^{466}$ Nicht zuletzt diese Quelle belegt die in der Literatur bisweilen nicht vollständig konsistent vorgenommene Abgrenzung zwischen Angaben aus dem internen und externen Rechnungswesen.

\subsubsection{Cost-Income-Ratio}

Ebenso wie die Bruttogewinnspanne ist auch die Cost-Income-Ratio (CIR) eine entscheidende Kennziffer zur Messung der nachhaltigen Ertragskraft von Banken. ${ }^{467}$ Es handelt sich um eine Beziehungszahl. ${ }^{468}$ CHRISTIANs schätzt besonders an der CIR, dass diese Effizienzkennzahl bilanzpolitisch nicht manipulierbar sei, ${ }^{469}$ wie es etwa das Betriebsergebnis aus normaler Geschäftstätigkeit aufgrund der abgezogenen Risikoaufwendungen wäre, oder auch der Reingewinn vor Steuern aufgrund des berücksichtigten außerordentlichen Ergebnisses. Die CIR ist ein Indikator für die Effizienz von Unternehmen, und setzt den Allgemeinen Verwaltungsaufwand in das Verhältnis zu den operativen Erträgen. Deshalb spricht man im Deutschen auch vom Aufwand-Ertrag-Verhältnis. ${ }^{470}$

$$
C I R=\frac{\text { Aufwand }}{\text { Ertrag }}
$$

Je geringer dieses Verhältnis ausfällt, umso weniger Aufwand muss eine Bank betreiben, um einen Euro an Ertrag zu generieren. Aus diesem Grund sind kleinere Werte als vorteilhaft anzusehen.

Der folgende Abschnitt soll eruieren, wie der Wert der Cost-Income-Ratio auf Veränderungen der Eingangsgrößen reagiert. Bei aufwandsseitigen Veränderungen (im Zähler) ändert sich die Cost-Income-Ratio linear: Eine Aufwandssteigerung um $1 \%$ führt zu einer CIR-

\footnotetext{
464 Vgl. Abschnitt 3.5.1.

${ }^{465} \mathrm{Vgl}$. Schierenbeck (2003b), S. 425

${ }^{466}$ Vgl. Botsis et al. (2012), S. 81.

467 Vgl. Bremke et al. (2004), S. 303.

${ }^{468}$ Vgl. Abschnitt 3.5.1.

${ }^{469}$ Vgl. Christians (2010), S. 144.

${ }^{470}$ Vgl. Bundesbank (2012b), S. 1.
} 
Steigerung von $1 \%$. Dies lässt sich auch analytisch über die Ableitung der CIR nach dem Aufwand begründen:

$$
\frac{\partial C I R}{\partial A u f w a n d}=\frac{1}{\text { Ertrag }}
$$

Das Resultat ist eine Konstante, welche 1 im Zähler und den Ertrag im Nenner enthält. Diese Konstante repräsentiert die Steigung der Geraden, welche die Abhängigkeit der CIR vom Aufwand abbildet.

Hingegen ist der Zusammenhang bei ertragsseitigen Veränderungen (im Nenner) nicht linear. Bei einer Erhöhung der Erträge um $1 \%$ verringert sich die Cost-Income-Ratio um mehr als $1 \%$. Bei größeren Steigerungsraten bzw. bei der Betrachtung höherer Unterschiede fällt die Abweichung entsprechend deutlicher aus. Liegen die Erträge von Institut A ceteris paribus - um $25 \%$ über denen von Institut B, so liegt $C_{I} R_{B}$ nur um $20 \%$ ter $C I R_{A}$ :

$$
C I R_{B}=\frac{1}{1+25 \%}=0,80
$$

Weist Institut B jedoch - ceteris paribus - um $25 \%$ kleinere Aufwendungen aus als Institut A, so liegt $C I R_{B}$ gar um $33 \%$ über $C I R_{A}$ :

$$
C I R_{B}=\frac{1}{1-25 \%}=1,33
$$

Als analytische Begründung folgt die Ableitung der CIR nach dem Ertrag:

$$
\frac{\partial C I R}{\partial E r t r a g}=-\frac{\text { Aufwand }}{\text { Ertrag }}
$$

In diesem Fall besteht die Ableitung sowohl aus einer Konstanten im Zähler als auch aus der Variablen Ertrag, nach der abgeleitet wurde, im Nenner. Die Höhe der Erträge spielt also eine Rolle, indem sie umgekehrt quadratisch in das Differential eingeht. Bei hohen Erträgen ist die Steigung betragsmäßig gering und negativ. Bei geringen Erträgen wird die Ableitung vom Betrag her größer, bleibt jedoch negativ. Die beiden beispielhaft errechneten CIR-Werte für um $25 \%$ gestiegene bzw. um $25 \%$ gefallene Erträge konnten daher analytisch untermauert werden.

Kurzum erfasst die CIR-Kennzahl Aufwandsänderungen linear. Bei Ertragsänderungen tritt hingegen eine Hebelwirkung ein: Ertragserhöhungen senken die CIR unterlinear, Ertragssenkungen steigern die CIR überlinear. Die CIR kann daher zu den vorsichtigen Erfolgsmaßen gezählt werden. 
Es soll nun genauer diskutiert werden, welche Positionen der Gewinn- und Verlustrechnung genau in die Ermittlung der Cost-Income-Ratio einfließen - denn hierüber herrscht keineswegs Eintracht. Selbst von der DEUTSCHEN BundESBANK werden regelmäßig, im Bericht Zur Ertragslage der deutschen Kreditinstitute, zwei Varianten der Cost-Income-Ratio veröffentlicht. ${ }^{471}$ Variante 1 verwendet die Rechenvorschrift

$$
C I R_{1}=\frac{\text { Allgemeine Verwaltungsaufwendungen }}{\text { Rohertrag }}
$$

Die Allgemeinen Verwaltungsaufwendungen im Zähler beziehen sich auf die Position 10 der Gewinn- und Verlustrechnung. Diese untergliedert sich in 10. a) Personalaufwand und in 10. b) andere Verwaltungsaufwendungen. Der Rohertrag im Nenner setzt sich zusammen aus Zinsüberschuss (GuV-Position 1 abzgl. GuV-Position 2) und Provisionsüberschuss (GuVPosition 5 abzgl. GuV-Position 6). ${ }^{472}$ Die CIR $_{1}$ der Kreditgenossenschaften für das Jahr 2011 beträgt $65,5 .^{473}$

Variante 2 der CIR errechnet sich wie gemäß

$$
C I R_{2}=\frac{\text { Allgemeine Verwaltungsaufwendungen }}{\text { Operative Erträge }} .
$$

Während die Allgemeinen Verwaltungsaufwendungen im Zähler zur CIR-Variante 1 identisch sind, unterscheidet sich der Nenner. Statt lediglich den aus Zins- und Provisionsüberschuss bestehenden Rohertrag anzusetzen, verwendet Variante 2 zusätzlich noch sämtliche Ertragskomponenten des operativen Geschäfts. ${ }^{474}$ Dazu zählen also noch das Nettoergebnis des Handelsbestands (GuV-Position 7) und sonstige betriebliche Erträge (GuV-Positionen $4,8,9,12,15,16,17,18)$. Aus diesem Grund fallen die nach Variante 2 errechneten CIRWerte stets etwas geringer aus, die $\mathrm{CIR}_{2}$ der Kreditgenossenschaften für das Jahr 2011 beträgt $63,9 .{ }^{475}$ CHRISTIANS orientiert sich an Variante $2 .{ }^{476}$

Burger ET AL. kritisieren, dass Preiskomponenten, wie Zinssätze, Provisionen und Faktorkosten, die Ermittlung der Erträge und der Aufwendungen beeinflussen und die Aussagekraft der CIR somit stark verzerren. ${ }^{477}$ Beispielsweise werden die Erträge berechnet, indem Absatz- oder Bestandsmengen mit Preisen bewertet werden. ${ }^{478}$ Jedoch folgen Preise den Marktbedingungen, und höhere Preise seien kein Indiz für höhere Produktivität. ${ }^{479}$ Besser wäre demnach eine Betrachtung der reinen Absatz- und Bestandsmengen, wie

${ }^{471}$ Vgl. Bundesbank (2014a), S. 1.

472 Vgl. Bundesbank (2014a), S. 1.

473 Vgl. Bundesbank (2014a), S. 1.

${ }^{474}$ Vgl. Burger et al. (2008), S. 7.

475 Vgl. Bundesbank (2014a), S. 1.

476 Vgl. Christians (2010), S. 133.

477 Vgl. Burger et al. (2008), S. 7.

${ }^{478}$ Vgl. Burger et al. (2008), S. 7.

${ }^{479}$ Vgl. Burger et al. (2008), S. 7 f. 
bereits oben empfohlen. ${ }^{480}$ Aufgrund fehlender öffentlich verfügbarbarer Informationen ist jedoch die Ermittlung einer mengenorientierten Produktivität kaum möglich. ${ }^{481}$

Eine hohe Cost-Income-Ratio repräsentiert auf den ersten Blick eine geringe Effizienz. Jedoch kann in ihr auch eine hohe Fairness in der Preisgestaltung gesehen werden. Dies ist dann der Fall, wenn die Banken ihre Produkte mit Preisen versehen, die nur moderat über den anfallenden Kosten liegen, und die Gewinnmarge somit gering halten. Speziell bei der Anwendung auf Genossenschaftsbanken und dem von ihnen zu erfüllenden Auftrag ist dieser Einfluss nicht zu unterschätzen.

Es folgt die Prüfung, ob die Kriterien 1 bis 4 des Abschnittes 3.5.3 von der Cost-IncomeRatio erfüllt werden.

Ad 1. Die Cost-Income-Ratio lässt sich in einer einzigen Größe ausdrücken.

Ad 2. Die Cost-Income-Ratio errechnet sich je nach Variante aus den bedeutendsten oder aus sämtlichen wesentlichen Erträgen und Aufwendungen des Kerngeschäftes. Sie setzt sich aus den Aufwendungen im Zähler und den Erträgen im Nenner zusammen. Zu den Aufwendungen zählen:

- 2. Zinsaufwendungen,

- 6. Provisionsaufwendungen,

- 10. a) Personalaufwand,

- 10. b) Andere Verwaltungsaufwendungen,

- 11. Abschreibungen und Wertberichtigungen auf immaterielle Anlagewerte und Sachanlagen sowie

- 12. Sonstige betriebliche Aufwendungen.

Zu den Erträgen zählen:

- 1. a) Zinserträge aus Kredit- und Geldmarktgeschäften,

- 1. b) Zinserträge aus festverzinslichen Wertpapieren und Schuldbuchforderungen,

- 3. Laufende Erträge aus

a) Aktien und anderen nicht festverzinslichen Wertpapieren,

b) Beteiligungen und Geschäftsguthaben bei Genossenschaften,

c) Anteilen an verbundenen Unternehmen,

- 4. Erträge aus Gewinngemeinschaften, Gewinnabführungs- oder Teilgewinnabführungsverträgen,

- 5. Provisionserträge,

- 7. Nettoertrag/-aufwand des Handelsbestands sowie

- 8. Sonstige betriebliche Erträge.

${ }^{480} \mathrm{Vgl}$. Abschnitt 3.5.2.

${ }^{481}$ Vgl. Burger et al. (2008), S. 9. 
Wie oben wurde hier auf die Nummerierung gemäß Formblatt 3 zur KreditinstitutsRechnungslegungsverordnung - Gewinn- und Verlustrechnung in der Staffelform abgestellt. $^{482}$

Ad 3. Wie bereits bei der Bruttogewinnspanne sind auch bei der Cost-Income-Ratio folgende Positionen ausdrücklich nicht in der Berechnung enthalten, um Beeinflussungsmöglichkeiten zu minimieren:

- 13. Abschreibungen und Wertberichtigungen auf Forderungen und bestimmte Wertpapiere sowie Zuführungen zu Rückstellungen im Kreditgeschäft,

- 14. Erträge aus Zuschreibungen zu Forderungen und bestimmten Wertpapieren sowie aus der Auflösung von Rückstellungen im Kreditgeschäft,

- 15. Abschreibungen und Wertberichtigungen auf Beteiligungen, Anteile an verbundenen Unternehmen und wie Anlagevermögen behandelte Wertpapiere,

- 16. Erträge aus Zuschreibungen zu Beteiligungen, Anteilen an verbundenen Unternehmen und wie Anlagevermögen behandelten Wertpapieren sowie

- 17. Aufwendungen aus Verlustübernahme.

Ad 4. Die Vergleichbarkeit über verschiedene Institute hinweg ist bei der Cost-Income-Ratio bereits per se gewährleistet. Eine Normierung auf Basis der durchschnittlichen Bilanzsumme ist also nicht erforderlich.

Somit werden alle genannten Kriterien von der Cost-Income-Ratio erfüllt. Dennoch bleibt insgesamt die mangelnde wissenschaftliche Fundierung der CIR festzuhalten. Ein Vergleich von Instituten erscheint lediglich bei identischem Geschäftsvolumen und bei identischer Struktur sinnvoll. Daher setzt die vorliegende Arbeit bei dem Erfolgsmaß auf den Bruttogewinn aus normaler Geschäftstätigkeit und die daraus ermittelte Bruttogewinnspanne.

\subsubsection{Margen und Spannen}

Die Marge bildet die zentrale Informationsquelle bei der Kalkulation zinsabhängiger Bankgeschäfte. ${ }^{483}$ Sie kann sowohl auf Ebene eines einzelnen Geschäftes, auf Ebene einer Geschäftseinheit als auch auf Ebene der gesamten Bank bestimmt werden. ${ }^{484}$

In der Literatur werden die Begriffe Marge und Spanne weitestgehend synonym verwendet. Eine Spanne setzt in aller Regel eine GuV-Position in das Verhältnis zur durchschnittlichen Bilanzsumme. Einzelne Beispiele werden in Abschnitt 3.6.3 erläutert.

\subsection{Jahresabschluss}

Der Jahresabschluss ist das zentrale Element der externen Rechnungslegung für Banken. ${ }^{485}$ $\mathrm{Zu}$ unterscheiden ist zwischen dem steuerrechtlichen und dem handelsrechtlichen Jahresabschluss (HGB), welche durch abweichende Ansatz- und Bewertungsvorschriften gekennzeichnet sind. ${ }^{486}$ Alle folgenden Ausführungen beziehen sich auf den handelsrechtli-

\footnotetext{
${ }^{482}$ Vgl. BaFin (2012b).

${ }^{483}$ Vgl. Schierenbeck, Lister und Kirmße (2014), S. 51.

${ }^{484}$ Vgl. Schierenbeck et al. (2014), S. 51.

485 Vgl. Becker und Peppmeier (2013), S. 540.

${ }^{486}$ Vgl. Becker und Peppmeier (2013), S. 540.
} 
chen Abschluss. ${ }^{487}$ Des Weiteren muss zwischen Einzel- und Konzernabschluss unterschieden werden. ${ }^{488}$ Die vorliegende Arbeit befasst sich mit den einzelnen Kreditgenossenschaften und konzentriert sich auf den Einzelabschluss. ${ }^{489}$ Zur Identifikation der mit der Rechnungslegung einhergehenden Pflichten kommen folgende Rechtsvorschriften zur Anwendung:

1. Genossenschaftsbanken gelten nach $\ 6$ Abs. 2 HGB als Kaufmann kraft Rechtsform und fallen daher unter das Handelsrecht. In Bezug auf die Erstellung des Jahresabschlusses gelten somit als erstes die Regelungen der $\iint 238$ bis 263 HGB.

2. Alle Kreditinstitute müssen im Sinne des $₫ 1$ Abs. 1 KWG nach $\ 340 a$ Abs. 1 HGB die Vorschriften der $\$ \int 264$ bis 289a HGB anwenden, die für große Kapitalgesellschaften gelten. Dies gilt unabhängig von Größe und Rechtsform.

3. Es gelten die ergänzenden Vorschriften für Kreditinstitute und Finanzdienstleitungsinstitute nach $\int 340$ bis 340 o HGB.

4. Die Verordnung über die Rechnungslegung der Kreditinstitute und Finanzdienstleistungsinstitute (RechKredV) ist zu beachten.

Gemäß \ 242 Abs. 3 HGB besteht der Jahresabschluss aus der Bilanz und der Gewinn- und Verlustrechnung. Jeder Kaufmann, zu denen im handelsrechtlichen Sinne auch die Kreditinstitute zählen, hat nach $\ 242$ Abs. 1 HGB ,zu Beginn seines Handelsgewerbes und für den Schluss eines jeden Geschäftsjahrs" einen Jahresabschluss aufzustellen. Dabei sollen Eröffnungs- und Schlussbilanz das Verhältnis zwischen sämtlichen Vermögens- und Schuldgegenständen darstellen. ${ }^{400}$ Eine Gewinn- und Verlustrechnung nach \242 Abs. 2 HGB hat der Kaufmann für den Schluss eines jeden Geschäftsjahrs als Gegenüberstellung der Aufwendungen und Erträge des Geschäftsjahrs aufzustellen.

Laut $\ 264$ Abs. 2 Satz 1 haben Kapitalgesellschaften den Jahresabschluss um einen Anhang zu erweitern. Da nach \340a Abs. 1 HGB für Kreditinstitute die Vorschriften für große Kapitalgesellschaften gelten, greift diese Pflicht zur Erweiterung um den Anhang ebenso für Kreditgenossenschaften. Weil die Vorschriften für große Kapitalgesellschaften zur Anwendungen kommen, gelten die Erleichterungen für kleine oder mittelgroße Kapitalgesellschaften nach $\ 288$ HGB nicht, wonach auf einige Angaben verzichtet werden könnte. $\mathrm{Da}$ es sich um ein Kreditinstitut handelt, muss der Anhang um weitere Angaben ergänzt werden. Ein Beispiel bildet der $\int 340$ a Abs. 4 HGB.

Nach \340a Abs. 1 Satz 2 HGB haben Kreditinstitute außerdem einen Lagebericht gemäß der Bestimmungen des $₫ 289$ HGB neben dem Jahresabschluss aufzustellen.

Jahresabschluss und Lagebericht von Genossenschaftsbanken müssen nach $\ 340 \mathrm{k}$ Abs. 2 HGB, unabhängig von der Größe der Institute, von einem Prüfungsverband geprüft werden. Die Prüfung wird von dem Prüfungsverband durchgeführt, in dem das Institut Mitglied ist. ${ }^{491}$

\footnotetext{
487 Vgl. Becker und Peppmeier (2013), S. 540.

488 Vgl. Becker und Peppmeier (2013), S. 540.

${ }^{489}$ Vgl. Becker und Peppmeier (2013), S. 540.

490 Vgl. \ 242 Abs. 1 Satz 2 HGB.

${ }^{491}$ Vgl. $\int 340 \mathrm{k}$ Abs. 2 HGB.
} 


\section{Funktionen des Jahresabschlusses}

Der Jahresabschluss erfüllt Informations-, Gläubigerschutz- und Ausschüttungsbemessungsfunktionen. ${ }^{492} \mathrm{Zu}$ den grundlegenden Informationsfunktionen zählen einerseits die Schuldendeckungsfähigkeit (Fähigkeit zur Deckung der Schulden durch Vermögen zu einem Stichtag) und andererseits die Ermittlung des Periodenerfolgs (Differenz zwischen Erträgen und Aufwendungen eines Geschäftsjahres) zur Kontrolle der Entwicklung der Rentabilität. ${ }^{493}$ Zur Gläubigerschutzfunktion zählen das Vorsichtsprinzip, nach welchem die Lage nicht besser dargestellt werden darf, als sie tatsächlich ist, und bilanzpolitische Handlungsspielräume, etwa nach \340f HGB zur Bildung stiller Reserven im Rahmen der Risikovorsorge. ${ }^{494}$ Die Ausschüttungsbemessungsfunktion wird erfüllt, indem im ersten Schritt der Erfolg der Periode ermittelt wird, und im zweiten Schritt über die Verwendung des Ergebnisses, also Höhe der Ausschüttung bzw. Thesaurierung, entschieden wird. ${ }^{495}$

\subsubsection{Bilanzaktiva}

Auf der Aktivseite werden die Vermögensgegenstände des Kreditinstitutes zum letzten Tag eines Geschäftsjahres erfasst. ${ }^{496}$ Es lässt sich daraus die Verwendung der finanziellen Mittel ersehen, in einem Zeitpunkt betrachtet. ${ }^{497}$ Die Bilanzgliederung für Kreditinstitute weicht von jener nach $\int 266$ HGB für Nichtbanken ab. Für Kreditinstitute erfolgt zum einen keine Differenzierung zwischen Anlage- und Umlaufvermögen. Zum anderen richtet sich bei Kreditinstituten die Reihenfolge der Aktiva nach dem Grad ihrer Liquidität. Die liquideste Position 1. Barreserve beinhaltet sowohl den Kassenbestand als auch die Guthaben bei der Deutschen BundesBAnk. ${ }^{498}$ Tabelle 2 enthält die Aktiva der Bankbilanz gemäß Formblatt $1 .{ }^{499}$

\subsubsection{Bilanzpassiva}

Die Passiva werden, ebenso wie die Aktiva, am letzten Tag eines Geschäftsjahres im Rahmen einer Zeitpunktbetrachtung erfasst und nach ihrer Fälligkeit geordnet, wobei die am frühesten fälligen Positionen zuerst aufgeführt werden. ${ }^{500}$ Position 1 sind hier die Verbindlichkeiten gegenüber Kreditinstituten, unter die in a) täglich fällig und b) mit vereinbarter Laufzeit oder Kündigungsfrist eingeteilt werden. Es folgen 2. Verbindlichkeiten gegenüber Kunden. Als letzte Position wird das Eigenkapital geführt, welches zum langfristigen Verbleib im Institut designiert ist und daher die längste denkbare Frist verkörpert. Tabelle 3 enthält die Passiva der Bankbilanz gemäß Formblatt 1 inklusive der Positionen unterhalb des Bilanzstriches. ${ }^{501}$

\footnotetext{
492 Vgl. Becker und Peppmeier (2013), S. 542.

${ }^{493}$ Vgl. Becker und Peppmeier (2013), S. 543.

${ }^{494}$ Vgl. Becker und Peppmeier (2013), S. 543.

495 Vgl. Becker und Peppmeier (2013), S. 543.

${ }^{496}$ Vgl. Becker und Peppmeier (2013), S. 544.

497 Vgl. Becker und Peppmeier (2013), S. 544.

498 Vgl. BaFin (2012a), S. 1.

${ }^{499}$ Vgl. BaFin (2012a), S. 1 f.

${ }^{500}$ Vgl. Becker und Peppmeier (2013), S. 544.

501 Vgl. BaFin (2012a), S. 1 f.
} 
Aktivseite

1. Barreserve
a) Kassenbestand
b) Guthaben bei Zentralnotenbanken darunter: bei der Deutschen Bundesbank
c) Guthaben bei Postgiroämtern

2. Schuldtitel öffentlicher Stellen und Wechsel, die zur Refinanzierung bei Zentralnotenbanken zugelassen sind

a) Schatzwechsel und unverzinsliche Schatzanweisungen sowie ähnliche Schuldtitel öffentlichen Stellen darunter: Bei der Deutschen Bundesbank refinanzierbar

b) Wechsel

3. Forderungen an Kreditinstitute
a) täglich fällig
b) andere Forderungen

4. Forderungen an Kunden darunter: durch Grundpfandrechte gesichert Kommunalkredite

5. Schuldverschreibungen und andere festverzinsliche Wertpapiere

a) Geldmarktpapiere

aa) von öffentlichen Emittenten darunter: beleihbar bei der Deutschen Bundesbank

ab) von anderen Emittenten darunter: beleihbar bei der Deutschen Bundesbank

b) Anleihen und Schuldverschreibungen

ba) von öffentlichen Emittenten darunter: beleihbar bei der Deutschen Bundesbank

bb) von anderen Emittenten darunter: beleihbar bei der Deutschen Bundesbank

c) eigene Schuldverschreibungen Nennbetrag

6. Aktien und andere nicht festverzinsliche Wertpapiere

6a. Handelsbestand

7. Beteiligungen darunter: an Kreditinstituten an Finanzdienstleistungsinstituten

8. Anteile an verbundenen Unternehmen darunter: an Kreditinstituten an Finanzdienstleistungsinstituten

9. Treuhandvermögen, darunter: Treuhandkredite

10. Ausgleichsforderungen gegen die öffentliche Hand einschließlich Schuldverschreibungen aus deren Umtausch

11. Immaterielle Anlagewerte:

a) selbst geschaffene gewerbliche Schutzrechte und ähnliche Rechte und Werte

b) entgeltlich erworbene Konzessionen, gewerbliche Schutzrechte und ähnliche Rechte und Werte sowie Lizenzen an solchen Rechten und Werten

c) Geschäfts- oder Firmenwert

d) geleistete Anzahlungen

12. Sachanlagen

13. Eingefordertes, noch nicht eingezahltes Kapital

14. Sonstige Vermögensgegenstände

15. Rechnungsabgrenzungsposten ${ }^{5}$

16. Aktive latente Steuern

17. Aktiver Unterschiedsbetrag aus der Vermögensverrechnung 18. Nicht durch Eigenkapital gedeckter Fehlbetrag

Summe der Aktiva
Tabelle 2:
Aktiva des Formblattes $1^{502}$ 


\section{Passivseite}

1. Verbindlichkeiten gegenüber Kreditinstituten

a) täglich fällig

b) mit vereinbarter Laufzeit oder Kündigungsfrist

2. Verbindlichkeiten gegenüber Kunden

a) Spareinlagen

aa) mit vereinbarter Kündigungsfrist von drei Monaten

ab) mit vereinbarter Kündigungsfrist von mehr als drei Monaten

b) andere Verbindlichkeiten

ba) täglich fällig

bb) mit vereinbarter Laufzeit oder Kündigungsfrist

3. Verbriefte Verbindlichkeiten

a) begebene Schuldverschreibungen

b) andere verbriefte Verbindlichkeiten

darunter: Geldmarktpapiere

3a. Handelsbestand

eigene Akzepte und Solawechsel im Umlauf

4. Treuhandverbindlichkeiten

darunter: Treuhandkredite

5. Sonstige Verbindlichkeiten

6. Rechnungsabgrenzungsposten

6a. Passive latente Steuern

7. Rückstellungen

a) Rückstellungen für Pensionen und ähnliche Verpflichtungen

b) Steuerrückstellungen

c) andere Rückstellungen

8. (weggefallen)

9. Nachrangige Verbindlichkeiten

10. Genussrechtskapital

darunter: vor Ablauf von zwei Jahren fällig

11. Fonds für allgemeine Bankrisiken

12. Eigenkapital

a) Gezeichnetes Kapital

b) Kapitalrücklage

c) Gewinnrücklagen

ca) gesetzliche Rücklage

cb) Rücklage für Anteile an einem herrschenden oder

mehrheitlich beteiligten Unternehmen

cc) satzungsmäßige Rücklagen

cd) andere Gewinnrücklagen

d) Bilanzgewinn/Bilanzverlust

1. Eventualverbindlichkeiten

a) Eventualverbindlichkeiten aus weitergegebenen abgerechneten

Wechseln

b) Verbindlichkeiten aus Bürgschaften und Gewährleistungsverträgen

c) Haftung aus der Bestellung von Sicherheiten für fremde Verbindlichkeiten

2. Andere Verpflichtungen

a) Rücknahmeverpflichtungen aus unechten Pensionsgeschäften

b) Platzierungs- und Übernahmeverpflichtungen

c) Unwiderrufliche Kreditzusagen

Tabelle 3: $\quad$ Passiva des Formblattes $1^{503}$ 


\subsubsection{Gewinn- und Verlustrechnung}

Die Gewinn- und Verlustrechnung beinhaltet die Aufwendungen und Erträge, die im Laufe eines Geschäftsjahres angefallen sind.$^{504}$ Deshalb handelt es sich um eine Zeitraumbetrachtung. ${ }^{505}$ Ziel ist die Ermittlung des handelsrechtlichen Gewinns oder Verlusts eines Instituts. ${ }^{506}$ Das Saldierungsverbot nach $\ 246$ Abs. 2 S. 1 HGB ist Voraussetzung für die oben genannte Informationsfunktion des Jahresabschlusses. Im Falle einer Saldierung würde der Abschluss keine Informationen zu den Ertrags- und Aufwandsquellen geben können. Aus den einzelnen Positionen lässt sich auf die Herkunft der Erträge schließen und somit die Entstehung des Ergebnisses erklären. ${ }^{507}$ Tabelle 4 enthält die Positionen der Gewinn- und Verlustrechnung gemäß Formblatt 3.

\subsubsection{Zinserträge}

Bei den Zinserträgen handelt es sich um die erste Position, die gemäß Formblatt 3 in der Gewinn- und Verlustrechnung von Kreditinstituten ausgewiesen wird. ${ }^{508}$ Zunächst soll geklärt werden, was genau unter dem Begriff des Zinses zu verstehen ist. BIEG definiert den Zins als Entgelt oder Preis für die Überlassung von Kapital über Zeit. ${ }^{509} \mathrm{Im}$ Gegensatz dazu seien Provisionen das Entgelt oder der Preis für die Erbringung von Dienstleistungen. ${ }^{510}$ Die in $\ 30$ Abs. 1 RechKredV aufgezählten Beispiele werden im Abschnitt über Provisionserträge behandelt. ${ }^{511}$ Jedoch ergeben sich in der Praxis trotzdem Abgrenzungschwierigkeiten. Einerseits werden bisweilen Entgelte für Dienstleistungen mit über den Zins abgegolten, wodurch keine Aufteilungsmöglichkeit in Zinsen und Provisionen mehr möglich ist. Andererseits besitzen zinsähnliche Erträge in der Praxis häufig die Bezeichnungen Provision, Gebühr oder ähnliche, obwohl es sich um Entgelte für die Kapitalüberlassung handelt. Diese sind daher in den Zinserträgen auszuweisen. ${ }^{512}$ BIEG fasst zusammen, dass es für die Zuordnung letztlich entscheidend ist, ob der Ertrag oder der Aufwand primär auf der Überlassung von Kapital auf Zeit beruht, oder aufgrund einer Dienstleistung entstanden ist. $^{513}$

Formblatt 3 führt diejenigen Positionen, zwischen denen ein sachlicher Bezug besteht, unmittelbar nacheinander auf. ${ }^{514}$ Eine nähere Umschreibung des Posteninhalts Zinserträge findet sich in $\ 28$ Abs. 1 RechKredV. Der Posten Zinserträge umfasst die Zinserträge selbst, ähnliche Erträge aus dem Bankgeschäft sowie Erträge aus dem Factoringgeschäft; ihm werden die Zinserträge folgender Bilanzposten zugeordnet:

\footnotetext{
${ }^{504}$ Vgl. Becker und Peppmeier (2013), S. 566.

505 Vgl. Becker und Peppmeier (2013), S. 566.

506 Vgl. Becker und Peppmeier (2013), S. 566.

507 Vgl. Becker und Peppmeier (2013), S. 566.

508 Vgl. BaFin (2012b), S. 1.

${ }^{509}$ Vgl. Bieg (2010a), S. 334.

${ }^{510}$ Vgl. Bieg (2010a), S. 334.

511 Vgl. Abschnitt 3.6.3.4.

512 Vgl. Bieg (2010a), S. 334.

${ }^{513}$ Vgl. Bieg (2010a), S. 335.

514 Vgl. Scharpf und Schaber (2011), S. 909.
} 


\section{Gewinn- und Verlustrechnung}

1. Zinserträge aus

a) Kredit- und Geldmarktgeschäften

b) festverzinslichen Wertpapieren und Schuldbuchforderungen

2. Zinsaufwendungen

3. Laufende Erträge aus

a) Aktien und anderen nicht festverzinslichen Wertpapieren

b) Beteiligungen

c) Anteilen an verbundenen Unternehmen

4. Erträge aus Gewinngemeinschaften, Gewinnabführungs- oder Teilgewinnabführungsverträgen

5. Provisionserträge

6. Provisionsaufwendungen

7. Nettoertrag oder Nettoaufwand des Handelsbestands

8. Sonstige betriebliche Erträge

9. (weggefallen)

10. Allgemeine Verwaltungsaufwendungen

a) Personalaufwand

aa) Löhne und Gehälter

ab) Soziale Abgaben und Aufwendungen für Altersversorgung und für Unterstützung darunter: für Altersversorgung

b) andere Verwaltungsaufwendungen

11. Abschreibungen und Wertberichtigungen auf immaterielle Anlagewerte und Sachanlagen

12. Sonstige betriebliche Aufwendungen

13. Abschreibungen und Wertberichtigungen auf Forderungen und bestimmte Wertpapiere sowie Zuführungen zu Rückstellungen im Kreditgeschäft

14. Erträge aus Zuschreibungen zu Forderungen und bestimmten Wertpapieren sowie aus der Auflösung von Rückstellungen im Kreditgeschäft

15. Abschreibungen und Wertberichtigungen auf Beteiligungen, Anteile an verbundenen Unternehmen und wie Anlagevermögen behandelte Wertpapiere

16. Erträge aus Zuschreibungen zu Beteiligungen, Anteilen an verbunden Unternehmen und wie Anlagevermögen behandelten Wertpapieren

17. Aufwendungen aus Verlustübernahme

18. (weggefallen)

19. Ergebnis der normalen Geschäftstätigkeit

20. Außerordentliche Erträge

21. Außerordentliche Aufwendungen

22. Außerordentliches Ergebnis

23. Steuern vom Einkommen und vom Ertrag

24. Sonstige Steuern, soweit nicht unter Posten 12 ausgewiesen

25. Erträge aus Verlustübernahme

26. Auf Grund einer Gewinngemeinschaft, eines Gewinnabführungs- oder eines Teilgewinnabführungsvertrags abgeführte Gewinne

27. Jahresüberschuss / Jahresfehlbetrag

28. Gewinnvortrag / Verlustvortrag aus dem Vorjahr

29. Entnahmen aus der Kapitalrücklage

30. Entnahmen aus Gewinnrücklagen

a) aus der gesetzlichen Rücklage

b) aus der Rücklage für Anteile an einem herrschenden oder mehrheitlich beteiligten Unternehmen

c) aus satzungsmäßigen Rücklagen

d) aus anderen Gewinnrücklagen

31. Entnahmen aus Genussrechtskapital

32. Einstellungen in Gewinnrücklagen

a) in die gesetzliche Rücklage

b) in die Rücklage für Anteile an einem herrschenden oder mehrheitlich beteiligten Unternehmen

c) in satzungsmäßige Rücklagen

d) in andere Gewinnrücklagen

33. Wiederauffüllung des Genussrechtskapitals

34. Bilanzgewinn / Bilanzverlust

Tabelle 4: $\quad$ Positionen der Gewinn- und Verlustrechnung des Formblattes $3^{515}$ 
- $\quad$ "Barreserve" (Aktivposten Nr. 1),

- $\quad$ "Schuldtitel öffentlicher Stellen und Wechsel, die zur Refinanzierung bei Zentralnotenbanken zugelassen sind" (Aktivposten Nr. 2),

- "Forderungen an Kreditinstitute" (Aktivposten Nr. 3),

- "Forderungen an Kunden" (Aktivposten Nr. 4) und

- "Schuldverschreibungen und andere festverzinsliche Wertpapiere" (Aktivposten Nr. 5). ${ }^{516}$

Bei diesem Ausweis von Erträgen ist die Form der Berechnung nicht erheblich. ${ }^{517}$ Hierunter ist zu verstehen, dass $\ 28$ Abs. 1 Satz 1 RechKredV alle Erträge der eben aufgeführten Bilanzpositionen als Zinserträge ansieht. Aus diesem Grund müssen alle diese Erträge auch als Zinsen ausgewiesen werden. ${ }^{518}$ Hiervon ausgenommen sind jedoch Erträge aus zinsbedingten Zuschreibungen zinstragender Wertpapiere sowie Einlösegewinne zinstragender Wertpapiere bei einem Kauf unter pari stattgefundenen Kauf. ${ }^{519}$

Unter ähnlichen Erträgen nach $\ 28$ Abs. 1 Satz 2 RechKredV (s. o.) gelten auch alle Gebühren und Provisionen mit Zinscharakter, welche entweder nach dem Zeitablauf oder nach der Höhe der Forderung errechnet werden. Aus diesem Grund sind hier auch folgende Einnahmen auszuweisen. ${ }^{520}$

- Bereitstellungsprovisionen,

- Kreditprovisionen,

- Überziehungsprovisionen,

- Zessionsgebühren.

Weiter dürfen unter diesem Posten nur Zinserträge aus bankgeschäftlichen Forderungen erfasst werden. Zinserträge aus nichtbankgeschäftlichen Forderungen werden in den sonstigen betrieblichen Erträgen ausgewiesen. ${ }^{521}$ Auszuweisen sind auch

- Diskontabzüge,

- Ausschüttungen auf Genussrechte und Gewinnschuldverschreibungen im Bestand,

- Erträge mit Zinscharakter, die im Zusammenhang mit der zeitlichen Verteilung des Unterschiedsbetrages bei unter dem Rückzahlungsbetrag erworbenen Vermögensgegenständen entstehen,

- Zuschreibungen aufgelaufener Zinsen zu Null-Kupon-Anleihen im Bestand,

- Erträge mit Zinscharakter, die sich aus gedeckten Termingeschäften ergeben und auf die tatsächliche Laufzeit verteilt werden,

- Gebühren und Provisionen mit Zinscharakter, die nach dem Zeitablauf oder nach der Höhe der Forderung berechnet werden. ${ }^{522}$

518 Vgl. Scharpf und Schaber (2011), S. 914

${ }^{519}$ Vgl. Scharpf und Schaber (2011), S. 914.

${ }^{520}$ Vgl. Bieg (2010a), S. 336.

521 Vgl. Scharpf und Schaber (2011), S. 910. 
Die Zinserträge werden in zwei Unterposten a) und b) untergliedert: ${ }^{523}$

1. Zinserträge aus

a) Kredit- und Geldmarktgeschäften

b) festverzinslichen Wertpapieren und Schuldbuchforderungen

Der Unterposten a) enthält alle Zinserträge aus den Bilanzaktivposten Nr. 1. bis 4 und aus den Geldmarktpapieren, die im Bilanzaktivposten Nr. 5. a) erfasst wurden, sowie die Erträge aus dem Factoringgeschäft. Zinsen aus Wertpapieren im Rahmen von Geldmarktgeschäften sind den Geldmarktgeschäften und somit der GuV-Position 1. a) zuzuordnen, da das Kriterium „Geldmarkt“ schwerer wiegt als das Kriterium „Wertpapier““. ${ }^{524}$

Der Unterposten 1. b) beinhaltet laufende Zinserträge aus festverzinslichen Wertpapieren und Schuldbuchforderungen. ${ }^{52}$ Dies umfasst die Titel, welcher unter dem Bilanzaktivposten Nr. 5 b) subsumiert werden. ${ }^{526}$ Auch Erträge aus dem Aktivposten „10. Ausgleichsforderungen gegen die öffentliche Hand einschließlich Schuldverschreibungen aus deren Umtausch" sind hier im Unterposten 1. b) zu erfassen. ${ }^{527}$

Im Folgenden soll der Bezug der Zinserträge zu den relevanten Bilanzpositionen hergestellt werden, um eine relationale Kennziffer bilden zu können. Hierzu werden die fünf Bilanzaktivposten, deren Zinserträge im GuV-Posten 1. a) erfasst werden, genauer untersucht:

- Die Barreserve (Aktivposten Nr. 1) liefert typischerweise keine Zinserträge. Dies ist darauf zurückzuführen, dass keine der drei Komponenten dieser Barreserve (Kassenbestand, Guthaben bei Zentralnotenbanken, Guthaben bei Postgiroämtern) regelmäßig Zinserträge abwirft. ${ }^{528}$

- Schuldtitel öffentlicher Stellen und Wechsel, die zur Refinanzierung bei Zentralnotenbanken zugelassen sind (Aktivposten Nr. 2) beinhaltet Diskonterträge aus dem Ankauf von Wechseln, sowie Schatzwechseln, unverzinslichen Schatzanweisungen und sonstigen diskontierten Geldmarktpapieren, die zur Refinanzierung bei Zentralnotenbanken zugelassen sind. ${ }^{529}$

- Die Forderungen an Kreditinstitute (Aktivposten Nr. 3) führen zu Zinserträgen aus Kontokorrentguthaben, Tagesgeldern, Termingeldern und dem Geldhandel. AuBerdem sind Erträge aus gedeckten Devisentermingeschäften möglich, ${ }^{530}$ wobei nicht sicher ist, ob diese Möglichkeit flächendeckend von allen Instituten genutzt wird.

- Die Forderungen an Kunden (Aktivposten Nr. 4) repräsentieren regelmäßig einen großen Anteil an der Aktivseite der Bilanz einer durchschnittlichen Bank. ${ }^{531}$ Zinserträge werden in den folgenden Bereichen erzielt: Kontokorrentforderungen, Überziehungsprovisionen, Warenforderungen, Ratenkredite, Sonderkredite, Weiterlei-

\footnotetext{
522 I 28 RechKredV.

${ }^{523}$ Vgl. Scharpf und Schaber (2011), S. 922

524 Vgl. Scharpf und Schaber (2011), S. 922; vgl. Bieg (2010a), S. 336

525 Vgl. BaFin (2012b), S. 1.

${ }^{526}$ Vgl. Bieg (2010a), S. 336.

527 Vgl. Scharpf und Schaber (2011), S. 923

528 Vgl. Scharpf und Schaber (2011), S. 914.

${ }^{529}$ Vgl. Scharpf und Schaber (2011), S. 914.

${ }^{530}$ Vgl. Scharpf und Schaber (2011), S. 914.

531 Vgl. Abschnitt 4.4.3.
} 
tungskredite, Darlehen, Disagioerträge, Bearbeitungsgebühren, Bereitstellungsprovisionen, Kreditprovisionen, Vorfälligkeitsentschädigungen, Wechseldiskonterträge und Factoringgebühren. ${ }^{532}$

- Die Geldmarktpapiere (Aktivposten Nr. 5a) werden untergliedert in solche von öffentlichen und solche von anderen Emittenten. ${ }^{533}$ Die Zinserträge fallen an in Form von Diskonterträgen aus Schatzwechseln und aus unverzinslichen Schatzanweisungen. ${ }^{534}$

Es folgt der Bezug zu der Bilanzposition, deren Erträge im GuV-Posten 1. b) ausgewiesen werden:

- Die Anleihen und Schuldverschreibungen (Aktivposten Nr. 5b) werden im Bilanzausweis ebenfalls untergliedert in solche von öffentlichen und solche von anderen Emittenten. Als dritte Unterscheidungsart kommen hier noch eigene Schuldverschreibungen hinzu, die naturgemäß netto keine Zinserträge generieren. Zinserträge fallen an aus börsenfähigen Schuldtiteln wie Anleihen, Schuldverschreibungen, Pfandbriefen, Obligationen und Nullkuponanleihen.

- Die Ausgleichsforderungen gegen die öffentliche Hand (Aktivposten Nr. 10) werden hier nachrichtlich erwähnt. Es handelt sich um Ausgleichsforderungen aus der Währungsunion im Jahre 1990. Hintergrund der Existenz dieses Posten ist die Abwertung von Forderungen zu einem höheren Zinssatz als Verbindlichkeiten. ${ }^{535}$

\subsubsection{Zinsaufwendungen}

Als zweite Position nach den Zinserträgen sind auf Formblatt 3 die Zinsaufwendungen aufgeführt. ${ }^{536} \mathrm{Zu}$ erfassen sind hier Zinsaufwendungen und ähnliche Aufwendungen aus dem Bankgeschäft. ${ }^{537}$ Dies beinhaltet auch wieder Aufwendungen aus dem Factoringgeschäft. ${ }^{538}$ Konkret sind jene Aufwendungen aufzuführen, die für folgende Posten der Bilanz entstanden sind:

- "Verbindlichkeiten gegenüber Kreditinstituten" (Passivposten Nr. 1),

- "Verbindlichkeiten gegenüber Kunden" (Passivposten Nr. 2),

- "Verbriefte Verbindlichkeiten" (Passivposten Nr. 3) und

- "Nachrangige Verbindlichkeiten" (Passivposten Nr. 9). ${ }^{539}$

Wie bei den Zinserträgen ${ }^{540}$ ist die Form der Berechnung für den Ausweis nicht erheb$\operatorname{lich}^{541}$, das heißt, alle Aufwendungen der vorgenannten Passivposten 1, 2, 3 und 9 sind als Zinsaufwendungen anzusehen und demnach auch als solche auszuweisen. ${ }^{542}$ Diskontabzüge sowie weitere Aufwendungen sind analog zu den Zinserträgen auszuweisen. ${ }^{543}$ Diskont-

\footnotetext{
532 Vgl. Scharpf und Schaber (2011), S. 914

${ }^{533}$ Vgl. BaFin (2012a), S. 1.

${ }^{534}$ Vgl. Scharpf und Schaber (2011), S. 914

535 Vgl. Bieg (2010a), S. 256.

536 Vgl. BaFin (2012b), S. 1

53728 RechKredV.

$538 \int 28$ RechKredV.

$539 \int 28$ RechKredV.

540 Vgl. Abschnitt 3.6.3.1.

541 \ 29 Satz 1 RechKredV.

${ }^{542}$ Vgl. Scharpf und Schaber (2011), S. 927.

543 Vgl. § 28 RechKredV.
} 
abzüge sind Zinsaufwendungen, die aus dem Verkauf von Wechseln, Schatzwechseln und unverzinslichen Schatzanweisungen herrühren. ${ }^{544}$

Es folgt die Zuordnung einzelner Geschäfte zu den 4 betreffenden Passivpositionen:

- Für Verbindlichkeiten gegenüber Kreditinstituten (Passivposten Nr. 1) entstehen Zinsaufwendungen für laufende Rechnung (Kontokorrent) inklusive Überziehungsprovisionen, für Tagesgeldaufnahmen, für Refinanzierungen z. B. von Weiterleitungskrediten und für aufgenommene Darlehen. Dazu sind Abschreibungen auf Disagio sowie Bereitstellungsprovisionen möglich. ${ }^{545}$

- Für Verbindlichkeiten gegenüber Kunden (Passivposten Nr. 2) entstehen Zinsaufwendungen für Spareinlagen, für Kontokorrenteinlagen, für befristete Verbindlichkeiten, für Wachstumszertifikate und Stufenzinsprodukte; dazu kommen Disagioabschreibungen für Einlagen wie Sparbriefe, falls unter Rückzahlungswert hereingenommen. ${ }^{546}$

- Für verbriefte Verbindlichkeiten (Passivposten Nr. 3) entstehen Zinsaufwendungen für begebene Schuldverschreibungen; dazu kommen Disagioabschreibungen für Schuldverschreibungen, falls unter dem Nennwert ausgegeben. ${ }^{547}$

- Für nachrangige Verbindlichkeiten (Passivposten Nr. 9) entstehen Zinsaufwendungen für Darlehen, Schuldverschreibungen und andere nachrangige Verbindlichkeiten sowie ggfs. Disagioabschreibungen. ${ }^{548}$

\subsubsection{Laufende Erträge}

Die laufenden Erträge aus Aktien, anderen nicht festverzinslichen Wertpapieren, Beteiligungen und Anteilen an verbundenen Unternehmen (Position 3 auf dem Formblatt $3^{549}$ ) werden bei der Ermittlung des Zinsüberschusses mit einbezogen. ${ }^{550}$ Häufig werden von den Genossenschaftsbanken Spezialfonds eingesetzt. Dies ermöglicht dem Treasurer eine hohe Flexibilität bei der Steuerung der Erträge. Dies ist darauf zurückzuführen, dass der Zeitpunkt der Ertragsausschüttung geplant werden kann, woraus sich gewisse Gestaltungsmöglichkeiten ergeben. Die Erträge aus Spezialfonds werden selbst dann in dieser GuV-Position erfasst, wenn diese innerhalb des Fonds zu $100 \%$ aus festverzinslichen Wertpapieren stammen. Dazu kommen noch Dividenden und Erträge aus anderen Publikumsfonds. Aufgrund des strengen Niederstwertprinzips des HGB können keine ertragswirksamen Zuschreibungen über den Anschaffungswert hinaus getätigt werden. ${ }^{551}$ Jedoch können aufwandswirksame Abschreibungen auf einen temporär gesunkenen Marktpreis vorgenommen werden, sofern dies durch die Liquiditätsreserve gedeckt ist.

\footnotetext{
${ }^{544}$ Vgl. Bieg (2010a), S. 340.

545 Vgl. Scharpf und Schaber (2011), S. 927.

546 Vgl. Scharpf und Schaber (2011), S. 927.

547 Vgl. Scharpf und Schaber (2011), S. 927

548 Vgl. Scharpf und Schaber (2011), S. 927.

${ }^{549}$ Vgl. BaFin (2012b), S. 1.

${ }^{550}$ Vgl. Schierenbeck (2003b), S. 424.

551 \ 253 Abs. 4 S. 2 HGB.
} 


\subsubsection{Provisionserträge}

Die Provisionserträge werden in der Position 5 der Gewinn- und Verlustrechnung erfasst. ${ }^{552}$ Gemäß der RechKredV sind hier Provisionen und ähnliche Erträge aus Dienstleistungsgeschäften auszuweisen. ${ }^{553}$ Es sind jedoch nur Erträge auszuweisen, die aus bankgeschäftlichen Dienstleistungen im weiteren Sinne resultieren. ${ }^{54}$ Hingegen sind Erträge aus nicht bankgeschäftlichen Dienstleistungen als sonstige betriebliche Erträge in der GuV-Position Nr. $8^{555}$ zu erfassen. ${ }^{556}$

$\mathrm{Zu}$ den Dienstleistungsgeschäften zählen die im Folgenden aufgelisteten Geschäfte. ${ }^{557}$ Nach dem jeweiligen Geschäft werden die wichtigsten Gebühren- und Provisionen aufgeführt. ${ }^{558}$

- Zahlungsverkehr und Kontoführung: Umsatzprovisionen, Kontoführungsgebühren (insbesondere Postengebühren, Überweisungsgebühren, Rückruf von Überweisungen, Dauerauftragsgebühren), Einzugsprovision für Schecks, Lastschriften und Wechsel, Gebühren im Kassenverkehr (Nachttresoreinzahlungen, Geldtransporte, Auszahlungen an Geldautomaten an Kunden anderer Kreditinstitute, Bereitstellung von Münzen, Einzahlungen von Nichtbankkunden zugunsten Dritter);

- Außenhandelsgeschäft: Gebühren aus Akkreditiven, Inkassos und dem Auslandszahlungsverkehr;

- Wertpapierkommissionsgeschäft: An- und Verkaufsprovisionen, Provisionen im Wertpapieremissionsgeschäft (Konsortialprovisionen, Börseneinführungsprovisionen), für die Effektenauslieferung, für die Einlösung von Zins- und Dividendenscheinen;

- Depotgeschäft: Depotgebühren, Stahlfachmieten;

- Treuhand- und Verwaltungskreditgeschäft: Obwohl die Marge als Zins berechnet wird, handelt es sich um ein Entgelt für eine Dienstleistung;

- Veräußerung von Devisen, Sorten und Edelmetallen: An- und Verkaufsprovisionen;

- Vermittlung von Kredit-, Spar-, Bauspar- und Versicherungsverträgen: Provisionen;

- Platzierung von Wertpapieren;

- Avalgeschäft (Bürgschaftsprovisionen, Verwaltungsprovisionen von Kreditsicherheiten, Kreditbearbeitungsprovisionen). ${ }^{559}$

BIEG ergänzt die Provisionen folgender Geschäfte:

- Vermögensberatung und Vermögensverwaltung,

- Einzugsgeschäft,

\footnotetext{
${ }^{552}$ Vgl. BaFin (2012b), S. 1.

553 S 30 Abs. 1 Satz 1 RechKredV.

554 Vgl. Scharpf und Schaber (2011), S. 942.

555 Vgl. BaFin (2012b), S. 1.

556 Vgl. Scharpf und Schaber (2011), S. 942.

$557 \int 30$ Abs. 1 Satz 1 RechKredV.

558 Vgl. Bieg (2010a), S. 346-349.

559 \& 30 Abs. 1 Satz 2 RechKredV.
} 
- Versicherungsgeschäft,

- Finanzielle Beratung inkl. Unternehmensberatung,

- Immobilienvermittlung,

- Beteiligungsvermittlung sowie

- EDV- und Finanzservice $e^{500}$

\subsubsection{Provisionsaufwendungen}

Die Provisionsaufwendungen werden in Position 6 der Gewinn- und Verlustrechnung angegeben. ${ }^{51}$ Gemäß der RechKredV sind in diesem Posten Provisionen und ähnliche Aufwendungen der genannten und bereits in Abschnitt 3.6.3.4 aufgelisteten Dienstleistungsgeschäfte auszuweisen. ${ }^{562}$

\subsubsection{Nettoertrag/-aufwand des Handelsbestands}

In der GuV-Position 7. Nettoertrag/-aufwand des Handelsbestands werden als Saldo die Erträge und Aufwendungen erfasst, die sich aus Geschäften mit Wertpapieren des Handelsbestands, mit Finanzinstrumenten, mit Devisen und mit Edelmetallen ergeben. ${ }^{563}$ Laut $\int 340 \mathrm{c}$ Abs. 1 S. 1 HGB ist für diese Position zwingend ein saldierter Ausweis vorgesehen. Dies steht im Gegensatz zum Zins- und zum Provisionsgeschäft, bei denen Erträge und Aufwendungen einzeln ausgewiesen werden, und stellt einen Durchbruch des allgemeinen Saldierungsverbots des HGB dar. ${ }^{564}$ Das Saldierungsgebot des \340c Abs. 1 S. 1 HGB verpflichtet zur Verrechnung von Erträgen und Aufwendungen aus Finanzgeschäften. ${ }^{565}$ Zinsen oder Dividenden dürfen jedoch nicht verrechnet werden. ${ }^{566}$ Eine Begründung für diese Ausnahme ist weder im Gesetz noch in der verwendeten einschlägigen Literatur enthalten. ${ }^{567}$ Die Position 7 der Gewinn- und Verlustrechnung trägt entweder den Titel „Nettoertrag des Handelsbestands“ oder „Nettoaufwand des Handelsbestands“.568

\subsubsection{Personalaufwand und Personalaufwandsspanne}

Die GuV-Position 10 Allgemeine Verwaltungsaufwendungen gliedert sich in a) Personalaufwand und b) andere Verwaltungsaufwendungen ${ }^{569}$ Der Teil a) Personalaufwand umfasst alle Geld- und Sachleistungen für Angestellte, gewerbliche Arbeitnehmer und Mitglieder der Geschäftsführung. ${ }^{570}$ Er untergliedert sich weiter in aa) Löhne und Gehälter und ab) Soziale Abgaben und Aufwendungen für Altersversorgung und für Unterstützung ${ }^{571}$, während die in $a b$ ) enthaltenen Aufwendungen „für Altersversorgung“ separat ausgewiesen werden müssen. ${ }^{572}$ Die Löhne und Gehälter werden in aa) teilen sich derart auf, dass Angestellte und die

\footnotetext{
${ }^{560}$ Vgl. Bieg (2010a), S. 344 f.

561 Vgl. BaFin (2012b), S. 1

$562 \int 30$ Abs. 2 RechKredV.

563 Vgl. Schierenbeck (2003b), S. 424

564 Vgl. Botsis et al. (2012), S. 21.

565 Vgl. Eisele und Knobloch (2010), S. 108, vgl. Bieg (2010a), S. 353

566 Vgl. Beyer (2012), S. 61.

567 Vgl. S 340c Abs. 1 S. 1 HGB; vgl. Everling und Goedeckemeyer (2015), S. 239; vgl. Eisele und Knobloch (2010), S. 108, vgl. Bieg (2010a), S. 353; vgl. Scharpf und Schaber (2011), S. 259 f.

568 Vgl. Bieg (2010a), S. 349 f.

${ }^{569} \mathrm{Vgl}$. BaFin (2012b), S. 1.

570 Vgl. Bieg (2010a), S. 374.

571 Vgl. Botsis et al. (2012), S. 79.

572 Vgl. BaFin (2012b), S. 1.
} 
Geschäftsführung Gehälter beziehen, während gewerbliche Arbeitnehmer Lohn erhalten. ${ }^{573}$ $\mathrm{Zu}$ den Löhnen und Gehältern und aa) zählen auch sogenannte Nebenbezüge und Sachbezüge. ${ }^{574}$ Nebenbezüge umfassen Gratifikationen, Gewinnbeteiligungen, Vergütungen für Verbesserungsvorschläge, während in die Sachbezüge z. B. die unentgeltliche Dienstwagennutzung oder Aufwendungen für Essensmarken fallen, von denen die Mitarbeiter des Instituts profitieren. ${ }^{575}$ Die in $\mathrm{ab}$ ) ausgewiesenen sozialen Abgaben beinhalten die gesetzlichen Pflichtabgaben: Arbeitgeberanteile der Sozialversicherungsbeiträge und die Beiträge an die Berufsgenossenschaft. ${ }^{576}$ Freiwillige Leistungen des Arbeitgebers werden je nach Typ entweder den Löhnen und Gehältern, den Aufwendungen für Altersvorsorge oder als Aufwendungen für Unterstützung erfasst. ${ }^{577}$ Abbildung 10 enthält eine Gliederung der allgemeinen Verwaltungsaufwendungen.

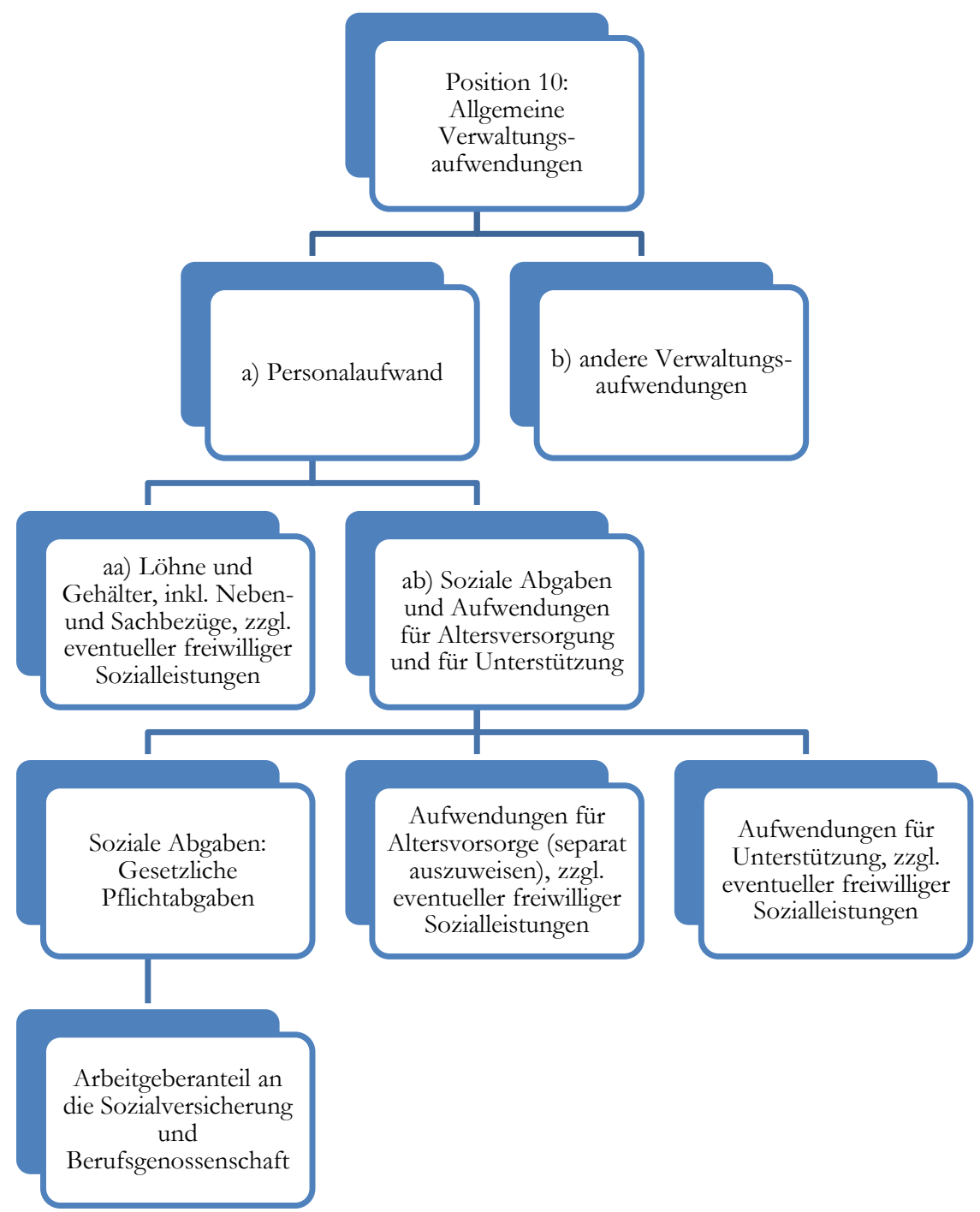

Abbildung 10: Gliederung der allgemeinen Verwaltungsaufwendungen ${ }^{578}$

\footnotetext{
${ }^{573}$ Vgl. Bieg (2010a), S. 374.

574 Vgl. Bieg (2010a), S. 374.

575 Vgl. Bieg (2010a), S. 374.

576 Vgl. Bieg (2010a), S. 375

577 Vgl. Bieg (2010a), S. 375.

${ }^{578}$ Eigene Darstellung.
} 


\subsubsection{Sachaufwand}

Der Sachaufwand wird in der Gewinn- und Verlustrechnung unterteilt in Position 10. b) andere Verwaltungsaufwendungen sowie in Position 11. Abschreibungen und Wertberichtigungen auf immaterielle Anlagewerte und Sachanlagen. ${ }^{579}$ Häufig werden die Abschreibungen auch mit in die Sachaufwendungen hineingerechnet. ${ }^{580}$

Die Position 10. b) andere Verwaltungsaufwendungen gehört zum technisch-organisatorischen Bereich des Kreditinstituts und enthält die gesamten Aufwendungen sachlicher Art. ${ }^{581}$ Dazu zählen Raumaufwendungen, Bürobetriebsaufwendungen, Kommunikationsaufwendungen, Kraftfahrzeugsbetriebsaufwendungen, Aufwendungen für Werbung und Repräsentation, Aufwendungen für Verbandsbeiträge einschließlich Einlagensicherung und Anlegerentschädigungseinrichtung, Aufwendungen für Rechtsberatung, andere Beratungen und Prüfungsleistungen, Vergütungen für Mitglieder eines Aufsichtsrates sowie eines Beirates und ähnliche Aufwendungen. ${ }^{582}$

Die Position 11. Abschreibungen und Wertberichtigungen auf immaterielle Anlagewerte und Sachanlagen enthält alle Abschreibungen von Bilanzaktivposten 11. Immaterielle Anlagewerte und Bilanzaktivposten 12. Sachanlagen. ${ }^{583}$ Hierbei sind den immateriellen Anlagewerten selbst geschaffene oder entgeltlich erworbene gewerbliche Schutzrechte sowie zugehörige Lizenzen zuzurechnen, wie etwa EDV-Software. ${ }^{584}$ Standardisierte Anwendersoftware jedoch ist im folgend genannten Bilanzaktivposten 12 anzugeben. ${ }^{585} \mathrm{Zu}$ diesen Sachanlagen zählen Grundstücke, Gebäude sowie die Betriebs- und Geschäftsausstattung. ${ }^{586}$

Inhaltlich verwandt ist GuV-Position 12. Sonstige betriebliche Aufwendungen. ${ }^{587}$ Hier werden sämtliche Aufwendungen der normalen Geschäftstätigkeit angegeben, die keinem anderen Aufwandsposten zugeordnet sind, bzw. die nicht mit Erträgen zu verrechnen sind. ${ }^{588}$

\subsubsection{Risikovorsorge}

Die Positionen 13 und 14 der Gewinn- und Verlustrechnung dienen der Vorsorge für allgemeine Bankrisiken. ${ }^{589}$ Die Risikovorsorge umfasst den Kreditbereich und auch große Teile des Wertpapierbereichs, also die zentralen Geschäftsfelder der Kreditinstitute. ${ }^{590}$

\ 253 Abs. 1 Satz 1 HGB regelt generell zur Bilanzierung, dass Vermögensgegenstände höchstens mit den Anschaffungs- oder Herstellungskosten anzusetzen sind. Es sind jedoch definierte Abschreibungen nach $₫ 253$ Abs. 2 und 3 HGB erlaubt.

Über $₫ 253$ hinaus erlaubt es $₫ 340 \mathrm{f}$ Abs. 1 Satz 1 HGB den Kreditinstituten, einige Aktiva zu einem niedrigeren Wert zu bilanzieren. Zu diesen Aktiva zählen:

\footnotetext{
${ }^{579}$ Vgl. BaFin (2012b), S. 1.

${ }^{580}$ Vgl. Schierenbeck (2003b), S. 424

581 Vgl. $\int 31$ Abs. 2 RechKredV.

582 Vgl. Bieg (2010a), S. 374; vgl. § 31 Abs. 2 RechKredV.

583 Vgl. Winkler (2004), S. 302.

584 Vgl. Bieg (2010a), S. 261; vgl. Bieg (2010b), S. 16.

${ }^{585}$ Vgl. Bieg (2010a), S. 261; vgl. Bieg (2010b), S. 16.

${ }^{586}$ Vgl. Bieg (2010a), S. 261.

587 Vgl. Bieg (2010b), S. 16.

588 Vgl. Winkler (2004), S. 303.

${ }^{589}$ Vgl. Bieg (2010a), S. 366.

${ }^{590}$ Vgl. Bieg (2010a), S. 370.
} 
- Forderungen an Kreditinstitute,

- Forderungen an Kunden,

- Schuldverschreibungen und andere festverzinsliche Wertpapiere, die weder wie Anlagevermögen behandelt werden noch Teil des Handelsbestands sind, also zur Liquiditätsreserve zählen ${ }^{591}$ sowie

- Aktien und andere nicht festverzinsliche Wertpapiere, die weder wie Anlagevermögen behandelt werden noch Teil des Handelsbestands sind, also zur Liquiditätsreserve zählen. ${ }^{52}$

Gemäß der Formulierung des $\ 340 f$ Abs. 1 Satz 1 HGB gilt die Risikovorsorge nur für Umlaufvermögen. Laut BIEG sind Kreditgewährungen, lang- sowie kurzfristig, als wesentliche Bestandteile des Bankgeschäfts anzusehen. ${ }^{593}$ Daher zählen alle Forderungen an Kreditinstitute und Kunden zum Umlaufvermögen. ${ }^{59}$

Als Bedingung für diesen niedrigeren Wertansatz gilt, dass dies „,nach vernünftiger kaufmännischer Beurteilung zur Sicherung gegen die besonderen Risiken des Geschäftszweigs der Kreditinstitute notwendig ist. ${ }^{\text {“595 }}$ Die so vorgenommenen Abschreibungen sind jedoch nicht im individuellen Risiko einer jeden einzelnen Forderung begründet. Deshalb werden sie auch als Globalabschreibungen bezeichnet. ${ }^{596}$ Die gebildeten Vorsorgereserven dürfen maximal vier Prozent des Bilanzwertes betragen. ${ }^{597}$

Nach \253 Abs. 3 Satz 5 HGB müssen bei voraussichtlich dauernder Wertminderung von Vermögensgegenständen des Anlagevermögens außerplanmäßige Abschreibungen vorgenommen werden. Erleichternd können nach $\ 253$ Abs. 3 Satz 6 HGB bei Finanzanlagen außerplanmäßige Abschreibungen auch dann vorgenommen werden, wenn es sich um eine voraussichtlich nicht dauernde Wertminderung handelt. Generell darf gemäß \253 Abs. 5 Satz 1 HGB ein niedrigerer Wertansatz nicht beibehalten werden, wenn die Gründe dafür nicht mehr bestehen. Auch hier erlaubt $\int 340$ f Abs. 1 Satz 3 HGB eine Erleichterung für Kreditinstitute, denn diese dürfen einen niedrigeren Wertansatz beibehalten. ${ }^{598}$ Durch den ausweisbaren Aufwand für die Risikovorsorge verringert sich der Jahresüberschuss, was sich wiederum im Berichtsjahr steuermindernd auswirkt.

\subsubsection{Anhang}

Laut $\ 264$ Abs. 2 HGB müssen im Anhang zusätzliche Angaben gemacht werden, falls der „Jahresabschluss ein den tatsächlichen Verhältnissen entsprechendes Bild im Sinne des Satzes 1 nicht vermittelt“. Der Anhang ist als dritter Bestandteil des handelsrechtlichen Jahresabschlusses zu sehen und steht gleichberechtigt neben Bilanz und Gewinn- und Verlustrechnung. ${ }^{599}$ Alle drei Elemente bilden eine untrennbare Einheit. ${ }^{600}$ Während Bilanz und Gewinn- und Verlustrechnung vor allem dem Jahresabschlusszweck der Erfolgsermitt-

\footnotetext{
${ }^{591}$ Vgl. Bieg (2010a), S. 367.

${ }^{592}$ Vgl. Bieg (2010a), S. 367.

593 Vgl. Bieg (2010a), S. 116.

${ }^{594}$ Vgl. Bieg (2010a), S. 370

595 Vgl. 340 f Abs. 1 Satz 1 HGB.

${ }_{596}$ Vgl. Bieg (2010a), S. 366.

597 Vgl. 340 f Abs. 1 Satz 2 HGB.

${ }^{598}$ Vgl. S 340 f Abs. 1 Satz 3 HGB; vgl. Philipps (2010), S. 338; vgl. Padberg, Padberg und Werner (2010).

${ }^{599}$ Vgl. Bieg (2010a), S. 689.

${ }^{600}$ Vgl. Bieg (2010a), S. 689.
} 
lung dienen, kommt dem Anhang bei der Informationsvermittlung eine Schlüsselrolle zu und bietet eine verbale Informationsmöglichkeit. ${ }^{601}$

\subsubsection{Lagebericht}

Der Geschäftsverlauf und die Lage der Gesellschaft sind im Lagebericht in einer Art darzustellen, welche ein tatsächliches Bild der Vermögens-, Finanz- und Ertragslage vermittelt. ${ }^{602}$ Ähnlich wie der Anhang hat der Lagebericht eine reine Informationsfunktion. ${ }^{603}$ Der Lagebericht gehört nicht zum Jahresabschluss und ist daher nicht an dessen inhaltliche Vorgaben gebunden. ${ }^{604}$ Obwohl ein Ermessensspielraum bei der Strukturierung der Informationen besteht, müssen die Grundsätze ordnungsgemäßer Buchführung beachtet werden. ${ }^{605}$ Ziel des Lageberichts ist die Darstellung der Bank innerhalb der Branche und die Gewährung eines Zukunftsausblicks. ${ }^{606}$

\subsubsection{Jahresabschlusspolitik}

Dieser Abschnitt thematisiert die Beeinflussungsmöglichkeiten des Jahresabschlusses, genauer von einzelnen Positionen der Gewinn- und Verlustrechnung sowie der Bilanz. Unter Jahresabschlusspolitik versteht man nach COENENBERG die ,zielgerichtete Gestaltung der externen Rechnungslegung durch das Management im Rahmen der Möglichkeiten, die unter Einhaltung der Regeln des jeweils zur Anwendung kommenden Normensystems bestehen. “607 Dabei werde das Ziel verfolgt, das Urteil der Informationsempfänger bzw. Rechtsfolgen zu beeinflussen. ${ }^{608}$ COENENBERG sieht die Jahresabschlusspolitik als ein sinnvolles und nützliches Instrument, um übergeordnete Zielsetzungen zu erreichen. ${ }^{609}$

\section{Ziele der Jahresabschlusspolitik}

Bei näherer Betrachtung der Ziele des Jahresabschlusses ${ }^{610}$ ergeben sich Ausgangspunkte für die Ziele der Jahresabschlusspolitik. Diese beinhalten etwa die Steuerung der Höhe der Ausschüttung an die Gesellschafter, die Ergebnisglättung im Zeitablauf und eine Minimierung des Barwerts der Steuerzahlungen. ${ }^{611}$ Auch nutzen Geschäftsführer und Vorstände die Bankbilanzpolitik, um ihre eigene Managementleistung zu ihrem Vorteil hervorzuheben. ${ }^{612}$ Beispielsweise zeigen empirische Studien, dass Geschäftsführer nach ihrem Antritt die wirtschaftliche Lage eines Unternehmens als eher schlecht darstellen, indem sie etwa hohe Aufwendungen oder Verluste ausweisen. ${ }^{613}$ Dies ermöglicht ihnen in den Folgejahren den Ausweis einer positiven Geschäftsentwicklung und steigender Gewinne. ${ }^{614}$

\footnotetext{
${ }^{601}$ Vgl. Bieg (2010a), S. 691.

${ }^{602}$ Vgl. Bieg (2010a), S. 518

${ }^{603}$ Vgl. Bieg (2010a), S. 827.

${ }^{604}$ Vgl. Bieg (2010a), S. 827.

${ }^{605}$ Vgl. Bieg (2010a), S. 827.

${ }^{606}$ Vgl. Bieg (2010a), S. 827.

${ }^{607}$ Coenenberg (2012), S. 997.

608 Vgl. Coenenberg (2012), S. 997.

${ }^{609}$ Vgl. Coenenberg (2012), S. 997.

${ }^{610}$ Vgl. Abschnitt 3.6.

${ }^{611}$ Vgl. Coenenberg (2012), S. 997.

${ }^{612}$ Vgl. Coenenberg (2012), S. 998.

613 Vgl. Coenenberg (2012), S. 998.

${ }^{614}$ Vgl. Coenenberg (2012), S. 998
} 
Investoren sind häufig daran interessiert, dass Überschüsse regelmäßig anfallen. ${ }^{615}$ Durch geeignete Bilanzierungswahlrechte kann das Ergebnis über die Jahre geglättet werden, beispielsweise durch die Aktivierung von Entwicklungskosten und Abschreibung über die folgenden Jahre, anstatt im ersten Jahr ein schlechtes Ergebnis auszuweisen. ${ }^{616}$ Mit dem Wissen über den Einbezug bestimmter Bilanzpositionen in Ratings und deren Steuerung kann ein Unternehmen Einfluss auf die eigene Bonität und damit auf die erforderlichen Finanzierungskosten nehmen. ${ }^{617}$ Steht ein Unternehmensverkauf an, ermöglicht die Jahresabschlusspolitik eine besonders positive Darstellung der Lage und des Erfolgs. ${ }^{618}$ Umgekehrt kann durch eine negative Darstellung eine drohende Übernahme verhindert oder etwa die Abfindung eines Gesellschafters gedrückt werden. ${ }^{619}$

Die Jahresabschlusspolitik unterscheidet zunächst zwischen der Sachverhaltsgestaltung und der Sachverhaltsabbildung. Die Sachverhaltsgestaltung umfasst Instrumente, deren Einsatz bis vor dem Abschlussstichtag entschieden werden muss. ${ }^{620}$ Die sachverhaltsabbildenden Maßnahmen hingegen werden erst nach dem Abschlussstichtag eingesetzt. Dabei muss das Rechtsgeschäft von den Beteiligten ernsthaft gewollt sein, was insbesondere bei Pensionsgeschäften zu einer problematischen Abgrenzung führen kann. ${ }^{621}$ Außerdem müssen sich die erbrachte Leistung und die erhaltene Gegenleistung entsprechen, sodass die Konditionen des Geschäfts als angemessen beurteilt werden. ${ }^{622}$ Insbesondere bei Geschäften zwischen Konzerngesellschaften können hier Probleme auftreten. ${ }^{623}$

Der Anspruch der vorliegenden Arbeit liegt jedoch nicht darin, sämtliche in den Jahresabschlüssen genutzten Beeinflussungsmöglichkeiten zu identifizieren und zu quantifizieren; hierzu wäre auch die detaillierte Betrachtung von Anhang und Lagebericht vonnöten. Jedoch muss der Leser bei der Interpretation der Jahresabschlüsse diese Möglichkeiten der Jahresabschlusspolitik kennen und sich bewusst sein, dass diese eventuell angewendet wurden.

\subsection{ROI-Analyse}

Bei der ROI-Analyse handelt es sich um ein systematisches Verfahren zur Auswertung von Jahresabschlüssen. ${ }^{624}$ Sie wurde ursprünglich zur Bilanzanalyse von Industrieunternehmen entwickelt. Durch einige spezielle Anpassungen ist es jedoch auch zur Analyse von Bankbilanzen geeignet. ${ }^{625}$ ROI steht dabei für Return on Investment. ${ }^{626}$ Grundsätzlich unterscheidet man zwischen zwei verschiedenen Auswertungsdimensionen. ${ }^{627}$ In der ersten Dimension werden die Daten auf Ebene einzelner Geschäfte für die Kalkulation herangezogen. ${ }^{628}$ Diese Daten liegen jedoch nur bankintern vor. Daher verwendet die vorliegende Arbeit die

\footnotetext{
${ }^{615}$ Vgl. Coenenberg (2012), S. 998.

${ }^{616}$ Vgl. Coenenberg (2012), S. 998.

${ }^{617}$ Vgl. Coenenberg (2012), S. 998.

${ }^{618}$ Vgl. Coenenberg (2012), S. 998 f.

${ }^{619}$ Vgl. Coenenberg (2012), S. 999.

${ }^{620}$ Vgl. Bieg (2010a), S. 980.

${ }^{621}$ Vgl. Bieg (2010a), S. 980.

${ }^{622}$ Vgl. Bieg (2010a), S. 980.

${ }^{623}$ Vgl. Bieg (2010a), S. 980.

${ }^{624}$ Vgl. Schierenbeck (2003a), S. 81 f.

${ }^{625}$ Vgl. Schierenbeck (2003a), S. 81 f.

${ }^{626}$ Vgl. Schierenbeck (2003b), S. 304.

${ }^{627}$ Vgl. Schierenbeck (2003b), S. 304

${ }^{628}$ Vgl. Schierenbeck (2003b), S. 304.
} 
zweite Dimension, nämlich die gesamtbankbezogenen Daten des externen Rechnungswesens. ${ }^{629}$

Im ROI-Grundschema bildet die Eigenkapitalrentabilität die oberste Bezugs- und Steuergröße. ${ }^{630}$ Durch die Erfolgsspaltung werden die einzelnen Komponenten des Ergebnisses systematisch zu dieser Eigenkapitalrentabilität zusammengeführt. ${ }^{631}$ Eine grafische Darstellung des ROI-Grundschemas findet sich in Abbildung 11. Den angegebenen Werten liegt ein fiktiver Beispielfall zugrunde, in dem das außerordentliche Ergebnis und die Position Sonstige Erträge zur AOSE-Spanne zusammengefasst wurden. ${ }^{632}$

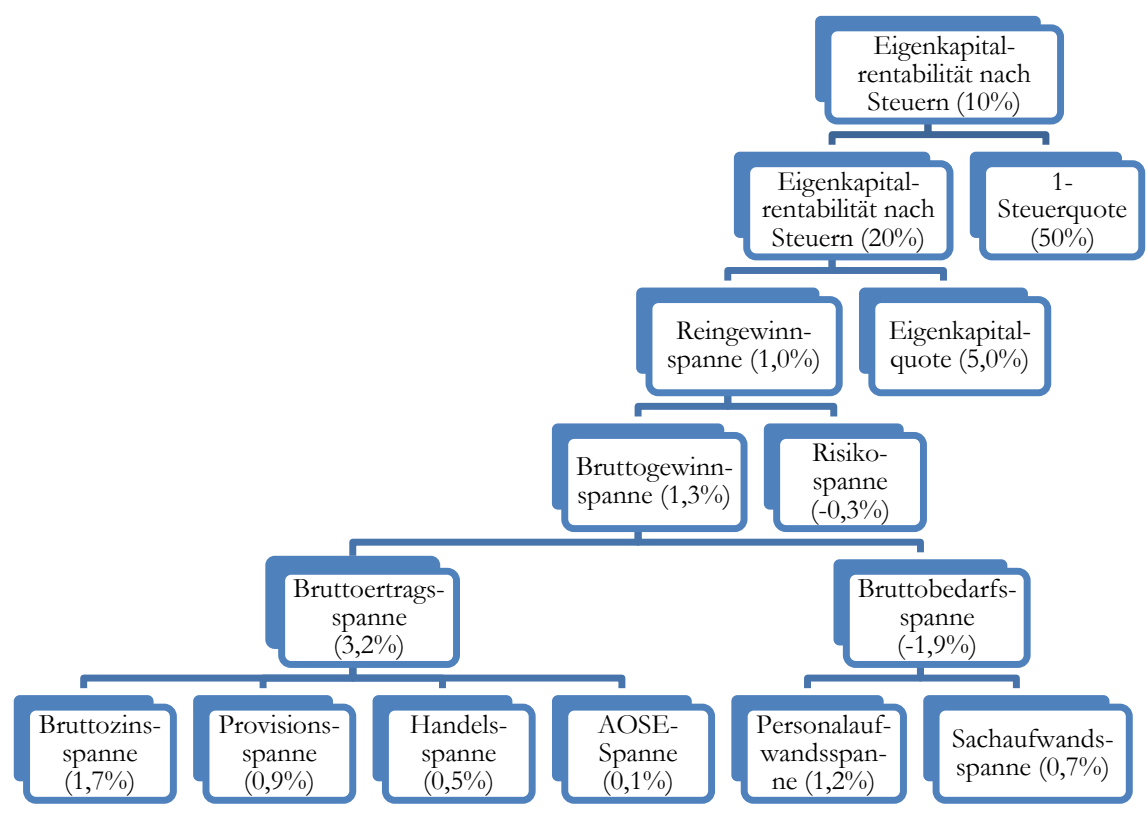

Abbildung 11: ROI-Grundschema zur Verknüpfung gesamtbankbezogener Aufwands-, Ertrags- und Ergebniskennzahlen ${ }^{633}$

Schon BÄSCH setzte die inhaltlich stark ähnelnde „Quellen- und strukturorientierte Erfolgsanalyse" ein, um die Komponenten und Einflussfaktoren des Jahreserfolgs von Banken transparent zu machen. ${ }^{634}$ Sein Ziel war ebenso, den Erfolg vergangener Rechnungsperioden zu erklären. ${ }^{635}$

Die folgenden Abschnitte erläutern die Berechnung und den Ausweis einzelner Ergebnisbereiche. Die Angaben entstammen den Gewinn- und Verlustrechnungen der einzelnen Banken, welche der Gliederung nach Formblatt $3^{636}$ folgen.

\subsubsection{Bruttozinsspanne}

Die Berechnung des Zinsüberschusses erfolgt durch Bilden des Saldos aus den Zinserträgen und den Zinsaufwendungen. Zusätzlich zu addieren sind die laufenden Erträge aus

\footnotetext{
${ }^{629}$ Vgl. Schierenbeck (2003b), S. 422.

${ }^{630} \mathrm{Vgl}$. Schierenbeck (2003b), S. 428

${ }^{631}$ Vgl. Schierenbeck (2003b), S. 428

${ }^{632}$ Vgl. Schierenbeck (2003b), S. 428.

${ }^{633}$ Eigene Darstellung, in Anlehnung an Schierenbeck (2003b), S. 429 und an Mugler (2014), S. 104

${ }^{634}$ Vgl. Bäsch (1992), S. 295

${ }^{635}$ Vgl. Bäsch (1992), S. 295.

636 Abbildung A1 im Anhang enthält Formblatt 3.
} 
Wertpapieranlagen, Beteiligungen und Anteilen an verbundenen Unternehmen ${ }^{637}$, welche allesamt in den Unterpunkten von Position 3 der GuV erfasst sind. ${ }^{638}$ Obwohl es sich hierbei um Dividenden handelt, werden diese im Rahmen der ROI-Analyse aus Gründen der Praktikabilität trotzdem zum Zinsüberschuss gezählt. ${ }^{639}$

Setzt man den erhaltenen Zinsüberschuss in das Verhältnis zur jahresdurchschnittlichen Bilanzsumme, erhält man die Zinsspanne ${ }^{640}$, welche bisweilen auch als Zinsmarge oder Bruttozinsspanne ${ }^{641}$ bezeichnet wird. Die Beziehung

$$
\text { Bruttozinsspanne (Zinsspanne) }=\frac{\text { Zinsüberschuss }}{D B S}
$$

enthält die entsprechende Berechnungsvorschrift. Die Zinsspanne wird in Prozent angegeben und liegt in der Regel im unteren einstelligen Bereich.

\subsubsection{Provisionsspanne}

Der Provisionsüberschuss errechnet sich als Differenz zwischen aus den Provisionserträgen ${ }^{642}$ und den Provisionsaufwendungen ${ }^{643}$. Der Provisionsüberschuss repräsentiert den Erfolg im nicht zinstragenden Geschäft. ${ }^{644}$ Bisweilen ist anstatt Provision auch der Begriff Kommission zu finden. ${ }^{645}$ Provisionen können bei allen Bankgeschäften anfallen. ${ }^{646}$ Dazu zählen nicht nur der Wertpapierhandel und die Vermögensverwaltung ${ }^{647}$, sondern auch der Zahlungsverkehr, das Außenhandelsgeschäft, das Treuhandgeschäft, das Emissionsgeschäft, das Devisen- und Sortengeschäft sowie die Vermittlung von Versicherungsverträgen, Immobilienanlagen oder Investmentfonds. ${ }^{648}$ Der Provisionsüberschuss ist nach dem Zinsüberschuss die zweitwichtigste Einnahmequelle von Kreditinstituten. ${ }^{649}$ Vergleicht man die Höhe der Provisionsspanne mit der Höhe der Zinsspanne, so wird eine relativ hohe Provisionsspanne ceteris paribus von externen Betrachtern in der Regel positiv gesehen werden. ${ }^{60}$ Dies ist darauf zurückzuführen, dass das Provisionsgeschäft weitgehend unabhängig von der Zinsentwicklung und von der Konjunktur ist, und dadurch als beständiger angesehen wird. ${ }^{651}$ Bei Genossenschaftsbanken, ebenso wie bei Sparkassen, liegt das Provisionsergebnis häufig deutlich über dem Zinsüberschuss. ${ }^{652}$ Dennoch kommt dem Provisionsergebnis eine entscheidende Rolle für das Jahresergebnis der Bank zu. ${ }^{653}$

\footnotetext{
${ }^{637}$ Vgl. Schierenbeck (2003b), S. 424

${ }^{638}$ Vgl. BaFin (2012b), S. 1.

${ }^{639}$ Vgl. Bäsch (1992), S. 314; vgl. Botsis et al. (2012), S. 75 f.

${ }^{640}$ Vgl. Bundesbank (2013c), S. 20.

${ }^{641}$ Vgl. Schierenbeck et al. (2014), S. 80; vgl. Botsis et al. (2012), S. 77.

${ }^{642}$ Vgl. Abschnitt 3.6.3.4.

${ }^{643}$ Vgl. Abschnitt 3.6.3.5.

${ }^{644}$ Vgl. Botsis et al. (2012), S. 78.

${ }^{645}$ Vgl. Schierenbeck (2003b), S. 424.

${ }^{646}$ Vgl. Schierenbeck (2003b), S. 424.

${ }^{647}$ Vgl. Botsis et al. (2012), S. 78.

${ }^{648} \mathrm{Vgl}$. Schierenbeck (2003b), S. 424.

${ }^{649}$ Vgl. Botsis et al. (2012), S. 78.

${ }^{650} \mathrm{Vgl}$. Bieg (2010a), S. 958.

${ }^{651}$ Vgl. Bieg (2010a), S. 958.

${ }^{652}$ Padberg (2005).

${ }^{653}$ Padberg (2005).
} 
Analog zur oben beschriebenen Zinsspanne lässt sich die Provisionsspanne ermitteln, indem man den Provisionsüberschuss in das Verhältnis zur durchschnittlichen Bilanzsumme setzt. Die Berechnung erfolgt anhand der Beziehung

$$
\text { Provisionsspanne }=\frac{\text { Provisionsüberschuss }}{D B S} .
$$

Synonym zum Begriff Provisionsspanne wird auch Provisionsmarge verwendet.

\subsubsection{Handelsspanne}

Die Nettoerträge aus dem Handelsgeschäft werden auch als Handelsergebnis bezeichnet. ${ }^{654}$ Sie werden in Position 7 des Formblatts 3 erfasst. ${ }^{655}$ Die Errechnung der Handelsspanne erfolgt gemäß der folgenden Beziehung, indem die genannten Nettoerträge in das Verhältnis zur durchschnittlichen Bilanzsumme gesetzt werden:

$$
\text { Handelsspanne }=\frac{\text { Nettoerträge aus dem Handelsgeschäft }}{D B S} .
$$

\subsubsection{Sonstige Ertragsspanne}

Die in der ROI-Analyse berechnete Position Sonstige betriebliche Erträge (netto) beinhaltet alle Ertrags- und Aufwandsposten aus der Gewinn- und Verlustrechnung, die weder zum Zinsüberschuss noch zum Provisionsüberschuss noch zum Handelsgeschäft gerechnet werden können. ${ }^{65}$ Obwohl diese ROI-Position eine ähnliche Bezeichnung trägt wie die GuVPosition 8 Sonstige betriebliche Erträge, ist sie inhaltlich ausdrücklich nicht deckungsgleich, sondern umfasst, wie beschrieben, alle übrigen, in der ROI-Analyse noch nicht erfassten Erträge und Aufwendungen auf der Gewinn- und Verlustrechnung. ${ }^{657}$ Dies beinhaltet im Einzelnen die Positionen 4, 8, 9, 12, 15, 16, 17 und 18 aus Formblatt $3 .^{658}$

\subsubsection{Bruttoertragsspanne}

Die Summe aus Zinsüberschuss, Provisionsüberschuss, Handelsergebnis und den sonstigen betrieblichen Erträgen (netto) fasst die ROI-Analyse zusammen als Gesamterträge aus normaler Geschäftstätigkeit. Die entsprechende Bruttoertragsspanne errechnet sich anhand der Beziehung

$$
\text { Bruttoertragsspanne }=\frac{\text { Gesamterträge aus normaler Geschäftstätigkeit }}{D B S} .
$$

\footnotetext{
${ }^{654} \mathrm{Vgl}$. Schierenbeck (2003b), S. 425.

${ }^{655}$ Vgl. Schierenbeck (2003b), S. 424.

${ }^{656}$ Vgl. Schierenbeck (2003b), S. 424.

${ }^{657}$ Vgl. Schierenbeck (2003b), S. 424.

${ }^{658}$ Vgl. BaFin (2012b); vgl. Schierenbeck (2003b), S. 424.
} 


\subsubsection{Bruttobedarfsspanne}

Die Betriebsaufwendungen setzen sich zusammen aus den Personalaufwendungen aus der GuV-Position 10. a), den Sachaufwendungen aus der GuV-Position 10. b) sowie den Abschreibungen und Wertberichtigungen auf immaterielle Anlagewerte und Sachanlagen aus der GuV-Position 11. ${ }^{659}$ Die Errechnung der Personalaufwandsspanne ${ }^{660}$ und der Sachaufwandsspanne ${ }^{661}$ erfolgt anhand der Beziehungen

$$
\text { Personalaufwandsspanne }=\frac{\text { Personalaufwendungen }}{D B S}
$$

und

$$
\text { Sachaufwandsspanne }=\frac{\text { Sachaufwendungen }}{D B S} .
$$

Wie bereits oben erwähnt, werden die Abschreibungen häufig in die Sachaufwendungen eingerechnet. ${ }^{622}$ Die Spanne, welche die Betriebsaufwendungen abbildet, wird als Bruttobedarfsspanne bezeichnet und ergibt sich durch ${ }^{663}$

$$
\text { Bruttobedarfsspanne }=\frac{\text { Betriebsaufwendungen }}{D B S} .
$$

\subsubsection{Bruttogewinn aus normaler Geschäftstätigkeit}

Durch Saldierung der Gesamterträge aus normaler Geschäftstätigkeit mit den Betriebsaufwendungen erhält man den Bruttogewinn aus normaler Geschäftstätigkeit. Dieser wird im Rahmen der vorliegenden Arbeit als Erfolgsmessgröße eingesetzt. ${ }^{664}$ In das Verhältnis zur durchschnittlichen Bilanzsumme gesetzt, errechnet sich die Bruttogewinnspanne gemäß

$$
\text { Bruttogewinnspanne }=\frac{\text { Bruttogewinn aus normaler Geschäftstätigkeit }}{D B S} .
$$

\subsubsection{Betriebsergebnis aus normaler Geschäftstätigkeit}

Aus dem Bruttogewinn aus normaler Geschäftstätigkeit erhält man, nach Abzug der Risikoaufwendungen, das Betriebsergebnis aus normaler Geschäftstätigkeit. ${ }^{665}$ Als Verhältniszahl zur durch-

\footnotetext{
${ }^{659} \mathrm{Vgl}$. Schierenbeck (2003b), S. 424.

${ }^{660}$ Vgl. Botsis et al. (2012), S. 79.

661 Vgl. Botsis et al. (2012), S. 80

${ }^{662} \mathrm{Vgl}$. Abschnitt 3.6.3.8.

${ }^{663}$ Vgl. Mugler (2014), S. 86

${ }^{664} \mathrm{Vgl}$. Abschnitt 3.5.3.1.

${ }^{665}$ Vgl. Schierenbeck (2003b), S. 425.
} 
schnittlichen Bilanzsumme wird es als Nettogewinnspanne bezeichnet und mit der Beziehung

$$
\text { Nettogewinnspanne }=\frac{\text { Betriebsergebnis aus normaler Geschäftstätigkeit }}{D B S}
$$

errechnet.

\subsubsection{Zusammenfassung ROI-Analyse}

Die ROI-Analyse erlaubt ferner die Berücksichtigung des außerordentlichen Ergebnisses und von Gewinnsteuern. ${ }^{666} \mathrm{Da}$ beide Punkte für die vorliegende Arbeit nicht näher von Belang sind, erfolgen hierzu keine weiteren Ausführungen.

Das rechnerische Vorgehen der ROI-Analyse wird in Tabelle 5 zusammengefasst.

\begin{tabular}{|l|l|l|}
\hline \multicolumn{2}{|l|}{$\begin{array}{l}\text { Erfolgsspaltung in der } \\
\text { Gewinn- und Verlustrechnung }\end{array}$} & $\begin{array}{l}\text { Begriffskategorien } \\
\text { der ROI-Analyse }\end{array}$ \\
\hline+ & $\begin{array}{l}\text { Überschuss im zinsabhängigen Geschäft (Zinsüberschuss) } \\
\text { (Provisionsüberschuss) }\end{array}$ & Bruttozinsspanne \\
\hline \pm & Nettoerträge aus dem Handelsgeschäft (Handelsergebnis) & Provisionsspanne \\
\hline \pm & Sonstige betriebliche Erträge (netto) & Sonstige Ertragsspanne \\
\hline$=$ & Gesamterträge aus normaler Geschäftstätigkeit & Bruttoertragsspanne \\
\hline- & $\begin{array}{c}\text { Betriebsaufwendungen } \\
\bullet \quad \begin{array}{l}\text { Personalaufwendungen } \\
\text { Sachaufwendungen }\end{array}\end{array}$ & $\begin{array}{l}\text { Bruttobedarfsspanne } \\
\text { Personalaufwandsspanne } \\
\text { Sachaufwandsspanne }\end{array}$ \\
\hline$=$ & Bruttogewinn aus normaler Geschäftstätigkeit & Bruttogewinnspanne \\
\hline- & Risikoaufwendungen & Risikospanne \\
\hline$=$ & Betriebsergebnis aus normaler Geschäftstätigkeit & Nettogewinnspanne \\
\hline
\end{tabular}

Tabelle 5: $\quad$ Erfolgsspaltung in der Gewinn- und Verlustrechnung sowie Begriffskategorien der ROI-Analyse ${ }^{667}$ 


\subsection{Zu untersuchende Erfolgsfaktoren}

Die folgenden Faktoren (Erfolgsfaktoren) werden als unabhängige Variablen daraufhin untersucht, ob sie einen Einfluss auf abhängige Variablen haben, welche wiederum als Erfolgsmessgrößen angesehen werden.

- Lage nach Städtisch/Halbstädtisch/Ländlich

- Personal- und Sachaufwand

- Lage nach Bundesländern

- Abschreibungen auf Forderungen

- Eigenkapitalquote

- Kreditquote

- Betriebsgröße der Bank

- Zukunftsfähigkeit des Sitzkreises

- Arbeitnehmerentgelt bzw. Bruttolöhne und -gehälter

- Bruttoinlandsprodukt je Einwohner bzw. je Erwerbstätiger

- Anteil grundpfandrechtlich gesicherter Kredite an den Kundenforderungen

- Wachstum des Bruttoinlandsprodukts in der Vergangenheit

- Anzahl der Mitarbeiter der Bank

- Anzahl der Zweigstellen der Bank

- Im Geschäftsjahr stattgefundene Fusion bzw. Fusionen

- Niveau der Zinsstrukturkurve

- Steilheit der Zinsstrukturkurve

- Anteil älterer Menschen an der Bevölkerung des Sitzkreises

Obgleich die Lage für ein Kreditinstitut nicht beeinflussbar ist, und das Geschäftsgebiet, wenn überhaupt, nur in engen Grenzen erweitert werden kann, ist davon auszugehen, dass es sich bei der Lage um einen sehr wichtigen Einflussfaktor auf den Erfolg handelt. Dies konnte durch einige empirische Arbeiten belegt werden. ${ }^{668}$

\subsection{Ableitung der Forschungshypothesen}

Im nächsten Schritt werden die Forschungshypothesen aus den identifizierten Forschungslücken ${ }^{69}$ und den Forschungsfragen ${ }^{670}$ abgeleitet und in Kategorien gegliedert. Es sei darauf hingewiesen, dass jeweils nur die Nullhypothese $\mathrm{H}_{0}$ angegeben wird. Die entsprechende

\footnotetext{
${ }^{668}$ Vgl. Richter (2014); vgl. Christians und Gärtner (2014); vgl. Hummel und Pedrotti (2013); vgl. Tischer (2011); vgl. Reichel (2011); vgl. Christians (2010); vgl. Conrad et al. (2009); vgl. Thiry (2009); vgl. Auerbach (2009); vgl. Varmaz (2006); vgl. Padberg (2005); vgl. Riekeberg (2003); vgl. Wutz (2002); vgl. Tebroke (1993); vgl. Bäsch (1992); vgl. Karmann et al. (2013).

${ }^{669} \mathrm{Vgl}$. Abschnitt 2.2.

${ }^{670}$ Vgl. Abschnitt 2.3.
} 
Alternativhypothese $\mathrm{H}_{1}$ ist stets gleich der Negation der jeweiligen Nullhypothese. Dabei kommt ein Signifikanzniveau von $\alpha=0,05$ zur Anwendung. ${ }^{671}$

\subsubsection{Hypothesenkategorie A: Nach der Lage von Banken}

H1: Banken in ländlichen Regionen unterscheiden sich hinsichtlich ihres Erfolges von Banken in urbanen Gebieten.

Aus der Differenzierung nach den Komponenten der Bruttogewinnspanne ergeben sich folgende Hypothesen $\mathrm{H} 2$ bis $\mathrm{H} 9$ :

H2: Banken in ländlichen Regionen unterscheiden sich hinsichtlich ihrer Zinserträge aus Kredit- und Geldmarktgeschäften von Banken in urbanen Gebieten. Unterschiede bestehen weiter im Anteil risikoreicher Kundenforderungen.

H3: Banken in ländlichen Regionen unterscheiden sich hinsichtlich ihrer Zinserträge aus festverzinslichen Wertpapieren und Schuldbuchforderungen von Banken in urbanen Gebieten.

H4: Banken in ländlichen Regionen unterscheiden sich hinsichtlich ihrer Zinsaufwendungen von Banken in urbanen Gebieten.

H5: Banken in ländlichen Regionen unterscheiden sich hinsichtlich ihrer laufenden Erträge von Banken in urbanen Gebieten.

H6: Banken in ländlichen Regionen unterscheiden sich hinsichtlich ihrer Zinsüberschüsse von Banken in urbanen Gebieten.

H7: Banken in ländlichen Regionen unterscheiden sich hinsichtlich ihres Provisionsüberschusses von Banken in urbanen Gebieten

H8: Banken in ländlichen Regionen unterscheiden sich hinsichtlich ihres Personalaufwands von Banken in urbanen Gebieten.

H9: Banken in ländlichen Regionen unterscheiden sich hinsichtlich ihres Sachaufwands von Banken in urbanen Gebieten.

H10: Banken in ländlichen Regionen unterscheiden sich hinsichtlich ihrer Größe von Banken in urbanen Gebieten.

H11: Die Höhe des Erfolgs hängt davon ab, in welchem Bundesland eine Bank liegt.

\subsubsection{Hypothesenkategorie B: Nach Positionen der Gewinn- und Verlustrech- nung und nach der Bilanzstruktur}

H12: Abschreibungen auf Forderungen und im Kreditgeschäft stehen im Zusammenhang mit der Bruttogewinnspanne.

H13: Banken mit einer hohen Eigenkapitalquote sind erfolgreicher.

H14: Banken mit einer hohen Kreditquote sind erfolgreicher. 


\subsubsection{Hypothesenkategorie C: Nach der Größe von Banken}

H15: Bezogen auf ihre Bilanzsumme weisen größere Banken eine höhere Bruttogewinnspanne als kleinere Banken auf. Nach Überschreiten einer kritischen Bilanzsumme führen andere Einflüsse jedoch zur Umkehrung dieses Effekts.

H16: Es existieren Regionen, in denen besonders viele, dafür aber sehr kleine Genossenschaftsbanken gehäuft existieren.

\subsubsection{Hypothesenkategorie D: Nach regionalen Wirtschaftsdaten}

H17: Erfolgreiche Banken liegen in Gegenden mit guter Zukunftsfähigkeit.

H18: Ein hohes Arbeitnehmerentgelt in der Region begünstigt den Erfolg regionaler Banken.

H19: Erfolgreiche Banken liegen in Regionen mit hohem Bruttoinlandsprodukt je Einwohner.

H20: Erfolgreiche Banken liegen in Regionen mit hohem Bruttoinlandsprodukt je Erwerbstätiger.

H21: In Regionen mit hohem durchschnittlichem Wachstum des Bruttoinlandsprodukts erzielen Banken eine relativ hohe Bruttogewinnspanne.

Zwischen den Hypothesenkategorien sind inhaltliche Überlappungen möglich, da sich die Teilgebiete nicht vollständig trennen lassen. Insbesondere seien hier Hypothese 15 zur Bankgröße und Hypothese 21 zum Wachstum des Bruttoinlandsprodukts genannt. 
Die empirische Analyse bildet den Kern der vorliegenden Arbeit, da sie die Erfolgsfaktoren untersucht. Im Rahmen der Analyse werden die in Abschnitt 3.9 aufgestellten Forschungshypothesen geprüft und die Resultate interpretiert.

\subsection{Methodik der Datenerhebung}

Zur Datenerhebung der Jahresabschlüsse sind verschiedene Ansätze denkbar. Eine erste Möglichkeit stellt der Bundesanzeiger ${ }^{672}$ dar, welcher vormals als Elektronischer Bundesanzeiger firmierte. Beginnend mit dem Geschäftsjahr 2005 enthält er sämtliche Jahresabschlüsse deutscher Unternehmen, die zur Veröffentlichung verpflichtet sind, und somit auch die der Kreditgenossenschaften. Diese Abschlüsse sind dort im Teil Rechnungslegung/Finanz̧berichte abrufbar. Der Abruf geschieht einzeln für jedes Geschäftsjahr und für jede Bank in Textform, eine maschinelle Verarbeitung ist seitens des Bundesanzeigers nicht vorgesehen. Eine weitere Möglichkeit stellt der sogenannte ,Jährliche Betriebsvergleich“ dar, welchen der Bundesverband der Deutschen Volksbanken und Raiffeisenbanken (BVR) jährlich von allen seinen Mitgliedern erhebt. ${ }^{673}$ In Ermangelung der Erhältlichkeit dieser Daten für die wissenschaftliche Forschung erfolgte die Erhebung der Daten durch eine dritte Möglichkeit, der Abfrage einer Bilanzdatenbank.

\subsection{Methodik der Datenanalyse und -auswertung}

Zur Identifikation von Zusammenhängen stehen verschiedene statistische Analysen und Methoden zur Verfügung, welche im Folgenden vor deren Einsatz erläutert werden.

\subsubsection{Formale vs. sachliche Abhängigkeit}

Mit statischen Methoden können formale Abhängigkeiten untersucht werden, das heißt, zahlenmäßige Zusammenhänge werden identifiziert. ${ }^{674}$ Diese formale Abhängigkeit ist dabei von der kausalen (sachlichen) Abhängigkeit zu unterscheiden.

Unter kausaler Abhängigkeit versteht man einen eindeutigen Zusammenhang, ob die Höhe eines Merkmals ursächlich für die Höhe eines anderen Merkmals ist. Das Vorliegen einer kausalen Abhängigkeit muss gesondert untersucht werden, zusätzlich zur reinen statistischen Betrachtung. ${ }^{675}$ Der Nachweis von Kausalzusammenhängen kann nur durch sachlogische Überlegungen geschehen. ${ }^{676}$ Durch statistische Verfahren werden nie Kausalzusammenhänge ermittelt. ${ }^{677}$

Ein Beispiel: Der Zusammenhang zwischen dem Zinsüberschuss und dem Bruttogewinn aus normaler Geschäftstätigkeit kann formal auf statistischem Wege nachgewiesen werden. Kausal, also sachlich, erfolgt der Nachweis des Zusammenhangs über die Argumentation, dass sich aufgrund einer Erhöhung des Zinsüberschusses auch der Bruttogewinn aus normaler Geschäftstätigkeit erhöht und vice versa.

\footnotetext{
${ }^{672} \mathrm{Vgl}$. Bundesanzeiger

${ }^{673}$ Vgl. Richter (2014), S. 417.

${ }^{674}$ Vgl. Bourier (2012), S. 198.

${ }^{675}$ Vgl. Bourier (2012), S. 198.

${ }^{676}$ Vgl. Fahrmeir, Künstler, Pigeot und Tutz (2007), S. 148.

${ }^{677}$ Vgl. Atteslander (2010), S. 315.
} 
Weiter unterscheidet BOURIER ${ }^{678}$ zwischen folgenden Fällen, in denen ein formaler $\mathrm{Zu}$ sammenhang nachgewiesen werden kann, sachlich aber keine Begründung möglich ist:

- Inhomogenitätskorrelation: der formale Zusammenhang liegt in der inhomogenen Zusammensetzung der Gruppe begründet. Beispielhaft sei hier ein Zusammenhang zwischen Körpergröße und Einkommen Erwachsener aufgeführt: je größer eine Person, desto mehr Einkommen erzielt diese. Die Begründung liegt jedoch in der Zusammensetzung der Gesamtgruppe Erwachsene aus männlichen und weiblichen Probanden. Frauen sind im Durchschnitt kleiner als Männer und verdienen im Mittel auch weniger. ${ }^{679}$ In Ergänzung zu diesen Ausführungen nach BOURIER wird darauf hingewiesen, dass das Deutsche Institut für Wirtschaftsforschung e.V. (DIW) im Jahr 2004 speziell für Männer einen Einfluss der Körpergröße auf den Verdienst aufzeigen konnte. ${ }^{600}$

- Gemeinsamkeitskorrelation: diese liegt vor, wenn der formale Zusammenhang von $\mathrm{X}$ und $\mathrm{Y}$ darauf zurückzuführen ist, dass sowohl $\mathrm{X}$ als auch $\mathrm{Y}$ von einem dritten Merkmal $\mathrm{Z}$ abhängig sind. ${ }^{681}$ Ist also formal etwa ein Zusammenhang zwischen dem Kraftfahrzeugbestand und dem Bestand an Eigentumswohnungen nachweisbar, so sind beide auf das Merkmal Wohlstand zurückzuführen. ${ }^{682}$

- „Unsinns“-Korrelation: liegt dann vor, wenn der formale Zusammenhang zufällig ist oder zumindest sachlogisch nicht begründet werden kann. ${ }^{63}$

In allen drei der vorgenannten Fälle spricht man von einer Scheinkorrelation. Hier ist der formale Zusammenhang sachlich nicht begründbar, das heißt inhaltlich nicht gerechtfertigt ist. $^{684}$

\subsubsection{Test auf Normalverteilung}

Das Vorliegen der Normalverteilung ist eine Voraussetzung für die Anwendbarkeit zahlreicher Tests. Die Normalverteilung ist die bekannteste und gebräuchlichste Wahrscheinlichkeitsverteilung in der Statistik. ${ }^{685}$ Zur Überprüfung, ob eine solche Verteilung vorliegt, stehen folgende grundlegende Arten von Möglichkeiten zur Disposition, welche je nach ihrem methodischen Ansatz zusammengefasst werden.

1) Grafische Methoden wie Histogramm, Q-Q-Plot und Boxplot ${ }^{686}$ zur Ersteinschätzung

2) Numerische Methoden (Momententests) mit Kennzahlen von Schiefe und Kurtosis (Wölbung) zum Vergleich der empirischen Momente mit den theoretischen Momenten der Normalverteilung ${ }^{687}$

3) Formale EDF-Tests, welche die empirische Verteilungsfunktion (Empirical Distribution Function) der Stichprobe mit der theoretischen Normalverteilung verglei-

\footnotetext{
${ }^{678}$ Vgl. Bourier (2012), S. 198.

${ }^{679}$ Vgl. Bourier (2012), S. 198.

${ }^{680} \mathrm{Vgl}$. Deutsches Institut für Wirtschaftsforschung e.V. (2004).

${ }^{681}$ Vgl. Bourier (2012), S. 198.

${ }^{682}$ Vgl. Bourier (2012), S. 198.

${ }^{683}$ Vgl. Bourier (2012), S. 198.

${ }^{684}$ Vgl. Fahrmeir et al. (2007), S. 148f; vgl. Bourier (2012), S. 198.

${ }^{685}$ Vgl. Gohout und Specht (2014), S. 544.

${ }^{686}$ Vgl. Gohout und Specht (2014), S. 548.

${ }^{687}$ Vgl. Gohout und Specht (2014), S. 544, 546 f.
} 
chen. Hierzu zählen Tests wie SHAPIRO-WILK ${ }^{688}$, ANDERSON-DARLING ${ }^{689}$, LILLIEFORS $^{690}$, KOLMOGOROW-SMIRNOV ${ }^{691}$ sowie der Chi-QuadratAnpassungstest ${ }^{692}$.

Eine schematische Darstellung der Methoden findet sich in Abbildung 12.

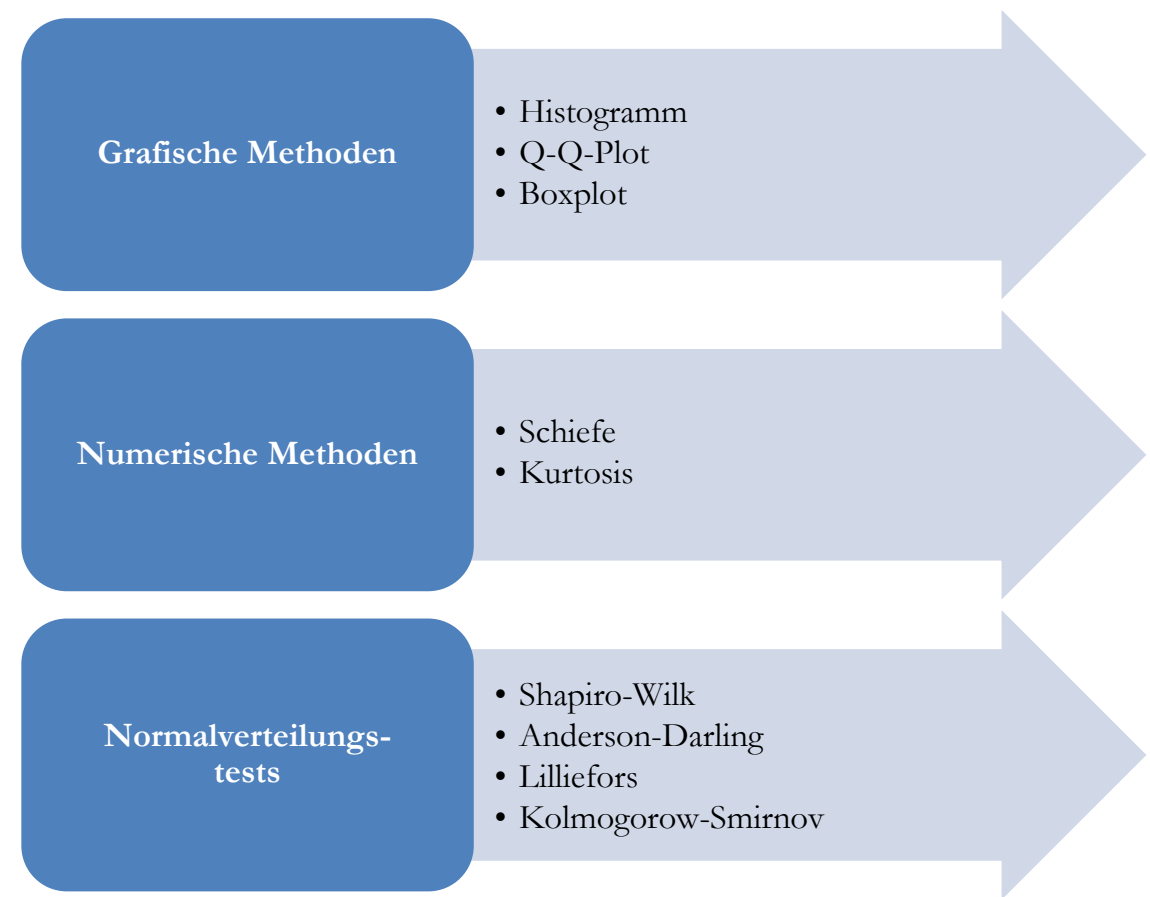

Abbildung 12: Methoden zur Prüfung auf Normalverteilung

In einem Vergleichstest stellten RAZALI und WAH vier der fünf genannten Tests gegenüber. ${ }^{693}$ Sie konnten herausfinden, dass von allen vier Tests SHAPIRO-WILK die höchste Güte $^{694}$ besitzt. $^{695}$ Auf den Rängen folgen ANDERSON-DARLING, LILLIEFORS und Kolmogorow-SMirnov. ${ }^{696}$ KESKIN bestätigt dieselbe Rangfolge. ${ }^{697}$ Auch GOHOUT und SPECHT raten von der Verwendung von KOLMOGOROW-SMIRNOV ab. ${ }^{698}$ Die vorliegende Arbeit wird daher den SHAPIRO-WILK-Test zur Evaluation der Normalverteilung verwenden.

\subsubsection{Test auf Varianzhomogenität}

Für einige Tests ist das Vorliegen von Varianzhomogenität erforderlich. Dies bedeutet, dass sich die Varianzen zwischen den einzelnen Gruppen nicht unterscheiden. Es muss Homoskedastizität vorliegen, das heißt, die Varianzen müssen homogen sein. Beim Vorlie-

\footnotetext{
${ }^{688}$ Vgl. Shapiro und Wilk (1965), S. 591.

${ }^{689} \mathrm{Vgl}$. Anderson und Darling (1954), S. 765

${ }^{690}$ Vgl. Lilliefors (1967), S. 399.

691 Vgl. Kolmogorov (1933), S. 83.

${ }^{692}$ Der Chi-Quadrat-Anpassungstest wird wegen seiner geringen statistischen Trennschärfe nicht weiter verfolgt.

${ }^{693}$ Vgl. Razali und Wah (2011), S. 1.

${ }^{694}$ Im Rahmen der Beurteilung der statistischen Güte wird die Höhe des Betafehlers betrachtet. Der Betafehler (Fehler 2. Art) tritt beispielsweise auf, wenn ein tatsächlich vorhandener Unterschied zwischen zwei Populationen durch einen Test zweier Stichproben nicht identifiziert wird. Vgl. Bortz und Schuster (2010), S. 100.

${ }^{695}$ Vgl. Razali und Wah (2011), S. 1.

${ }^{696}$ Vgl. Razali und Wah (2011), S. 1.

${ }^{697}$ Vgl. Keskin (2006), S. 297.

${ }^{698}$ Vgl. Gohout und Specht (2014), S. 545.
} 
gen von Heteroskedastizität muss ein entsprechender anderer Test durchgeführt werden. Als Testverfahren kann unter anderem der Levene-Test eingesetzt werden. ${ }^{699}$ Die Nullhypothese $H_{0}$ des Levene-Tests lautet, es liege Varianzhomogenität vor. Wie allgemein üblich bei der Bearbeitung vergleichbarer Aufgabenstellungen ${ }^{700}$ wurde ein Konfidenzniveau von 0,95 bzw. eine Irrtumswahrscheinlichkeit von 0,05 verwendet. Bei einem Testwert von unter 0,05 wird der Test signifikant und die Nullhypothese der Varianzhomogenität ist abzulehnen. ${ }^{701}$ Dann ist auf diesem Signifikanzniveau davon auszugehen, dass sich die Varianzen zwischen den einzelnen Stichproben signifikant unterscheiden.

\subsubsection{Boxplot}

Wie bereits im Abschnitt 4.2.2 (Test auf Normalverteilung) beschrieben, kann die Verteilung der einzelnen Merkmalsausprägungen mittels eines Boxplots dargestellt werden. Bisweilen wieder auch als Box-Whisker-Plot bezeichnet. Die Box beinhaltet die mittleren $50 \%$ aller Werte, das heißt vom $25 \%$-Quantil bis zum $75 \%$-Quantil. Sie wird durch eine starke Trennlinie an der Stelle des Medians unterteilt. ${ }^{702}$ Nach oben und unten schließen sich Linien an, welche die Lage der übrigen Werte bis einschließlich des Minimums bzw. des Maximums darstellen. Ausreißer werden durch o, Extremwerte mit * gekennzeichnet. ${ }^{703}$ Als AusreiBer werden Werte verstanden, die zwischen 1,5 und 3 Boxlängen außerhalb der Box liegen. ${ }^{704}$ Extremwerte (auffallende Ausreißer) liegen mehr als 3 Boxlängen außerhalb der Box. ${ }^{705}$ Ein Beispiel eines Boxplots wird in Abbildung 13 dargestellt.

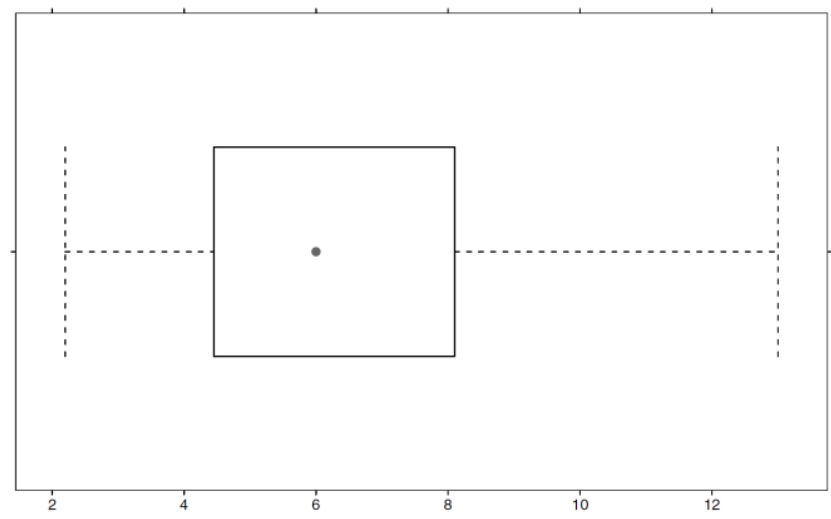

Abbildung 13: Beispiel eines Boxplots ${ }^{706}$

\subsubsection{Mittelwerttests}

Mittelwerttests erlauben einen Vergleich zwischen zwei oder mehr Grundgesamtheiten. Es kann untersucht werden, ob zwischen diesen Grundgesamtheiten ein signifikanter Unterschied bezüglich des Mittelwertes besteht. ${ }^{707}$ Die Einteilung in die Grundgesamtheiten

\footnotetext{
${ }^{699}$ Vgl. Huber, Meyer und Lenzen (2014), S. 65; vgl. Bortz und Schuster (2010), S. 129 f.

${ }^{700}$ Vgl. Richter (2014), S. 428; vgl. Hummel und Pedrotti (2013), S. 12; vgl. Christians (2010), S. 76.

701 Vgl. Kähler (2011), S. 439.

702 Vgl. Bortz und Schuster (2010), S. 44f; vgl. Bühl (2012), S. 1011.

${ }^{703}$ Vgl. Bühl (2012), S. 1011.

${ }^{704}$ Vgl. Bühl (2012), S. 1011

705 Vgl. Hartung, Elpelt und Klösener (2005), S. 835.

706 Übernommen aus Fahrmeir et al. (2007), S. 69.

${ }^{707}$ Dazu ist zunächst sicherzustellen, dass Varianzhomogenität vorliegt. Danach kann das Vorhandensein einer Normalverteilung geprüft werden. Mit der Verteilungsannahme eruiert schließlich in einem dritten Schritt ein parametrischer Test, ob die Mittelwerte signifikant voneinander abweichen.
} 
erfolgt in der Regel mittels eines qualitativen Merkmals, beispielsweise der Zugehörigkeit zu einer der Lagekategorien Städtisch, Halbstädtisch oder Ländlich. ${ }^{708}$

Das zu untersuchende Merkmal, beispielsweise die kardinal skalierte Bruttogewinnspanne, ist quantitativer Natur. Im Rahmen des Tests werden schließlich die arithmetischen Mittel von allen Grundgesamtheiten miteinander verglichen. ${ }^{709}$

Grundsätzlich wäre alternativ auch ein direkter Vergleich der Mittelwerte der Grundgesamtheiten möglich. Jedoch werden diese Mittelwerte in der Regel auf Basis von Stichproben ermittelt. Aufgrund von Zufallsschwankungen können sich diese Stichprobenmittelwerte selbst dann noch voneinander unterscheiden, wenn die Grundgesamtheiten identisch sind. Aus diesem Grunde muss geprüft werden, ob sich die Abweichung in den Mittelwerten signifikant von Null unterscheidet. Dies leisten die Mittelwerttests. ${ }^{710}$

\subsubsection{Der $t$-Test}

Der $t$-Test gehört zu den Mittelwerttests. ${ }^{711}$ Mit dem $t$-Test für unabhängige Stichproben kann überprüft werden, ob zwei Stichproben aus Grundgesamtheiten stammen, die sich hinsichtlich des Mittelwertes auf einem statistisch signifikanten Niveau unterscheiden. ${ }^{712}$

Sind mehr als zwei Grundgesamtheiten auf ihre Unterschiede zu prüfen, ist es zunächst denkbar, den t-Test paarweise zwischen allen Kombinationen von Stichproben durchzuführen. Liegen etwa 3 Grundgesamtheiten vor, so würde man folglich 1 mit 2, 1 mit 3 und 2 mit 3 vergleichen. Jedoch ist der $t$-Test ungeeignet, wenn mehr als zwei Grundgesamtheiten zur Untersuchung vorliegen, denn er ist grundsätzlich nur für unabhängige Stichproben geeignet. ${ }^{713}$ Mit steigender Anzahl durchgeführter Einzelvergleiche erhöht sich die Wahrscheinlichkeit, dass der $t$-Test fälschlicherweise einen signifikanten Unterschied der Mittelwerte anzeigt. ${ }^{714}$ An dieser Stelle kommt die Varianzanalyse zur Anwendung.

\subsubsection{Varianzanalyse (ANOVA)}

Der Begriff ANOVA (Analysis of Variance) entstammt der englischen Bezeichnung für die Varianzanalyse. Ursprung dieser Bezeichnung ist die Tatsache, dass sich die betrachtete Prüfgröße als Quotient zweier Varianzen errechnet. Denn obwohl die Bezeichnung Varianzanalyse suggeriert, dass das Verfahren die Varianzen vergleiche, prüft es eine Hypothese über die Gleichheit zweier oder mehrerer Mittelwerte. ${ }^{715}$ Das Verfahren zerlegt die Varianz, aus dieser Dekomposition lässt sich die Prüfstatistik ableiten. ${ }^{716}$ Eine Varianzanalyse, die zum Vergleich zweier Mittelwerte eingesetzt wird, führt zu demselben Resultat wird der $t$ Test. $^{717}$

Grundsätzlich erlaubt es die Varianzanalyse, $N$ Gruppen von Individuen daraufhin zu untersuchen, ob sich diese anhand ihres Mittelwertes signifikant voneinander unterscheiden:

\footnotetext{
${ }^{708}$ Vgl. Schulze und Porath (2012), S. 530.

${ }^{709}$ Vgl. Schulze und Porath (2012), S. 530.

${ }^{710}$ Vgl. Schulze und Porath (2012), S. 530.

711 Vgl. Bortz und Schuster (2010), S. 117.

712 Vgl. Bortz und Schuster (2010), S. 120.

${ }^{713}$ Ein Wechsel im Vorstand oder in der Strategie einer Bank kann zu einem Bruch der Zeitreihe führen.

714 Vgl. Kähler (2011), S. 435.

715 Vgl. Schulze und Porath (2012), S. 539; Bamberg, Baur und Krapp (2012), S. 184.

${ }^{716}$ Vgl. Auer und Rottmann (2015), S. 473.

717 Vgl. Abschnitt 4.2.5.1.
} 
$\mu_{1}$ : Mittelwert Gruppe 1

$\mu_{2}$ : Mittelwert Gruppe 2

$\mu_{3}$ : Mittelwert Gruppe 3

..

$\mu_{N}$ : Mittelwert Gruppe N

Die Nullhypothese der Varianzanalyse lautet, dass die Erwartungswerte aller $N$ betrachteten Kategorien identisch sind. ${ }^{718}$

Nullhypothese $H_{0}: \mu_{1}=\mu_{2}=\mu_{3}=\ldots=\mu_{N}$

Die Alternativhypothese $H_{1}$ besagt, dass es beim paarweisen Vergleich aller $N$ Kategorien untereinander mindestens bei einem Vergleich einen Unterschied gibt.

Alternativhypothese $H_{1}: \mu_{i} \neq \mu_{j}$ für mindestens ein $i, j=1 \ldots N(i \neq j)$

Die Stichproben müssen folgende Voraussetzungen erfüllen: ${ }^{719}$

1. Die Stichproben müssen voneinander unabhängig sein.

2. Die Stichproben müssen normalverteilt sein.

3. Die Varianz in allen Stichproben muss gleich sein.

Ad Voraussetzung 1: Es liegt Unabhängigkeit zwischen Zufallsgrößen vor, wenn für jede Auswahl von maximal $N$ Ereignissen Unabhängigkeit vorliegt. ${ }^{720}$ Die Erhebung einer Beobachtung in einer Kategorie hat hierbei keinen Einfluss auf die Erhebung von Beobachtungen, die in anderen Kategorien liegen. Es lassen sich die Kategorien als Kriterien zur Abgrenzung der Stichproben verwenden. ${ }^{721}$ In den Fällen, die in der vorliegenden Arbeit untersucht werden, kann davon ausgegangen werden, dass die Voraussetzung unabhängiger Stichproben erfüllt ist, da sich die Stichproben nicht überschneiden. Eine eindeutige Zuordnung zu den Kategorien ist möglich.

Ad Voraussetzung 2: Zur Überprüfung, ob eine Normalverteilung vorliegt, kann ein entsprechender Test durchgeführt werden. ${ }^{722}$ Sollte keine Normalverteilung vorliegen, erfolgt eine gesonderte Kennzeichnung. Einige Autoren schlagen für diesen Fall vor, als Alternative zur Varianzanalyse den H-Test von KRUSKAL und WALLIS ${ }^{723}$ einzusetzen. Hierbei handelt es sich um eine Weiterentwicklung des Mann-Whitney-U-Tests. ${ }^{724}$ Der Kruskal-WallisH-Test vergleicht mehr als zwei unabhängige Stichproben. Im Gegensatz zur Normalverteilung, die mit den Parametern $\mu$ und $\sigma$ arbeitet, ist er nichtparametrisch ${ }^{725}$ und verwendet eine gemeinsame Rangreihe der Werte aller Stichproben. ${ }^{726}$ Auf diese Art setzt er nicht das Vorliegen einer bestimmten Verteilung voraus. Zu diesen Autoren zählen $\mathrm{BOSCH}^{727}$,

\footnotetext{
718 Vgl. Auer und Rottmann (2015), S. 353 f.

${ }^{719}$ Vgl. Tebroke (1993), S. 115.

${ }^{720}$ Vgl. Hartung et al. (2005), S. 108.

721 Vgl. Schulze und Porath (2012), S. 539 f.

722 Vgl. Abschnitt 4.2.2.

${ }^{723}$ Vgl. Kruskal und Wallis, W. Allen (1952), S. 586 f.

${ }^{724}$ Vgl. Bühl (2012), S. 395.

${ }^{725}$ Vgl. Vargha und Delaney (1998), S. 170.

${ }^{726}$ Vgl. Bühl (2012), S. 395.

${ }^{727}$ Vgl. Bosch (1998), S. 613.
} 
BAMBERG ET AL. ${ }^{728}$, BÜHL ${ }^{729}$, HARTUNG ET AL. ${ }^{730}$ und TRENKLER UND BÜNING ${ }^{731}$. Jedoch geht aus der wissenschaftlichen Diskussion hervor, dass eine Überlegenheit nicht zwangsläufig besteht. Dies liegt als erstes daran, dass die Nachteile der Varianzanalyse nicht so stark wiegen und wirken. Die Varianzanalyse ist ein sehr robustes Verfahren. Bei Abweichungen von der Normalverteilungsvoraussetzung reagiert es nicht empfindlich und toleriert diese Abweichungen gut, wie Tests mit flachen, spitzgipfligen, schiefen und sogar bimodalen Verteilungen zeigen. ${ }^{732}$ Als zweites ist der Kruskal-Wallis-H-Test ein parametrisches Verfahren, bei dem Messwerte durch Rangzahlen ersetzt werden. Hier kann nicht ausgeschlossen werden, dass nützliche Informationen verlorengehen. Aus diesen Gründen empfehlen MCDONALD ${ }^{733}$ sowie LUND und LUND ${ }^{734}$ explizit, auch bei Fehlen der Normalverteilung die normale Varianzanalyse durchzuführen, und auf die Verwendung des Kruskal-Wallis-Tests zu verzichten. Auch KÄHLER hebt die Robustheit der Varianzanalyse hervor. Liegt Varianzhomogenität vor, aber keine Normalverteilung, lässt sich der Test laut KÄHLER trotzdem durchführen, sofern die Stichproben einen hinreichend großen Umfang aufweisen und die Abweichung von der Normalverteilung nicht erheblich ist. ${ }^{735}$ Als hinreichend groß werden je nach Autor Stichproben ab mindestens 15 bzw. mehr als 30 Elementen angesehen. ${ }^{736}$

Ad Voraussetzung 3: Zur Überprüfung der Homoskedastizität wird, wie bereits erwähnt, der Levene-Test angewendet. ${ }^{737}$ Weichen die Varianzen der einzelnen Stichproben signifikant voneinander ab, empfiehlt KäHLER trotzdem die Durchführung der Varianzanalyse, sofern die Normalverteilungsannahme bestätigt wird und die Stichproben einen hinreichend großen Umfang aufweisen. Dabei verweist er abermals auf die Robustheit des Tests, also die bereits beschriebene Unempfindlichkeit gegenüber der Verletzung von Annahmen. ${ }^{738}$ JANSSEN empfiehlt den Brown-Forsythe-Test und den Welch-Test, falls sich die Varianzen der Vergleichsgruppen unterscheiden. ${ }^{739}$ Auch MCDONALD empfiehlt den Welch-Test, falls die Voraussetzung der Varianzhomogenität verletzt ist. ${ }^{74}$ LuND und LUND raten bei Varianzheterogenität zum Welch-Test und zum Brown-Forsythe-Test. Beide Tests prüfen, ob sich die Mittelwerte signifikant unterscheiden, obwohl unterschiedliche Varianzen vorliegen. Alternativ wird zwar hier auch der Kruskal-Wallis-H-Test genannt, jedoch bringe der Welch-Test in den meisten Situationen die besten Ergebnisse. ${ }^{741}$ Liefern Brown-Forsythe und Welch widersprüchliche Ergebnisse bezüglich der Signifikanz, sollte dem Ergebnis des Welch-Tests der Vorzug erteilt werden. ${ }^{742}$

Abbildung 14 enthält eine Übersicht, welcher Test in welcher Situation angewendet wird. Mit einem Stern $\left(^{*}\right)$ sind die oben beschriebenen Verfahren in ihren jeweiligen Anwendungsszenarien markiert.

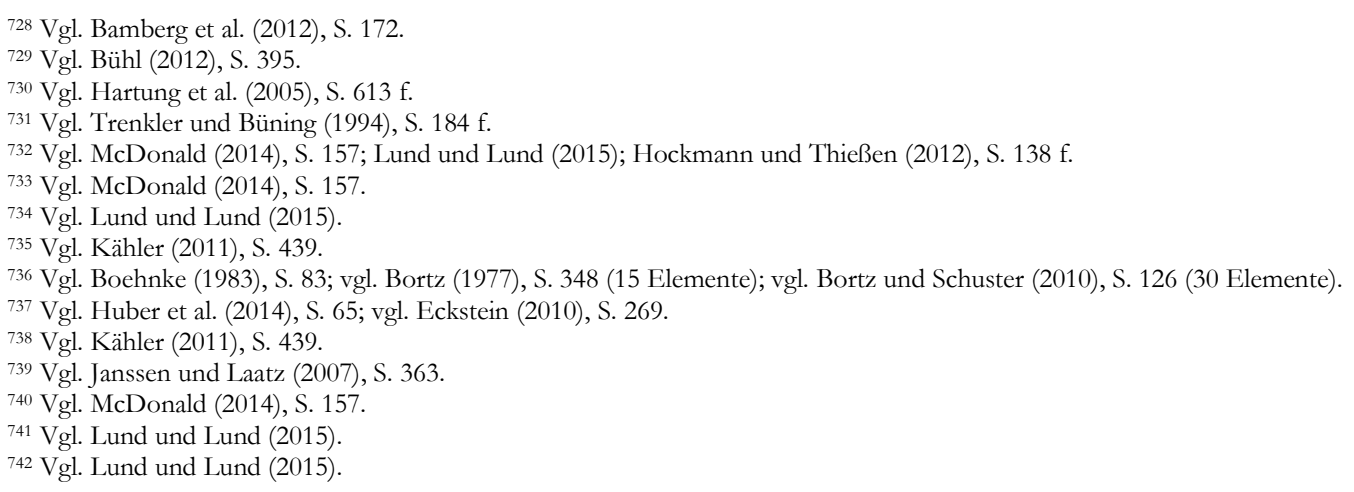




\section{Unverbundene (unabhängige) Stichproben}
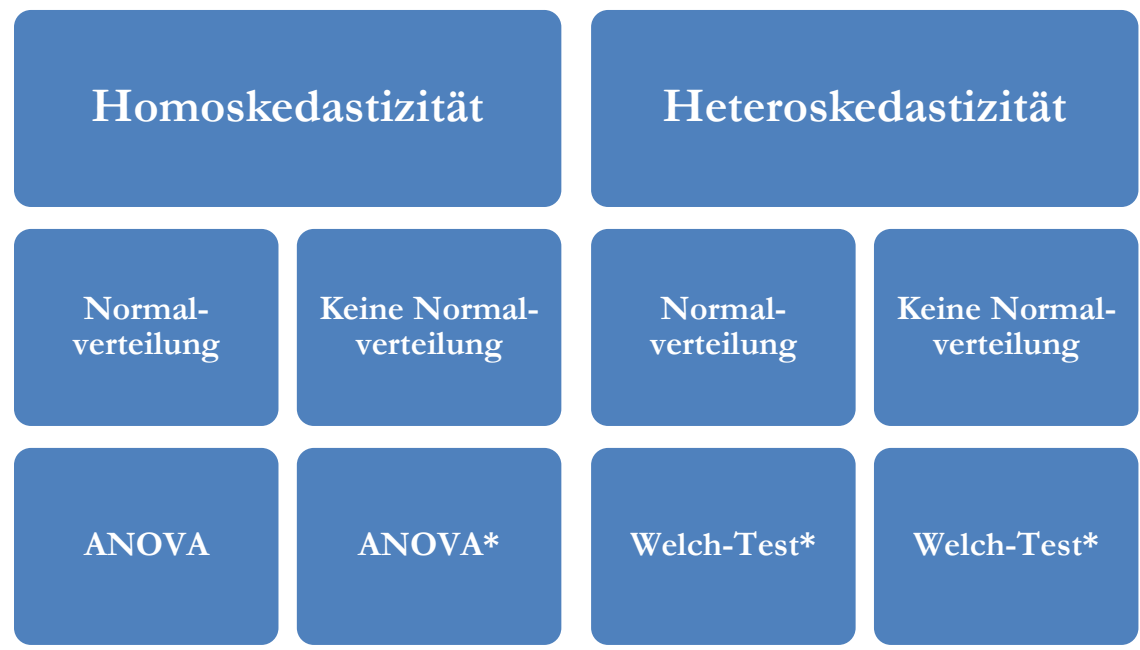

Abbildung 14: Verfahren zum Vergleich von mehr als zwei unabhängigen Stichproben ${ }^{743}$

Nach der Feststellung, dass überhaupt ein signifikanter Unterschied besteht, kommt ein Post-hoc-Test zur Anwendung. Dieser hat die Aufgabe herauszufinden, zwischen welchen Gruppenpaaren jeweils ein Unterschied besteht. Hier wird Tukey-HSD (honestly significant difference) verwendet.

\subsubsection{Korrelationsanalyse}

Die Korrelationsanalyse erlaubt es, den Zusammenhang bzw. die Beziehung zwischen zwei Variablen zu messen. Daher wird sie bisweilen auch bivariate Korrelation genannt.

Der Korrelationskoeffizient nach PEARSON beschreibt die Höhe des linearen Zusammenhangs bei einer kardinalen Skalierung. Als Alternativen gibt es die Korrelationskoeffizienten nach SPEARMAN sowie nach KENDALL für ordinale Skalierung, falls die Voraussetzungen für PEARSON nicht erfüllt sind. Die vorliegende Arbeit setzt die Kennzahl nach PEARSON ein, sofern die Voraussetzungen dafür erfüllt sind. ${ }^{744}$

Die erste Voraussetzung für die Anwendung des Korrelationskoeffizienten nach PEARSON ist, dass die Werte eine Normalverteilung aufweisen. ${ }^{745}$ Dies wird in der vorliegenden Arbeit mit dem SHAPIRO-WILK-Test untersucht. ${ }^{746}$ Falls mindestens eine der variablen nicht normalverteilt ist, so kann alternativ der Rangkorrelationskoeffizient nach SPEARMAN oder KENDALLS Tau bestimmt werden. ${ }^{747}$ Als zweite Voraussetzung sollte der gesuchte Zusammenhang linear oder zumindest quasi-linear sein, da der Korrelationskoeffizient nach PEARSON die Stärke des linearen Zusammenhangs ausdrückt. Als quasi-linear wird dabei ein Regressionsansatz bezeichnet, der in seinen Koeffizienten zwar nicht linear ist, sich jedoch durch eine geeignete Transformation linearisieren lässt. ${ }^{74}$ Zur Beurteilung polyno-

\footnotetext{
743 Eigene Darstellung, in Anlehnung an Kähler (2011), S. 460.

744 Vgl. Bühl (2012), S. 421.

745 Vgl. Bühl (2012), S. 420.

${ }^{746}$ Vgl. Abschnitt 4.2.2.

747 Vgl. Bühl (2012), S. 420.

${ }^{748}$ Vgl. Egert (2013), S. 29.
} 
mialer, logarithmischer oder exponentieller Zusammenhänge ist der Korrelationskoeffizient nach PEARSON ungeeignet.

Der Korrelationskoeffizient $r$ ist normiert und liegt stets im Wertebereich $[-1 ;+1]$. Gilt $r=1$, so liegt ein perfekter positiver linearer Zusammenhang vor: Jede Erhöhung der einen Variablen ist mit einer Erhöhung der anderen Variable verbunden. Gilt dagegen $r=$ gen $r=-1$, so handelt es sich um einen perfekten negativen Zusammenhang. Liegt $r$ nahe 0 , so existiert kein linearer Zusammenhang. ${ }^{749}$ Je nach der Höhe des Betrages von $r$ wird von verschieden hohen Korrelationen gesprochen. BÜHL unterscheidet zwischen sehr geringer Korrelation (bis 0,2), geringer Korrelation (bis 0,5), mittlerer Korrelation (bis 0,7), hoher Korrelation (bis 0,9) und sehr hoher Korrelation (über 0,9). ${ }^{750}$ Das in Beziehung stehende Bestimmtheitsmaß $R^{2}$ wird unten erläutert. ${ }^{751}$

Zusätzlich zu $r$ wird das Signifikanzniveau angegeben, um $r$ gegen Null abzusichern. Beim Vorliegen von Signifikanz kann davon ausgegangen werden, dass sich der Korrelationskoeffizient $r$ signifikant von Null unterscheidet. ${ }^{752}$

\subsubsection{Regressionsanalyse}

Die Korrelationsanalyse dient dazu, die Stärke eines Zusammenhangs zu quantifizieren, welcher zwischen zwei Variablen besteht. ${ }^{753}$ Im Gegensatz dazu wird die Regressionsanalyse eingesetzt, um die Art des Zusammenhangs aufzudecken und um den Wert einer abhängigen Variable vorherzusagen, wenn der Wert der unabhängigen Variablen vorliegt. ${ }^{754}$ Der einfachste Fall ist hier ein linearer Zusammenhang, welcher durch die einfache lineare Regression ${ }^{755}$ oder die multiple lineare Regression ${ }^{756}$ identifiziert werden kann.

Eine einfache lineare Regression wird angewendet, wenn die abhängige Variable $y$ nur von einer unabhängigen Variablen $x$ bestimmt wird. ${ }^{757}$ Untersucht man mehrere unabhängige Variable $x_{1}, x_{2}$ und $x_{3}$ auf den Einfluss hin, den diese auf die abhängige Variable $y$ besitzen, so kommt die multiple lineare Regression zur Anwendung. ${ }^{758}$ Im Gegensatz dazu spricht man von einer multivariaten Regression, wenn mehrere abhängige Variable gleichzeitig untersucht werden. Die multivariate Regression wird in der vorliegenden Arbeit nicht angewendet. KOMLOS und SÜsSMUTH empfehlen zunächst eine grafische Darstellung anhand eines Streudiagramms, um das Problem zu veranschaulichen. ${ }^{759}$

Man geht für eine Grundgesamtheit von einem linearen Zusammenhang zwischen einer unabhängigen und einer abhängigen Variable aus. ${ }^{760}$ Dieser Zusammenhang wird additiv von einer Zufallsvariable überlagert. ${ }^{761}$ Daneben gibt es noch weitere Einflussgrößen, die sich jedoch nur geringfügig positiv oder negativ auf die abhängige Variable auswirken, und

\footnotetext{
${ }^{749}$ Vgl. Bühl (2012), S. 422.

${ }^{750}$ Vgl. Bühl (2012), S. 422.

${ }^{751}$ Vgl. Abschnitt 4.2.8.

752 Vgl. Bühl (2012), S. 422.

${ }^{753}$ Vgl. Bühl (2012), S. 433.

${ }^{754}$ Vgl. Bühl (2012), S. 433.

755 Vgl. Bühl (2012), S. 434

${ }^{756}$ Vgl. Bühl (2012), S. 442.

757 Vgl. Janssen und Laatz (2007), S. 415.

${ }^{758}$ Vgl. Janssen und Laatz (2007), S. 415.

759 Vgl. Komlos und Süssmuth (2010), S. 59.

${ }^{760}$ Vgl. Janssen und Laatz (2007), S. 419.

${ }^{761}$ Vgl. Janssen und Laatz (2007), S. 419.
} 
daher in ihrer Summe als eine Zufallsvariable gesehen werden können. ${ }^{762}$ Das Regressionsmodell wird durch

$$
y_{i}=b_{0}+b_{1} x_{i}+\epsilon_{i}
$$

beschrieben, wobei die Regressionskoeffizienten $b_{0}$ und $b_{1}$ so bestimmt werden, dass die abhängige Variable $y_{i}$ bestmöglich durch die unabhängige Variable $x_{i}$ erklärt wird. Der hierdurch nicht erklärte Teil geht in die Residuen $\epsilon_{i}$ ein, die auch als Störterm bezeichnet werden. Ist der lineare Zusammenhang zwischen $x_{i}$ und $y_{i}$ plausibel, so weisen alle $\epsilon_{i}$ einen zufälligen Charakter auf, sind also unabhängig und identisch normalverteilt mit einem Erwartungswert von Null und der konstanten Varianz $\sigma^{2}$. Des Weiteren dürfen die Residuen nicht autokorreliert sein.

Die Variable $y_{i}$ besteht somit aus einer systematischen Komponente $\widehat{y}_{i}=b_{0}+b_{1} x_{i}$, die die Vorhersage für $y_{i}$ durch die Regressionsgleichung darstellt, wenn $x_{i}$ gegeben ist. ${ }^{763}$ Dieser prognostizierte Wert $\hat{y}_{i}$ unterscheidet sich in der Regel vom tatsächlich beobachteten Wert $y_{i}$ um den Fehler $\epsilon_{i}{ }^{764}$ Als Residualwert wird die Abweichung $\epsilon_{i}=y_{i}-\hat{y}_{i}$ bezeichnet. ${ }^{765}$ Die Schätzung der Parameter $b_{0}$ und $b_{1}$ beruht auf der Methode der kleinsten Quadrate (MKQ). ${ }^{766}$ Mit dieser wird versucht, die Summe der quadrierten vertikalen Abstände jeweils zwischen einem beobachteten Wert $y_{i}$ und dem für das entsprechende $x_{i}$ berechnete $\hat{y}_{i}$ zu minimieren. ${ }^{767}$

Für eine multiple Regression muss die Anzahl der Variablen entsprechend erhöht werden. Das Regressionsmodell für $J$ Variablen lautet dann:

$$
y_{i}=b_{0}+b_{1} x_{1}+b_{2} x_{2}+\ldots+b_{J} x_{J}+\epsilon_{i}
$$

Der Regressionsansatz wird durch die folgende Gleichung beschrieben. ${ }^{768}$

$$
\hat{y}_{i}=b_{0}+b_{1} x_{1}+b_{2} x_{2}+\ldots+b_{J} x_{J}
$$

Die Regressionskoeffizienten $b_{0}, b_{1}, b_{2}, \ldots, b_{J}$ werden durch die Methode der kleinsten Quadrate bestimmt. ${ }^{769}$

In manchen Fällen vermutet man, dass die Zugehörigkeit zu einer Gruppe (qualitatives Merkmal) einen Einfluss auf die Höhe einer anderen Eigenschaft hat (quantitatives Merkmal). Um herauszufinden, wie groß dieser partielle Einfluss der Gruppenzugehörigkeit ist,

\footnotetext{
762 Vgl. Janssen und Laatz (2007), S. 419.

${ }^{763}$ Vgl. Janssen und Laatz (2007), S. 415.

${ }^{764}$ Vgl. Janssen und Laatz (2007), S. 415.

765 Vgl. Janssen und Laatz (2007), S. 415.

${ }^{766}$ Vgl. Hartung et al. (2005), S. 574f; Winker (2010), S. 138f; Engel und Reinecke (1994), S. 16.

${ }^{767}$ Vgl. Janssen und Laatz (2007), S. 416 f.

${ }^{768}$ Vgl. Backhaus (2006), S. 60.

${ }^{769}$ Vgl. Backhaus (2006), S. 60.
} 
wird die Regressionsanalyse mit Dummyvariablen angewendet. ${ }^{770}$ Dabei kann eine Dummyvariable etwa den Wert 1 annehmen, wenn eine bestimmte Eigenschaft erfüllt ist, und den Wert 0, wenn die Eigenschaft nicht erfüllt ist. Dummyvariablen können beispielsweise für die Zugehörigkeit zu Gruppen oder für Datensätze aus bestimmten Jahren vergeben werden.

\subsubsection{Bestimmtheitsmaß $\mathbf{R}^{2}$}

Das Bestimmtheitsmaß $R^{2}$ ist ein Indikator dafür, welcher Anteil der gesamten Varianz (bzw. Streuung) durch das jeweilige statistische Verfahren erklärt wird.

$$
R^{2}=\frac{\text { erklärte Varianz }}{\text { Gesamtvarianz }}
$$

Das Bestimmtheitsmaß ist eine normierte Größe. ${ }^{771}$ Sein Wertebereich liegt zwischen 0 und $1 .^{772}$ Wird die gesamte Streuung erklärt, so nimmt $R^{2}$ den Wert 1 an. Lässt sich die beobachtete Streuung gar nicht durch das Modell erklären, so gilt $R^{2}=0 .{ }^{773}$

Das Bestimmtheitsmaß ist abhängig von der Anzahl der Regressoren, das heißt von den unabhängigen bzw. erklärenden Variablen. ${ }^{774}$ Lässt man eine gegebene Stichprobengröße konstant, erhöht jedoch die Anzahl der Regressoren, so kann sich $\mathrm{R}^{2}$ erhöhen, jedoch nicht verringern. ${ }^{775}$ Möglicherweise steigt aber der Erklärungsgehalt durch den zusätzlich aufgenommenen Regressor nicht an, oder dieser zusätzliche Erklärungsgehalt ist zufällig bedingt. Die Schätzeigenschaften des Modells verschlechtern sich besonders bei einer geringen Anzahl von Freiheitsgraden, das heißt bei kleinen Stichproben. ${ }^{776}$

Das korrigierte Bestimmtheitsmaß (englisch adjusted $R$-squared) verbessert $R^{2}$ in folgender Hinsicht: Es subtrahiert einen Korrekturterm. ${ }^{777}$ In der Folge sinkt das korrigierte Bestimmtheitsmaß mit jedem zusätzlichen Regressor und mit abnehmender Zahl der Freiheitsgrade. ${ }^{778}$

\subsubsection{Paneldatenanalyse}

Paneldaten sind verwandt mit Zeitreihen- und mit Querschnittsdaten. Unter Zeitreihendaten (englisch time series data) versteht man die Erhebung der Größenordnung eines Sachverhalts im Zeitablauf, oft in festen Zeitabständen wie z. B. jährlich. Wird etwa die Bilanzsumme einer einzelnen Bank für eine Reihe von Jahren immer am Jahresende erhoben, stellt dies eine Zeitreihe dar. Durch Auswertung kann dann die Entwicklung dieser einen Größe über die Zeit erforscht werden.

Unter Querschnittsdaten (englisch cross section data) versteht man eine Menge an Daten, die zur Beschreibung eines Sachverhaltes zum selben Zeitpunkt erhoben wurden. Das können

\footnotetext{
${ }^{770}$ Vgl. Schulze und Porath (2012), S. 521.

771 Vgl. Backhaus (2006), S. 66.

772 Vgl. Assenmacher (2002), S. 121 und Backhaus (2006), S. 66.

${ }^{773}$ Vgl. Backhaus (2006), S. 66.

${ }^{774}$ Vgl. Backhaus (2006), S. 68.

775 Vgl. Backhaus (2006), S. 68 und Assenmacher (2002), S. 121.

${ }^{776}$ Vgl. Backhaus (2006), S. 68.

777 Vgl. Backhaus (2006), S. 68 und Assenmacher (2002), S. 121.

${ }^{778}$ Vgl. Backhaus (2006), S. 68 und Assenmacher (2002), S. 121.
} 
etwa alle Positionen einer Jahresschlussbilanz einer Bank sein, oder auch die Bilanzsummen aller Banken zu einem Zeitpunkt. Auch hier können Zusammenhänge identifiziert werden, die im selben Zeitpunkt begründet liegen.

Paneldaten besitzen innerhalb der empirischen Forschung eine besondere Qualität. Sie vereinen Zeitreihendaten und Querschnittsdaten. Ein Panel beinhaltet Angaben zu einer Menge von Messdaten, z. B. Bilanzsummen mehrerer Banken, über einen Zeitraum von mindestens zwei Messpunkten, oft jedoch mehrere Jahre. Daher werden sie in der Literatur auch als time series cross section data bezeichnet. ${ }^{779}$

Paneldaten sind auch speziell gegenüber Trendstudien abzugrenzen: Panelstudien befragen wiederholt dieselben Untersuchungseinheiten. ${ }^{780}$ Trendstudien hingegen ziehen wiederholt unabhängige Zufallsauswahlen aus derselben statistischen Population und beinhalten daher nicht dieselben Untersuchungseinheiten. ${ }^{781}$

Zur Auswertung von Paneldaten werden besondere Verfahren angewendet. Diese erlauben es vor allem, diesen dynamischen Aspekt zu berücksichtigen und eine Entwicklung im Zeitablauf über alle Eingangsparameter zu erklären. ${ }^{782}$ Je nachdem, welche Voraussetzungen vorliegen, fällt die Wahl auf ein bestimmtes Verfahren.

Bei Fixed Effects (FE) werden die Niveauunterschiede zwischen den Einheiten des Datensatzes eliminiert. ${ }^{783}$ Dazu wird zunächst der einheitenspezifische Mittelwert bestimmt, und dieser dann von allen Beobachtungen subtrahiert. ${ }^{784}$ Übrig bleibt eine Schwankung um den Nullpunkt, wobei jedoch die absolute Höhe der Schwankung erhalten bleibt. Als Beispiel enthält Abbildung 15 einen Zeitreihenplot von drei Personen. Für diese wurde die Höhe des Einkommens in verschiedenen Zeitpunkten nach ihrem Schulabschluss bestimmt. Bestimmt werden soll der Einfluss des Zeitabstands in Jahren seit dem Schulabschluss auf die Höhe des Einkommens. Auf Basis des linearen Regressionsmodells aus Gleichung (29) wird die Beziehung zwischen dem Gehalt $y_{t}{ }^{(j)}$ von Person $j$ und der Zeit $t$ seit dem Schulabschluss durch

$$
y_{i}^{(j)}=b_{0}^{(j)}+b_{1}^{(j)} x_{i}+\epsilon_{i}^{(j)}
$$

beschrieben. Für die Residuen gelten die oben getroffenen Annahmen. Der Parameter $b_{0}{ }^{(j)}$ kann als Startgehalt nach Beendigung des Schulabschlusses interpretiert werden. Nach Schätzung der Parameter sowohl für jede einzelne Person als auch für alle drei gemeinsam ergeben sich die in Abbildung 15 dargestellten Regressionsgeraden. Die in schwarz eingezeichnete allgemeine Regressionsgerade fällt mit einem Steigungskoeffizienten von $b_{1}{ }^{\text {allgemein }}=44$ deutlich flacher als jede der Regressionsgeraden der drei Personen aus, deren Steigungskoeffizienten bei ${b_{1}}^{(1)}=113, b_{1}{ }^{(2)}=72, b_{1}{ }^{(3)}=116$ liegen. $^{785}$

\footnotetext{
${ }^{779}$ Vgl. Giesselmann und Windzio (2012), S. 9.

${ }^{780}$ Vgl. Engel und Reinecke (1994), S. 3.

${ }^{781}$ Vgl. Engel und Reinecke (1994), S. 3.

782 Vgl. Giesselmann und Windzio (2012), S. 10.

${ }^{783}$ Vgl. Giesselmann und Windzio (2012), S. 40.

${ }^{784}$ Vgl. Giesselmann und Windzio (2012), S. 40.

${ }^{785}$ Vgl. Giesselmann und Windzio (2012), S. 38.
} 


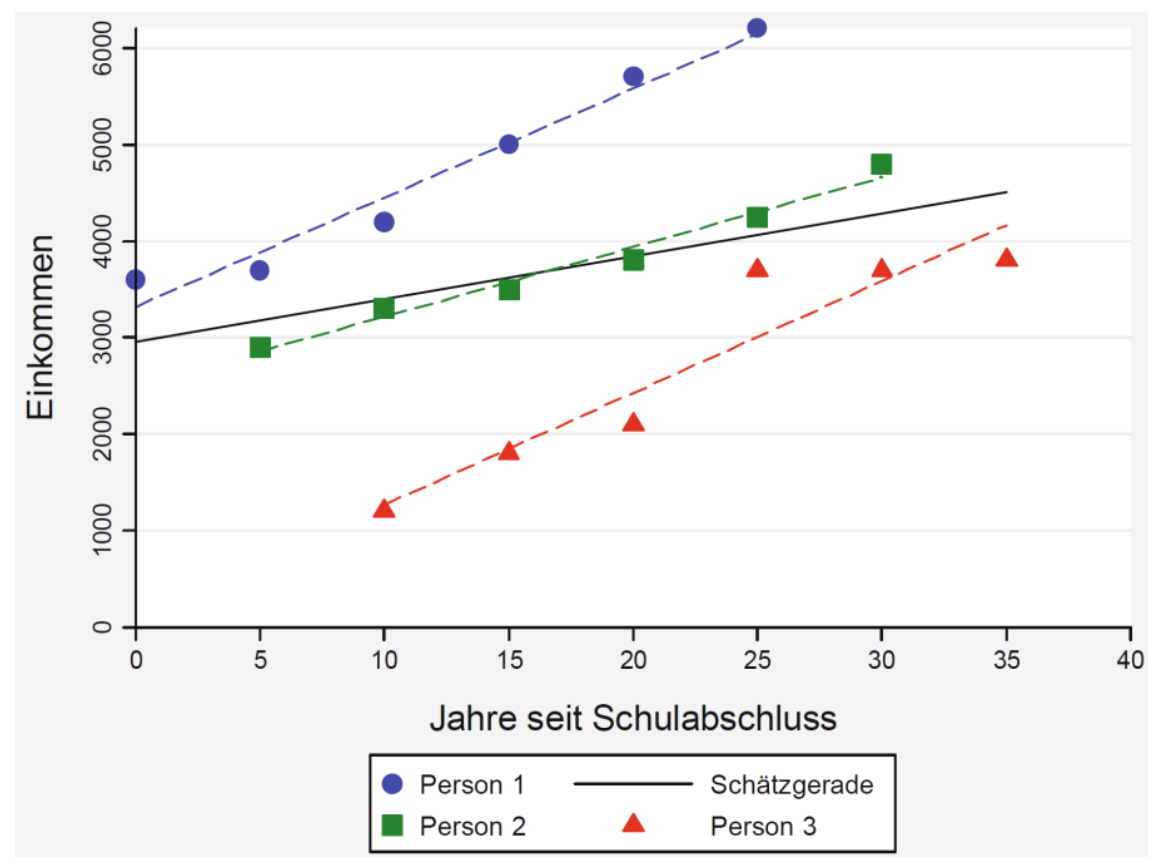

Abbildung 15: Zeitreihenplot der Verteilung von „Jahre seit Schulabschluss“ und „Einkommen“"786

Nach Anwendung des Fixed Effects-Verfahrens der Paneldatenanalyse lautet der Steigungskoeffizient $b_{\text {allgemein }}=100$ und ist damit auch visuell deutlich realistischer. ${ }^{787}$ Abbildung 16 enthält die grafische Darstellung des Resultats, die allgemeine Regressionsgerade wird als gestrichelte Linie dargestellt.

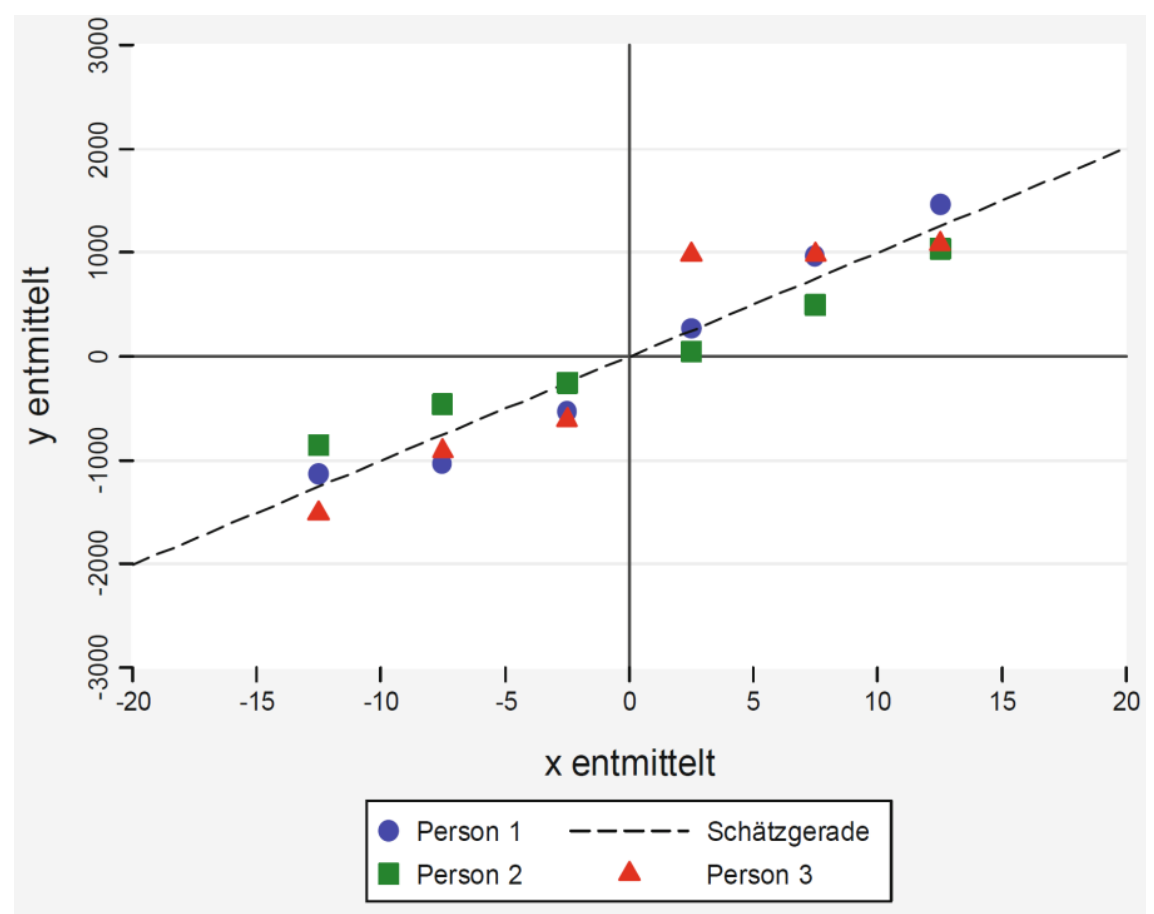

Abbildung 16: Streudiagramm der Verteilung von „Jahre seit Schulabschluss“ und „Einkommen“ (Fixed Effects) ${ }^{788}$

\footnotetext{
${ }^{786}$ Giesselmann und Windzio (2012), S. 38.

787 Vgl. Giesselmann und Windzio (2012), S. 42.

788 Giesselmann und Windzio (2012), S. 42.
} 
Auch hier wird die erwähnte Streuung um die Abszissenachse sichtbar. Zeitkonstante Variablen können nicht mit dem Fixed Effects-Modell untersucht werden. ${ }^{789}$ In diesem Fall kann auf eine OLS-Regression zurückgegriffen werden, bei der alle Werte gleichzeitig regressiert werden. Die OLS-Regression wird daher auch als Pooled-Methode bezeichnet. ${ }^{790}$ Zeitkonstant sind alle diejenigen Variablen, die sich im Zeitablauf nicht verändern, also unveränderliche Eigenschaften von Individuen. Beispiele hierfür sind das Geschlecht einer Person oder die Lage einer Bank in einem der drei Lagebereiche städtisch, halbstädtisch oder ländlich. Random Effects (RE) kann zur Anwendung kommen, um die Effizienz der Schätzung im Vergleich zur einfachen OLS-Regression bei unbalancierten Daten zu erhöhen. ${ }^{791}$ Jedoch müssen die impliziten Annahmen beachtet werden. ${ }^{792}$ GIESSELMANN UND WINDZIO kritisieren die Bezeichnung Random Effects als irreführend, da sie ein Abgrenzungskriterium suggeriert, welches auch auf FE oder OLS zutrifft. ${ }^{793}$ Das Kriterium sei, dass der Einheiteneffekt als Realisation einer Zufallsgröße aufgefasst werden kann. ${ }^{794}$

Der Hausman-Test ${ }^{795}$ hilft als Kriterium bei der Entscheidung zwischen Random Effects und Fixed Effects. ${ }^{796}$ Hierzu überprüft der Test die Nullhypothese, dass die mit Random Effects errechneten Koeffizienten den mit Fixed Effects ermittelten entsprechen. ${ }^{797}$ Fällt der Hausman-Test signifikant aus, sollte man sich gegen Random Effect entscheiden und auf Fixed Effects hin prüfen.

\subsection{Datenbasis}

\subsubsection{Datenerhebung}

Im Rahmen der Datenerhebung wurden folgende Datensätze gewonnen:

- Bilanz- und GuV-Daten der Genossenschaftsbanken

- Bankliste der Deutschen Bundesbank

- Bankliste des BVR

- Gemeindeverzeichnis des Statistischen Bundesamtes

- Regionale Wirtschaftsdaten auf Kreisebene

- Bewertung der Zukunftsfähigkeit nach KRÖHNERT

Der Datensatz Genossenschaftsbanken wurde im Jahr 2013 mit Hilfe der Datenbank Bankscope erhoben. Enthalten sind die Positionen der Aktivseite, der Passivseite, der Gewinn- und Verlustrechnung sowie die Anzahl der Mitarbeiter und Angaben zu Fusionen aus dem Lagebericht. Eine detaillierte Vorstellung der einzelnen Positionen erfolgt unten in den Abschnitten 4.3.3.3 und 4.3.3.4. Der Betrachtung der GuV-Positionen kommt im Rahmen der vorliegenden Arbeit eine besondere Bedeutung zu. Nach Abschluss der Erhe-

\footnotetext{
${ }^{789}$ Vgl. Kopp und Lois (2009), S. 23.

790 Vgl. Giesselmann und Windzio (2012), S. 107 f.

791 Vgl. Giesselmann und Windzio (2012), S. 79.

${ }^{792}$ Vgl. Giesselmann und Windzio (2012), S. 79.

${ }^{793}$ Vgl. Giesselmann und Windzio (2012), S. 79.

794 Vgl. Giesselmann und Windzio (2012), S. 79.

795 Vgl. Hausman, J. A. (1978), S. 1251 f.

${ }^{796}$ Vgl. Giesselmann und Windzio (2012), S. 109f; Vgl. Kopp und Lois (2009), S. 54 f.

${ }^{797}$ Vgl. Allison (2009), S. 23.
} 
bung erfolgte ein umfangreicher Datenvalidierungsprozess, der in Abschnitt 4.3.2 gesondert beschrieben wird.

Der Datensatz der regionalen Wirtschaftsdaten in den kreisfreien Städten und Landkreisen Deutschlands enthält unter anderem folgende Angaben jeweils im Zeitraum 2005 bis 2011:

- Bruttoinlandsprodukt pro Kopf

○ Arbeitnehmerentgelt je Arbeitnehmer

○ Bruttolöhne und -gehälter

- Einkommen der privaten Haushalte

\subsubsection{Aufbereitung und Validierung}

Der gesamte Datensatz zu den Jahresabschlüssen der Banken umfasst 1.662 Institute. Unter diesen befinden sich 955 aktive Banken. Hierbei bedeutet aktiv, dass die jeweilige Bank zum Zeitpunkt der Erhebung aktiv tätig war. Jedoch liegen nicht für alle tatsächlich aktiven Banken in jedem betrachteten Geschäftsjahr alle Daten vor. Auch existieren 707 Datensätze von Instituten, die im Zuge einer Fusion von einer anderen Bank absorbiert wurden. Die absorbierten Institute werden als nicht mehr aktiv gekennzeichnet. ${ }^{798}$ Die Untersuchung zieht für jedes Geschäftsjahr sämtliche verfügbare von den insgesamt bestehenden Datensätzen heran. Die gesamte Stichprobe deckt 7.133 Bankenjahre ab, die sieben Geschäftsjahren entstammen. In der Untersuchung werden jedoch nur regionale Genossenschaftsbanken betrachtet. Grundsätzlich betreiben alle regionalen Genossenschaftsbanken in Deutschland vorwiegend einen Geschäftstypus, welcher häufig als das „klassische Einlagen- und Kreditgeschäft" beschrieben wird und sich aus deren Geschäftspolitik ergibt. Dies geschieht überall - unabhängig von der Lage der einzelnen Bank.

Einige Banken gehören zwar zur Kategorie der Genossenschaftsbanken, weichen in ihrem Geschäftsmodell jedoch von demjenigen regionaler Genossenschaftsbanken ab und werden daher nicht für Berechnungen in der vorliegenden Arbeit herangezogen. Die Darstellung erfolgt in Tabelle 6.

Es verbleiben 1.637 Fälle. Im anschließenden Prozess der Datenvalidierung erfolgte ein Abgleich mit anderen Datenbanken. Dazu zählen insbesondere das Gemeindeverzeichnis $^{799}$, das Verzeichnis der Kreditinstitute der DeutsChen BundeSBANK ${ }^{800}$ sowie die Liste aller Volksbanken und Raiffeisenbanken vom Bundesverband der Deutschen Volksbanken und Raiffeisenbanken. ${ }^{801}$

\footnotetext{
${ }^{798}$ Fortgeführt wird lediglich die Zeitreihe des absorbierenden Instituts.

${ }^{799}$ Vgl. Statistisches Bundesamt (2012a).

800 Vgl. Bundesbank (2012c).

${ }^{801}$ Vgl. Bülles (2012), S. 1-18.
} 


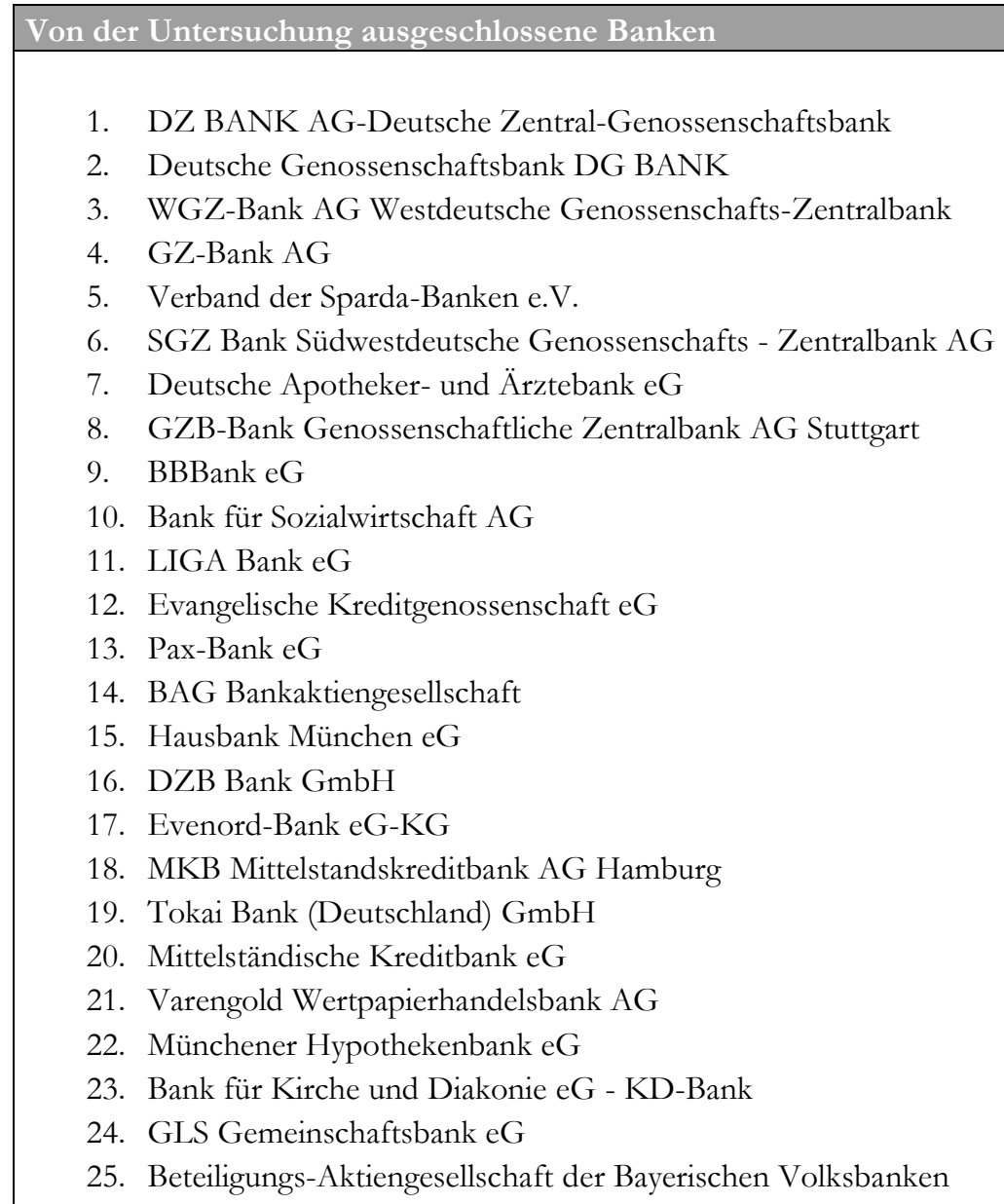

Tabelle 6: $\quad$ Von der Untersuchung ausgeschlossene Banken

\subsubsection{Beschreibung der Datenbasis}

\subsubsection{Gemeindeverzeichnis und Regionalatlas}

Die Identifizierung, ob eine Bank eine städtische, halbstädtische oder ländliche Lage aufweist, geschieht auf Grundlage der Stadt-/Landgliederung der Europäischen Union. ${ }^{802}$ Diese Gliederung wiederum basiert auf dem Kriterium der Bevölkerungsdichte, nach welchem drei Gebietstypen festgelegt werden:

1. Städtische Gebiete: Diese sind dicht besiedelt und sie weisen eine Bevölkerungsdichte von mehr als 500 Einwohnern je Quadratkilometer auf. Zusätzlich beherbergen die Gemeinden einzeln oder zusammenhängend mit Nachbargemeinden mindestens 50.000 Einwohner. $^{803}$

2. Halbstädtische Gebiete: Diese weisen eine mittlere Besiedlungsdichte auf, welche zwischen 100 und 500 Einwohnern je Quadratkilometer liegt. Zusätzlich weisen die Gemeinden einzeln oder zusammenhängend mit Nachbargemeinden mindestens 50.000 Einwohner auf. ${ }^{804}$

\footnotetext{
802 Statistisches Bundesamt (2014). 
3. Ländliche Gebiete: Diese sind gering besiedelt und haben eine Bevölkerungsdichte von weniger als 100 Einwohnern je Quadratkilometer. ${ }^{805}$

Gemeinden mit einer Fläche von weniger als 100 Quadratkilometer werden einer höheren Kategorie zugeordnet, falls sie vollständig von Gemeinden dieser höheren Kategorie umschlossen sind. Daher kann zur Ermittlung des Gebietstyps einer Gemeinde nicht einfach auf die Bevölkerungsdichte als Kriterium zurückgegriffen werden.

Tabelle 7 stellt dar, wie sich die Fläche und die Bevölkerung auf die drei Regionalkategorien aufteilen. Städtische Gebiete beherbergen auf nur knapp $5 \%$ der Fläche mehr als $35 \%$ aller Einwohner. ${ }^{806}$

\begin{tabular}{|c|r|c|c|c|c|}
\hline $\begin{array}{c}\text { Stadt- } \\
\text { Land- } \\
\text { Gliederung }\end{array}$ & $\begin{array}{c}\text { Fläche in } \\
\mathbf{k m}^{2}\end{array}$ & $\begin{array}{c}\text { Fläche } \\
\text { in } \\
\text { Prozent }\end{array}$ & Bevölkerung & $\begin{array}{c}\text { Bevölke- } \\
\text { rung in } \\
\text { Prozent }\end{array}$ & $\begin{array}{c}\text { Bevölke- } \\
\text { rungsdichte } \\
\text { in EW/ } / \mathbf{k m}^{2}\end{array}$ \\
\hline 01 & $17.815,86$ & 4,99 & 28.399 .014 & 35,27 & 1.594 \\
\hline 02 & $101.150,97$ & 28,32 & 33.450 .802 & 41,54 & 331 \\
\hline 03 & $238.201,11$ & 66,69 & 18.673 .930 & 23,19 & 78 \\
\hline Gesamt & $\mathbf{3 5 7 . 1 6 7 , 9 9}$ & $\mathbf{1 0 0 , 0 0}$ & $\mathbf{8 0 . 5 2 3 . 7 4 6}$ & $\mathbf{1 0 0 , 0 0}$ & $\mathbf{2 2 5}$ \\
\hline
\end{tabular}

Tabelle 7: $\quad$ Aufteilung der Fläche Deutschlands nach der Stadt-/Landgliederung807

Auf Basis dieser dreiteiligen Gliederung wurden zunächst alle Städte und Gemeinden Deutschlands klassifiziert. Datenquelle für die Eingliederung einer Stadt oder Gemeinde in eine der drei Gliederungsebenen ist das Gemeindeverzeichnis. ${ }^{808}$ Die verwendete Fassung enthält Angaben zu 11.252 Gemeinden, darunter 125 in städtischer, 2.646 in halbstädtischer und 8.481 in ländlicher Lage. Abbildung 17 beinhaltet eine grafische Darstellung aller Gemeinden in Deutschland. Je nach Gebietstyp wurden die Gemeinden farbig markiert. Hell markierte Gebiete besitzen eine ländliche Lage, mittel steht für halbstädtisch, dunkel für städtisch.

Die in der vorliegenden Arbeit untersuchten Banken befinden sich allesamt - gemäß der Stadt-/Landgliederung - entweder in einer städtischen, in einer halbstädtischen oder in einer ländlichen Lage. Zur Bestimmung der Lagekategorie einer Bank wurde als Indikator die Stadt des Hauptsitzes derjenigen Bank herangezogen. Für Genossenschaftsbanken herrscht das in Absatz 3.2.1 beschriebene Regionalprinzip. Bezüglich des jeweiligen Geschäftsgebiets wird die Annahme getroffen, dass dieses in derselben Kategorie wie die Stadt des Hauptsitzes der Bank liegt. Hintergrund ist, dass sich das Geschäftsgebiet einer Genossenschaftsbank in der Regel auf die unmittelbare geografische Umgebung des Hauptsitzes erstreckt. Es wird daher davon ausgegangen, dass hinsichtlich der Stadt-/Landgliederung eine Identität zwischen der Lage der Stadt des Hauptsitzes und der Lage des Geschäftsgebietes besteht. Diese Annahme ist vonnöten, um die Herstellung der entsprechenden Beziehungen überhaupt zu ermöglichen. Es sei jedoch darauf hinwiesen, dass es vor allem bei Genossenschaftsbanken mit relativ großen Geschäftsgebieten zu Abweichungen kommen kann. Am wahrscheinlichsten sind derartige Divergenzen für Mecklenburg-

\footnotetext{
805 Statistisches Bundesamt (2014).

${ }^{806}$ Statistisches Bundesamt (2013a).

${ }^{807}$ Eigene Darstellung, basierend auf Statistisches Bundesamt (2013a).

${ }^{808}$ Vgl. Statistisches Bundesamt (2012a).
} 
Vorpommern, Brandenburg und Sachsen-Anhalt. Zusätzlich werden aus dem Gemeindeverzeichnis die Flächen der 16 deutschen Bundesländer entnommen. ${ }^{809}$

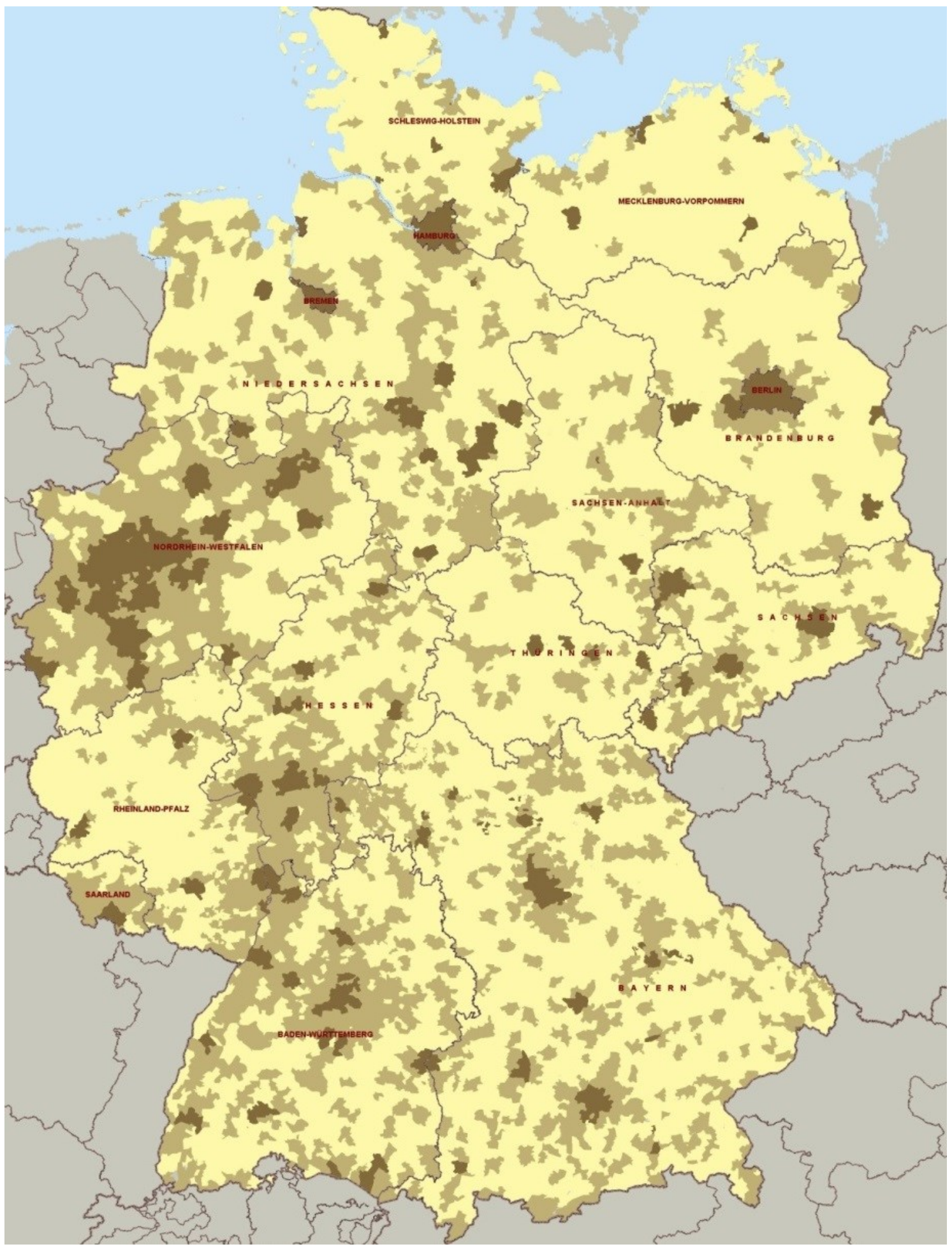

Abbildung 17: $\quad$ Stadt-Land-Gliederung Deutschlands nach Gemeinden ${ }^{810}$ 


\subsubsection{Grundgesamtheit und Stichprobe}

Signifikante Ergebnisse von Hypothesentests lassen generell den Schluss von einer Stichprobe auf die Grundgesamtheit zu und erlauben allgemein gültige Aussagen. ${ }^{811}$ Der entsprechende Teilbereich der Statistik wird auch schließende oder Interferenzstatistik genannt. ${ }^{812}$ Das Schließen auf die Grundgesamtheit ist in vielen Bereichen entscheidend, da die Erhebung der Daten aller Individuen nicht möglich ist.

Eine Stichprobe ist immer dann repräsentativ, wenn sie in der Verteilung aller untersuchungsrelevanten Merkmale der Grundgesamtheit entspricht, das heißt ein zwar verkleinertes, aber sonst wirklichkeitsgetreues Abbild der Grundgesamtheit darstellt. ${ }^{813}$ Das Vorhandensein dieser Repräsentativität soll im Folgenden ausgehend von der räumlichen Verteilung der Institute untersucht werden. Zur Überprüfung, ob die gewünschte Gleichverteilung vorliegt, wird die Stichprobe nach Quoten untersucht. ${ }^{814}$ Als Separierungskriterium kommt die erste Ziffer der Postleitzahl zur Anwendung, welche die Gebiete zu sogenannten Postleitzonen zusammenfasst. Zunächst wird für jede Postleitzone geprüft, welchen Anteil die dort tatsächlich per Ende 2011 vorhandenen Banken an der Gesamtzahl der deutschen Genossenschaftsbanken von 1.121 ausmachen. Diese Angabe stellt die Vergleichsbasis dar. In einem zweiten Schritt wird für jede Postleitzone ermittelt, welchen Anteil die in der Stichprobe enthaltenen Institute an der Gesamtzahl der Banken der Stichprobe darstellen. Idealerweise sollten sich nur geringe Abweichungen ergeben. Wünschenswert ist, dass sich die abgedeckten und somit auch die fehlenden Institute gleichmäBig über die Fläche der Bundesrepublik verteilen. Die Verteilung wird in Abbildung 18 dargestellt.

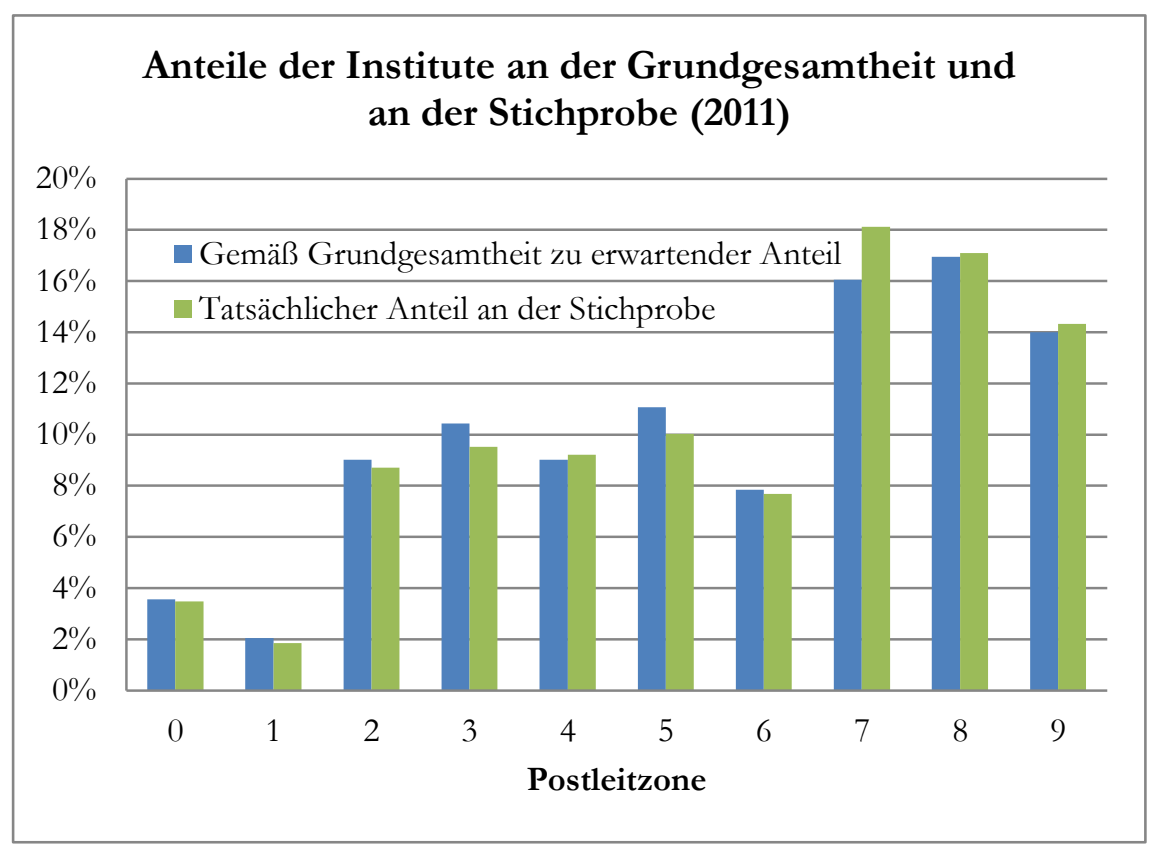

Abbildung 18: Anteile der Institute an der Grundgesamtheit und an der Stichprobe (2011) nach Postleitzonen

\footnotetext{
811 Vgl. Bortz und Schuster (2010), S. 79.

812 Vgl. Bortz und Schuster (2010), S. 79.

${ }^{813}$ Vgl. Berekoven, Eckert und Ellenrieder (2006), S. 51.

${ }^{814}$ Es handelt sich im vorliegenden Fall jedoch nicht direkt um eine Quotenauswahl, da die Auswahl an sich bereits durch den Datenbankanbieter selbst bzw. durch von ihm nicht zu vertretende Umstände zustande kam.
} 
Es stellt sich heraus, dass nur geringe Abweichungen des tatsächlichen Anteils von Banken an der Stichprobe vom erwarteten Anteil existieren. Beispielsweise lagen per 31.12.2011 etwa $11 \%$ aller Genossenschaftsbanken in Postleitzone 5. Bezogen auf den betrachteten Datensatz liegt der Anteil von Postleitzone 5 bei $10 \%$. Ein Chi-Quadrat-Anpassungstest (,Goodness of Fit"-Test) ${ }^{815}$ zeigt keine Abweichung der vorhandenen von den erwarteten Häufigkeiten für jede Postleitzone an. ${ }^{816}$ Es kann daher die Schlussfolgerung gezogen werden, dass sich die Auswahl regional gleichmäßig über das Bundesgebiet verteilt.

Auch eine Detailbetrachtung auf Ebene der aus den ersten zwei Ziffern der Postleitzahl bestehenden, sogenannten Postleitregionen untermauert die Ergebnisse. Von den 94 deutschen Postleitregionen, in denen Genossenschaftsbanken existieren, wurden 36 Stück zu $100 \%$ mit vorhandenen Datensätzen abgedeckt. Bei weiteren 11 lag die Abdeckung bei mindestens $90 \%$. 22 sind zu mindestens $80 \%$ abgedeckt, $11 \mathrm{zu}$ mindestens $70 \%, 8 \mathrm{zu}$ mindestens $60 \%$, nur 6 lagen darunter. Es ist jedoch von extern nicht nachvollziehbar, weshalb einige Banken in der Datenbank nicht abgebildet sind. Ein Chi-QuadratAnpassungstest ${ }^{817}$ zeigt auf Ebene der Postleitregionen keine Abweichung der vorhandenen von den erwarteten Häufigkeiten. Aus der gezeigten annähernden Gleichverteilung kann schließlich auf die Repräsentativität geschlossen werden.

Zusätzlich zur dieser Repräsentativität der Stichprobe ist zu erwähnen, dass der Datensatz einen sehr hohen Anteil der Grundgesamtheit aller existierenden Banken abdeckt. Beispielsweise liegen für das Jahr 2011 Daten für 963 der 1.121 Institute vor, was einer Abdeckungsquote von $86 \%$ entspricht. ${ }^{818}$ Die Abdeckungsquoten für alle untersuchten Geschäftsjahre finden sich in Tabelle 8 .

Es besteht durchweg eine sehr hohe Abdeckungsquote der Stichprobe. Zusammen mit der gezeigten Repräsentativität ist es statistisch treffender, im Weiteren von einer Vollerhebung auszugehen. ${ }^{819}$ Signifikanztests werden normalerweise auf Stichproben angewendet, können aber auch bei Vollerhebungen verwendet werden. ${ }^{820}$

\begin{tabular}{|c|c|c|c|}
\hline \multirow{2}{*}{ Jahr } & \multicolumn{2}{|c|}{ Anzahl der Genossenschaftsbanken } & Abdeckungsquote \\
& in Deutschland & im Datensatz & \\
\hline 2005 & 1.290 & 1.077 & $83 \%$ \\
\hline 2006 & 1.255 & 1.088 & $87 \%$ \\
\hline 2007 & 1.232 & 1.074 & $87 \%$ \\
\hline 2008 & 1.197 & 1.048 & $88 \%$ \\
\hline 2009 & 1.156 & 1.014 & $88 \%$ \\
\hline 2010 & 1.138 & 1.002 & $88 \%$ \\
\hline 2011 & 1.121 & 963 & $86 \%$ \\
\hline
\end{tabular}

Tabelle 8: $\quad$ Abdeckungsquote der vorliegenden Daten nach Jahren ${ }^{821}$

\footnotetext{
815 Chi-Quadrat-Wert 4,81; nicht signifikant.

${ }^{816}$ Vgl. Janssen und Laatz (2007), S. 561; vgl. Bühl (2012), S. 406 f.

817 Chi-Quadrat-Wert 20,46; nicht signifikant.

${ }^{818}$ Vgl. BVR (2015a) und Bundesbank (2012c).

${ }^{819}$ Vgl. Weis (2012), S. 203.

${ }^{820}$ Vgl. Broscheid und Gschwend (2005), S. 24

${ }^{821}$ Eigene Berechnungen, basierend auf BVR (2015a).
} 


\subsubsection{Bilanz und Bilanzsumme}

Die originäre Datenquelle, die alle Jahresabschlüsse beinhaltet, ist der elektronische Bundesanzeiger. ${ }^{822}$ In der Regel werden dort alle Werte der Bilanz und der Gewinn- und Verlustrechnung auf Euro und Cent genau angegeben. Die verwendete Datenbank enthält hingegen alle Positionen in Millionen mit einer Nachkommastelle, das heißt auf Vielfache von 100.000 Euro gerundet. Aufgrund dieser Rundung kommt es zu Abweichungen in Bezug zur Originärquelle. $\mathrm{Da}$ die Positionen jedoch in der Regel deutlich größer sind als die Rundungsgenauigkeit, sind hieraus keine substanziellen Abweichungen zu erwarten.

\begin{tabular}{|c|c|c|}
\hline Pos. & Positionen Bilanz (HGB) & Positionen Balance Sheet \\
\hline 1. & $\begin{array}{l}\text { Barreserve } \\
\text { a) Kassenbestand } \\
\text { b) Guthaben bei Zentralnotenbanken } \\
\text { c) Guthaben bei Postgiroämtern } \\
\end{array}$ & Cash and Due From Banks \\
\hline 2. & $\begin{array}{l}\text { Schuldtitel öffentlicher Stellen und Wechsel, die zur Refinanzie- } \\
\text { rung bei Zentralnotenbanken zugelassen sind } \\
\text { a) Schatzwechsel und unverzinsliche Schatzanweisungen sowie } \\
\text { ähnliche Schuldtitel öffentlicher Stellen } \\
\text { b) Wechsel }\end{array}$ & Keine Entsprechung \\
\hline 3. & $\begin{array}{l}\text { Forderungen an Kreditinstitute } \\
\text { a) täglich fällig } \\
\text { b) andere Forderungen }\end{array}$ & Loans and Advanced to Banks \\
\hline 4. & $\begin{array}{l}\text { Forderungen an Kunden, darunter: } \\
\text { Durch Grundpfandrechte gesichert } \\
\text { Kommunalkredite } \\
\text { Alle übrigen Forderungen an Kunden } \\
\end{array}$ & $\begin{array}{l}\text { Net Loans } \\
\text { Residential Mortgage Loans } \\
\text { Corporate and Commercial Loans } \\
\text { Other Loans } \\
\end{array}$ \\
\hline 5. & $\begin{array}{l}\text { Schuldverschreibungen und andere festverzinsliche Wertpapiere } \\
\text { a) Geldmarktpapiere } \\
\text { b) Anleihen und Schuldverschreibungen } \\
\text { ba) von öffentlichen Emittenten } \\
\text { bb) von anderen Emittenten } \\
\text { c) eigene Schuldverschreibungen }\end{array}$ & $\begin{array}{l}\text { Other Securities } \\
\text { ba) einzeln ausgewiesen unter "Memo: } \\
\text { Government Securities included Above", }\end{array}$ \\
\hline 6. & Aktien und andere nicht festverzinsliche Wertpapiere & \\
\hline $6 a$. & Handelsbestand & $\begin{array}{l}\text { Trading Securities and at Fair Value } \\
\text { through Income }\end{array}$ \\
\hline 7. & Beteiligungen und Geschäftsguthaben bei Genossenschaften & At-equity Investments in Associates \\
\hline 8. & Anteile an verbundenen Unternehmen & \\
\hline 9. & Treuhandvermögen & Keine Entsprechung \\
\hline 10. & $\begin{array}{l}\text { Ausgleichsforderungen gegen die öffentliche Hand einschließlich } \\
\text { Schuldverschreibungen aus deren Umtausch }\end{array}$ & Keine Entsprechung \\
\hline 11. & Immaterielle Anlagewerte & Other Intangibles \\
\hline 12. & Sachanlagen & Fixed Assets \\
\hline 13. & Sonstige Vermögensgegenstände & Other Assets \\
\hline 14. & Rechnungsabgrenzungsposten & \\
\hline 16. & Aktiver Unterschiedsbetrag aus der Vermögensverrechnung & Keine Entsprechung \\
\hline & Summe der Aktiva & Total Assets \\
\hline
\end{tabular}

822 Bundesanzeiger 
Alle Kreditgenossenschaften nehmen ihre Bilanzierung nach $\int \mathbb{S} 340-340 \mathrm{~g}$ HGB i. V. m.

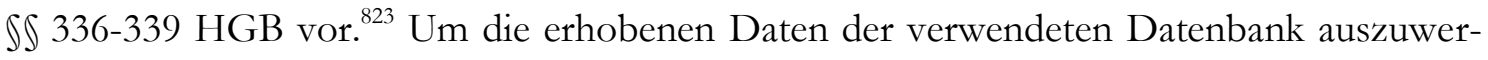
ten, müssen sie den Positionen der Bilanz nach HGB zugeordnet werden. Zunächst erfolgt die Zuordnung für die Aktivseite der Bilanz gemäß Tabelle 9.

Hierbei wird darauf hingewiesen, dass die Posten 2. Schuldtitel öffentlicher Stellen und Wechsel und 5. a) Geldmarktpapiere für alle erhobenen Kreditinstitute und Jahre stets null sind. Folgend werden in Tabelle 10 die Positionen der Passivseite zugeordnet.

\begin{tabular}{|c|c|c|}
\hline Pos. & Positionen Bilanz (HGB) & $\begin{array}{l}\text { Positionen Balance Sheet } \\
\text { (Datenquelle) }\end{array}$ \\
\hline 1. & Verbindlichkeiten gegenüber Kreditinstituten & Deposits from Banks \\
\hline \multirow[t]{5}{*}{2.} & Verbindlichkeiten gegenüber Kunden & Total Customer Deposits \\
\hline & a) Spareinlagen & Customer Deposits - Savings \\
\hline & b) andere Verbindlichkeiten & \\
\hline & ba) täglich fällig & Customer Deposits - Current \\
\hline & bb) mit vereinbarter Laufzeit oder Kündigungsfrist & Customer Deposits - Term \\
\hline 3. & Verbriefte Verbindlichkeiten & Other Funding \\
\hline 3. & a) Handelsbestand & \\
\hline 4. & Treuhandverbindlichkeiten & Keine Entsprechung \\
\hline 5. & Sonstige Verbindlichkeiten & Other Liabilities \\
\hline 6. & Rechnungsabgrenzungsposten & Other Deferred Liabilities \\
\hline 6. & a) Passive latente Steuern & Deferred Tax Liabilities \\
\hline \multirow[t]{2}{*}{7.} & $\begin{array}{l}\text { Rückstellungen } \\
\text { a) Rückstellungen für Pensionen u. ähnliche Verpflichtungen } \\
\text { c) andere Rückstellungen }\end{array}$ & Reserves for Pensions and Other \\
\hline & b) Steuerrückstellungen & Current Tax Liabilities \\
\hline 8. & [gestrichen] & \\
\hline 9. & Nachrangige Verbindlichkeiten & Subordinated Borrowing \\
\hline 10. & Genussrechtskapital & $\begin{array}{l}\text { Pref. Shares and Hybrid Capital } \\
\text { accounted for as Debt }\end{array}$ \\
\hline 11. & Fonds für allgemeine Bankrisiken & Common Equity \\
\hline \multirow[t]{2}{*}{12.} & Eigenkapital & \\
\hline & Summe der Passiva & Total Liabilities and Equity \\
\hline
\end{tabular}

\section{Bilanzsumme}

Die vorliegende Untersuchung erstreckt sich über die Jahre 2005 bis 2011. Zur Ermittlung der durchschnittlichen Bilanzsumme (DBS) eines Jahres - für eine adäquate Berücksichtigung der Positionen aus der Gewinn- und Verlustrechnung - ist stets auch der Wert des Vorjahres notwendig. ${ }^{824}$ Speziell für 2005, das erste Jahr der Zeitreihe, wäre die Summe der Eröffnungsbilanz des Jahres 2005 vonnöten, um schließlich die durchschnittliche Bilanzsumme zu ermitteln. Diese Eröffnungsbilanzsumme 2005 ist jedoch nur in Form der Schlussbilanzsumme aus 2004 denkbar. Entsprechende Daten stehen nicht zur Verfügung. Um dennoch auf die Auswertung der Daten aus 2005 nicht verzichten zu müssen, wird für 
dieses Jahr ausnahmsweise nicht die DBS, sondern die echte Bilanzsumme am Jahresende verwendet. In der Regel liegt die Schlussbilanzsumme leicht über der Eröffnungsbilanzsumme. Dies führt zu einer minimalen Verzeichnung, die jedoch in diesem einen Fall akzeptiert werden kann. Des Weiteren sind alle als Quotienten ermittelten Werte des Jahres 2005 für die Positionen der Gewinn- und Verlustrechnung untereinander valide vergleichbar. Sie sind lediglich um einen Faktor verzeichnet, welcher sehr nahe an der Zahl eins liegt.

\subsubsection{Gewinn- und Verlustrechnung}

Wie bei den Bilanzpositionen werden nun die korrespondieren Werte aus der verwendeten Datenbank den Werten aus den jeweiligen Jahresabschlüssen zugeordnet. Tabelle 11 enthält eine entsprechende Aufstellung.

\begin{tabular}{|c|c|c|}
\hline Pos. & $\begin{array}{l}\text { Positionen Gewinn- und Verlustrech- } \\
\text { nung (HGB) }\end{array}$ & $\begin{array}{l}\text { Positionen Income State- } \\
\text { ment (Datenquelle) }\end{array}$ \\
\hline 1.a) & Zinserträge aus Kredit- und Geldmarktgeschäften & Interest Income on Loans \\
\hline 1.b) & $\begin{array}{l}\text { Zinserträge aus festverzinslichen Wertpapieren } \\
\text { und Schuldbuchforderungen }\end{array}$ & Other Interest Income \\
\hline \multirow[t]{2}{*}{2.} & Zinsaufwendungen & Total Income Expense \\
\hline & Zinsüberschuss & Net Interest Income \\
\hline 3. & $\begin{array}{l}\text { Laufende Erträge aus } \\
\text { a) Aktien und anderen nicht festverzinslichen } \\
\text { Wertpapieren } \\
\text { b) Beteiligungen und Geschäftsguthaben bei } \\
\text { Genossenschaften } \\
\text { c) Anteilen an verbundenen Unternehmen }\end{array}$ & Dividend Income \\
\hline 4. & $\begin{array}{l}\text { Erträge aus Gewinngemeinschaften, Gewinnab- } \\
\text { führungs- oder Teilgewinnabführungsverträgen }\end{array}$ & - \\
\hline 5., 6. & Provisionserträge abzgl. Provisionsaufwendungen & Net Fees and Commissions \\
\hline 7. & Nettoertrag/-aufwand des Handelsbestands & - \\
\hline 8. & Sonstige betriebliche Erträge & Other Operating Income \\
\hline 9. & [gestrichen] & \\
\hline 10.a) & $\begin{array}{l}\text { aa) Löhne und Gehälter } \\
+ \text { ab) Soziale Abgaben und Aufwendungen für } \\
\text { Altersversorgung und für Unterstützung } \\
=\text { Personalaufwand }\end{array}$ & Personnel Expenses \\
\hline $\begin{array}{l}10 . b) \\
+11 . \\
+12 .\end{array}$ & $\begin{array}{l}\text { andere Verwaltungsaufwendungen } \\
+ \text { Abschreibungen und Wertberichtigungen auf } \\
\text { immaterielle Anlagewerte und Sachanlagen } \\
+ \text { Sonstige betriebliche Aufwendungen }\end{array}$ & Other Operating Expenses \\
\hline & $\begin{aligned}=\text { Bruttogewinn aus normaler } \\
\text { Geschäftstätigkeit }\end{aligned}$ & $\begin{array}{r}=\text { Pre-Impairment } \\
\text { Operating Profit }\end{array}$ \\
\hline
\end{tabular}




\begin{tabular}{|c|c|c|}
\hline Pos. & $\begin{array}{l}\text { Positionen Gewinn- und Verlustrech- } \\
\text { nung (HGB) }\end{array}$ & $\begin{array}{l}\text { Positionen Income State- } \\
\text { ment (Datenquelle) }\end{array}$ \\
\hline $\begin{array}{l}13 . \\
-14 .\end{array}$ & $\begin{array}{l}\text { Abschreibungen und Wertberichtigungen auf } \\
\text { Forderungen und bestimmte Wertpapiere sowie } \\
\text { Zuführungen zu Rückstellungen im Kreditge- } \\
\text { schäft } \\
\text { abzüglich } \\
\text { Erträge aus Zuschreibungen zu Forderungen und } \\
\text { bestimmten Wertpapieren sowie aus der Auflö- } \\
\text { sung von Rückstellungen im Kreditgeschäft }\end{array}$ & Loan Impairment Charge \\
\hline $\begin{array}{l}15 . \\
-16 \\
+17\end{array}$ & $\begin{array}{l}\text { Abschreibungen und Wertberichtigungen auf } \\
\text { Beteiligungen, Anteile an verbundenen Unter- } \\
\text { nehmen und wie Anlagevermögen behandelte } \\
\text { Wertpapiere } \\
\text { abzüglich } \\
\text { Erträge aus Zuschreibungen zu Beteiligungen, } \\
\text { Anteilen an verbundenen Unternehmen und wie } \\
\text { Anlagevermögen behandelten Wertpapieren } \\
\text { zuzüglich } \\
\text { Aufwendungen aus Verlustübernahme }\end{array}$ & $\begin{array}{l}\text { Securities and Other Credit } \\
\text { Impairment Charges }\end{array}$ \\
\hline 19. & $=$ Ergebnis der normalen Geschäftstätigkeit & Operating Profit (EBIT) \\
\hline 20. & Außerordentliche Erträge & Non-recurring income \\
\hline 21. & Außerordentliche Aufwendungen & Non-recurring expense \\
\hline $\begin{array}{l}22 . \\
=20 .- \\
21 .\end{array}$ & Außerordentliches Ergebnis & \\
\hline $\begin{array}{l}23 . \\
+24 .\end{array}$ & $\begin{array}{l}\text { Steuern von Einkommen und vom Ertrag } \\
\text { zuzüglich } \\
\text { Sonstige Steuern, soweit nicht unter Posten } 12 \\
\text { ausgewiesen }\end{array}$ & Tax expense \\
\hline $24 \mathrm{a}$. & Einstellung in Fonds für allgemeine Bankrisiken & $t^{-1}$ \\
\hline 25. & $=$ Jahresüberschuss & Net Income \\
\hline
\end{tabular}

Tabelle 11: $\quad$ Zuordnung von Positionen der Gewinn- und Verlustrechnung zu Positionen des Income Statements

Die einzelnen Positionen in Tabelle 11 orientieren sich an Formblatt 3 zur KreditinstitutsRechnungslegungsverordnung (RechKredV) - Gewinn- und Verlustrechnung. ${ }^{825}$

\subsubsection{Fusionen}

Liegt die Bilanzsumme deutlich über dem Wert des Vorjahres, so ist zu prüfen, ob ein Unternehmenszusammenschluss stattfand. Dennoch ist selbst ein hohes Bilanzsummenwachstum kein Beleg für eine erfolgte Fusion, und ein geringes Wachstum kein Indiz dagegen. Deshalb werden Angaben zur Bankhistorie separat auf Fusionen hin ausgewertet. Fand im betreffenden Jahr in einer Bank eine Fusion statt, so wird dies im Rahmen der Panelanalyse durch eine Dummyvariable gekennzeichnet. ${ }^{826}$ 


\subsubsection{Verteilung der untersuchten Banken in Deutschland}

Abbildung 19 stellt die räumliche Anordnung der untersuchten Banken dar. Datengrundlage sind hierbei alle Banken, deren Jahresabschlüsse per 31.12.2011 vorliegen. Wie bereits oben in Abbildung 17 werden die Farben Gelb, Hellbraun und Dunkelbraun zur Hervorhebung ländlicher, halbstädtischer und städtischer Regionen verwendet. Grüne, rote und schwarze Nadelkuppen stellen Banken in ländlicher, halbstädtischer bzw. städtischer Lage dar.

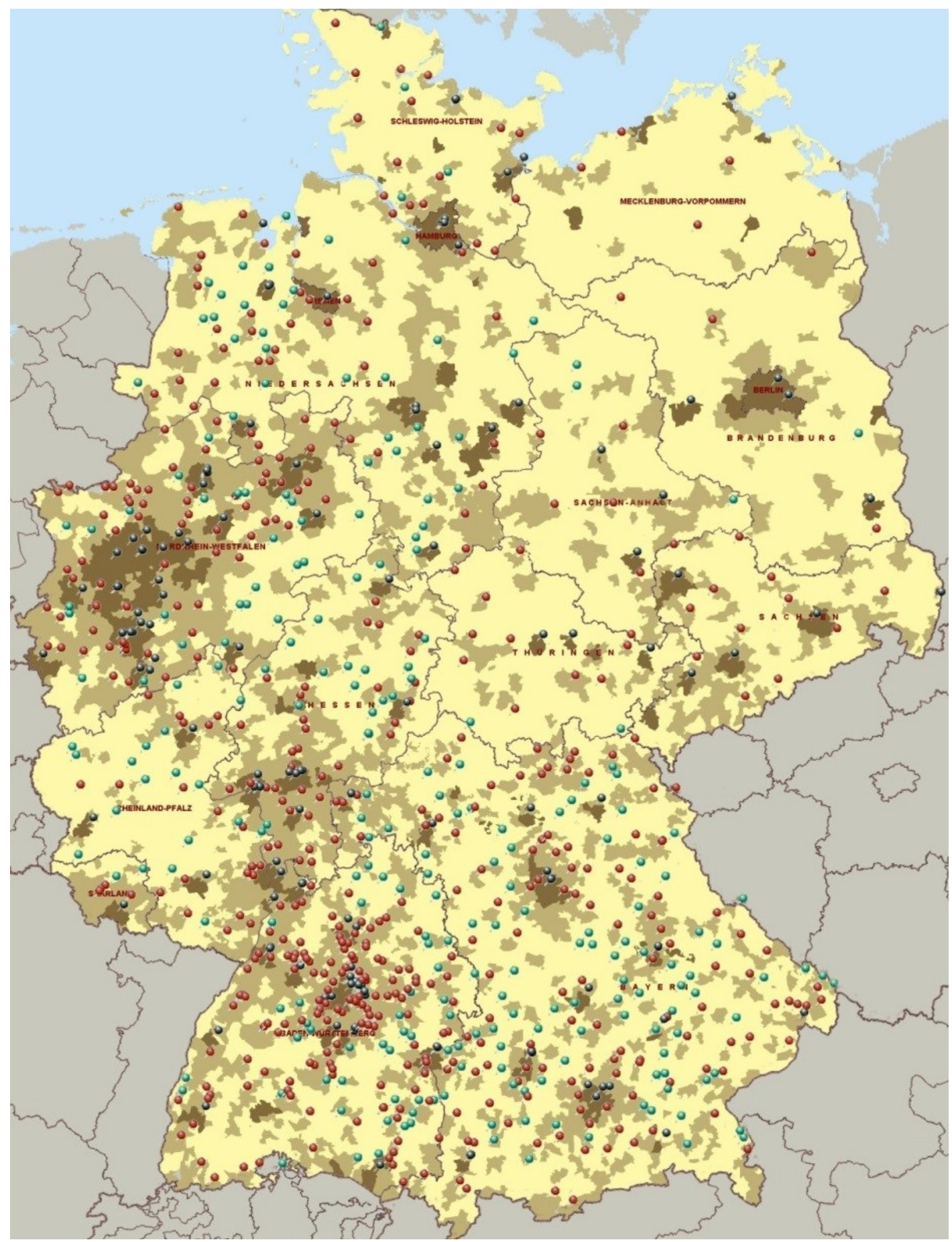

Abbildung 19: Verteilung der untersuchten Banken ${ }^{827}$

${ }^{827}$ Eigene Darstellung, basierend auf Statistisches Bundesamt (2012a). 


\subsubsection{Regionale Wirtschaftsdaten}

Um regionale Abhängigkeiten herausstellen zu können, greift die vorliegende Arbeit auf regionale Wirtschaftsdaten auf Kreisebene zurück. Diese wurden durch den Arbeitskreis Volkswirtschaftliche Gesamtrechnungen der Länder (VGRdL) veröffentlicht, welcher 1954 gegründet wurde. ${ }^{82}$ Herangezogen wurden drei Datensätze mit den Bezeichnungen $\mathrm{R} 2 \mathrm{~B} 1^{829}, \mathrm{R} 2 \mathrm{~B} 2^{830}$ und R2B33 ${ }^{831}$. Weitere Angaben auf Kreisebene werden vom VGRdL aktuell nicht veröffentlicht. Die starke Aufgliederung bis hinunter zur Kreisebene ermöglicht es, regionale Zusammenhänge im Detail zu ergründen. Die Gliederung basiert auf der Systematik der Gebietseinheiten für die Statistik ${ }^{832}$ der EU.

NUTS $^{833}$ gliedert jedes EU-Mitgliedsland auf bis zu drei Ebenen:

- NUTS 1: sozioökonomische Großregionen

- NUTS 2: Basisregionen für regionalpolitische Maßnahmen

- NUTS 3: kleine Regionen für spezifische Diagnosen. ${ }^{834}$

Die Daten des VGRdL liegen auf folgenden Ebenen vor:

1. Ebene der 16 Bundesländer (NUTS 1)

2. Ebene von 38 Regierungsbezirken/Statistischen Regionen (NUTS 2)

3. Ebene von 402 Kreisen und kreisfreien Städten (NUTS 3).

Der Datensatz R2B1 enthält folgende Angaben auf Kreisebene. ${ }^{835}$

1. Bruttoinlandsprodukt in jeweiligen Preisen (in EUR)

○ Bruttoinlandsprodukt in jeweiligen Preisen (in Prozent, Veränderung gegenüber jeweils ausgewiesenem Vorjahr)

- Bruttoinlandsprodukt in jeweiligen Preisen (in Prozent, Anteil am Bundesland)

2. Bruttowertschöpfung in jeweiligen Preisen (in EUR), insgesamt und aufgegliedert nach Land- und Forstwirtschaft, Fischerei; Produzierendes Gewerbe; Produzierendes Gewerbe ohne Baugewerbe; Verarbeitendes Gewerbe; Baugewerbe; Dienstleistungsbereiche; Handel, Verkehr und Lagerei, Gastgewerbe, Information und Kommunikation; Finanz-, Versicherungs- und Unternehmensdienstleister, Grundstücks- und Wohnungswesen; Öffentliche und sonstige Dienstleister, Erziehung und Gesundheit, Private Haushalte mit Hauspersonal

\footnotetext{
${ }^{828}$ Vgl. Arbeitskreis Volkswirtschaftliche Gesamtrechnungen der Länder VGRdL (2014c).

${ }^{829}$ Vgl. Arbeitskreis Volkswirtschaftliche Gesamtrechnungen der Länder VGRdL (2014a).

${ }^{830}$ Vgl. Arbeitskreis Volkswirtschaftliche Gesamtrechnungen der Länder VGRdL (2014b), Arbeitsblatt Vorwort.

831 Vgl. Arbeitskreis Volkswirtschaftliche Gesamtrechnungen der Länder VGRdL (2014c).

832 Vgl. Eurostat (2014).

${ }^{833}$ Die Abkürzung NUTS entstammt dem Französischen und steht für Nomenclature des unités territoriales statistiques.

${ }^{834}$ Vgl. Eurostat (2014).

${ }^{835}$ Vgl. Arbeitskreis Volkswirtschaftliche Gesamtrechnungen der Länder VGRdL (2014a).
} 
3. Erwerbstätige (Inland) in Personen insgesamt und aufgegliedert nach Land- und Forstwirtschaft, Fischerei; Produzierendes Gewerbe; Produzierendes Gewerbe ohne Baugewerbe; Verarbeitendes Gewerbe; Baugewerbe; Dienstleistungsbereiche; Handel, Verkehr und Lagerei, Gastgewerbe, Information und Kommunikation; Finanz-, Versicherungs- und Unternehmensdienstleister, Grundstücks- und Wohnungswesen; Öffentliche und sonstige Dienstleister, Erziehung und Gesundheit, Private Haushalte mit Hauspersonal

4. Bruttoinlandsprodukt in jeweiligen Preisen je Erwerbstätigen (Inland; in EUR)

5. Anzahl der Einwohner

6. Bruttoinlandsprodukt in jeweiligen Preisen je Einwohner (in EUR)

7. Standardarbeitsvolumen der Erwerbstätigen (Inland; in Arbeitsstunden)

8. Bruttoinlandsprodukt in jeweiligen Preisen je Arbeitsstunde der Erwerbstätigen (Inland; in EUR)

Der Datensatz R2B2 enthält folgende Angaben auf Kreisebene: ${ }^{836}$

1. Arbeitnehmerentgelt (insgesamt; davon produzierendes Gewerbe; jeweils Werte in Euro, Veränderung gegenüber Vorjahr in Prozent, Anteil am Bundesland in Prozent)

2. Bruttolöhne und -gehälter (insgesamt; davon produzierendes Gewerbe; jeweils Werte in Euro, Veränderung gegenüber Vorjahr in Prozent, Anteil am Bundesland in Prozent)

3. Arbeitnehmerentgelt je Arbeitnehmer pro Jahr (in Euro)

4. Bruttolöhne und -gehälter je Arbeitnehmer pro Jahr (in Euro)

5. Anzahl der Arbeitnehmer

6. Arbeitnehmerentgelt je Arbeitsstunde der Arbeitnehmer (in Euro)

7. Bruttolöhne und -gehälter je Arbeitsstunde der Arbeitnehmer (in Euro)

8. Standardarbeitsvolumen der Arbeitnehmer (in Arbeitsstunden)

Der Datensatz R2B3 enthält folgende Angaben auf Kreisebene: ${ }^{837}$

1. Primäreinkommen der privaten Haushalte (je Haushalt in Euro, Veränderung gegenüber Vorjahr in Prozent, Anteil am Bundesland in Prozent, je Einwohner in Euro)

2. Verfügbares Einkommen der privaten Haushalte (je Haushalt in Euro, Veränderung gegenüber Vorjahr in Prozent, Anteil am Bundesland in Prozent, je Einwohner in Euro)

3. Anzahl der Einwohner

${ }^{836}$ Vgl. Arbeitskreis Volkswirtschaftliche Gesamtrechnungen der Länder VGRdL (2014b).

${ }^{837}$ Vgl. Arbeitskreis Volkswirtschaftliche Gesamtrechnungen der Länder VGRdL (2014c). 
Das Bruttoinlandsprodukt gilt als der gebräuchlichste Indikator für Gesamtrechnungen. ${ }^{838}$ Es umfasst den „Wert aller innerhalb eines Wirtschaftsgebietes während einer bestimmten Periode produzierten Waren und Dienstleistungen $[\ldots]^{6839}$, und bildet die Ausgangsgröße für Konjunkturanalysen und -prognosen. Bruttowertschöpfung und Arbeitnehmerentgelt erlauben umfangsreiche Strukturanalysen. ${ }^{840}$ Die „Bruttowertschöpfung [...] ergibt sich aus dem Bruttoproduktionswert zu Herstellungspreisen abzüglich der Vorleistungen zu Anschaffungspreisen.“ ${ }^{\text {841 }}$ Das Arbeitnehmerentgelt beinhaltet „sämtliche Geld- und Sachleistungen, die den innerhalb eines Wirtschaftsgebietes beschäftigten Arbeitnehmern aus den Arbeits- oder Dienstverhältnissen zugeflossen sind. ${ }^{6842}$ Es setzt sich zusammen aus den „Bruttolöhnen und -gehältern sowie den tatsächlichen und unterstellten Sozialbeiträgen der Arbeitgeber. ${ }^{c 643}$ Das verfügbare Einkommen schließlich erlaubt Rückschlüsse auf die Kaufkraft von Regionen und hilft so Unternehmen bei Standortentscheidungen. ${ }^{844}$ Abbildung 20 enthält eine schematische Darstellung der wichtigsten Begriffe in der volkswirtschaftlichen Gesamtrechnung.

\subsubsection{Regionales Wirtschaftswachstum}

Um das regionale wirtschaftliche Wachstum der einzelnen Kreise und kreisfreien Städte abzubilden, wurde ein entsprechender Indikator gesucht. In der Literatur wird zur Darstellung des Wachstums häufig auf die Veränderung des Bruttoinlandsprodukts (BIP) zurückgegriffen. ${ }^{845}$ Als Proxy verwendet die vorliegende Arbeit das BIP der einzelnen Kreise und kreisfreien Städte im Betrachtungszeitraum 2005 bis 2011. ${ }^{846}$ Aus den entsprechenden Werten wurde für jeden Kreis die Rate ermittelt, mit welcher die Wirtschaft im jeweiligen Gebiet im Durchschnitt pro Jahr des Zeitraums gewachsen ist. Die entsprechende Variable lautet $g$, die Beziehung zur Berechnung lautet

$$
g=\sqrt[6]{\frac{B I P_{2011}}{B I P_{2005}}}-1
$$

Die Zuordnung der Gemeinden zu den Kreisen geschieht anhand der geografischen Lage. Der geringste Wert aller 11.252 Gemeinden liegt bei -1,7\% (Schrumpfung), das $10 \%$ Quantil bei 1,4\% der Median bei 2,7\%, das $90 \%$-Quantil bei 3,9 \% und der Maximalwert bei $8,8 \%$ p. a. und die Standardabweichung beträgt 1,1\%. Für 0,9\% der Gemeinden konnte eine Schrumpfung nachgewiesen werden.

\footnotetext{
${ }^{838}$ Vgl. Arbeitskreis Volkswirtschaftliche Gesamtrechnungen der Länder VGRdL (2014c).

839 Arbeitskreis Volkswirtschaftliche Gesamtrechnungen der Länder VGRdL (2014c).

${ }^{840} \mathrm{Vgl}$. Arbeitskreis Volkswirtschaftliche Gesamtrechnungen der Länder VGRdL (2014c).

${ }^{841}$ Arbeitskreis Volkswirtschaftliche Gesamtrechnungen der Länder VGRdL (2014c).

${ }^{842}$ Arbeitskreis Volkswirtschaftliche Gesamtrechnungen der Länder VGRdL (2014c).

${ }^{843}$ Arbeitskreis Volkswirtschaftliche Gesamtrechnungen der Länder VGRdL (2014c).

${ }^{844}$ Vgl. Arbeitskreis Volkswirtschaftliche Gesamtrechnungen der Länder VGRdL (2014c).

${ }^{845}$ Vgl. Arbeitskreis Volkswirtschaftliche Gesamtrechnungen der Länder VGRdL (2014a), Arbeitsblatt Vorwort.

${ }^{846}$ Vgl. Arbeitskreis Volkswirtschaftliche Gesamtrechnungen der Länder VGRdL (2014a).
} 


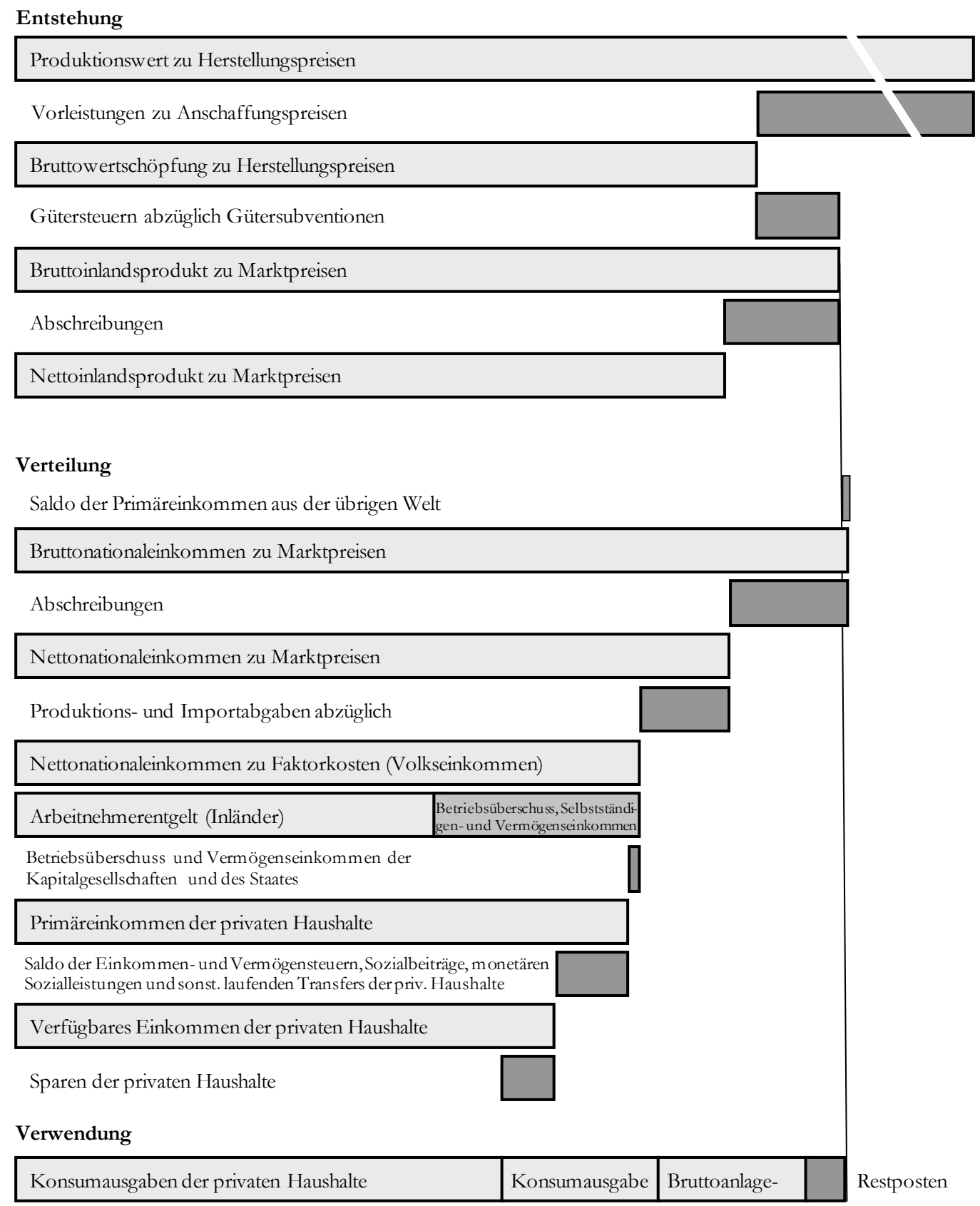

Abbildung 20: Schematische Darstellung der wichtigsten VGR-Begriffe ${ }^{847}$

Im nächsten Schritt wurden alle Gemeinden gemäß der jahresdurchschnittlichen Wachstumsrate in Quintile, das heißt $20 \%$-Abschnitte, eingeteilt. Folgende Farben wurden verwendet, um die fünf Wachstumsquintile zu kennzeichnen:

- Quintil 1: sehr schwaches Wachstum - dunkelblau

- Quintil 2: schwaches Wachstum - hellblau

- Quintil 3: durchschnittliches Wachstum - gelb

- Quintil 4: starkes Wachstum - rosa

- Quintil 5: sehr starkes Wachstum - rot

${ }^{847}$ Arbeitskreis Volkswirtschaftliche Gesamtrechnungen der Länder VGRdL (2014c), Arbeitsblatt Schaubild. 


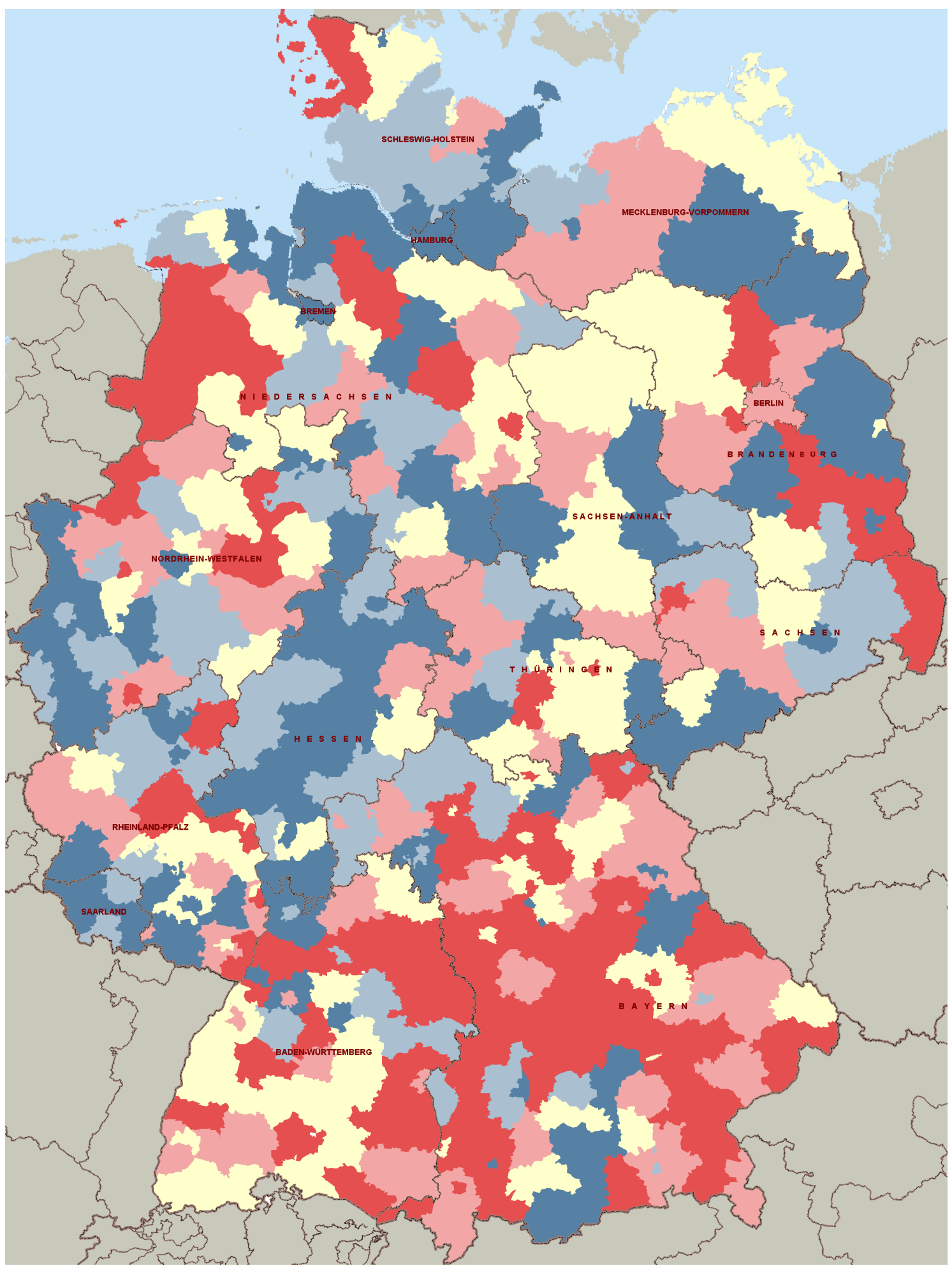

Abbildung 21: Jahresdurchschnittliches BIP-Wachstum 2005-2011 auf Kreisebene ${ }^{848}$

Die verwendeten Farben orientieren sich an der Darstellung des Bundesinstituts für Bau-, Stadt- und Raumforschung. ${ }^{849}$ Die grafische Darstellung auf der Karte in Abbildung 21 basiert auf allen 11.252 einzelnen Gemeinden Deutschlands. CHRISTIANS und HARTL

${ }^{848}$ Eigene Darstellung, basierend auf Arbeitskreis Volkswirtschaftliche Gesamtrechnungen der Länder VGRdL (2014a) in Verbindung
mit Statistisches Bundesamt (2012a).

${ }^{849}$ Vgl. Bundesinstitut für Bau-, Stadt- und Raumforschung (2014). 
verwenden eine ähnliche Darstellung des genannten Instituts, jedoch zur Verdeutlichung der zukünftigen Entwicklung der Bevölkerung. ${ }^{850}$

Unter den Kreisen und kreisfreien Städten gibt es einzelne Gebiete mit vergleichsweise hohen anhaltenden Wachstumsraten, wie die Landkreise Spree-Neiße, Pfaffenhofen an der Ilm und Böblingen, ferner die kreisfreien Städte Coburg, Ingolstadt, Wolfsburg und Erlangen. Die zehn wachstumsstärksten Gebiete sind zusammen mit ihrer jährlichen durchschnittlichen Wachstumsrate in Tabelle 12 dargestellt:

\begin{tabular}{|c|l|c|}
\hline Nr. & $\begin{array}{l}\text { Landkreis bzw. kreisfreie Stadt } \\
\text { (Bundesland) }\end{array}$ & $\begin{array}{l}\text { Durchschnittliche jährliche } \\
\text { Wachstumsrate 2005 bis 2011 }\end{array}$ \\
\hline 1 & Spree-Neiße, Landkreis (BB) & $8,77 \%$ \\
\hline 2 & Pfaffenhofen a. d. Ilm, Landkreis (BY) & $8,44 \%$ \\
\hline 3 & Coburg, Kreisfreie Stadt (BY) & $7,63 \%$ \\
\hline 4 & Ingolstadt, Kreisfreie Stadt (BY) & $7,30 \%$ \\
\hline 5 & Böblingen, Landkreis (BW) & $6,81 \%$ \\
\hline 6 & Dahme-Spreewald, Landkreis (BB) & $6,06 \%$ \\
\hline 7 & Wolfsburg, Kreisfreie Stadt (NI) & $5,02 \%$ \\
\hline 8 & Germersheim, Landkreis (RP) & $5,74 \%$ \\
\hline 9 & Heilbronn, Landkreis (BW) & $5,74 \%$ \\
\hline 10 & Erlangen, Kreisfreie Stadt (BY) & $5,69 \%$ \\
\hline
\end{tabular}

Tabelle 12: $\quad$ Landkreise und kreisfreie Städte mit den höchsten durchschnittlichen Wachstumsraten von 2005 bis $2011^{851}$

Hingegen existieren auch Gebiete, in denen die Wachstumsraten über den Zeitraum 2005 bis 2011 im Mittel über alle Jahre sehr niedrig waren. Dazu zählen die Kreise Sömmerda, Anhalt-Bitterfeld, aber auch der Hochtaunuskreis. Bei den kreisfreien Städten sind Eisenach, Suhl und Offenbach am Main zu nennen. Die zehn wachstumsschwächsten Gebiete werden in Tabelle 13 dargestellt. Bei den ersten neun von diesen liegt für den Zeitraum eine Schrumpfung vor.

\begin{tabular}{|c|l|c|}
\hline Nr. & $\begin{array}{l}\text { Landkreis bzw. kreisfreie Stadt } \\
\text { (Bundesland) }\end{array}$ & $\begin{array}{l}\text { Durchschnittliche jährliche } \\
\text { Wachstumsrate 2005 bis 2011 }\end{array}$ \\
\hline 1 & Sömmerda, Kreis (TH) & $-1,71 \%$ \\
\hline 2 & Eisenach, Kreisfreie Stadt (TH) & $-1,43 \%$ \\
\hline 3 & Suhl, Kreisfreie Stadt (TH) & $-0,72 \%$ \\
\hline 4 & Anhalt-Bitterfeld (ST) & $-0,47 \%$ \\
\hline 5 & Hochtaunuskreis (HE) & $-0,46 \%$ \\
\hline 6 & Offenbach am Main, Kreisfreie Stadt (HE) & $-0,26 \%$ \\
\hline 7 & Teltow-Fläming, Landkreis (BB) & $-0,22 \%$ \\
\hline 8 & Schwabach, Kreisfreie Stadt (BY) & $-0,14 \%$ \\
\hline 9 & Krefeld, Kreisfreie Stadt (NW) & $-0,08 \%$ \\
\hline 10 & Herzogtum Lauenburg, Landkreis (SH) & $0,10 \%$ \\
\hline
\end{tabular}

Tabelle 13: $\quad$ Landkreise und kreisfreie Städte mit den geringsten durchschnittlichen Wachstumsraten von 2005 bis $2011^{852}$

${ }^{850}$ Vgl. Christians und Hartl (2015), S. 14.

${ }^{851}$ Eigene Berechnungen, basierend auf Arbeitskreis Volkswirtschaftliche Gesamtrechnungen der Länder VGRdL (2014a). 


\subsubsection{Regionaldatenbank}

Die Regionaldatenbank Deutschland wird von den statistischen Ämtern des Bundes und der Länder herausgegeben. Enthalten sind tief gegliederte Ergebnisse der amtlichen Statistik. ${ }^{853}$ Die vorliegende Arbeit stellt auf den Altersaufbau der Bevölkerung ab. Dieser wird auf Ebene der Kreise und kreisfreien Städte ausgewertet. Auf diese Art kann ein räumlicher Bezug zu den untersuchten Banken hergestellt werden. Verwendet wird die Fortschreibung des Bevölkerungsstandes (Statistik 12411) zum 31.12.2011. ${ }^{854}$ Jedoch betrifft dies lediglich 2,1 \% der Banken, wodurch keine bedeutenden Verzerrungen zu erwarten sind.

\subsubsection{Zukunftsfähigkeit}

KRÖHNERT ET AL. untersuchen in ihrer Studie das zukünftige Entwicklungspotenzial der einzelnen Landkreise und kreisfreien Städte Deutschlands. ${ }^{855}$ Die verwendeten 22 Kriterien stammen aus den Bereichen Demografie, Wirtschaft, Integration, Bildung sowie Familienfreundlichkeit, die in Abbildung 22 dargestellt werden.

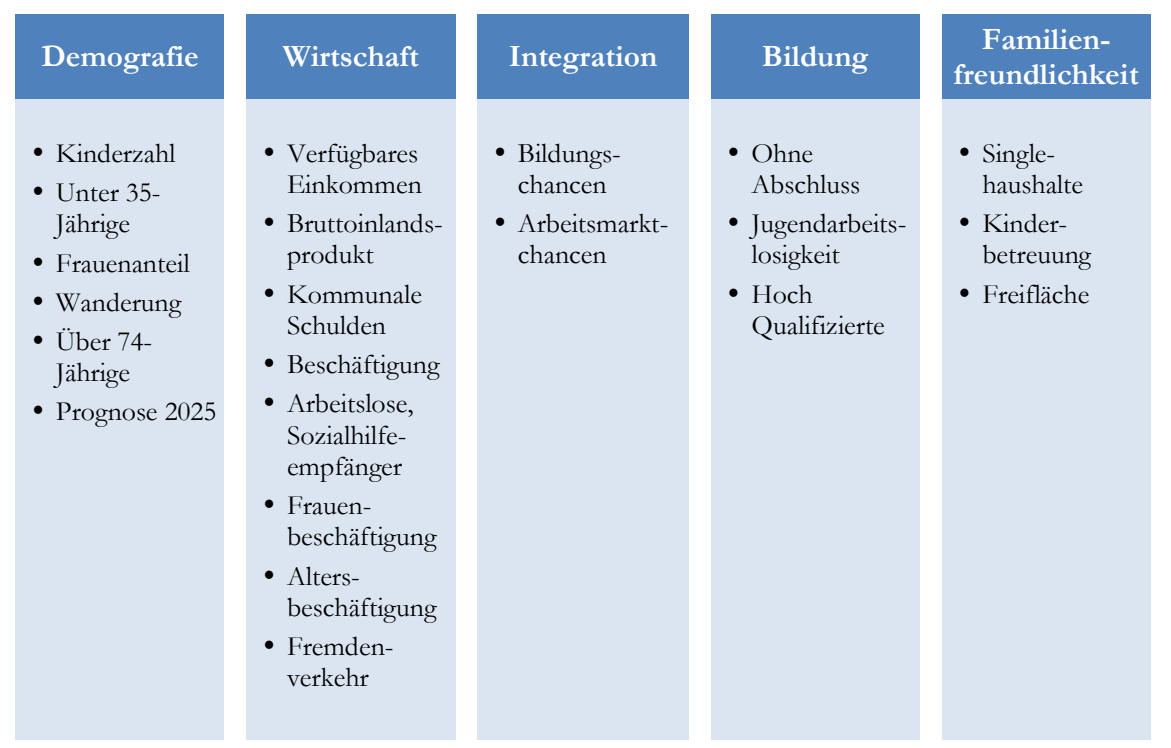

Abbildung 22: Bereiche und Kriterien zur Ermittlung der Zukunftsfähigkeit ${ }^{856}$

Die Bewertung der Kriterien erfolgte nach dem Schulnotenprinzip, 1 ist der beste Wert, 6 hingegen der schlechteste. Je Bereich wurde von den Autoren auch ein Mittelwert gebildet. In die Gesamtnote gehen alle 22 Einzelkriterien in arithmetischer Mittelung ein. Insofern wiegt der Wirtschaftsbereich mit acht Einzelkriterien deutlich schwerer als die Integration oder die Familienfreundlichkeit mit zwei oder drei Einzelkriterien. Die Gesamtnote entspricht also dem Kondensat aus allen Bereichen und ist als eine Beurteilung der Zukunftsfähigkeit eines Kreises bzw. einer kreisfreien Stadt zu interpretieren.

Leider steht keine jährliche Neubewertung der Zukunftsfähigkeit zur Verfügung, sodass die Werte zunächst prinzipiell auf das Erhebungsjahr 2010 zu beziehen sind. Jedoch handelt es

\footnotetext{
${ }^{852}$ Eigene Berechnungen, basierend auf Arbeitskreis Volkswirtschaftliche Gesamtrechnungen der Länder VGRdL (2014a).

${ }^{853} \mathrm{Vgl}$. Statistische Ämter des Bundes und der Länder (2013).

${ }^{854}$ Hinweis: Für das Bundesland Sachsen sind keine Werte verfügbar.

${ }^{855}$ Vgl. Kröhnert et al. (2011).

${ }^{856}$ Eigene Darstellung auf Basis von Kröhnert et al. (2011), S. 12 f.
} 
sich bei den Kriterien in der Regel um Eigenschaften, die sich nur langsam und über relativ lange Zeiträume verändern. Daher kann in zeitlicher Hinsicht von Stationarität ausgegangen werden, was letztlich Schlussfolgerungen auf den gesamten Betrachtungszeitraum der vorliegenden Studie (2005-2011) zulässt.

Neben der EU-Stadt/Landgliederung ${ }^{857}$ steht mit der Zukunftsfähigkeit eine zweite Möglichkeit zur Verfügung, um das Bundesgebiet kleinteilig aufzugliedern. Diese Aufteilung ermöglicht es, weitere relevante Erklärungsvariablen zu identifizieren. Ein kurzer Pre-Test vergleicht die Mittelwerte der Zukunftsfähigkeit aller drei Lagebereiche Städtisch, Halbstädtisch und Ländlich miteinander. Einbezogen werden alle 402 deutschen Kreise und kreisfreien Städte. Die Mittelwerte der drei Lagebereiche liegen bei 3,59, 3,46 und 3,60. Halbstädtische Bereiche werden etwas besser bewertet als ländliche bzw. städtische Bereiche. Der Median der Zukunftsfähigkeit aller Gemeinden liegt bei 3,58. Ein direkter Zusammenhang kann hier nicht abgeleitet werden, in allen drei Lagebereichen gibt es Gemeinden mit guter, mittlerer und auch schlechter Zukunftsfähigkeit. Abbildung 23 enthält eine grafische Darstellung der Verteilung anhand eines Boxplots.

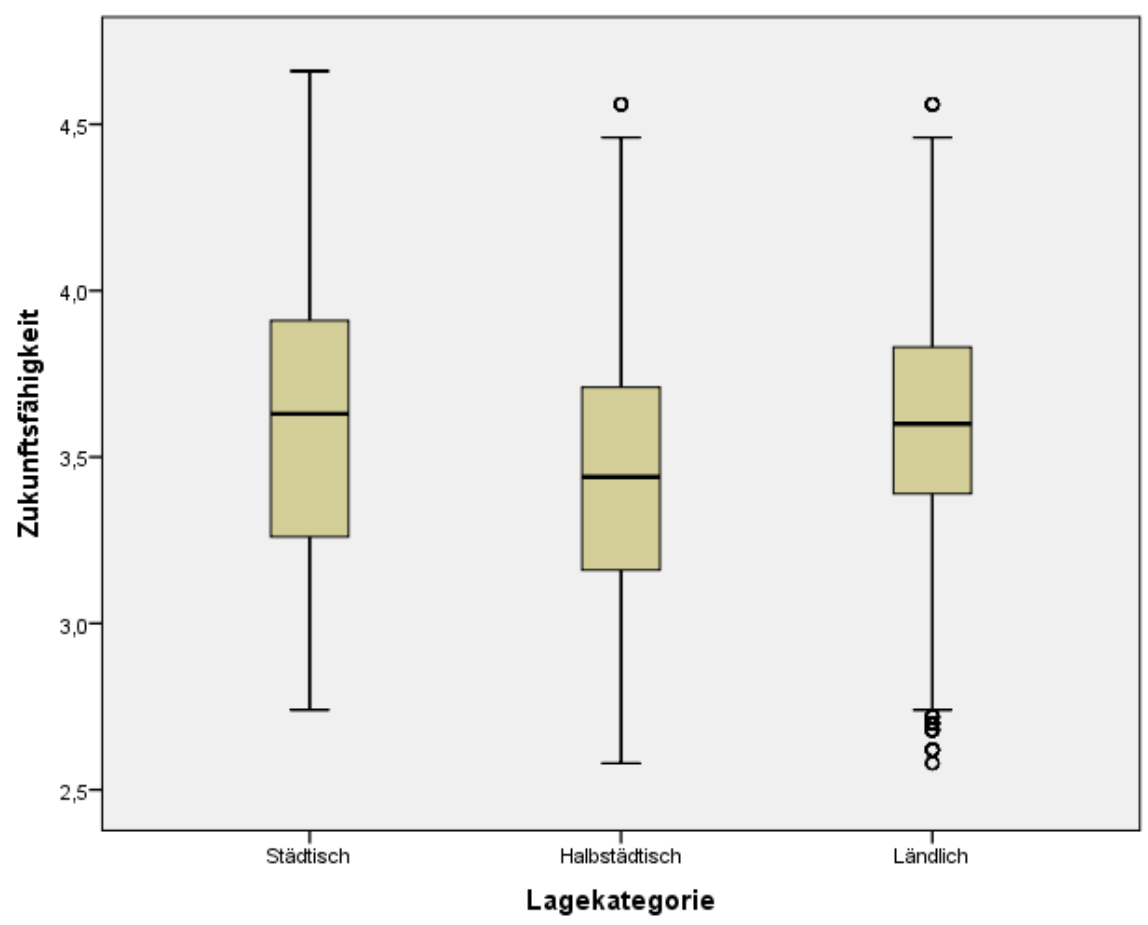

Abbildung 23: Einfluss der Lage auf die Zukunftsfähigkeit

Auch der geringe Korrelationskoeffizient von 0,14 belegt, dass kein funktionaler linearer Zusammenhang zu den Lagebereichen, ebenso wenig Multikollinearität zwischen Zukunftsfähigkeit und Lagebereich zu erwarten ist. Abbildung 24 stellt sämtliche Kreise Deutschlands dar. Die Farbe der Füllung repräsentiert die Zukunftsfähigkeit. Petrol und grün stehen für die Kreise mit den besten Aussichten, rot und rotbraun für die Kreise mit der pessimistischsten Einschätzung. 


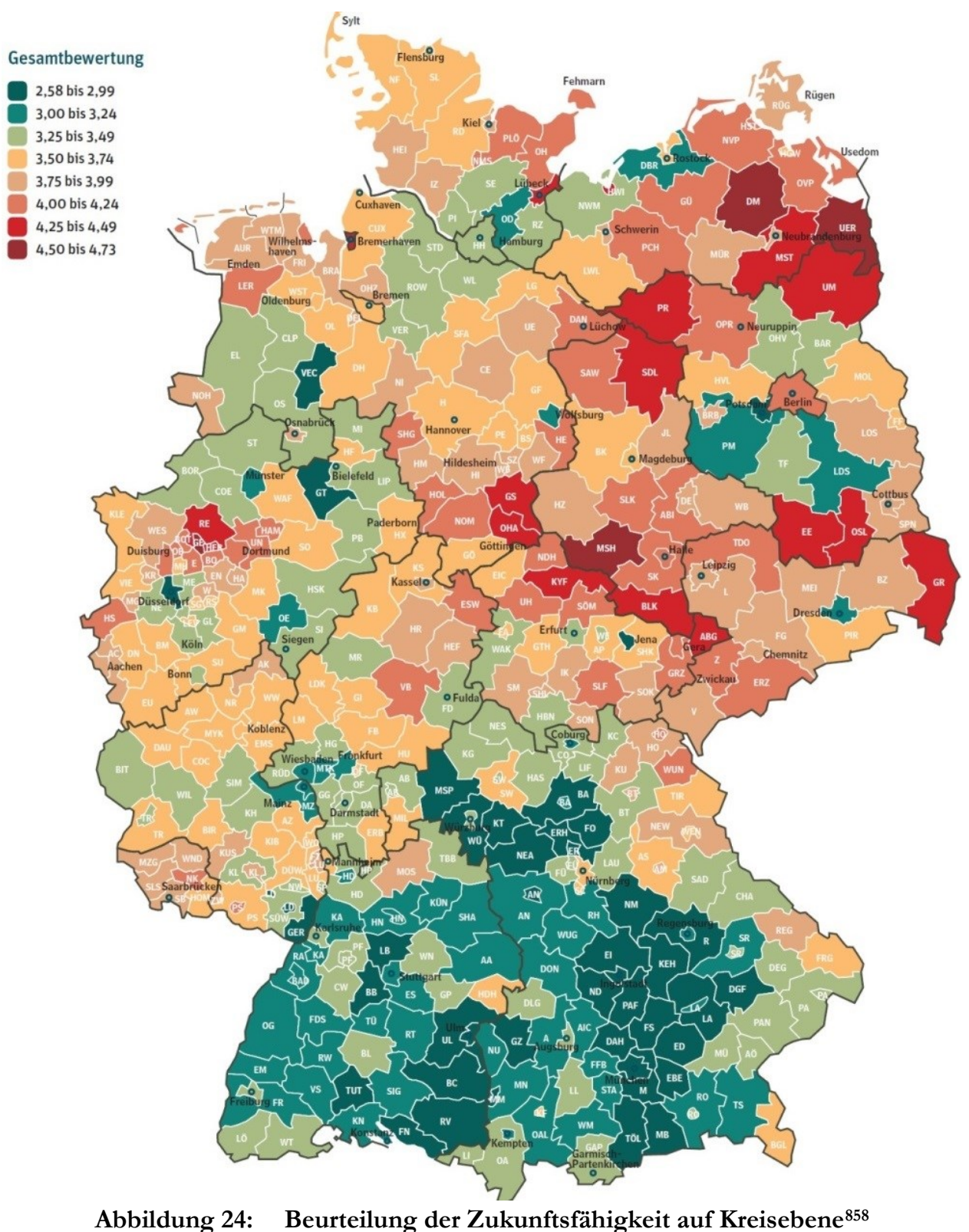

\subsubsection{Zinsentwicklung}

Wie häufig in der Literatur vorzufinden ${ }^{859}$, wird die Entwicklung der Marktzinsen über verschiedene Laufzeiten durch die Verwendung von Swapsätzen nachgezeichnet. Die bisweilen auch als Swap-Zinssätze bezeichneten Werte können als nahezu risikofrei angesehen werden, was auf die Art der Handelsabwicklung zurückzuführen ist. ${ }^{860}$ Gegenüber Zinssätzen von Staatsanleihen haben sie die Vorteile hoher Liquidität und geringer GeldBrief-Spannen. ${ }^{861}$ Sie werden der Datenbank Thomson Reuters EIKON entnommen. ${ }^{862}$ Konkret werden die Laufzeiten 2 Jahre, 5 Jahre und 10 Jahre in die Panelanalyse einbezogen. ${ }^{863}$

${ }^{858}$ Kröhnert et al. (2011), S. 7.

${ }^{859}$ Vgl. Richter (2014), S. 418 f.

${ }^{860} \mathrm{Vgl}$. GDV (2009), S. 3.

861 Vgl. GDV (2009), S. 3.

862 Thomson Reuters EIKON (2015).

${ }^{863} \mathrm{Vgl}$. Abschnitt 4.4.22. 


\subsection{Auswertung und empirische Analysen}

Das Ziel der vorliegenden Arbeit ist es, mögliche Einflussgrößen auf den Geschäftserfolg von Genossenschaftsbanken in Deutschland genau zu untersuchen und, sofern realisierbar, die Höhe des Einflusses zu quantifizieren.

Als abhängige (zu erklärende) Variable kommt hierbei die aggregierte Größe Bruttogewinn aus normaler Geschäftstätigkeit des jeweils zu untersuchenden Geschäftsjahres in Frage. Die einzelnen Positionen der Gewinn- und Verlustrechnung, die in den Bruttogewinn aus normaler Geschäftstätigkeit einfließen, werden ebenfalls durchleuchtet. Um eine Vergleichbarkeit zwischen größeren und kleineren Instituten herzustellen, werden diese Größen in Relation zur durchschnittlichen Bilanzsumme des betreffenden Geschäftsjahres gesetzt. Hieraus ergeben sich verschiedene Spannen, z. B. die Bruttogewinnspanne aus dem Bruttogewinn aus normaler Geschäftstätigkeit. Diese und weitere Spannen wurden bereits in Abschnitt 3.6.3 bzw. in Abschnitt 3.7 erläutert.

Die unabhängigen Variablen (Ursachen) sind Daten zur räumlichen Lage, zur regionalen Wirtschaft, aber auch Positionen der Bilanz und der Gewinn- und Verlustrechnung. Ebenfalls wird die konjunkturelle Entwicklung mit einbezogen. Dies geschieht beispielsweise durch die Berücksichtigung des regionalen Bruttoinlandsprodukts (BIP) auf Kreisebene, welches in den regionalen Wirtschaftsdaten enthalten ist oder über den Index der Zukunftsfähigkeit der Kreise.

Wie bereits erwähnt, wird in der vorliegenden Arbeit für den bankenübergreifenden Erfolgsvergleich die Bruttogewinnspanne eingesetzt. Alternativ verwenden einige Autoren auch die Cost-Income-Ratio. ${ }^{864}$ Für alle sieben betrachteten Geschäftsjahre konnte in einem PreTest eine hohe negative Korrelation zwischen Bruttogewinnspanne und Cost-Income-Ratio bestätigt werden. Der Korrelationskoeffizient $r$ liegt im Bereich $-0,80$ bis $-0,84$. Dies ist kausal darauf zurückzuführen, dass hohe Betriebsaufwendungen (Personal und Sachaufwendungen) die CIR erhöhen, während die Bruttogewinnspanne sinkt (und vice versa). Im Gegensatz hierzu führen hohe Erträge zu einer sinkenden CIR, während sich die Bruttogewinnspanne erhöht (und vice versa). ${ }^{865}$

\subsubsection{ROI-Analyse des Genossenschaftsbankensektors}

Das Verfahren zur ROI-Analyse wurde bereits in Abschnitt 3.7 beschrieben. Den Einstieg in die empirischen Untersuchungen bildet die ROI-Analyse, welche auf alle im Datensatz enthaltenen Genossenschaftsbanken angewendet wird. Hierbei wird zunächst für jedes Geschäftsjahr die aggregierte durchschnittliche Bilanzsumme aller Institute als absoluter Wert ermittelt. Danach erfolgt die Aufsummierung der Beträge je Einzelkomponente über alle Banken und Jahre als Absolutwert. Im dritten Schritt werden die Komponenten in das Verhältnis zur durchschnittlichen Bilanzsumme gesetzt und so die erforderlichen Spannen ermittelt.

Tabelle 14 stellt die Resultate der ROI-Analyse dar. Die ersten vier Zeilen repräsentieren die Ertragsquellen, die Bruttoertragsspanne ist hiervon die Summe. Es folgen die Aufwendungen für Personal und Verwaltung, deren Summe die Bruttobedarfsspanne ist. Die 
Bruttogewinnspanne ergibt sich durch Saldierung dieser Erträge und Aufwendungen, sie entspricht dem Bruttogewinn aus normaler Geschäftstätigkeit bezogen auf die durchschnittliche Bilanzsumme. Die Entwicklung der vier Komponenten der Bruttoertragsspanne im Zeitverlauf der Betrachtungsperiode wird in Abbildung 25 dargestellt.

\begin{tabular}{l|ccccccc} 
ROI-Komponenten & $\mathbf{2 0 0 5}$ & $\mathbf{2 0 0 6}$ & $\mathbf{2 0 0 7}$ & $\mathbf{2 0 0 8}$ & $\mathbf{2 0 0 9}$ & $\mathbf{2 0 1 0}$ & $\mathbf{2 0 1 1}$ \\
\hline Bruttozinsspanne & $2,51 \%$ & $2,39 \%$ & $2,22 \%$ & $2,09 \%$ & $2,29 \%$ & $2,40 \%$ & $2,32 \%$ \\
Provisionsspanne & $0,70 \%$ & $0,72 \%$ & $0,73 \%$ & $0,68 \%$ & $0,64 \%$ & $0,66 \%$ & $0,64 \%$ \\
Handelsspanne & $0,01 \%$ & $0,01 \%$ & $0,01 \%$ & $0,00 \%$ & $0,01 \%$ & $0,00 \%$ & $0,00 \%$ \\
Sonstige Ertragsspanne & $0,15 \%$ & $0,56 \%$ & $0,18 \%$ & $0,18 \%$ & $0,13 \%$ & $0,15 \%$ & $0,17 \%$ \\
Bruttoertragsspanne & $\mathbf{3 , 3 6} \%$ & $\mathbf{3 , 6 7} \%$ & $\mathbf{3 , 1 4} \%$ & $\mathbf{2 , 9 5} \%$ & $\mathbf{3 , 0 7} \%$ & $\mathbf{3 , 2 1} \%$ & $\mathbf{3 , 1 3} \%$ \\
Personalaufwandsspanne & $1,44 \%$ & $1,45 \%$ & $1,36 \%$ & $1,29 \%$ & $1,31 \%$ & $1,23 \%$ & $1,19 \%$ \\
Sachaufwandsspanne & $1,00 \%$ & $0,99 \%$ & $0,93 \%$ & $0,86 \%$ & $0,82 \%$ & $0,88 \%$ & $0,86 \%$ \\
Bruttobedarfsspanne & $\mathbf{2 , 4 4} \%$ & $\mathbf{2 , 4 4} \%$ & $\mathbf{2 , 2 8} \%$ & $\mathbf{2 , 1 5} \%$ & $\mathbf{2 , 1 3} \%$ & $\mathbf{2 , 1 1} \%$ & $\mathbf{2 , 0 6} \%$ \\
Bruttogewinnspanne & $\mathbf{0 , 9 2} \%$ & $\mathbf{1 , 2 3} \%$ & $\mathbf{0 , 8 6} \%$ & $\mathbf{0 , 8 0} \%$ & $\mathbf{0 , 9 3} \%$ & $\mathbf{1 , 0 9} \%$ & $\mathbf{1 , 0 7 \%}$ \\
Risikospanne & $0,46 \%$ & $0,69 \%$ & $0,41 \%$ & $0,43 \%$ & $0,33 \%$ & $0,31 \%$ & $0,01 \%$ \\
Nettogewinnspanne & $0,47 \%$ & $0,54 \%$ & $0,45 \%$ & $0,37 \%$ & $0,60 \%$ & $0,78 \%$ & $1,06 \%$ \\
\hline
\end{tabular}

Tabelle 14: ROI-Analyse aller Banken über den Betrachtungszeitraum

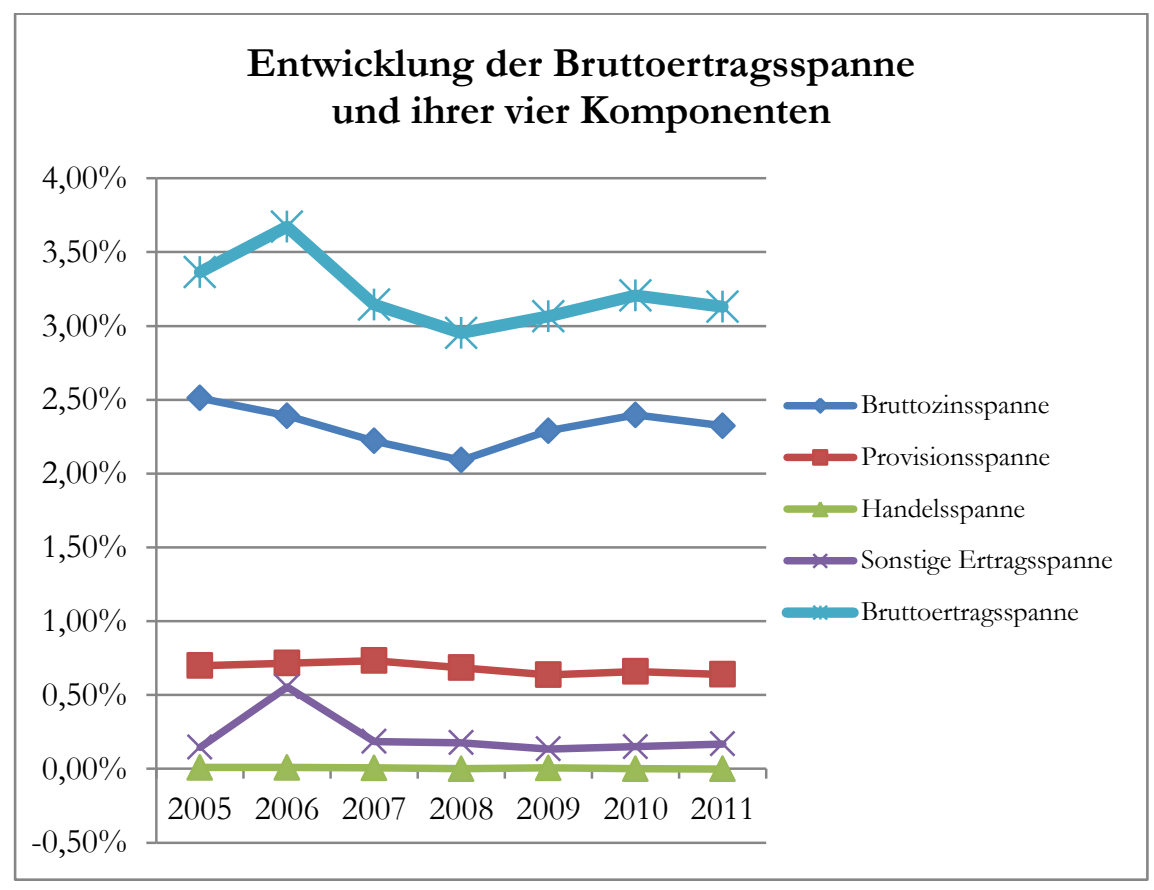

Abbildung 25: Entwicklung der Bruttoertragsspanne und ihrer vier Komponenten

Es wird deutlich, dass die Bruttozinsspanne in jedem Geschäftsjahr die bedeutendste Ertragsquelle darstellt. Die Werte dieser Spanne rangieren im Betrachtungszeitraum zwischen 2,09 und 2,51\%. Die zweite bedeutende Ertragsquelle ist die Provisionsspanne, die Werte zwischen 0,51 und 0,64\% annimmt. Von der Deutschen BundesBank werden diese Positionen als die „beiden tragenden Säulen und Stabilitätsfaktoren im Ertragsprofil““ bezeichnet. ${ }^{866}$ Die Handelsspanne und die sonstige Ertragsspanne spielen bei den Genos- 
senschaftsbanken eine untergeordnete Rolle. Tabelle 15 beleuchtet den prozentualen Beitrag der einzelnen Ertragspannen zur Bruttoertragsspanne.

\begin{tabular}{l|rrrrrrr} 
ROI-Komponenten & \multicolumn{1}{|c}{$\mathbf{2 0 0 5}$} & \multicolumn{1}{c}{$\mathbf{2 0 0 6}$} & \multicolumn{1}{c}{2007} & \multicolumn{1}{c}{$\mathbf{2 0 0 8}$} & \multicolumn{1}{c}{$\mathbf{2 0 0 9}$} & \multicolumn{1}{c}{$\mathbf{2 0 1 0}$} & \multicolumn{1}{c}{$\mathbf{2 0 1 1}$} \\
\hline Bruttozinsspanne & $74,6 \%$ & $65,1 \%$ & $70,6 \%$ & $70,8 \%$ & $74,7 \%$ & $74,7 \%$ & $74,2 \%$ \\
Provisionsspanne & $20,8 \%$ & $19,5 \%$ & $23,3 \%$ & $23,2 \%$ & $20,8 \%$ & $20,6 \%$ & $20,4 \%$ \\
Handelsspanne & $0,3 \%$ & $0,2 \%$ & $0,2 \%$ & $0,0 \%$ & $0,2 \%$ & $0,0 \%$ & $0,0 \%$ \\
Sonstige Ertragsspanne & $4,3 \%$ & $15,1 \%$ & $5,9 \%$ & $6,0 \%$ & $4,4 \%$ & $4,7 \%$ & $5,4 \%$ \\
\hline
\end{tabular}

Tabelle 15:

Entwicklung der Beiträge zur Bruttoertragsspanne

Auch hier wird die große Bedeutung der Zinsüberschüsse deutlich, die durch das Kerngeschäft der Kreditinstitute zum Erfolg beigesteuert werden.

\subsubsection{Hypothese 1: Bruttogewinnspanne}

Die Hypothese lautet:

Banken in ländlicben Regionen unterscheiden sich binsichtlich ibres Erfolges von Banken in urbanen Gebieten.

Als Messgröße für die Höhe des Erfolgs kommt hier der Bruttogewinn aus normaler Geschäftstätigkeit in Form der Bruttogewinnspanne zum Einsatz, welche einschließlich der Gründe für ihre Anwendung bereits in Abschnitt 3.5.3.1 beschrieben wurde.

Wie bereits in Abschnitt 4.3.3.5 erwähnt, können Fusionen zu Sondereffekten in der empirischen Untersuchung führen. Um mögliche Verzerrungen auszuschließen, werden Datensätze von Genossenschaftsbanken von der Berechnung ausgenommen, wenn im betreffenden Geschäftsjahr eine Fusion stattgefunden hat.

Der Boxplot in Abbildung 26 verdeutlicht die Unterschiede für alle Geschäftsjahre im Untersuchungszeitraum 2005 bis 2011 grafisch. Für jedes Jahr ist die Verteilung der Bruttogewinnspannen getrennt für die drei Lagebereiche dargestellt, um die Daten zunächst einzuordnen.

Jeweils alle Banken in städtischer, halbstädtischer und ländlicher Lage werden statistisch betrachtet. Zur Anwendung kommt eine einfaktorielle Varianzanalyse (ANOVA). ${ }^{867}$ Zunächst werden die Anwendungsvoraussetzungen hierfür geprüft:

1. Die Varianzen unterscheiden sich für nahezu alle Geschäftsjahre nicht signifikant. Der Levene-Test auf Varianzhomogenität findet lediglich für das Geschäftsjahr 2010 einen signifikanten Unterschied ${ }^{868}$ zwischen den einzelnen Gruppen hinsichtlich der Varianz. Jedoch sind die Stichprobenumfänge groß. Die Varianzanalyse ist daher trotzdem zulässig. ${ }^{869}$

2. Der Shapiro-Wilk-Test lehnt eine Normalverteilung nur für halbstädtisch in 2011 und $2010 \mathrm{ab}^{870}$ Für alle übrigen Jahre und Lagebereiche ist das Vorliegen der Normalverteilung gesichert.

\footnotetext{
${ }^{867}$ Vgl. Abschnitt 4.2.2.

${ }^{868} p=0,025$.

${ }^{869}$ Vgl. Abschnitt 4.2.2.

${ }^{870}$ Für $\alpha=0,05$.
} 


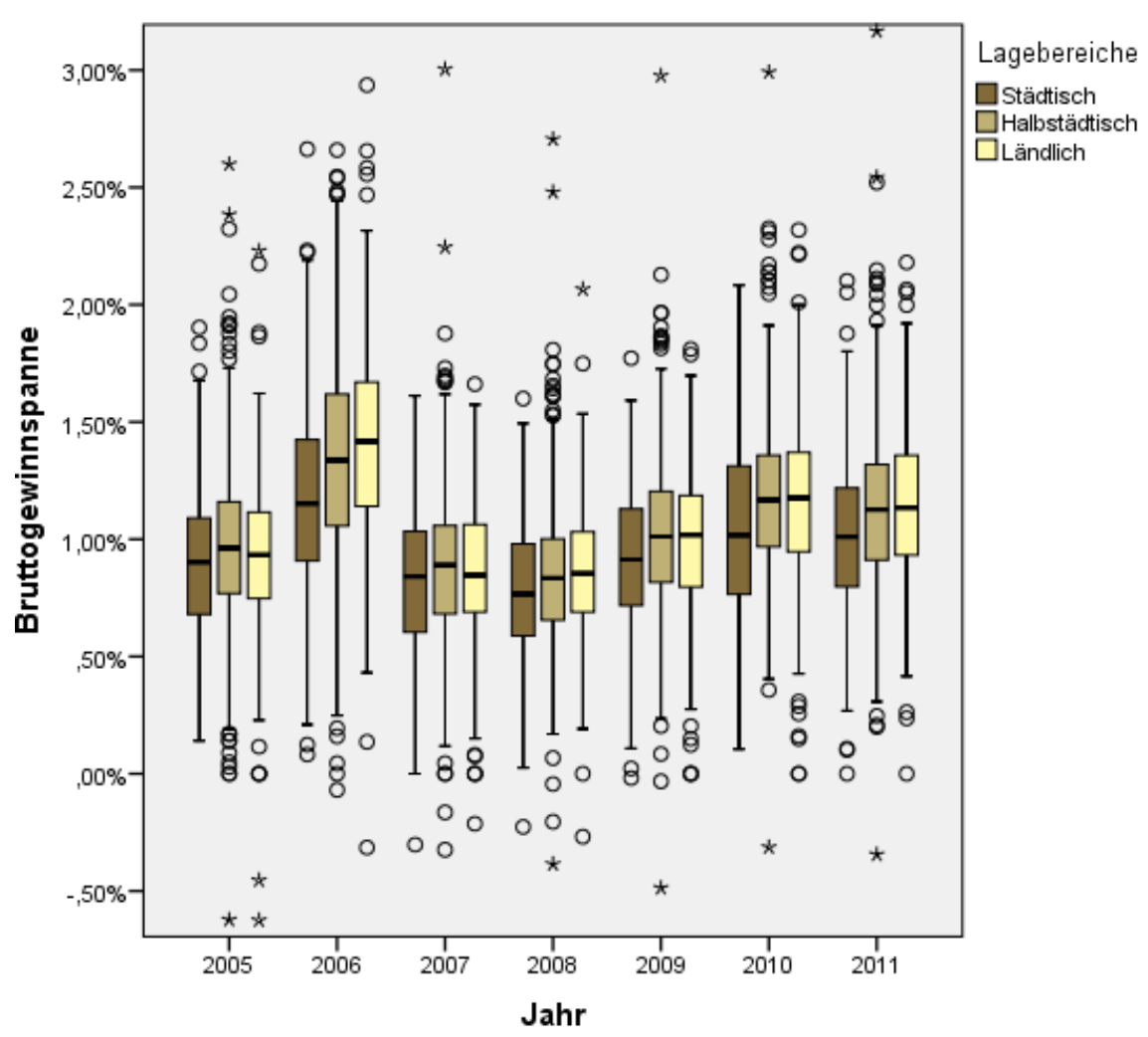

Abbildung 26: Verteilung der Bruttogewinnspanne nach Lagebereichen

Tabelle 16 enthält die Ergebnisse der empirischen Untersuchung. Auf Basis von ANOVA bzw. Welch-Test konnte gezeigt werden, dass signifikant unterschiedliche Mittelwerte zwischen den Lagebereichen vorliegen.

\begin{tabular}{|c|c|c|c|c|c|}
\hline & $\begin{array}{c}\text { Städtisch } \\
(\mathrm{n}=151-161)\end{array}$ & $\begin{array}{c}\text { Halbstädtisch } \\
(\mathrm{n}=518-580)\end{array}$ & $\begin{array}{c}\text { Ländlich } \\
(\mathrm{n}=260-298)\end{array}$ & $\begin{array}{c}\mathbf{F} \\
(\mathrm{ANOVA}) \\
\end{array}$ & $\begin{array}{c}\mathbf{F} \\
\text { (Welch) }\end{array}$ \\
\hline 2005 & $0,90 \%$ & $0,96 \%$ & $0,93 \%$ & 2,20 & \\
\hline 2006 & $1,16 \% \mathrm{o}^{\mathrm{a}}$ & $1,34 \%{ }^{\mathrm{b}}$ & $1,39 \%{ }^{b}$ & $15,64 * * *$ & \\
\hline 2007 & $0,83 \%$ & $0,88 \%$ & $0,86 \%$ & 1,39 & \\
\hline 2008 & $0,76 \% \mathrm{o}^{\mathrm{a}}$ & $0,84 \% \mathrm{ob}^{\mathrm{b}}$ & $0,84 \%{ }^{\mathrm{b}}$ & $4,05^{*}$ & \\
\hline 2009 & $0,92 \% \mathrm{o}^{\mathrm{a}}$ & $1,02 \%{ }^{b}$ & $1,00 \%{ }^{\mathrm{b}}$ & $5,79 * *$ & \\
\hline 2010 & $1,05 \% \mathrm{o}^{\mathrm{a}}$ & $1,17 \% \mathrm{o}^{\mathrm{b}}$ & $1,16 \%{ }^{\mathrm{b}}$ & & $6,55^{* *}$ \\
\hline 2011 & $1,02 \% \mathrm{a}^{\mathrm{a}}$ & $1,14 \% \mathrm{ob}^{\mathrm{b}}$ & $1,15 \% \mathrm{ob}^{\mathrm{b}}$ & $6,93 * *$ & \\
\hline
\end{tabular}

Im Jahr 2006 beispielsweise erzielten Banken in städtischer Lage im Mittel eine Bruttogewinnspanne von 1,16 \%. In halbstädtischer bzw. ländlicher Lage liegen mit 1,34 \% und 1,39\% bessere Werte vor. Auch in allen übrigen betrachteten Geschäftsjahren erzielen Banken in halbstädtischer oder ländlicher Lage im Mittel höhere Bruttogewinnspannen als Banken in städtischer Lage. Abgesehen von 2005 und 2007 konnte für alle betrachteten Geschäftsjahre ein signifikanter Unterschied zwischen den drei Lagebereichen Städtisch, Nichtstädtisch und Ländlich

${ }^{871}$ Darstellung nach Rossmann (2010), S. 5. 
gefunden werden. Bei der Interpretation der Werte von Tabelle 16 sollte besonderes Augenmerk auf die hochgestellten Kennbuchstaben der einzelnen Mittelwerte gelegt werden. Diese Kennbuchstaben kennzeichnen innerhalb eines Jahres die einzelnen Gruppen, welche sich untereinander signifikant auf dem $5 \%$-Niveau unterscheiden. Der Tukey-Posthoc-Test liefert schließlich ein einheitliches Bild: Wieder mit Ausnahme von 2005 und 2007 bestehen signifikante Unterschiede zwischen Banken in städtischer Lage ${ }^{872}$ einerseits und Banken in halbstädtischer und ländlicher Lage ${ }^{873}$ andererseits.

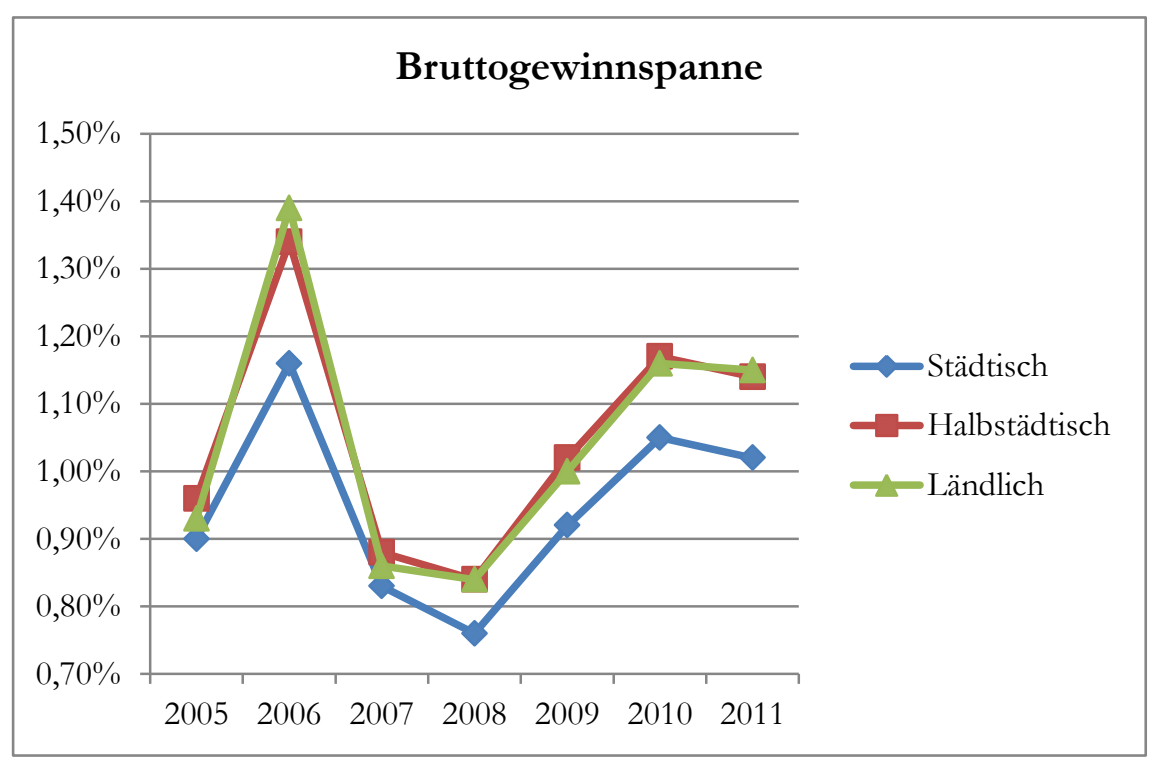

Abbildung 27: Entwicklung der Bruttogewinnspanne nach Lagebereichen

Zur Verdeutlichung der gezeigten Unterschiede stellt Abbildung 27 die Werte aus Tabelle 16 grafisch dar.

Dieser Hypothese liegt die Tatsache zu Grunde, dass es in ländlichen Regionen generell weniger Filialen unterschiedlicher Banken gibt. In kleinen Gemeinden sind regelmäßig nur Filialen von Genossenschaftsbanken und Sparkassen zu finden. Die meisten anderen national und international tätigen Banken beschränken sich bei der filialbasierten Markterschließung auf größere Städte. Dort finden sich häufig Filialen der Deutschen Bank, Commerzbank, HypoVereinsbank, Postbank und weiteren Banken. CHRISTIANS begründet dies damit, dass private Banken sich auf Gebiete mit den höchsten Erfolgsaussichten konzentrieren. ${ }^{874}$ Kreditgenossenschaften und Sparkassen bieten Bankleistungen, gemäß ihrem jeweiligen Auftrag, flächendeckend an. Durch die geringere Bankdiversität existiert im ländlichen Bereich folglich weniger Wettbewerb, daher können Banken dort im gleichen Geschäftsjahr höhere Margen erzielen als Banken in urbanen Ballungszentren. Wie bereits oben beschrieben, kann der relative Marktanteil als Erfolgsfaktor verstanden werden. ${ }^{875}$ Teilen sich - so wie im ländlichen Bereich - nur wenige Institute den Markt, so kann ein jedes von ihnen einen hohen relativen Marktanteil für sich beanspruchen. Neben dem Marktanteil an sich bietet die Wettbewerbslage weiteres Potenzial zur Durchsetzung vor-

872 In Tabelle 16 mit dem Kennbuchstaben a markiert.

${ }^{873}$ In Tabelle 16 mit dem Kennbuchstaben b markiert.

${ }^{874} \mathrm{Vgl}$. Abschnitt 2.1.2.

${ }^{875}$ Vgl. Abschnitt 3.1.3.2. 
teilhafter Konditionen. Beides wirkt sich positiv auf den Geschäftserfolg ländlicher Institute aus, welcher hier anhand der Bruttogewinnspanne gemessen wird. PEEMÖLLER sieht für Genossenschaften allgemein, und damit auch für Genossenschaftsbanken speziell, auch zukünftig beste Marktchancen. ${ }^{876}$ Er betont dabei Bereiche mit Marktversagen, in denen der Markt den Wirtschaftsteilnehmern keine geeigneten Angebote zur Verfügung stellt, und den ländlichen Raum. ${ }^{877}$

GÄRTNER gelang der Nachweis einer ähnlichen Gesetzmäßigkeit in Bezug auf Sparkassen. Konkret wies er einen umso größeren Erfolg nach, je schlechter die regionalwirtschaftliche Situation war. ${ }^{878}$

Die hohe Wettbewerbsintensität wird auch von SPATH, BAUER UND ENGSTLER angeführt. Als Gründe nennen sie die hohe Anbieterdichte im deutschen Markt einerseits und die Expansionsbestrebungen von Direktbanken andererseits. ${ }^{879}$ Diese Aussage über die gesamte deutsche Bankenlandschaft, verknüpft mit der Tatsache einer überdurchschnittlichen Anbieterdiversität in Städten, untermauert die Wettbewerbsthese argumentativ. STRAUß leitet aus einer hohen Anzahl von Hauptstellen der Wettbewerber im Geschäftsgebiet einen negativen Einfluss auf das Erfolgsmaß der Institute her. ${ }^{880}$ CHRISTIANS sieht zusätzlich den Konditionenwettbewerb mit den Direktbanken als Grund für zukünftig geringere Margen. ${ }^{881}$ Dies trifft neben den Genossenschaftsbanken auch auf die Sparkassen und die Kreditbanken zu.

Nach 2007 ist in Tabelle 16 eine Phase beobachtbar, in der sich das Signifikanzniveau des Unterschiedstests wieder schrittweise erhöht, d. h, die Unterschiede werden signifikanter. Hier wird ein Zusammenhang zur Banken- und Finanzkrise vermutet. ${ }^{82} 2007$ fiel es den Banken sowohl in städtischer als auch in nichtstädtischer Lage schwer, eine hohe Bruttogewinnspanne zu erzielen. Absolute Unterschiede sind zwar erkennbar, jedoch fallen diese nicht so deutlich aus wie in den Vergleichsjahren.

Den städtischen Banken kann jedoch aufgrund dieses Ergebnisses nicht generell attestiert werden, dass sie eine geringere Effizienz hätten. WUTZ begründet dies damit, dass Banken bei hohem Wettbewerb etwa ein breites Serviceangebot sichern müssten oder sich dem grundsätzlich teuren Ausbau bzw. der Verteidigung von Marktanteilen widmeten. ${ }^{883}$

Es kann davon ausgegangen werden, dass es sich bei den Messreihen um Vollerhebungen handelt. Daher ist die Beurteilung nicht von einem signifikanten Ergebnis abhängig. Selbst der deskriptive Bezug auf die Mittelwerte ist bereits aussagekräftig. ${ }^{884}$ Dies gilt auch für alle folgenden Hypothesentests.

Um im Detail zu untersuchen, warum genau Institute in ländlicher Lage Vorteile bei der Bruttogewinnspanne haben, folgt nun die Betrachtung der wichtigsten Subkomponenten, aus denen sie sich errechnet, jeweils mit Verweis auf die GuV-Position. Hierzu zählen:

${ }^{876}$ Vgl. Peemöller (2005), S. 422.

877 Vgl. Peemöller (2005), S. 422.

${ }^{878}$ Vgl. Gärtner (2010), S. 217.

${ }^{879}$ Vgl. Spath, Bauer und Engstler (2008), S. 5.

${ }^{880}$ Vgl. Strauß (2006), S. 216.

881 Vgl. Christians (2010), S. 145

${ }^{882}$ Vgl. Bundesbank (2013a).

883 Vgl. Wutz (2002), S. 14.

${ }^{884}$ Vgl. Abschnitt 4.3.3.2. 
- 1. a) Zinserträge aus Kredit- und Geldmarktgeschäften

- 1. b) Zinserträge aus festverzinslichen Wertpapieren und Schuldbuchforderungen

- 2. Zinsaufwendungen

- 3. Laufende Erträge

- Zinsüberschüsse als Saldo aus 1. a), 1. b), 2. und 3.

- Provisionsüberschüsse als Saldo aus 5. und 6.

- 10. a) Personalaufwand

- 10. b) Sachaufwand

Zusätzlich wird folgende Komponente beleuchtet:

- Abschreibungen auf Forderungen von Banken (Saldo aus 13. und 14.)

\subsubsection{Hypothese 2: Zinserträge aus Kredit- und Geldmarktgeschäften}

Die Hypothese lautet:

Banken in ländlichen Regionen unterscbeiden sich binsichtlich ibrer Zinserträge aus Kredit- und Geldmarktgeschäften von Banken in urbanen Gebieten. Unterschiede bestehen weiter im Anteil risikoreicher Kundenforderungen.

Für alle Geschäftsjahre kann anhand einer Varianzanalyse gezeigt werden, dass Banken in städtischer Lage stets die niedrigsten Zinserträge gemäß Position 1. a) der Gewinn- und Verlustrechnung erzielten. Hingegen weisen die Abschlüsse der ländlichen Banken in fast allen Fällen die höchsten Zinserträge dieser Kategorie auf. Wie in Tabelle 17 dargestellt, ergeben sich für nahezu alle Geschäftsjahre signifikante Unterschiede. Lediglich für 2008 und 2011 konnte keine Signifikanz für die bestehenden Unterschiede nachgewiesen werden.

\begin{tabular}{|c|c|c|c|c|c|}
\hline & $\begin{array}{c}\text { Städtisch } \\
(\mathrm{n}=151-157)\end{array}$ & $\begin{array}{c}\text { Halbstädtisch } \\
(\mathrm{n}=512-580)\end{array}$ & $\begin{array}{c}\text { Ländlich } \\
(\mathrm{n}=259-300)\end{array}$ & $\begin{array}{c}\mathbf{F} \\
(\mathrm{ANOVA}) \\
\end{array}$ & $\begin{array}{c}\mathbf{F} \\
\text { (Welch) }\end{array}$ \\
\hline 2005 & $3,79 \% 0^{a}$ & $4,01 \%{ }^{\mathrm{b}}$ & $3,97 \%$ b & & $6,07 * * *$ \\
\hline 2006 & $3,73 \% \mathrm{a}^{\mathrm{a}}$ & $3,92 \%{ }^{\mathrm{b}}$ & $3,91 \% \mathrm{~b}$ & & $4,76^{* *}$ \\
\hline 2007 & $3,79 \% \mathrm{a}^{\mathrm{a}}$ & $3,96 \% \mathrm{o}^{\mathrm{b}}$ & $3,97 \% \mathrm{~b}$ & $5,03^{* *}$ & \\
\hline 2008 & $3,89 \% \mathrm{a}^{\mathrm{a}}$ & $4,02 \% \mathrm{~b}^{\mathrm{b}}$ & $4,01 \% \mathrm{o}^{\mathrm{b}}$ & & 2,64 \\
\hline 2009 & $3,47 \% \mathrm{o}^{\mathrm{a}}$ & $3,61 \%{ }^{\mathrm{b}}$ & $3,63 \%$ b & & $3,42^{*}$ \\
\hline 2010 & $3,17 \% \mathrm{o}^{\mathrm{a}}$ & $3,29 \% \mathrm{ob}^{\mathrm{b}}$ & $3,31 \% \mathrm{~b}$ & & $3,48^{*}$ \\
\hline 2011 & $3,06 \%$ & $3,15 \%$ & $3,16 \%$ & & 1,97 \\
\hline \multicolumn{6}{|c|}{$\begin{array}{c}\text { Basis: } \mathrm{n}=923-1037 \text {, einfaktorielle Varianzanalyse bzw. Welch-Test (Post-Hoc-Test: Tukey-HSD } \\
\text { ***, **, }{ }^{*} \text { signifikant auf dem 0,1 \%-, } 1 \% \text { - bzw. } 5 \% \text {-Niveau. Gruppen mit unterschiedlichen } \\
\text { Kennbuchstaben }(\mathrm{a}, \mathrm{b}) \text { unterscheiden sich signifikant auf dem } 5 \% \text {-Niveau. }\end{array}$} \\
\hline \multicolumn{2}{|c|}{ Tabelle 17: } & \multicolumn{4}{|c|}{$\begin{array}{l}\text { Entwicklung der Zinserträge aus Kredit- und } \\
\text { Geldmarktgeschäften nach Lagebereichen }\end{array}$} \\
\hline
\end{tabular}

Eine grafische Darstellung der Testresultate findet sich in Abbildung 28. Der starke Einbruch der Zinserträge nach 2008 ist auf die zugrundeliegende volkswirtschaftliche Entwicklung zurückzuführen. Immerhin senkte die Europäische Zentralbank den Hauptrefinanzierungssatz von 4,25\% im Herbst 2008 schrittweise bis auf 1,00 \% im Frühjahr 2009 ab. 


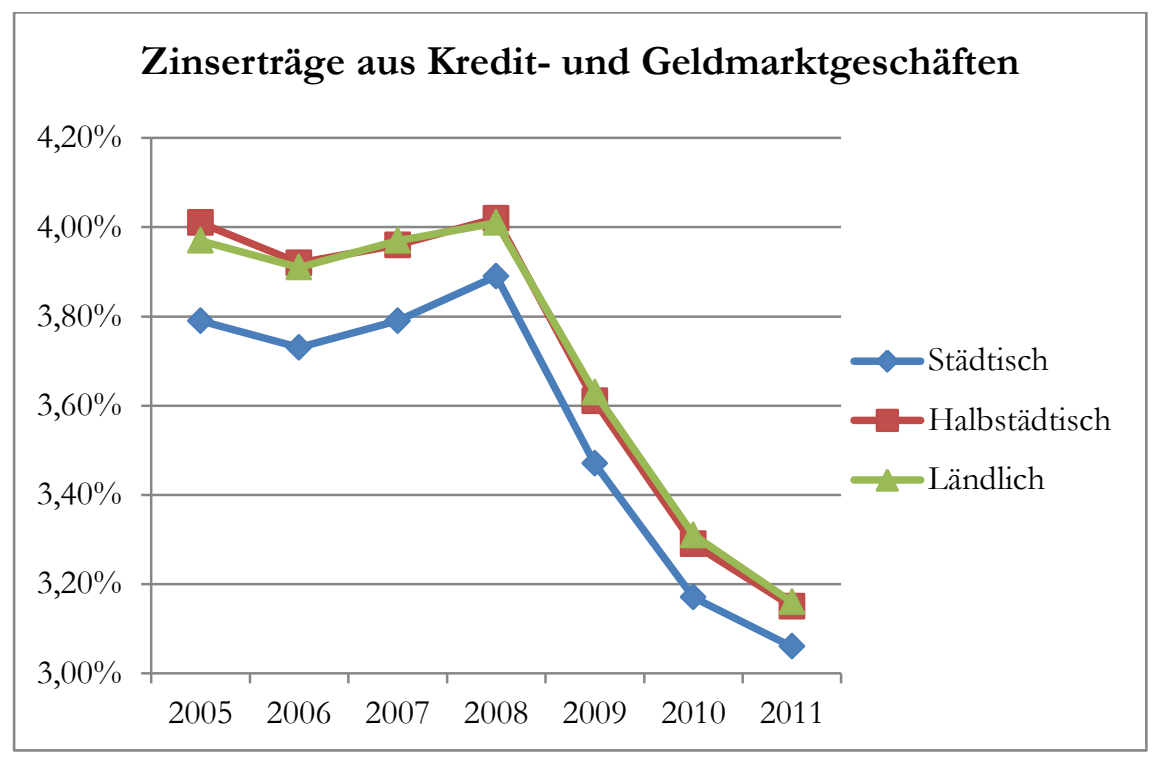

Abbildung 28: Entwicklung der Zinserträge aus Kredit- und Geldmarktgeschäften nach Lagebereichen

Zur Quantifizierung der Abweichungshöhe zwischen den einzelnen Lagebereichen wurde die lineare OLS-Regression durchgeführt. ${ }^{85}$ In diesem Pooled-Verfahren werden die Werte aller sieben betrachteten Geschäftsjahre gleichzeitig regressiert. Die Ergebnisse sind in Tabelle 18 dargestellt.

\begin{tabular}{|c|c|}
\hline VARIABLE & $\begin{array}{c}(2) \\
\text { ze1s }\end{array}$ \\
\hline st & $\begin{array}{c}-0,155^{* * *} \\
(0,0252)\end{array}$ \\
\hline ha & $\begin{array}{c}-0,00342 \\
(0,0185)\end{array}$ \\
\hline o.la & - \\
\hline Konstante & $\begin{array}{c}3,724 * * * \\
(0,0150)\end{array}$ \\
\hline $\begin{array}{l}\text { Beobachtungen } \\
\mathrm{R}^{2}\end{array}$ & $\begin{array}{l}7.087 \\
0,007\end{array}$ \\
\hline
\end{tabular}

Tabelle 18: $\quad$ Einfluss der Lage auf die Zinserträge aus Kredit- und Geldmarktgeschäften (OLS)

Die OLS-Regression bestätigt die Ergebnisse der Varianzanalyse. Die Variable la für den ländlichen Lagebereich wurde aufgrund von Kollinearität übersprungen und bildet gleichzeitig die Basislinie dieser Regression. Bei städtischen Banken liegt die Spanne der Zinserträge aus Kredit- und Geldmarktgeschäften im Mittel 15,5 Basispunkte bzw. 0,155 Prozentpunkte unter diesem Niveau, wie anhand des Koeffizienten für st gezeigt werden kann. Dies deckt sich mit der grafischen Darstellung in Abbildung 28. Die Höhe des Koeffizienten für die halbstädtische Lage $h a$ ist hingegen nicht signifikant von null verschieden und liegt auf etwa 
demselben Niveau wie bei Banken in ländlicher Lage. Es kann wieder anhand von Abbildung 28 nachvollzogen werden, dass die Werte für die halbstädtische Lage nahezu deckungsgleich zu den Werten der ländlichen Lage liegen. Das Bestimmtheitsmaß $R^{2}$ fällt in derartigen Partialanalysen stets sehr gering aus. Durch den Einbezug aller möglichen Variablen lässt sich prinzipiell eine Steigerung von $R^{2}$ erreichen. Jedoch kommt es dadurch auch zu einer Verzerrung der Koeffizienten für $s t, b a$ und $l a$, weswegen im Rahmen dieser und der folgenden Partialanalysen hierauf verzichtet wird.

Wie bereits oben beschrieben ${ }^{886}$, enthält der hier untersuchte Unterposten a) die Zinserträge aus folgenden Bilanzpositionen.

- Barreserve: Der Kassenbestand wird ebenso wenig verzinst wie die Guthaben bei Zentralnotenbanken zur Gewährleistung der Mindestreserve. ${ }^{877}$ Guthaben bei Postgiroämtern sind ungebräuchlich, deren Ausweis kommt nicht mehr zu Anwendung. ${ }^{888}$

- Schuldtitel öffentlicher Stellen und Wechsel: Die Bilanzbestände in dieser Position sind bei den untersuchten Genossenschaftsbanken im untersuchten Zeitraum in der Regel Null, weshalb hieraus keine nennenswerten Zinserträge zu erwarten sind.

- Forderungen an Kreditinstitute

- Forderungen an Kunden

- Geldmarktpapiere ${ }^{889}$

Es soll nun für die Gesamtheit aller Banken untersucht werden, welcher Teil der erklärten Zinserträge aus welchem Aktivgeschäft herrührt. Zu diesem Zwecke werden stellvertretend am Beispiel des Geschäftsjahres 2011 die Größenordnungen der betreffenden Aktivpositionen analysiert. ${ }^{890}$ Hierfür wird zunächst die aggregierte Bilanzsumme aller untersuchten Banken gebildet. Im nächsten Schritt erfolgte die Aggregierung der einzelnen Aktivpositionen über alle Banken. Durch Bildung von Gliederungszahlen ${ }^{891}$ kann schließlich der Anteil jeder einzelnen Position bestimmt werden.

Die Resultate dieser Betrachtung sind in Abbildung 29 grafisch dargestellt. Die Barreserven machen lediglich 2,2\% der aggregierten Bilanzsumme aus. Sie setzen sich zusammen aus dem Kassenbestand, dem Guthaben bei Zentralnotenbanken und Guthaben bei Postgiroämtern. ${ }^{892}$ Keine dieser Positionen wird verzinst, daher ist hier keine Kontribution zu den Zinserträgen zu erwarten.

Auf die Forderungen an Kreditinstitute entfallen immerhin 11,6 \% der aggregierten Bilanzsumme. ${ }^{893}$ Jedoch ist hier aufgrund der Marktgegebenheiten damit zu rechnen, dass die Banken durch die Vergabe von Krediten an andere Banken keine nennenswerten Beiträge zu dem gesamten Zinsüberschuss dieser Position 1. a) erwirtschaften. Dies ist auf sehr niedrige Zinssätze im Interbankengeschäft zurückzuführen. Des Weiteren wird auf das

886 Vgl. Abschnitt 3.6.3.1.

887 Vgl. Abschnitt 3.6.3.1.

888 Vgl. Hartmann-Wendels, Weber und Pfingsten (2007), S. 763.

889 Anm.: Von den untersuchten Genossenschaftsbanken wurden nur sporadisch Geldmarktpapiere in der Bilanzposition 5. a) ausgewiesen. Quasi das gesamte Aufkommen der Aktivposition 5 rührt aus der Unterposition 5. b) her.

${ }^{890}$ Dieses Jahr wurde gewählt, weil es sich um das jüngste der Betrachtungsperiode handelt.

891 Vgl. Abschnitt 3.5.1.

892 Vgl. BaFin (2012a), S. 1.

${ }^{893}$ Vgl. Hartmann-Wendels et al. (2007), S. 757. 
möglicherweise profitablere Geschäft mit Banken in geringeren Ratingkategorien per se verzichtet.

Die Forderungen an Kunden sind die bedeutendste Position mit einem Anteil von 57,7 \%. Sie können weiter in durch Grundpfandrechte gesicherte Forderungen (26,7 \%), Kommunalkredite $(1,5 \%)$ und übrige Kredite $(29,6 \%)$ unterteilt werden.

Aus dieser Analyse folgt, dass grundpfandrechtlich gesicherte Kredite sowie übrige Kredite die bedeutendsten Treiber des Zinsertrags aus Kredit- und Geldmarktgeschäften sind.

Durch Grundpfandrechte gesicherte Darlehen umfassen häufig Immobilienkredite, aber auch Finanzierungen in der Landwirtschaft sowie Hypothekenpfandbriefe (Covered Bonds).

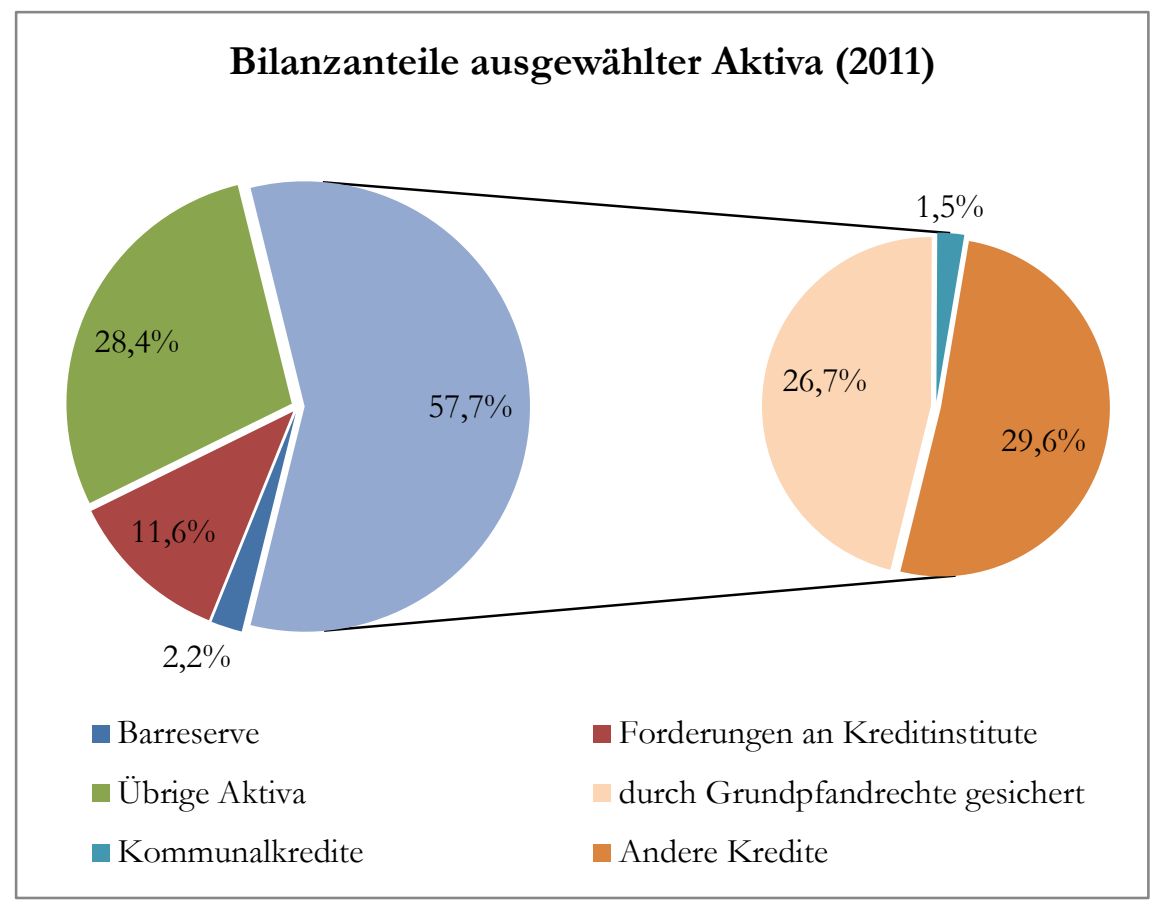

Abbildung 29: Bilanzanteile ausgewählter Aktiva über alle analysierten Genossenschaftsbanken (2011)

Kommunalkredite sind in der Regel Schuldscheindarlehen, welche an die einzelnen Gliederungsebenen der Gebietskörperschaften in Deutschland unterhalb der Bundesebene vergeben werden. Kreditnehmer sind alle öffentlichen Gliederungsebenen unterhalb des Bundes, das heißt die Bundesländer, Landkreise, Städte und Kommunen. Trotzdem weisen Kommunalkredite einen sehr geringen Anteil am Gesamtkreditportfolio auf. Dies ist darauf zurückzuführen, dass vorrangig die Sparkassen die Finanzierung der einzelnen Gebietskörperschaften übernehmen.

Wie aus Abbildung 28 hervorgeht, erzielten Institute in städtischer Lage in jedem Geschäftsjahr niedrigere Erträge als solche in den übrigen Lagebereichen. Kausal ist hier wieder auf das Wettbewerbsargument abzustellen, welches bereits erläutert wurde. ${ }^{894}$ Für Immobilien- 
kredite und übrige Kredite befinden sich die Institute in städtischer Lage in einem stärkeren Wettbewerb als ländliche Banken.

Die beiden eben genannten speziellen Kreditarten, durch Grundpfandrechte gesicherte Kredite und Kommunalkredite, sind nur mit einem geringen Kredit- bzw. Ausfallrisiko behaftet. ${ }^{895}$ Andere Kredite, wie z. B. Konsumentendarlehen und Firmenkredite, weisen hingegen ein höheres Risiko auf. Dies führt zu nachweisbaren Unterschieden zwischen den einzelnen Lagebereichen. Die Abbildung 30 stellt die Entwicklung der Anteile dieser risikoreichen Kundenforderungen dar, aufgegliedert nach städtischer, halbstädtischer und ländlicher Lage.

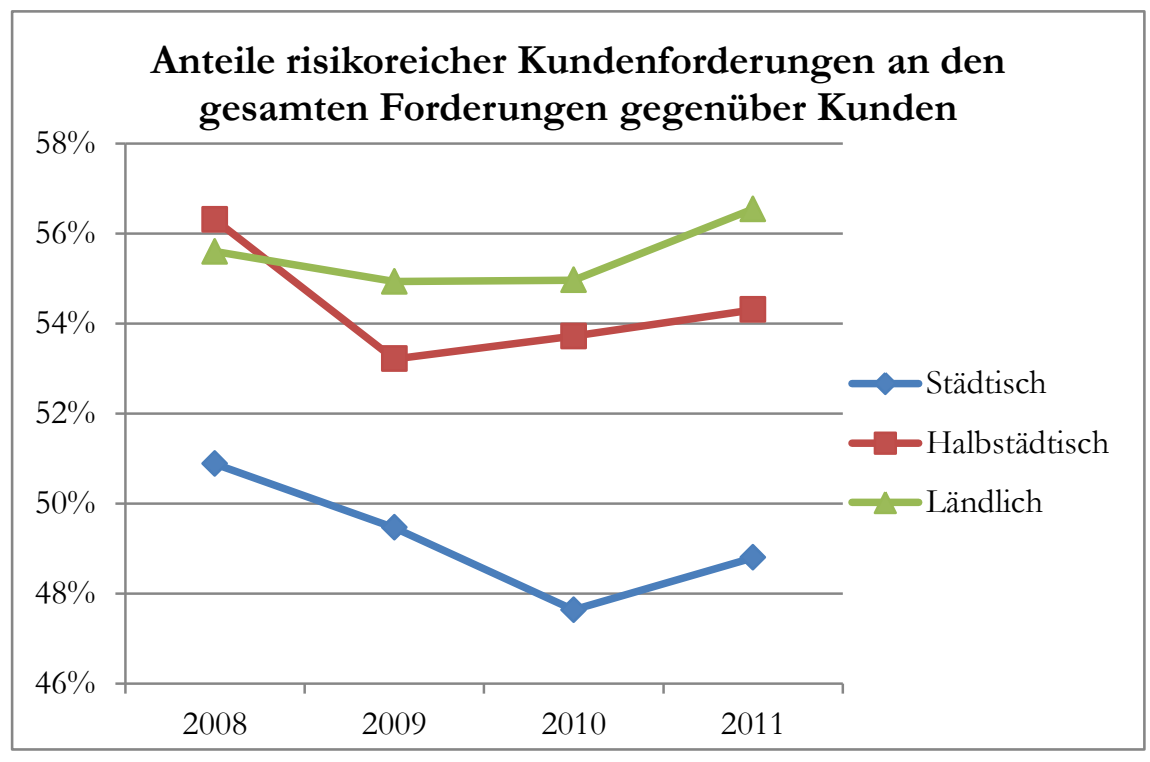

Abbildung 30: Entwicklung der Anteile risikoreicher Kundenforderungen nach Lagebereichen

Es wird sichtbar, dass halbstädtische Banken im Mittel einen um 5,2 Prozentpunkte höheren Anteil an risikoreichen Kundenforderungen aufweisen als städtische Banken. Ländliche Banken liegen noch weitere 1,1 Prozentpunkte darüber. Dies ist darauf zurückzuführen, dass das besicherte Kreditgeschäft mit Wohnimmobilien in den Städten stärker ausgeprägt ist. Es beinhaltet dort neben Einfamilienhäusern vor allem auch Eigentumswohnungen, deren Finanzierungsvolumen pro Objekt häufig in der Nähe ganzer Einfamilienhäuser rangiert. Zudem kann davon ausgegangen werden, dass die Umschichtungsfrequenz im städtischen Bereich höher liegt. Städtische Genossenschaftsbanken weisen daher häufig einen hohen Immobilienanteil auf, der durch die Besicherung durch Grundstücke geringere Risiken trägt. Die risikoreichen Anteile, beispielsweise Konsumentenkredite oder Firmenkredite zur Finanzierung von Kleinunternehmern, verwässern bei dem hohen Immobilienanteil städtischer Institute. Eine separate Betrachtung von Privat- und Firmenkundengeschäft ist auf Basis der vorliegenden Daten nicht möglich. Da hier noch aufschlussreiche Ergebnisse zu erwarten sind, besteht hier noch Raum für weitere Forschungen auf Grundlage eines entsprechenden Datensatzes.

Bezüglich der Anteile risikoreicher Kundenforderungen (Abbildung 30) ist nach dem Jahr 2008 in allen drei Lagebereichen, in Folge der Finanzkrise, ein Rückgang zu verzeichnen. In 
Städten und im halbstädtischen Bereich kann hier zudem ein deutlich stärkerer Rückgang nachgewiesen werden als im ländlichen Bereich, wo die Werte nahezu konstant blieben. Die Konsumentendarlehen und Firmenkredite von städtischen Banken reagieren - durch den höheren Einfluss der Marktteilnehmer - wesentlich sensibler.

Wie bereits aus Abbildung 28 hervorgeht, weisen halbstädtische und ländliche Institute einen höheren Zinsüberschuss aus als städtische Institute. Es ist davon auszugehen, dass zumindest ein Teil dieses Mehrertrags auf den höheren Anteil risikoreicher Kundenforderungen zurückzuführen ist. Denn bei Verträgen mit höheren Risiken lassen sich, zusätzlich zu den Refinanzierungs- und Kreditrisikokosten, auch höhere Margen generieren. Kreditinstitute haben zumindest generell die Möglichkeit, Darlehen risikoadäquat zu bepreisen.

\subsubsection{Hypothese 3: Zinserträge aus festverzinslichen Wertpapieren und Schuld- buchforderungen}

Die Hypothese lautet:

Banken in ländlichen Regionen unterscheiden sich binsicbtlich ibrer Zinserträge aus festverzinslichen Wertpapieren und Schuldbuchforderungen von Banken in urbanen Gebieten.

Neben 1. a) Zinserträgen aus Kredit- und Geldmarktgeschäften unterscheidet die Gewinn- und Verlustrechnung bei Zinserträgen noch in 1. b) Zinserträge aus festverzinslichen Wertpapieren und Schuldbuchforderungen. Als Untersuchungsmethode zur Aufweckung der Unterschiede zwischen städtischen, halbstädtischen und ländlichen Regionen wird wieder die Varianzanalyse gewählt, die Darstellung der Ergebnisse erfolgt in Tabelle 19.

\begin{tabular}{|c|c|c|c|c|c|}
\hline & $\begin{array}{c}\text { Städtisch } \\
(\mathrm{n}=146-156)\end{array}$ & $\begin{array}{c}\text { Halbstädtisch } \\
(\mathrm{n}=518-555)\end{array}$ & $\begin{array}{c}\text { Ländlich } \\
(\mathrm{n}=260-293)\end{array}$ & $\begin{array}{c}\mathbf{F} \\
\text { (ANOVA) } \\
\end{array}$ & $\begin{array}{c}\mathbf{F} \\
\text { (Welch) } \\
\end{array}$ \\
\hline 2005 & $0,67 \%$ & $0,69 \% \mathrm{a}^{\mathrm{a}}$ & $0,76 \% \mathrm{o}^{\mathrm{b}}$ & & $4,05^{*}$ \\
\hline 2006 & $0,66 \%$ & $0,66 \%{ }^{a}$ & $0,72 \%$ b & & $3,07 *$ \\
\hline 2007 & $0,70 \%$ & $0,69 \%$ & $0,74 \%$ & & 1,05 \\
\hline 2008 & $0,80 \%$ & $0,88 \%$ & $0,86 \%$ & 0,81 & \\
\hline 2009 & $0,80 \% 0^{a}$ & $0,86 \%$ & $0,96 \% 0^{\mathrm{b}}$ & & $5,79 * *$ \\
\hline 2010 & $0,73 \% \mathrm{o}^{\mathrm{a}}$ & $0,83 \%$ & $0,95 \%$ b & & $11,30 * * *$ \\
\hline 2011 & $0,70 \% 0^{\mathrm{a}}$ & $0,80 \%$ & $0,88 \%{ }^{\mathrm{b}}$ & $5,74^{* *}$ & \\
\hline
\end{tabular}

Tabelle 19: $\quad$ Entwicklung der Zinserträge aus festverzinslichen Wertpapieren und Schuldbuchforderungen nach Lagebereichen

Insgesamt lässt sich auch bei festverzinslichen Wertpapieren und Schuldbuchforderungen ein Gefälle von ländlich über halbstädtisch zu städtisch nachweisen. Die größten Erträge in dieser GuVPosition liefern in der Regel Institute in ländlicher Lage, die geringsten Erträge werden von Kreditgenossenschaften in städtischer Lage erwirtschaftet. Eine grafische Darstellung findet sich in Abbildung 31. 


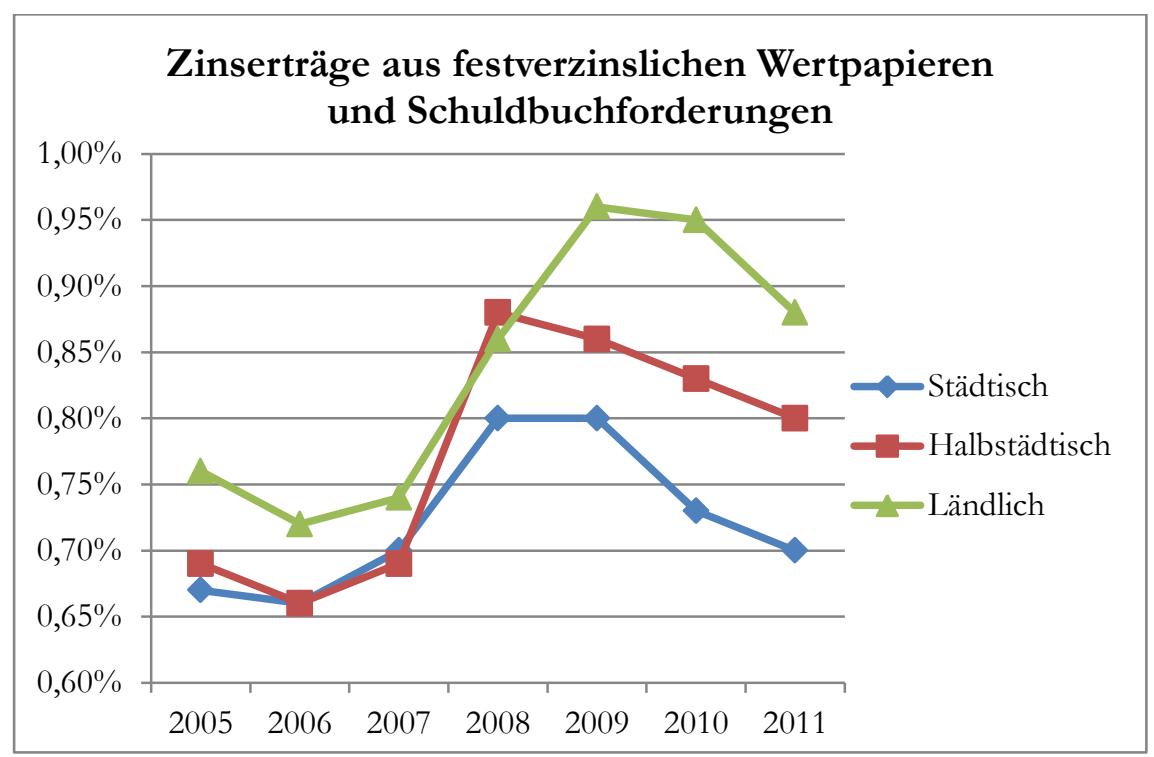

Abbildung 31: Entwicklung der Zinserträge aus festverzinslichen Wertpapieren und Schuldbuchforderungen nach Lagebereichen

Die Ergebnisse einer OLS-Regression in Tabelle 20 zeigen, wie ha um 5,13 Basispunkte (0,0513 Prozentpunkte) und la um 11,60 Basispunkte (0,1160 Prozentpunkte) jeweils im Mittel nach oben von der Basislinie st abweichen. Dies deckt sich mit der Darstellung in Abbildung 31 .

Bevor hier als Begründung erneut das Wettbewerbsargument in Betracht kommt, soll die Herkunft der Erträge näher beleuchtet werden. Wie bereits oben erläutert ${ }^{896}$, werden in dieser GuV-Position 1. b) die Zinserträge aus börsenfähigen Schuldtiteln wie Anleihen, Schuldverschreibungen, Pfandbriefen, Obligationen, Nullkuponanleihen etc. erfasst. Fraglich ist, warum es ländlichen Instituten im Mittel in allen Jahren gelingt, ein besseres Ergebnis als städtische Institute zu erzielen. Alle Institute sollten schließlich über denselben Marktzugang zu den genannten Anlageobjekten verfügen.

\begin{tabular}{lc}
\hline \multirow{2}{*}{ VARIABLE } & $(3)$ \\
ze2s
\end{tabular}

Tabelle 20: $\quad$ Einfluss der Lage auf die Zinserträge aus festverzinslichen Wertpapieren und Schuldbuchforderungen (OLS) 
Zunächst sind bei der Mittelherkunft sowohl städtische als auch ländliche Genossenschaftsbanken auskömmlich mit günstigen Einlagen versorgt. ${ }^{897}$ Jedoch verfügen ländliche Regionen, vor allem in den neuen Bundesländern, häufig nur über eine sehr dünne Gewerbeinfrastruktur. Dies hat zweierlei Folgen: Einerseits sind durch einen Mangel an Betrieben die Möglichkeiten zur Vergabe von Firmenkrediten begrenzt. Andererseits ist auch das nachgefragte Volumen privater Kredite limitiert, da im Geschäftsgebiet der Kreditgenossenschaft speziell eingeschränkte Beschäftigungsmöglichkeiten für Arbeitnehmer bestehen, sowie allgemein eine geringe Bevölkerungsdichte. Die Unterschiede im Bruttoinlandsprodukt je Einwohner zwischen den Lagekategorien städtisch, halbstädtisch und ländlich sind enorm. ${ }^{898}$ Aus beiden Gründen verfügen die ländlichen Regionalbanken über begrenzte Möglichkeiten auf der Seite der Mittelverwendung. An dieser Stelle kommt das Eigengeschäft zum Tragen. Dieses ist zwar nicht gleichsam lukrativ wie eine Kreditvergabe an Firmen- oder Privatkunden, aber dennoch eine lohnende Ertragsquelle. In der Gewinn- und Verlustrechnung werden die Erträge in diesem Posten 1. b) erfasst, daher fällt dieser bei den ländlichen Banken überdurchschnittlich aus.

Die Entwicklung der Zinserträge aus festverzinslichen Wertpapieren und Schuldbuchforderungen im Verlauf über den Betrachtungszeitraum ist in Abbildung 32 dargestellt. Auf eine Differenzierung nach den Lagebereichen städtisch, halbstädtisch und ländlich wird verzichtet, um einen aggregierten Überblick zu erhalten.

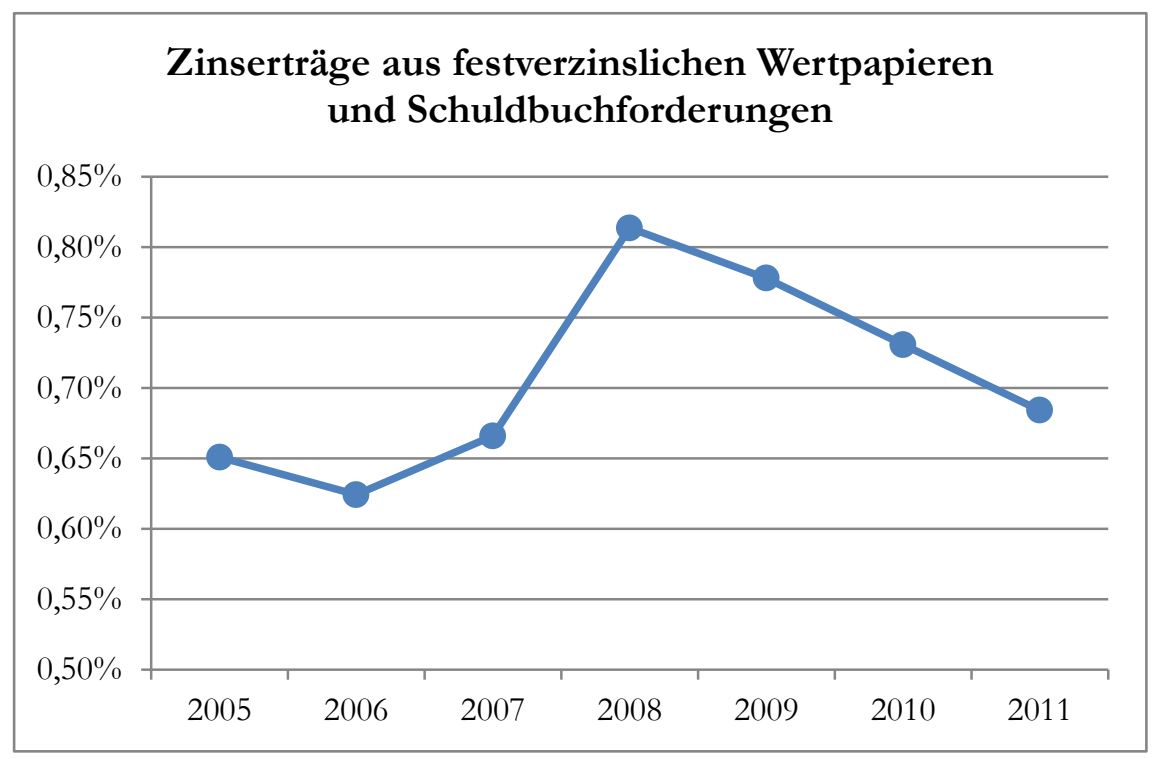

Abbildung 32: Entwicklung der Zinserträge aus festverzinslichen Wertpapieren und Schuldbuchforderungen aller Banken

Die hier untersuchten Zinserträge unterliegen primär einer Abhängigkeit vom Hauptrefinanzierungssatz der Europäischen Zentralbank. Nachdem dieser Zinssatz in der Eurozone von Mitte 2003 bis Ende 2005 bei 2,00 \% lag, kam es im Anschluss bis Mitte 2008 zu einer Anhebung bis auf 4,25\%. Auslaufende festverzinsliche Wertpapiere der Bilanzaktivposition 5. ba) und 5. bb) wurden in dieser Zeit durch höherverzinsliche Papiere mit idealerweise 
längerer Laufzeit ersetzt. Ende 2008 darauf bis Mitte 2009 erfolgte eine rasche Absenkung bis hinunter auf 1,00\%. Im Bestand befindliche höherverzinsliche Papiere lieferten auch in den Jahren nach 2008 noch einen guten Ertrag, jedoch reduzierte sich deren Anteil im Zeitablauf aufgrund des Auslaufens, weswegen wieder in Papiere zum dann gültigen, niedrigeren Zinssatz investiert wurde.

\subsubsection{Hypothese 4: Zinsaufwand}

Die Hypothese lautet:

Banken in ländlicben Regionen unterscheiden sich binsichtlich ibrer Zinsaufwendungen von Banken in urbanen Gebieten.

Zur Untersuchung dieser Hypothese wird wieder die Varianzanalyse eingesetzt. Diese vergleicht die Zinsaufwendungen anhand der Zinsaufwandsspanne über die drei Lagebereiche anhand der jeweiligen Mittelwerte und Jahre. Die Ergebnisse sind in Tabelle 21 dargestellt.

\begin{tabular}{|c|c|c|c|c|}
\hline & $\begin{array}{c}\text { Städtisch } \\
(\mathrm{n}=151-163)\end{array}$ & $\begin{array}{l}\text { Halbstädtisch } \\
(\mathrm{n}=517-576)\end{array}$ & $\begin{array}{c}\text { Ländlich } \\
(\mathrm{n}=260-299)\end{array}$ & $\begin{array}{c}\mathbf{F} \\
\text { (Welch) }\end{array}$ \\
\hline 2005 & $2,23 \% \mathrm{o}^{\mathrm{a}}$ & $2,09 \% \mathrm{~b}^{\mathrm{b}}$ & $2,06 \%{ }^{b}$ & $11,67 * * *$ \\
\hline 2006 & $2,27 \% \mathrm{a}^{\mathrm{a}}$ & $2,11 \% \mathrm{~b}^{\mathrm{b}}$ & $2,06 \% 0^{c}$ & $18,06^{* * *}$ \\
\hline 2007 & $2,55 \%^{a}$ & $2,37 \% \mathrm{o}^{\mathrm{b}}$ & $2,31 \% \mathrm{c}^{\mathrm{c}}$ & $23,17 * * *$ \\
\hline 2008 & $2,81 \% \mathrm{o}^{\mathrm{a}}$ & $2,63 \% \mathrm{ob}^{\mathrm{b}}$ & $2,54 \% \mathrm{c}^{\mathrm{c}}$ & $28,02^{* * *}$ \\
\hline 2009 & $2,16 \% \mathrm{a}^{\mathrm{a}}$ & $2,02 \% \mathrm{~b}^{\mathrm{b}}$ & $2,02 \% \mathrm{o}^{\mathrm{b}}$ & $5,82 * *$ \\
\hline 2010 & $1,70 \% \mathrm{a}^{\mathrm{a}}$ & $1,59 \% \mathrm{o}^{\mathrm{b}}$ & $1,59 \% \mathrm{ob}^{\mathrm{b}}$ & $3,50^{*}$ \\
\hline 2011 & $1,60 \% \mathrm{a}^{\mathrm{a}}$ & $1,49 \%{ }^{\mathrm{b}}$ & $1,48 \%{ }^{\mathrm{b}}$ & $4,46^{*}$ \\
\hline \multicolumn{5}{|c|}{$\begin{array}{l}\text { Basis: } n=930-1027 \text {, Welch-Test (Post-Hoc-Test: Tukey-HSD) } \\
\text { fikant auf dem 0,1\%-, } 1 \% \text { - bzw. } 5 \% \text {-Niveau. Gruppen mit unterschiedlicher } \\
\text { uchstaben }(a, b, c) \text { unterscheiden sich signifikant auf dem } 5 \% \text {-Niveau. }\end{array}$} \\
\hline Tabelle 21: & \multicolumn{4}{|c|}{ Entwicklung des Zinsaufwands nach Lagebereichen } \\
\hline
\end{tabular}

Banken in städtischer Lage müssen im Mittel höhere Aufwendungen für die Refinanzierung aufbringen. Mögliche Gründe hierfür sind, dass diese Banken ihren Kunden einerseits höhere Einlagenzinsen bieten müssen, da sie mit Filialen anderer Banken im Wettbewerb um die Kundeneinlagen stehen. Durch den Wettbewerb verfügen städtische Banken über eine geringere Preisfestsetzungsmacht als ländliche Institute, die ihrerseits häufig nur gegen die Sparkassen konkurrieren. Im ländlichen Bereich fällt es den Banken leichter, ihre Preisvorstellungen durchzusetzen.

Das reine für die Refinanzierung von Genossenschaftsbanken zur Verfügung stehende Volumen der Einlagen dürfte jedoch in allen Lagebereichen nicht knapp sein, HUMMEL und PEDROTTI sehen eine auskömmliche Versorgung mit günstigen Einlagen für alle Kreditgenossenschaften als gesichert an. ${ }^{899}$ Als zweite Quelle überdurchschnittlicherer Refinanzierungskosten sind höhere Zinsen am Interbankenmarkt für Institute in städtischer Lage denkbar. Hierfür ist dem Autor jedoch kein empirischer Beleg bekannt. Abbildung 33 enthält eine grafische Darstellung der Ergebnisse. 


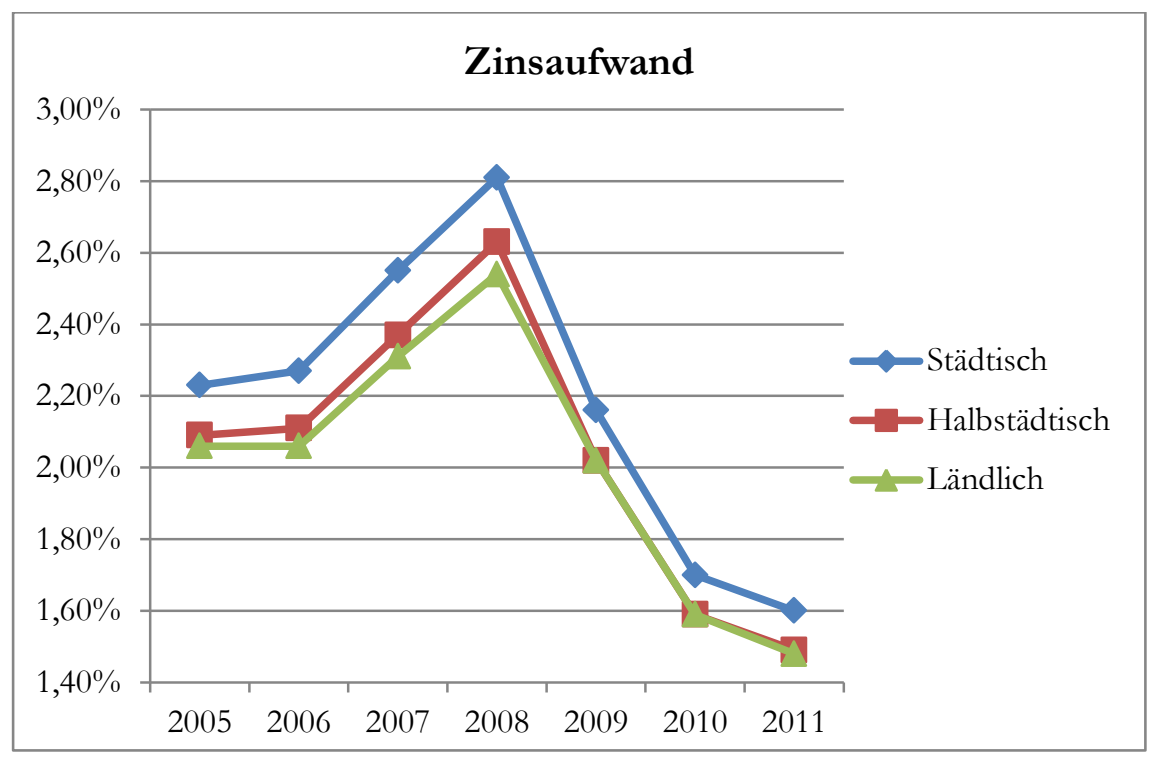

Abbildung 33: Entwicklung des Zinsaufwands nach Lagebereichen

Tabelle 22 enthält die Resultate einer bestätigenden OLS-Regression. Ausgehend vom Niveau der Zinsaufwandsspanne im ländlichen Bereich la als Basislinie, kann für städtische Banken st ein Aufschlag von 17 Basispunkten hochsignifikant nachgewiesen werden, während für die halbstädtische Lage la nur 3,14 Basispunkte Aufschlag signifikant sind.

\begin{tabular}{|c|c|}
\hline & (4) \\
\hline VARIABLE & zas \\
\hline st & $\begin{array}{c}0,170^{* * *} \\
(0,0176)\end{array}$ \\
\hline ha & $\begin{array}{l}0,0314^{*} \\
(0,0129)\end{array}$ \\
\hline o.la & - \\
\hline Konstante & $\begin{array}{c}2,018 * * * \\
(0,0105)\end{array}$ \\
\hline Beobachtungen & 7.095 \\
\hline $\mathrm{R}^{2}$ & 0,014 \\
\hline
\end{tabular}

Tabelle 22: $\quad$ Einfluss der Lage auf die Zinsaufwandsspanne (OLS)

Wie oben dargestellt ${ }^{900}$, fallen die Zinsaufwendungen für die Passivpositionen 1, 2, 3 und 9 an, durch welche sich die Banken hauptsächlich refinanzieren. PADBERG kritisiert die Verwendung der Bilanzsumme als Basis. Stattdessen wirbt er für die Verwendung der Summe der vier genannten Passivpositionen. Errechnet wird der sogenannte Durchschnittszinssatz der Verbindlichkeiten. ${ }^{901}$ Auch HARTMANN-WENDELS ET AL. argumentieren an einer Stelle für den Einsatz der gesamten Einlagen als Größenmaßstab. ${ }^{902}$ Wie aus der Darstellung in Tabelle 23 hervorgeht, bewegen sich die Unterschiede und auch deren

${ }^{900}$ Vgl. Abschnitt 3.6.3.2.

901 Vgl. Padberg (2005), S. 24

902 Vgl. Hartmann-Wendels et al. (2015), S. 52. 
Signifikanzen auf vergleichbarem Niveau wie bei der Verwendung der durchschnittlichen Bilanzsumme als Basis.

\begin{tabular}{|c|c|c|c|c|}
\hline & $\begin{array}{c}\text { Städtisch } \\
(\mathrm{n}=155-164)\end{array}$ & $\begin{array}{l}\text { Halbstädtisch } \\
(\mathrm{n}=525-591)\end{array}$ & $\begin{array}{c}\text { Ländlich } \\
(\mathrm{n}=263-302)\end{array}$ & $\begin{array}{c}\mathbf{F} \\
\text { (Welch) }\end{array}$ \\
\hline 2005 & $2,40 \% \mathrm{a}^{\mathrm{a}}$ & $2,27 \% \mathrm{o}^{\mathrm{b}}$ & $2,24 \%{ }^{\mathrm{b}}$ & $9,52^{* * *}$ \\
\hline 2006 & $2,42 \% \mathrm{a}^{\mathrm{a}}$ & $2,28 \%{ }^{\mathrm{b}}$ & $2,23 \%{ }^{b}$ & $14,68^{* * *}$ \\
\hline 2007 & $2,73 \% \mathrm{a}^{\mathrm{a}}$ & $2,55 \% \mathrm{o}^{\mathrm{b}}$ & $2,49 \% \mathrm{o}^{\mathrm{b}}$ & $22,62 * * *$ \\
\hline 2008 & $2,95 \% \mathrm{o}^{\mathrm{a}}$ & $2,78 \%{ }^{\mathrm{b}}$ & $2,71 \%{ }^{c}$ & $21,12^{* * *}$ \\
\hline 2009 & $2,28 \% \mathrm{a}^{\mathrm{a}}$ & $2,16 \% \mathrm{~b}^{\mathrm{b}}$ & $2,16 \% \mathrm{~b}$ & $4,71 * *$ \\
\hline 2010 & $1,81 \% \mathrm{a}^{\mathrm{a}}$ & $1,71 \% \mathrm{o}^{\mathrm{b}}$ & $1,71 \% \mathrm{o}^{\mathrm{b}}$ & 2,72 \\
\hline 2011 & $1,72 \% \mathrm{a}^{\mathrm{a}}$ & $1,60 \% \mathrm{~b}$ & $1,60 \% \mathrm{c}$ & $3,88^{*}$ \\
\hline $\begin{array}{l}* * \text { sign } \\
\text { Kenn }\end{array}$ & $\begin{array}{l}\mathrm{n}=943-1052, \mathrm{~V} \\
\text { uf dem } 0,1 \%- \\
\text { pen }(a, b, c) \text { unt }\end{array}$ & $\begin{array}{l}\text { ch-Test (Post-Hoc } \\
\% \text { - bzw. } 5 \% \text {-Nive } \\
\text { cheiden sich signi }\end{array}$ & $\begin{array}{l}\text { est: Tukey-HSI } \\
\text { Gruppen mit } \\
\text { ant auf dem } 5\end{array}$ & $\begin{array}{l}\text { chiedlichen } \\
\text { eau. }\end{array}$ \\
\hline \multicolumn{2}{|c|}{ Tabelle 23: } & $\begin{array}{l}\text { klung des Dur } \\
\text { bindlichkeiten }\end{array}$ & $\begin{array}{l}\text { schnittszinss } \\
\text { ch Lagebere }\end{array}$ & \\
\hline
\end{tabular}

Es folgt nun eine Betrachtung der Volumina, die den Instituten zur Refinanzierung zur Verfügung stehen. Hierzu wird die Passivseite beispielhaft anhand des Geschäftsjahres 2011 untersucht. Zugrunde liegen hierbei wieder die Gesamtvolumina der einzelnen Posten über alle Banken. Diese werden in das Verhältnis zu der über alle Institute aggregierten Bilanzsumme gesetzt. Auf diese Weise erhält man eine Durchschnittsverteilung auf Basis von Gliederungszahlen. ${ }^{903}$ Grafisch dargestellt ist diese Verteilung in Abbildung 34.

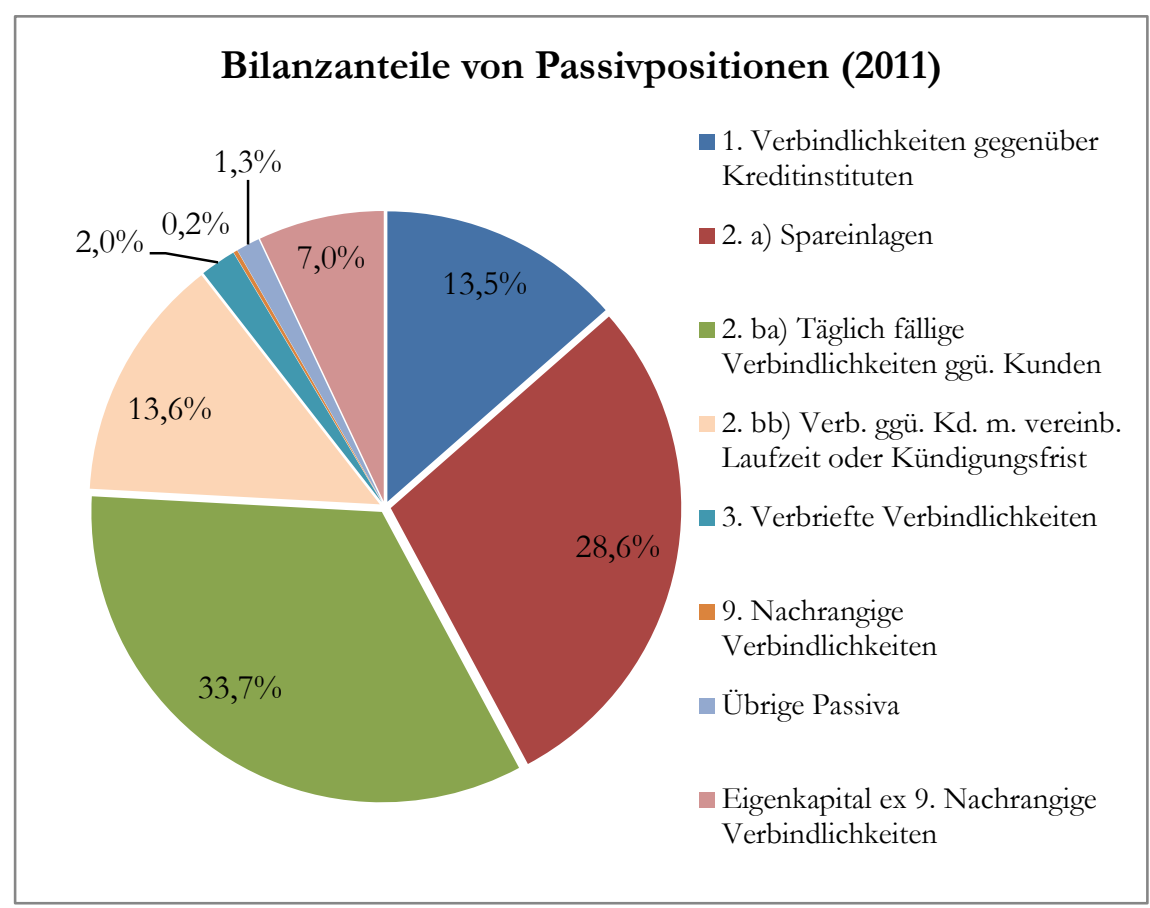

Abbildung 34: Bilanzanteile ausgewählter Passiva über alle analysierten Genossenschaftsbanken (2011) 
Der Anteil von Passivposten Nr. 1, der Verbindlichkeiten gegenüber Kreditinstituten, liegt bei 13,5\%. Dies spiegelt jedoch lediglich das unverbriefte Volumen wider; verbriefte Instrumente wie begebene Schuldverschreibungen werden in der Passivposition Nr. 3 erfasst - unabhängig davon, ob diese im Bestand eines Kreditinstitutes sind. Der mit insgesamt 75,9 \% größte und bedeutendste Anteil der Refinanzierung der Kreditgenossenschaften liegt im Bereich von Passivposten Nr. 2, der Verbindlichkeiten gegenüber Kunden, worunter Kundeneinlagen zu verstehen sind. Hiervon entfallen 28,6 \% auf Spareinlagen, welche im Unterposten 2. a) erfasst werden. Von diesen wiederum hat der überwiegende Teil eine Laufzeit von bis zu 3 Monaten; länger laufende Festgelder werden von den Kunden deutlich weniger nachgefragt. Unterposten 2. b) enthält diejenigen Einlagen, welche nicht die Voraussetzungen des $\ 21$ Abs. 4 RechKredV für Spareinlagen erfüllen ${ }^{904}$. Hierzu zählen einerseits die täglich fälligen Sichteinlagen mit einem Anteil von 33,7 \% an der aggregierten Bilanzsumme. Andererseits stellen Einlagen mit vereinbarter Laufzeit oder Kündigungsfrist einen Anteil von 13,6 \%. Alle übrigen Positionen spielen eher eine untergeordnete Rolle.

Bemerkenswert ist der hohe Anteil von Kundeneinlagen an dem Gesamtrefinanzierungsvolumen der Genossenschaftsbanken. Selbst während der Finanzkrise ab 2007 gab es stets eine stabile Refinanzierungsbasis durch Kundeneinlagen. Die Liquiditätsstörungen an den Geldmärkten tangierten die Kreditgenossenschaften somit wenig. ${ }^{905}$ Der hohe Einlagenanteil trägt maßgeblich zur Stabilität der Institute bei.

\subsubsection{Hypothese 5: Laufende Erträge}

Die Hypothese lautet:

Banken in ländlichen Regionen unterscheiden sich binsichtlich ibrer laufenden Erträge von Banken in urbanen Gebieten.

Grundsätzlich ist jedes Kreditinstitut bestrebt, die von Kunden entgegengenommenen Einlagen in Form von Krediten wieder auszureichen, da dieses Kerngeschäft die mit Abstand höchsten Erträge für das Institut verspricht. Der Bilanzanteil der Forderungen an Kunden wird als Kreditquote bezeichnet und in einer späteren Hypothese als Erfolgsfaktor untersucht. ${ }^{906}$ Ist die Ausreichung in Form von Krediten nicht im gewünschten Umfang möglich, z. B. mangels Nachfrage an Krediten, legen die Institute das Kapital in anderer Form an, um Erträge zu erzielen. Posten 3 der Gewinn- und Verlustrechnung enthält diese laufenden Erträge. Sie stammen a) aus Aktien und anderen nicht festverzinslichen Wertpapieren, b) aus Beteiligungen und Geschäftsguthaben bei Genossenschaften sowie c) aus Anteilen an verbundenen Unternebmen.

Viele vor allem kleinere Genossenschaftsbanken führen keinen Eigenhandel und weisen daher kein Depot A auf. Es handelt sich um sogenannte Nichthandelsbuchinstitute (NHI), bzw. um NHI mit beschränktem Eigenhandel, sofern ein Eigenhandel in limitiertem Umfang durchgeführt wird. Anstelle des Eigenhandels lassen die Institute die Mittel in sogenannten Spezialfonds durch die verbundeigene Fondsgesellschaft Union Investment verwalten. Jedoch wird diese Möglichkeit auch von größeren Instituten genutzt, zusätzlich zur Verwaltung durch eigene Fondsmanager. Die Ausschüttungen dieser Spezialfonds werden

904 Vgl. Scharpf und Schaber (2011), S. 727.

${ }^{905}$ Vgl. Reichel (2011), S. 33.

906 Vgl. Hypothese 14: Kreditquote in Abschnitt 4.4.15. 
in der GuV-Position 3. a) als Erträge erfasst. Obwohl diese Position die Bezeichnung Laufende Erträge aus Aktien und anderen nicht festverzinslichen Wertpapieren trägt, können hier dennoch Erträge aus festverzinslichen Wertpapieren ausgewiesen werden. Dies ist z. B. der Fall, wenn ein Spezialfonds teilweise oder vollständig in festverzinsliche Wertpapiere investiert. Begründet wird dies damit, dass der Spezialfonds selbst kein festverzinsliches Wertpapier ist, sondern eben allenfalls in solche Papiere investiert. Da die Höhe der Ausschüttungen gesteuert werden kann, verfügen die Institute so über eine Möglichkeit, die Höhe der Erträge in gewissen Grenzen zu beeinflussen. Das in Spezialfonds anlegte Vermögen wird in der Bilanzaktivposition 6 Aktien und andere nicht festverzinsliche Wertpapiere erfasst.

$\mathrm{Zu} \mathrm{b)} \mathrm{zählen} \mathrm{die} \mathrm{Erträge} \mathrm{aus} \mathrm{Anteilen,} \mathrm{die} \mathrm{jede} \mathrm{Kreditgenossenschaft} \mathrm{an} \mathrm{den} \mathrm{genossen-}$ schaftlichen Zentralinstituten hält, wie der DZ BANK AG, der Bausparkasse Schwäbisch Hall AG oder der Union Investment Asset Management Holding AG. Weitere mögliche Erträge stammen aus Immobilienbeteiligungen, Energiegenossenschaften oder Windparks. $\mathrm{Zu} \mathrm{c)} \mathrm{werden} \mathrm{Erträge} \mathrm{gerechnet,} \mathrm{die} \mathrm{aus} \mathrm{Tochterunternehmen} \mathrm{stammen,} \mathrm{an} \mathrm{denen} \mathrm{die}$ jeweilige Kreditgenossenschaft eine Mehrheit hält, das heißt mehr als $50 \%$ der Anteile innehat. ${ }^{907}$ In vielen Fällen werden $100 \%$ der Anteile gehalten. Hierbei kann es sich um eine Tochtergesellschaft handeln, die Immobiliengeschäfte betreibt und, etwa bei der Finanzierung, eng mit der Mutterbank zusammenarbeitet. Es ist daher mit einer positiven Korrelation der Ertragsquellen zu rechnen.

Die Ergebnisse der Varianzanalyse zu den Unterschieden zwischen den drei Lagekategorien sind in Tabelle 24 dokumentiert.

\begin{tabular}{|c|c|c|c|c|}
\hline & $\begin{array}{c}\text { Städtisch } \\
(\mathrm{n}=154-164)\end{array}$ & $\begin{array}{l}\text { Halbstädtisch } \\
(\mathrm{n}=525-589)\end{array}$ & $\begin{array}{c}\text { Ländlich } \\
(\mathrm{n}=262-299)\end{array}$ & $\begin{array}{c}\mathbf{F} \\
\text { (Welch) }\end{array}$ \\
\hline 2005 & $0,23 \%{ }^{a}$ & $0,16 \% \mathrm{o}^{\mathrm{b}}$ & $0,10 \%{ }^{c}$ & $18,34 * * *$ \\
\hline 2006 & $0,25 \% \mathrm{o}^{\mathrm{a}}$ & $0,17 \% \mathrm{o}^{\mathrm{b}}$ & $0,11 \%{ }^{\mathrm{c}}$ & $21,77 * * *$ \\
\hline 2007 & $0,27 \%{ }^{\mathrm{a}}$ & $0,17 \%{ }^{\mathrm{b}}$ & $0,11 \%{ }^{c}$ & 21,51 *** \\
\hline 2008 & $0,23 \% \mathrm{o}^{\mathrm{a}}$ & $0,16 \%{ }^{\mathrm{b}}$ & $0,10 \%{ }^{\mathrm{c}}$ & $18,97 * * *$ \\
\hline 2009 & $0,19 \%{ }^{\mathrm{a}}$ & $0,13 \% \mathrm{or}^{\mathrm{b}}$ & $0,07 \%{ }^{c}$ & $30,86 * * *$ \\
\hline 2010 & $0,19 \% \mathrm{o}^{\mathrm{a}}$ & $0,13 \%{ }^{\mathrm{b}}$ & $0,08 \%{ }^{\mathrm{c}}$ & $26,65^{* * *}$ \\
\hline 2011 & $0,18 \% \mathrm{a}^{\mathrm{a}}$ & $0,13 \% 0^{\mathrm{b}}$ & $0,08 \%{ }^{\mathrm{c}}$ & $20,66 * * *$ \\
\hline
\end{tabular}

Es zeigt sich, dass die laufenden Erträge innerhalb der ersten drei Positionen der Gewinnund Verlustrechnung die einzige ist, welche im Mittel zugunsten städtisch gelegener Banken ausfällt. Auf hohem signifikantem Niveau heben sich städtische Banken im Mittel von balbstädtischen ab, und diese ebenfalls wieder von den ländlichen Banken.

Zurückzuführen ist dieses Resultat auf die Größe der Institute. Wie eine folgende analoge deskriptive Untersuchung zeigt ${ }^{908}$, sind Banken in städtischer Lage im Mittel bedeutend größer als solche in halbstädtischer Lage, und diese wiederum als solche in ländlicher Lage. Durch die bereits oben in Abschnitt 3.4.3 beschriebenen Skaleneffekte ist der Aufbau und

907 Vgl. \ 271 HGB.

${ }^{908}$ Vgl. Abschnitt 4.4.11. 
die Durchführung einer professionellen und umfassenden Vermögensverwaltung desto lohnenswerter, je größer die Anlagesummen sind. Dies ist wiederum vor allen durch die hohen Fixkostenanteile zu begründen. Die Abbildung 35 beleuchtet dieses Resultat grafisch.

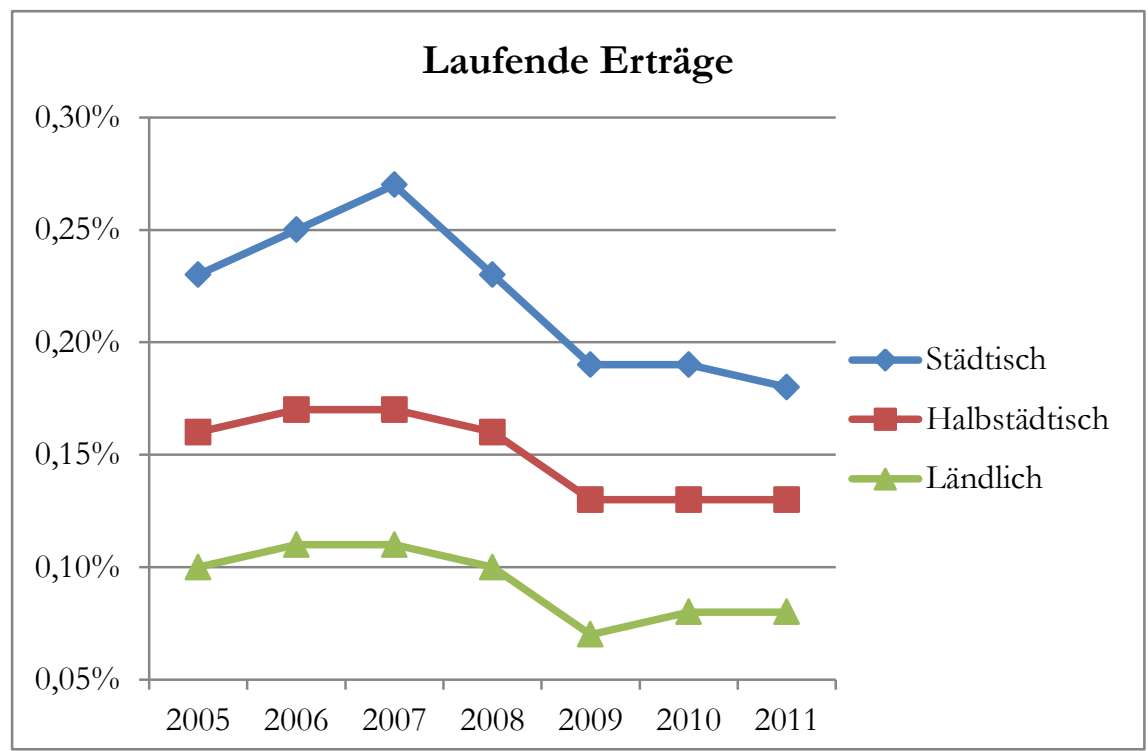

Abbildung 35: Entwicklung der laufenden Erträge nach Lagebereichen

Tabelle 25 stellt die Ergebnisse einer entsprechenden OLS-Regression dar, welche die Ergebnisse bestätigt. Die geringsten laufenden Erträge werden von Genossenschaftsbanken in ländlicher Lage la erzielt. Um 5,73 Basispunkte höhere laufende Erträge werden in halbstädtischer Lage ha erwirtschaftet, während Banken in städtischer Lage st sich bei den laufenden Erträgen im Mittel um 12,6 nach oben von den ländlichen Instituten abheben. Die Resultate weisen das höchste Signifikanzniveau auf.

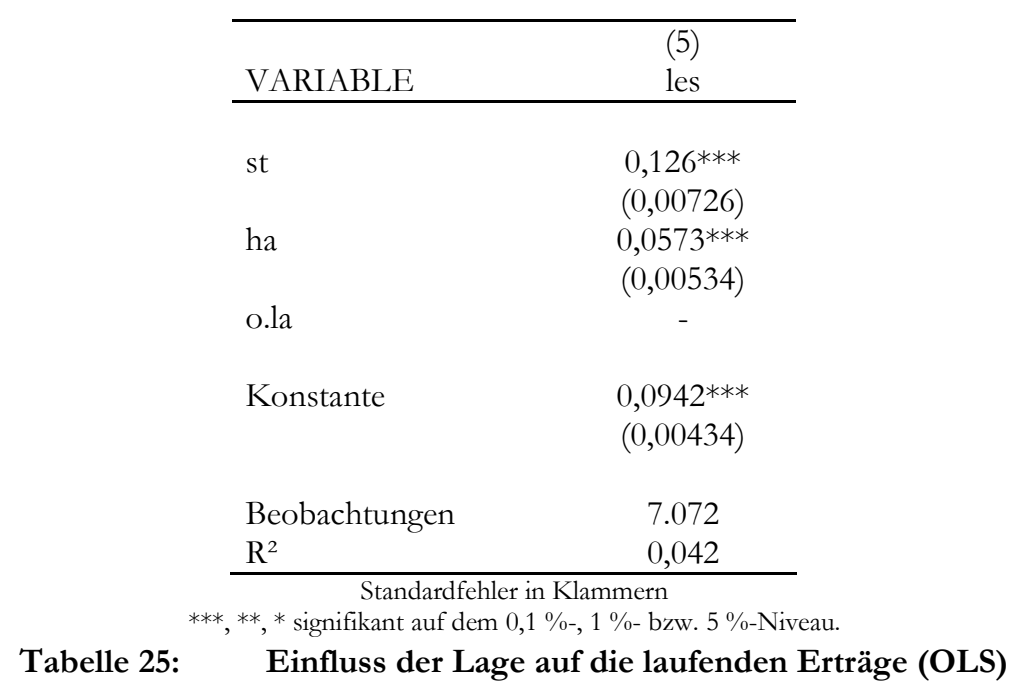

Die Entwicklung der laufenden Erträge im Zeitablauf ist in Abbildung 36 dargestellt. Hierzu wurden für jedes Geschäftsjahr die aggregierten laufenden Erträge über alle Banken in das Verhältnis gesetzt zur aggregierten Bilanzsumme aller Banken. 
Nach dem Beginn der Finanzmarktkrise 2007 nahmen die durchschnittlich über alle Genossenschaftsbanken pro Jahr erzielten laufenden Erträge ab. Das sinkende Zinsniveau ab 2008 ist einer der einflussreichsten Treiber für den Rückgang dieser laufenden Erträge. Der Einfluss der Steilheit der Zinsstruktur auf den Erfolg spielt auch eine Rolle. Dieser wurde durch RICHTER ${ }^{909}$ untersucht, und wird in einer späteren Betrachtung in dieser Arbeit weiter verfolgt.

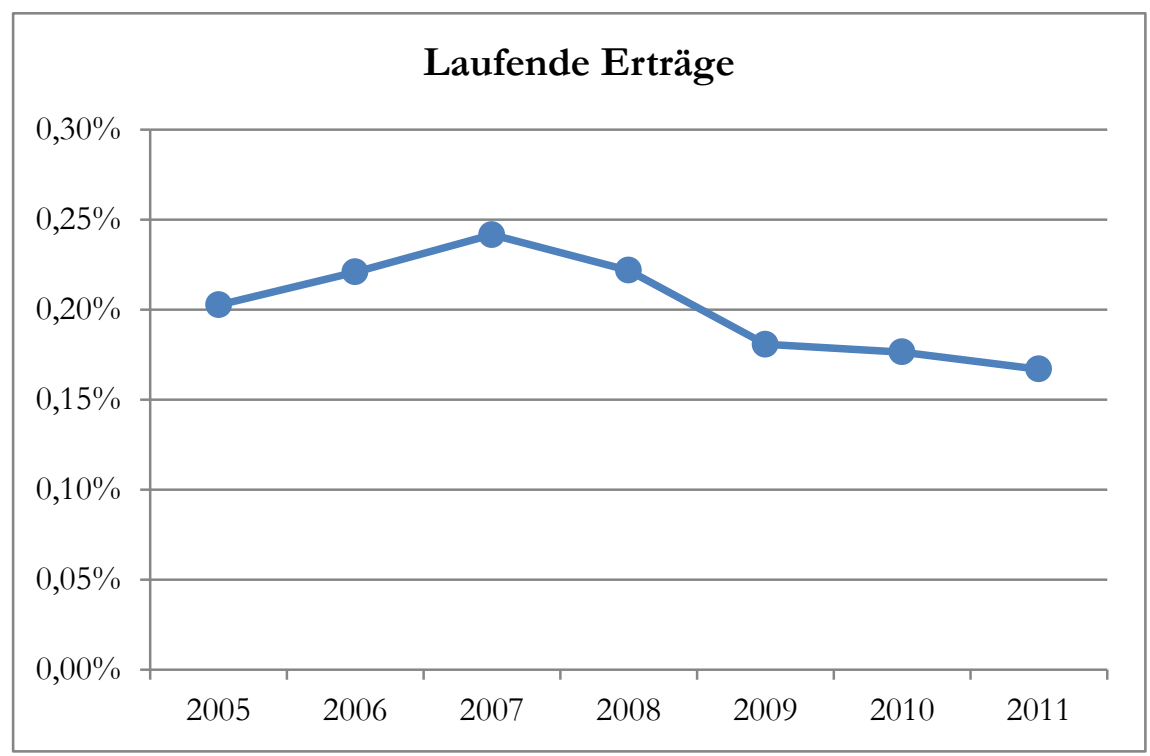

Abbildung 36: Entwicklung der laufenden Erträge aller Banken

\subsubsection{Hypothese 6: Zinsüberschuss}

Die Hypothese lautet:

Banken in ländlichen Regionen unterscheiden sich binsichtlich ibres Zinsüberschusses von Banken in urbanen Gebieten.

Diese Hypothese verwendet mit dem Zinsüberschuss eine aggregierte Kennzahl. Bezieht man in die Errechnung des Zinsüberschusses lediglich die GuV-Positionen 1. a), 1. b) und 2. ein, und lässt die laufenden Erträge außen vor, so ergibt sich erneut das bekannte Gefälle, welches in Tabelle 26 dargestellt wird.

Beim Vergleich der drei Lagebereiche Städtisch, Halbstädtisch und Ländlich fällt auf, dass sich die Unterschiede in den Mittelwerten in jedem Geschäftsjahr deutlich hervorheben. Die Signifikanzen untermauern dieses Bild, denn sie fallen durchgängig über alle Geschäftsjahre drastisch höher aus als bei der Betrachtung der drei einzelnen Komponenten. Dies ist darauf zurückzuführen, dass die bestehenden Unterschiede auf Basis von Zinsüberschuss und Zinsaufwand kumulierend verstärken. Ein hoher Unterschied wiederum resultiert bei der Varianzanalyse in einer hohen Signifikanz der Ergebnisse. Wie bereits oben bei der Bruttogewinnspanne erklärt ${ }^{910}$, fällt der Zinsüberschuss im ländlichen Bereich höher aus, da dort in der Regel weniger Wettbewerb herrscht. Dies deckt sich mit der Aussage von 
WuTZ, welcher gar konstatiert, dass die Bruttozinsspanne als Indikator für die Wettbewerbsintensität fungiere. ${ }^{911}$ Abbildung 37 enthält die grafische Darstellung der Resultate, welche in diesem ersten Schritt exklusive laufende Erträge ermittelt wurden.

\begin{tabular}{|c|c|c|c|c|}
\hline & $\begin{array}{l}\text { Städtisch } \\
(\mathrm{n}=146-155)\end{array}$ & $\begin{array}{l}\text { Halbstädtisch } \\
(\mathrm{n}=512-555)\end{array}$ & $\begin{array}{c}\text { Ländlich } \\
(\mathrm{n}=259-289)\end{array}$ & $\begin{array}{c}\mathbf{F} \\
\text { (Welch) }\end{array}$ \\
\hline 2005 & $2,21 \%^{a}$ & $2,60 \% \mathrm{o}^{\mathrm{b}}$ & $2,67 \%{ }^{c}$ & $37,43^{* * *}$ \\
\hline 2006 & $2,10 \% \mathrm{a}^{\mathrm{a}}$ & $2,47 \% \mathrm{o}^{\mathrm{b}}$ & $2,57 \%{ }^{c}$ & $41,28^{* * *}$ \\
\hline 2007 & $1,93 \% \mathrm{a}^{\mathrm{a}}$ & $2,27 \% \mathrm{~b}^{\mathrm{b}}$ & $2,39 \%{ }^{c}$ & $33,85^{* * *}$ \\
\hline 2008 & $1,86 \% \mathrm{a}^{\mathrm{a}}$ & $2,17 \% \mathrm{o}^{\mathrm{b}}$ & $2,29 \%{ }^{c}$ & $18,52^{* * *}$ \\
\hline 2009 & $2,11 \% \mathrm{o}^{\mathrm{a}}$ & $2,43 \% \mathrm{o}^{\mathrm{b}}$ & $2,56 \%{ }^{c}$ & $33,66^{* * *}$ \\
\hline 2010 & $2,19 \% \mathrm{a}^{\mathrm{a}}$ & $2,52 \% \mathrm{~b}^{\mathrm{b}}$ & $2,65 \%{ }^{c}$ & $37,65^{* * *}$ \\
\hline 2011 & $2,12 \% \mathrm{a}^{\mathrm{a}}$ & $2,43 \% \mathrm{~b}$ & $2,55 \%{ }^{c}$ & $35,56 * * *$ \\
\hline \multicolumn{5}{|c|}{$\begin{array}{l}\text { Basis: } \mathrm{n}=922-994 \text {, Welch-Test (Post-Hoc-Test: Tukey-HSD) } \\
\text { ***, **, * signifikant auf dem 0,1 \%-, } 1 \% \text { - bzw. } 5 \% \text {-Niveau. Gruppen mit unterschiedlichen } \\
\text { Kennbuchstaben (a, b, c) unterscheiden sich signifikant auf dem } 5 \% \text {-Niveau. }\end{array}$} \\
\hline \multicolumn{2}{|c|}{ Tabelle 26: } & $\begin{array}{l}\text { lung des Zinsü } \\
\text { nder Erträge n }\end{array}$ & $\begin{array}{l}\text { rschusses e } \\
\text { L Lageberei }\end{array}$ & \\
\hline
\end{tabular}

Die Unterschiede, welche PADBERG ${ }^{912}$ zwischen Instituten in städtischer und ländlicher Lage nachweisen konnte, wurden in großem Umfang bestätigt und auf Genossenschaftsbanken ausgeweitet. ${ }^{913}$

Gemäß der ROI-Analyse errechnet sich der Zinsüberschuss aus seinen Komponenten laut der Darstellung in Tabelle 27. ${ }^{114}$ Hierbei sind auch die laufenden Erträge mit einzubeziehen.

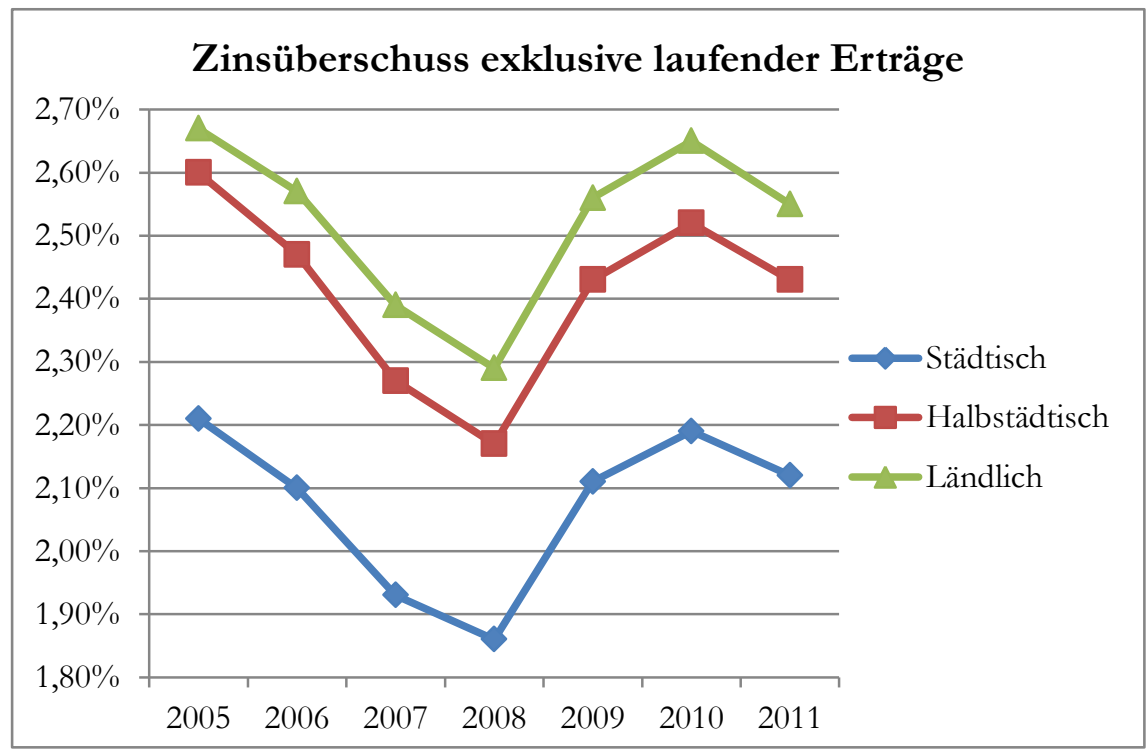

Abbildung 37: Entwicklung des Zinsüberschusses exklusive laufender Erträge nach Lagebereichen

\footnotetext{
${ }^{11}$ Vgl. Wutz (2002), S. 14.

912 Vgl. Padberg (2005), S. 25.

913 Vgl. Abschnitt 2.1.9.

${ }^{914}$ Vgl. Schierenbeck (2003b), S. 424; vgl. 3.7.1.
} 


\begin{tabular}{|l|}
\hline \multicolumn{1}{|c|}{ Berechnung des Zinsüberschusses } \\
\hline \multicolumn{1}{|c|}{ 1. a) Zinserträge aus Kredit- und Geldmarktgeschäften } \\
\hline$+\quad$ 1. b) Zinserträge aus festverzinslichen Wertpapieren und Schuldbuchforderungen \\
\hline$-\quad$ 2. Zinsaufwendungen \\
\hline$+\quad$ 3. a) Laufende Erträge aus Aktien und anderen nicht festverzinslichen Wertpapieren \\
\hline$+\quad$ 3. b) Laufende Erträge aus Beteiligungen und Geschäftsguthaben an Genossen- \\
schaftsbanken \\
\hline$+\quad$ 3. c) Laufende Erträge aus Anteilen an verbundenen Unternehmen \\
\hline Zinsüberschuss
\end{tabular}

Tabelle 27: Berechnung des Zinsüberschusses

Die Ergebnisse der Varianzanalyse sind in Tabelle 28 dargestellt.

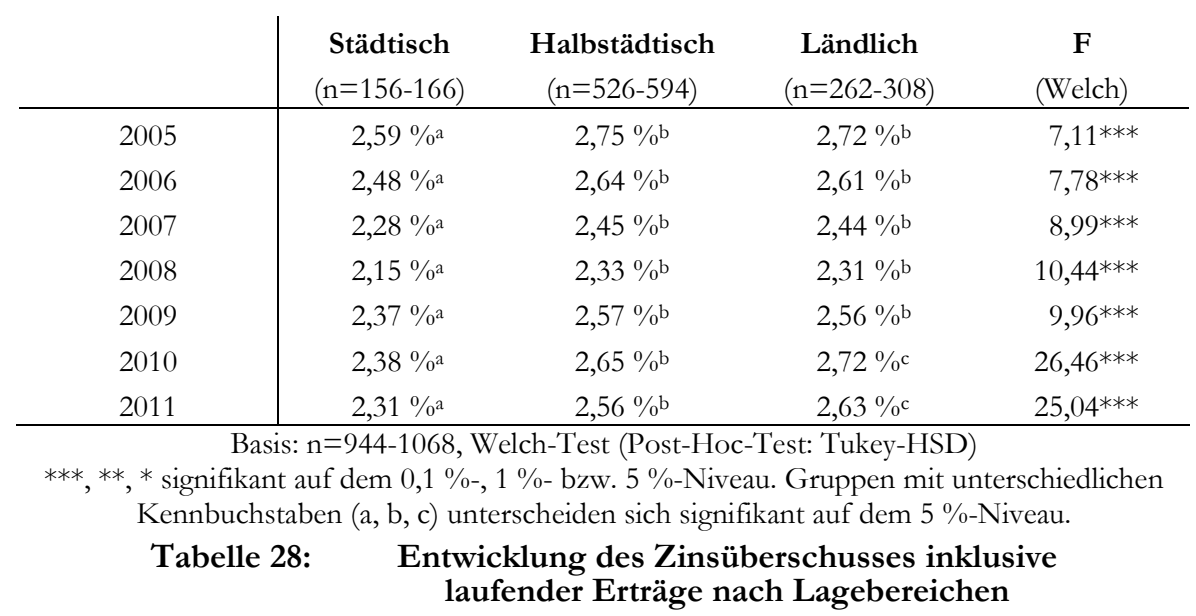

Die Bruttozinsspanne, so die Bezeichnung der Relation von Zinsüberschuss und durchschnittlicher Bilanzsumme, unterscheidet sich zwischen den Lagebereichen, es ergibt sich folgendes Bild: In jedem betrachteten Geschäftsjahr weisen die städtisch gelegenen Institute die geringste Bruttozinsspanne auf. Dennoch können städtische Banken durch den Einbezug laufender Erträge leicht aufholen: Wie oben gezeigt ${ }^{915}$ erlauben Skalenvorteile diesen in der Regel großen Instituten zumindest eine teilweise Kompensation dank der Erzielung laufender Erträge. Das Resultat fällt dennoch deutlich zugunsten von balbstädtischen und ländlichen Banken aus.

Auch die deutliche Überlegenheit bezüglich Zinserträgen und -aufwand, welche für ländliche Banken gegenüber halbstädtischen gezeigt werden konnten ${ }^{916}$, relativiert sich nach Einbezug der laufenden Erträge. Nur für 2010 und 2011 können signifikante Unterschiede zwischen beiden Lagebereichen in Bezug auf die Bruttogewinnspanne bestätigt werden. Eine grafische Darstellung findet sich in Abbildung 38. Auch die Entwicklung der Bruttozinsspanne über den Betrachtungszeitraum kann hier abgelesen werden. 


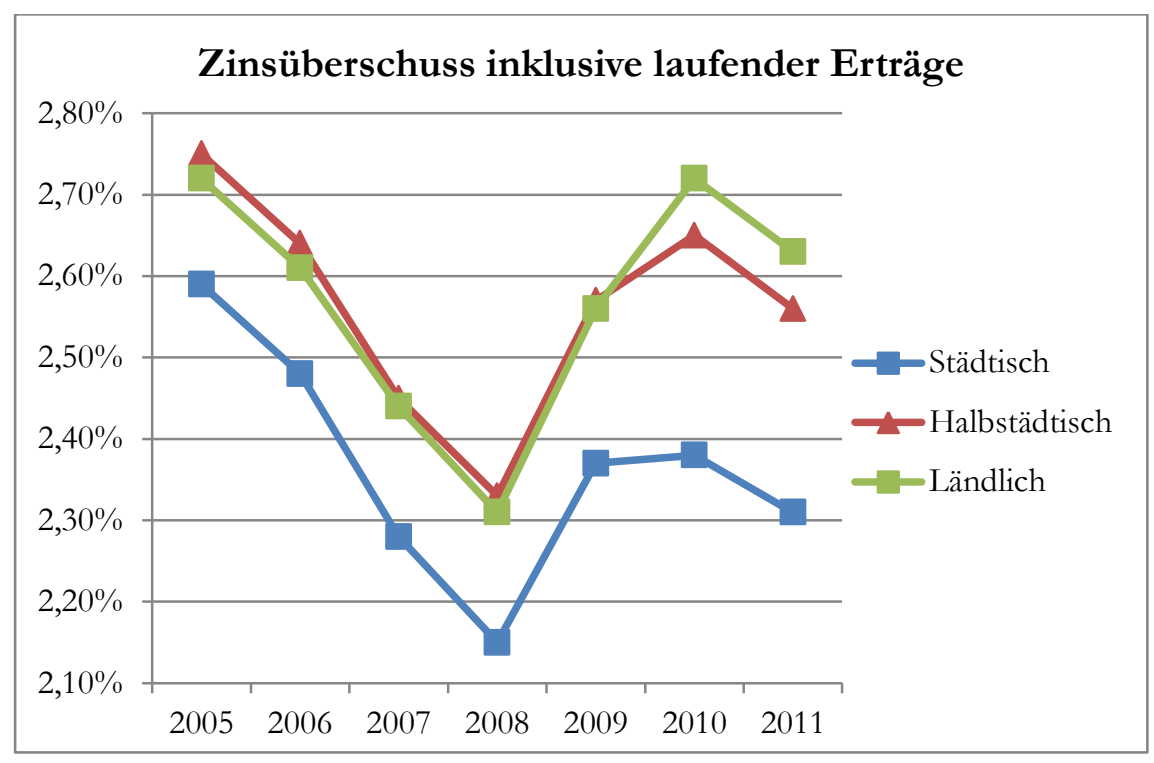

Abbildung 38: Entwicklung des Zinsüberschusses inklusive laufender Erträge nach Lagebereichen

Tabelle 29 stellt die bestätigenden und hochsignifikanten Resultate dar, welche sich durch eine OLS-Regression ergeben haben. Banken der städtischen Lagekategorie st bilden die Basislinie. Ausgehend von dieser weisen halbstädtische Banken ha im Mittel einen Aufschlag von 26 Basispunkten auf, während ländliche Banken la im Mittel gar um 31,4 Basispunkte höhere Zinsüberschüsse erwirtschaften.

Wie bereits in Abschnitt 4.4.1 ROI-Analyse herausgefunden wurde, ist insgesamt ein bedeutender Teil der Bankerträge auf die Zinsüberschüsse zurückzuführen. 2011 erwirtschafteten die Genossenschaftsbanken 74,2 \% ihrer Bruttoerträge im Zinsbereich, während auf den Provisionsbereich lediglich 20,4 \% entfielen. Die sonstigen Erträge machten 5,4 \% aus, während die Handelsspanne keinen bzw. keinen nennenswerten Beitrag zu den Erträgen aus der normalen Geschäftstätigkeit leistete.

\begin{tabular}{lc}
\hline VARIABLE & $(6)$ \\
o.st & bzs \\
\hline \multirow{2}{*}{ ha } & - \\
& $0,260^{* * *}$ \\
la & $(0,0128)$ \\
& $0,314^{* * *}$ \\
Konstante & $(0,0141)$ \\
& $2,300^{* * *}$ \\
& $(0,0113)$ \\
Beobachtungen & 7.132 \\
$\mathrm{R}^{2}$ & 0,070 \\
\hline
\end{tabular}

Standardfehler in Klammern

***, **, * signifikant auf dem 0,1\%-, 1 \%- bzw. 5 \%-Niveau.

Tabelle 29: $\quad$ Einfluss der Lage auf den Zinsüberschuss (OLS) 


\subsubsection{Hypothese 7: Provisionsüberschuss}

Die Hypothese lautet:

Banken in ländlichen Regionen unterscheiden sich binsichtlich ibres Provisionsüberschusses von Banken in urbanen Gebieten.

Dieser Hypothese liegt die Annahme zugrunde, dass sich die einzelnen Räume dahingehend unterscheiden, welche Arten und Volumina von Finanzprodukten nachgefragt und abgesetzt werden. Die Resultate der Varianzanalyse zur Identifikation der Unterschiede zwischen städtischer, halbstädtischer und ländlicher Lage sind in Tabelle 30 dargestellt und in Abbildung 39 abgebildet.

\begin{tabular}{|c|c|c|c|c|}
\hline & $\begin{array}{c}\text { Städtisch } \\
(\mathrm{n}=156-167)\end{array}$ & $\begin{array}{l}\text { Halbstädtisch } \\
(\mathrm{n}=525-595)\end{array}$ & $\begin{array}{c}\text { Ländlich } \\
(\mathrm{n}=262-305)\end{array}$ & $\begin{array}{c}\mathbf{F} \\
\text { (Welch) }\end{array}$ \\
\hline 2005 & $0,74 \%$ & $0,78 \% \mathrm{a}^{\mathrm{a}}$ & $0,70 \% \mathrm{~b}$ & $13,00^{* * *}$ \\
\hline 2006 & $0,76 \%$ & $0,80 \%{ }^{\mathrm{a}}$ & $0,71 \% \mathrm{o}^{\mathrm{b}}$ & $13,13^{* * *}$ \\
\hline 2007 & $0,77 \%$ & $0,82 \%{ }^{a}$ & $0,74 \% \mathrm{ob}^{\mathrm{b}}$ & $10,65^{* * *}$ \\
\hline 2008 & $0,72 \%{ }^{\mathrm{a}}$ & $0,77 \% \mathrm{~b}^{\mathrm{b}}$ & $0,71 \% 0^{\mathrm{a}}$ & $8,08^{* * *}$ \\
\hline 2009 & $0,67 \%{ }^{\mathrm{a}}$ & $0,72 \%{ }^{\mathrm{b}}$ & $0,65 \% \mathrm{a}^{\mathrm{a}}$ & $12,65^{* * *}$ \\
\hline 2010 & $0,68 \% 0^{\mathrm{a}}$ & $0,73 \% \mathrm{o}^{\mathrm{b}}$ & $0,66 \% 0^{\mathrm{a}}$ & $12,76^{* * *}$ \\
\hline 2011 & $0,65 \% 0^{\mathrm{a}}$ & $0,70 \% \mathrm{~b}$ & $0,65 \% 0^{a}$ & $9,38 * * *$ \\
\hline
\end{tabular}

Tabelle 30: $\quad$ Entwicklung des Provisionsüberschusses nach Lagebereichen

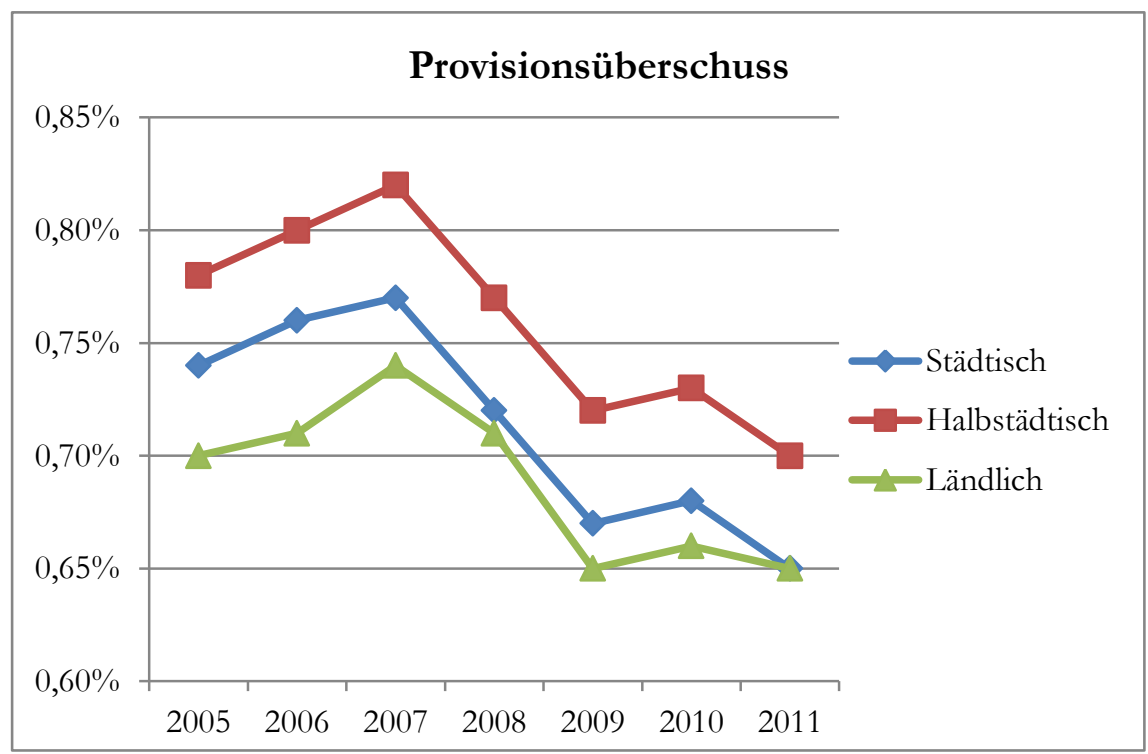

Abbildung 39: Entwicklung des Provisionsüberschusses nach Lagebereichen 
Tabelle 31 enthält die hochsignifikanten OLS-Resultate. Im Vergleich zur Basislinie der städtischen Banken st weisen halbstädtische Institute im Mittel um 5,4 Basispunkte höhere Provisionsspannen auf, während ländliche Banken im Mittel um 2,06 Basispunkte darunterbleiben.

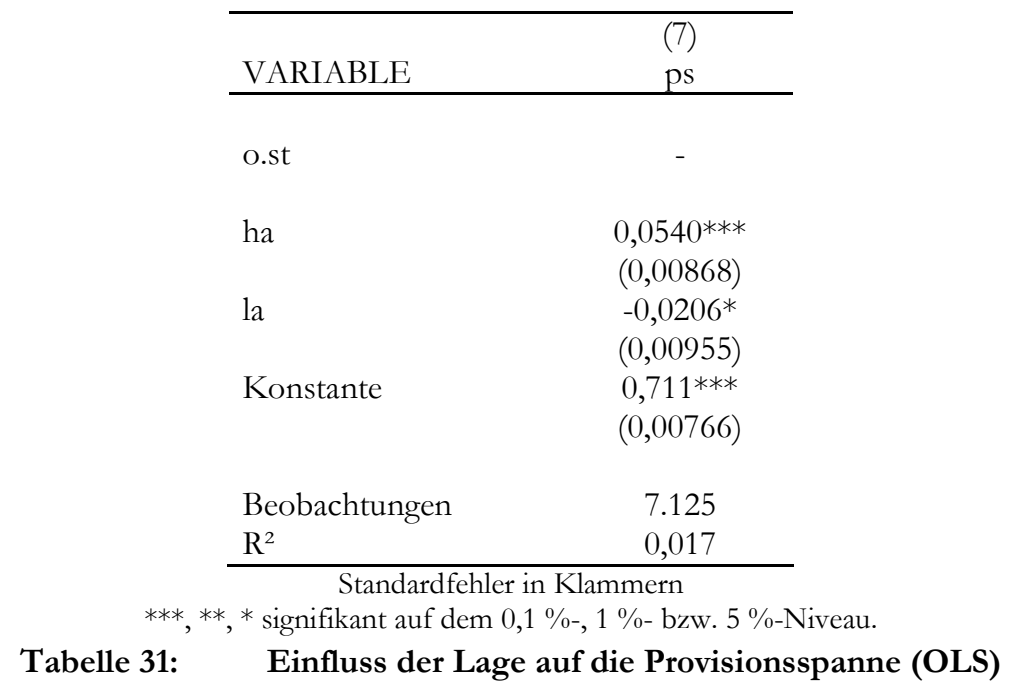

Im Gegensatz zu den vorangegangenen Positionen gibt es hier kein klares Gefälle von städtisch zu ländlich oder von ländlich zu städtisch. Stattdessen erzielten die Banken in jedem Geschäftsjahr im halbstädtischen Bereich die höchste Provisionsspanne, das heißt dort, wo die Bevölkerungsdichte zwischen 100 und 500 Einwohnern je Quadratkilometer liegt. ${ }^{917}$ Einerseits profitieren die Banken in diesem Bereich davon, dass die Intensität des Wettbewerbs nicht so hoch ist wie in städtischen Bereichen. Hierdurch lassen sich höhere Margen erzielen. Andererseits hebt sich der halbstädtische Bereich vom ländlichen Bereich ab. Die Hauptertragsquellen des Provisionsgeschäftes sind die Durchführung des qualifizierten Wertpapier- und Investmentfondsgeschäfts durch individuelle Beratung von Privatkunden inklusive Derivate und geschlossene Fonds, Versicherungen und Bausparverträge. Der Absatz hiervon ist jedoch in ländlichen Gebieten mangels Nachfrage als eher schwach einzuschätzen bzw. basiert auf geringeren Volumina. Daher kommt dort häufig allenfalls das Geschäft mit Standardfonds der genossenschaftlichen Investmentgesellschaft zur Anwendung, für welches eine Dokumentationsunterstützung durch die genossenschaftlichen Zentralbanken angeboten wird.

Die Entwicklung über den Ablauf der betrachteten Geschäftsjahre hinweg wird in Abbildung 40 dargestellt. Von 2005 bis 2007 sind zunächst steigende Provisionserträge zu verzeichnen. In Erwartung weiter steigender Kurse an den Aktienmärkten nahm die Kundennachfrage nach Fondsanlagen zu. Nach Beginn der Finanzkrise im Jahr 2007 ging die Höhe der Provisionsspanne bis 2009 zurück. 


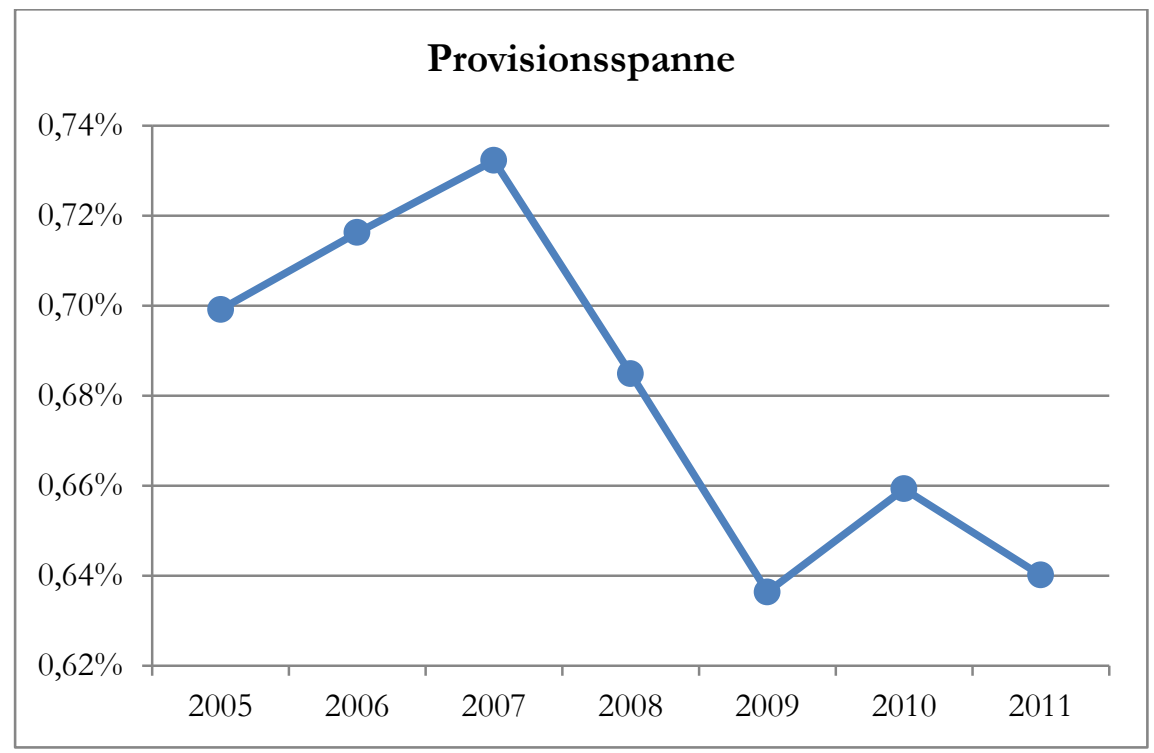

Abbildung 40: Entwicklung der Provisionsspanne aller Banken

Aufgrund stetig steigender Bilanzvolumina im gesamten Betrachtungszeitraum wird jedoch die Steilheit dieses Abstieges überzeichnet, in absoluten Werten ist der Rückgang geringer, wie anhand von Abbildung 41 nachvollzogen werden kann. Die Entwicklung der Bilanzsummen verlief auf diese Art etwa parallel in allen drei Lagebereichen.

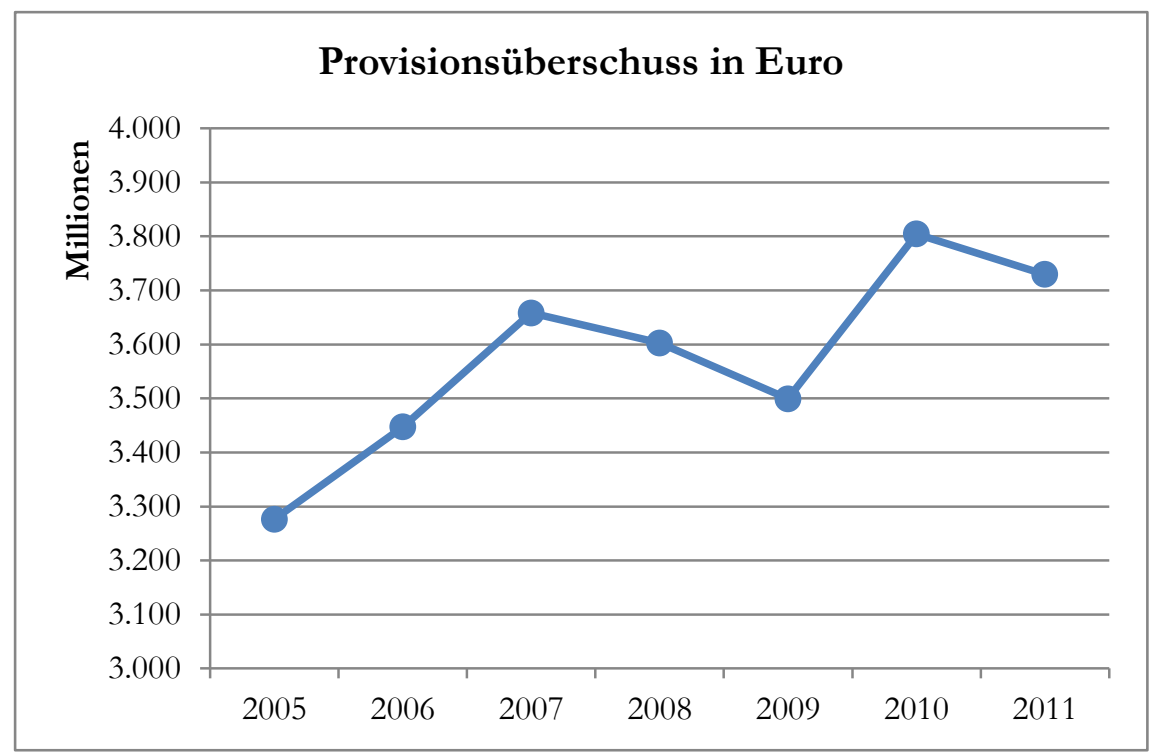

Abbildung 41: Entwicklung des über alle Banken kumulierten Provisionsüberschusses

PAdBerg kritisiert an der Provisionsspanne, dass die Normierung mit Hilfe der Bilanzsumme nicht sachgerecht sei, da kein sinnvoller Zusammenhang zwischen Provisionsergebnis und Bilanzsumme bestehe. ${ }^{918}$ Sie werde in Ermangelung anderer Normierungsmöglichkeiten häufig in der Literatur durchgeführt. ${ }^{919}$ Als einzige zweckmäßige Alternative für 
die externe Datenanalyse schlägt er vor, die Mitarbeiteranzahl für die Normierung einzusetzen. ${ }^{920}$ Mit Hilfe dieser Kennzahl kann bestimmt werden, welche Provisionserträge ein Mitarbeiter im Betrachtungszeitraum erwirtschaftet hat. ${ }^{921}$

Die Ermittlung des Provisionsüberschusses je Mitarbeiter (Abbildung 42) erfolgte für jedes Geschäftsjahr, indem einzeln für jeden Lagebereich die Provisionsüberschüsse aller Banken aggregiert wurden. Dieser Wert wurde dann in das Verhältnis zur Anzahl der Mitarbeiter im betreffenden Jahr im jeweiligen Lagebereich gesetzt. Es fällt auf, dass in allen drei Lagebereichen über den Betrachtungszeitraum hinweg insgesamt ein Wachstum des Provisionsüberschusses je Mitarbeiter erzielt werden konnte. Nach dem Beginn der Finanzkrise im Jahre 2007 ist je nach Lagebereich leichter bzw. stärkerer Einbruch festzustellen. Nach 2009 kam es überall wieder zu einem Ausbau der Provisionsgeschäfte. Ein möglicher Erklärungsansatz begründet dies damit, dass die Banken auf die Senkung des Hauptrefinanzierungssatzes der Europäischen Zentralbank reagieren. Die schrittweise Senkung von 4,25 \% im Herbst 2008 bis auf 1,00 \% im Frühjahr 2009 führte zu geschmälerten Erwartungen im zinsabhängigen Geschäft. Als Alternative hierzu bauten die Institute das Provisionsgeschäft aus, um in der Niedrigzinsphase wieder mehr Erträge generieren zu können.

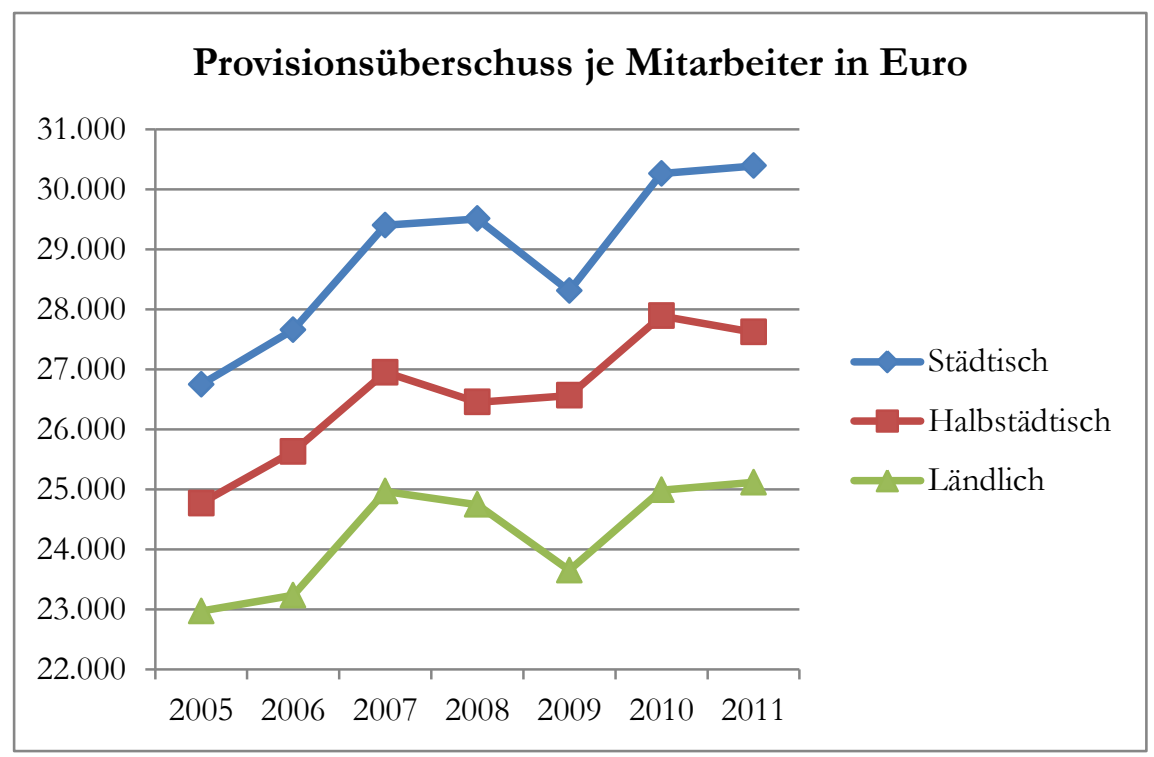

Abbildung 42: Entwicklung des Provisionsüberschusses je MA nach Lagebereichen

Bei der Betrachtung des insgesamt absolut erzielten Provisionsüberschusses in Euro von 2006 bis 2011 nach den drei Lagebereichen fällt auf, dass ländliche Banken hier im Wesentlichen eine Stagnation aufweisen. In halbstädtischen und städtischen Bereichen konnten die Genossenschaftsbanken ihr Provisionsergebnis über die Jahre ausbauen, lediglich in den Jahren 2007 bis 2009 blieb es, bedingt durch die Finanzkrise, bei nur einem leichten Wachstum des absoluten Provisionsüberschusses. Eine grafische Darstellung befindet sich in Abbildung 43. 


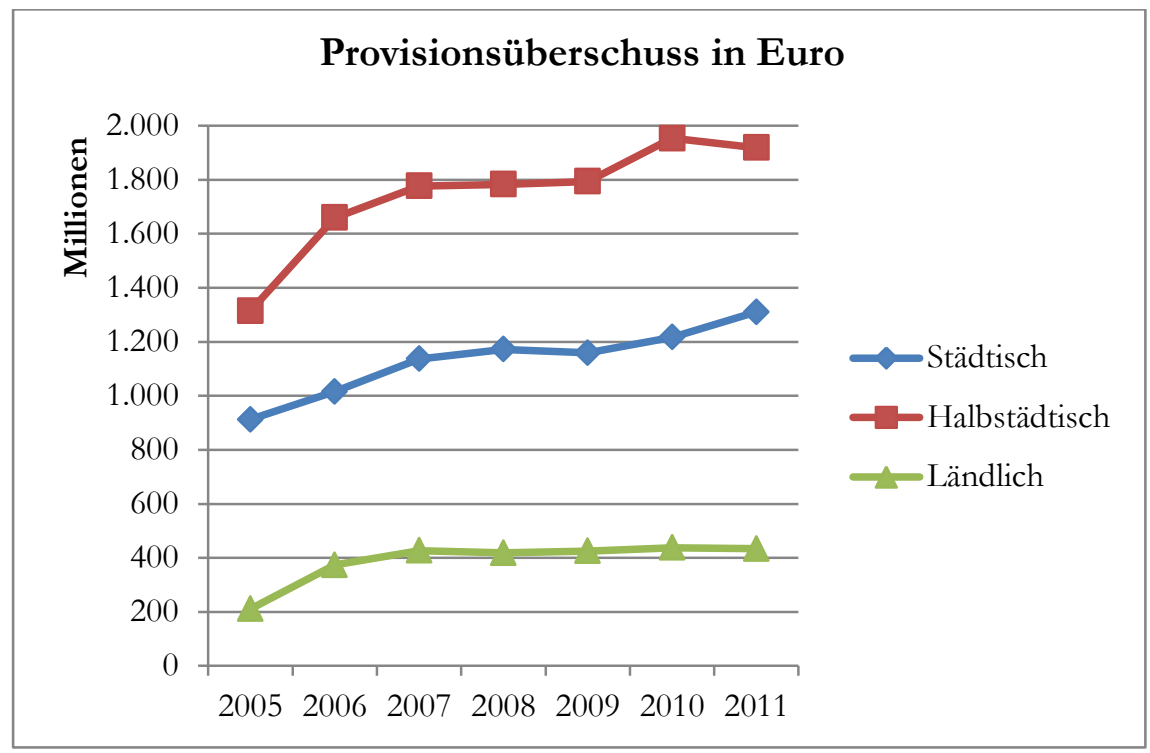

Abbildung 43: Entwicklung des nach Lagebereichen kumulierten Provisionsüberschusses

PADBERG ergänzt, dass neben der eigentlichen Höhe der Provisionserträge auch die Effizienz der Banken einen Einfluss auf die Kennzahl hat. ${ }^{922}$ Eine Vergleichbarkeit sei nur bei effizientem Mitteleinsatz durch die Banken gegeben. ${ }^{923}$

\subsubsection{Hypothese 8: Personalaufwand}

Die Hypothese lautet:

\section{Banken in ländlichen Regionen unterscheiden sich binsichtlich ibres Personalauf- wands von Banken in urbanen Gebieten.}

Zur Überprüfung dieser Hypothese wird der Personalaufwand herangezogen. Für den ersten Untersuchungsschritt wird dieser Personalaufwand in das Verhältnis zur durchschnittlichen Bilanzsumme gesetzt und in Gestalt der sogenannten Personalaufwandsspanne verwendet. ${ }^{924}$ Zum Vergleich der Personalaufwandsspanne über die drei Lagebereiche hinweg werden wieder die Mittelwerte aus den Personalaufwandsspannen aller Banken des jeweiligen Bereichs gebildet. Die Resultate der entsprechenden Varianzanalyse sind in Tabelle 32 dargestellt.

\begin{tabular}{|c|c|c|c|c|}
\hline & $\begin{array}{c}\text { Städtisch } \\
(n=151-159)\end{array}$ & $\begin{array}{l}\text { Halbstädtisch } \\
(\mathrm{n}=516-571)\end{array}$ & $\begin{array}{c}\text { Ländlich } \\
(\mathrm{n}=260-297)\end{array}$ & $\begin{array}{c}\text { F } \\
\text { (Welch) }\end{array}$ \\
\hline 2005 & $1,41 \% \mathrm{o}^{\mathrm{a}}$ & $1,68 \%{ }^{\mathrm{b}}$ & $1,72 \%{ }^{\mathrm{b}}$ & $22,41 * * *$ \\
\hline 2006 & $1,48 \% 0^{\mathrm{a}}$ & $1,70 \% \mathrm{o}^{\mathrm{b}}$ & $1,76 \%{ }^{\mathrm{b}}$ & $16,10^{* * *}$ \\
\hline 2007 & $1,35 \% \mathrm{a}^{\mathrm{a}}$ & $1,58 \%{ }^{\mathrm{b}}$ & $1,66 \%{ }^{c}$ & $26,67 * * *$ \\
\hline 2008 & $1,31 \% \mathrm{o}^{\mathrm{a}}$ & $1,50 \% \mathrm{o}^{\mathrm{b}}$ & $1,62 \%{ }^{c}$ & $30,27 * * *$ \\
\hline 2009 & $1,31 \% \mathrm{a}^{\mathrm{a}}$ & $1,51 \% \mathrm{o}^{\mathrm{b}}$ & $1,62 \%{ }^{c}$ & $27,60 * * *$ \\
\hline 2010 & $1,23 \% \mathrm{a}^{\mathrm{a}}$ & $1,42 \% \mathrm{~b}^{\mathrm{b}}$ & $1,51 \%{ }^{c}$ & $28,18^{* * *}$ \\
\hline 2011 & $1,19 \% \%^{a}$ & $1,38 \%$ b & $1,46 \%{ }^{c}$ & $28,38 * * *$ \\
\hline
\end{tabular}

Tabelle 32: $\quad$ Entwicklung der Personalaufwandsspanne nach Lagebereichen

\footnotetext{
922 Vgl. Padberg (2005), S. 47. 
Für alle betrachteten Jahre kann belegt werden, dass Banken in städtischer Lage im Mittel die niedrigste Personalaufwandsspanne haben. Dagegen weisen Banken in ländlicher Lage die höchsten Aufwendungen auf. Institute in halbstädtischer Lage liegen für alle betrachteten Zeiträume dazwischen. Folglich zeichnet sich hier ein deutliches Stadt-Land-Gefälle ab, für jedes Geschäftsjahr belegt durch ein sehr hohes Signifikanzniveau der Unterschiede. Eine Darstellung findet sich in Abbildung 44.

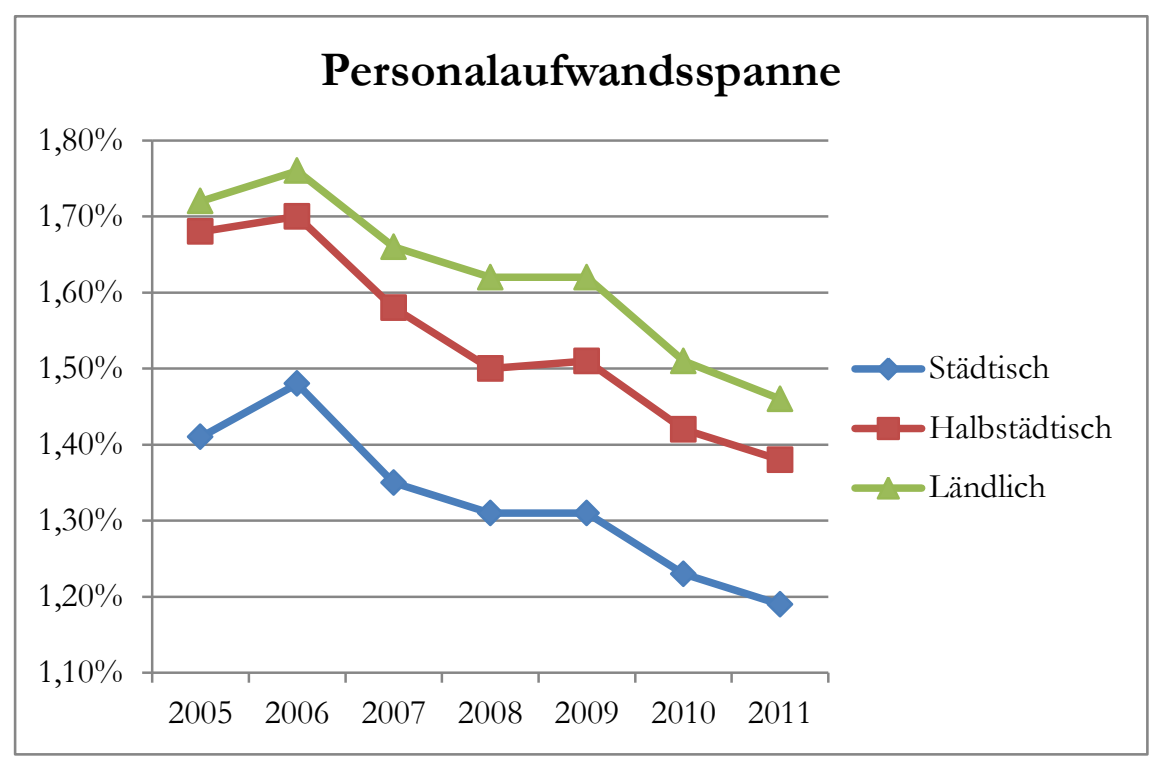

Abbildung 44: Entwicklung der Personalaufwandsspanne nach Lagebereichen

Tabelle 33 liefert die Resultate der OLS-Regression. Im Vergleich zu den städtischen Instituten st liegen die Personalausgaben der übrigen Institute höher. Banken im halbstädtischen Lagebereich ha zahlen im Mittel 20,7 Basispunkte mehr, im ländlichen Bereich la im Mittel gar 29,2 Basispunkte mehr.

\begin{tabular}{|c|c|}
\hline VARIABLE & $\begin{array}{l}\text { (8) } \\
\text { pks }\end{array}$ \\
\hline o.st & - \\
\hline ha & $\begin{array}{c}0,207 * * * \\
(0,0119)\end{array}$ \\
\hline la & $\begin{array}{c}0,292^{* * *} \\
(0,0131)\end{array}$ \\
\hline Konstante & $\begin{array}{l}1,336 \text { *** } \\
(0,0105)\end{array}$ \\
\hline Beobachtungen & 7.081 \\
\hline $\mathrm{R}^{2}$ & 0,066 \\
\hline
\end{tabular}

Tabelle 33:

Einfluss der Lage auf die Personalaufwandsspanne (OLS) 
In einem zweiten Untersuchungsschritt werden die Personalaufwendungen in das Verhältnis zur Anzahl der Mitarbeiter gesetzt. Der Quotient ist als Durchschnittswert der Aufwendungen pro Mitarbeiter im jeweiligen Geschäftsjahr zu interpretieren. ${ }^{925}$ Wie bereits oben beschrieben, zählen zu diesen Aufwendungen die Löhne und Gehälter von Mitarbeitern und Geschäftsführung sowie Sozialabgaben, Altersversorgung und sonstige Unterstützungsleistungen. ${ }^{926}$ Die Höhe der Personalaufwendungen hängt von der Qualifikation des Personals, von der Komplexität der Prozesse und der Produkte und auch von der Vergütungsstruktur der jeweiligen Bank ab. ${ }^{927}$ Die Resultate der Varianzanalyse werden in Tabelle 34 dargestellt.

\begin{tabular}{|c|c|c|c|c|c|}
\hline & $\begin{array}{c}\text { Städtisch } \\
(\mathrm{n}=130-153)\end{array}$ & $\begin{array}{l}\text { Halbstädtisch } \\
\qquad(\mathrm{n}=361-524)\end{array}$ & $\begin{array}{c}\text { Ländlich } \\
(\mathrm{n}=101-271)\end{array}$ & $\begin{array}{c}\mathbf{F} \\
(\mathrm{ANOVA})\end{array}$ & $\begin{array}{c}\mathbf{F} \\
\text { (Welch) }\end{array}$ \\
\hline 2005 & $54.783^{a}$ & $52.109^{b}$ & $51.581^{\mathrm{b}}$ & $5,93 * *$ & \\
\hline 2006 & $56.992^{\mathrm{a}}$ & $53.447 \mathrm{~b}$ & 55.030 & & $8,43^{* * *}$ \\
\hline 2007 & $54.537^{a}$ & $51.423^{b}$ & $53.675^{a}$ & & $10,09 * * *$ \\
\hline 2008 & $55.897^{a}$ & $52.303^{b}$ & $53.826^{\mathrm{b}}$ & & $11,48^{* * *}$ \\
\hline 2009 & $58.094^{a}$ & $55.415^{\mathrm{b}}$ & 56.074 & & $5,33^{* *}$ \\
\hline 2010 & $56.406^{a}$ & $52.947^{b}$ & $54.342^{\mathrm{b}}$ & & $10,90 * * *$ \\
\hline 2011 & $55.930^{\mathrm{a}}$ & $53.022^{\mathrm{b}}$ & 54.170 & & $8,94 * * *$ \\
\hline
\end{tabular}

Tabelle 34: Entwicklung des Personalaufwands je Mitarbeiter nach Lagebereichen

Durch eine abweichende Sichtweise ergibt sich hier ein anderes Bild. In jedem Geschäftsjahr können die höchsten Personalaufwendungen je Mitarbeiter für die städtische Lage nachgewiesen werden, häufig mit sehr hohen Signifikanzen der Unterschiede. Eine grafische Darstellung folgt in Abbildung 45.

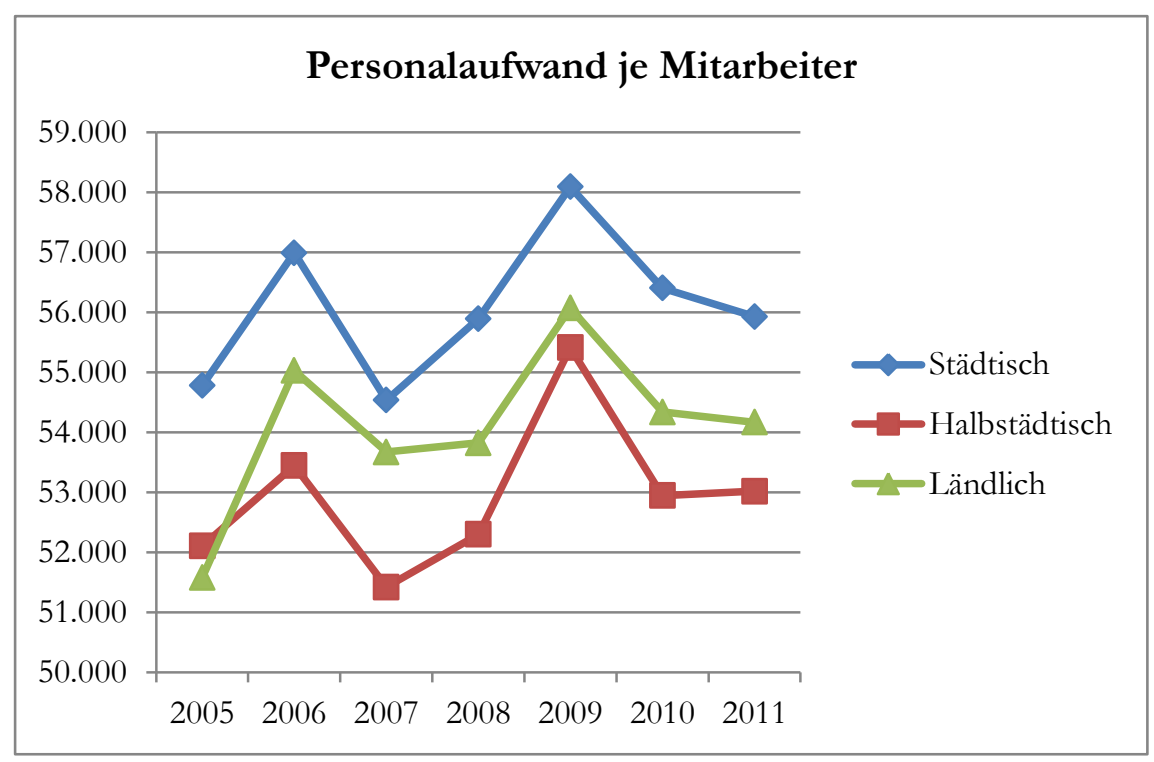

Abbildung 45: Entwicklung des Personalaufwands je Mitarbeiter nach Lagebereichen 
Ein dritter Untersuchungsschritt betrachtet für jedes Geschäftsjahr, welchen Bruttogewinn aus normaler Geschäftstätigkeit ein jeder Mitarbeiter im Durchschnitt erwirtschaftet hat. ${ }^{928}$ In den Städten fällt dieser Quotient deutlich höher aus als auf dem Lande, lediglich 2005 weicht ab. Der Zusammenhang ist in Abbildung 46 dargestellt.

Ein vierter Untersuchungsschritt erkundet, welcher Anteil der jahresdurchschnittlichen Bilanzsumme auf einen einzelnen Mitarbeiter entfällt. ${ }^{929}$ Auch hier liegt der Quotient städtischer Banken deutlich höher als der Quotient halbstädtischer oder ländlicher Banken. Dargestellt ist der Zusammenhang in Abbildung 47.

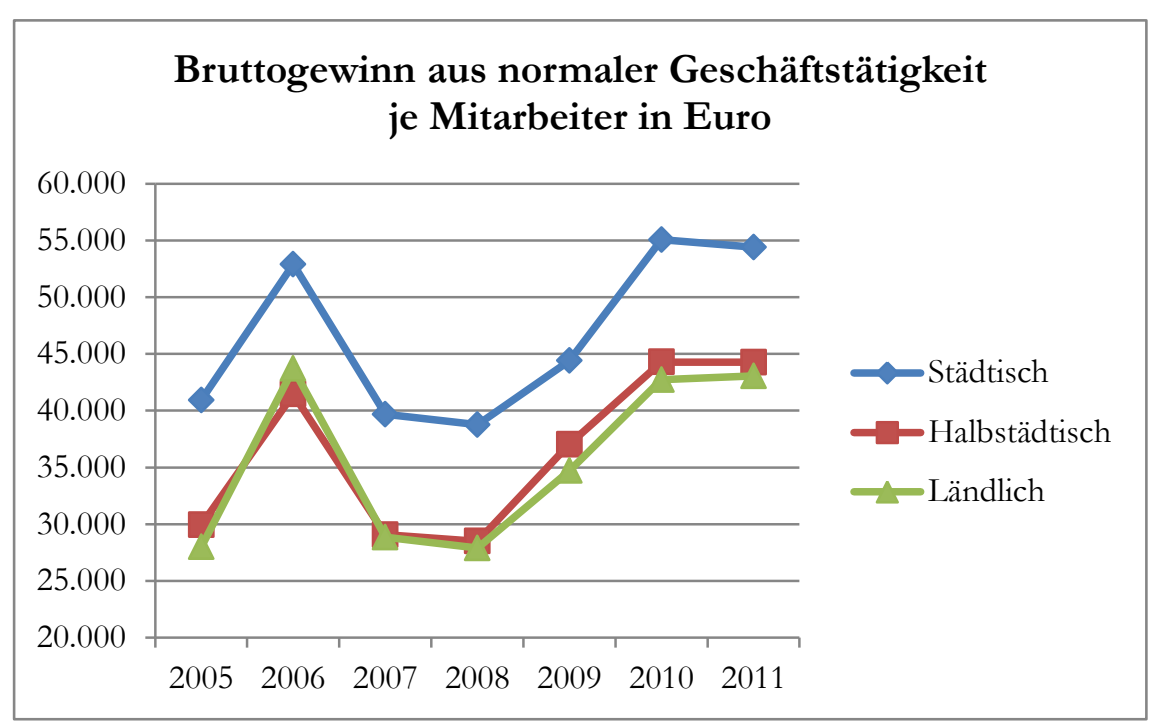

Abbildung 46: Entwicklung des Bruttogewinns aus normaler Geschäftstätigkeit je Mitarbeiter nach Lagebereichen

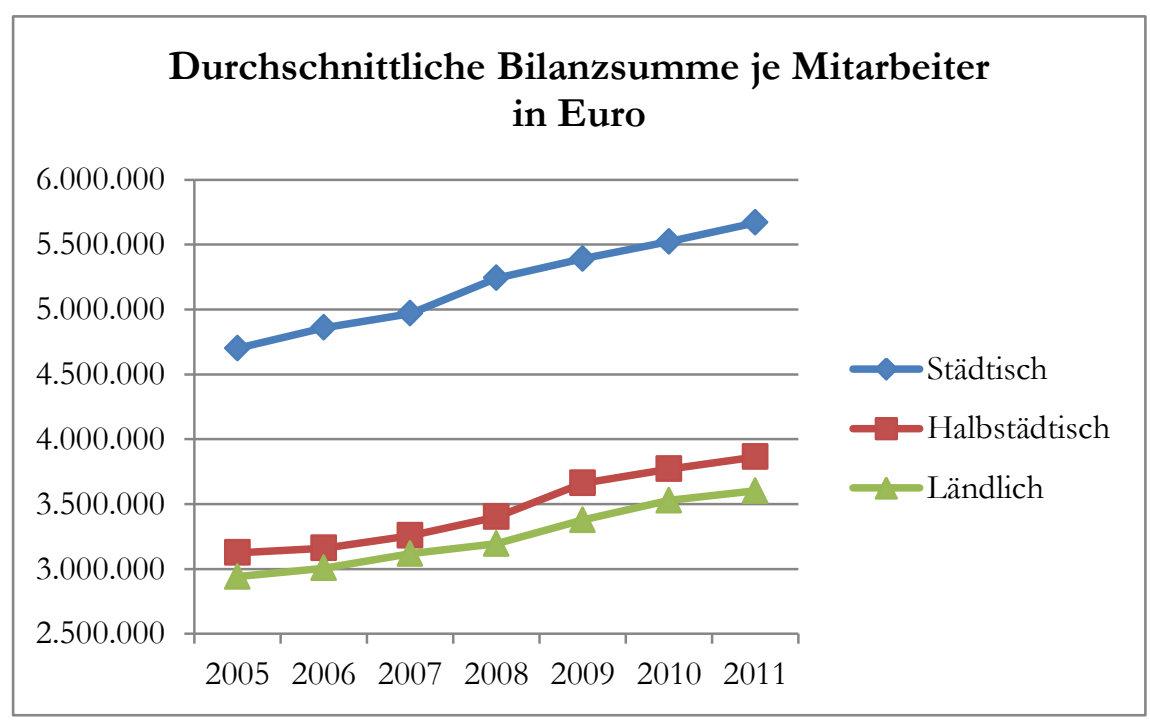

Abbildung 47: Entwicklung der durchschnittlichen Bilanzsumme je Mitarbeiter nach Lagebereichen 
Im Folgenden sollen die Resultate aller vier Untersuchungsschritte geschlossen interpretiert werden. Denn obwohl sich die Graphen der einzelnen Schritte zunächst zu widersprechen scheinen, lassen sich die Ergebnisse konsistent erklären.

Ad Abbildung 44: Untersuchungsschritt eins attestiert den ländlichen Instituten eine relativ hohe Personalaufwandsspanne, während sie in städtischen Instituten niedriger liegt. Das heißt, bezogen auf einen Euro durchschnittlicher Bilanzsumme wird auf dem Land mehr für das Personal ausgegeben. Zurückzuführen ist dies darauf, dass die Filialen im ländlichen Bereich im Mittel eine nicht so hohe Auslastung aufweisen, was an der geringeren Bevölkerungsdichte vor Ort liegt. Es gibt dort schlicht nicht so viele Kunden wie in städtischen Bereichen. Zusätzlich gibt es weniger Kunden mit hohen Guthaben. In ihren Geschäftsgebieten verfolgen die Genossenschaftsbanken das Ziel, die Wirtschaft ihrer Mitglieder zu fördern. ${ }^{930}$ Hierzu ist die Wahrung der Präsenz in der Fläche erforderlich. Aus geschäftspolitischen Gründen werden so auch unrentable Filialen auf dem Lande vorgehalten und weiter betrieben. Selbst in der kleinsten Filiale müssen allein zur Einhaltung der DGUVVorschrift $25^{931}$ (früher UVV Kassen) jederzeit mindestens zwei Mitarbeiter präsent sein. ${ }^{932}$ Den einerseits überdurchschnittlichen Personal- und auch Sachaufwendungen kleiner Filialen stehen jedoch andererseits höhere Zins- und Provisionsüberschüsse gegenüber, was insgesamt, wie bereits in Abbildung 27 dargestellt, sogar in einer höheren Bruttogewinnspanne von Instituten auf dem Lande resultiert. Durch den geringeren Wettbewerb und die hierdurch möglichen höheren Margen ist daher oft zumindest ein kostendeckender Betrieb der ländlich gelegenen Filialen möglich. Die Genossenschaftsbanken in den Städten hingegen haben im Mittel eine bessere Filialauslastung, da es eine hohe Anzahl von Personen im direkten Umkreis bzw. im Einzugsgebiet gibt. Per definitionem liegt die Bevölkerungsdichte in städtischen Regionen bei mehr als 500 Einwohnern je Quadratkilometer, häufig auch noch deutlich darüber. Etwa Deutschlands am dichtesten besiedelte Gemeinde München beherbergt im Mittel mehr als 4.000 Einwohner je Quadratkilometer. ${ }^{933}$ Laut THIRY deutet eine hohe Personalaufwandsspanne auf ein ineffizientes Management hin. ${ }^{934}$ Es sind jedoch darüber hinaus noch weitere Gründe für eine hohe Spanne möglich, wie etwa im Falle einer ungewöhnlich kleinen Bilanzsumme denkbar.

Ad Abbildung 45: Betrachtet man den Personalaufwand je Mitarbeiter, so fällt auf, dass dieser in Städten deutlich höher liegt als in anderen Lagen. In den Städten wird demnach ein höheres Gehalt gezahlt als auf dem Lande. Jedoch müssen die städtischen Banken ihren Mitarbeitern auch mehr Gehalt bieten, da in der Regel die Mieten und häufig auch die Lebenshaltungskosten in den Städten höher liegen. Der Personalaufwand je Mitarbeiter beinhaltet die Arbeitgeberanteile der Sozialabgaben ${ }^{935}$ und entspricht somit dem Arbeitnehmerentgelt aus der volkswirtschaftlichen Gesamtrechnung. Nicht nur Banken zahlen in Städten höhere Löhne und Gehälter als auf dem Lande. Wie die spätere Untersuchung zeigen wird ${ }^{936}$, besteht dieser Zusammenhang auch über alle übrigen Branchen hinweg. Dies wird anhand von Abbildung 69 belegt. PIEPER konnte zusätzlich für den Zeitraum 1992-2002 empirisch belegen, dass in Städten ein deutlich höherer Anteil der Bankbeschäf-

\footnotetext{
${ }^{930} \mathrm{Vgl}$. Abschnitt 3.1.1.

931 Vgl. Deutsche Gesetzliche Unfallversicherung e.V. [DGUV] (1997), S. 5.

932 Vgl. \ 18 Abs. 1 Satz 2 DGUV Vorschrift 25 - Kassen (bisher BGV C9/UVV Kassen).

933 Vgl. Statistisches Bundesamt (2012a).

934 Vgl. Thiry (2009), S. 158.

935 Vgl. Abschnitt 3.6.3.7.

936 Vgl. Abschnitt 4.4.19.
} 
tigten über einen Hochschulabschluss verfügt. ${ }^{937}$ Zudem nahm dieser Anteil in allen Lagebereichen über den Zeitraum hinweg stetig zu. PIEPER rechnet darüber hinaus mit einem weiter wachsenden Anteil. ${ }^{938}$ Es ist davon auszugehen, dass ein Hochschulabschluss auch zu einer höheren Entlohnung beiträgt, was mit höheren durchschnittlichen Personalaufwendungen bei städtischen Banken verbunden ist. Sind Bankmitarbeiter in städtischen Regionen also eher knapp ${ }^{939}$, so können sie eine höhere Vergütung aushandeln. Ceteris paribus kann für ländliche Regionen abgeleitet werden, dass dort eine bessere Verfügbarkeit, zumindest jedoch keine Knappheit von Bankmitarbeitern besteht. Vergleicht man die Personalaufwendungen je Mitarbeiter speziell zwischen balbstädtischen und ländlichen Regionen, so fällt auf, dass der Verdienst in ländlichen Regionen noch sichtbar über dem Verdienst in balbstädtischen Regionen liegt. ${ }^{940}$ Laut SPIES trifft die Landflucht von gut ausgebildeten Mitarbeitern die Filialbanken auf dem Lande. ${ }^{941}$ Möglicherweise ist die etwas höhere Entlohnung durch ländliche Banken Ausdruck des Versuchs, der Abwanderung von gut ausgebildeten Mitarbeitern zu begegnen.

Ad Abbildung 46: Der Bruttogewinn aus normaler Geschäftstätigkeit je Mitarbeiter spiegelt wider, welchen Beitrag ein jeder Mitarbeiter im Durchschnitt zur Bruttogewinnspanne geleistet hat. Die höchsten Ergebnisse liegen in allen Geschäftsjahren bei den städtischen Instituten. Zur Ermittlung der Werte wurde der über alle Genossenschaftsbanken im jeweiligen Lagebereich und Jahr aggregierte Bruttogewinn aus normaler Geschäftstätigkeit in das Verhältnis zur entsprechenden aggregierten Anzahl der Mitarbeiter gesetzt. Für einen Teil der Begründung kann wieder auf Abbildung 44 verwiesen werden, da ein auf dem Lande hoher Personalaufwand rechnerisch direkt zu einem niedrigeren Bruttogewinn je Mitarbeiter führt. Hinzu kommt, dass Kunden städtischer Banken im Mittel höhere Guthaben führen, wodurch der Verwaltungsaufwand je Euro Einlage tiefer liegt. Zudem werden in Städten auch anspruchsvollere Geschäftsarten nachgefragt, mit denen auf Bankseite eine höhere Marge generiert werden kann, z. B. durch qualifizierte Wertpapierberatungsgeschäfte. Daher kann mit einem Kunden einer städtischen Bank im Mittel ein höherer Deckungsbeitrag erwirtschaftet werden. Die höheren Personalaufwendungen je Mitarbeiter städtischer Banken (Abbildung 45) können so wieder eingespielt werden. Das durchschnittliche Guthaben von Kunden ländlicher Banken ist hingegen geringer. Bei geringeren durchschnittlichen Guthaben müssen jedoch entsprechend mehr Kunden betreut werden. Dieser höhere Kundenbetreuungsaufwand schlägt sich in niedrigeren Margen bzw. einem niedrigeren Bruttogewinn je Mitarbeiter nieder.

Ad Abbildung 47: Die Durchschnittsbilanzsumme je Mitarbeiter ist bei städtischen Banken höher. Diese Tatsache untermauert die Interpretation von Abbildung 46. Jeder Mitarbeiter eines städtischen Instituts betreut daher im Durchschnitt ein deutlich höheres Einlagebzw. Kreditvolumen. Dies wiederum ermöglicht es den Mitarbeitern städtischer Banken, im Mittel einen höheren Beitrag zum Bruttogewinn aus normaler Geschäftstätigkeit zu erzielen. Als Begründung kann hierbei auf die Skaleneffekte verwiesen werden. ${ }^{942}$ Häufig ist der Beratungs- und Betreuungsaufwand nicht stark abhängig vom Geschäftsumfang. Ver-

\footnotetext{
937 Vgl. Pieper (2005), S. 113.

938 Vgl. Pieper (2005), S. 114.

${ }^{939}$ Vgl. Breyer (2007), S. 87.

940 Vgl. Abbildung 45.

${ }_{941}$ Vgl. Spies (2015), S. 54.

${ }_{942}$ Vgl. Abschnitt 3.4.3.
} 
gleicht man etwa die Vergabe zweier Immobilienkredite mit 100.000 EUR und 500.000 EUR, so dürfte sich der Aufwand in einer ähnlichen Größenordnung bewegen. Die erzielte Marge jedoch ist in der Regel direkt proportional zum Geschäftsumfang. Doch nicht nur die Betrachtung der Durchschnittsbilanzsumme je Mitarbeiter fällt zugunsten der städtischen Banken aus. Auch bei der Untersuchung der reinen durchschnittlichen Bilanzsummen liegen die städtischen Institute weit vorn: Wie unten in Abbildung 52 dargelegt ${ }^{943}$, weisen Genossenschaftsbanken in städtischer Lage im Mittel deutlich höhere Durchschnittsbilanzsummen auf als solche in halbstädtischer oder ländlicher Lage. Das heißt, in den Städten sind weitaus größere Institute vorhanden als auf dem Lande.

\section{Die Veränderung der Personalaufwandsspanne von 2005 bis 2011}

Für jeden der drei Lagebereiche Städtisch, Halbstädtisch und Ländlich lässt sich anhand von Tabelle 32 sehen, dass die Personalaufwandsspanne von 2005 zu 2011 annähernd stetig abnahm. ${ }^{944}$ Somit verringert sich auch der über alle Banken aggregiert berechnete Mittelwert über den Betrachtungszeitraum hinweg, wie aus Tabelle 35 hervorgeht. Die Werte werden grafisch in Abbildung 48 dargestellt.

\begin{tabular}{|c|c|}
\hline Jahr & Personalaufwandsspanne, errechnet über alle Banken \\
\hline 2005 & $1,65 \%$ \\
\hline 2006 & $1,68 \%$ \\
\hline 2007 & $1,57 \%$ \\
\hline 2008 & $1,51 \%$ \\
\hline 2009 & $1,51 \%$ \\
\hline 2010 & $1,41 \%$ \\
\hline 2011 & $1,37 \%$ \\
\hline
\end{tabular}

Tabelle 35: $\quad$ Entwicklung der Personalaufwandsspanne über alle Banken

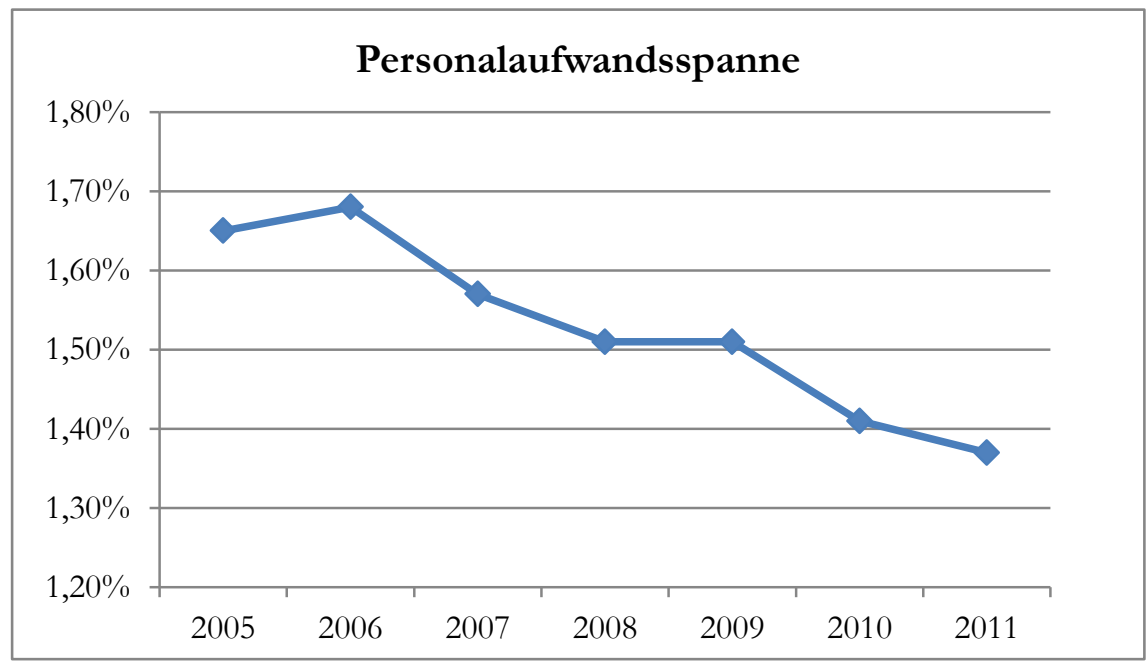

Abbildung 48: Entwicklung der Personalaufwandsspanne über alle Banken 
Die durchschnittliche Personalaufwandsspanne hat sich über den Betrachtungszeitraum 2005 bis 2011 hinweg von 1,65 \% auf 1,37\% reduziert, was einem Rückgang von 20,4 \% entspricht. Folgende Gründe für diesen Rückgang werden vermutet:

1. Die Anzahl der Mitarbeiter hat sich reduziert.

2. Die durchschnittliche Bilanzsumme hat sich erhöht.

3. Die Personalaufwendungen oder Gehälter haben sich verändert.

Das verwendete Erklärungsmodell arbeitet mit sogenannten Veränderungsfaktoren. Hierbei wird der Ausgangswert $A W$ mit verschiedenen Veränderungsfaktoren $V F_{1} \ldots V F_{n}$ multipliziert, um den Endwert $E W$ zu erklären:

$$
A W \cdot V F_{1} \cdot V F_{2} \cdot \ldots \cdot V F_{n}=E W
$$

Ad 1. Im Jahr 2005 arbeiteten 162.550 Mitarbeiter im Bankgeschäft der Kreditgenossenschaften. ${ }^{945} 2011$ lag dieser Wert bei 158.250, was einer Reduktion der Mitarbeiteranzahl von 2,65\% über den genannten Zeitraum entspricht ${ }^{946}$, bzw. einer Multiplikation mit dem Veränderungsfaktor $V F_{1}=0,9735$. Isoliert betrachtet führt dies zu einer Reduktion des Aufwands für die Mitarbeitervergütung.

Ad 2. Die Personalaufwandsspanne errechnet sich als Prozentanteil der Personalaufwendungen an der durchschnittlichen Bilanzsumme. Über die Jahre hinweg liegt jedoch für die meisten Banken ein Wachstum der durchschnittlichen Bilanzsumme vor. Ceteris paribus führt dieser Wachstumseffekt allein zu einer Verringerung der Personalaufwandsspanne. Wie Tabelle 36 zeigt, hat sich die Bilanzsumme aller Banken im Mittel tatsächlich von Jahr zu Jahr erhöht. Dies gilt für alle Geschäftsjahre im Betrachtungszeitraum 2006 bis 2011, jeweils im Vergleich zum Vorjahr. Betrachtet wurden alle vorhandenen Fälle eines Jahres, zu dessen Vorjahr ebenfalls Daten vorliegen. Um Verzerrungen durch Überzeichnungen auszuschließen, wurden Datensätze von denjenigen Jahren ausgenommen, in denen die Bilanzsumme durch eine Fusion anstieg. Neben dem Neukundengeschäft und fortgesetzter Ansparbemühungen seitens der Kunden ist ein weiterer Grund für das stetige Wachstum der Bilanzsummen, dass erzielte Zinserträge nicht von den jeweiligen Konten abgehoben werden. Auf diese Weise können sich diese über Jahre kumulieren. Durch Multiplikation der einzelnen Veränderungsfaktoren in Tabelle 36 ergibt sich $V F_{2}=0,8038$. Dabei liegt eine geometrische Berechnung zugrunde. 


\begin{tabular}{|c|c|c|c|}
\hline $\begin{array}{c}\text { Betrach- } \\
\text { tungsjahr }\end{array}$ & $\begin{array}{l}\text { Bilanzsummenwachstum im } \\
\text { Vergleich zum Vorjahr }\end{array}$ & $\begin{array}{l}\text { Veränderungs- } \\
\text { faktoren }\end{array}$ & $\begin{array}{l}\text { Anzahl } \\
\text { Datensätze }\end{array}$ \\
\hline 2006 & $2,23 \%$ & 0,9782 & 1022 \\
\hline 2007 & $2,99 \%$ & 0,9710 & 1028 \\
\hline 2008 & $5,62 \%$ & 0,9468 & 1000 \\
\hline 2009 & $4,19 \%$ & 0,9598 & 961 \\
\hline 2010 & $3,42 \%$ & 0,9669 & 965 \\
\hline 2011 & $3,83 \%$ & 0,9631 & 933 \\
\hline
\end{tabular}

Tabelle 36: Bilanzsummenwachstum im Vergleich zum Vorjahr

Ad 3. Betrachtet man die Entwicklung der absoluten Personalaufwendungen von Jahr zu Jahr im selben Zeitraum anhand von Tabelle 37, so fallen mehrere Aspekte auf. Zunächst schwanken die Personalaufwendungen von Jahr zu Jahr. Diese Schwankungen sind insbesondere auf variable Vergütungsmodelle zurückzuführen. Ermittelt man durch Multiplikation der einzelnen Veränderungsfaktoren den Gesamtwert, so ergibt sich $V F_{3}=1,0195$. Dieser Wert ist größer als eins und zeigt einen Anstieg der gesamten Personalaufwendungen, obwohl sich die Mitarbeiteranzahl gemäß $V F_{1}$ reduziert hat. Dies ist darauf zurückzuführen, dass sich die Gehälter in regelmäßigen Abständen leicht erhöhen, entweder durch den Aufstieg in eine höhere Tarifgruppe ${ }^{947}$ oder durch die Erhöhung der Vergütungssätze der einzelnen Tarifgruppen, i. d. R. mit jedem neuen Abschluss eines Tarifvertrages.

\begin{tabular}{|c|c|c|c|}
\hline $\begin{array}{c}\text { Betrach- } \\
\text { tungsjahr }\end{array}$ & $\begin{array}{l}\text { Personalaufwandsveränderung } \\
\text { im Vergleich zum Vorjahr }\end{array}$ & $\begin{array}{l}\text { Veränderungs- } \\
\text { faktoren }\end{array}$ & $\begin{array}{l}\text { Anzahl } \\
\text { Datensätze }\end{array}$ \\
\hline 2006 & $1,71 \%$ & 0,9832 & 929 \\
\hline 2007 & $-3,32 \%$ & 1,0343 & 954 \\
\hline 2008 & $0,95 \%$ & 0,9906 & 951 \\
\hline 2009 & $4,23 \%$ & 0,9594 & 902 \\
\hline 2010 & $-2,30 \%$ & 1,0235 & 938 \\
\hline 2011 & $0,85 \%$ & 0,9916 & 907 \\
\hline
\end{tabular}

Tabelle 37: Durchschnittliche Personalaufwandsveränderung im Vergleich zum Vorjahr

Zur Erklärung des Endwertes ergibt sich das multiplikative Modell

$$
1,65 \% \cdot 0,9735 \cdot 0,8038 \cdot 1,0195=1,32 \% \text {. }
$$

Es verbleibt eine geringe Differenz, welche mit den vorliegenden Faktoren nicht erklärt werden kann. Diese könnte aus dem fusionsbedingten Ausschluss von Fällen, aus einer nicht hundertprozentigen Datenverfügbarkeit sowie weiteren Gründen resultieren. 
Abschließend erfolgt in Abbildung 49 die Darstellung der Verteilung der Mitarbeiteranzahl beispielhaft am Geschäftsjahr 2011. Es ist unmittelbar sichtbar, dass in städtischer Lage zahlreiche Institute mit hoher Mitarbeiterzahl existieren, während die absolute Zahl städtischer Institute relativ gering ist. Für die übrigen beiden Lagebereiche halbstädtisch und ländlich ist dies umgekehrt.
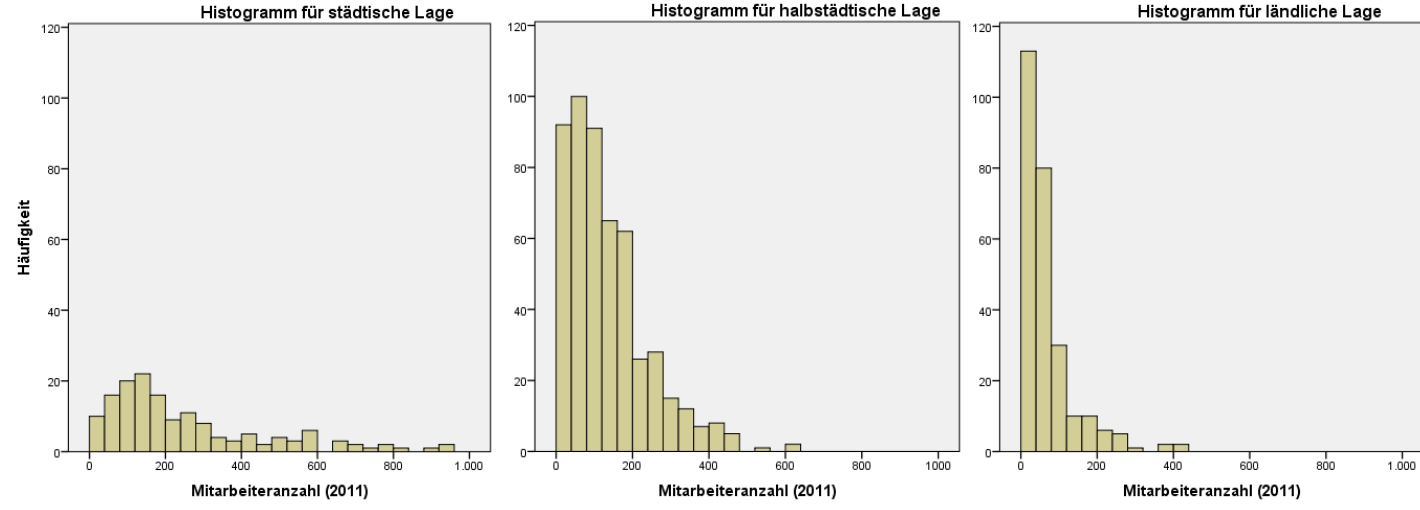

Abbildung 49: Lageabhängige Häufigkeitsverteilung der Bankgrößen nach Mitarbeiteranzahl (2011)

Aus dem Boxplot in Abbildung 50 lässt sich für das Geschäftsjahr 2011 die mittlere Mitarbeiteranzahl und deren Verteilung ablesen. Der Median liegt für städtische Institute bei 185, für halbstädtische bei 106 und für ländliche bei 46.

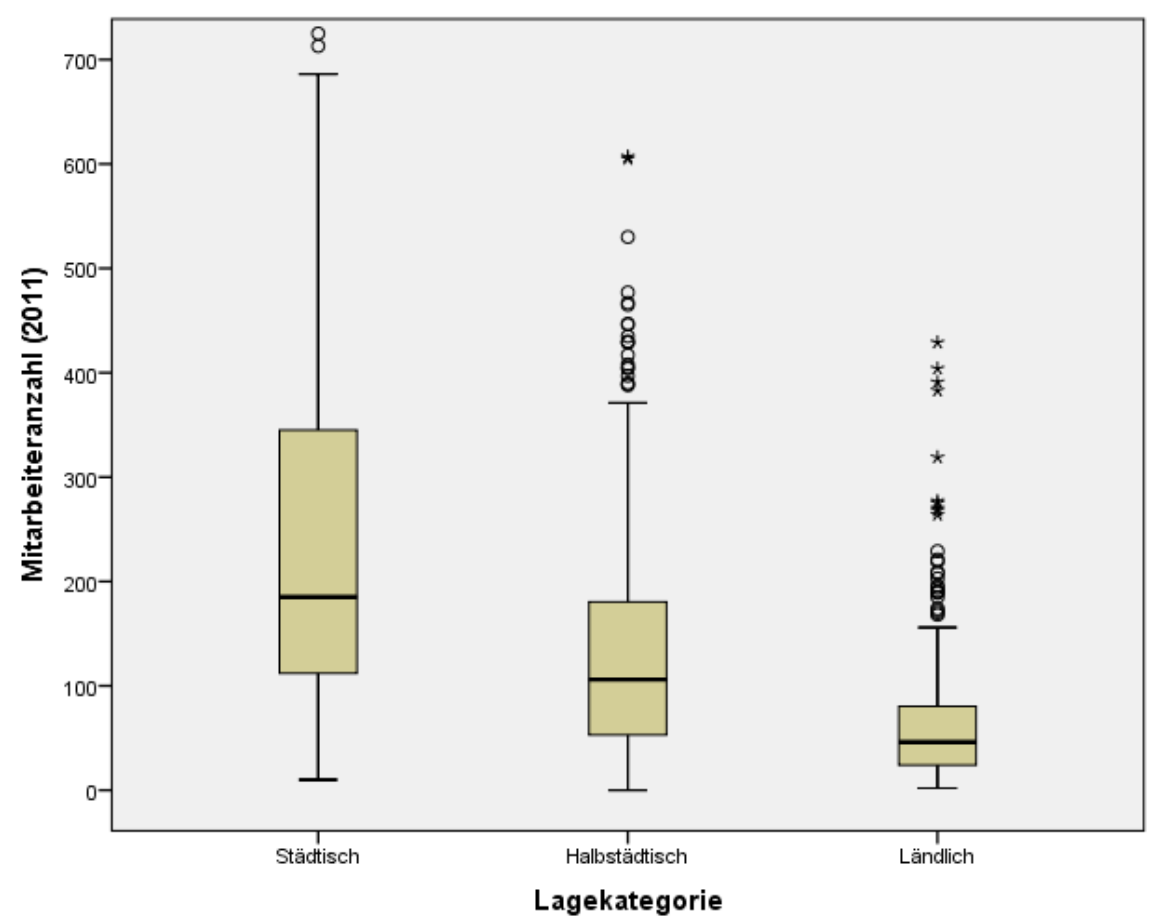

Abbildung 50: Einfluss der Lage auf die Mitarbeiteranzahl 


\subsubsection{Hypothese 9: Sachaufwand}

Die Hypothese lautet:

Banken in ländlichen Regionen unterscheiden sich binsichtlich ibres erweiterten Sachaufreands von Banken in urbanen Gebieten.

Dieser Hypothese liegt die Annahme zugrunde, dass sich die Zugehörigkeit zu einer der Lageklassen Städtisch, Halbstädtisch oder Ländlich auf die Höhe des Sachaufwands auswirkt. Hierbei kommt das Konstrukt des erweiterten Sachaufwands zum Einsatz, da die Volumina der Aufwandsposten 10. b), 11. und 12. als eine aggregierte Ziffer für die Auswertung zur Verfügung stehen. Jedoch besitzt Position 12 betragsmäßig ein geringes Volumen im Vergleich zu den Positionen 10. b) und 11., daher geht von der beschriebenen Handhabung keine nennenswerte Verzeichnung aus. Der Anteil von Position 12 am erweiterten Sachaufwand beträgt in der Regel etwa 2 bis 3 Prozent. Die Berechnung des erweiterten Sachaufwands geschieht anhand der in Tabelle 38 dargestellten Rechenvorschrift.

\begin{tabular}{|l|l|}
\hline \multicolumn{1}{|c|}{ Berechnung des erweiterten Sachaufwands } \\
\hline$+\quad \begin{array}{l}\text { 10. b) Andere Verwaltungsaufwendungen } \\
\text { Sachanlagen }\end{array}$ \\
\hline$+\quad$ 12. Sonstige betriebliche Aufwendungen \\
\hline$=\quad$ Erweiterter Sachaufwand
\end{tabular}

Tabelle 38: $\quad$ Berechnung des erweiterten Sachaufwands

$\mathrm{Zu} \mathrm{10.} \mathrm{b)} \mathrm{gehören} \mathrm{z.} \mathrm{B.} \mathrm{Aufwendungen} \mathrm{für} \mathrm{Geschäftsräume} \mathrm{oder} \mathrm{Kommunikation,} \mathrm{zu} 11$. zählen etwa Abschreibungen auf EDV-Software, Geschäftsgebäude oder Ausstattung und zu 12. werden Aufwendungen gerechnet, die zwar betrieblich sind, aber keinem anderen Aufwandsposten zugeordnet sind. ${ }^{948}$ Vereinfachend wird der erweiterte Sachaufwand im Folgenden als Sachaufwand bezeichnet.

Wie aus Tabelle 39 hervorgeht, befindet sich der Sachaufwand von Banken in halbstädtischer und in ländlicher Lage für alle betrachteten Geschäftsjahre im Mittel über dem Wert für Banken in städtischer Lage. In Bezug auf die durchschnittliche Bilanzsumme wenden Banken in halbstädtischer und ländlicher Lage also mehr für die Verwaltung auf.

Dies kann auf folgenden Grund zurückgeführt werden: Die Auslastung von Filialen ist im ländlichen Raum gewöhnlich geringer als in städtischer Lage. Hierdurch können Banken in ländlicher Lage weniger von der Fixkostendegression profitieren. ${ }^{949}$ Wie in Hypothese 8 untersucht, befinden sich größere Banken eher in städtischer Lage. Dort gelingt es ihnen eher, sich Größendegressionseffekte zunutze zu machen.

Wie aus der Darstellung der statistischen Ergebnisse in Tabelle 39 hervorgeht, kann für keines der betrachteten Geschäftsjahre ein signifikanter Zusammenhang zwischen der Lage und der Höhe des Sachaufwands festgestellt werden. Die nachgewiesenen Unterschiede sind hierfür betragsmäßig nicht groß genug. Da es sich bei der vorliegenden Untersuchung um

${ }^{948} \mathrm{Vgl}$. Abschnitt 3.6.3.8

${ }^{949}$ Vgl. Abschnitt 3.4.1. 
eine Vollerhebung handelt, ${ }^{950}$ ist das deskriptive Resultat jedoch auch ohne Vorliegen einer Signifikanz valide. Dennoch sind die Unterschiede gering.

\begin{tabular}{c|ccccc} 
& $\begin{array}{c}\text { Städtisch } \\
(\mathrm{n}=156-167)\end{array}$ & $\begin{array}{c}\text { Halbstädtisch } \\
(\mathrm{n}=526-596)\end{array}$ & $\begin{array}{c}\text { Ländlich } \\
(\mathrm{n}=262-305)\end{array}$ & $\begin{array}{c}\mathbf{F} \\
(\text { ANOVA })\end{array}$ & $\begin{array}{c}\text { F } \\
(\text { Welch })\end{array}$ \\
\hline 2005 & $1,03 \%$ & $1,10 \%$ & $1,08 \%$ & & 2,77 \\
2006 & $1,02 \%$ & $1,08 \%$ & $1,06 \%$ & & 1,65 \\
2007 & $0,97 \%$ & $1,04 \%$ & $1,01 \%$ & 3,02 & \\
2008 & $0,90 \%$ & $0,97 \%$ & $0,96 \%$ & 2,51 & \\
2009 & $0,88 \%$ & $0,92 \%$ & $0,91 \%$ & & 1,66 \\
2010 & $0,92 \%$ & $0,97 \%$ & $0,97 \%$ & 2,18 & \\
2011 & $0,90 \%$ & $0,95 \%$ & $0,93 \%$ & \\
\hline \multicolumn{7}{c}{ Basis: $\mathrm{n}=944-1068$, Welch-Test (Post-Hoc-Test: Tukey-HSD), n. s. }
\end{tabular}

Tabelle 39: $\quad$ Entwicklung des Sachaufwands nach Lagebereichen

Die Resultate werden in Abbildung 51 dargestellt.

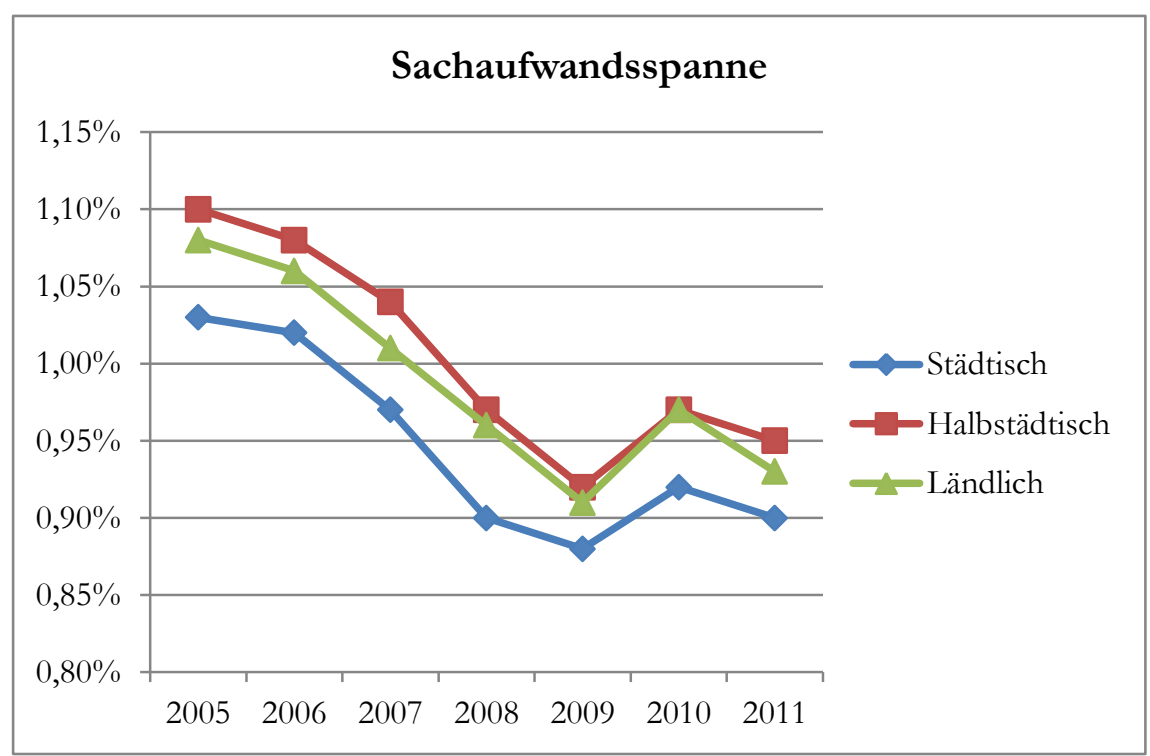

Abbildung 51: Entwicklung des Sachaufwands nach Lagebereichen

Tabelle 40 stellt die Ergebnisse der OLS-Regression dar, um die bestehenden Unterschiede zu quantifizieren. Ausgehend vom Niveau der Sachaufwandsspanne von städtischen Banken liegen halbstädtische Institute im Mittel um 5,86 Basispunkte darüber, ländliche Genossenschaftsbanken immerhin 4,38 Basispunkte.

Es kann zusammengefasst werden, dass die Lage als Erfolgsfaktor nachweisbar ist; dies gilt auch für alle vorher getesteten Hypothesen. Die Lage kann demnach eingesetzt werden, um die Höhe des Erfolgs zu erklären. Trotzdem ist sie aus der Sicht der jeweiligen Genossenschaftsbank nicht änderbar. Das liegt vor allem daran, dass das Geschäftsgebiet auf die unmittelbare Umgebung begrenzt ist. Aus diesem Grund zählt die Lage zu den exogenen Erfolgsfaktoren, mit denen sich ein Institut „arrangieren“ muss. 


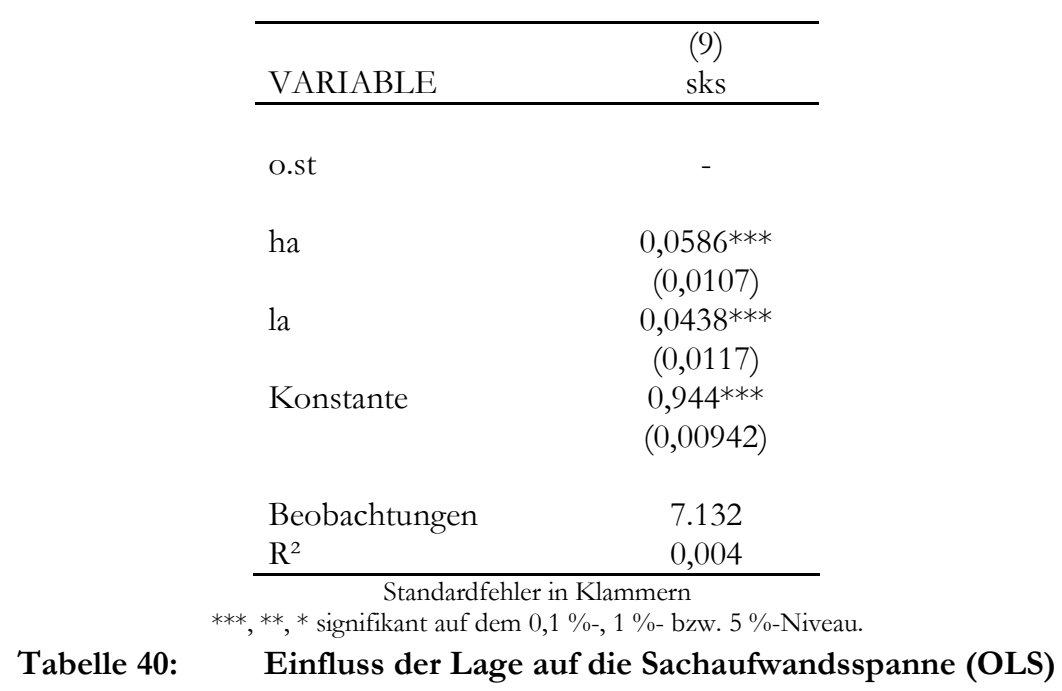

Dennoch ist ein Engagement mit Derivaten denkbar, die eine geschäftliche Betätigung über die Grenzen des Geschäftsgebietes hinaus ermöglichen. Hierzu zählen etwa Kreditderivate als Absicherungsinstrumente. Allerdings widmen sich Kreditgenossenschaften eher dem gewöhnlichen Einlagen- und Kreditgeschäft. Eine Expansion in diesen Geschäftsarten über die Grenzen des eigenen Geschäftsgebietes hinaus ist daher üblicherweise nicht vorgesehen, und wäre gleichsam aus Wettbewerbsüberlegungen heraus nicht ratsam. Immerhin würde eine Verstärkung des Wettbewerbs im halbstädtischen und vor allem im ländlichen Bereich zum Zusammenlaufen der Margen führen. Die Margen sind jedoch für die Aufrechterhaltung der Filialinfrastruktur und somit letzten Endes der Zielerfüllung von existenzieller Bedeutung. Durch hohe Raum- und Personalaufwendungen für Filialen sind ländliche Institute bereits hinreichend herausgefordert, auskömmliche Margen in der Fläche zu erzielen.

\subsubsection{Hypothese 10: Größe nach Stadt-Land-Lage}

Die Hypothese lautet:

Banken in ländlichen Regionen unterscheiden sich binsichtlich ibrer Größe von Banken in urbanen Gebieten.

Als Proxy für die Größe einer Bank wird hier wieder die Bilanzsumme eingesetzt. ${ }^{951}$ Vermutet wird, dass Banken in städtischer Lage eher groß sind, Banken in ländlicher Lage dagegen klein.

Die Ergebnisse der Varianzanalyse in Tabelle 41 bestätigen den vermuteten Zusammenhang. Für alle betrachteten Geschäftsjahre unterscheiden sich die Banken in städtischer Lage hinsichtlich ihrer mittleren Größe signifikant von denen in ländlicher Lage. Banken in halbstädtischer Lage weisen Werte im dazwischenliegenden Bereich auf. Dies ist darauf zurückzuführen, dass im Geschäftsgebiet städtischer Banken in der Regel eine höhere Anzahl von realen und potenziellen Kunden lebt. Städtische Gebiete sind per definitionem von einer höheren Einwohnerdichte gekennzeichnet. 


\begin{tabular}{|c|c|c|c|c|}
\hline Werte in Mio. $€$ & $\begin{array}{c}\text { Städtisch } \\
(\mathrm{n}=156-167)\end{array}$ & $\begin{array}{l}\text { Halbstädtisch } \\
(\mathrm{n}=527-597)\end{array}$ & $\begin{array}{c}\text { Ländlich } \\
(\mathrm{n}=262-307)\end{array}$ & $\begin{array}{c}\mathbf{F} \\
\text { (Welch) } \\
\end{array}$ \\
\hline 2005 & $1.153^{\mathrm{a}}$ & $376^{\mathrm{b}}$ & $179 c$ & $111,66^{* * *}$ \\
\hline 2006 & $1.188^{a}$ & $388^{\mathrm{b}}$ & $188^{c}$ & $103,96 * * *$ \\
\hline 2007 & $1.255^{\mathrm{a}}$ & $412^{\mathrm{b}}$ & $194^{c}$ & $106,94 * * *$ \\
\hline 2008 & $1.377^{\mathrm{a}}$ & $449^{\mathrm{b}}$ & $207 c$ & $108,22^{* * *}$ \\
\hline 2009 & $1.463^{\mathrm{a}}$ & $480^{\mathrm{b}}$ & $223^{c}$ & $101,10^{* * *}$ \\
\hline 2010 & $1.557^{\mathrm{a}}$ & $505^{\mathrm{b}}$ & $231^{c}$ & $96,66^{* * *}$ \\
\hline 2011 & $1.595^{\mathrm{a}}$ & $530^{\mathrm{b}}$ & $245^{\mathrm{c}}$ & $92,26 * * *$ \\
\hline \multicolumn{5}{|c|}{$\begin{array}{l}\text { Basis: } \mathrm{n}=945-1071 \text {, Welch-Test (Post-Hoc-Test: Tukey-HSD) } \\
\text { kant auf dem 0,1 \%-, } 1 \% \text { - bzw. } 5 \% \text {-Niveau. Gruppen mit unterschiedlichen } \\
\text { chstaben }(a, b, c) \text { unterscheiden sich signifikant auf dem } 5 \% \text {-Niveau. }\end{array}$} \\
\hline Tabelle 41: & \multicolumn{4}{|c|}{ Entwicklung der Bankgröße nach Lagebereichen } \\
\hline
\end{tabular}

Wie unten in Abbildung 69 dargestellt, weisen die Kunden der städtischen Institute auch ein höheres Einkommen auf, was ceteris paribus auch zu höheren Guthaben auf den Konten führt. Abbildung 52 enthält die grafische Darstellung der hier beschriebenen Ergebnisse.

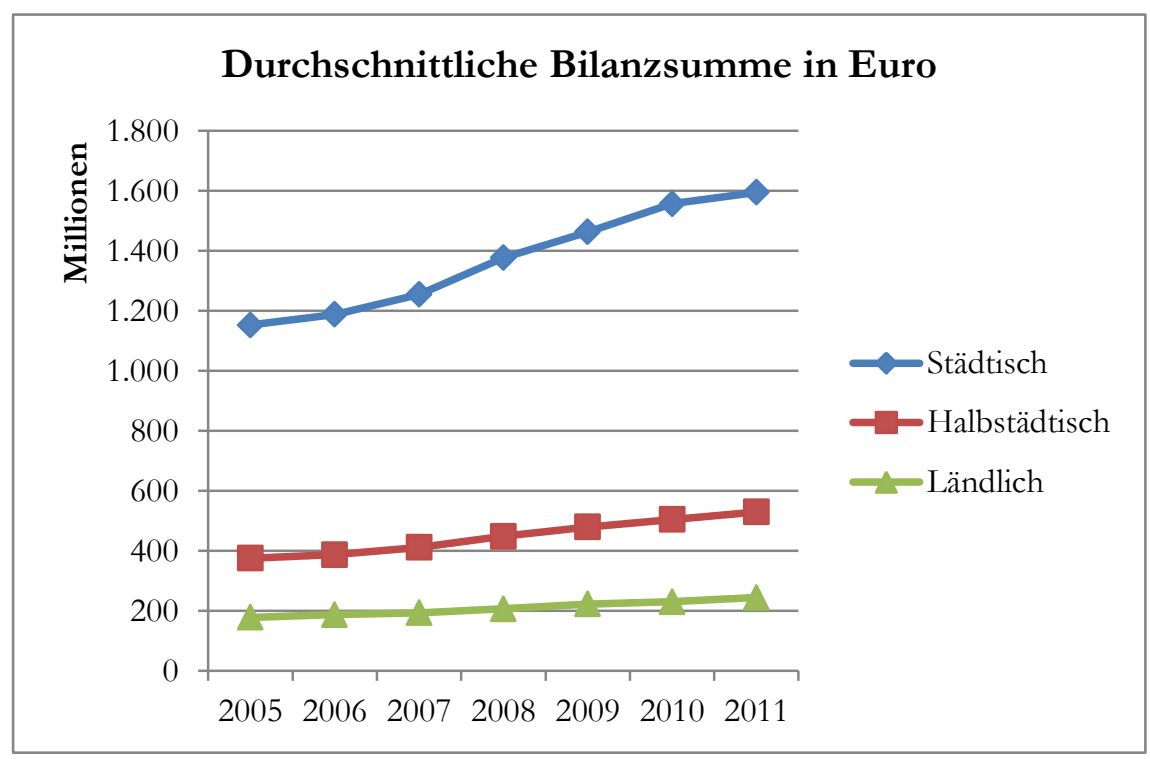

Abbildung 52: Entwicklung der Bankgröße nach Lagebereichen

Anhand von Abbildung 52 lässt sich das stetige Bilanzsummenwachstum von Jahr zu Jahr, welches im Abschnitt 4.4.8 thematisiert wurde, sehr gut ablesen. Für jeden der drei Lagebereiche liegt die durchschnittliche Bilanzsumme eines Jahres über dem Wert des Vorjahres. Als Folge der Finanzkrise kam es zu einer Verlagerung von Einlagen hin zu Instituten, die das Bankgeschäft lediglich konventionell betreiben. Hiervon konnten die Genossenschaftsbanken in besonderem Maße profitieren. Im Jahr 2008 lag die Bilanzsumme städtischer Institute im Mittel 9,7\% über dem Vorjahreswert. Bei Instituten in halbstädtischer Lage kam es immerhin zu einer Zunahme um 9,0\%. Die Wachstumsraten werden grafisch in Abbildung 53 dargestellt.

Ebenfalls für alle drei Lagebereiche lässt sich nachweisen, dass die über alle untersuchten Banken hinweg aggregierte Bilanzsumme einem stetigen Anstieg unterliegt. Dieser Sachverhalt wird in Abbildung 54 dargestellt. 


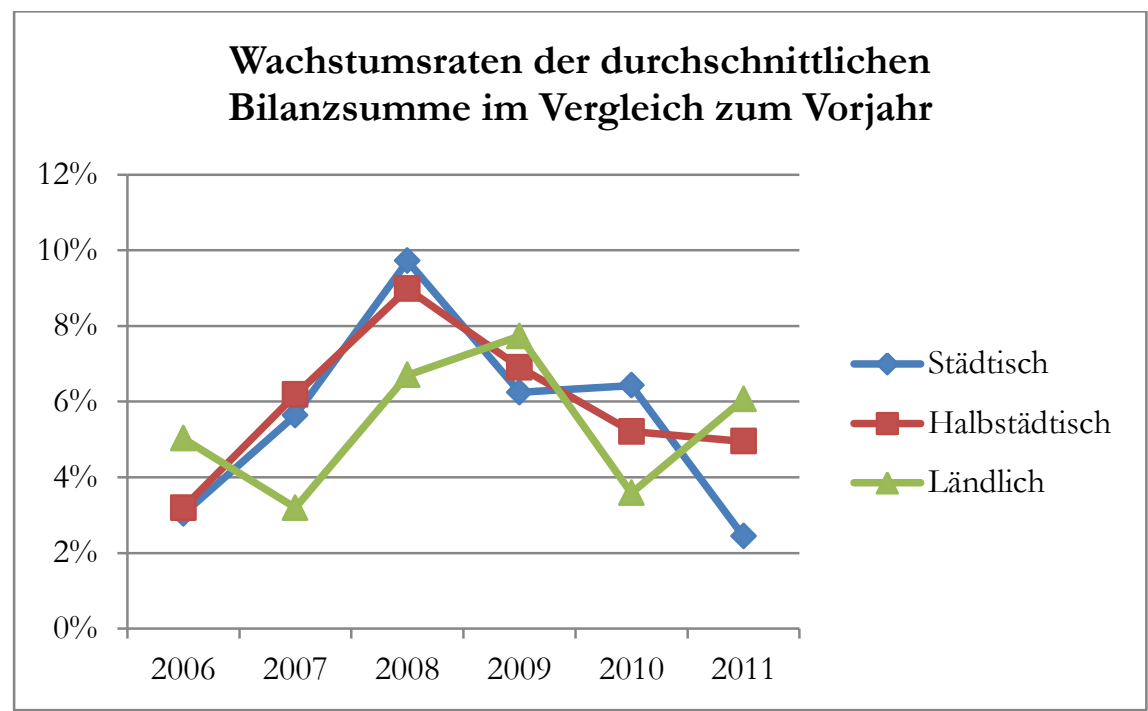

Abbildung 53: Wachstumsraten der durchschnittlichen Bilanzsumme im Vergleich zum Vorjahr

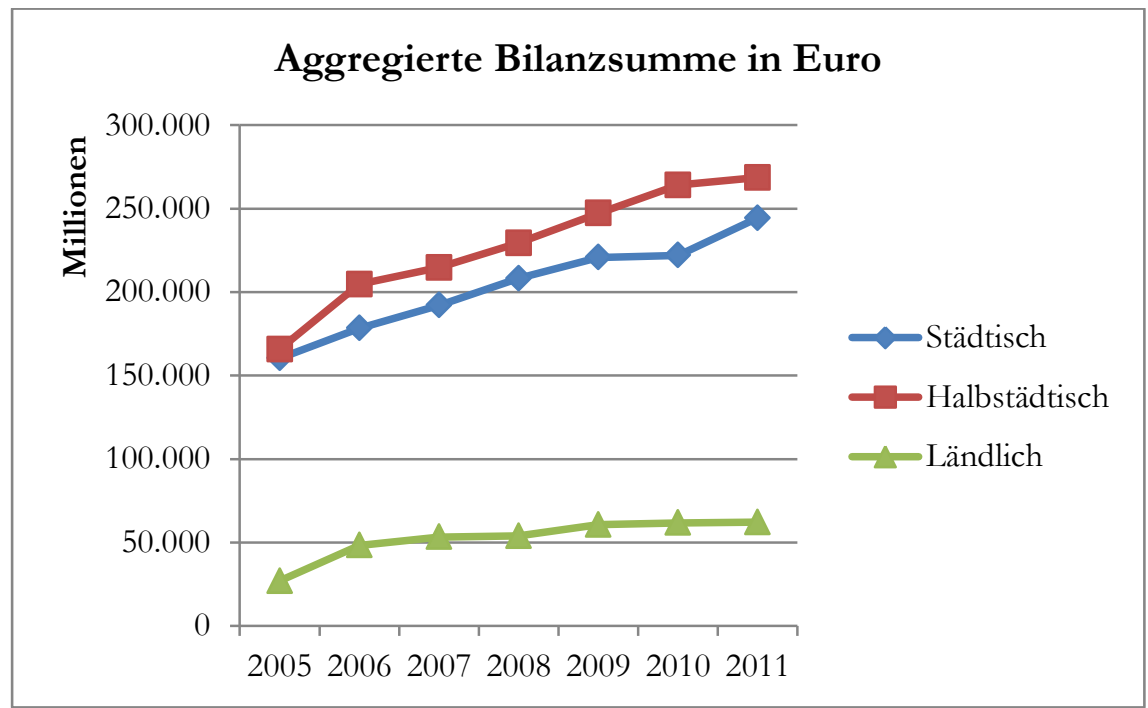

Abbildung 54: Aggregierte Bilanzsumme der untersuchten Banken nach den drei Lagebereichen

\subsubsection{Hypothese 11: Lage nach Bundesland}

Die Hypothese lautet:

Die Höbe des Erfolgs hängt davon ab, in welchem Bundesland eine Bank liegt.

Die Messung der Erfolgshöhe findet wieder anhand der Bruttogewinnspanne statt. Die Banken unterscheiden sich im Erfolg stark danach, in welchem Bundesland sie liegen. Diese Betrachtung wurde stellvertretend am Geschäftsjahr 2011 durchgeführt. Berlin wies mit $0,57 \%$ die geringste Bruttogewinnspanne auf, gefolgt von Hamburg mit 1,00 \% und Sachsen mit 1,03\%. In Thüringen waren die Banken mit 1,36\% im Mittel am erfolgreichsten, gefolgt von Mecklenburg-Vorpommern mit 1,33\% und Sachsen-Anhalt mit 1,23\%. Dennoch muss darauf hingewiesen werden, dass die durchschnittliche Bilanzsumme ungleich auf die Bundesländer verteilt ist. Alle Angaben inklusive der Anteile sind in Tabelle 42 dargestellt. 


\begin{tabular}{|l|r|r|c|c|}
\hline Bundesland & Erfolg & $\begin{array}{c}\text { An- } \\
\text { zahl }\end{array}$ & $\begin{array}{c}\text { Durchschnittliche } \\
\text { Bilanzsumme (DBS) }\end{array}$ & BS-Anteil \\
\hline Baden-Württemberg & $1,04 \%$ & 226 & 131.154 .750 .000 & $23,04 \%$ \\
\hline Bayern & $1,14 \%$ & 254 & 124.041 .523 .500 & $21,79 \%$ \\
\hline Berlin & $0,57 \%$ & 3 & 16.241 .500 .000 & $2,85 \%$ \\
\hline Brandenburg & $1,17 \%$ & 7 & 2.385 .150 .000 & $0,42 \%$ \\
\hline Bremen & $1,14 \%$ & 2 & 1.011 .450 .000 & $0,18 \%$ \\
\hline Hamburg & $1,00 \%$ & 4 & 4.429 .600 .000 & $0,78 \%$ \\
\hline Hessen & $1,08 \%$ & 65 & 52.072 .400 .000 & $9,15 \%$ \\
\hline Mecklenburg-Vorpommern & $1,33 \%$ & 8 & 2.834 .600 .000 & $0,50 \%$ \\
\hline Niedersachsen & $1,22 \%$ & 87 & 45.776 .400 .000 & $8,04 \%$ \\
\hline Nordrhein-Westfalen & $1,15 \%$ & 141 & 112.895 .850 .000 & $19,83 \%$ \\
\hline Rheinland-Pfalz & $1,16 \%$ & 51 & 37.452 .950 .000 & $6,58 \%$ \\
\hline Saarland & $1,11 \%$ & 9 & 7.094 .800 .000 & $1,25 \%$ \\
\hline Sachsen & $1,03 \%$ & 20 & 7.560 .900 .000 & $1,33 \%$ \\
\hline Sachsen-Anhalt & $1,23 \%$ & 11 & 3.290 .100 .000 & $0,58 \%$ \\
\hline Schleswig-Holstein & $1,16 \%$ & 29 & 16.086 .070 .700 & $2,83 \%$ \\
\hline Thüringen & $1,36 \%$ & 15 & 5.020 .650 .000 & $0,88 \%$ \\
\hline Gesamtergebnis & $\mathbf{1 , 1 2 \%}$ & $\mathbf{9 3 2}$ & $\mathbf{5 6 9 . 3 4 8 . 6 9 4 . 2 0 0}$ & $\mathbf{1 0 0 , 0 0 \%}$ \\
\hline
\end{tabular}

Tabelle 42: $\quad$ Einfluss der Lage nach Bundesland auf den Erfolg

In Abbildung 55 sind die Verteilungen der Erfolgswerte für die einzelnen Bundesländer als Boxplot dargestellt, in aufsteigender alphabetischer Ordnung. Die horizontale Linie wurde beim Mittelwert für Deutschland (1,12\%) eingezeichnet. Es existieren beträchtliche Unterschiede zwischen den Bundesländern. Dennoch ist die Aussagekraft der Ergebnisse von der Anzahl untersuchter Banken im jeweiligen Bundesland abhängig. Eine Ungleichverteilung ist auch anhand der Bilanzsummenanteile ersichtlich. So vereinen die 5 Länder BadenWürttemberg, Bayern, Nordrhein-Westfalen, Hessen und Niedersachsen nahezu $82 \%$ der repräsentierten Volumina.

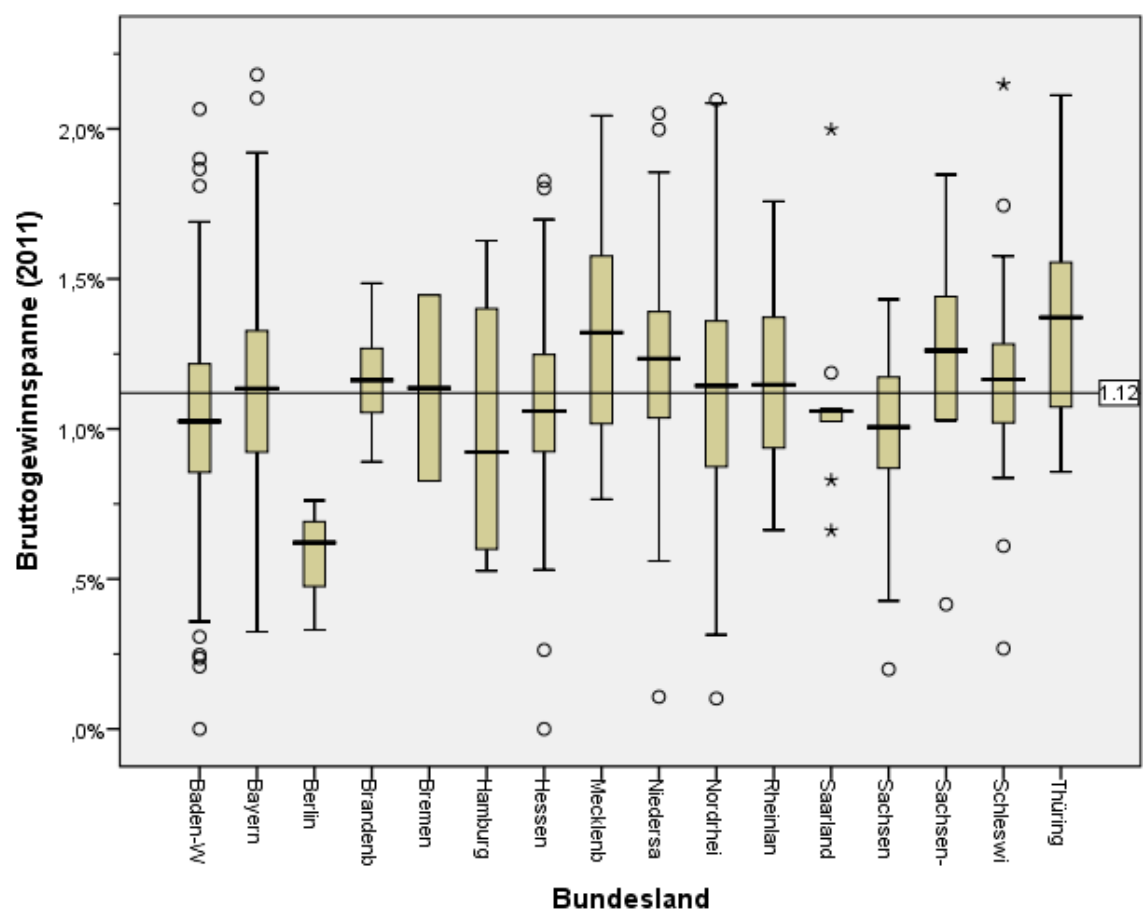

Abbildung 55: Einfluss der Lage nach Bundesland auf den Erfolg 


\subsubsection{Hypothese 12: Abschreibungen auf Forderungen}

Die Hypothese lautet:

\section{Abschreibungen auf Forderungen und im Kreditgeschäft steben im Zusammenbang mit dem Bruttogewinn aus normaler Geschäftstätigkeit.}

Der Bruttogewinn aus normaler Geschäftstätigkeit wird im Rahmen dieser Arbeit als Erfolgsmesszahl eingesetzt. ${ }^{952}$ Die Position Abschreibungen und Wertberichtigungen auf Forderungen und bestimmte Wertpapiere sowie Zufübrungen zu Rückstellungen im Kreditgeschäft sowie die Gegenposition Erträge aus Zuschreibungen zu Forderungen und bestimmte Wertpapiere sowie aus der Auflösung von Rückstellungen im Kreditgeschäft gehen nicht in dessen Berechnung ein, wie in Tabelle 43 dargestellt ist. Vielmehr werden diese Positionen erst nach Ermittlung des Bruttogewinns aus normaler Geschäftstätigkeit addiert. Wie bereits oben in den Absätzen 3.5.1 und 3.6.3.9 beschrieben, ist gerade die Nichteinbeziehung dieses Risikovorsorgebereichs in die Erfolgsmesszahl ein entscheidendes Argument, um eine möglichst hohe Vergleichbarkeit zwischen den Erfolgen einzelner Banken zu erreichen.

\begin{tabular}{|c|c|}
\hline Pos. & Positionen Gewinn- und Verlustrechnung \\
\hline & Bruttogewinn aus normaler Geschäftstätigkeit \\
\hline+13 & $\begin{array}{l}\text { Zuzüglich Abschreibungen und Wertberichtigungen auf Forderungen und be- } \\
\text { stimmte Wertpapiere sowie Zuführungen zu Rückstellungen im Kreditgeschäft }\end{array}$ \\
\hline-14. & $\begin{array}{l}\text { Abzüglich Erträge aus Zuschreibungen zu Forderungen und bestimmten Wertpa- } \\
\text { pieren sowie aus der Auflösung von Rückstellungen im Kreditgeschäft }\end{array}$ \\
\hline 15. & $\begin{array}{l}\text { Abschreibungen und Wertberichtigungen auf Beteiligungen, Anteile an verbunde- } \\
\text { nen Unternehmen und wie Anlagevermögen behandelte Wertpapiere }\end{array}$ \\
\hline-16. & $\begin{array}{l}\text { Abzüglich Erträge aus Zuschreibungen zu Beteiligungen, Anteilen an verbundenen } \\
\text { Unternehmen und wie Anlagevermögen behandelten Wertpapieren }\end{array}$ \\
\hline+17. & Zuzüglich Aufwendungen aus Verlustübernahme \\
\hline 19. & $=$ Betriebsergebnis aus normaler Geschäftstätigkeit \\
\hline
\end{tabular}

Betriebsergebnis aus normaler Geschäftstätigkeit

Obgleich der Risikovorsorgebereich nicht in die Berechnung des Bruttogewinns aus normaler Geschäftstätigkeit eingeht, kann trotzdem ein Zusammenhang vermutet werden. Zur Auflösung dieser Beziehung werden für ein jedes Geschäftsjahr die Erfolgsmesszahlen aller Banken (Bruttogewinnspanne) aufsteigend geordnet und anschließend 5 Quintile für jedes Jahr gebildet. Die $20 \%$ der schlechtesten Ergebnisse bilden jeweils das erste Quintil. Entsprechend bilden die $20 \%$ der besten Ergebnisse das fünfte Quintil. Abbildung 56 stellt diesen Zusammenhang für alle betrachteten Geschäftsjahre grafisch dar. 


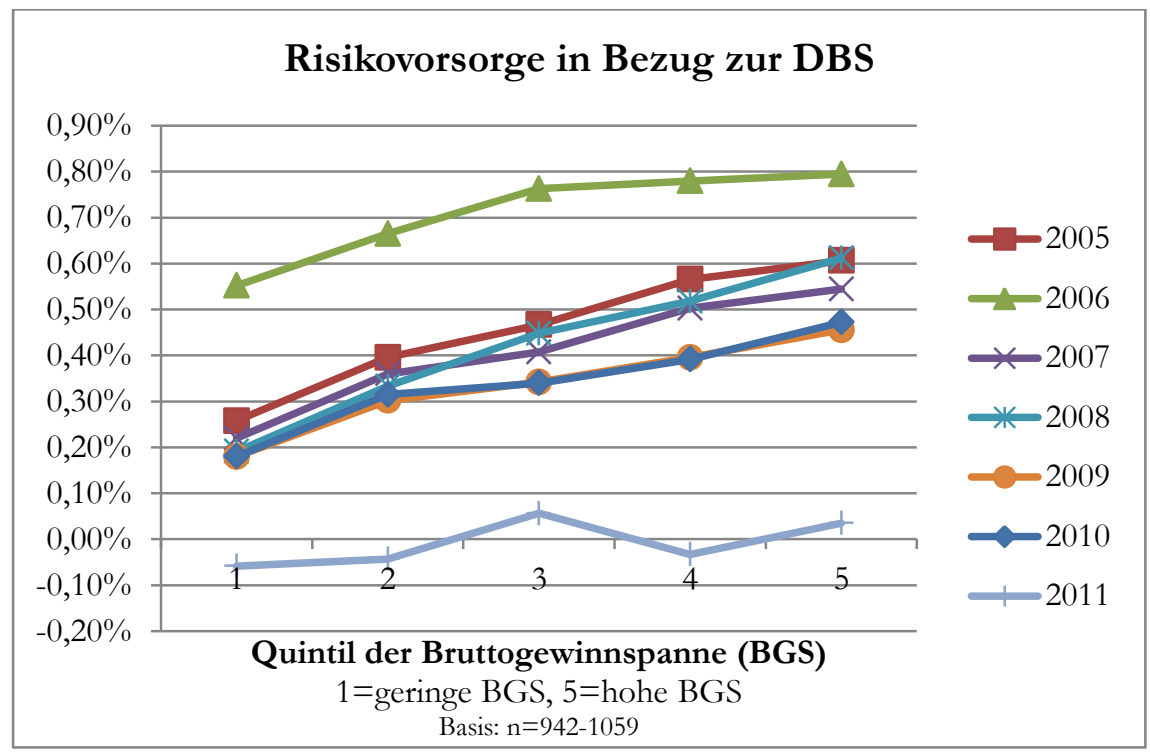

Abbildung 56: Zusammenhang zwischen Bruttogewinnspanne und Risikovorsorge

Ein Geschäftsjahr wird in Abbildung 56 dargestellt als fünf gleichfarbige Marker, die mittels einer Linie verbunden sind. Für alle sieben betrachteten Geschäftsjahre lässt sich zeigen, dass die ergebnisstärksten Banken (fünftes Quintil) stets einen höheren Aufwand für Risikovorsorge betreiben als die ergebnisschwächsten Banken (erstes Quintil). Die anderen Quintile liegen jeweils dazwischen. Eine Ausnahme, auch bezüglich des Niveaus der Risikovorsorge, ist für das Jahr 2011 beobachtbar: Die $20 \%$ Institute mit den schlechtesten Ergebnissen für 2011 entschieden sich gar für eine Nettoauflösung von Rückstellungen im Kreditgeschäft. Umgekehrt bildeten die $20 \%$ Institute mit den besten Ergebnissen für 2011 netto Rückstellungen im Kreditgeschäft, welche vom Niveau her deutlich unter den vorangegangenen Jahren lagen. Zurückzuführen ist dieser Sondereffekt auf eine durch die Institute durchgeführte zusätzliche Dotierung des Fonds für allgemeine Bankrisiken gemäß $\int 340 \mathrm{~g} \mathrm{HGB}$, aber auch durch die Vorsorge für allgemeine Bankrisiken nach \340f HGB.

Insgesamt führt der Ausweis hoher Abschreibungen auf Forderungen zu einer Verringerung des zu versteuernden Gewinns, wodurch die Zahlungen im Unternehmen verbleiben.

In den meisten Fällen sind die Unterschiede zwischen den einzelnen Quintilen statistisch signifikant, wie aus Tabelle 44 hervorgeht. Lediglich für 2011 ließen sich keine statistisch signifikanten Unterschiede nachweisen.

\begin{tabular}{c|cccccc} 
& $\begin{array}{c}\text { Quintil 1 } \\
(\mathrm{n}=189-\end{array}$ & $\begin{array}{c}\text { Quintil 2 } \\
(\mathrm{n}=188-\end{array}$ & $\begin{array}{c}\text { Quintil 3 } \\
\left(\mathrm{n}=188^{-}\right.\end{array}$ & $\begin{array}{c}\text { Quintil 4 } \\
\left(\mathrm{n}=188^{-}\right.\end{array}$ & $\begin{array}{c}\text { Quintil 5 } \\
(\mathrm{n}=189-\end{array}$ & $\begin{array}{c}\text { F } \\
\text { (Welch) }\end{array}$ \\
\hline 212$)$ & $212)$ & $211)$ & $212)$ & $212)$ & \\
\hline 2005 & $0,26^{\mathrm{a}}$ & $0,40^{\mathrm{b}}$ & $0,47^{\mathrm{b}}$ & $0,57^{\mathrm{c}}$ & $0,60^{\mathrm{c}}$ & $35,58^{* * *}$ \\
2006 & $0,55^{\mathrm{a}}$ & $0,66^{\mathrm{b}}$ & $0,76^{\mathrm{c}}$ & $0,78^{\mathrm{c}}$ & $0,79^{\mathrm{c}}$ & $12,76^{* * *}$ \\
2007 & $0,22^{\mathrm{a}}$ & $0,36^{\mathrm{b}}$ & $0,41^{\mathrm{b}}$ & $0,50^{\mathrm{c}}$ & $0,54^{\mathrm{c}}$ & $53,01^{* * *}$ \\
2009 & $0,19^{\mathrm{a}}$ & $0,33^{\mathrm{b}}$ & $0,45^{\mathrm{c}}$ & $0,52^{\mathrm{c}}$ & $0,61^{\mathrm{d}}$ & $55,58^{* * *}$ \\
2010 & $0,18^{\mathrm{a}}$ & $0,30^{\mathrm{b}}$ & $0,34^{\mathrm{b}}$ & $0,40^{\mathrm{c}}$ & $0,46^{\mathrm{c}}$ & $28,83^{* * *}$ \\
2011 & $0,66^{\mathrm{a}}$ & $0,97 \mathrm{~b}$ & $1,15^{\mathrm{c}}$ & $1,32^{\mathrm{d}}$ & $1,66^{\mathrm{e}}$ & $1543,03^{* * *}$ \\
\hline & $-0,06$ & $-0,04$ & 0,06 & $-0,03$ & 0,04 & 1,19 \\
$* * * * *, *$ & signifikant auf dem 0,1 \%-, 1 \%- bzw. 5\%-Niveau. Gruppen mit unterschiedlichen
\end{tabular}


Insgesamt zeichnen sich ergebnisschwache Banken durch geringere Abschreibungen bzw. durch eine geringe Risikovorsorge aus. Ergebnisstarke Banken wenden relativ viel für die Risikovorsorge auf. Wie bereits in den Absätzen 3.6.3.9 und 3.6.5 beschrieben, besitzen die Banken einigen Spielraum bei der Durchführung der Risikovorsorge. Es ist davon auszugehen, dass vor allem ergebnisstarke Banken die Risikovorsorgemechanismen nach \340f Abs. 1 Satz 3 HGB aktiv einsetzen, um ihre Steuerlast zu reduzieren bzw. um eine Steuerstundung zu erreichen, denn durch den Ansatz hoher Rückstellungen reduziert sich die Bemessungsgrundlage für die Ertragssteuern. In dieser Hinsicht kann auf Basis des vorliegenden Datensatzes eine gleichlautende These von CHRISTIANs belegt werden. ${ }^{953}$

Eine sorgfältige Auswahl bei Kreditengagements bzw. eine vorsichtige Vergabepraxis sind zusätzlich als Begründung für relativ geringe Abschreibungen ergebnisschwacher Institute denkbar. Jedoch wendet CHRISTIANS ein, dass ein besseres Bewertungsergebnis nicht voreilig, das heißt ohne Detailbetrachtung, mit qualitativ besseren Kreditportfolios begründet werden sollte. Auch dies führt er auf das Ausmaß der getroffenen Risikovorsorge zurück. ${ }^{954}$ Eine andere, aber dennoch in diesem Kontext annehmbare Begründung für diesen Zusammenhang führt PADBERG an. ${ }^{955}$ Er argumentiert für die in der Geschäftstätigkeit ganz ähnlich aufgestellten Sparkassen, dass eine geringe Kreditquote zu einem hohen Anteil festverzinslicher Wertpapiere im Aktivgeschäft führt. Durch die sehr geringen Ausfallraten dieser festverzinslichen Wertpapiere - zumindest wenn diese von öffentlichen Emittenten stammen - müssen auch weniger Abschreibungen vorgenommen werden. ${ }^{956}$ Auf der Ertragsseite jedoch führen die geringen Ausfallrisiken in der Regel auch zu niedrigen Erträgen, wodurch der hier untersuchte Zusammenhang begründet wird. ${ }^{957}$

Im Ergebnis lässt sich das Bewertungsergebnis nur begrenzt als Erfolgsfaktor verwenden und interpretieren. Hohe Abschreibungen treten zwar empirisch tendenziell bei einem hohen betrieblichen Vorbewertungserfolg auf. Aufgrund des oben beschriebenen Mechanismus kann eine Kausalität lediglich in der Gegenrichtung statiert werden.

Eine entsprechende Paneluntersuchung mit Fixed Effects zur Evaluierung der Höhe des Einflusses der Bruttogewinnspanne auf die Risikospanne liefert einen Koeffizienten von $0,35^{* * *}$ bei einem Standardfehler von 0,018. Dies bedeutet, eine Erhöhung der Bruttogewinnspanne um einen Basispunkt ( $z$. B. von $0,80 \%$ auf $0,81 \%$ ) führt im Mittel zu einem Anstieg der Risikovorsorgespanne um 0,35 Basispunkte (z. B. von 0,4616 \% auf 0,4651\%).

\subsubsection{Hypothese 13: Eigenkapitalquote}

Die Hypothese lautet:

\section{Banken mit einer hohen Eigenkapitalquote sind erfolgreicher.}

Generell ist eine angemessene Unterlegung mit Eigenkapital die Voraussetzung für eine solide Geschäftspolitik. ${ }^{958}$ Aus regulatorischer Sicht sind die Anforderungen an die Eigenkapitalunterlegung von Geschäften in den letzten Jahren gewachsen. ${ }^{959}$ Eigenkapital ist eine

\footnotetext{
953 Vgl. Christians (2010), S. 143.

954 Vgl. Christians (2010), S. 143.

955 Vgl. Padberg (2005), S. 23.

956 Vgl. Padberg (2005), S. 23.

957 Vgl. Padberg (2005), S. 23.

958 Vgl. Köppen (1966), S. 65.

${ }^{959}$ Vgl. Bartetzky und Volk (2012), S. 20.
} 
knappe Ressource, dessen Vorhalten Kosten für die Kreditinstitute verursacht, denn die Eigentümer verlangen eine risikoadäquate Rendite. Zumindest fallen Opportunitätskosten an. Aus diesem Grund kann man davon ausgehen, dass die Eigentümer aus eigenem Antrieb heraus dazu bestrebt sind, das Eigenkapital möglichst gering zu halten. Bei Genossenschaftsbanken setzt sich das haftende Eigenkapital vor allem aus den eingezahlten Geschäftsguthaben der Mitglieder, aus Gewinnrücklagen und einem sogenannten Haftsummenzuschlag zusammen. ${ }^{960}$ Abbildung 57 stellt anhand der Verteilung den Verlauf der durchschnittlichen Eigenkapitalquote über die betrachteten Geschäftsjahre hinweg dar. Seit dem Minimum im Jahr 2008 ist ein stetiges Wachstum bis zum Ende der Betrachtungsperiode erkennbar, welches nur durch die beiden Folgejahre der beginnenden Finanzkrise unterbrochen wurde. Dieses Wachstum setzt sich auch nach 2011 weiter fort. ${ }^{961}$

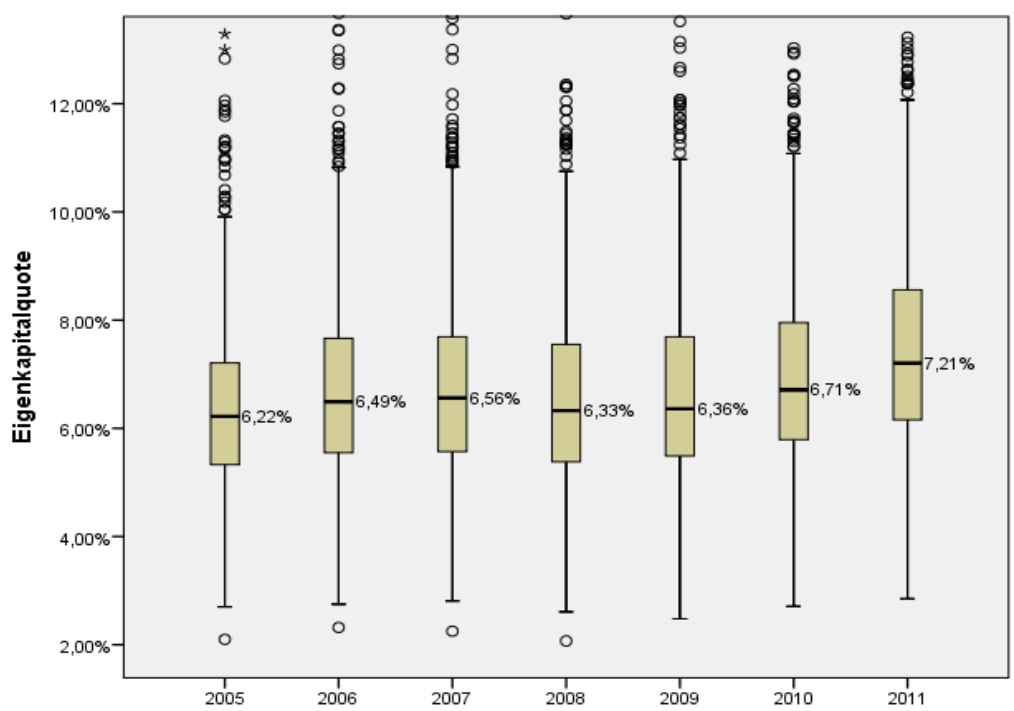

Abbildung 57: Entwicklung der mittleren Eigenkapitalquote im Zeitablauf

Die Grundüberlegung der Formulierung dieser Hypothese ist, dass solide wirtschaftende Institute erfolgreicher sind. Unter solide wirtschaftend soll hierbei eine Unterlegung mit Eigenkapital verstanden werden, welche nahezu allen denkbaren Geschäftsrisiken abfedert und den Fortbestand der Instituts auch in Krisenzeiten sichert.

Der Zusammenhang lässt sich empirisch belegen. Die $20 \%$ Institute mit den höchsten Eigenkapitalquoten weisen in jedem Geschäftsjahr im Mittel die höchste Bruttogewinnspanne auf. Gleichsam gilt, dass die $20 \%$ eigenkapitalschwächsten Institute nahezu immer die niedrigsten Bruttogewinnspannen ausweisen. Die übrigen Quintile liegen, ihrer Reihenfolge entsprechend, dazwischen. Der Zusammenhang wird in Abbildung 58 dargestellt. Die Mittelwerte und Ergebnisse der durchgeführten Varianzanalyse zum Mittelwertvergleich finden sich in Tabelle 45. 


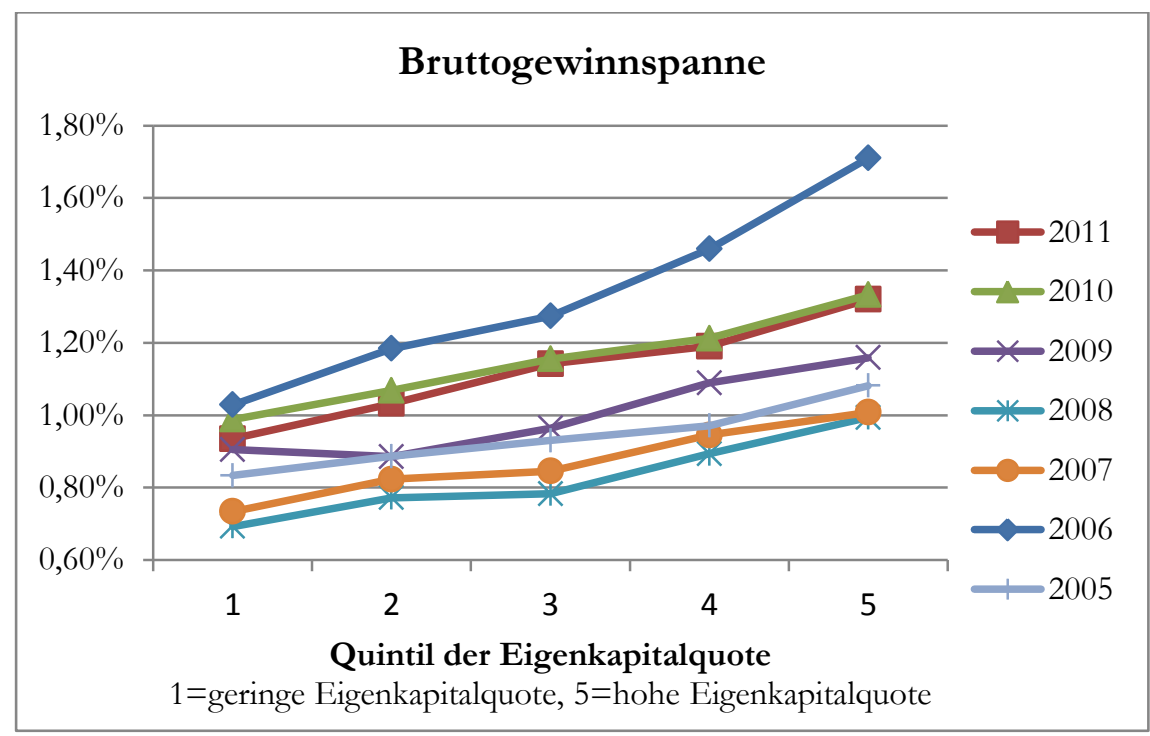

Abbildung 58: Einfluss der Eigenkapitalquote auf die Bruttogewinnspanne

\begin{tabular}{|c|c|c|c|c|c|c|}
\hline & $\begin{array}{c}\text { Quintil 1 } \\
(n=189-212)\end{array}$ & $\begin{array}{c}\text { Quintil 2 } \\
(n=188-213)\end{array}$ & $\begin{array}{c}\text { Quintil 3 } \\
(\mathrm{n}=189-213)\end{array}$ & $\begin{array}{c}\text { Quintil 4 } \\
(\mathrm{n}=188-214)\end{array}$ & $\begin{array}{c}\text { Quintil 5 } \\
(\mathrm{n}=190-214)\end{array}$ & $\begin{array}{c}\mathbf{F} \\
\text { (Welch) }\end{array}$ \\
\hline 2005 & $0,83 \% \mathrm{o}^{\mathrm{a}}$ & $0,87 \% \mathrm{o}^{\mathrm{a}}$ & $0,93 \%{ }^{\mathrm{b}}$ & $0,97 \% 0^{\mathrm{b}}$ & $1,08 \%{ }^{c}$ & $13,90 * * *$ \\
\hline 2006 & $1,03 \% \mathrm{a}^{\mathrm{a}}$ & $1,18 \%{ }^{\mathrm{b}}$ & $1,27 \% \mathrm{o}^{\mathrm{b}}$ & $1,46 \%{ }^{c}$ & $1,71 \% 0^{d}$ & $59,24 * * *$ \\
\hline 2007 & $0,73 \% \mathrm{a}^{\mathrm{a}}$ & $0,82 \%{ }^{\mathrm{b}}$ & $0,84 \% \mathrm{o}^{\mathrm{b}}$ & $0,95 \%{ }^{c}$ & $1,01 \%{ }^{c}$ & $9,71 * * *$ \\
\hline 2008 & $0,69 \% \mathrm{o}^{\mathrm{a}}$ & $0,77 \%{ }^{\mathrm{b}}$ & $0,78 \%{ }^{\mathrm{b}}$ & $0,89 \%{ }^{c}$ & $0,99 \% 0^{d}$ & $26,26 * * *$ \\
\hline 2009 & $0,90 \% \mathrm{a}^{\mathrm{a}}$ & $0,89 \% \mathrm{a}^{\mathrm{a}}$ & $0,96 \% \mathrm{o}^{\mathrm{a}}$ & $1,09 \%$ b & $1,16 \% \%^{\mathrm{b}}$ & $28,04 * * *$ \\
\hline 2010 & $0,99 \% \mathrm{a}^{\mathrm{a}}$ & $1,07 \% \mathrm{o}^{\mathrm{a}}$ & $1,16 \% \mathrm{o}^{\mathrm{b}}$ & $1,21 \%{ }^{\mathrm{b}}$ & $1,33 \%{ }^{c}$ & $24,99 * * *$ \\
\hline 2011 & $0,93 \% \mathrm{a}^{\mathrm{a}}$ & $1,03 \% 0^{b}$ & $1,14 \%{ }^{c}$ & $1,19 \% \mathrm{c}^{\mathrm{c}}$ & $1,31 \% \mathrm{~d}$ & $36,83^{* * *}$ \\
\hline
\end{tabular}

Tabelle 45: $\quad$ Einfluss der Eigenkapitalquote auf die Bruttogewinnspanne

Im Ergebnis lässt sich festhalten, dass sich eine hohe Eigenkapitalquote positiv auf die Bruttogewinnspanne auswirkt. Dies bestätigt die Ergebnisse von CHRISTIANS UND GÄRTNER. ${ }^{962}$ Auch ist der Einfluss der Bruttozinsspanne auf die Bruttogewinnspanne unstrittig, stellen die Zinsüberschüsse doch nahezu drei Viertel der Bruttoerträge. ${ }^{963}$ Eigenkapital lässt sich gewissermaßen als die Voraussetzung für den Ausbau der unternehmerischen Tätigkeit der Banken sehen. Zu diesen Geschäften zählt die Vergabe von Darlehen auf der Kreditseite, deren Ergebnisse sich wiederum in der Bruttozinsspanne niederschlagen. Banken mit schwacher Eigenkapitaldecke stoßen beim Ausbau ihrer Geschäfte an regulatorische Grenzen. Erst nach der Aufstockung der Eigenkapitalquote kann weiteres Geschäft eingegangen werden. Eine höhere Eigenkapitalquote eröffnet also die Möglichkeit, risikoreichere Geschäfte in höherem Umfang einzugehen, von denen wiederum höhere Margen erwartet werden können. Beispielsweise können bei ausreichendem Eigenkapital Kunden mit einem hohen Blankoanteil kreditiert werden, welcher wiederum zu $100 \%$ mit Eigenkapital zu unterlegen ist.

962 Vgl. Christians und Gärtner (2014), S. 14; vgl. Abschnitt 2.1.2.

963 Vgl. Abschnitt 4.4.7. 
Eine hohe Eigenkapitalquote ist nicht nur für das Kundengeschäft, sondern auch für die Eigenanlagen einer Bank von Vorteil. Eigenkapital, welches nicht durch aufsichtsrechtliche Anforderungen im Rahmen von Basel III in der Säule I gebunden ist, kann im Going Concern-Ansatz für Risiken in der Säule II zur Verfügung gestellt werden. ${ }^{964}$ Dies erhöht die Risikotragfähigkeit eines Instituts. ${ }^{965}$ Verfolgt eine Bank hingegen Geschäfte, deren Erträge substanziell auf der Fristentransformation beruhen, so ist hierfür keine direkte aufsichtsrechtliche Eigenkapitalunterlegung erforderlich. Jedoch muss eine Zinsschockmeldung nach Basel II durchgeführt werden. ${ }^{966}$ Die Institute sind zu einer Meldung an die Aufsicht verpflichtet, sobald der entsprechende Indikator einen gewissen Schwellenwert überschreitet. ${ }^{967}$ Zur Durchführung von Fristentransformation benötigt die Bank ein entsprechendes Risikobudget bzw. freies Eigenkapital. Eine robuste Risikotragfähigkeit und eine entsprechend dazu ausgestaltete Marktpreisrisikostrategie zum Management der Eigenanlagen ermöglichen wiederum höhere Erträge aus der Fristentransformation.

Insgesamt bleibt für Genossenschaftsbanken festzuhalten, dass Eigenkapital nur durch Erfolg gebildet werden kann. Ein hohes Eigenkapital ermöglicht seinerseits einen weiteren Geschäftserfolg. Unter diesen Prämissen kann hier von einem selbstverstärkenden Effekt gesprochen werden. Dies ist ein weiterer Grund für den Zusammenhang, dass hohe Eigenkapitalquoten tendenziell bei erfolgreichen Banken zu beobachten sind.

\subsubsection{Hypothese 14: Kreditquote}

Die Hypothese lautet:

\section{Banken mit einer boben Kreditquote sind erfolgreicher.}

Die Kreditvergabe gehört zum Kerngeschäft der Kreditinstitute. Gleichzeitig ist die Kreditvergabe auch eine der lukrativsten Verwendungsformen für Einlagen. Es ist daher davon auszugehen, dass Genossenschaftsbanken mit einer hohen Kreditquote erfolgreicher sind. Unter der Kreditquote versteht man nach VARMAZ das Verhältnis der Bilanzaktivposition 4: Forderungen an Kunden zur Bilanzsumme. ${ }^{968}$ Zur Überprüfung dieser Hypothese werden Quintile über die Kreditquote aller Banken gebildet, und anschließend für jedes Quintil der Mittelwert der Bruttogewinnspanne errechnet. Das Resultat ist in Abbildung 59 dargestellt. Es zeigt sich empirisch, dass eine hohe Kreditquote förderlich für den Erfolg von Banken ist. Es kann empirisch belegt werden, dass die $20 \%$ Banken mit der höchsten Kreditquote auch die größte Bruttogewinnspanne erwirtschaften. Kausal lässt sich dies darauf zurückführen, dass die Vergabe von Krediten im Normalfall mit der Generierung von Zinsüberschüssen einhergeht, welche ihrerseits positiv auf die Bruttogewinnspanne wirken. ${ }^{969}$ Jedoch sind wegen der risikoadäquaten Bepreisung von Krediten Abweichungen möglich. Gelingt den Instituten die Ausreichung der Mittel in Form von Krediten nur unzureichend, führen sie als Alternative häufig Kreditersatzgeschäft durch. Hierunter sind die zahlreichen

\footnotetext{
964 Vgl. Bundesbank (2013b), S. 34.

965 Vgl. Bundesbank (2013b), S. 34.

966 Vgl. \25a Abs. 1 S. 7 KWG i. V. m. \ 24 Abs. 1 Nr. 14 KWG; vgl. Bundesbank (2012d), S. 58.

967 Vgl. Bundesbank (2012d), S. 58.

${ }^{66}$ Vgl. Varmaz (2006), S. 96; vgl. BaFin (2012a), S. 1. BOTSIS ET AL. (Botsis et al. (2012), S. 130) verwenden hier die alternative Bezeichnung Forderungen Netto im Verbältnis zur durchschnittlichen Bilanzsumme. Jedoch sollte hinterfragt werden, warum sie die durchschnittliche Bilanzsumme im Nenner einsetzen. Die Bestandsgröße der Forderungen an Kunden am Geschäftsjahresende müsste vielmehr zur korrespondierenden Bilanzsumme desselben Zeitpunktes in das Verhältnis gesetzt werden.

${ }^{969}$ Vgl. Abschnitt 3.7.1.
} 
übrigen Verwendungsmöglichkeiten für die Mittel zusammengefasst. Kreditersatzgeschäft wird jedoch im Vergleich zum originären Kreditgeschäft als weniger lukrativ angesehen. ${ }^{970}$

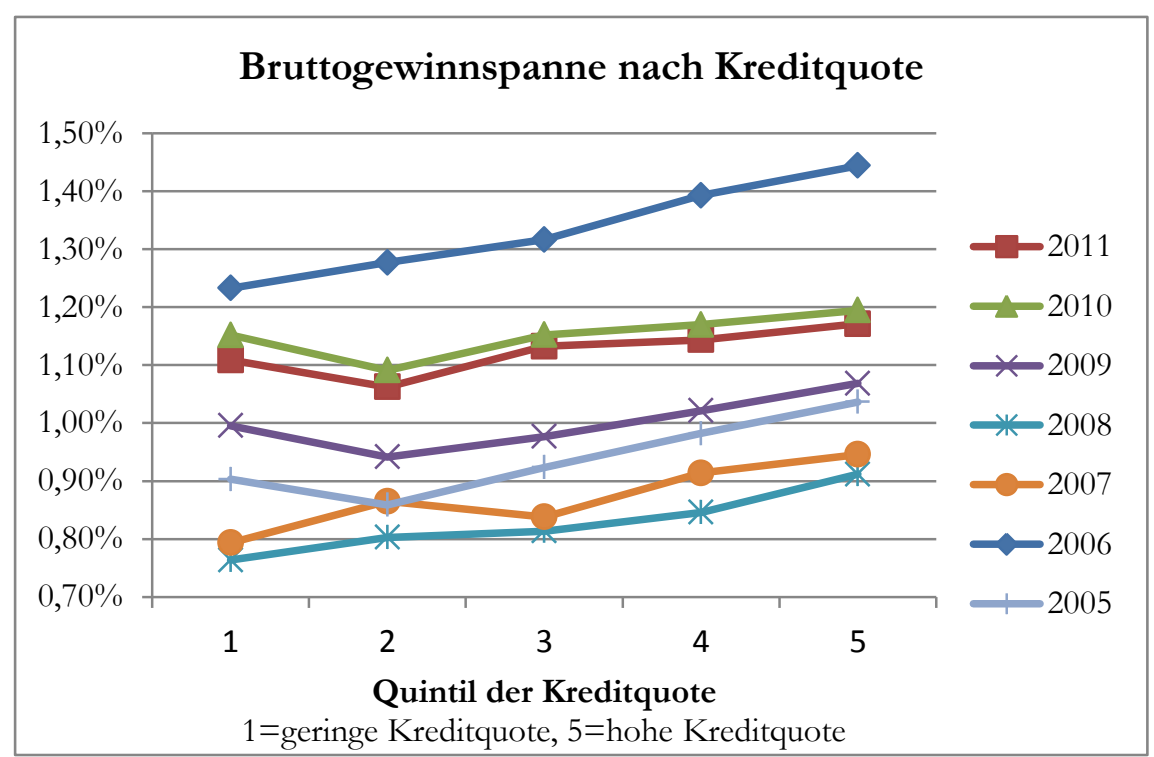

Abbildung 59: Zusammenhang zwischen Kreditquote und Bruttogewinnspanne

Die Entwicklung der Kreditquoten im Zeitablauf wird in Abbildung 60 dargestellt. Hierfür wurden die über alle im Datensatz enthaltenen Genossenschaftsbanken aggregierten Kundenkredite in das Verhältnis zur aggregieren Bilanzsumme gesetzt. Die so für jedes Jahr ermittelten Kreditquoten geben die Entwicklung im gesamten Genossenschaftssektor wider.

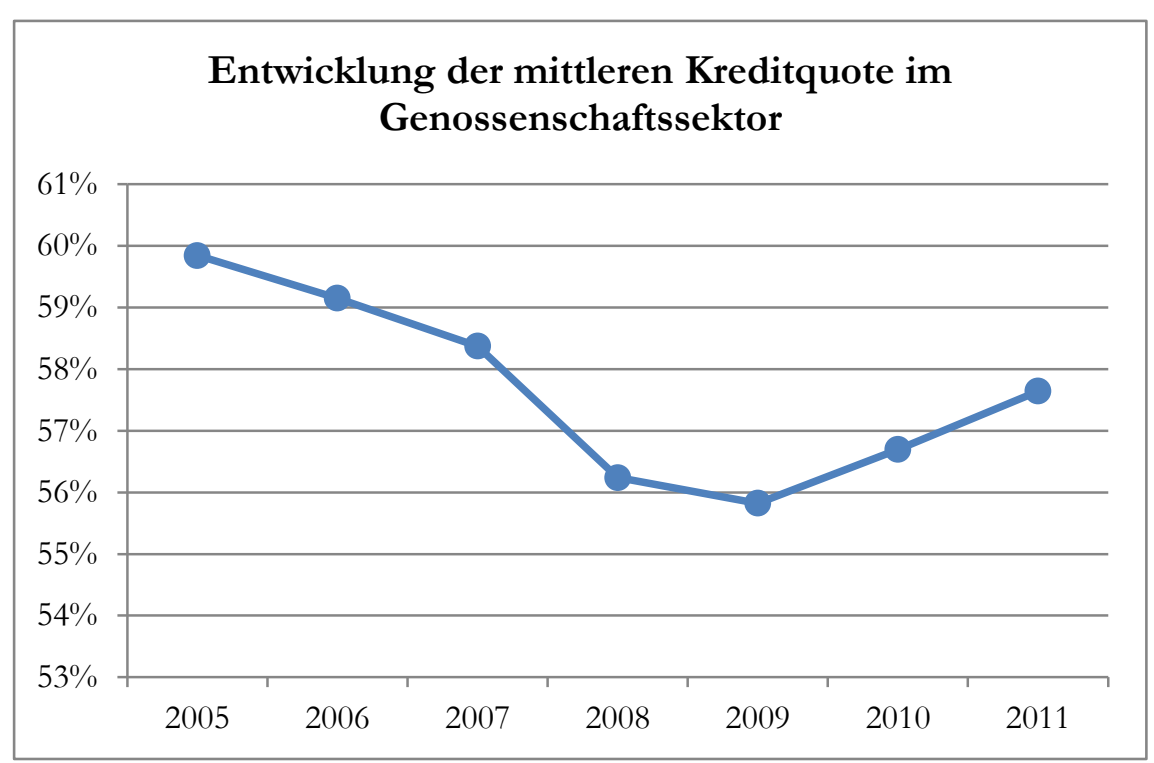

Abbildung 60: Entwicklung der mittleren Kreditquote im Genossenschaftssektor 
Die mittlere Kreditquote betrug 59,8 \% im Jahr 2005. In den folgenden Jahren sank sie ab und erreichte 2009 mit 55,8\% ein Minimum, wonach sie bis 2011 wieder bis auf 57,6 \% anstieg. Jedoch ist zu bemerken, dass im Zeitraum 2005 bis 2011 sowohl die aggregierte Bilanzsumme aller untersuchten Genossenschaftsbanken von jedem Jahr zum Folgejahr anstieg, als auch die aggregierte Kreditsumme. Beide Anstiege unterscheiden sich lediglich in ihren Wachstumsraten. Im Zeitraum 2005 bis 2009 wuchs die aggregierte Bilanzsumme stärker als die aggregierte Kreditsumme, was zu einer Verwässerung des Kreditanteils und somit einer sinkenden Kreditquote führte. In den Jahren 2009 bis 2011 setzte der umgekehrte Effekt ein und resultierte in einer steigenden Kreditquote. Grafisch dargestellt werden die Verläufe beider Kurven in Abbildung 61.

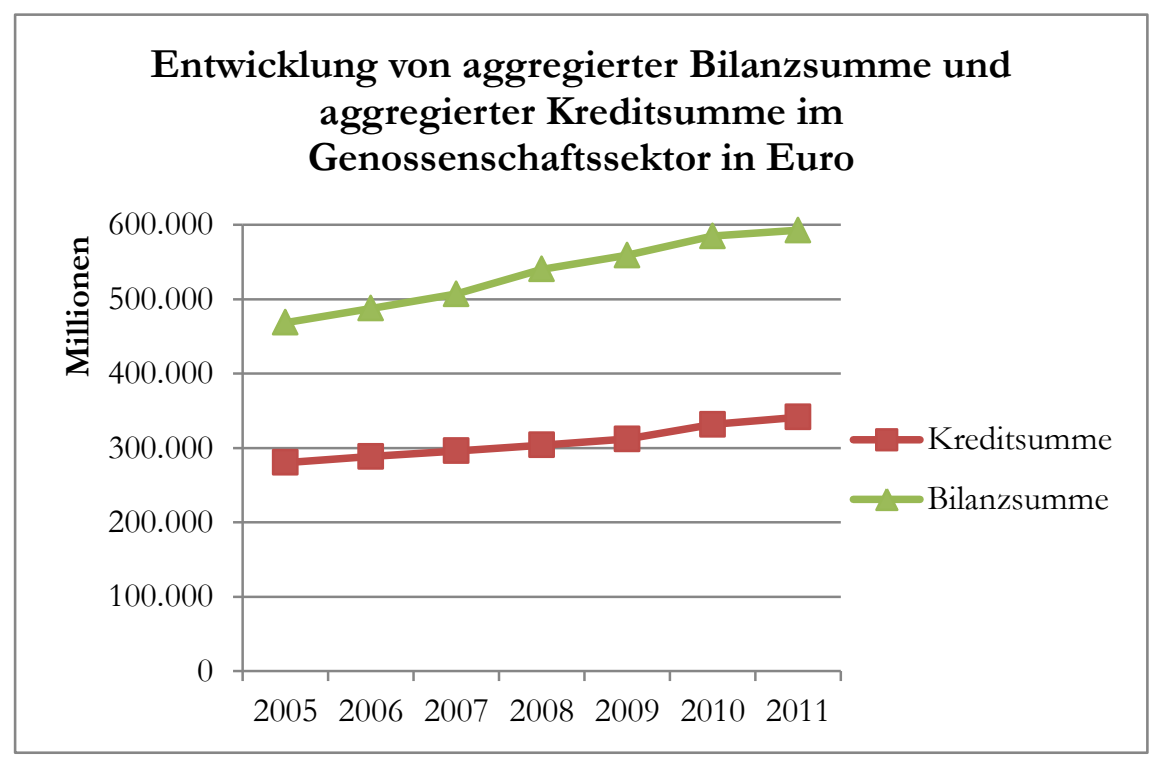

Abbildung 61: Entwicklung von aggregierter Bilanzsumme und aggregierter Kreditsumme im Genossenschaftssektor

Abbildung 62 illustriert die Entwicklung der mittleren Kreditquote über den Betrachtungszeitraum, und stellt dabei die Werte der einzelnen Bundesländer dar. Hierbei fällt zunächst deutlich auf, dass Genossenschaftsbanken in den neuen Bundesländern im Vergleich sehr niedrige Kreditquoten aufweisen. Diese sind wieder auf den oben in Abschnitt 4.4.4 erläuterten Mangel an Gewerbeinfrastruktur zurückzuführen. Durch Einlagen entgegengenommenes Kapital konnte 2011 nur zwischen 32 und 50\% wieder in Form von Krediten ausgereicht werden, da weniger Firmenkredite und auch Privatkredite nachgefragt werden als in den alten Bundesländern. Dort weisen die Institute deutlich höhere Werte auf. Im Jahr 2011 lag die Kreditquote in Bayern bei $56 \%$, während Rheinland-Pfalz gar $66 \%$ erzielen konnte. 


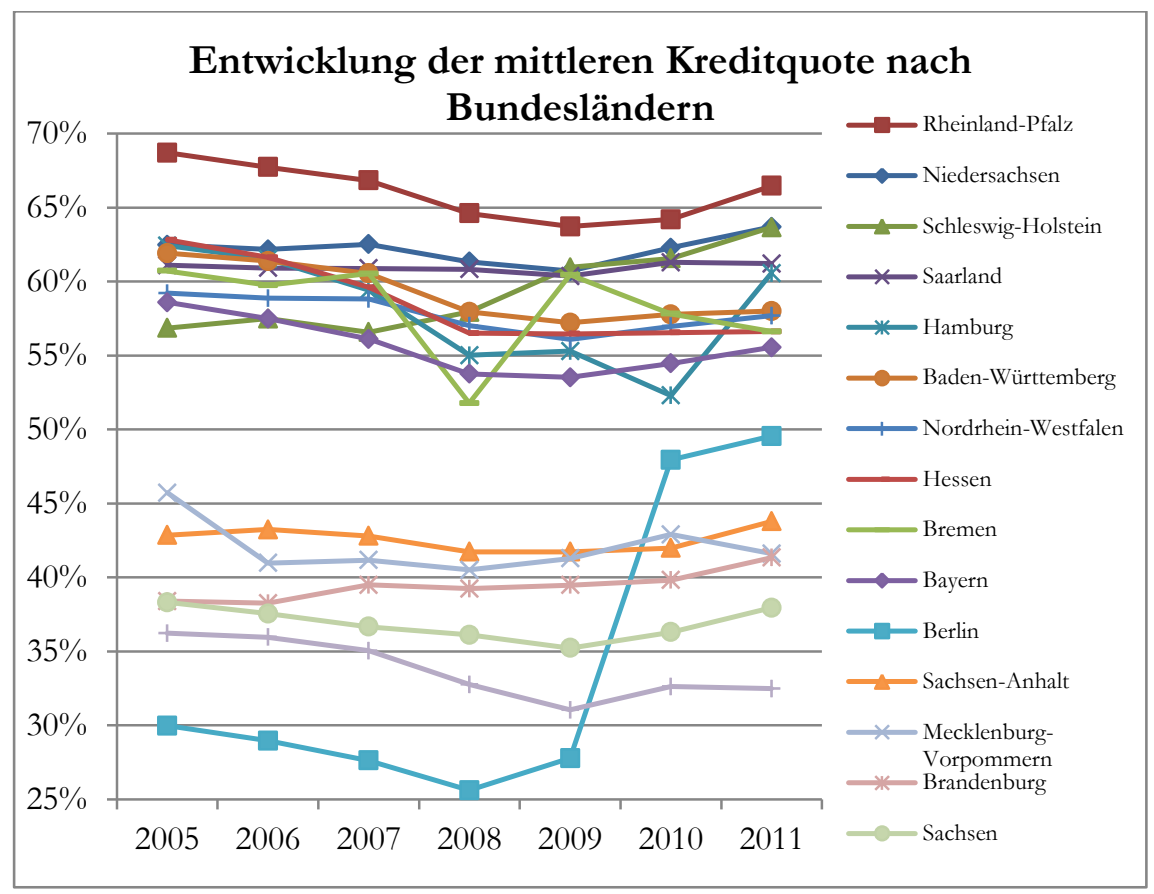

Abbildung 62: Entwicklung der mittleren Kreditquote nach Bundesländern

\subsubsection{Hypothese 15: Effiziente Betriebsgröße}

Die Hypothese lautet:

Bezogen auf ibre Bilanzsumme weisen größere Banken eine böbere Bruttogewinnspanne als kleinere Banken auf. Nach Überschreiten einer kritischen Bilanzsumme fübren andere Einflüsse jedoch zur Umkebrung dieses Effekts.

Dieser Hypothese liegt die folgende Argumentation zugrunde: Begründet durch Skaleneffekte kann man davon ausgehen, dass Banken mit zunehmender Bilanzsumme erfolgreicher sind. ${ }^{971}$ Dies ist darauf zurückzuführen, dass die durchschnittlichen Kosten mit zunehmender Ausbringungsmenge sinken. ${ }^{972}$ Jedoch ist das Erfolgsmaß bei sehr großen Bilanzsummen wieder rückläufig. Gemessen wird der Erfolg anhand der Bruttogewinnspanne, welche dem Bruttogewinn aus normaler Geschäfstätigkeit in Bezug zur durchschnittlichen Bilanzsumme entspricht. Die Eignung der durchschnittlichen Bilanzsumme zur Messung der Betriebsgröße wurde oben in Abschnitt 3.5.2 diskutiert.

Zur Veranschaulichung der untersuchten Bankgrößen dient Abbildung 63 mit einem Histogramm der Bilanzsummenverteilung beispielhaft für das Geschäftsjahr 2011. Hieraus wird ersichtlich, dass eine hohe Anzahl von Instituten eine Bilanzsumme von weniger als 500 Mio. Euro aufweist. Der Median liegt bei 341 Mio. Euro. 


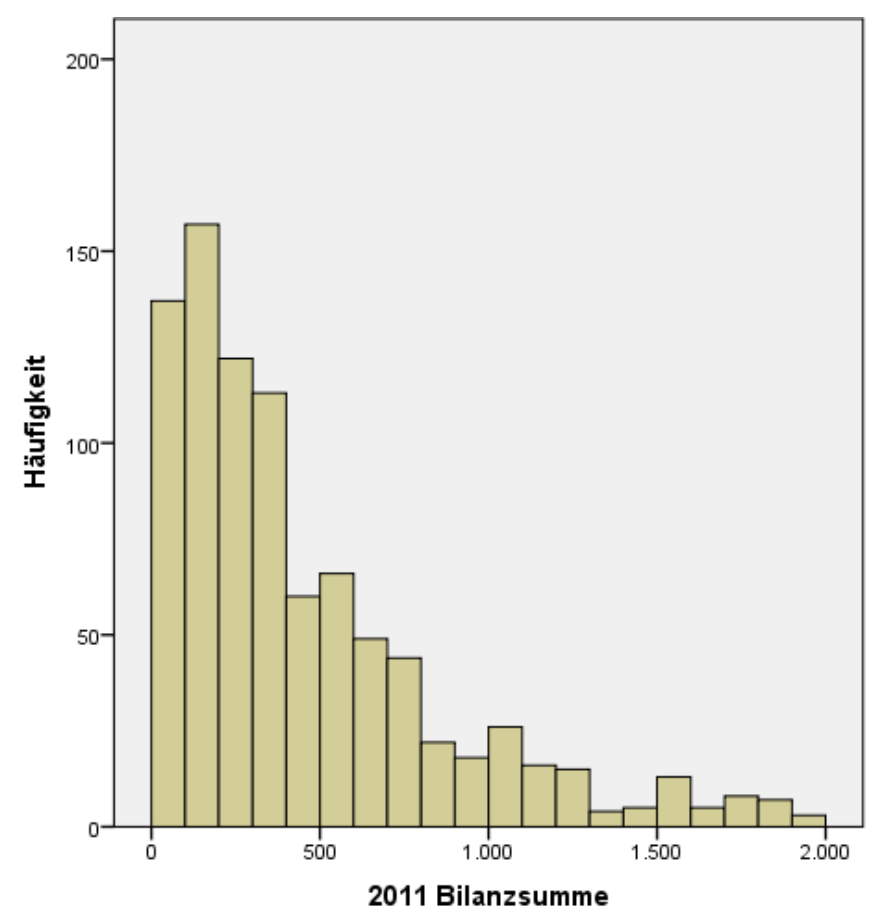

Abbildung 63: Histogramm zur Häufigkeit von Banken im jeweiligen Intervall ${ }^{973}$

Der folgende Boxplot in Abbildung 64 illustriert die Verteilung für alle Geschäftsjahre im Betrachtungszeitraum 2005 bis 2011. Ein leichtes Wachstum der Bilanzsumme von Jahr zu Jahr kann abgelesen werden.

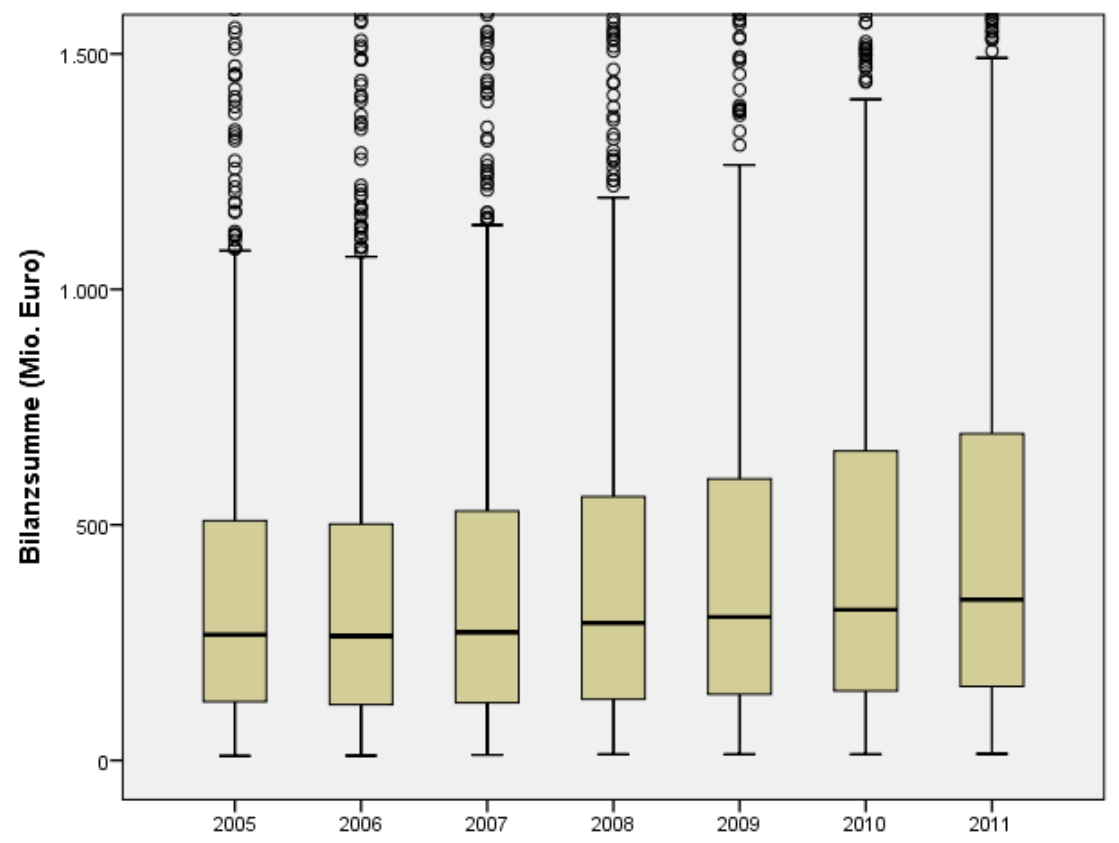

Abbildung 64: Boxplot zur Verteilung der Bilanzsummen

Bereits in vorangegangenen Hypothesentests wurden Quintile für die Verdeutlichung einsetzt. In Abbildung 65 findet sich eine grafische Darstellung der Erfolgsverteilungen

\footnotetext{
973 Mittelwert: 611 Mio. Euro; n=932; Bilanzsummenwerte oberhalb von 2 Mrd. Euro existieren sporadisch und wurden darstellungsbedingt abgeschnitten.
} 
aller Banken über die einzelnen Jahre. Die Bilanzsummen der einzelnen Institute wurden aufsteigend nach der Größe sortiert und in Quintile eingeteilt. Anschließend wurde die mittlere Bruttogewinnspanne aller Institute im jeweiligen Quintil und Jahr ermittelt.

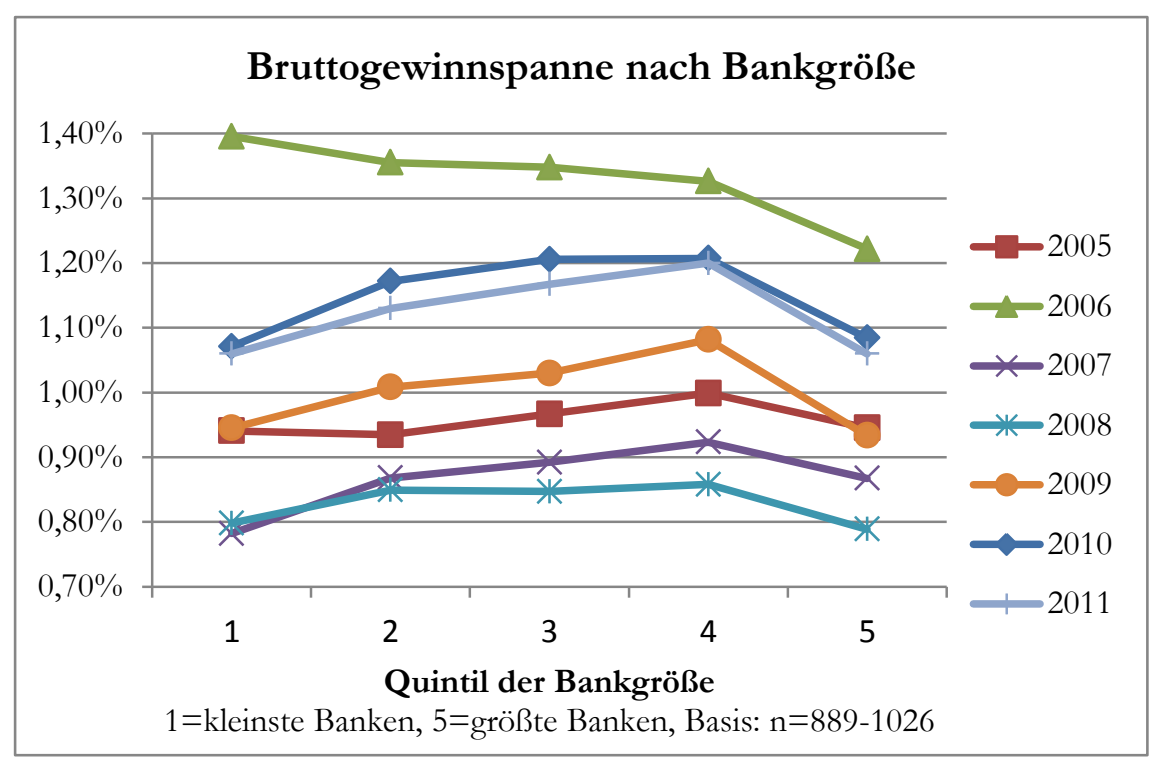

Abbildung 65: Zusammenhang zwischen Bankgröße und Bruttogewinnspanne

Wie man in Abbildung 65 sehen kann, weisen größere Banken eine höhere Bruttogewinnspanne als kleinere Banken auf. Dies ist insbesondere auf Skaleneffekte zurückzuführen. An einer bestimmten Stelle bzw. in einem bestimmten Bereich liegt dann folgend eine kritische Bilanzsumme. Bei dieser handelt es sich um die gesuchte optimale Betriebsgröße, bei welcher die Bruttogewinnspanne maximal ist. Nach Überschreiten dieser kritischen Bilanzsumme führen andere Einflüsse zur Umkehrung bzw. zur Überkompensation der Skaleneffekte. ${ }^{974}$ Dies könnten Kosten sein, die im Zusammenhang mit der komplexeren Unternehmensstruktur stehen, welche durch die Größe bedingt wird. ${ }^{975}$ Diese Komplexitätskosten begründen sich beispielsweise durch höhere Aufwendungen bei der Organisation des Unternehmens wie durch eine Finanzierung einer höheren Anzahl von Hierarchieebenen. ${ }^{976}$ Daher sind sprunghafte Kostenverläufe möglich. Auch ist denkbar, dass große Institute kostenintensivere Geschäftsfelder bedienen können und auch bedienen. Dies führt ceteris paribus zwar einerseits zu einem Anstieg der Bilanzsumme, andererseits jedoch nur zu einem unterproportionalen Wachstum der Bruttogewinnspanne.

Es kann für nahezu alle untersuchten Geschäftsjahre der beschriebene Zusammenhang klar belegt werden: Das vierte Bankgrößenquintil beinhaltet die höchste Bruttogewinnspanne. Nur im Jahr 2006 wird ein abweichendes Resultat diagnostiziert: Die kleinsten Institute erzielten damals die besten Ergebnisse. Größere Institute blieben dahinter zurück. Es muss erwähnt werden, dass 2006 - im Jahr vor Ausbruch der Finanzkrise - viele Institute bei weitem die höchsten Bruttogewinnspannen im Betrachtungszeitraum erzielten. ${ }^{977}$ Daher kann hier von einem Ausnahmejahr gesprochen werden. 
Das im Folgenden beschriebene explorative Verfahren wird genutzt, um den empirischen Gesetzmäßigkeiten näher beizukommen. Dieses Verfahren hat zum Ziel, die effiziente Betriebsgröße in Euro zu identifizieren. Es trägt dem oben unterstellten, nichtlinearen Verlauf Rechnung, der sich hier zwischen dem Erfolg und der Institutsgröße abzeichnet. Das Verfahren basiert auf der Regressionsanalyse und liefert in der Regel eine Funktion, welche bei einem geringen Wert für das erste Quantil beginnt. Über die nächsten Quantile erfolgt ein Anstieg bis hin zu einem Maximalwert. Nach diesem Maximalwert kommt es wieder zu einer Verringerung des Erfolgsmaßes, bis einschließlich zum letzten gebildeten Quantil. Zur genaueren Identifizierung dieses Maximalwertes werden die nach Größe geordneten Betriebsgrößen zunächst in fünf, dann schrittweise in mehr Quantile eingeteilt. Danach wird aufgeklärt, in welchem Quantil sich der Maximalwert der Bruttogewinnspanne befindet. Schließlich wird für dieses Quantil die Quantilsmitte bestimmt und errechnet, welcher Bilanzsumme dieser Wert entspricht. Je kleiner dabei die Quantile gewählt werden, desto präziser lässt sich die erfolgsmaximale Bilanzsumme lokalisieren. Im Extremfall kann man für jedes Geschäftsjahr genau eine Bilanzsumme mit der höchsten Bruttogewinnspanne ermitteln. ${ }^{978} \mathrm{Um}$ sinnvolle Ergebnisse sicherzustellen, muss jedoch eine nennenswerte Anzahl von Instituten in einem Quantil liegen.

Tabelle 46 enthält für jedes Jahr die Resultate der entsprechenden Berechnungen. Zusätzlich wurde für jedes Jahr das arithmetische Mittel der Werte berechnet Mittlere erfolgsmaximale Betriebsgröße). Hiervon ausgenommen wurden insgesamt sieben Werte der Jahre 2006 und 2008 aufgrund der starken Abweichungen von den restlichen Daten. Systematische Fehler können ausgeschlossen werden. ${ }^{979}$ Ein Ausreißer-Test ergab, dass diese Werte nicht zur präzisierten Stichprobe zu rechnen sind. ${ }^{900}$ Um eine Verfälschung der Ergebnisse zu vermeiden, sind die Werte von der Auswertung auszuschließen. ${ }^{981}$

Der errechnete Mittelwert ist als erfolgsmaximale Betriebsgröße zu interpretieren. Aufgrund der empirischen Evidenz konnte gezeigt werden, dass Institute im Bereich dieser Betriebsgröße die höchste Effizienz aufwiesen.

\begin{tabular}{|c|c|c|c|c|c|c|}
\hline \multirow[b]{2}{*}{ Geschäftsjahr } & \multicolumn{5}{|c|}{ Anzahl der verwendeten Quantile } & \multirow{2}{*}{$\begin{array}{c}\text { Mittlere er- } \\
\text { folgsmaximale } \\
\text { Betriebsgröße }\end{array}$} \\
\hline & 5 & 10 & 20 & 25 & 50 & \\
\hline 2005 & 441,5 & 509,0 & 479,4 & 492,3 & 486,7 & 481,8 \\
\hline 2006 & $(59,4)$ & $(118,6)$ & $(278,7)$ & $(124,5)$ & $(87,3)$ & $(133,7)$ \\
\hline 2007 & 451,5 & 529,2 & 487,2 & 504,4 & 492,6 & 493,0 \\
\hline 2008 & 489,4 & 559,6 & $(117,0)$ & 546,5 & $(109,5)$ & 531,8 \\
\hline 2009 & 520,6 & 597,2 & 416,2 & 520,6 & 501,5 & 511,2 \\
\hline 2010 & 555,4 & 489,1 & 526,0 & 555,4 & 541,7 & 533,5 \\
\hline 2011 & 597,1 & 525,1 & 554,1 & 597,1 & 581,0 & 570,9 \\
\hline
\end{tabular}

Tabelle 46:

Erfolgsmaximale Betriebsgröße in Mio. Euro

\footnotetext{
${ }_{978}$ Dies würde jedoch zum Overfitting des Modells führen und keine weiteren induktiven Schlussfolgerungen zulassen.

${ }^{979} \mathrm{Vgl}$. Hartung et al. (2005), S. 343. Das Abweichen ist nicht durch Mess-, Rechen-, Schreib- oder Datenerfassungsfehler zu erklären.

${ }^{980}$ Der Wert liegt unterhalb der unteren Ausreißergrenze. Vgl. Hartung et al. (2005), S. 343; vgl. Bortz und Schuster (2010), S. 44; vgl. Abschnitt 4.2.4.

981 Vgl. Hartung et al. (2005), S. 343.
} 
Die Interpretation soll beispielhaft für das Jahr 2011 erklärt werden. Hier wurden die aufsteigend sortierten Bilanzsummen unter anderem in zehn Quantile eingeteilt. Das siebente Quantil, welches sich von 60 bis $70 \%$ erstreckt, enthält den höchsten Mittelwert, das heißt, es setzt sich im Mittel aus den höchsten Bruttogewinnspannen zusammen. Die Quantilsmitte $(65 \%)$ entspricht rechnerisch einer Bilanzsumme von 525,1 Mio. Euro. Eine grafische Darstellung der erfolgsmaximalen Betriebsgrößen enthält Abbildung 66.

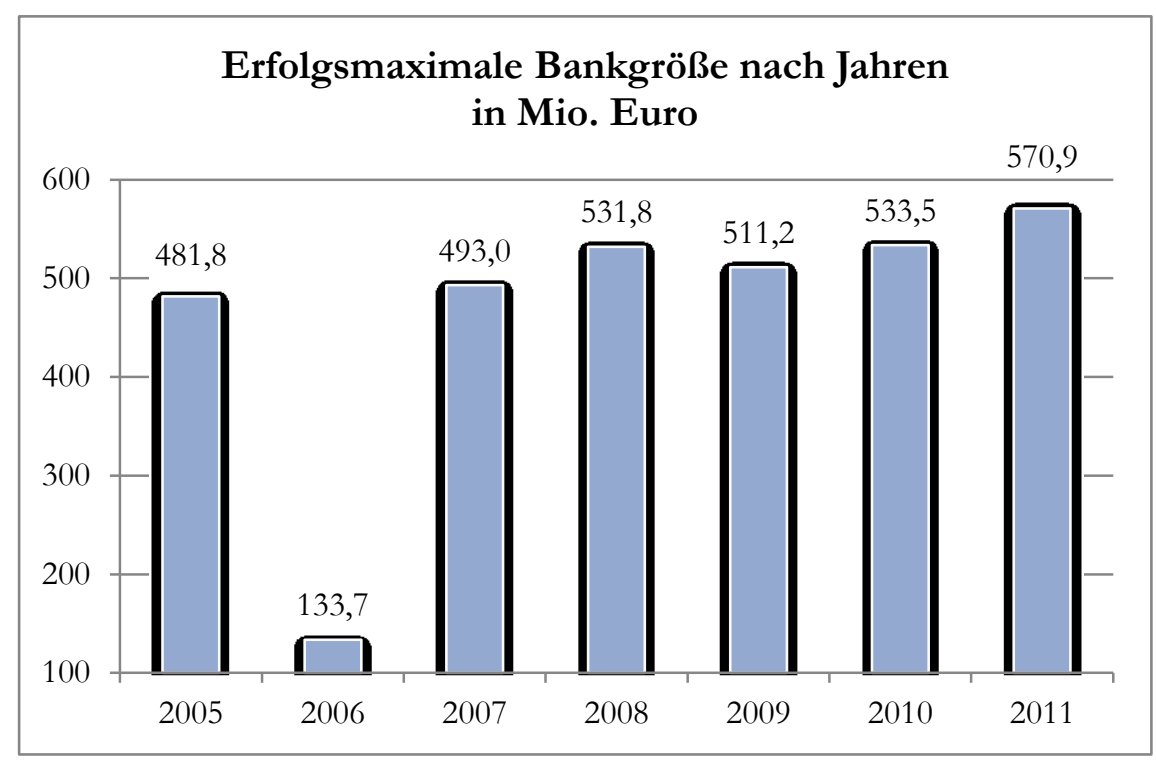

Abbildung 66: Erfolgsmaximale Bankgröße nach Jahren in Mio. Euro

Über den Verlauf der Jahre 2005 bis 2011 lässt sich ein Anstieg der erfolgsmaximalen Betriebsgröße feststellen. Nach ausreißerbegründetem Ausschluss des Ausnahmejahres 2006 lässt sich folgender linearer funktionaler Zusammenhang postulieren, welcher die erfolgsmaximale Bankgröße $G_{\max }$ in Abhängigkeit des interessierenden Jahres ermittelt:

$$
G_{\max }(\mathrm{Jahr})=13,365 \cdot(\mathrm{Jahr}-2004)+462,46 .
$$

Das Bestimmtheitsmaß $\mathrm{R}^{2}$ dieser Regressionsfunktion liegt bei 0,80 und erklärt somit $80 \%$ der Gesamtvariation. ${ }^{982}$ Im nächsten Schritt wird die Funktion näher beschrieben. Es soll gezeigt werden, dass unterhalb der erfolgsmaximalen Größe $G_{\max }$ ein positiver Zusammenhang zwischen Erfolg und Institutsgröße besteht, und oberhalb $G_{\max }$ entsprechend ein negativer Zusammenhang. Daher wird die Datenreihe in der Mitte des erfolgsmaximalen Quantils aufgeteilt. Die jeweils trennende, erfolgsmaximale Bilanzsumme geht oben aus Tabelle 46 hervor. Wie aus Abbildung 65 ersichtlich, enthält das vierte Quintil, am Beispiel des Geschäftsjahre 2011, die erfolgsmaximale Institutsgröße. Der Mittelwert aller in die Berechnung eingehenden Werte für die Bruttogewinnspanne liegt in diesem Quintil liegt bei ca. $1,20 \%$. Bildet man anstatt fünf Quintilen nun 50 Quantile, so beträgt die Quantilsbreite $2 \%$. Das erfolgsmaximale Quantil ist jenes im Wertebereich von 68 bis $70 \%$. Die der Quantilsmitte von $69 \%$ entsprechende Bilanzsumme beträgt 581,0 Mio. Euro. 
Bevor die Korrelationsanalyse nach PEARSON zum Einsatz kommen kann, muss überprüft werden, ob die Voraussetzungen für deren Einsatz erfüllt sind. ${ }^{983}$ Hierzu wird zuerst die Normalverteilung überprüft. Für alle Geschäftsjahre konnte anhand des SHAPIRO-WILKTests gezeigt werden, dass die Werte für das Erfolgsmaß Bruttogewinnspanne normalverteilt sind. ${ }^{984}$ Als zweites muss geklärt werden, ob ein linearer oder quasi-linearer Zusammenhang vermutet werden kann. Für die beiden Abschnitte, die jeweils unterhalb bzw. oberhalb der effizienten Betriebsgröße liegen, ist dies der Fall.

Wie aus Tabelle 47 ablesbar ist, ließ sich zeigen, dass unterhalb der erfolgsmaximalen GröBe $G_{\max }$ stets eine positive Korrelation herrscht, das heißt, mit zunehmender Bilanzsumme steigt auch das Erfolgsmaß. Bei $G_{\max }$ liegt der maximale Erfolg. Nach Überschreiten von $G_{\max }$ bis zum Maximalwert der Betriebsgröße am Ende der Datenreihe konnte stets ein negativer Zusammenhang nachgewiesen werden. Betrachtet man also Institute, deren Größe jenseits von $G_{\max }$ liegen, so ist für diese mit einem Rückgang des Erfolgsmaßes zu rechnen. Nahezu alle Korrelationskoeffizienten unterscheiden sich signifikant von Null. ${ }^{985}$

Als eine neue Erkenntnis der vorliegenden Arbeit kann subsumiert werden, dass es erstmals gelungen ist, eine optimale Bankgröße für Kreditgenossenschaften in Euro zu quantifizieren. Für das Geschäftsjahr 2015 liegt die erfolgsmaximale Größe in Mio. Euro bei

$$
G_{\max }(2015)=13,365 \cdot(2015-2004)+462,46=609,48 .
$$

\begin{tabular}{c|cccc}
\multirow{2}{*}{} & \multicolumn{2}{|c}{ Unterhalb von $\mathbf{G}_{\max }$} & \multicolumn{2}{c}{ Oberhalb von $\mathbf{G}_{\max }$} \\
& Korrelation & Basis & Korrelation & Basis \\
\hline 2005 & 0,037 & 649 & $-0,149 *$ & 240 \\
2006 & 0,019 & 174 & $-0,154 * * *$ & 846 \\
2007 & $0,145^{* * *}$ & 749 & $-0,169 * * *$ & 277 \\
2008 & 0,023 & 731 & $-0,108$ & 267 \\
2009 & $0,132^{* * *}$ & 665 & $-0,284 * * *$ & 295 \\
2010 & $0,156^{* * *}$ & 666 & $-0,188^{* *}$ & 299 \\
2011 & $0,147^{* * *}$ & 643 & $-0,220^{* * *}$ & 289 \\
$* * *, * *, *$ Korrelationskoeffizient auf dem 0,1 \%-, 1 \%- bzw. 5\%-Niveau signifikant unterschiedlich von Null. \\
Tabelle 47: Korrelationen unterhalb und oberhalb der effizienten Bankgröße
\end{tabular}

Es folgt eine deskriptive Betrachtung des erfolgsmaximalen 4. Quintils, dessen Banken im Jahr 2011 Bilanzsummen zwischen 449,3 und 794,8 Mio. Euro aufwiesen. Zu klären ist, inwiefern sich die Zusammensetzung des Quintils vom Aufbau der Grundgesamtheit mit allen untersuchten Instituten unterscheidet. Die durchschnittliche Bruttogewinnspanne aller Institute des Quintils beträgt 1,21\% und liegt damit über dem Durchschnitt aller Institute von 1,12\%. In Bezug auf die Lagebereiche fällt mit $70 \%$ ein höherer Anteil halbstädtischer Institute im Quintil auf als in der Grundgesamtheit mit $56 \%$. Auch der Anteil der Institute mit städtischer Lage liegt mit $19 \%$ etwas höher als in der Grundgesamtheit (17\%). Hingegen sind mit $11 \%$ gegenüber $28 \%$ deutlich weniger ländliche Institute enthalten. Auch in Bezug auf die Lage nach Bundesländern sind kleinere Unterschiede erkennbar. Das 4. Quintil enthält überproportional viele Institute aus Niedersachsen und dem Saar-

\footnotetext{
${ }^{983} \mathrm{Vgl}$. Abschnitt 4.2.6.

${ }_{984}$ Die Irrtumswahrscheinlichkeit liegt für alle Geschäftsjahre bei unter 0,001***

${ }^{985} \mathrm{Vgl}$. Abschnitt 4.2.6.
} 
land, dagegen einen unterproportionalen Anteil aus Baden-Württemberg und NordrheinWestfalen.

Trotz einer quantifizierten erfolgsmaximalen Größe sollte man jedoch nicht sofort die Schlussfolgerung ziehen, dass alle größenmäßig darunterliegenden Institute fusionieren und sich größere Institute aufspalten müssen. Es können Abweichungen durch regionale Besonderheiten auftreten, die das verwendete Verfahren nicht abzubilden vermag. Außerdem konnte in der Literatur gezeigt werden, dass sich räumliche Nähe und Kenntnis der lokalen Märkte auszahlen und äußert sich nicht zuletzt in einer beachtlichen Krisenfestigkeit des deutschen dezentralen Bankwesens. ${ }^{986}$

Bevor kleinere Institute fusionieren können, ist zunächst eine sorgfältige Vorbereitung notwendig. Hierbei stellt für die Kunden auch die Identifikation mit der Bank ein wichtiges Kriterium dar. In Instituten mit einem flächenmäßig großen Geschäftsgebiet ist eine Identifikation des Kunden mit der Bank einerseits und die Identifikation der Bank mit der Region andererseits nicht mehr so stark ausgeprägt möglich, das Regionalprinzip wandelt sich zu einem überregionalen Prinzip. REICHLING argumentiert, dass eine Fusion naturgemäß einen größeren räumlichen Abstand der Kunden und Mitarbeiter zur aufnehmenden Bank bringt. ${ }^{987}$ Eine Fusion berge daher einen Verlust der regionalen Identität der kleineren Genossenschaftsbanken in sich. ${ }^{988}$

Umgekehrt kann die Fusion von Kreditgenossenschaften mit sich überschneidenden Geschäftsgebieten kosteneffizient sein. Diese Fusion trägt zu der oben von STAATS beschriebenen Wettbewerbsvermeidung zwischen Kreditgenossenschaften bei. ${ }^{989}$ Dieser Wettbewerb würde in der Realität zu sinkenden Margen bei allen involvierten Instituten führen. Die Erhaltung der Präsenz in der Fläche ist an sich sehr kostenintensiv und bisweilen wenig ertragreich, sie sollte daher nicht unnötig durch derartige margenschmälernde Aktivitäten gefährdet werden, wie sie in sich überschneidenden Geschäftsgebieten ceteris paribus stattfinden. Vorteilhaft ist es in diesem Fall, wenn bei der Aufnahme der zweiten Bank keine neuen Verwaltungsstrukturen erschaffen werden. Zu bevorzugen ist ein reines Ankoppeln an bestehende Hierarchieebenen.

Auch dieser Zusammenhang resultiert in der Empfehlung, ein Größenwachstum in der Fläche genau zu hinterfragen. Die erfolgsmaximale Größe ist daher eher als eine Empfehlung anzusehen, bei deren Erreichen weitere Fusionen besonders kritisch überprüft werden sollten, bevor ineffizienter Größenordnungen entstehen. Dennoch befindet sich eine große Anzahl von Instituten noch weit unterhalb dieser erfolgsmaximalen Größe, weshalb unter anderem von einer weiter rasch voranschreitenden Konsolidierung ausgegangen werden kann.

Strebt ein sehr großes Institut eine erfolgsmaximale Betriebsgröße an, die unter der aktuellen Größe liegt, so ist dies umgekehrt durch eine Aufspaltung zu erreichen. Dies kann jedoch dazu führen, dass bestimmte Geschäftsarten, für die eine große Bilanzsumme vonnöten ist, nicht mehr durchgeführt werden können. Es ist daher hierin keine explizite Handlungsempfehlung zur Verkleinerung von Instituten zu sehen.

\footnotetext{
986 Vgl. Christians und Gärtner (2014), S. 2 und 4.

987 Vgl. Reichling (2004), S. 152.

988 Vgl. Abschnitt 3.3.5; vgl. Reichling (2004), S. 152.

${ }^{989}$ Vgl. Abschnitt 3.3.3.
} 
Im Zusammenhang mit der Bankgröße wurde die Anzahl der Zweigstellen betrachtet. Eine entsprechende Panelanalyse liefert folgendes Ergebnis: Je größer die Bilanzsumme einer Bank in Relation zur Anzahl der Zweigstellen, umso geringer ist empirisch die Sachaufwandsspanne der Bank. Der Koeffizient einer entsprechenden univariaten Fixed EffectsPanelanalyse beträgt $-0,0020^{* * *} \cdot{ }^{990}$ Für jede Million Euro mehr, welche auf eine Zweigstelle entfällt, sinkt die Sachaufwandsspanne im Mittel um 0,20 Basispunkte. ${ }^{91}$ Das Resultat ist nachvollziehbar, denn bei einer höheren Arbeitsauslastung einer Zweigstelle kann diese effizienter arbeiten.

\subsubsection{Hypothese 16: Häufung kleiner Genossenschaftsbanken}

Die Hypothese lautet:

Es existieren Regionen mit besonders vielen, dafür aber sebr kleinen Genossenschaftsbanken.

Zur Überprüfung dieser Hypothese werden stellvertretend die Angaben zu den untersuchten Genossenschaftsbanken aus dem Jahr 2011 zugrunde gelegt; in den übrigen Jahren ergibt sich ein analoges Bild. Beim Vergleich der Bundesländer untereinander fällt zunächst auf, dass einige Flächenländer eine hohe absolute Zahl an Genossenschaftsbanken aufweisen. Dazu zählen Bayern (257), Baden-Württemberg (228) und Nordrhein-Westfalen (143). Aussagekräftiger wird der Vergleich durch den Einbezug der Fläche, die die Genossenschaftsbanken unter sich als Geschäftsgebiet aufteilen. Hierzu muss die Annahme getroffen werden, dass die Geschäftsgebiete der einzelnen Institute, oder zumindest der durchgeführten Geschäfte, sich nicht überlappen. Im Wesentlichen sollte diese Annahme mit der Realität vereinbar sein. Tabelle 48 enthält zu den einzelnen Bundesländern die Anzahl der untersuchten Institute sowie die Fläche, welche rechnerisch auf eine Genossenschaftsbank entfällt. Das dichteste Netz regionaler Kreditgenossenschaften existiert in BadenWürttemberg, wo auf jedes Institut lediglich $157 \mathrm{~km}^{2}$ an Fläche entfallen. BadenWürttemberg kann man attestieren, dass dort die genossenschaftliche Idee am stärksten angekommen und gelebt wird. Sieht man von den Stadtstaaten Hamburg und Bremen ab, so folgen Nordrhein-Westfalen mit 239 und Bayern mit 275 Quadratkilometer je Institut. Dieses sehr eindeutige Bild steht im Einklang mit Abbildung 19, in welcher die hohe Dichte der Genossenschaftsbanken anhand der eingezeichneten Nadeln zu erkennen ist. ${ }^{992}$ Auffällig ist der deutliche Bruch zwischen alten und neuen Bundesländern. Letztere weisen relativ wenige Genossenschaftsbanken auf, wodurch die auf eine einzelne Bank entfallende Fläche deutlich höher ist als in den alten Bundesländern. Kausal kann dies einerseits wieder auf die starke Rolle der Sparkassen als Wettbewerber zurückgeführt werden, die diese Institute noch immer in den neuen Bundesländern spielen. Andererseits muss auf den Mangel an Gewerbeinfrastruktur abgestellt werden, der zusammen mit dem vorgenannten Aspekt in Folge erforderlicher Anpassungen zur Bildung relativ großflächiger Institute beigetragen hat.

\footnotetext{
${ }^{990}$ Der Standardfehler liegt bei 0,00019.

${ }^{991}$ Die Bilanzsumme wurde hier in Mio. Euro berücksichtigt.

992 Siehe Seite 107.
} 


\begin{tabular}{|c|c|c|}
\hline Bundesland & $\begin{array}{l}\text { Anzahl untersuchter } \\
\text { Institute (2011) }\end{array}$ & $\begin{array}{l}\text { Fläche je Genossen- } \\
\text { schaftsbank (in } \mathrm{km}^{2} \text { ) }\end{array}$ \\
\hline Baden-Württemberg & 228 & 157 \\
\hline Hamburg & 4 & 189 \\
\hline Bremen & 2 & 210 \\
\hline Nordrhein-Westfalen & 143 & 239 \\
\hline Bayern & 257 & 275 \\
\hline Saarland & 9 & 285 \\
\hline Berlin & 3 & 297 \\
\hline Hessen & 66 & 320 \\
\hline Rheinland-Pfalz & 53 & 375 \\
\hline Schleswig-Holstein & 30 & 527 \\
\hline Niedersachsen & 89 & 535 \\
\hline Sachsen & 20 & 921 \\
\hline Thüringen & 15 & 1.078 \\
\hline Sachsen-Anhalt & 11 & 1.859 \\
\hline Mecklenburg-Vorpommern & 8 & 2.902 \\
\hline Brandenburg & 7 & 4.236 \\
\hline
\end{tabular}

Tabelle 48: $\quad$ Fläche je Genossenschaftsbank nach Bundesländern ${ }^{993}$

Die große Anzahl relativ kleiner Banken birgt ein hohes Potenzial für Fusionen. Dennoch gibt es viele Banken, die nicht zu Fusionen tendieren - die Fusionsbereitschaft ist in einigen Bundesländern nicht besonders ausgeprägt. Jedoch muss dies nicht zwingend als negativ oder gar rückständig beurteilt werden. Viele kleine Banken sind sehr auf den Erhalt der Eigenständigkeit und Regionalität bedacht. Wie bereits in Abschnitt 4.4.16 dargelegt, spielt der Bezug zur Region im Rahmen des Geschäftsmodells eine wichtige Rolle bei der Kundenbindung. Die Kunden legen Wert darauf, dass sich eine Bank mit der Region identifiziert. Bearbeiten Banken hingegen ein relativ großes Geschäftsgebiet, ist ein abnehmender Bezug zur Region die Folge.

\subsubsection{Hypothese 17: Zukunftsfähigkeit}

Die Hypothese lautet:

\section{Erfolgreiche Banken liegen in Gegenden mit guter Zukunftsfäbigkeit.}

Das Konzept der Zukunftsfähigkeit nach KRÖHNERT wurde bereits oben beschrieben. ${ }^{994}$ Im Rahmen dieser Hypothese soll überprüft werden, ob eine Abhängigkeit des Bankenerfolgs von der Zukunftsfähigkeit besteht, also von Demografie, Wirtschaft, Integration, Bildung und Familienfreundlichkeit. Hierzu wird wieder eine Quintilsbetrachtung durchgeführt. Als unabhängige Variable bzw. Erfolgsfaktor dient die Zukunftsfähigkeit in Form von fünf Quintilen. Als abhängige Variable wird die Bruttogewinnspanne untersucht. Es zeigt sich ein positiver Zusammenhang: Je höher die Zukunftsfähigkeit, desto höher fällt in der Regel die Bruttogewinnspanne aus. Die Ergebnisse werden in Abbildung 67 dargestellt. Jedoch wird die Zukunftsfähigkeit in Schulnoten bemessen. ${ }^{995}$ Daher sind Banken in Gegenden mit tendenziell schlechteren Zukunftsaussichten erfolgreicher, sofern man die Bruttogewinnspanne als Messkriterium einsetzt. Dies kann erneut im Wesentlichen mit

\footnotetext{
${ }^{993}$ Eigene Berechnungen nach Daten von Statistisches Bundesamt (2013b). 
dem Wettbewerbsargument begründet werden, denn hohe Margen lassen sich vor allem dort erzielen, wo es weniger Wettbewerb gibt. Die Wettbewerber hingegen fokussieren eher Gebiete mit günstigen Entwicklungsaussichten.

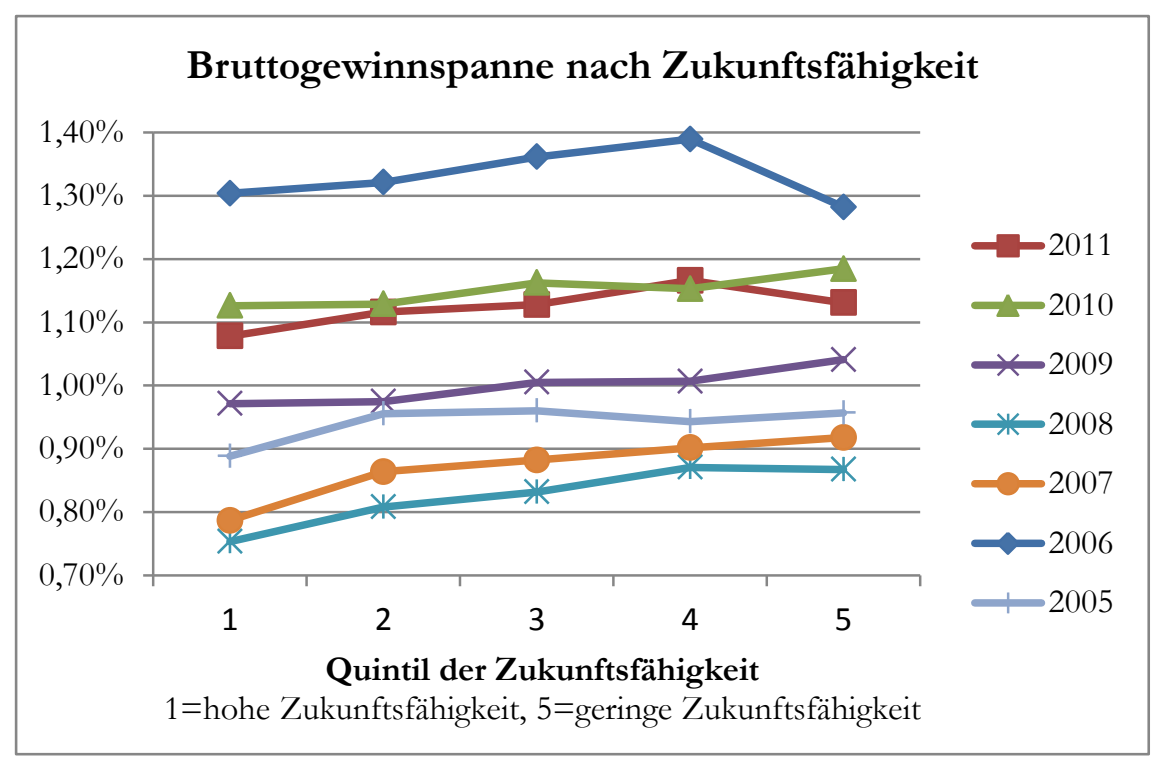

Abbildung 67: Zusammenhang zwischen Zukunftsfähigkeit und Bruttogewinnspanne

\subsubsection{Hypothese 18: Arbeitnehmerentgelt}

Die Grundlage für diese sowie für die folgenden drei Hypothesen bildet die Überlegung, dass erfolgreiche Banken in Gegenden mit vorteilhaften regionalen Wirtschaftsdaten liegen. In dieser Hypothese wird der Zusammenhang zum Arbeitnehmerentgelt je Arbeitnehmer untersucht.

Die Hypothese lautet:

Ein bobes Arbeitnebmerentgelt in der Region begünstigt den Erfolg regionaler Banken.

Das Arbeitnehmerentgelt umfasst „sämtliche Geld- und Sachleistungen, die den innerhalb eines Wirtschaftsgebietes beschäftigen Arbeitnehmern aus den Arbeits- oder Dienstverhältnissen zugeflossen sind. Das Arbeitnehmerentgelt (ANE) setzt sich zusammen aus den Bruttolöhnen und -gehältern sowie den tatsächlichen und unterstellten Sozialbeiträgen der Arbeitgeber. ${ }^{\text {‘996 }}$ Es handelt sich beim Arbeitnehmerentgelt also um den Entlohnungsaufwand inklusive aller Arbeitgeberanteile. Die Errechnung des Arbeitnehmerentgelts ist oben in Abbildung 20 schematisch dargestellt. ${ }^{997}$

Hinter dieser Hypothese steckt folgender vermuteter Zusammenhang: Ein hohes privates Einkommen führt ceteris paribus zum Wachstum der verfügbaren privaten Mittel der Einwohner. Diese Mittel liegen regelmäßig in Form von Einlagen auf Bankkonten und werden aus Sicht der jeweiligen Bank als Bilanzpassivposten 2 Verbindlichkeiten gegenüber Kunden geführt. Nun kann - aufgrund bestehender Geschäftsverhältnisse der Kunden zu anderen Banken sowie der Wettbewerbssituation - keineswegs davon ausgegangen werden,

\footnotetext{
${ }_{996}$ Arbeitskreis Volkswirtschaftliche Gesamtrechnungen der Länder VGRdL (2014b), Arbeitsblatt Definitionen.

${ }_{997}$ Siehe S. 111.
} 
dass alle diese Mittel auf Konten bei Genossenschaftsbanken gelagert werden. Es ist vielmehr davon auszugehen, dass sich die Mittel im Verhältnis der Marktanteile auf die jeweils vor Ort aktiven Banken verteilen. Die Banken können das durch Einlagen erhaltene Kapital beispielsweise in Form von Darlehen an andere Kunden ausreichen oder zu einem höheren Zins am Markt anlegen. Hierdurch erzielen sie Zinserträge aus Kredit- und Geldmarktgeschäften (Position 1. a) der GuV) bzw. Zinserträge aus festverzinslichen Wertpapieren und Schuldbuchforderungen (Position 1. b) der GuV). Parallel ist noch das Provisionsgeschäft über den Vertrieb von Vermögensanlageprodukten möglich, aus dem Provisionserträge (Position 5 der $\mathrm{GuV}$ ) entstehen. Beide Ertragsarten sind erfolgswirksam und führen schließlich zur Verbesserung des Bruttogewinns aus normaler Geschäftstätigkeit.

Zur Evaluierung dieses Zusammenhangs werden zunächst für jedes Geschäftsjahr alle verfügbaren Werte für das Arbeitnehmerentgelt in fünf Quintile eingeordnet. Dabei enthält Quintil eins die geringsten Entgelte, Quintil fünf hingegen die höchsten Entgelte. Eine grafische Darstellung der durchschnittlichen Bruttogewinnspannen der fünf Quintile findet sich in Abbildung 68.

Es stellt sich für die meisten Geschäftsjahre heraus, dass ergebnisschwache Banken eher in Regionen mit hohem Arbeitnehmerentgelt liegen. Hingegen liegen erfolgreiche Banken tendenziell in Gebieten mit niedrigem Arbeitnehmerentgelt. Für die Jahre 2008, 2009, 2010 und 2011 gilt, dass sich die übrigen Quintile zwei bis vier dazwischen entsprechend ihrer Reihenfolge einordnen. Hierbei ist die Bruttogewinnspanne die zu erklärende Variable. Das Quintil des Arbeitnehmerentgelts ist die erklärende Variable, deren Funktion als Erfolgsfaktor hier untersucht wird. Die Ergebnisse deuten darauf hin, dass ein umgekehrter Zusammenhang besteht: Ein hohes Arbeitnehmerentgelt resultiert in einer niedrigen Bruttogewinnspanne, ein geringes Arbeitnehmerentgelt führt zu einer hohen Bruttogewinnspanne. Dies steht der zugrundeliegenden Erwartung entgegen.

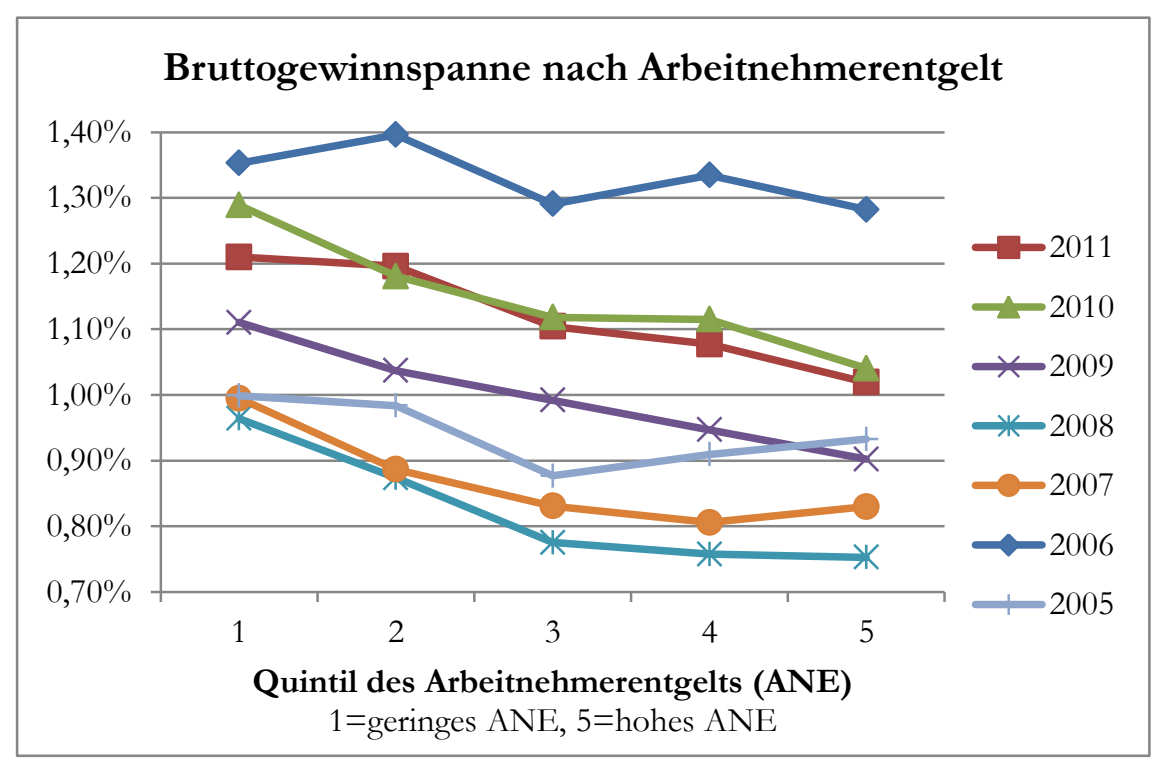

Abbildung 68: Einfluss des Arbeitnehmerentgelts auf die Bruttogewinnspanne

Im nächsten Untersuchungsschritt soll die Begründung für den so gearteten Zusammenhang geliefert werden. Es steht zu vermuten, dass es sich hierbei um eine Scheinkorrelation handelt. Wie bereits in Abschnitt 4.2.1 beschrieben, liegt eine Scheinkorrelation vor, wenn 
beide Größen von einer dritten abhängen. In Hypothese 1: Bruttogewinnspanne nach Stadt/Land-Lage (Abschnitt 4.4.2) wurde bereits die Abhängigkeit des Erfolgs von der Lage aufgezeigt. Möglicherweise hängt die Höhe des Arbeitnehmerentgelts ebenso von der Lage ab. Zur Untersuchung wird eine Varianzanalyse durchgeführt, um die Mittelwerte der drei Lagekategorien zu vergleichen. Tabelle 50 beinhaltet die Ergebnisse, eine grafische Darstellung folgt in Abbildung 69.

Es zeichnen sich für jedes Geschäftsjahr hochsignifikante Unterschiede zwischen städtischer Lage einerseits und halbstädtischer/ländlicher Lage andererseits ab. Institute in städtischer Lage weisen stets ein höheres Arbeitnehmerentgelt auf.

\begin{tabular}{|c|c|c|c|c|}
\hline Werte in $€$ & $\begin{array}{l}\text { Städtisch } \\
(\mathrm{n}=156)\end{array}$ & $\begin{array}{l}\text { Halbstädtisch } \\
\quad(\mathrm{n}=527)\end{array}$ & $\begin{array}{l}\text { Ländlich } \\
(\mathrm{n}=262)\end{array}$ & $\begin{array}{c}\text { F } \\
\text { (Welch) }\end{array}$ \\
\hline 2005 & $34.652^{\mathrm{a}}$ & $31.934^{b}$ & $31.344^{\mathrm{b}}$ & $36,60 * * *$ \\
\hline 2006 & $34.974^{a}$ & $32.452^{\mathrm{b}}$ & $31.857^{\mathrm{b}}$ & $28,83^{* * *}$ \\
\hline 2007 & $35.238^{a}$ & $32.719^{\mathrm{b}}$ & $32.138^{\mathrm{b}}$ & $27,69 * * *$ \\
\hline 2008 & $35.946^{\mathrm{a}}$ & $33.373^{b}$ & $32.827^{\mathrm{b}}$ & $26,87 * * *$ \\
\hline 2009 & $36.041^{\mathrm{a}}$ & $33.215^{b}$ & $32.759^{\mathrm{b}}$ & $33,13^{* * *}$ \\
\hline 2010 & $36.838^{a}$ & $34.179^{\mathrm{b}}$ & $33.720^{\mathrm{b}}$ & $29,07 * * *$ \\
\hline 2011 & $37.925^{\mathrm{a}}$ & $35.221^{\mathrm{b}}$ & $34.679^{\mathrm{b}}$ & $28,03^{* * *}$ \\
\hline
\end{tabular}

Tabelle 49: $\quad$ Entwicklung des Arbeitnehmerentgelts in Euro nach Lagebereichen

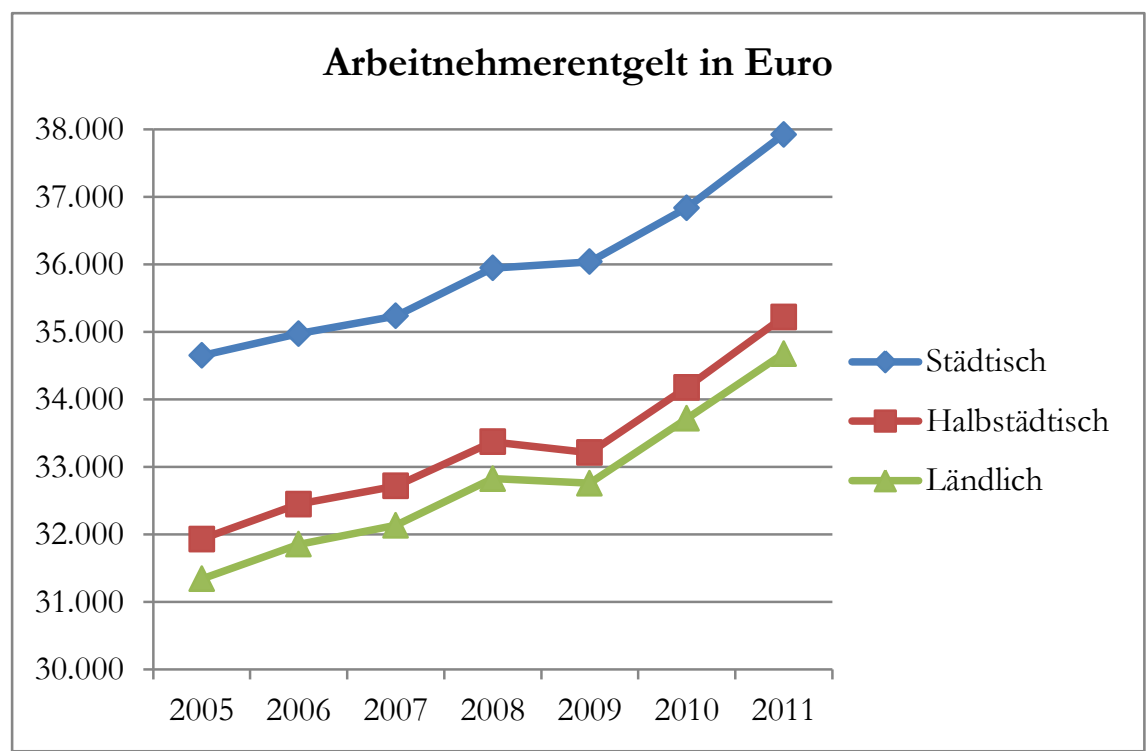

Abbildung 69: Entwicklung des Arbeitnehmerentgelts in Euro nach Lagebereichen

Der Erfolg der Institute ist folglich wesentlich stärker wettbewerbsinduziert als vom Arbeitnehmerentgelt abhängig, wie noch eingangs dieser Hypothese vermutet. Gemäß Abbildung 68 führt ein hohes Arbeitnehmerentgelt zu einer geringen Bruttogewinnspanne. Tatsächlich ist dies darauf zurückzuführen, dass Institute mit Sitz in einer Region mit hohem Arbeitnehmerentgelt tendenziell eine städtische Lage aufweisen, wo wiederum - durch den Wettbewerb begründet - nur eine geringe Bruttogewinnspanne erzielt werden kann.

Zusätzlich wurde eine Paneluntersuchung durchgeführt, um die Art der Abhängigkeit der Bruttogewinnspanne vom regionalen Arbeitnehmerentgelt näher zu durchleuchten bzw. zu un- 
termauern. Hierbei handelt es sich um eine Längsschnittfragestellung, das heißt, es wird die Variation im Zeitablauf der jeweiligen sieben Geschäftsjahre untersucht. ${ }^{998}$ Speziell ist es hier die unabhängige Variable Arbeitnehmerentgelt, welche sich im Zeitablauf ändert. Die regionalen Arbeitnehmerentgelte werden quasi als Eigenschaft der jeweiligen Bank betrachtet. Es sollen ausschließlich die Unterschiede innerhalb einer Bank verwendet werden, um die Koeffizienten zu ermitteln. ${ }^{999}$ Folglich werden Fixed Effects betrachtet. Der HAUSMANTest ${ }^{1000}$ bestätigt mit einem Signifikanzniveau von 0,95 , dass die Untersuchung von Fixed Effects angemessen ist. ${ }^{1001}$ Eine Hinzunahme von Dummyvariablen - beispielsweise für die Lageparameter städtisch, halbstädtisch und ländlich - ist nicht möglich, da es sich hierbei um zeitkonstante Variablen handelt, die vom Fixed Effects-Modell per se nicht berücksichtigt werden können. ${ }^{1002}$

Die Betrachtung der Quintile in Abbildung 68 lieferte einen umgekehrt proportionalen Zusammenhang: je höher die regionalen Löhne und Gehälter, desto geringer der Bankerfolg. Die Paneluntersuchung liefert hier jedoch einen positiven Koeffizienten von 0,0206 für die Variable ane, siehe Tabelle 50.

Das Arbeitnehmerentgelt wurde in Tausend Euro einbezogen, die Bruttogewinnspanne bgs als Prozentwert des Bruttogewinns aus normaler Geschäftstätigkeit in Bezug zur durchschnittlichen Bilanzsumme. Der Koeffizient ist auf folgende Weise zu interpretieren: Bei einer Erhöhung des Arbeitnehmerentgelt in der Region einer Bank um 1.000 EUR ist im Mittel ceteris paribus mit einem Anstieg der Bruttogeninnspanne um 0,0206 Prozentpunkte zu rechnen.

Die Variable lfdnr trägt die eindeutige Kennnummer einer jeden Bank. Obwohl das Arbeitnehmerentgelt einen statistisch signifikanten Einfluss auf den Erfolg hat, erklärt es nur 0,65 \% der Varianz des Erfolgs (within- $\mathrm{R}^{2}$ ).

\begin{tabular}{lc}
\hline VARIABLE & $(1)$ \\
bne & $0,0206^{* * *}$ \\
& $(0,00330)$ \\
Konstante & $0,347 * * *$ \\
& $(0,111)$ \\
Beobachtungen & 7.095 \\
Anzahl von lfdnr & 1.092 \\
$\mathrm{R}^{2}$ & 0,006 \\
\hline \multicolumn{2}{c}{ Standardfehler in Klammern } \\
$* * *, * * *$ signifikant auf dem $0,1 \%-, 1 \%-$ bzw. $5 \%$-Niveau.
\end{tabular}

Tabelle 50: $\quad$ Einfluss des regionalen Arbeitnehmerentgelts auf die Bruttogewinnspanne

Naturgemäß herrscht eine sehr hohe Korrelation von nahe eins zwischen dem Arbeitnehmerentgelt (ANE) und den Bruttolöhnen und -gebältern (BLG) einer Region. Dies kann auf

\footnotetext{
998 Vgl. Kopp und Lois (2009), S. 19.

${ }_{999}$ Vgl. Giesselmann und Windzio (2012), S. 107 f

1000 Vgl. Hausman, J. A. (1978), S. 1251 ff.

1001 Vgl. Torres-Reyna (2007), S. 29.

1002 Vgl. Abschnitt 4.2.8.
} 
Ebene der Kreise und kreisfreien Städte für die Jahre 2005 bis 2011 ( $n=446)$ gezeigt werden. Die Korrelationskoeffizienten $r_{A N E ; B L G}$ der einzelnen Jahre rangieren im Bereich 0,9977 bis 0,9985. Die Betrachtung der Bruttolöhne und -gehälter führt folglich zu einem vergleichbaren Resultat wie in der aktuellen Hypothese. Dies trifft auch für die durchgeführte Panelanalyse zu. Auf eine Darstellung und eine Diskussion wird daher verzichtet.

\subsubsection{Hypothese 19: BIP je Einwohner}

Die Hypothese lautet:

Erfolgreiche Banken liegen in Regionen mit bobem Bruttoinlandsprodukt je Einwobner.

Hinter dieser Hypothese steckt folgender vermuteter Zusammenhang:

Eine hohe regionale Produktivität, gemessen pro Einwohner, führt zu mehr Möglichkeiten für Genossenschaftsbanken, ihr Kerngeschäft zu betreiben. Inbegriffen sind hier insbesondere die Entgegennahme von Einlagen zur Refinanzierung und die Ausreichung der Mittel in Form von Darlehen für Firmen- und Privatkunden. Zusätzlich bietet auch das Provisionsgeschäft Margenchancen, beispielsweise durch den Vertrieb von Finanzanlagen.

Wie bereits vorher werden den Quintilen des Bruttoinlandsprodukts die mittleren Bruttogewinnspannen der jeweiligen Genossenschaftsbanken zugeordnet. Die Werte für das Bruttoinlandsprodukt liegen detailliert auf Kreisebene vor und werden der jeweiligen Bank vor Ort zugeordnet, um einen größtmöglichen regionalen Bezug sicherzustellen. ${ }^{1003}$ Eine grafische Darstellung findet sich in Abbildung 70.

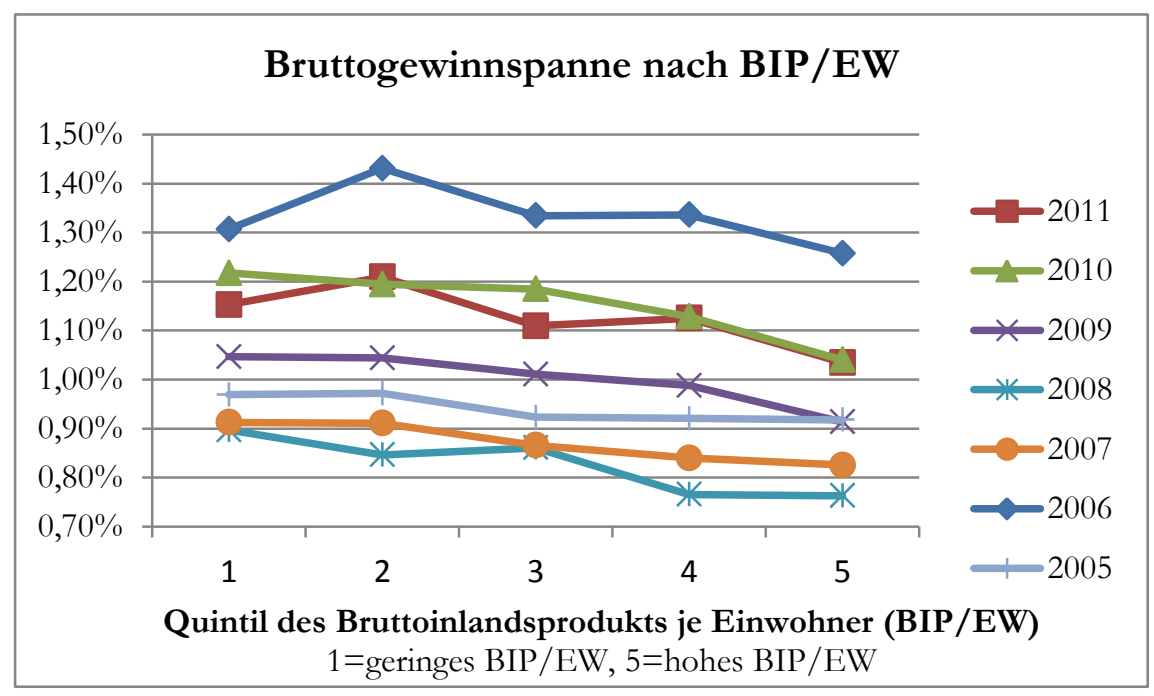

Abbildung 70: Zusammenhang zwischen regionalem BIP je Einwohner und der Bruttogewinnspanne

$\mathrm{Zu}$ beobachten ist für alle betrachteten Geschäftsjahre, dass in Regionen mit relativ hohem BIP je Einwohner (Quintil 5) die erfolgsschwächsten Banken liegen. Umgekehrt gilt für die meisten Regionen mit relativ geringem BIP je Einwohner (Quintil 1), dass dort die erfolgreichsten Banken ansässig sind. Die übrigen Werte liegen etwa dazwischen. 
Unterstellt man eine direkte Beziehung zwischen den beiden Größen, so sind hierfür zwei Richtungen des Einflusses denkbar. Einerseits könnte ein schwaches BIP als Ursache zu erfolgreichen Genossenschaftsbanken führen. Dies ist jedoch unwahrscheinlich. Andererseits könnte ein Mangel an Wettbewerbern dazu führen, dass es den Genossenschaftsbanken systematisch gelingt, hohe Margen gegenüber ihren regionalen Kunden durchzusetzen. So sind die Banken ihrerseits erfolgreich, während etwa teure Kreditfinanzierungen die regionale Produktivität bremsen, was sich schließlich auf die Entwicklung des regionalen Bruttoinlandsprodukts auswirkt. Jedoch scheint dieser kausale Begründungsansatz zu pessimistisch.

Am wahrscheinlichsten ist hingegen wieder eine Wirkung zusammen mit einer dritten Variablen. Von der Lagekategorie kann auf die Intensität des Wettbewerbs geschlossen werden. Tabelle 51 enthält die Resultate der Varianzanalyse zur Evaluation der Unterschiede des BIP je Einwohner zwischen den einzelnen Lagekategorien. Es fällt auf, dass die in städtischen Lagen das BIP je Einwohner drastisch über dem in halbstädtischen und ländlichen Bereichen liegt. In Städten herrscht zwar eine hohe Einwohnerdichte, diese wird also stark durch die Wirtschaftsstärke der dort ansässigen Unternehmen überkompensiert. Die Resultate werden in Abbildung 71 grafisch dargestellt. Eine Paneluntersuchung mit Fixed Effects führt nicht zu signifikanten Koeffizienten für das BIP je Einwohner.

\begin{tabular}{c|cccc} 
Werte in $€$ & $\begin{array}{c}\text { Städtisch } \\
(\mathrm{n}=156)\end{array}$ & $\begin{array}{c}\text { Halbstädtisch } \\
(\mathrm{n}=527)\end{array}$ & $\begin{array}{c}\text { Ländlich } \\
(\mathrm{n}=262)\end{array}$ & $\begin{array}{c}\mathbf{F} \\
(\text { Welch})\end{array}$ \\
\hline 2005 & $37.669^{\mathrm{a}}$ & $23.932^{\mathrm{b}}$ & $22.495^{\mathrm{b}}$ & $88,53^{* * *}$ \\
2006 & $39.256^{\mathrm{a}}$ & $25.100^{\mathrm{b}}$ & $23.528^{\mathrm{b}}$ & $86,22^{* * *}$ \\
2007 & $41.066^{\mathrm{a}}$ & $26.467^{\mathrm{b}}$ & $24.712^{\mathrm{b}}$ & $86,16^{* * *}$ \\
2008 & $41.515^{\mathrm{a}}$ & $27.072^{\mathrm{b}}$ & $25.505^{\mathrm{b}}$ & $84,08^{* * *}$ \\
2009 & $39.735^{\mathrm{a}}$ & $25.804^{\mathrm{b}}$ & $24.596^{\mathrm{b}}$ & $86,71^{* * *}$ \\
2010 & $41.812^{\mathrm{a}}$ & $27.463^{\mathrm{b}}$ & $26.144^{\mathrm{b}}$ & $79,04^{* * *}$ \\
2011 & $43.123^{\mathrm{a}}$ & $28.789^{\mathrm{b}}$ & $27.464^{\mathrm{b}}$ & $70,53^{* * *}$ \\
\hline \multicolumn{5}{c}{ Basis: $\mathrm{n}=945$, Welch-Test (Post-Hoc-Test: Tukey-HSD) } \\
Kennbuchstaben (a, b, c) unterscheiden sich signifikant auf dem 5 \%-Niveau.
\end{tabular}

Tabelle 51: $\quad$ Einfluss der Lage auf das regionale Bruttoinlandsprodukt je Einwohner

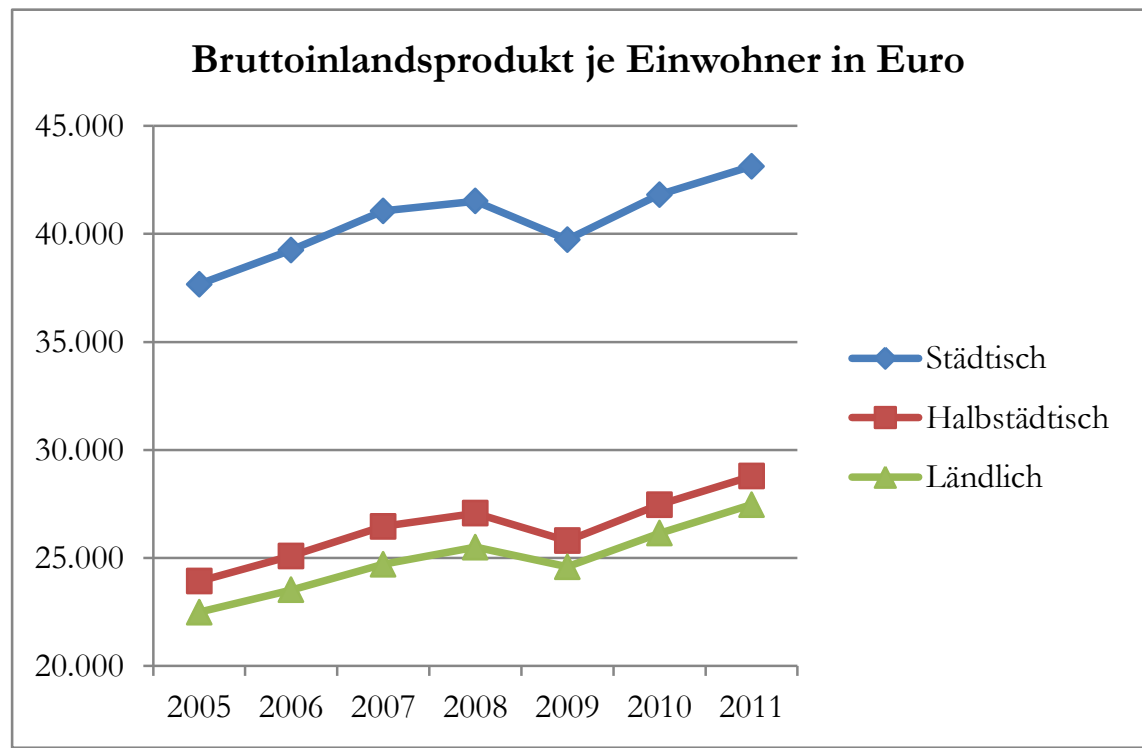

Abbildung 71: Entwicklung des regionalen BIP je Einwohner nach Lagebereichen 


\subsubsection{Hypothese 20: BIP je Erwerbstätiger}

Die Hypothese lautet:

Erfolgreiche Banken liegen in Regionen mit hobem Bruttoinlandsprodukt je Erwerbstätiger.

Die Korrelation zwischen dem Bruttoinlandsprodukt je Einwohner und dem Bruttoinlandsproduk.t je Erwerbstätiger rangiert - je nach Geschäftsjahr - zwischen 71,8 und 74,7 \%. Dies ist zwar ein hoher Wert, dennoch liegt dieser in deutlicher Entfernung zu 1, weshalb der Zusammenhang wieder im Detail betrachtet wird. Fraglich ist, ob das BIP je Enverbstätiger als Erfolgsfaktor für die Genossenschaftsbanken in Frage kommt. Das Bruttoinlandsprodukt wurde wieder auf Kreisebene der Bruttogewinnspanne der Institute zugeordnet, um den regionalen Bezug so genau wie möglich abzubilden. Wie anhand von Abbildung 72 dargestellt, führt in den meisten Geschäftsjahren ein höheres Bruttoinlandsprodukt je Erwverbstätiger $\mathrm{zu}$ einer niedrigeren Bruttogewinnspanne. In nahezu allen Geschäftsjahren sind diejenigen $20 \%$ Banken im Mittel am erfolgsreichsten, welche in Regionen mit schwachem BIP je Erwerbstätiger liegen. Lediglich 2006 stellt wieder ein Ausnahmejahr dar.

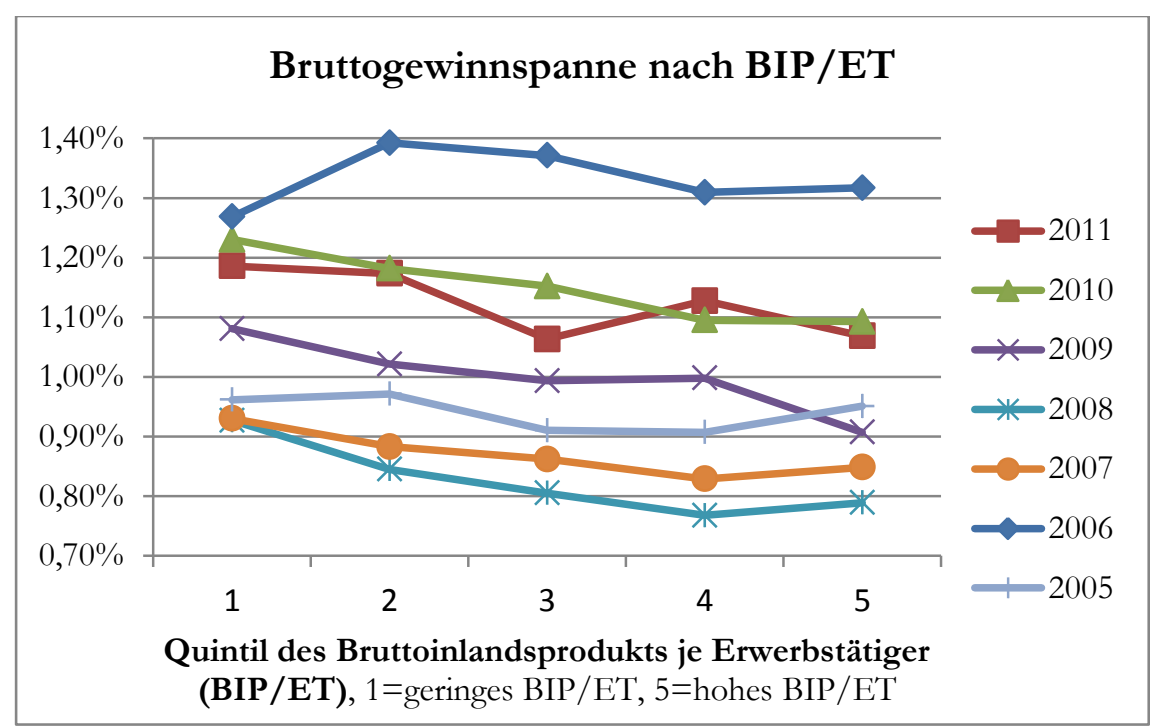

Abbildung 72: Zusammenhang zwischen der Bruttogewinnspanne und regionalem BIP je Erwerbstätiger

\begin{tabular}{|c|c|c|c|c|}
\hline Werte in $€$ & $\begin{array}{l}\text { Städtisch } \\
(n=156)\end{array}$ & $\begin{array}{l}\text { Halbstädtisch } \\
\qquad(\mathrm{n}=527)\end{array}$ & $\begin{array}{l}\text { Ländlich } \\
(\mathrm{n}=262)\end{array}$ & $\begin{array}{c}\mathbf{F} \\
\text { (Welch) }\end{array}$ \\
\hline 2005 & $59.151^{a}$ & $55.318^{\mathrm{b}}$ & $53.735^{\mathrm{b}}$ & $18,90^{* * *}$ \\
\hline 2006 & $61.244^{a}$ & $57.616^{\mathrm{b}}$ & $55.670^{c}$ & $19,23 * * *$ \\
\hline 2007 & $63.321^{\mathrm{a}}$ & $59.537^{\mathrm{b}}$ & $57.235^{c}$ & $21,20^{* * *}$ \\
\hline 2008 & $63.450^{a}$ & $60.030^{\mathrm{b}}$ & $58.058^{c}$ & $17,19 * * *$ \\
\hline 2009 & $60.752^{a}$ & $57.139^{\mathrm{b}}$ & $55.729^{\mathrm{b}}$ & $17,73^{* * *}$ \\
\hline 2010 & $63.607^{a}$ & $60.371^{\mathrm{b}}$ & $58.667^{\mathrm{b}}$ & $13,96^{* * *}$ \\
\hline 2011 & $65.036^{\mathrm{a}}$ & $62.124^{b}$ & $60.402^{\mathrm{b}}$ & $11,57 * * *$ \\
\hline
\end{tabular}

Tabelle 52: $\quad$ Einfluss der Lage auf das regionale Bruttoinlandsprodukt je Erwerbstätiger 
Wie bereits beim Bruttoinlandsprodukt je Einwohner kann dies auf eine Scheinkorrelation mit der Lagekategorie hindeuten. In Tabelle 52 werden die Ergebnisse einer entsprechenden Varianzanalyse numerisch aufgelistet und in Abbildung 73 grafisch dargestellt. Der vorangegangenen Hypothese zum BIP gleich zeigt sich in der Relation zur Anzahl Erwerbstätiger ein ähnliches, wenn auch nicht so stark ausgeprägtes, Gefälle von Stadt- zu Landlage. Die Begründung verläuft analog zur vorangegangenen Hypothese.

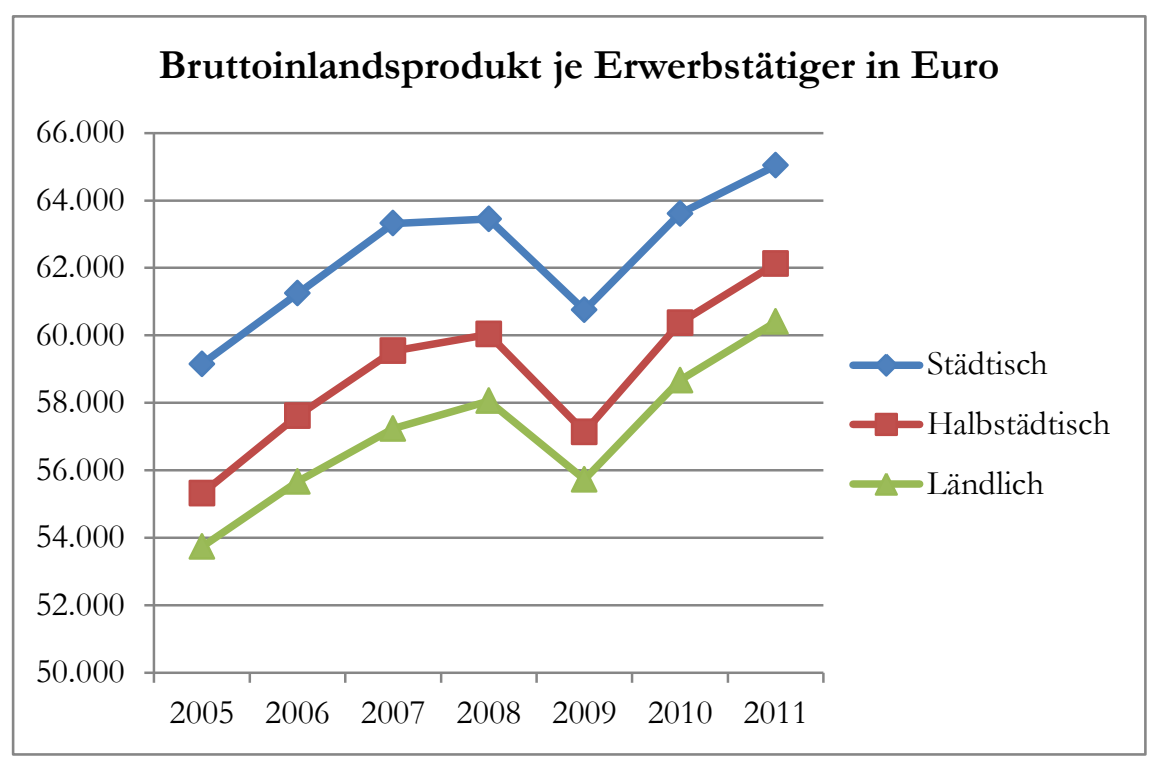

Abbildung 73: Zusammenhang zwischen der Lage und regionalem BIP je Erwerbstätiger

\subsubsection{Panelanalyse}

Im Nachgang zur Einzelbetrachtung möglicher Erfolgsfaktoren für Genossenschaftsbanken erfolgt nun eine Panelanalyse. Wie bereits oben beschrieben ${ }^{1004}$, handelt es sich bei Paneldaten um Daten, welche einerseits im Querschnitt über alle Banken vorliegen, und andererseits im Längsschnitt über mehrere Beobachtungszeitpunkte erhoben wurden. Für den Längsschnitt werden hier Daten von sieben Jahren verwendet. Die Panelanalyse untersucht den Einfluss von Erfolgsfaktoren auf den Erfolg.

Bei einigen Faktoren konnte in vorangegangenen Hypothesentests einzeln nachgewiesen werden, dass ein Einfluss auf die Bruttogewinnspanne als Erfolgsmaß besteht. Die nun folgenden Panelanalysen betrachten alle Faktoren integriert, um ein Gesamtbild der Abhängigkeiten zu erstellen. 
Wie bereits oben beschrieben ${ }^{1005}$, gehen mehrere Aufwands- und Ertragsgrößen in die Berechnung der Bruttogewinnspanne (bgs) ein. Um die Höhe des Einflusses zu quantifizieren, werden die wichtigsten der betreffenden Größen untersucht. Hierzu zählen:

- Zinserträge aus Kredit- und Geldmarktgeschäften (ze1s)

- Zinserträge aus festverzinslichen Wertpapieren und Schuldbuchforderungen (₹e2s)

- Laufende Erträge aus Aktien, Beteiligungen und Anteilen (les)

- Zinsaufwendungen (zas)

- Zinsüberschuss $(b ə s)$

- Provisionsüberschuss $(p s)$

- Sonstige betriebliche Erträge (ses)

- Personalaufwand ( $p k s)$

- Sachaufwand (skes)

Die Größen wurden im Rahmen einer Panelanalyse mit Fixed-Effects auf die Höhe des Einflusses hin untersucht. Die Koeffizienten, Signifikanzniveaus und Standardfehler sind in Tabelle 53 dargestellt.

\begin{tabular}{lc}
\hline \multirow{2}{*}{ VARIABLE } & $(1)$ \\
\hline \multirow{2}{*}{ ze1s } & bgs \\
& $1,006^{* * *}$ \\
\multirow{2}{*}{ ze2s } & $(0,00283)$ \\
& $1,005^{* * *}$ \\
les & $(0,00328)$ \\
& $1,000^{* * *}$ \\
zas & $(0,00515)$ \\
& $-1,002^{* * *}$ \\
& $(0,00223)$
\end{tabular}

Tabelle 53: $\quad$ Ergebnisse der Paneluntersuchung (Fixed Effects) 
Es wird deutlich, dass die Größen entweder einen Koeffizienten sehr nahe +1 oder sehr nahe -1 aufweisen. Andere Werte wurden nicht ermittelt. Aufgrund der Koeffizienten kann gemäß BIEG auf eine sehr hohe betragsmäßige Korrelation geschlossen werden. ${ }^{1006}$ Diese ist auf die direkte Abhängigkeit auf Grundlage der Berechnungsvorschrift zurückzuführen. So führen die Erträge ze1s, ze2s, les, $p s$ und ses direkt zu einem Anstieg der Bruttogewinnspanne, während die Aufwendungen zas, pks und sks direkt die Bruttogewinnspanne verringern. Die Variable $b \underset{s}{s}$ wurde übersprungen ( 0 . für omitted), da hier eine lineare Abhängigkeit zur Summe aus ze1s, ze2s, les und zas besteht. Im Ergebnis konnten die in der Berechnungsvorschrift immanenten Beziehungen empirisch belegt werden.

Es folgt eine Panelanalyse mit Fixed Effects. Die folgenden Variablen werden daraufhin untersucht, ob sie einen Einfluss auf die Höhe der Bruttogewinnspanne (bgs) haben:

- Anzahl der Bankmitarbeiter (ma),

- Bilanzsumme pro Mitarbeiter (bsma),

- Eigenkapitalquote (ekq),

- Kreditquote (kq),

- Bilanzsumme als natürlicher Logarithmus (bsln),

- Fusion (fus),

- Bruttoinlandsprodukt pro Einwohner als natürlicher Logarithmus (bipewln),

- Steilheit der Zinsstrukturkurve ( $(s w 2 s)$ und jeweils alternativ die absolute Lage der Zinssätze für 2-, 5- und 10-jährige Laufzeiten (sw2, sw5 und sw10),

- Alle betrachteten Jahre einzeln als Dummyvariablen (j2005 bis j2011).

Die Resultate der Analyse sind in Tabelle 54 dargestellt.

Die Anzahl der Bankmitarbeiter ( $m a$ ) ist negativ mit der Bruttogewinnspanne (bgs) korreliert. Unterscheidet sich die Mitarbeiteranzahl zwischen zwei Banken, so weist die Bank mit mehr Mitarbeitern im Mittel je Mitarbeiter eine um etwa 0,05 Basispunkte bzw. 0,0005 Prozentpunkte niedrigere Bruttogewinnspanne aus. Dies deckt sich von der Wirkungsrichtung her im Wesentlichen mit dem Resultat der oben in Abschnitt 4.4.9 durchgeführten Analyse. Es sei jedoch auf die differenzierte Betrachtung im Rahmen der Prüfung der entsprechenden Hypothese verwiesen.

Die Variable bsma ist der Quotient aus der Bilanzsumme in Mio. Euro und der Anzahl der Mitarbeiter einer Bank. Sie beschreibt die Höhe der Bilanzsumme, welche auf einen einzelnen Mitarbeiter entfällt. Aus Effizienzgesichtspunkten ist es günstig, wenn jeder Mitarbeiter ein hohes Volumen an Bilanzsumme betreut. Für jede Million Euro an Geschäftsvolumen mehr, welche im Durchschnitt auf einen Mitarbeiter kommt, steigt die Bruttogewinnspanne um 1,68 Basispunkte bzw. um 0,0168 Prozentpunkte. Wie bereits oben in Abschnitt 4.4.9 dargelegt, gibt es auch Regionen, in welchen die Institute trotz einer ungünstigen bsmaRelation gute Ergebnisse erzielen. Banken in Städten und auch Banken im ländlichen Bereich sind oftmals sehr gut an die herrschenden Bedingungen angepasst und haben sich mit den Gegebenheiten arrangiert. 


\begin{tabular}{|c|c|}
\hline VARIABLE & $\begin{array}{l}(1) \\
\text { bgs }\end{array}$ \\
\hline $\mathrm{ma}$ & $\begin{array}{c}-0,000549 * * * \\
(0,000155)\end{array}$ \\
\hline bsma & $\begin{array}{l}0,0168^{* *} \\
(0,00731)\end{array}$ \\
\hline ekq & $\begin{array}{c}0,0491 * * * \\
(0,00732)\end{array}$ \\
\hline $\mathrm{kq}$ & $\begin{array}{c}0,00466^{* * *} \\
(0,00109)\end{array}$ \\
\hline bsln & $\begin{array}{l}0,121 * * \\
(0,0475)\end{array}$ \\
\hline fus & $\begin{array}{c}-0,135^{* * * *} \\
(0,0238)\end{array}$ \\
\hline bipewln & $\begin{array}{c}0,354 * * * \\
(0,0933)\end{array}$ \\
\hline sw2s & $\begin{array}{c}0,0723 * * * \\
(0,00770)\end{array}$ \\
\hline$(s w 2)$ & $\begin{array}{c}-0,0771 * * * \\
(0,00482)\end{array}$ \\
\hline$(\operatorname{sw} w)$ & $\begin{array}{c}-0,0792 * * * \\
(0,00940)\end{array}$ \\
\hline$(s w 10)$ & $\begin{array}{c}-0,110^{* * *} \\
(0,0130)\end{array}$ \\
\hline j2005 & $\begin{array}{c}0,0736 * * * \\
(0,0162)\end{array}$ \\
\hline j2006 & $\begin{array}{c}0,463 * * * \\
(0,0116)\end{array}$ \\
\hline o.j2007 & - \\
\hline j2008 & $\begin{array}{c}-0,0592 * * * \\
(0,0113)\end{array}$ \\
\hline o.j2009 & - \\
\hline j2010 & $\begin{array}{c}0,119 * * * \\
(0,0124)\end{array}$ \\
\hline j2011 & $\begin{array}{c}0,0692 * * * \\
(0,0161)\end{array}$ \\
\hline Konstante & $\begin{array}{c}-5,708^{* * *} \\
(1,293)\end{array}$ \\
\hline Beobachtungen & 6.304 \\
\hline Anzahl der Banken & 1.090 \\
\hline $\mathrm{R}^{2}$ & 0,374 \\
\hline
\end{tabular}

Die Variable ek q spiegelt die Eigenkapitalquote einer Bank in Prozent wider. Diese wurde bereits oben in Abschnitt 4.4.14 untersucht. Der dort erhobene positive Zusammenhang zwischen der Eigenkapitalquote und der Bruttogewinnspanne (bgs) kann durch die Paneluntersuchung belegt werden. Das arithmetische Mittel von $e k q$ beträgt 6,82. Steigt die Eigenkapitalquote um einen Prozentpunkt, z. B. auf 7,82, so ist im Mittel mit einer Verbesserung der Bruttogewinnspanne um 4,91 Basispunkte bzw. 0,0491 Prozentpunkte auszugehen. Dies könnte ein Anstieg von 1,0309 (arithmetisches Mittel aller bgs-Werte) auf 1,08 sein. Diese Reagibilität ist als hoch einzustufen. 
Die Kreditquote $k q$ wurde bereits oben in Abschnitt 4.4.15 untersucht. Sie spiegelt wider, welcher Anteil der Bilanzsumme in Form von Darlehen wieder ausgereicht werden konnte und beträgt im Mittel aller Institute 57,66. Bei einer Erhöhung der Kreditquote einer Bank um einen Prozentpunkt, z. B. von 61 auf 62, ist im Mittel von einem Anstieg der Bruttogewinnspanne um 0,47 Basispunkte bzw. 0,0047 Prozentpunkte auszugehen. Dies könnte ein Anstieg von 1,0309 auf 1,0358 sein.

Die Variable $b s \ln$ repräsentiert den natürlichen Logarithmus der Bilanzsumme. Der arithmetische Mittelwert von bsln beträgt 19,47. Ein um 1 auf 20,47 erhöhter Wert bedeutet eine Multiplikation der Ausgangsbilanzsumme in Euro mit dem Faktor 2,71. In solch einem Fall liegt die Bruttogewinnspanne bgs um 12,1 Basispunkte bzw. um 0,1210 Prozentpunkte höher. Dies könnte ein Anstieg von 1,0309 auf 1,1519 sein. Vergleicht man Banken generell anhand ihrer Bilanzsumme, so fällt auf, dass größere Banken im Mittel höhere Bruttogewinnspannen aufweisen. RICHTER wies in seinem Panelmodell mit Fixed Effects ebenfalls einen positiven Einfluss der Bankgröße auf den Bankerfolg nach. ${ }^{1007}$ Gleichsam wurde dieser Zusammenhang bereits oben in Abschnitt 4.4.16 untersucht. Das dort angewendete investigative Verfahren hat für kleinere Bankgrößen eine positive Beziehung zwischen Bankgröße und Bruttogewinnspanne zu Tage gefördert, während sich für große Institute der umgekehrte Zusammenhang herausstellte. Die hier vorliegende Panelanalyse kann jedoch keine Umkehr der Wirkungsrichtung darstellen.

Die Variable fus zeigt an, ob im jeweiligen Geschäftsjahr eine Fusion in einer Bank stattgefunden hat. Betrachtet wird dies aus Sicht der absorbierenden Bank. Die andere Bank bzw. die anderen Banken, welche im Rahmen einer Fusion in der absorbierenden Bank aufgehen, liefern nach der Fusion keine weiteren Abschlussdaten. Es handelt sich bei fus um eine Dummyvariable: Im Falle des Vorliegens einer Fusion nimmt diese den Wert eins an, ansonsten den Wert null. Der negative Wert von -0,135 sagt aus, dass bei Vorliegen einer Fusion ceteris paribus mit einer um 13,5 Basispunkte bzw. 0,135 Prozentpunkte geringeren Bruttogewinnspanne zu rechnen ist. Dies könnte eine Reduktion von 1,0309 auf 0,8959 sein. Ohne eine Fusion tritt der genannte Abschlag nicht auf. Die insgesamt berücksichtigte Anzahl von Fusionsfällen beträgt 178. Die negative Abweichung vom Durchschnitt ist im Jahr der Fusion sehr gut nachzuvollziehen. Zahlreiche Mitarbeiter sind zur Betreuung der Fusion abgestellt bzw. nehmen Sonderaufgaben im Zusammenhang mit der Fusion wahr. Daher können sie im gleichen Zeitraum ihrer eigentlichen Beschäftigung nicht oder nur beschränkt nachgehen, was zu weniger margenträchtigen Geschäften führt. Des Weiteren können im Rahmen der Fusion auch stille Lasten abgewickelt werden oder sonstige Maßnahmen der Bilanzbereinigung ergriffen werden, beispielsweise in Zusammenhang mit einer strategischen Neuausrichtung, was zu weiteren Verzerrungen durch besondere Aufwendungen führt. Das Untersuchungsverfahren stellt den Erfolgsunterschied zwischen in Fusionen befindlichen Banken und normal operierenden Banken sehr deutlich heraus. Langfristig sind jedoch durch Kosteneinsparungen Effizienzgewinne bei fusionierten Instituten gut möglich.

Die jeweilige Aufnahme des logarithmierten Arbeitnehmerentgelts aneln, der logarithmierten Bruttolöhne und -gehälter blgln oder des logarithmierten Bruttoinlandsprodukts je 
Erwerbstätiger bipewln in die Panelanalyse führt zu keinem signifikanten Ergebnis für den Koeffizienten.

Die Variable bipewln enthält das Bruttoinlandsprodukt je Einwohner als logarithmierten Wert. Der Koeffizient 0,354 zeigt hier einen positiven Zusammenhang bei hoher Signifikanz an. Je größer das Bruttoinlandsprodukt je Einwohner, desto größer sei die Bruttogewinnspanne der vor Ort operierenden Bank bzw. Banken. In Abschnitt 4.4.20 wurde hierzu hingegen ein umgekehrter Zusammenhang identifiziert.

Die Variable $s w 2 s$ bildet die Steilheit der Zinsstrukturkurve ab. Die Beurteilung der Steilheit erfolgt anhand jeweils gültiger langfristiger und kurzfristiger Zinssätze. Als langfristiger Zinssatz wurde eine Laufzeit von 10 Jahren gewählt, als kurzfristiger Zinssatz kommt hingegen eine Laufzeit von 2 Jahren zu Anwendung. Als Datenbasis wurden die entsprechenden Euro-Swapsätze aller Geschäftstage eines Kalenderjahres verwendet. ${ }^{1008}$ Die Angabe der Steilheit ergibt sich für jeden Geschäftstag aus der Differenz zwischen dem 2jährigen und dem 10-jährigen Swapsatz. Als jahresdurchschnittliche Steilheit der Zinsstrukturkurve wurde das arithmetische Mittel aller für das Jahr vorliegenden Swapsatzdifferenzen ermittelt. Die in Prozent angegebene Steilheit betrug im Mittel über den Betrachtungszeitraum 0,9356. Die geringste Steilheit lag im Jahr 2007 mit 0,1395 Prozent vor, während die im Jahresmittel größte Steilheit der Zinsstrukturkurve für das Jahr 2009 mit 1,7753 Prozent berechnet wurde. Der Koeffizient 0,0723 der Variable sw2s ist wie folgt zu interpretieren: bei einem Anstieg der Steilheit um 1 Prozentpunkt, z. B. von 0,9356 auf 1,9356, nimmt die Bruttogewinnspanne der Banken um 7,23 Basispunkte bzw. um 0,0723 Prozentpunkte zu. Dies könnte ein Anstieg von 1,0309 auf 1,1032 sein. Das Resultat ist hochsignifikant. Als nächstes soll dieser Zusammenhang kausal begründet werden. Bei einer steilen Zinsstrukturkurve bietet sich den Banken eine bessere Möglichkeit, durch die Fristentransformation Margen zu erzielen. Während einerseits für kurzlaufende Einlagen relativ niedrige Zinssätze gezahlt werden, können andererseits Darlehen zu höheren langfristigen Zinssätzen vergeben werden. Somit konnte der bereits von RICHTER ${ }^{1009}$ identifizierte Zusammenhang, dass große Steilheit der Zinsstrukturkurve von Vorteil für die Institute ist, von der Wirkungsrichtung her bestätigt werden. Es sei darauf verwiesen, dass die Abflachung einer existierenden steilen Zinsstrukturkurve für die Institute ein Zinsrisiko birgt, da dann neu abgeschlossenes, langfristiges Aktivgeschäft geringere Margen verspricht als das bestehende Aktivgeschäft.

Im Austausch zur Variable sw2s wurde jeweils einzeln geprüft, welcher Einfluss auf die Bruttogewinnspanne von der Höhe der Swapsätze mit 2-jähriger, 5-jähriger und 10-jähriger Laufzeit ausgeht. Die Prüfung der Swapsätze sw2, sw5 und sw10 wurde im Modell jeweils einzeln und ohne die Einbeziehung der Steigung $s w 2 s$ durchgeführt, um Multikollinearität zwischen den Variablen zu vermeiden. ${ }^{1010}$ Für jede der drei Variablen konnte hochsignifikant ein Zusammenhang mit einem negativen Koeffizienten belegt werden. Dieses Resultat bedeutet, dass bei höheren Marktzinssätzen eine geringere Bruttogewinnspanne zu erwarten ist. Sinken die Marktzinssätze hingegen, darf eine höhere Bruttogewinnspanne erwartet werden. Bezogen auf den 2-jährigen Swapsatz ist das Resultat wie folgt zu interpretieren: Bei einem Anstieg der 2-jährigen Zinsen um 1 Prozentpunkt, z. B. von 1,8582 (Wert des

1008 Vgl. Thomson Reuters EIKON (2015); vgl. Abschnitt 4.3.3.11.

1009 Vgl. Abschnitt 2.1.1.

1010 Vgl. Backhaus (2006), S. 89f; vgl. Winker (2010), S. 163 f. 
Jahres 2011) auf 2,8582 Prozent, nimmt die Bruttogewinnspanne der Institute um 7,71 Basispunkte bzw. um 0,0771 Prozentpunkte ab. Dies könnte ein Rückgang von 1,0309 auf 0,9586 Prozent sein. Bei der Variation der 10-jährigen Zinsen ist eine noch stärkere Reaktion der Bruttogewinnspanne zu erwarten. Zumindest aus empirischer Sicht kann daher kein nachteiliger Zusammenhang zwischen dem aktuell niedrigen Zinsniveau und den Bruttogewinnspannen der Banken konstatiert werden - empirisch begünstigen niedrige Zinsen die Bruttogewinnspanne. (Hinzu kommt, dass sinkende Zinsen zu höheren Kursen bei festverzinslichen Wertpapieren führen, was wiederum in einem verbesserten Bewertungsergebnis resultiert. ${ }^{1011}$ Daraufhin wird ein geringerer Aufwand für die Risikovorsorge geringer. Jedoch wirkt dieser Mechanismus erst nach der hier betrachteten Bruttogewinnspanne.) Die Untersuchung der Zinssätze bestätigt die Ergebnisse von RICHTER ${ }^{1012}$, welcher für 5-jährige und für 10-jährige Laufzeiten ebenfalls signifikante Koeffizienten mit derselben Wirkungsrichtung bestimmen konnte. Dieser Zusammenhang muss noch explizit aus Sicht des Bankbetriebs interpretiert werden. Die Genossenschaftsbanken refinanzieren sich auf der Passivseite zu großen Teilen (geschätzt 60 bis $80 \%$ ) variabel verzinslich, dies geschieht über Referenzzinsmodelle und Spareinlagen. Umgekehrt werden die Mittel jedoch auf der Aktivseite zu großen Teilen (geschätzt $90 \%$ ) festverzinslich verwendet. Diese Konstellation führt zu zweierlei Effekten in Bezug zur Elastizität der Aktiv- und der Passivseite. Bei steigenden Marktzinsen wächst der Aufwand zur Refinanzierung, während die Zinserträge auf der Aktivseite relativ konstant bleiben. Dies liegt daran, dass für vergebene Darlehen in der Regel feste Zinssätze mit den Kreditnehmern vereinbart werden, und dass vergebene Darlehen auch deutlich längere Laufzeiten aufweisen als die zur Refinanzierung eingesetzten Mittel, welche deshalb wiederum rasch auf Zinsänderungen reagieren. Dies führt, wie oben dargelegt, zu einem Rückgang der Bruttogewinnspanne und stellt daher ein Zinsänderungsrisiko dar. ${ }^{1013}$ Bei sinkenden Marktzinsen hingegen bleiben die Zinserträge des Aktivgeschäfts ebenfalls relativ konstant, während die Aufwendungen für die Refinanzierung auf der Passivseite zurückgehen. Aus diesem Grund kommt es zu einer Ausweitung der Bruttogewinnspanne. Durch die aktive Durchführung von Fristentransformation verstärkt sich der beschriebene Effekt in beiden Richtungen der Zinsentwicklung. (Den Einfluss der Fristentransformation auf den Zinsüberschuss untersuchen auch ENTROP ET AL. in ihrem Werk Determinants of bank interest margins: Impact of maturity transformation. ${ }^{1014}$ ) Die Aussage von RICHTER ${ }^{1015}$, dass sinkende Zinsen keine Nachteile implizieren, geht jedoch nicht weit genug. Diese Aussage ist die logische Konsequenz bei einer lediglich kurzfristigen Betrachtung auf Ebene von einzelnen Jahresscheiben, wie es mit dem Fixed EffectsVerfahren möglich ist. Betrachtet man die Geschäfte bei schwankenden Zinsen über ihre gesamte Laufzeit hinweg, ergibt sich ein abweichendes Bild. Zunächst soll hier eine Betrachtung für sinkende Marktzinsen durchgeführt werden. In Euro oder in Basispunkten gerechnet, kann die Bank dann weniger verdienen, während die Verwaltungsaufwendungen relativ konstant bleiben. Bei einem Marktzins von beispielsweise 3\% darf eine Marge von $1 \%$ als für die Banken auskömmlich betrachtet und beim Kunden als durchsetzbar gewertet werden. Bei einem jedoch aktuell derart niedrigen Marktzins, beispielsweise 0,5\%, ist die Generierung einer auskömmlichen Marge deutlich schwerer, was zu sinkenden Zins-

\footnotetext{
1011 Vgl. ZfgK (2015), S. 60.

1012 Vgl. Richter (2014), S. 428.

1013 Vgl. Walter (2013), S. 294.

1014 Vgl. Entrop, Memmel, Ruprecht und Wilkens (2015), S. 1 f.

1015 Vgl. Richter (2014), S. 428.
} 
überschüssen führt. Der Rückgang der Marktzinsen führt schließlich zu sinkenden Margen. In diesem Zusammenhang muss auch auf die Reaktionsträgheit der Aktivseite verwiesen werden, die sich aus der in der Regel langen Laufzeit der vergebenen Darlehen ableitet. Hingegen reagiert die Passivseite - je nach Fristigkeit der verwendeten Instrumente relativ kurzfristig auf die veränderten Marktzinsen. Wie bereits oben in diesem Abschnitt erwähnt, wirkt sich dies in Zeiten steigender Zinssätze nachteilig auf die Bruttogewinnspanne aus: Die Refinanzierungskosten nehmen rasch zu, während die Erträge aus bestehendem Aktivgeschäft nur sehr langsam über die Zeit ansteigen. Dies ist darauf zurückzuführen, dass es einige Zeit dauert, bis der bestehende Block des in Zeiten niedriger Zinsen abgeschlossenen Aktivgeschäfts abgeschmolzen ist und durch ausreichend Neugeschäft zu den höheren Kreditkonditionen ersetzt wurde. Dieser Zusammenhang kann, je nach dem Fristentransformationsprofil, sehr schnell zu Ertragsproblemen führen. Zur Reduktion dieses Risikos können Banken versuchen, sich stärker durch länger laufende Spareinlagen zu refinanzieren und somit das Ausmaß der durchgeführten Fristentransformation verringern. Dies ist jedoch bei der herrschenden Nachfragestruktur ebenfalls schwierig, da sich die Kunden zu geringen Zinssätzen nicht über lange Zeit in einer Anlage binden lassen. Alternativ ist eine aktive Steuerung des Fristentransformationsbeitrags mittels Zinsswaps möglich. Die Marge (Konditionsbeitrag) kann jedoch nicht beliebig gesteuert werden. Diese ist in erster Linie abhängig von der Wettbewerbssituation und dem bestehenden Zinsniveau, wie bereits oben dargestellt.

Die Dummyvariablen j2005 bis j2011 zeigen an, ob ein Datensatz aus dem zugehörigen Jahr stammt. Für fünf der sieben untersuchten Jahre konnte ein Koeffizient ermittelt werden, nur 2007 und 2009 wurden aufgrund von Kollinearität ausgeschlossen. Der mit 0,463 größte Koeffizient wurde für das Jahr 2006 ermittelt. Wie bereits oben beschrieben, handelt es sich bei diesem Jahr um ein Ausnahmejahr in vielerlei Hinsicht. ${ }^{1016}$ Für 2006 liegt die Bruttogewinnspanne eines Instituts um 0,4630 Prozentpunkte über einer imaginären jahresunabhängigen Basislinie. Dies könnte eine Abweichung von 1,0309 nach oben auf 1,4939 sein. Der geringste Koeffizient wurde für das Jahr 2008 mit -0,0592 ermittelt, was einer Abweichung nach unten von 1,0309 auf 0,9717 entsprechen könnte. Alle Jahreskoeffizienten sind auf höchstem Niveau signifikant.

Eine Panelanalyse mit Fixed Effects kann nur diejenigen Werte berücksichtigen, die sich im Zeitablauf verändern. Das Fixed Effects-Modell rechnet eingangs alle Variablen um: Von jedem Wert wird der Mittelwert der Variable abgezogen. Das Ergebnis sind also Abweichungen, die um den Variablenmittelwert streuen. Einige der untersuchten Erfolgsfaktoren sind jedoch unveränderlich. Hierzu zählen:

- die Lagekategorie städtisch, halbstädtisch oder ländlich,

- die Zukunftsfähigkeit (Werte aus 2010 stellvertretend für den Betrachtungszeitraum),

- der Anteil der Einwohner mit einem Lebensalter von 65 Jahren oder höher,

- die Wachstumsrate des Bruttoinlandsprodukts im Zeitraum 2005 bis 2011,

- die Lage nach Bundesländern, 
- die Anzahl der Zweigstellen (Werte aus 2010 stellvertretend für den Betrachtungszeitraum).

Um den Einfluss dieser zeitkonstanten Werte dennoch zu bestimmen, kann eine OLSRegression ${ }^{1017}$ durchgeführt werden. Eine mögliche Veränderung im Zeitablauf wird hierbei ausgeblendet, alle Werte werden als gleichzeitig betrachtet und zusammen regressiert. Dargestellt werden die Testresultate in Tabelle 55.

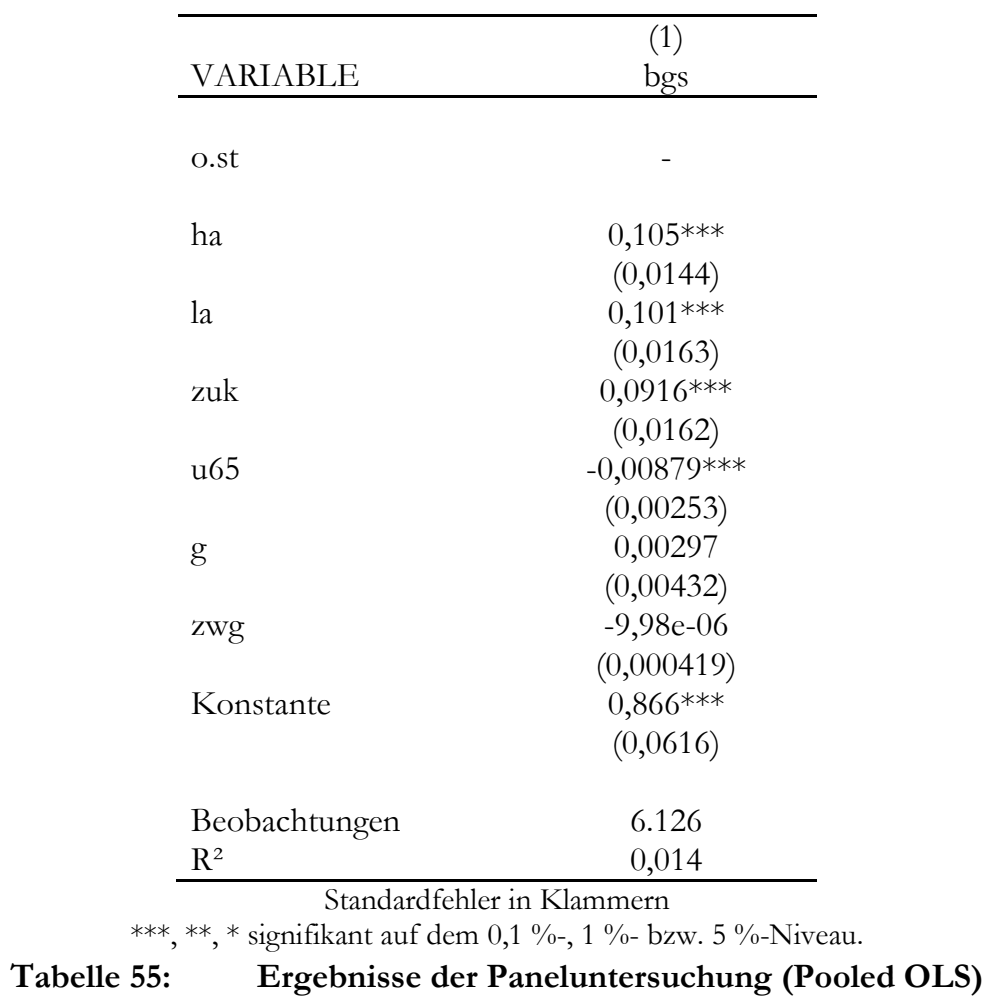

Bei den ersten zeitkonstanten Variablen handelt es sich um st, ha und la, welche die Lagekategorien nach städtisch, halbstädtisch und ländlich repräsentieren. Für st wurde kein Koeffizient ermittelt, da diese Variable aufgrund von Kollinearität ${ }^{1018}$ übergangen wird. Dieser Umstand bestätigt, dass eine jede Bank eindeutig einer der drei genannten Lagekategorien zugeordnet ist. Das Einflussniveau städtischer Lage ist als Basislinie zu betrachten. Banken in halbstädtischer und auch in ländlicher Lage weisen im Vergleich dazu eine im Mittel höhere Bruttogewinnspanne bgs aus, was sich aus den Koeffizienten für ba und la ergibt. Im Mittel führt eine halbstädtische Lage zu einem Aufschlag von 10,5 Basispunkten bzw. 0,105 Prozentpunkten. Für ländliche Lage wurde ein Koeffizient von 10,1 Basispunkten bzw. 0,101 Prozentpunkten ermittelt. Es wurde ein sehr hohes Signifikanzniveau erreicht. Die genannten Aufschläge bestätigen die Konsistenz der Ergebnisse mit Abschnitt 4.4.2. Bereits aus Abbildung 27 wird ersichtlich, dass Banken in halbstädtischer und ländlicher Lage im Mittel über alle Zeiträume eine etwa um 0,1 Prozentpunkte höhere Bruttogewinnspanne ausweisen.

\footnotetext{
1017 Vgl. Dreger, Kosfeld und Eckey (2014), S. 24.

1018 Dreger et al. (2014), S. 66.
} 
Der Einfluss der Zukunftsfähigkeit wird durch die nächste Variable zuk abgebildet. Die Zukunftsfähigkeit nach KRÖHNERT ${ }^{1019}$ verkörpert die Bewertungsbereiche Demografie, Wirtschaft, Integration, Bildung sowie Familienfreundlichkeit ${ }^{1020}$ als eine kondensierte Kennzahl. Sie wird nach dem Schulnotenprinzip auf einer Skala von 1 (sehr gut) bis 6 (sehr schlecht) gemessen. Die Höhe des Einflusses der Zukunftsfähigkeit quk auf die Bruttogewinnspanne bgs der vor Ort tätigen Banken wird durch den Koeffizienten beziffert. Der ermittelte Wert liegt bei 9,16 Basispunkten bzw. 0,0916 Prozentpunkten. Eine Verschlechterung um eine ganze Schulnote führt zu einer Erhöhung der Bruttogewinnspanne bgs um 0,0916, beispielsweise von 1,0309 auf 1,1225, und vice versa. Der Zusammenhang ist auf höchster Ebene signifikant. Jedoch ist hierzu zu bemerken, dass die Spannweite der Werte - das heißt die Differenz zwischen dem Minimum 2,58 und dem Maximum 4,66 gerade einmal 2,08 beträgt. Die Größenordnung des Zusammenhangs ist daher eher gering. In Regionen mit ungünstigen Zukunftsaussichten erzielen die Banken also eine höhere Bruttogewinnspanne. Kausal lässt sich dieser Zusammenhang wieder mit dem Wettbewerb begründen: Privatwirtschaftliche Wettbewerber der Genossenschaftsbanken ziehen sich aus den Regionen zurück, in denen die Zukunftsfähigkeit als eher schwach eingeschätzt wird. Eine Korrelation zwischen der Zukunftsfähigkeit und der Lagekategorie kann jedenfalls nicht nachgewiesen werden. Die Korrelationskoeffizienten liegen unter 0,1 und sind damit als gering zu bewerten. ${ }^{1021}$ Erneut kann hiermit die These der Anpassungsfähigkeit von Genossenschaftsbanken an die örtlichen Gegebenheiten untermauert werden. Das Resultat bestätigt ebenfalls die Ergebnisse von RICHTER ${ }^{1022}$, welcher auch anhand einer OLSRegression einen gleichgerichteten Einfluss der Zukunftsfähigkeit auf den Erfolg von Genossenschaftsbanken nachweisen konnte. Als Kriterium für die Messung des Erfolgs verwendet er die Cost-Income-Ratio.

Als ein weiterer möglicher Erfolgsfaktor wurde der Anteil älterer Menschen an der Bevölkerung untersucht. Speziell wird hier auf den Anteil von Personen mit einem Alter von 65 Jahren oder mehr an der Bevölkerung des Bezugskreises zum Stichtag 31.12.2011 abgestellt. Die Variable $u 65$ enthält diesen Anteil als Prozentwert, der Median beträgt 25,86. Der ermittelte Koeffizient lautet -0,88 Basispunkte bzw. -0,0088 Prozentpunkte. Für jeden zusätzlichen Prozentpunkt älterer Menschen im Geschäftsgebiet ist mit einer Abnahme der Bruttogewinnspanne zu rechnen, beispielsweise von 1,0309 auf 1,0221. Zurückzuführen ist dies auf den Kanon an Produkten und Volumina, welche von der genannten Zielgruppe nachgefragt werden. Im Alter wird weniger margenstarke Finanzierung nachgefragt, stattdessen vermehrt eine Geldanlage.

Die Variable $g$ enthält die durchschnittliche jährliche Wachstumsrate des Bruttoinlandsprodukts über den Zeitraum 2005 bis 2011. Der Wert wurde für jeden Kreis sowie für jede kreisfreie Stadt ermittelt und mit den einzelnen Banken verknüpft. ${ }^{1023} \mathrm{Zu}$ vermuten ist gemäß Hypothese 21, dass sich eine positive wirtschaftliche Entwicklung der Region auf den Erfolg der vor Ort ansässigen Regionalbanken niederschlägt. Im Ergebnis lässt sich jedoch kein spezifischer oder gar signifikanter Einfluss auf die Höhe der Bruttogewinn-

\footnotetext{
${ }^{1019}$ Vgl. Kröhnert et al. (2011).

1020 Vgl. Abschnitt 4.3.3.10.

1021 Vgl. Abschnitt 4.2.6.

1022 Vgl. Richter (2014), S. 430

1023 Vgl. Abschnitt 4.3.3.7.
} 
spanne von Banken nachweisen. ${ }^{1024}$ Zur detaillierten Klärung des Zusammenhangs enthält Abbildung 74 hierzu eine Darstellung, welche die Höhe der Bruttogewinnspanne in Abhängigkeit vom Quintil der durchschnittlichen Wachstumsrate des Bruttoinlandsprodukts über den Zeitraum 2005-2011 darstellt. Auch hier ist keine klare Richtung erkennbar, die Höhe der Bruttogewinnspanne ist nicht vom Wachstum des Bruttoinlandsprodukts vor Ort abhängig.

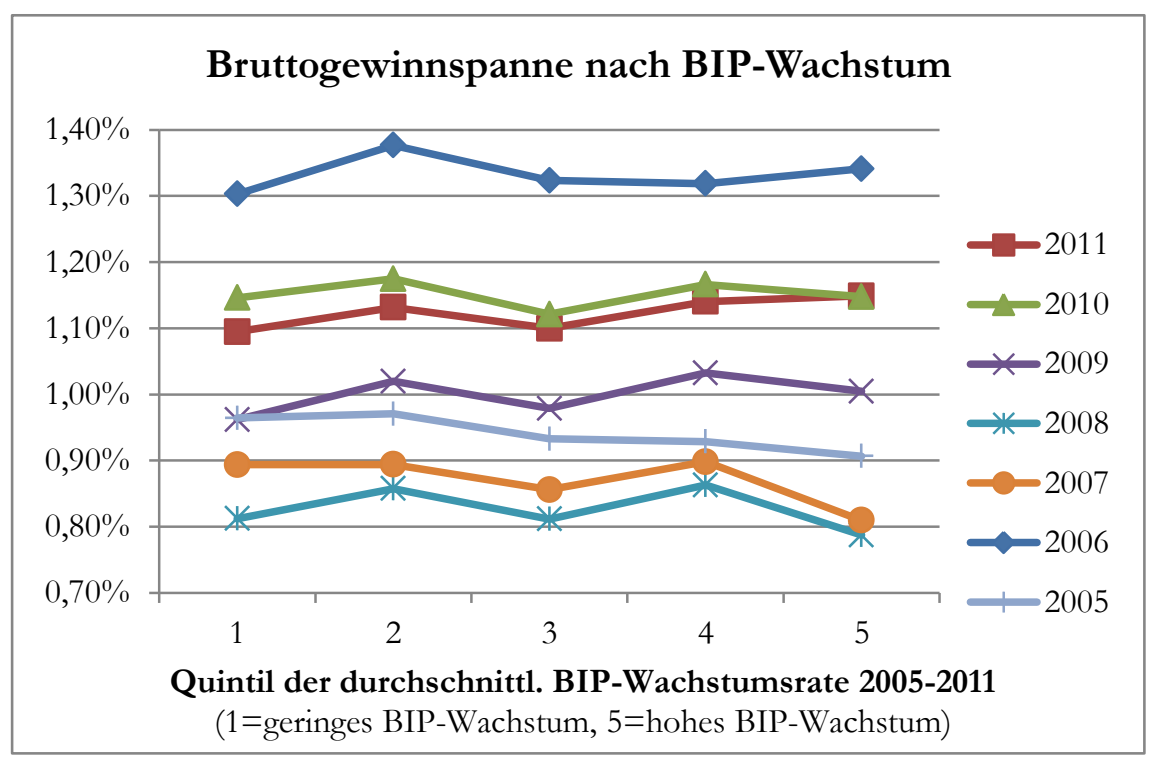

Abbildung 74: Einfluss des BIP-Wachstums auf die Bruttogewinnspanne

Auch in Hinblick auf die Anzahl der von den Banken unterhaltenen Zweigstellen zing konnte im Rahmen der OLS-Regression keine Regelmäßigkeit zu Tage gefördert werden. ${ }^{1025}$ Es sei jedoch auf die Resultate einer früheren Hypothese verwiesen. ${ }^{1026}$

Die Aufnahme der 16 zeitlich unveränderlichen Dummyvariablen zur Lage nach Bundesländern führt zu keiner Verbesserung der Aussagekraft. Die ermittelten Koeffizienten für die einzelnen Bundesländer erweisen sich nur sporadisch als signifikant und lassen keinen regelmäßigen Einfluss auf die Höhe des Erfolgs der Genossenschaftsbanken erkennen. Dennoch sei an dieser Stelle darauf verwiesen, dass bereits in einer vorangegangenen Hypothese eine entsprechende Detailbetrachtung erfolgreich existierende Unterschiede zwischen den Bundesländern herausstellen konnte. ${ }^{1027}$ 
Das Ziel der vorliegenden Arbeit ist die Identifikation und Analyse von Faktoren, die den Erfolg von Genossenschaftsbanken beeinflussen. Das Fundament bildet hierzu die theoretische Analyse in Bezug auf die zugrundeliegenden Teilgebiete: Regionalität, Fusionen, Skaleneffekte, optimale Betriebsgröße, Erfolgsmessung, Bilanzierung und ROI-Analyse. Es schließt sich die empirische Analyse von Jahresabschlüssen der Geschäftsjahre 2005 bis 2011 an. Diese Analyse konnte für einige Faktoren den Einfluss auf den Erfolg von Genossenschaftsbanken nachweisen. Der Erfolg wird anhand der Bruttogewinnspanne bemessen, welche sich aus dem Quotienten des Bruttoergebnisses aus normaler Geschäftstätigkeit und der durchschnittlichen Bilanæsumme errechnet. Der Bruttogewinn aus normaler Geschäftstätigkeit im Zähler wurde als Erfolgsmaß eingesetzt, um die Beeinflussungsmöglichkeiten durch den Ersteller des Jahresabschlusses möglichst gering zu halten. Die durchschnittliche Bilanzsumme im Nenner trägt dazu bei, die Werte verschieden großer Institute vergleichbar zu machen.

Obgleich sich die vorliegende Arbeit auf den Betrachtungszeitraum 2005 bis 2011 bezieht, soll sie hier auch die jüngste und aktuelle Debatte zu den untersuchten Bereichen aufgreifen und einen Ausblick gewähren.

Ein bedeutender Teil der empirischen Analyse befasst sich mit dem Einfluss der Lage auf den Erfolg von Genossenschaftsbanken. In städtischen Bereichen konkurrieren diese neben Sparkassen und anderen Geschäftsbanken um die Einlagen von Kunden, um die Nachfrage nach Darlehen und um das Provisionsgeschäft. Sowohl im Aktiv- als auch Passivgeschäft können dadurch geringere Margen erzielt werden. Dagegen konnten die Vorzüge einer halbstädtischen bzw. ländlichen Lage herausgestellt werden, wo Genossenschaftsbanken höhere Margen erzielen als im städtischen Bereich. Wie sowohl die Einzelanalyse als auch die Panelanalyse zeigen konnten, besitzt die Wettbewerbssituation vor Ort einen großen Einfluss auf das Ergebnispotenzial der Banken: Die Bruttogewinnspanne liegt bei ländlichen Banken durchweg höher als bei städtischen Instituten. Dieses Bild ergibt sich vor allem, weil das Zinsergebnis stets deutlich zum Vorteil ländlicher Banken ausfällt: Die Zinserträge liegen in der Regel höher, Zinsaufwendungen niedriger als bei Instituten in städtischer Lage. Das wesentliche Argument zur Erläuterung dieses Sachverhalts ist die Wettbewerbsintensität. In ländlichen Regionen sind häufig nur Genossenschaftsbanken und Sparkassen mit ihren Filialen vertreten. Durch die geringere Dichte an Wettbewerbern lassen sich dort höhere Margen erzielen. Dieser Umstand ist jedoch nicht nur vorteilhaft für die ländlichen Institute, sondern gleichzeitig auch von essenzieller Bedeutung für den Fortbestand derselben. Die überdurchschnittlichen Überschüsse sind ein entscheidender Baustein, um das Angebot von Finanzdienstleistungen in der breiten Fläche überhaupt sicherzustellen. Denn vor allem im Personalbereich, aber auch im Sachbereich fallen dort höhere Aufwendungen an. Dies ist im Wesentlichen auf das Vorhalten von Personal und Infrastruktur zurückzuführen, welche jedoch im ländlichen Bereich naturgemäß nicht annähernd so gut ausgelastet werden können wie in Städten. Ländliche Banken verfügen in der Regel über ein Filialnetz, dessen einzelne Filialen nicht so stark ausgelastet sind wie jene in städtischer Lage. Auch sind die Losgrößen für Geschäfte auf beiden Seiten der Bilanz bei ländlichen Instituten häufig kleiner als in städtischen Bereichen, wodurch pro Geschäft ein höherer Personal- und Sachaufwand anfällt. Daher verteilen sich die unabhängig von der 
Auslastung anfallenden Fixkosten weniger gut auf die mit Kunden abgeschlossenen Geschäfte. Die nachgewiesenen Margen sind also am ehesten eine logische Konsequenz der Gegebenheiten. Es kann die Schlussfolgerung gezogen werden, dass Banken in den einzelnen Bereichen an ihre jeweilige Situation gut angepasst sind und mit den unterschiedlichsten Bedingungen zurechtkommen.

Bedeutend nicht nur für ländliche Bereiche, sondern auch für Städte, ist eine klare Abgrenzung des Geschäftsgebietes. Überschneidungen müssen vermieden werden, um sich nicht durch einen unter Genossenschaftsbanken selbst geschaffenen Wettbewerb langfristig die Existenzgrundlage zu entziehen. Eine fortgesetzte Margen- und Ergebnisschwäche kann durchaus in einer unfreiwilligen Fusion mit einer Bank mit angrenzendem Geschäftsgebiet resultieren.

Die Betriebsgröße ist als ein weiterer Erfolgsfaktor derart zu sehen, dass Genossenschaftsbanken mit einer Bilanzsumme um etwa 500 bis 600 Mio. Euro im Mittel zu den erfolgreichsten zählen. Während kleinere Banken noch Potenzial für Skalen- und Verbundeffekte bergen, führen die komplexeren Verwaltungsaufwendungen größerer Institute ebenfalls zu einer geringeren Erfolgsmesszahl. Aktuell existiert aufgrund der niedrigen Zinsen wieder ein erhöhter Fusionsdruck. Dennoch kann es günstig sein, spezialisierte Institute zu erhalten, auch wenn diese klein sind. Zudem erscheint es trotz erfolgversprechender langfristiger Kostenvorteile aus Diversifikationsgesichtspunkten sinnvoll für die Stabilität des gesamten Bankenwesens, eine hohe Anzahl relativ kleiner Institute zu erhalten. Nehmen Banken dennoch eine Fusion in Angriff, sollten keine falschen Erwartungen über den Eintritt der Wirtschaftlichkeit gehegt werden. Eine Fusion führt im Jahr der Durchführung zu signifikant geringeren Erträgen. Auch in den Folgejahren rechnen sich Fusionen häufig noch nicht. Erfolgt die Fusion als Reaktion auf die aktuelle Niedrigzinsphase, so kann jedoch eine frühzeitige Umsetzung sinnvoll sein, da Kostenvorteile bestenfalls nach einigen Jahren zu erwarten sind.

Eine hohe Eigenkapitalquote wirkt sich positiv auf den Erfolg aus, da sie die Durchführung von margenträchtigen Geschäften unterstützt. Bei fehlendem Eigenkapital kann der Ausbau von profitablem Geschäft an Grenzen stoßen. Institute mit hoher Eigenkapitalquote zählen hingegen zu den erfolgreichsten. Zukünftig müssen Banken aufgrund neuer Anforderungen noch mehr Eigenkapital vorhalten. Hierzu zählen z. B. der Kapitalerhaltungspuffer und der antizyklische Kapitalpuffer (beide aus Basel III), sowie die Regularien nach CRR (Capital Requirements Regulation) und SREP (Supervisory Review and Evaluation Process). Es ist davon auszugehen, dass eine erhöhte Eigenkapitalquote auch künftig einen positiven Einfluss auf den Erfolg ausüben wird.

Die Genossenschaftsbanken können sich in der Regel komfortabel durch Einlagen refinanzieren. Werden die entgegengenommenen Einlagen in Form von Darlehen wieder ausgereicht, handelt es sich um das margenreichste Geschäft der Institute. Eine hohe Kreditquote zeugt von einer guten Lage im Kreditgeschäft, welche sich positiv auf die Bruttogewinnspanne auswirkt.

Die Zukunftsaussichten spiegeln sich ebenso wider: In Regionen mit guten Entwicklungsaussichten sind die Margen geringer, bedingt durch den dort herrschenden Wettbewerb mit anderen Instituten. Hohe Margen sind hingegen eher dort zu erwarten, wo es weniger 
Wettbewerb gibt. Dies ist tendenziell in Regionen mit schlechteren Zukunftsaussichten der Fall.

Hohe Arbeitnehmerentgelte sowie Bruttolöhne und -gebälter finden sich tendenziell in städtischer Lage und koinzidieren dort mit einer wettbewerbsbedingt geringen Bruttogewinnspanne. Die Höhe des Bruttoinlandsprodukts, gleichsam ob je Einwohner oder je Erwerbstätiger gemessen, steht in umgekehrtem Verhältnis zum realisierten Bankerfolg. Erneut kommt hier das Wettbewerbsargument zum Tragen, welches in strukturschwachen Regionen einen höheren Erfolg begründet.

Eine große Mitarbeiteranzabl in einem Institut führt empirisch ceteris paribus zu einer kleineren Bruttogewinnspanne, da ein höherer Personalaufwand diese Spanne senkt.

Für die Bruttogewinnspanne günstig ist eine möglichst große Steilheit der Zinsstrukturkurve, was darauf schließen lässt, dass die Häuser mit Fristentransformation Geld verdienen. Das Niveau der Zinsen spielt ebenfalls eine signifikante Rolle. Bei der Betrachtung von Jahresscheiben kann man zwar niedrige Zinsen empirisch signifikant zusammen mit hohen Bruttogewinnspannen beobachten. Jedoch würde diese Schlussfolgerung zu kurz greifen, denn in Zeiten stabiler Zinsen auf hohem Niveau lassen sich höhere Margen generieren als bei stabil niedrigen Zinsen. Das aktuell niedrige Zinsniveau stellt sämtliche Institute des Genossenschaftssektors zukünftig vor große Herausforderungen. Im Moment sind die meisten Institute zwar dank Überschüssen und stiller Reserven widerstandsfähig. Auch ist davon auszugehen, dass in den nächsten Jahren die aktivischen Konditionenbeiträge leicht stabilisierend wirken. Jedoch sind die passivischen Konditionenbeiträge stark unter Druck, da der Refinanzierungszinssatz von Sichteinlagen nicht unter null sinken kann. ${ }^{1028}$

Ein hoher Anteil älterer Menschen an den Einwohnern wirkt sich ungünstig auf die Bruttogewinnspanne aus, da im Alter weniger für die Banken margenreiche Darlehen nachgefragt werden. Die demografische Entwicklung wird künftig für ländliche Regionen erhebliche Auswirkungen haben. In Reaktion auf die dort in vielen Fällen zu beobachtende Überalterung müssen Konzepte entwickelt werden, um wieder mehr junge Kunden auf dem Land zu gewinnen und zu binden.

Von einigen Faktoren konnte nicht gezeigt werden, ob sie den Erfolg beeinflussen. Hierzu zählt etwa das BIP-Wachstum, aus dem kein spezifisch positiver oder negativer Zusammenhang zur Bruttogewinnspanne hervorgeht. Auch von der Anzahl der Zweigstellen je Bank war kein direkter Einfluss erkennbar. Es existieren sowohl Institute, die mit einer kleinen Anzahl von Zweigstellen wirtschaftlich arbeiten, als auch solche, die dies mit einer großen Anzahl tun. Bezieht man jedoch die Größe des Instituts mit ein, so fällt die Sachaufwandsspanne umso geringer aus, je mehr Geschäftsvolumen auf eine Filiale entfällt. Eine hohe Auslastung der Geschäftsstellen ist daher als Erfolgsfaktor zu sehen. In Bezug auf die aktuell geführte Debatte ${ }^{1029}$, ob und in welchem Umfang Filialschließungen durchgeführt werden sollen, lässt sich zwar zunächst keine generell gültige Empfehlung aussprechen. Jedoch kann es für eine Bank günstig sein, ihr Geschäft auf eine geringere Anzahl von Filialen zu konzentrieren. Die DZ BANK rechnet bis zum Jahr 2030 mit einem deutlichen Rückgang der Filialen aller Banken in Deutschland. ${ }^{1030}$ Sie geht von einer Schrumpfung um

1028 Vgl. Bundesbank (2015).

${ }^{1029}$ Vgl. Dowideit (2015); vgl. Reuters (2015).

${ }^{1030}$ Dowideit (2015). 
$40 \%$ aus, auf dann unter 20.000 Filialen. ${ }^{1031}$ Als Ursache wird der Wettbewerb mit reinen Onlinebanken genannt, und zusätzlich die rückläufige Rentabilität der gesamten Bankbranche, im Wesentlichen determiniert durch die geringen Zinsspannen, welche auch aus den niedrigen Marktzinsen resultieren. ${ }^{1032}$

Es ist davon auszugehen, dass noch weitere Erfolgsfaktoren mit einem signifikanten Einfluss auf den Geschäftserfolg existieren. Möglicherweise können bankinterne Daten oder eine qualitative Untersuchung noch mehr Aufschluss bringen. Auch ein noch durchzuführender deutschlandweiter Vergleich mit dem Sparkassensektor lässt interessante Ergebnisse aufwarten. 



\section{A1.1 Formblatt 1}

Jahresbilanz zum

der

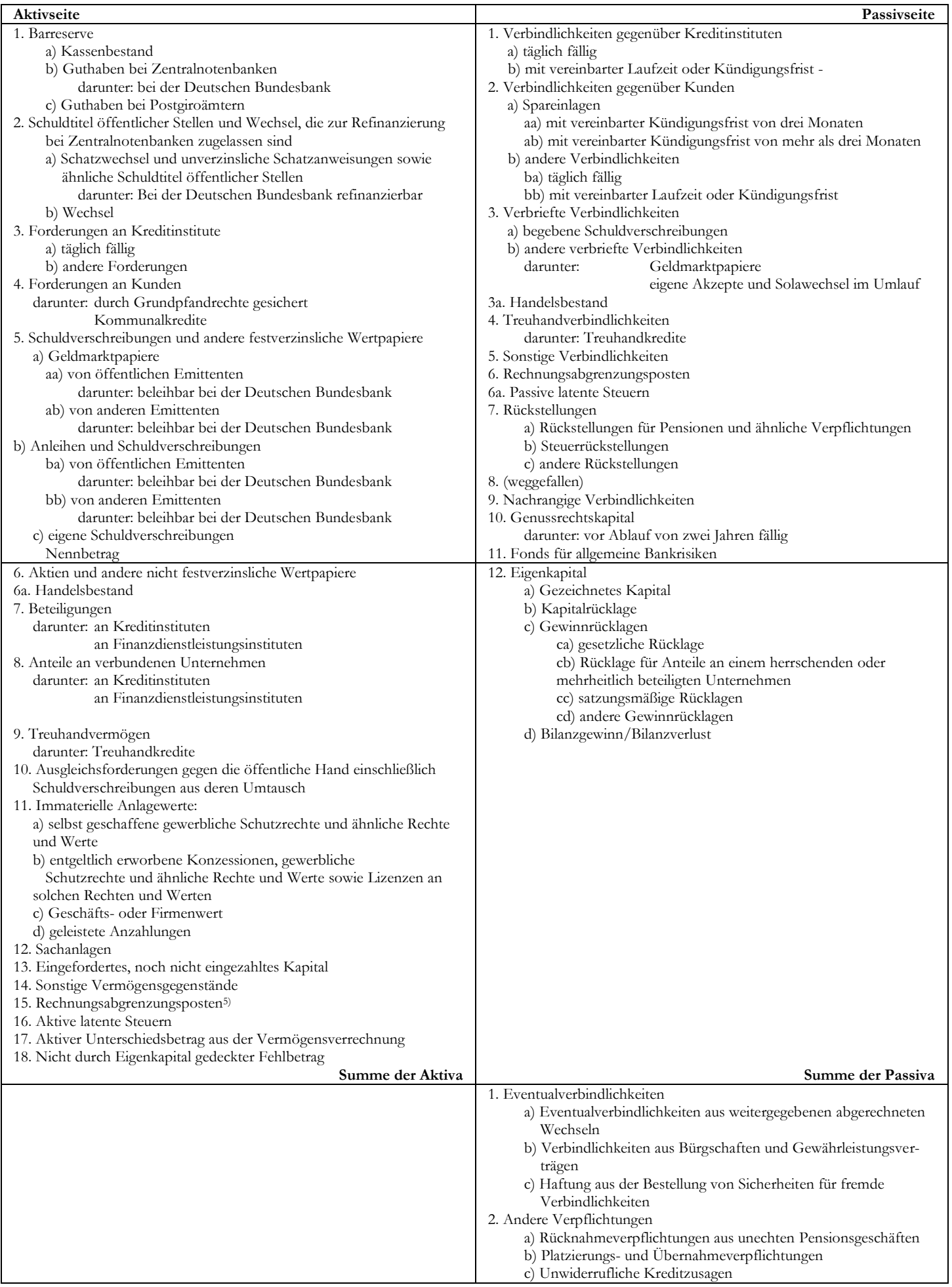

Tabelle A1:

Formblatt 1 


\section{A1.2 Formblatt 3 (Staffelform)}

Gewinn- und Verlustrechnung

der

für die Zeit vom

bis.

1. Zinserträge aus

a) Kredit- und Geldmarktgeschäften

b) festverzinslichen Wertpapieren und Schuldbuchforderungen

2. Zinsaufwendungen

3. Laufende Erträge aus

a) Aktien und anderen nicht festverzinslichen Wertpapieren

b) Beteiligungen

c) Anteilen an verbundenen Unternehmen

4. Erträge aus Gewinngemeinsch., Gewinnabführungs- oder Teilgewinnabführungsverträgen

5. Provisionserträge

6. Provisionsaufwendungen

7. Nettoertrag oder Nettoaufwand des Handelsbestands

8. Sonstige betriebliche Erträge

9. (weggefallen)

10. Allgemeine Verwaltungsaufwendungen

a) Personalaufwand

aa) Löhne und Gehälter

ab) Soziale Abgaben und Aufw. f. Altersversorg. u. f. Unterstützg.

darunter:

b) andere Verwaltungsaufwendungen

11. Abschreibungen und Wertberichtigungen auf immaterielle Anlagewerte und Sachanlagen

12. Sonstige betriebliche Aufwendungen

13. Abschreibungen und Wertberichtigungen auf Forderungen und bestimmte Wertp. sowie Zuführungen zu Rückstellungen im Kreditgeschäft

14. Erträge aus Zuschreibungen zu Forderungen und bestimmten Wertpapieren sowie aus der Auflösung von Rückstellungen im Kreditgeschäft

15. Abschreibungen und Wertberichtigungen auf Beteiligungen, Anteile an verbundenen Unternehmen und wie Anlagevermögen behandelte Wertpapiere

16. Erträge aus Zuschreibungen zu Beteiligungen, Anteilen an verbunden Unternehmen und wie Anlagevermögen behandelten Wertpapieren

17. Aufwendungen aus Verlustübernahme

18. (weggefallen)

19. Ergebnis der normalen Geschäftstätigkeit

20. Außerordentliche Erträge

21. Außerordentliche Aufwendungen

22. Außerordentliches Ergebnis

23. Steuern vom Einkommen und vom Ertrag

24. Sonstige Steuern, soweit nicht unter Posten 12 ausgewiesen

25. Erträge aus Verlustübernahme

26. Auf Grund einer Gewinngemeinschaft, eines Gewinnabführungs- oder eines Teilgewinn abführungsvertrags abgeführte Gewinne

27. Jahresüberschuss / Jahresfehlbetrag

28. Gewinnvortrag / Verlustvortrag aus dem Vorjahr

29. Entnahmen aus der Kapitalrücklage

30. Entnahmen aus Gewinnrücklagen

a) aus der gesetzlichen Rücklage

b) aus der Rücklage für Anteile an einem herrschenden oder mehrheitlich beteiligten Unternehmen

c) aus satzungsmäßigen Rücklagen

d) aus anderen Gewinnrücklagen

31. Entnahmen aus Genussrechtskapital

32. Einstellungen in Gewinnrücklagen

a) in die gesetzliche Rücklage

b) in die Rücklage für Anteile an einem herrschenden oder mehrheitlich

beteiligten Unternehmen

c) in satzungsmäßige Rücklagen

d) in andere Gewinnrücklagen

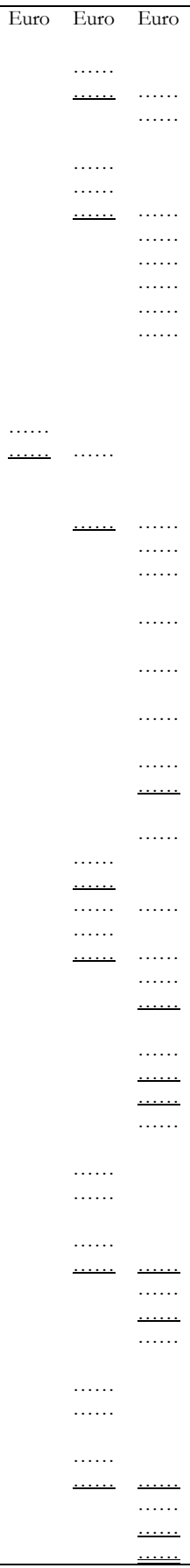

33. Wiederauffüllung des Genussrechtskapitals

34. Bilanzgewinn / Bilanzverlust

Tabelle A2:

Formblatt 3 (Staffelform) 


\section{LITERATURVERZEICHNIS}

Allison, P. D. (2009). Fixed effects regression models (Quantitative applications in the social sciences, no. 07-160). Los Angeles: SAGE.

Anderson, C. R. \& Paine, F. T. (1978). PIMS: A Reexamination. The Academy of Management Review, 3 (3), 602-612. Zugriff am 05.12.2014. Verfügbar unter http://www.jstor.org/ stable/257549

Anderson, T. W. \& Darling, D. A. (1954). A Test of Goodness of Fit. Journal of the American Statistical Association, 49 (268), 765-769.

Arbeitskreis Volkswirtschaftliche Gesamtrechnungen der Länder VGRdL. (2014a). Ergebnisse der Revision 2011, Kreisergebnisse für Deutschland, R2B1. Verfügbar unter http://www. vgrdl.de/VGRdL/tbls/RV2011/R2B1.zip

Arbeitskreis Volkswirtschaftliche Gesamtrechnungen der Länder VGRdL. (2014b). Ergebnisse der Revision 2011, Kreisergebnisse für Deutschland, R2B2. Verfügbar unter http://www. vgrdl.de/VGRdL/tbls/RV2011/R2B2.zip

Arbeitskreis Volkswirtschaftliche Gesamtrechnungen der Länder VGRdL. (2014c). Ergebnisse der Revision 2011, Kreisergebnisse für Deutschland, R2B3. Verfügbar unter http://www. vgrdl.de/VGRdL/tbls/RV2011/R2B3.zip

Assenmacher, W. (2002). Einfübrung in die Ökonometrie (6., vollständig überarbeitete und erweiterte Auflage). München: Oldenbourg.

Atteslander, P. (2010). Methoden der empirischen Sozialforschung (ESV basics, 13., neu bearb. und erw. Aufl). Berlin: Schmidt.

Auer, B. R. \& Rottmann, H. (2015). Statistik und Ökonometrie für Wirtschaftswissenschaftler. Eine anwendungsorientierte Einfübrung (3. Auflage). Wiesbaden: Gabler.

Auerbach, C. M. (2009). Fusionen deutscher Kreditinstitute. Erfolg und Erfolgsfaktoren am Beispiel von Sparkassen und Kreditgenossenschaften (Gabler Research : Schriften zum europäischen Management, 1. Aufl). Wiesbaden: Gabler.

Backhaus, K. (2006). Multivariate Analysemethoden. Eine anwendungsorientierte Einfübrung (11., überarb. Aufl). Berlin: Springer.

BaFin. (2012a). Formblatt 1 zur Kreditinstituts-Rechnungslegungsverordnung - Bilan₹, BaFin. Verfügbar unter http://www.bafin.de/SharedDocs/Downloads/DE/Formular/BA/dl_ rechkredv_formblatt1_ba.html

BaFin. (2012b). Formblatt 3 zur Kreditinstituts-Rechnungslegungsverordnung - Gewinn- und Verlustrechnung (Staffelform), BaFin. Verfügbar unter http://www.bafin.de/SharedDocs/Down loads/DE/Formular/BA/dl_rechkredv_formblatt3_ba.doc?_blob=publicationFile\& $\mathrm{v}=6$

Bamberg, G., Baur, F. \& Krapp, M. (2012). Statistik (Lehr- und Handbücher der Wirtschafts- und Sozialwissenschaften, 17. Aufl.). München: Oldenbourg.

Bartetzky, P. \& Volk, T. (2012). Praxis der Gesamtbanksteuerung. Methoden, Anforderungen der Aufsicht, Fallstudien. Stuttgart: Schäffer-Poeschel. 
Bäsch, H. (1992). Jahresabschlussanalyse bei Universalbanken. Grenzen und Möglichkeiten im Lichte neuer Rechnungslegungsvorschriften. Wiesbaden: Deutscher Universitäts-Verlag.

Baxmann, U. G. (1995a). Betriebsgrößen- und Fusionseffekte. Sparkasse (10), 470. Verfügbar unter http://www.wiso-net.de/webcgi?START=A60\&DOKV_DB=ZECU\& DOKV_NO=SPAR965012F90B33060FA8BCEF711B59B010\&DOKV_HS $=0 \& P P=1$

Baxmann, U. G. (1995b). Kreditwirtschaftliche Betriebsgrößen (Recht, Wirtschaft, Finanzen : Finanzen). Stuttgart: Dt. Sparkassenverl. Verfügbar unter http://digitool.hbz-nrw. de: $1801 /$ webclient $/$ DeliveryManager?pid $=1545025 \&$ custom $\% 5$ Fatt $\% 5 F 2=$ simple $\% 5 F$ viewer

Baxmann, U. G. (1999). Hintergründe und Facetten der Bankenkonzentration im Überblick. In U. G. Baxmann (Hrsg.), Konzentrationsprozesse in der Kreditwirtschaft. [2. kreditwirtschaftliches Kontaktforum ; Bankseminar Lüneburg] (S. 1-32). Frankfurt: Bankakademie-Vlg.

Becker, H. P. (2012). Investition und Finanzierung. Grundlagen der betrieblichen Finanzwirtschaft (Lehrbuch, 5., überarbeitete und erweiterte Auflage). Wiesbaden: Gabler Verlag.

Becker, H. P. \& Peppmeier, A. (2013). Bankbetriebslehre (Lernen, 9., aktualisierte Aufl). Herne: Kiehl.

Berekoven, L., Eckert, W. \& Ellenrieder, P. (2006). Marktforschung. Methodische Grundlagen und praktische Anwendung (Gabler Lehrbuch, 11., überarb. Aufl). Wiesbaden: Gabler.

Berger, A. N. \& Humphrey, D. B. (1997). Efficiency of financial institutions: International survey and directions for future research. European Journal of Operational Research, 98 (2), 175-212. Verfügbar unter http://www.sciencedirect.com/science/article/pii/ S0377221796003426

Berger, A. N. \& Mester, L. J. (2003). Explaining the Dramatic Changes in Performance of US banks: Technological Change, Deregulation, and Dynamic Changes in Competition. Journal of Financial Intermediation, 12 (1), 57-95. Verfügbar unter http://www. sciencedirect.com/science/article/pii/S1042957302000062

Bergmann, M. (2007). Qualitätsmanagement. In D. Bartmann, A. Burger, T. Burkhardt, W. Folz, T. Hartmann-Wendels, T. Heidorn et al. (Hrsg.), Knapps ensyklopädisches Lexikon des Geld-, Bank- und Börsenwesens (5., neu bearb. Aufl.). Frankfurt, M.: Knapp.

Beuthien, V. (2013). Die eingetragene Genossenschaft. Idee und Wirklichkeit (Marburger Schriften zur genossenschaftlichen Kooperation, Bd. 112, 1. Aufl). Baden-Baden: Nomos.

Beuthien, V. \& Hanrath, S. (2008). Den Förderauftrag prüfen - wie soll der Prüfer das machen? Zeitschrift für das gesamte Genossenschaftswesen, 58 (2), 85-97.

Beyer, D. (2012). Kompensationen in der Gewinn- und Verlustrechnung, Genoakademie. Verfügbar unter https://www.genoakademie.de/fileadmin/user_upload/documents/BankColleg/ Teilnehmerdownload_Fachwirt/2012/Skripte/BW_1Sem_LE_6-8_Teil2.pdf

Bieg, H. (2010a). Bankbilanzierung nach HGB und IFRS (Vahlens Handbücher der Wirtschafts- und Sozialwissenschaften, 2., Aufl). München: Vahlen, Franz.

Bieg, H. (2010b). Bankbilanzierung nach HGB und IFRS (Formblätter). Formblätter der Bilanz und Gewinn- und Verlustrechnung nach der RechKredV (2. Aufl.). Zugriff am 20.02.2015. Verfüg- 
bar unter http://www.vahlen.de/fachbuch/studentenmaterial/bankbilanzierung_form blaetter-rechkredv.pdf

Bode, I. \& Wilke, F. (2014). Private Vorsorge als Illusion. Rationalitätsprobleme des neuen deutschen Rentenmodells (1. Aufl). Frankfurt am Main: Campus.

Boehnke, K. (1983). Der Einfluss verschiedener Stichprobencharakteristika auf die Effizienz. der parametrischen und nichtparametrischen V arianzanalyse (Medizinische Informatik und Statistik, Bd. 42). Berlin: Springer.

Bortz, J. (1977). Lehrbuch der Statistik. für Sozialwissenschaftler. Berlin: Springer.

Bortz, J. \& Schuster, C. (2010). Statistik für Human- und Sozialwissenschaftler (SpringerLehrbuch, 7., vollst. überarb. u. erw. Aufl). Berlin: Springer.

Bosch, K. (1998). Statistik-Taschenbuch (3., verb. Aufl). München: Oldenbourg.

Botsis, D., Rock, T. \& Kaiser, B. (2012). Kennzablen und Kennzablensysteme für Banken (1. Auflage). Wiesbaden: Gabler.

Bourier, G. (2012). Beschreibende Statistik. Praxisorientierte Einführung (SpringerLink : Bücher, 10., aktualisierte Auflage). Mit Aufgaben und Lösungen. Wiesbaden: Gabler Verlag.

Brealey, R. A., Myers, S. C. \& Allen, F. (2014). Principles of Corporate Finance (The McGrawHill/Irwin series in finance, insurance, and real estate, 11th ed). New York: McGrawHill Irwin.

Bremke, K., Wagener, J. \& West, A. (2004). Fit für 2005: ein Programm zur Verbesserung der Wettbewerbsfähigkeit von Landesbanken. In O. Everling \& K.-H. Goedeckemeyer (Hrsg.), Bankenrating. Kreditinstitute auf dem Prüfstand (S. 291-322). Wiesbaden: Gabler Verlag.

Breyer, F. (2007). Mikroökonomik. Eine Einführung (Springer-Lehrbuch, 3., verb. Aufl). Berlin [u.a.]: Springer.

Broscheid, A. \& Gschwend, T. (2005). Zur statistischen Analyse von Vollerhebungen. Politische Vierteljahresschrift, 46 (1), O16.

Brösel, G. (2012). Bilanzanalyse. Unternehmensbeurteilung auf der Basis von HGB- und IFRSAbschlüssen (ESV basics, 14., neu bearb. u. erw. Aufl). Berlin: Schmidt.

Bühl, A. (2012). SPSS 20. Einführung in die moderne Datenanalyse (Scientific tools, 13., aktualisierte Aufl). München [u.a.]: Pearson.

Bülles, D. (Bundesverband der Deutschen Volksbanken und Raiffeisenbanken, Hrsg.). (2012). Alle Volksbanken und Raiffeisenbanken per Ende 2011, Bundesverband der Deutschen Volksbanken und Raiffeisenbanken. Zugriff am 03.06.2015.

Bundesanzeiger. Bundesanzeiger. Verfügbar unter www.bundesanzeiger.de

Bundesbank. (2012a). Die Ertragslage der deutschen Kreditinstitute im Jahr 2011. Verfügbar unter https://www.bundesbank.de/Redaktion/DE/Downloads/Veroeffentlichungen/Mo natsberichtsaufsaetze/2012/2012_09_ertragslage_kreditinstitute.pdf?_blob=publica tionFile 
Bundesbank. (2012b). Glossar aus dem Bereich der GuV-Statistik, Bundesbank. Verfügbar unter http://www.bundesbank.de/Redaktion/DE/Downloads/Statistiken/Banken_Und_An dere_Finanzielle_Institute/Banken/glossar_guv_statistik.pdf?_blob=publicationFile

Bundesbank. (2012c). Verzeichnis der Kreditinstitute. und ibrer Verbände sowie der Treubänder für Kreditinstitute in der Bundesrepublik Deutschland. Zugriff am 14.12.2012. Verfügbar unter https://www.bundesbank.de/Redaktion/DE/Downloads/Bundesbank/Aufgaben_ und_Organisation/verzeichnis_der_kreditinstitute_und_ihrer_verbaende.pdf?_blob= publicationFile

Bundesbank. (2012d). Die Rolle des "Baseler Zinsschocks" bei der bankaufsichtlichen Beurteilung von Zinsänderungsrisiken im Anlagebuch, Bundesbank. Monatsbericht. Verfügbar unter https://www.bundesbank.de/Redaktion/DE/Downloads/Veroeffentlichungen/ Monatsberichtsaufsaetze/2012/2012_06_zinsschock.pdf?_blob=publicationFile

Bundesbank. (2013a). Das Banken- und Finanzsystem. Die Finanz-, Wirtschafts- und Staatsschuldenkrise: Ein kurzer Überblick. Verfügbar unter http://www.bundesbank.de/Redaktion/ DE/Dossier/Service/schule_und_bildung_kapitel_4.html?notFirst=true\&docId= 147560

Bundesbank. (2013b). Bankinterne Methoden zur Ermittlung und Sicherstellung der Risikotragfäbigkeit und ibre bankaufsichtliche Bedeutung.

Bundesbank. (2013c). Die Ertragslage der deutschen Kreditinstitute im Jahr 2012. Monatsbericht: 13. Verfügbar unter http://www.bundesbank.de/Redaktion/DE/Downloads/Veroef fentlichungen/Monatsberichtsaufsaetze/2013/2013_09_ertragslage_kreditinstitute.pdf ?_blob=publicationFile

Bundesbank (Bundesbank, Hrsg.). (2014a). Die Ertragslage der deutschen Kreditinstitute. Aufwand/Ertrag-Relation nach Bankengruppen, Bundesbank. Verfügbar unter http://www. bundesbank.de/Redaktion/DE/Downloads/Statistiken/Banken_Und_Andere_Finan zielle_Institute/Banken/GuV_Statistik/guv_tab4.pdf?_blob=publicationFile

Bundesbank (Bundesbank, Hrsg.). (2014b). Genossenschaftsbank, Bundesbank. Zugriff am 09.12.2014. Verfügbar unter http://www.bundesbank.de/Navigation/DE/Service/ Glossar/Functions/glossar.html?lv2=32032\&lv3=62128

Bundesbank. (2014c). Die Ertragslage der deutschen Kreditinstitute im Jahr 2013. Monatsbericht: 13. Verfügbar unter https://www.bundesbank.de/Redaktion/DE/Downloads/Veroef fentlichungen/Monatsberichtsaufsaetze/2014/2014_09_ertragslage_kreditinstitute.pdf? _blob=publicationFile

Bundesbank. (2015). Ertragslage und Widerstandsfähigkeit deutscher Kreditinstitute im Niedrigzinsumfeld. Pressegespräch mit A. Dombret und R. Röseler am 18.September 2015. Verfügbar unter https://www.bundesbank.de/Redaktion/DE/Pressemitteilungen/BBK/2015/2015_ 09_18_bafin_bbk.html

Bundesinstitut für Bau-, Stadt- und Raumforschung, Milbert, A. (Mitarbeiter). (2014). Wachsende und schrumpfende Gemeinden in Deutschland. Relatives, am bundesweiten Trend gemessenes Wachstum/Schrumpfung, Bundesamt für Bauwesen und Raumordnung. Verfügbar unter http://www.bbsr.bund.de/BBSR/DE/Raumbeobachtung/Raumabgrenzungen/ Wachs_Schrumpf_gem/Wachs_Schrumpf_Gemeinden_node.html 
Burger, A., Frohmüller, K. P. \& Moormann, J. (2008). Produktivität in Banken: warum die CIR in die Irre führt. Bank-Archiv : Zeitschrift für das gesamte Bank- und Börsenwesen, 56 (12, (12)).

Büschgen, H. E. (2001). Strukturen des europäischen Bankwesens - Unterschiede und Besonderheiten. In B. Rolfes \& T. Fischer (Hrsg.), Handbuch der europäischen Finanzdienstleistungsindustrie (S. 13-23). Frankfurt am Main: Knapp.

Busse von Colbe, Walther. (1964). Die Planung der Betriebsgröße. Wiesbaden.

Buzzell, R. D. (2004). The PIMS program of strategy research. Journal of Business Research, 57 (5), 478-483.

Buzzell, R. D. \& Gale, B. T. (1987). The PIMS principles. Linking strategy to performance. New York: Free Press; Collier Macmillan.

BVR. (2013). Mitarbeiter (Köpfe) in den Kreditgenossenschaften seit 1990 in Gesamtdeutschland, Bundesverband der Deutschen Volksbanken und Raiffeisenbanken. Zugriff am 08.05.2015. Verfügbar unter http://www.bvr.de/p.nsf/0/92520CFE9AF551C3C1257D0A00561 E4E/\$file/BVR_Table_Beschaeftigte_SB.pdf

BVR. (2015a). Entwicklung der Volksbanken und Raiffeisenbanken ab 1970, Bundesverband der Deutschen Volksbanken und Raiffeisenbanken. Zugriff am 08.05.2015. Verfügbar unter http://www.bvr.de/p.nsf/0/F0F8A6D1636D3A1CC1257D0A00540564/\$file/3_Ent wicklung-seit-1970-2014.pdf

BVR. (2015b). Friedrich Wilhelm Raiffeisen (1818-1888), Bundesverband der Deutschen Volksbanken und Raiffeisenbanken. Verfügbar unter http://www.bvr.de/Presse/Bild datenbank/Geschichte

Casu, B., Molyneux, P. \& Girardone, C. (2006). Introduction to banking. London: Prentice Hall Financial Times.

Christians, U. (2010). Der Erfolg ostdeutscher Kreditinstitute. Region und Profitabilität am Beispiel der Sparkassen und Kreditgenossenschaften in den neuen Bundesländern. Berlin: Logos-Verl.

Christians, U. \& Gärtner, S. (2014). Einfluss regionaler Bankenmärkte auf dezentrale Banken: Demographie, Bankenwettbewerb und Kreditportfolio (02/2014), Gelsenkirchen. Verfügbar unter http://hdl.handle.net/10419/92940

Christians, U. \& Hartl, F. (2015). Effirienz von regionalen Kreditinstituten. Eine Studie zur Dynamik der Effizienz, von Regionalkreditinstituten am Beispiel ostdeutscher Sparkassen und Kreditgenossenschaften 2007-2012 auf Basis von Data Envelopment und Hauptkomponentenanalyse (1. Aufl). Berlin: Berliner Wissenschafts-Verlag.

Coelli, T. J., Prasada Rao, D., O'Donnell, C. J. \& Battese, G. E. (2005). An Introduction to Efficiency and Productivity Analysis (2nd ed.). New York: Springer.

Coenenberg, A. G. (2012). Jahresabschluß und Jahresabschlußanalyse. Betriebswirtschaftliche, handelsund steuerrechtliche Grundlagen (Studienbibliothek Betriebswirtschaft, [2,1], 22. überarb. Aufl). Landsberg am Lech: Verl. Moderne Industrie.

Conrad, A. (2008). Banking in schrumpfenden Regionen. Auswirkungen von Alterung und Abwanderung auf Regionalbanken. 
Conrad, A., Neuberger, D. \& Trigo Gamarra, L. (2009). Der Einfluss regionaler und demographischer Umfeldfaktoren auf die Kosten- und Ertragssituation von Sparkassen: Eine Effizienzanalyse (Nr. 107). : University of Rostock, Institute of Economics. Verfügbar unter http://EconPapers.repec.org/RePEc:zbw:roswps:107

Conrad, A., Neuberger, D. \& Trigo Gamarra, L. (2014). The Impact of Regional Economic Conditions on the Efficiency of Savings Banks in the Light of Demographic Change. Credit and Capital Markets - Kredit und Kapital, 47 (4), 533-570. Verfügbar unter http:// ejournals.duncker-humblot.de/doi/pdf/10.3790/ccm.47.4.533

Cortez, B. \& Vogel, T. (2012). Grundzüge der Bilanzanalyse. Vorgehensweise, Methoden, Fallstricke. Steuer + Studium (1), 42-48.

DBV. (2012). Gehaltstabelle in den Genossenschaftsbanken 2013 und 2014. Verfügbar unter http://www.dbv-gewerkschaft.de/fileadmin/user_upload/pdf/Gehalts tabelle_in_Geno-Banken_2013_und_2014.pdf

Deelmann, T. (2015). Meilensteine und Trends der Betriebswirtschaft. Grundlagen, Geschichte und Geschichten der BWL (ESVbasics, 2., neu bearb. und wesentlich erw. Aufl). Berlin: Schmidt.

Deppe, H.-D. (1969). Bankbetriebliches Wachstum. Funktionaliusammenbänge und Operations Research in Kreditinstituten. Stuttgart.

Deutsche Gesetzliche Unfallversicherung e.V. (1997). DGUV Vorschrift 25 - Kassen. bisher: $B G V$ C9. Verfügbar unter http://www.arbeitssicherheit.de/media/pdfs/bgv_c9.pdf

Deutsches Institut für Wirtschaftsforschung e.V. (2004). Gehaltszuschlag für große Männer: Neue Studie belegt erstmals Zusammenhang zwischen Körpergröße und Verdienst in Deutschland, Deutsches Institut für Wirtschaftsforschung e.V. Verfügbar unter http://www.diw.de/ de/diw_02.c.242689.de/pressemitteilungen.html? id=diw_01.c.10431.de

Disselbeck, K. (2007). Die Industrialisierung von Banken am Beispiel des Outsourcings. Frankfurt am Main: Knapp.

Dowideit, M. (2015). DZ Bank warnt vor massivem Filialsterben. Handelsblatt. Verfügbar unter http://www.handelsblatt.com/unternehmen/banken-versicherungen/bankenzweigstellen-dz-bank-warnt-vor-massivem-filialsterben/11825408.html

Dreger, C., Kosfeld, R. \& Eckey, H.-F. (2014). Ökonometrie. Grundlagen - Methoden - Beispiele (SpringerLink : Bücher, 5., überarb. u. akt. Aufl. 2014). Wiesbaden: Imprint: Springer Gabler.

Dullenkopf, I. (2001). Förderauftragsbezogenes Benchmarking in Kreditgenossenschaften (Veröffentlichung / Forschungsinstitut für Genossenschaftswesen an der Universität ErlangenNürnberg, Bd. 41). Nürnberg: Das Forschungsinstitut.

Dymski, G. A. (1999). The Bank Merger Wave. The Economic Causes and Social Consequences of Financial Consolidation (Issues in money, banking, and finance). Armonk, N.Y.: M.E. Sharpe.

Eckstein, P. P. (2010). Statistik für Wirtschaftswissenschaftler. Eine realdatenbasierte Einführung mit SPSS (Lehrbuch, 2., aktualisierte und erw. Aufl). Wiesbaden: Gabler. 
Egert, C. (2013). Lineare statistische Modellierung und Interpretation in der Praxis. München: Oldenbourg.

Eisele, W. \& Knobloch, A. P. (2010). Technik des betrieblichen Rechnungswesens. Buchfübrung und Bilanzierung, Kosten- und Leistungsrechnung, Sonderbilanzen (Vahlens Handbücher der Wirtschafts- und Sozialwissenschaften, 8. Aufl). München: Vahlen.

Engel, U. \& Reinecke, J. (1994). Panelanalyse. Grundlagen, Techniken, Beispiele (De Gruyter Lehrbuch). Berlin: Walter de Gruyter.

Entrop, O., Memmel, C., Ruprecht, B. \& Wilkens, M. (2015). Determinants of bank interest margins: Impact of maturity transformation. Journal of Banking \& Finance, 54, 1-19.

Eurostat. (2014). NUTS - Systematik der Gebietseinheiten für die Statistik. Nomenclature des unités territoriales statistiques. Verfügbar unter http:/ / ec.europa.eu/eurostat/web/nuts/overview

Everling, O. \& Goedeckemeyer, K.-H. (2015). Bankenrating. Normative Bankenordnung in der Finanzmarktkrise (2. Auflage). Wiesbaden: Springer Fachmedien Wiesbaden.

Fahrmeir, L., Künstler, R., Pigeot, I. \& Tutz, G. (2007). Statistik (Springer-Lehrbuch, 6., überarbeitete Auflage). Berlin: Springer.

Fechtrup, B. (1994). Die Deutsche Genossenschaftsbank. Rechtsgrundlagen, Entwicklung und Tätigkeit. Dissertation, Westfälische Wilhelms-Universität zu Münster. Münster.

Fischer, T. \& Rolfes, B. (2001). Finanzdienstleistungen im vereinten Europa. In B. Rolfes \& T. Fischer (Hrsg.), Handbuch der europäischen Finanzdienstleistungsindustrie (S. 1-10). Frankfurt am Main: Knapp.

Gärtner, S. (2009). Lehren aus der Finan₹krise: Räumliche Nähe als stabilisierender Faktor, Westfälische Wilhelms-Universität zu Münster; Institut Arbeit und Technik. Forschung Aktuell: 08/2009. Verfügbar unter http://hdl.handle.net/10419/57247

Gärtner, S. (2010). Die räumliche Dimensionierung im Bankgeschäft: Regionale Finanzintermediäre in strukturschwachen Räumen. In U. Christians (Hrsg.), Unternebmensfinanzierung und Region. Finanzierungsprobleme mittelständischer Unternebmen und Bankpolitik in peripheren Wirtschaftsräumen (Schriftenreihe Finanzmanagement, Bd. 70, S. 205-230). Hamburg: Kovač.

GDV. (2009). Positionspapier zur Bestimmung der risikofreien Zinsstrukturkurve unter Solvency II, Gesamtverband der Deutschen Versicherungswirtschaft e. V. Verfügbar unter http://www.gdv.de/wp-content/uploads/2010/01/Positions papier_Zinsstrukturkurve.pdf

Giesselmann, M. \& Windzio, M. (2012). Regressionsmodelle zur Analyse von Paneldaten (Studienskripten zur Soziologie, 1., neue Ausg). Wiesbaden: VS Verlag für Sozialwissenschaften.

Gilligan, T., Smirlock, M. \& Marshall, W. (1984). Scale and Scope Economies in the MultiProduct Banking Firm. Journal of Monetary Economics, 13 (3), 393-405. Verfügbar unter http://www.sciencedirect.com/science/article/pii/0304393284900412

Gischer, H. (2014). Wie bedroblich sind dauerbaft niedrige Zinsen für die Banken?, Bayreuther Finanzmarktsymposium. Verfügbar unter http://www.giw.uni-bayreuth.de/de/Finanz marktsymposium/ressourcen/141106_Bayreuther-Finanzmarktsymposium_HG.pdf 
Gohout, W. \& Specht, K. (2014). Tests zur Normalverteilung. Das Wirtschaftsstudium : wisu; Zeitschrift für Ausbildung, Prüfung, Berufseinstieg und Fortbildung, Zeitschrift für Ausbildung, Prüfung, Berufseinstieg und Fortbildung. - Lange, ISSN 0340-3084, ZDB-ID 1202844. - Vol. 43.2014, 4, p. 544-550 (4, (4)).

Götze, U. (2010). Kostenrechnung und Kostenmanagement (Springer-Lehrbuch, 5., verb. Aufl). Berlin: Springer.

Grant, R. M. (2010a). Contemporary strategy analysis (7th ed.). Hoboken, NJ: John Wiley \& Sons.

Grant, R. M. (2010b). Contemporary strategy analysis. Text and cases (7th ed.). Hoboken, N.J.: Wiley.

Grill, W. \& Perczynski, H. (2011). Wirtschaftslehre des Kreditwesens (Gehlenbuch, Bd. 303, 45. Aufl.). Bad Homburg vor d. Höhe: Gehlen.

Grüger, W. (2007). Genossenschaftsbanken: Grundlagen. In D. Bartmann, A. Burger, T. Burkhardt, W. Folz, T. Hartmann-Wendels, T. Heidorn et al. (Hrsg.), Knapps ensyklopädisches Lexikon des Geld-, Bank- und Börsenwesens (5., neu bearb. Aufl.). Frankfurt, M.: Knapp.

Hanker, P. (2007). Volksbanken und Raiffeisenbanken: Gut für Stand und Land? (08), 24 26.

Hartmann-Wendels, T., Pfingsten, A. \& Weber, M. (2010). Bankbetriebslehre (5., überarb. Aufl). Berlin: Springer.

Hartmann-Wendels, T., Pfingsten, A. \& Weber, M. (2015). Bankbetriebslehre (Lehrbuch, 6. Aufl). Berlin: Springer Gabler.

Hartmann-Wendels, T., Weber, M. \& Pfingsten, A. (2007). Bankbetriebslebre. Mit 148 Tabellen (4., überarb. Aufl). Berlin: Springer.

Hartung, J., Elpelt, B. \& Klösener, K.-H. (2005). Statistik. Lehr- und Handbuch der angewandten Statistik ; mit zablreichen, vollständig durchgerechneten Beispielen (14., unwesentlich veränd. Aufl.). München: Oldenbourg.

Hausman, J. A. (1978). Specification Tests in Econometrics. Econometrica, 46 (6), 12511271. Verfügbar unter http://www.jstor.org/stable/1913827

Heffernan, S. A. (2005). Modern banking. Chichester, West Sussex, England: John Wiley \& Sons.

Helten, E. (2004). Fusionen in der Assekuranz. In G. Kürble \& H. Reichling (Hrsg.), Fusionen von Banken und Versicherungen (Zweibrücker Reihe, Bd. 1, S. 1-18). Karlsruhe: VVW.

Hockmann, H.-J. \& Thießen, F. (2012). Investment Banking (3., überarb. u. erw. Aufl). Stuttgart: Schäffer-Poeschel.

Holtmann, C. F. \& Morales, W. (2010). Ostdeutsche Sparkassen im Wandel der Zeit. In D. Puchta (Hrsg.), Deutschland 20 Jahre nach dem Mauerfall. Rückblick und Ausblick (1. Aufl, S. S. 163-178). Wiesbaden: Gabler.

Huber, F., Meyer, F. \& Lenzen, J. M. (2014). Grundlagen der Varianzanalyse. Konzeption, Durchfübrung, Auswertung (Lehrbuch). Wiesbaden: Springer Gabler. 
Hummel, D. \& Pedrotti, M. (2013). Zinsmarge und Effizienz von Kreditgenossenschaften. In J. Brazda, M. Dellinger \& D. Rößl (Hrsg.), Genossenschaften im Fokus einer neuen Wirtschaftspolitik. Bericht der XVII. Internationalen Genossenschaftstagung (IGT) 2012 in Wien (S. 345-357). Teilband I. Wien: LIT.

Humphrey, D. B. (1992). Flow versus Stock Indicators of Banking Output: Effects on Productivity and Scale Economy Measurement. Journal of Financial Services Research, 6 (2), 115-135. Verfügbar unter http://search.ebscohost.com/login.aspx?direct=true\& $\mathrm{db}=$ eoh\&AN $=0274835 \&$ site $=$ ehost-live

Hunter, W. C. \& Timme, S. G. (1995). Core Deposits and Physical Capital: A Reexamination of Bank Scale Economies and Efficiency with Quasi-Fixed Inputs. Journal of Money, Credit and Banking, 27 (1), 165-185. Verfügbar unter http://www.jstor.org/stable/ 2077857

Hutzschenreuter, T. (2009). Allgemeine Betriebswirtschaftslebre. Grundlagen mit zablreichen Praxisbeispielen; [Bachelor geeignet!] (Lehrbuch, 3., überarb. und erw. Aufl). Wiesbaden: Gabler.

Jacobsen, L. K. (2003). Bestimmungsfaktoren für Erfolg im Entrepreneurship. Entwicklung eines umfassenden theoretischen Modells. Dissertation, FU Berlin. Berlin.

Janssen, J. \& Laatz, W. (2007). Statistische Datenanalyse mit SPSS für Windows. Eine anwendungsorientierte Einführung in das Basissystem und das Modul exakte Tests ; mit 193 Tabellen (6., neu bearb. und erw. Aufl). Berlin [u.a.]: Springer.

Kähler, W.-M. (2011). Statistische Datenanalyse. Verfahren verstehen und mit SPSS gekonnt einsetzen (OnlinePlus, 7., aktualisierte Aufl.) [S.l.]: Vieweg+Teubner.

Kalhöfer, C. (2004). Entwicklung, Ursachen und Erfolge von Bankfusionen. In G. Kürble \& H. Reichling (Hrsg.), Fusionen von Banken und Versicherungen (Zweibrücker Reihe, Bd. 1, Bd. 1, S. 77-85). Karlsruhe: VVW.

Karmann, A., Buehn, A. \& Pedrotti, M. (2013). What Determines the Interest Margin? An Analysis of the German Banking System. Beiträge zur Jahrestagung des Vereins für Socialpolitik 2013: Wettbewerbspolitik und Regulierung in einer globalen Wirtschaftsordnung - Session: Banking, No. A19-V1. Credit and Capital Markets - Kredit und Kapital, 46 (4), 467-494. Verfügbar unter http://hdl.handle.net/10419/80029

Keskin, S. (2006). Comparison of Several Univariate Normality Tests Regarding Type I Error Rate and Power of the Test in Simulation Based Small Samples. Journal of Applied Science Research, 296-300.

Kipker, I. (2010). VR-Bankfabrik 2015. In I. Hallberg-Fritz (Hrsg.), Zukunft der Produktionsbank - Produktionsbank der Zukunft. ADG-Perspektive (ADG-Perspektiven, 1. Aufl, S. 168175). Wiesbaden: DG-Verl.

Klee, J. (2002). Qualitätsmanagementsysteme in Banken. Konzeption und Implementierung im filialgebundenen Standardgeschäft unter besonderer Berücksicbtigung der Qualitätsmessung. Lohmar: Eul.

Klein, J. (2003). Das Sparkassenwesen in Deutschland und Frankreich. Entwicklung, aktuelle Rechtsstrukturen und Möglichkeiten einer Annäherung (Untersuchungen über das Spar-, Giro- und Kreditwesen. Abteilung B, Rechtswissenschaft, Bd. 147). Berlin: Duncker \& Humblot. 
Klemm-Bax, S. (2000). Erfolgsfaktoren. ein unscharfer Modellansatz zur Bestimmung des Unternehmenserfolgs auf der Grundlage empirischer Daten /. Paderborn.

Kobmann, W. (1996). Die Auswirkungen des Lean Management auf die Zweigstellen- und Abteilungsorganisation der Genossenschaftsbanken (Europäische Hochschulschriften. Reihe V, Volks- und Betriebswirtschaft Publications universitaires européennes. Série V, Sciences économiques, gestion d'entreprise European university studies. Series V, Economics and management, vol. 1959). Frankfurt am Main: P. Lang.

Kolmogorov, A. N. (1933). Sulla determinazione empirica di una legge di distruzione. Giornale dell'Instituto Italiano degli Attuari (4), 83-91.

Komlos, J. \& Süssmuth, B. (2010). Empirische Ökonomie. Eine Einführung in Methoden und Anwendungen (Springer-Lehrbuch). Berlin: Springer.

Kopp, J. \& Lois, D. (2009). Einführung in die Panelanalyse, Institut für empirische Sozialforschung, TU Chemnitz. Verfügbar unter https://www.tu-chemnitz.de/hsw/soziologie/ Professuren/Empirische_Sozialforschung/download/Einfuehrung_Panelanalyse.pdf

Köppen, J. (1966). Das Eigenkapital der Kreditinstitute (Schriftenreihe für Kreditwirtschaft und Finanzierung, Bd. 7). Wiesbaden: Gabler Verlag. Verfügbar unter http://dx.doi.org/ 10.1007/978-3-322-98896-6

Kositzki, A. (2004). Das öffentlich-rechtliche Kreditgewerbe. Eine empirische Analyse zur Struktureffizienz. und Unternehmensgröße im Sparkassensektor (Gabler Edition Wissenschaft, 1. Aufl). Wiesbaden: Dt. Univ.-Verl.

Kröhnert, S., Klingholz, R., Sievers, F., Großer, T. \& Friemel, K. (2011). Die demografische Lage der Nation. Was freiwilliges Engagement für die Regionen leistet. Berlin: Berlin-Institut für Bevölkerung und Entwicklung.

Kruskal, W. H. \& Wallis, W. Allen. (1952). Use of Ranks in One-Criterion Variance Analysis. Journal of the American Statistical Association, 47 (260), 583-621. Verfügbar unter http://www.jstor.org/stable/2280779

Küting, K. \& Weber, C.-P. (2012). Die Bilanzanalyse. Beurteilung von Abschlüssen nach HGB und IFRS (10., überarb. Aufl). Stuttgart: Schäffer-Poeschel.

Lang, G. \& Welzel, P. (1996). Efficiency and technical progress in banking Empirical results for a panel of German cooperative banks. Journal of Banking \& Finance, 20 (6), 1003-1023. Verfügbar unter http://www.sciencedirect.com/science/article/pii/03784 26695000402

Lilliefors, H. W. (1967). On the Kolmogorov-Smirnov Test for Normality with Mean and Variance Unknown. Journal of the American Statistical Association, 62 (318), 399-402.

Linseisen, A. (1995). Lean-Banking. Die Anwendbarkeit des Lean-Management bei deutschen Kreditinstituten (Gabler Edition Wissenschaft : Bank- und Finanzwirtschaft). Wiesbaden: Dt. Univ.-Verl.; Gabler.

Lund, A. \& Lund, M. (2015). One-way ANOVA, Leard Statistics. Verfügbar unter https://statistics.laerd.com/statistical-guides/one-way-anova-statistical-guide-3.php

Mathweis, R. (1996). Die Fusion von Kreditgenossenschaften. Potentielle einzelwirtschaftliche Auswirkungen der Fusion von Kreditgenossenschaften der Primärstufe (Kölner Genossenschaftswissen- 
schaft). Regensburg: Transfer. Verfügbar unter http://digitool.hbz-nrw.de:1801/ webclient $/$ DeliveryManager?pid $=1085253 \&$ custom $\% 5$ Fatt $\% 5 F 2=$ simple $\% 5$ Fviewer

Matz, S. (2007). Erfolgsfaktoren im Innovationsmanagement von Industriebetrieben (Gabler Edition Wissenschaft). Wiesbaden: Deutscher Universitäts-Verlag / GWV Fachverlage GmbH, Wiesbaden.

McDonald, J. H. (2014). Handbook of Biological Statistics. Kruskal-Wallis test (3. Auflage), University of Delaware. Verfügbar unter http://www.biostathandbook.com/kruskal wallis.html

Mitchell, K. \& Onvural, N. M. (1996). Economies of Scale and Scope at Large Commercial Banks: Evidence from the Fourier Flexible Functional Form. Journal of Money, Credit and Banking, 28 (2), 178-199. Verfügbar unter http://www.jstor.org/stable/2078022

Mugler, A. (2014). Das deutsche Bankensystem im internationalen Vergleich. Vergleich der Bankensysteme Deutschlands, $\operatorname{der}$ US A, Japans und Großbritanniens. Hamburg: Disserta Verl.

Obst, G. \& Hagen, J. v. (2000). Geld-, Bank- und Börsenwesen. Handbuch des Finanzsystems (40., völlig überarb. Aufl. / hrsg. von Jürgen von Hagen .). Stuttgart: Schäffer-Poeschel.

Padberg, C., Padberg, T. \& Werner, T. (2010). Das neue HGB. Bilanærechtsmodernisierungsgesetz. (BilMoG) - Kurzkommentierung (2., völlig neu bearb. und erw. Aufl.). Berlin: Erich Schmidt.

Padberg, T. (2005). Analyse der Jahresabschlïsse von Genossenschaftsbanken und Sparkassen (Banking \& finance aktuell, Bd. 23, 1. Aufl.). Frankfurt am Main: Bankakademie-Verl.

Park, M. H. (2010). Practical Guides To Panel Data Analysis, The University Information Technology Services (UITS) Center for Statistical and Mathematical Computing, Indiana University.

Peemöller, V. H. (2005). Genossenschaften als "historische" und moderne Form der Kooperation. In J. Zentes, B. Swoboda \& D. Morschett (Hrsg.), Kooperationen, Allianzen und Netzwerke. Grundlagen - Ansätže - Perspektiven (2., überarb. und erw. Aufl., S. 405-424). Wiesbaden: Gabler.

Perridon, L., Steiner, M. \& Rathgeber, A. W. (2012). Finanzwirtschaft der Unternehmung (Vahlens Handbücher der Wirtschafts- und Sozialwissenschaften, 16. Aufl). München: Vahlen.

Philipps, H. (2010). Rechnungslegung nach BilMoG. Kurzkommentar zum Jahresabschluss und Lagebericht nach neuem Bilanzrecht (1. Aufl). Wiesbaden: Gabler.

Pieper, C. (2005). Banken im Umbruch. Strukturwandel im deutschen Bankensektor und regionalwirtschaftliche Implikationen (Wirtschaftsgeographie, Bd. 30). Münster: LIT.

Rappaport, A. (1998). Creating Shareholder Value. A Guide For Managers And Investors. New York: The Free Press.

Razali, N. M. \& Wah, Y. B. (2011). Power comparisons of Shapiro-Wilk, KolmogorovSmirnov, Lilliefors and Anderson-Darling tests. Journal of Statistic Modeling and Analytics, 2 (1), 21-33. 
Reichel, R. (2011). Der Beitrag der Kreditgenossenschaften zur Finanzstabilität. Zeitschrift für das gesamte Kreditwesen (18), 959. Verfügbar unter https://www.wiso-net.de/ document/ZFGK_091115018

Reichling, H. (2004). Die regionale Identität der Genossenschaftsbanken. Geht die Geschichte fusionierter Genossenschaftsbanken verloren? In G. Kürble \& H. Reichling (Hrsg.), Fusionen von Banken und Versicherungen (Zweibrücker Reihe, Bd. 1, Bd. 1, S. 149158). Karlsruhe: VVW.

Reuters (2015). Genossen wollen das Filialgeschäft an sich reißen. Handelsblatt. Verfügbar unter http://www.handelsblatt.com/unternehmen/banken-versicherungen/volks-undraiffeisenbanken-genossen-wollen-das-filialgeschaeft-an-sich-reissen/11548650.html? nlayer=Organisation_11804700

Richard, W. \& Mühlmeyer, J. (2011). Betriebslehre der Banken und Sparkassen (Wirtschaftswissenschaftliche Bücherei für Schule und Praxis, 35. Aufl). Rinteln: Merkur-Verl.

Richter, F. (2014). Produktivität und ihre Einflussfaktoren - Eine empirische Analyse für Kreditgenossenschaften. Credit und Capital Markets, 47 (3), 415-437.

Riekeberg, M. (2003). Erfolgsfaktoren bei Sparkassen. Kausalanalytische Untersuchung mittels linearer Strukturgleichungsmodelle (Neue betriebswirtschaftliche Forschung, Bd. 307, 1. Aufl). Wiesbaden: Dt. Univ.-Verl.

Rolfes, B. (2008). Gesamtbanksteuerung. Risiken ertragsorientiert steuern (Handelsblatt-Bücher, 2., vollst. überarb. u. erw. Aufl). Stuttgart: Schäffer-Poeschel.

Rossmann, C. (2010). Darstellung von Tabellen in Präsentationen und Forschungsberichten. : Universität Erfurt. Zugriff am 25.02.2015.

Sassen, R. (2012). Optimierung des Fördererfolgsreporting und der Prüfung von Genossenschaften. Vorschläge zur Änderung des Genossenschaftsrechte. Zeitschrift für das gesamte Genossenschaftswesen, 62 (1), 35-50.

Scharpf, P. \& Schaber, M. (2011). Handbuch Bankbilanz (4., aktualisierte und erweiterte Auflage). Düsseldorf: IDW.

Schax, E. (2008). Strategieorientierte Personalentwicklung in Genossenschaftsbanken. Eine empirische Untersuchung zur betrieblichen Weiterbildung (Wirtschaftswissenschaft, 1. Aufl.). Wiesbaden: Deutscher Univ.-Verl.

Schierenbeck, H. (2003a). Grundzüge der Betriebswirtschaftslehre (16., vollst. überarb. und erw. Aufl). München [u.a.]: Oldenbourg.

Schierenbeck, H. (2003b). Ertragsorientiertes Bankmanagement. Band 1: Grundlagen, Marktzinsmethode und Rentabilitäts-Controlling (Gabler-Lehrbuch, Bd. 1, 8., überarb. und erw. Aufl). Wiesbaden: Gabler.

Schierenbeck, H., Lister, M. \& Kirmße, S. (2014). Ertragsorientiertes Bankmanagement. Band 1: Messung von Rentabilität und Risiko im Bankgeschäft (Bd. 1, 9., vollst. überarb. und erw. Aufl). Wiesbaden: Springer Gabler.

Schneider, G., Geiger, I. K. \& Scheuring, J. (2008). Prozess- und Qualitätsmanagement. Grundlagen der Prozessgestaltung und Qualitätsverbesserung mit zablreichen Beispielen, Repetitionsfragen und 
Antworten (Betriebswirtschaftslehre, 1. Aufl., Ausg.: N0088). Zürich: Compendio Bildungsmedien.

Schulze, P. M. \& Porath, D. (2012). Statistik mit Datenanalyse und ökonometrischen Grundlagen (7., überarbeitete Auflage). München: Oldenbourg, R.

Sealey, C. W. \& Lindley, J. T. (1977). Inputs, Outputs, and a Theory of Production and Cost at Depository Financial Institutions. Journal of Finance, 32 (4), 1251-1266. Verfügbar unter http://search.ebscohost.com/login.aspx?direct $=$ true $\& d b=$ eoh\&AN=0098439\& site $=$ ehost-live

Shapiro, S. S. \& Wilk, M. B. (1965). An Analysis of Variance Test for Normality. Biometrika, $52(3), 591-611$.

Spath, D., Bauer, W. \& Engstler, M. (2008). Innovationen und Konzepte für die Bank der Zukunft. Mit modernen Vertriebslösungen und optimierten Wertschöpfungsprozessen künftigen Herausforderungen begegnen (SpringerLink : Bücher). Wiesbaden: Gabler Verlag / GWV Fachverlage GmbH, Wiesbaden.

Spies, R. (2015). Herausforderungen auf dem Land. Bankmagażin, 64 (4), 54-56. Verfügbar unter http://www.springerprofessional.de/herausforderungen-auf-dem-land/5637908. html

Staats, S. (2006). Fusionen bei Sparkassen und Landesbanken. Eine Untersuchung zu den Möglichkeiten der Vereinigung öffentlich-rechtlicher Kreditinstitute. Fusionen bei Sparkassen und Landesbanken.

Statistische Ämter des Bundes und der Länder (Hrsg.). (2013). Regionaldatenbank Deutscbland. Bevölkerungsstand. Verfügbar unter https://www.regionalstatistik.de

Statistisches Bundesamt. (2012a). Gemeindeverzeichnis. Verfügbar unter https://www. destatis.de/DE/ZahlenFakten/LaenderRegionen/Regionales/Gemeindeverzeichnis/ Administrativ/Archiv/Administrativ.html

Statistisches Bundesamt. (2012b). Regionalatlas Deutschland. Indikatoren des Themenbereichs "Bevölkerung", Statistisches Bundesamt. Verfügbar unter http://www.destatis.de/ regionalatlas

Statistisches Bundesamt. (2013a). EU-Stadt-Land-Gliederung am 31.12.2012. Stadt-LandGliederung nach Fläche und Bevölkerung auf Grundlage des ZENSUS 2011 und Bevölkerungsdichte, Statistisches Bundesamt. Verfügbar unter https://www.destatis.de/DE/Zahlen Fakten/LaenderRegionen/Regionales/Gemeindeverzeichnis/NichtAdministrativ/Ar chiv/Standardtabellen/33STLVorjahr.xls?_blob=publicationFile

Statistisches Bundesamt. (2013b). Gemeindeverzeichnis. Bundesländer mit Hauptstädten nach Fläche und Bevölkerung. Verfügbar unter https://www.destatis.de/DE/ZahlenFakten/Laender Regionen/Regionales/Gemeindeverzeichnis/Administrativ/Aktuell/02Bundeslaender. xls?_blob=publicationFile

Statistisches Bundesamt. (2014). Stadt-/Landgliederung nach der Zuordnung von Eurostat 2000, Statistisches Bundesamt. Verfügbar unter https://www.destatis.de/DE/ZahlenFakten/ LaenderRegionen/Regionales/ 
Gemeindeverzeichnis/Administrativ/Archiv/Definition_STL_bis_30122011.pdf?_blo $\mathrm{b}=$ publicationFile

Stiftung GIZ. (2015). Hermann Scbulze-Deliţsch (1808-1883), Genossenschaftshistorisches Informationszentrum. Verfügbar unter http://www.bvr.de/Presse/Bilddatenbank/ Veranstaltungen

Strauß, M.-R. (2006). Erfolsfaktoren von Banken im Firmenkundengeschäft. Empirische Analyse und konzeptionelle Anwendung. Wiesbaden: DUV.

Swoboda, B. (2005). Kooperation: Erklärungsperspektiven grundlegender Theorien, Ansätze und Konzepte im Überblick. In J. Zentes, B. Swoboda \& D. Morschett (Hrsg.), Kooperationen, Allianzen und Netzwerke. Grundlagen - Ansätze - Perspektiven (2., überarb. und erw. Aufl., S. 35-64). Wiesbaden: Gabler.

Tebroke, H.-J. (1993). Größe und Fusionserfolg von Genossenschaftsbanken. Eine theoretische und empirische Analyse der Auswirkungen von Betriebsgröße und fusionsbedingter Betriebsgrößenervveiterung auf die Ergebnisstruktur von Kreditgenossenschaften (Reihe Finanzierung, Steuern, Wirtschaftsprüfung, Bd. 17). Köln: Müller Botermann.

Thiry, D. (2009). Eine empirische Analyse der Marktdisziplinierung deutscher Sparkassen (Gabler Research, Band 19). Wiesbaden: Gabler Verlag / GWV Fachverlage GmbH, Wiesbaden.

Thomson Reuters EIKON. (2015). Euro-Swapsätze für 2 Jabre, 5 Jahre und 10 Jahre. Verfügbar unter RICs: EUR2YZ=R, EUR5YZ=R und EUR10YZ=R

Tischer, M. (2011). Effizienzmessung im Sparkassensektor am Beispiel regionaler Cluster (Schriftenreihe Finanzierung und Banken, Bd. 18). Sternenfels: Verl. Wissenschaft \& Praxis.

Tolkmitt, V. (2007). Neue Bankbetriebslehre. Basiswissen zu Finanzprodukten und Finanzdienstleistungen (Lehrbuch, 2., überarb. Aufl.). Wiesbaden: Gabler.

Torres-Reyna, O. (2007). Panel Data Analysis. Fixed and Random Effects using Stata, Princeton University. Verfügbar unter http://www.princeton.edu/ otorres/Panel101.pdf

Trenkler, G. \& Büning, H. (1994). Nichtparametrische statistische Methoden (De Gruyter Lehrbuch, 2. Aufl., 2. erw. u. völlig überarb. Auf). Berlin: De Gruyter. Verfügbar unter http://www.degruyter.com/search?f_0=isbnissn\&q_0=9783110902990\&searchTitles= true

Vargha, A. \& Delaney, H. D. (1998). The Kruskal-Wallis Test and Stochastic Homogeneity. Journal of Educational and Behavioral Statistics, 23 (2), 170-192.

Varmaz, A. (2006). Rentabilität im Bankensektor. Identifizierung, Quantifizierung und Operationalisierung werttreibender Faktoren (Gabler Edition Wissenschaft, 1. Aufl.). Wiesbaden: Dt. Univ.-Verl.

Volksbank Stuttgart eG. (2011). Sat̨ung. Verfügbar unter https://www.volksbankstuttgart.de/content/dam/f0054-0/pdf/wir_ueber_uns/satzung/satzung_vbs.pdf

VR Bank Main-Kinzig-Büdingen eG. (2012). Fusionshistorie der VR Bank Main-KinæigBüdingen $e G$, VR Bank Main-Kinzig-Büdingen eG. Verfügbar unter http://upload. wikimedia.org/wikipedia/de/e/e9/Stammbaum_VR_Bank_MKB.jpg 
Walter, K.-F. (2013). Fristentransformation als Bestandteil einer nachhaltigen Geschäftsstrategie einer Genossenschaftsbank? Zeitscbrift für das gesamte Kreditwesen (06), 294.

Watson, D. \& Head, A. (2010). Corporate Finance. Principles and practice (5th ed). Harlow, England: Financial Times/Prentice Hall.

Weimer, T. \& Wißkirchen, C. (1999). Sechs Thesen zur Fusionswelle im Bankenbereich. Die Bank : Zeitschrift für Bankpolitik und Praxis (11).

Weis, H. C. (2012). Marketing (Kompendium der praktischen Betriebswirtschaft, 16., verbesserte und aktualisierte Auflage). Herne, Westf: NWB Verlag.

Wieser, F. (2003). Social economics (Routledge library editions. The economics series). London: Routledge.

Wimmer, K. (2013). Moderne Bankkalkulation. Grundlagen und Anwendungsmöglichkeiten (Betrieb : Controlling, 4., überarb. Aufl). Stuttgart: Dt. Sparkassenverl.

Winker, P. (2010). Empirische Wirtschaftsforschung und Ökonometrie (Springer-Lehrbuch, 3., aktual. Aufl.). Berlin [u.a.]: Springer.

Winkler, H. (2004). Prüfungsbericht von Kredit- und Finanzdienstleistungsinstituten. Eine betriebswirtschaftliche Analyse deutscher und internationaler Prüfungskonventionen (Gabler Edition Wissenschaft : Schriften zu Wirtschaftsprüfung, Steuerlehre und Controlling, 1. Aufl.). Wiesbaden: Dt. Univ.-Verl.

Wöhe, G. \& Döring, U. (2013). Einführung in die allgemeine Betriebswirtschaftslehre (Vahlens Handbücher der Wirtschafts- und Sozialwissenschaften, 25., überarb. und aktualisierte Aufl). München: Vahlen.

Wutz, A. (2000). Einfluß der Modellierung auf die Effiqien₹ der Bank - Produktions- und Intermediationsansatz, im Vergleich, Universität Augsburg. Volkswirtschaftliche Diskussionsreihe: 198. Verfügbar unter http://www.wiwi.uni-augsburg.de/vwl/institut/paper/198.pdf

Wutz, A. (2002). Wie beeinflusst das Umfeld einer Bank die Effizienz? - Eine DEA-Analyse für die Bayerischen Genossenschaftsbanken, Institut für Volkswirtschaftslehre, Fakultät für Wirtschaftswissenschaften, Universität Augsburg. Verfügbar unter http://www.wiwi.uniaugsburg.de/vwl/institut/paper/215.pdf

ZfgK. (2015). Kreditgenossenschaften. "Wenn es mal gut läuft ...". Zeitschrift für das gesamte Kreditwesen (2), 59-60. Verfügbar unter https://www.wiso-net.de/document/ZFGK 011515009 



\section{Selbständigkeitserklärung}

Hiermit erkläre ich ehrenwörtlich, dass ich die Dissertation selbst angefertigt habe und keine Unterstützung durch einen Promotionsberater in Anspruch genommen habe. Alle wörtlich oder inhaltlich übernommenen Stellen der von mir benutzen Quellen habe ich in der Arbeit angegeben.

Thomas Maurer

Chemnitz, 15.08.2016 